\title{
DEVELOPMENT AND STUDY OF CLOSURE STRIP BETWEEN PRECAST DECK PANELS IN ACCELERATED BRIDGE CONSTRUCTION
}

\author{
By \\ Mahmoud Shaaban Sayed Ahmed \\ B.Sc., Higher Institute of Engineering, Egypt, 2000 \\ M.A.Sc., Ryerson University, Canada, 2011
}

\author{
A Dissertation \\ Presented to Ryerson University \\ In partial fulfillment of the \\ Requirement for the degree of \\ Doctor of Philosophy \\ In the program of \\ Civil Engineering
}

Toronto, Ontario, Canada, 2016

(C) Mahmoud Shaaban Sayed Ahmed 2016 


\section{AUTHOR'S DECLARATION}

I hereby declare that I am the sole author of this dissertation. This is a true copy of the dissertation, including any required final revisions, as accepted by my examiners.

I authorize Ryerson University to lend this dissertation to other institutions or individuals for the purpose of scholarly research.

I further authorize Ryerson University to reproduce this dissertation by photocopying or by other means, in total or in part, at the request of other institutions or individuals for the purpose of scholarly research.

I understand that my dissertation may be made electronically available to the public. 


\title{
DEVELOPMENT AND STUDY OF CLOSURE STRIP BETWEEN PRECAST DECK PANELS IN ACCELERATED BRIDGE CONSTRUCTION
}

\author{
Mahmoud Shaaban SAYED AHMED \\ Ph.D. Civil Engineering, Department of Civil Engineering, Ryerson University, \\ Toronto, Ontario, Canada, 2016
}

\begin{abstract}
This research investigates the use of glass fiber reinforced polymer (GFRP) bars in bridge decks and ultra-high performance fibre-reinforced concrete (UHPFRC) as filling materials in (i) panelto-panel closure strips between transverse precast full-depth deck panels (FDDPs) supported over girders and (ii) the shear pockets for the panel-to-girder connection. The experimental research program included three phases. Phase I examined pullout strength of straight-end and headed-end GFRP bars embedded into UHPFRC to determine the required closure strip width to develop bar full strength. Phase II included the development and study of closure strip details incorporating UHPFRC as joint-filling materials and GFRP bars projecting into the joint. Three joints of width $200 \mathrm{~mm}$ between precast FDDPs were developed, namely: angle-shape joint (Ajoint), C-shape joint (C-shape), and zigzag-shape joint (Z-joint), with 175-mm projecting length of GFRP bars into the joint. Two series of 2500x600x200 mm one-way slabs were cast to investigate the flexural strength of the jointed precast slabs compared to cast-in-place slabs. Two types of concrete were used to fabricate the precast FDDPs, namely: normal concrete (NSC) and high-performance concrete (HPC). Correlation between experimental results and available design equations for moment and shear capacities, as well as CHBDC and AASHTO-LRFD applied factored design moments, was performed. All specimens failed in either flexural or flexural-shear mode outside the UHPFRC-filled joint. Phase III included testing three pairs of 3700x2500x200 mm laterally-restrained precast FDDPs incorporating the three developed joint details in the transverse direction of the girders. Each pair of specimens was tested under 600x250 mm wheel loading located beside the closure strip, considering (i) constant amplitude fatigue (CAF) loading up to 4 million cycles followed by increasing static loading to-collapse, and (ii) incremental variable amplitude fatigue (VAF) loading to-collapse. The failure mode of the tested slabs was punching shear, with the transverse UHPFRC joint diverting the extension of the punching shear plane to the adjacent precast FDDP segment. Results of fatigue load tests on
\end{abstract}


the three-jointed pairs of slabs showed high fatigue performance. A new prediction model for fatigue life of the GFRP-reinforced, UHPFRC-filled jointed deck slabs was developed.

Keywords: Accelerated Bridge Construction (ABC), Precast full-depth deck panels (FDDPs), Transverse joint, GFRP bars, Ultra-high performance fiber-reinforced concrete (UHPFRC), Monotonic and fatigue Loading, Bridge code, Pullout strength. 


\section{ACKNOWLEDGEMENTS}

The author would like to acknowledge the assistance of Professor Dr. Khaled Sennah for his constant support and valuable supervising. The author appreciates his family members who were patient and helped him to complete the research work. The author acknowledges the financial support to this research from his supervisor; Ryerson Graduate Awards and Scholarships; The Norman Esch Foundation; Transportation Association of Canada (TAC) Foundation Scholarship sponsored by SNC-Lavalin in celebration of TAC's centennial; Ontario Graduate Scholarship (OGS) and Ontario Ministry of Transportation's Highway Infrastructure Innovation Funding program (MTO-HIIFP). The author would like to thank the following companies for supplied materials for research: V-Rod Canada Inc., Pultrall Inc. Schoeck Canada Inc., Lafarge North America Inc. and St. Marys / Canada Building Materials (CBM). Opinions expressed in this dissertation are those of the author and do not necessarily reflect the views and policies of Ministry of Transportation of Ontario (MTO). 


\section{Table of Contents}

Author's Declaration $\quad$ ii

Abstract $\quad$ iii

Acknowledgements $\quad$ V

List of Tables $\quad$ viii

List of Figures $\quad$ xi

Abbreviations $\quad$ xvi

1 Chapter I

Introduction 1

1.1 General 1

1.2 The Problem 3

1.3 Objectives of Research 7

1.4 Scope of Research 8

1.5 Contents and Arrangements of the Thesis 9

References $\quad 9$

2 Chapter II

Bond Strength of High-Modulus Glass Fiber Reinforced Polymer Bars

Embedded into Unconfined Ultra-high Performance Concrete 17

$2.1 \quad$ General 17

2.2 Background of Research 18

2.3 Experimental Program 23

2.3.1. Description of Specimens $\quad 23$

2.3.2. Material Properties $\quad 24$

2.3.3. Test Setup and Instrumentation 25

2.4 Experimental Results for Ribbed-Surface GFRP Bars 26

2.5 Experimental Results for Sand-Coated GFRP Bars 28

2.6 Analytical Study of GFRP Bar Bond Strength and Development Length 32

2.6.1. Analytical Results for Ribbed-Surface GFRP Bars 35

2.6.2. Analytical Results for Sand-Coated GFRP Bars 36

2.6.3. Development of Modification Factor for Bond Strength Based on $\begin{array}{ll}\text { Concrete Cover } & 38\end{array}$

2.7 Recommendations for Minimum GFRP Development Length 39

$\begin{array}{lll}2.8 & \text { Conclusions } & 39\end{array}$

References $\quad 40$

3 Chapter III

Structural Behavior of NSC Full-Depth Deck Panels with Closure Strips Reinforced with Glass Fiber Reinforced Polymer Bars and filled with UltraHigh Performance Fiber Reinforced Concrete 88

3.1 General $\quad 88$

3.2 Research Background 89

3.2.1. Bridge Deck Slabs and Accelerated Bridge Construction 89

3.2.2. Background on Ultra-High Performance Fibre-Reinforced Concrete 93

$\begin{array}{lll}3.3 & \text { New Connection Details } & 94\end{array}$

3.4 Experimental Program 96

3.5 Test Results 99 
Bars in UHPFRC

3.6 Conclusions

References

Appendix: Design Data

4 Chapter IV

Mechanical Behavior of HPC Full-Depth Precast Bridge Deck Panel with Transverse Joint and GFRP Lap-Spliced Bars Embedded into UHPFRC

4.1 General

4.2 Research Background

4.3 New Connection Details

4.4 Experimental Program

144

4.5 Test Results

4.5.1. Behavior of the Tested Slabs

4.5.2. Analysis of Test Data for Flexural and Shear Resistance

4.5.3. Moment-Strain Relationship and Associated Bond Stress for GFRP

Bars in UHPFRC

4.5 Conclusions

References

Appendix: Design Data

Fatigue and Ultimate Strength Evaluation of GFRP-Reinforced Full-Depth Precast Deck Panels with UHPFRC-Filled Transverse Closure Strips

5.1 General

5.2 Research Background

5.3 New Connection Details

5.4 Experimental Program 198

$\begin{array}{lll}5.5 & \text { Test Results } & 200\end{array}$

5.5.1 Behavior of the A-Jointed Precast FDDP 201

5.5.1.1 Constant Amplitude Fatigue Loading 201

5.5.1.2 Variable Amplitude Fatigue Loading 202

5.5.1.3 Stiffness Degradation 203

5.5.2 Behavior of the C-Jointed Precast FDDP 204

5.5.2.1 Constant Amplitude Fatigue Loading 204

5.5.2.2 Variable Amplitude Fatigue 205

5.5.2.3 Stiffness Degradation 206

5.5.3 Behavior of the Z-Jointed Precast FDDP 206

5.5.3.1 Constant Amplitude Fatigue Loading 207

5.5.3.2 Variable Amplitude Fatigue Loading 208

$\begin{array}{ll}\text { 5.5.3.3 Stiffness Degradation } & 209\end{array}$

5.6 Life Estimation of Fatigue of GFRP-Reinforced Precast FDDP 209

$\begin{array}{ll}5.7 & \text { Punching Shear Strength } \\ & 211\end{array}$

5.7.1. Developed Punching Shear Model 216 
$\begin{array}{lll}5.8 & \text { Conclusions } & 216\end{array}$

5.8.1 A-Jointed Precast FDDP 216

$\begin{array}{ll}\text { 5.8.2 C-Jointed Precast FDDP } & 217\end{array}$

5.8.3 The Z-Jointed Precast FDDP 218

5.8.4. Developed Punching Shear Model
nces

$6 \quad \begin{aligned} & \text { Chapter VI } \\ & \text { Conclusions and Recommendations for Future Research }\end{aligned}$

$\begin{array}{lll}6.1 & \text { Summary } & 290\end{array}$

6.1.1. Phase I: Pullout Mechanism of GFRP Bars Embedded into UHPFRC 291

6.1.2. Phase II: Development and Study of Closure Strip Details 291

Incorporating UHPFRC as Joint-Filling Materials and GFRP Bars

Projecting into the Joint

6.1.3. Phase III: Monotonic and Cyclic Load Tests on Laterally-Restrained 291 Deck Slabs

6.2 Conclusions 292

6.3 Recommendations for Future Research 297 


\section{List of Tables}

$\begin{array}{llll}\text { Table } & 2.1 & \text { Pullout test matrix } & 43\end{array}$

Table $2.2 \quad$ Proportion of UHPFRC (Ductal) constituent materials 43

Table $2.3 \quad$ UHPFRC (Ductal JS1000) physical properties (Lafarge Canada Inc., 43 2009)

Table $\quad 2.4 \quad$ Material properties 15M and 20M straight ribbed-surface GFRP bar 44 (Schoeck Canada, 2013)

Table $2.5 \quad$ Mechanical properties 15M and 20M straight sand-coated GFRP bars 44 (VROD - Pultrall, 2013)

Table 2.6 Pullout test results for 15M ribbed-surface GFRP bars with centric bar 45 location

Table $\quad 2.7 \quad$ Pullout test results for 15M ribbed-surface GFRP bars with $40 \mathrm{~mm} \quad 46$ eccentric bar location

Table $\quad 2.8 \quad$ Pullout test results for 15M ribbed-surface GFRP bars with $60 \mathrm{~mm} \quad 47$ eccentric bar location

Table $\quad 2.9 \quad$ Pullout test results for 20M ribbed-surface GFRP bars with centric bar 48 location

Table $2.10 \quad$ Pullout test results for 20M ribbed-surface GFRP bars with $40 \mathrm{~mm}$ eccentric bar location

Table $2.11 \quad$ Pullout load - slip modeling for ribbed-surface GFRP bars 50

Table $2.12 \quad$ Bond stress and experimental coefficients for ribbed-surface GFRP bars 51

Table 2.13 Pullout load versus the embedment length equations for ribbed-surface 52 GFRP bars

Table $\quad 2.14 \quad$ Bond stress versus the embedment length equations for ribbed-surface 52 GFRP bars

Table $2.15 \quad$ Concrete and reinforcement location factor (bond factor) for ribbed- 53 surface GFRP bars

Table 2.16 Reinforcement location factor for ribbed-surface GFRP bars 53

Table $2.17 \quad$ ACI basic factor kexp for ribbed-surface GFRP bars 54

Table $2.18 \quad$ Pullout test results for 15M sand-coated GFRP bars with centric bar 55 location

Table $2.19 \quad$ Pullout test results for 15M sand-coated GFRP bars with $40 \mathrm{~mm}$ of 56 eccentric bar location

$\begin{array}{llll}\text { Table } & 2.20 & \text { Pullout test results for 15M sand-coated GFRP bars with } 60 \mathrm{~mm} & 57\end{array}$ eccentric bar location

Table $\quad 2.21 \quad$ Pullout test results for 20M sand-coated GFRP bars with centric bar $\quad 58$ location

Table $\quad 2.22 \quad$ Pullout test results for 20M sand-coated GFRP bars with $40 \mathrm{~mm}$ eccentric bar location

Table $\quad 2.23 \quad$ Pullout test results for 20M sand-coated GFRP bars with $60 \mathrm{~mm} \quad 60$ eccentric bar location

Table $\quad 2.24 \quad$ Pullout load - slip modeling for sand-coated GFRP bar 61

$\begin{array}{llll}\text { Table } & 2.25 & \text { Bond stress and experimental coefficients for sand-coated GFRP bars } \quad 62\end{array}$

Table $2.26 \quad$ Pullout load versus the embedment length equations for sand-coated 63 GFRP bars 


\begin{tabular}{|c|c|c|c|}
\hline Table & 2.27 & $\begin{array}{l}\text { Bond stress versus the embedment length equations for sand-coated } \\
\text { GFRP bars }\end{array}$ & 63 \\
\hline Table & 2.28 & Concrete and reinforcement location factors for sand-coated GFRP bars & 64 \\
\hline Table & 2.29 & Reinforcement location factor for sand-coated GFRP bars & 64 \\
\hline Table & 2.30 & ACI basic factor $k \exp$ for the sand-coated HM GFRP bars & 65 \\
\hline Table & 2.31 & $\begin{array}{l}\text { Bond factors, } \mathrm{k} \text {, and development length, ld, of GFRP bars embedded } \\
\text { into UHPFRC }\end{array}$ & 65 \\
\hline Table & 3.1 & Name coding & 114 \\
\hline Table & 3.2 & Properties of GFRP bars considered in slab testing & 114 \\
\hline Table & 3.3 & Typical UHPFRC Composition & 114 \\
\hline Table & 3.4 & Characteristic values of UHPFRC Type JS1000 for the design of joint & 114 \\
\hline Table & 3.5 & Summary of slab configurations and test results & 115 \\
\hline Table & 3.6 & Test results & 116 \\
\hline Table & 3.7 & Shear and bending moment values & 117 \\
\hline Table & 3.8 & Experimental parameters for the moment-deflection equation & 118 \\
\hline Table & 3.9 & Maximum micro-strain readings for the tested slabs & 118 \\
\hline Table & 3.10 & $\begin{array}{l}\text { Calculated pullout force of bottom GFRP bars embedded in the } \\
\text { UHPFRC-filled joint based on experimental data }\end{array}$ & 119 \\
\hline Table & 4.1 & Name Coding & 160 \\
\hline Table & 4.2 & Properties of GFRP bars considered in slab testing & 160 \\
\hline Table & 4.3 & Typical UHPFRC Composition & 160 \\
\hline Table & 4.4 & Characteristic values of UHPFRC Type JS1000 for the design of joint & 160 \\
\hline Table & 4.5 & Summary of slab configurations and test results & 161 \\
\hline Table & 4.6 & Test $\mathrm{r}$ & 162 \\
\hline Table & 4.7 & d bendir & 163 \\
\hline Table & 4.8 & Experimental parameters for the Moment-Deflection & 164 \\
\hline Table & 4.9 & Maximum micro-strain readings for the tested slabs & 164 \\
\hline Table & 4.10 & Pullout force from the bottom reinforcement & 165 \\
\hline Table & 5.1 & sed in this study & 224 \\
\hline Table & 5.2 & ry of fatigue tested slab configurations & 224 \\
\hline Table & 5.3 & Static Load Configuration & 224 \\
\hline Table & 5.4 & Summary of test results & 225 \\
\hline Table & 5.5 & deoradotion of the tected slohe & 226 \\
\hline Table & 5.6 & gradation of the tested slabs with C-type joint & 227 \\
\hline Table & 5.7 & Stiffness degradation of the tested slabs with Z-shape joint & 228 \\
\hline Table & 5.8 & Fatigue parameters & 228 \\
\hline Table & 5.9 & $\begin{array}{l}\text { Loading history of the tested slab } \mathrm{S} 2 \text { of type A-joint with equivalent } \\
\text { constant amplitude fatigue load segments }\end{array}$ & 229 \\
\hline Table & 5.10 & $\begin{array}{l}\text { Loading history of the tested slab S4 of type C-joint with equivalent } \\
\text { constant amplitude fatigue load segments }\end{array}$ & 229 \\
\hline $\mathrm{Tab}$ & 5.11 & $\begin{array}{l}\text { Loading history of the tested slab S6 of type Z-joint with equivalent } \\
\text { constant amplitude fatigue load segments }\end{array}$ & 230 \\
\hline Table & 5.12 & Critical sections due to punching shear & 231 \\
\hline Table & 5.13 & $\begin{array}{l}\text { Correlation between the punching shear strength of the tested slabs } \\
\text { obtained experimentally and from available equation in the literature }\end{array}$ & 232 \\
\hline
\end{tabular}


Table 5.14 Comparison between the developed punching shear equation and

experimental data

Table A.5.1 Fatigue Equations for the $\mathrm{d}-\mathrm{N}$ for the precast FDDPs

Table A.5.2 Fatigue parameters for the $\mathrm{d}-\mathrm{N}$ for the precast FDDPs

Table A.5.3 Fatigue Equations for the $\epsilon-\mathrm{N}$ for for the precast FDDPs

Fatigue parameters for the $\epsilon-\mathrm{N}$ for concrete and GFRP bars embedded amplitude fatigue load segments

Table A.5.6 C-jointed precast FDDP deflection history for the equivalent constant amplitude fatigue load segments

Table A.5.7 Z-jointed precast FDDP deflection history for the equivalent constant amplitude fatigue load segments

Table A.5.8 A-jointed precast FDDP longitudinal bar strain history for the equivalent constant amplitude fatigue load segments

Table A.5.9 A-jointed precast FDDP transverse bar strain history for the equivalent 282 constant amplitude fatigue load segments

Table A.5.10 A-jointed precast FDDP concrete strain history for the equivalent constant amplitude fatigue load segments

Table A.5.11 C-jointed precast FDDP longitudinal bar strain history for the equivalent constant amplitude fatigue load segments

Table A.5.12 C-jointed precast FDDP transverse bar strain history for the equivalent constant amplitude fatigue load segments

Table A.5.13 C-jointed precast FDDP concrete strain history for the equivalent constant amplitude fatigue load segments

Table A.5.14 Z-jointed precast FDDP longitudinal bar strain history for the equivalent constant amplitude fatigue load segments

Table A.5.15 Z-jointed precast FDDP transverse bar strain history for the equivalent constant amplitude fatigue load segments

Table A.5.16 Z-jointed precast FDDP concrete strain history for the equivalent constant amplitude fatigue load segments 


\section{List of Figures}

Fig. $\quad 1.1 \quad$ Precast full-depth deck panels (FDDPs) placed transversally over girders 11

Fig. $\quad 1.2$ Views of full-depth, full width, deck panels (FDDPs) placed 12 transversally on girders

Fig. $\quad 1.3 \quad$ Schematic view of precast bulb-tee girders with closure strips oriented in 13 girder direction

Fig. $\quad 1.4 \quad$ Prefabricated bulb-tee bridge girder system in Moose Creek Bridge 14 (source: Hossain and Lam, 2006)

Fig. $\quad 1.5 \quad$ Typical elevation for an integral abutment bridge in the longitudinal 15 direction

Fig. $\quad 1.6 \quad$ Typical bending moment diagram in the longitudinal direction of an 15 integral abutment bridge

Fig. $\quad 1.7 \quad$ Cross-section analysis in the negative moment regions showing stress 16

Fig. $\quad 2.1 \quad$ Ribbed-surface and sand-coated GFRP bars considered in this study 66

Fig. $\quad 2.2 \quad$ Possible failure modes of GFRP bar embedded in concrete 67

$\begin{array}{lll}\text { Fig. } & 2.3 & \text { Concentric and eccentric cube specimens }\end{array}$

Fig. $\quad 2.4 \quad$ Rheological model for the UHPC (Ductal JS1000) 68

$\begin{array}{llll}\text { Fig. } & 2.5 & \text { Pullout test setup } & 69\end{array}$

$\begin{array}{lll}\text { Fig. } & 2.6 \quad \text { Typical failure of the } 15 \mathrm{M} \text { ribbed-surface bar with embedment length } & 70\end{array}$ equal 4 times the diameter showing bar slip and sheared lugs

Fig. $\quad 2.7 \quad$ Views of splitting cracks after pullout test for the 20M ribbed-surface 71 GFRP bars with embedment length of 4 and 6 times the bar diameters

$\begin{array}{llll}\text { Fig. } & 2.8 & \text { Typical pullout failure of the 20M ribbed surface GFRP bar with } & 72\end{array}$ embedment length of 4 times the bar diameter

Fig. $\quad 2.9 \quad$ Typical bar slippage failure from its anchorage head for the $15 \mathrm{M}$ ribbed $\quad 73$ surface GFRP bar embedded in UHPC block with $60 \mathrm{~mm}$ concrete cover

Fig. $\quad 2.10 \quad$ Experimental pullout load for the ribbed-surface GFRP bars embedded 74 in UHPC cube specimens

Fig. $\quad 2.11 \quad$ Normalized bond stress, $\mu$, for the ribbed-surface GFRP bars embedded 74 in UHPC cube specimens

Fig. $\quad 2.12$ Combined bond factor, B, for the ribbed-surface GFRP bars 75

Fig. $2.13 \quad$ Bar stress ratio, $\varphi$, for the ribbed-surface GFRP bars $\quad 75$

Fig. $\quad 2.14 \quad$ Comparison between predicted and experimental embedment length and $\quad 77$ pullout-load for the ribbed-surface GFRP bar respectively

Fig. $\quad 2.15 \quad$ Typical failure of the 15M sand-coated GFRP bar due to: (i) 78 interlaminar shear failure of sand coating and (ii) shear-off failure of sand coating from bar surface

Fig. 2.16 Typical failure of the 15M sand-coated GFRP bar with 40 and $60 \mathrm{~mm}$ concrete cover due to: (i) interlaminar shear failure of sand coating and (ii) shear-off failure of sand coating from bar surface

Fig. $\quad 2.17 \quad$ Typical failure of the 20M sand-coated GFRP bar due to: (i) interlaminar shear failure of sand coating and (ii) shear-off failure of sand coating from bar surface

Fig. $\quad 2.18$ Typical failure of the 20M sand-coated GFRP bar with 40 and $60 \mathrm{~mm}$ concrete cover due to: (i) interlaminar shear failure of sand coating and 
(ii) shear-off failure of sand coating from bar surface

Fig. $\quad 2.19$ Typical failure of the 15M sand-coated, headed-end, GFRP bar with

different concrete cover due to slippage of the bar from the anchorage

head as a result of shear failure along the grooves at the interface

between the bar and the anchorage inner surface

Fig. $\quad 2.20 \quad$ Typical failure of the 20M sand-coated, headed-end, GFRP bar with

different concrete cover due to slippage of the bar from the anchorage

head as a result of shear failure along the grooves at the interface

between the bar and the anchorage inner surface

Fig. 2.21 Experimental pullout load for the sand-coated GFRP bars embedded in UHPC cube specimens

Fig. $\quad 2.22 \quad$ Normalized bond stress, $\mu$, for the sand-coated GFRP bars embedded in 84 UHPC cube specimens

Fig. $\quad 2.23$ Combined bond factor, B, for the sand-coated GFRP bars 85

Fig. $\quad 2.24 \quad$ Bar stress ratio, $\varphi$, for the sand-coated GFRP bars 85

Fig. $\quad 2.25 \quad$ Comparisons between predicted and experimental embedment length and $\quad 87$

pullout-load for the sand-coated GFRP bar respectively

Fig. $\quad 3.1 \quad$ Plan view of a slab-on-girder bridge with full-depth precast deck panels 120

Fig. $\quad 3.2 \quad$ Schematic diagrams of the three proposed joints between precast deck 122 panels

Fig. $\quad 3.3 \quad$ Schematic diagram showing location of simulated wheel load at the joint 123 between precast deck panels

Fig. $\quad 3.4 \quad$ Reinforcement details and locations of strain gauges and potentiometers 124 (POTs)

Fig. $\quad 3.5 \quad$ Views of reinforcement layout and form work for slab specimens $\quad 125$

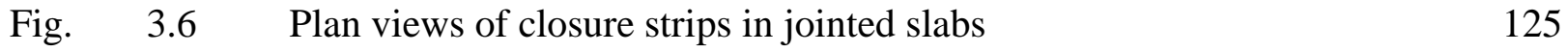

$\begin{array}{llll}\text { Fig. } & 3.7 & \text { Test setup and crack pattern at failure for cast-in-place specimen S1 } & 126\end{array}$

(C.ST.C) reinforced with steel bars

$\begin{array}{llll}\text { Fig. } & 3.8 & \text { Test setup and crack pattern at failure of cast-in-place specimen S2 } & 126\end{array}$

(C.GFRP.C) reinforced with GFRP bars

Fig. $\quad 3.9 \quad$ Test setup and crack pattern at failure of jointed slab S3 (J.C.C) and $\quad 127$ centric loading over the joint

Fig. $\quad 3.10 \quad$ Test setup and crack pattern at failure of jointed slab S4 (J.C.E) with 127 eccentric loading at the joint

Fig. $\quad 3.11 \quad$ Test setup and crack pattern at failure of slab S5 (J.Z.E) with zigzag- $\quad 128$ shape joint under eccentric loading

Fig. $\quad 3.12$ Test setup and crack pattern at failure of slab S6 (J.A.C) with angle- 128 shape joint under centric loading

Fig. $\quad 3.13 \quad$ Test setup and crack pattern at failure of slab S6 (J.A.E) with angleshape joint under eccentric loading

Fig. $\quad 3.14 \quad$ Load-deflection relationships obtained at mid-span of the slab specimens

Fig. $\quad 3.15$ Applied moment-deflection curves for the tested specimens

Fig. $\quad 3.16$

Structural analysis of the tested slabs

Fig. $\quad 3.17$

Whitney rectangular stress distribution for flexural design

Fig. $\quad 3.18$

UHPFRC Internal stress behavior

Fig. $\quad 3.19$

Control CIP steel slab S1 (C.ST.C) under centric load

133

134

Fig. $\quad 3.20 \quad$ Control CIP GFRP slab S2 (C.GFRP.C) under centric load 
Fig. $\quad 3.21 \quad$ Jointed GFRP-reinforced FDDP S3 (JCC) under centric load

Fig. $\quad 3.22$ Jointed GFRP-reinforced FDDP S4 (JCE) under eccentric load

Fig. $\quad 3.23$

Jointed GFRP-reinforced FDDP S5 (JZE) under eccentric load

135

Fig. $\quad 3.24$

Jointed GFRP-reinforced FDDP S6 (JAC) under centric load

135

Fig. $\quad 3.25$

Jointed GFRP-reinforced FDDP S7 (JAE) under eccentric load

136

Fig. $\quad 4.1$

Plan view of a slab-on-girder bridge with full depth precast deck panels

166

Fig. $\quad 4.2$

Schematic diagrams of the three proposed joints between precast deck panels

Fig. $\quad 4.3 \quad$ Schematic diagram showing location of simulated wheel load at the joint between precast deck panels

Fig. $\quad 4.4 \quad$ Reinforcement details and locations of strain gauges and potentiometers (POTs)

Fig. $\quad 4.5 \quad$ Views of reinforcement layout and form work for slab specimens

Fig. $\quad 4.6 \quad$ Plan views of closure strip in jointed slabs

Fig. $\quad 4.7 \quad$ Test setup and crack pattern at failure for cast-in-place specimen S1

172 (C.ST.C) reinforced with steel bars

Fig. $\quad 4.8 \quad$ Test setup and crack pattern at failure of cast-in-place specimen S2 (C.GFRP.C) reinforced with GFRP bars

Fig. $\quad 4.9 \quad$ Test setup and crack pattern at failure of jointed slab S3 (J.C.C) and centric loading over the joint

Fig. $\quad 4.10 \quad$ Test setup and crack pattern at failure of jointed slab S4 (J.C.E) with eccentric loading at the joint

Fig. $\quad 4.11$ Test setup and crack pattern at failure of slab S5 (J.Z.E) with zigzagshape joint under eccentric loading

Fig. $\quad 4.12 \quad$ Test setup and crack pattern at failure of slab S6 (J.A.C) with angleshape joint under centric loading

Fig. $\quad 4.13 \quad$ Test setup and crack pattern at failure of slab S6 (J.A.E) with angleshape joint under eccentric loading

Fig. $\quad 4.14$ Load-deflection relationships obtained at mid-span of slab specimens

Fig. $\quad 4.16$

Moment-deflection curves for the tested specimens

$\begin{array}{lll}\text { Fig. } & 4.17 & \text { Structural analysis of the tested slabs }\end{array}$

Fig. $\quad 4.18$

Whitney rectangular stress distribution for flexural design

Fig. $\quad 5.1 \quad$ Isometric views of precast full-depth, full width, deck panels placed

Fig. $\quad 5.2 \quad$ Cross-sections at the developed A-shape joint for precast slabs S1 and S2

Fig. $\quad 5.3 \quad$ Plan of precast FDDP arrangement for A-shape joints 
Fig. $\quad 5.4 \quad$ Cross-sections at the developed C-shape joint for precast slabs S3 and S4

Fig. $\quad 5.5 \quad$ Plan of precast FDDP arrangement for C-shape joints 235

Fig. $\quad 5.6 \quad$ Plan views of the developed zigzag-shape joint (Z-joint) for slabs S5 and 236 S6

Fig. $\quad 5.7 \quad$ Styrofoam prepared to form the zigzag-shape joint 236

Fig. $\quad 5.8 \quad$ Arrangement of GFRP bars projecting into the zigzag-shape joint (Z- 237 shape) for slabs S5 and S6

$\begin{array}{lll}\text { Fig. } & 5.9 & \text { Plan of precast FDDP arrangement for Z-shape joints }\end{array}$

Fig. $\quad 5.10 \quad$ Schematic diagrams of the steel twin-girder system supporting the 238

$\begin{array}{llll}\text { Fig. } & 5.11 & \text { View of the steel twin-girder system supporting the precast FDDPs } & 239\end{array}$

Fig. $\quad 5.12 \quad$ Views of M25 high-strength headed bolt and its nuts and washers used to connect the precast FDDPs to the steel girders through shear pockets

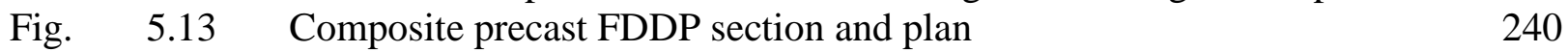

Fig. $\quad 5.14 \quad$ Panel-to-girder connection in the transverse direction 241

Fig. $\quad 5.15 \quad$ Panel-to-girder connection in the longitudinal direction at the edges of 241 the precast FDDP

Fig. $\quad 5.16$ Panel-to-girder connection in the longitudinal direction at the mid-width 242 of the precast FDDP

$\begin{array}{llll}\text { Fig. } & 5.17 & \text { Constant amplitude fatigue (CAF) loading history } & 243\end{array}$

Fig. $\quad 5.18 \quad$ Variable amplitude fatigue (VAF) loading history 243

Fig. $\quad 5.19$ Construction of the precast FDDP with A-shape join (slabs S1 and S2) 244

$\begin{array}{llll}\text { Fig. } & 5.20 \quad \text { View of the test setup for fatigue loading for specimens S1 and S2 } & 245\end{array}$

Fig. $\quad 5.21 \quad$ View of the test setup for the monotonic loading for slab S1

Fig. $\quad 5.22 \quad$ Static load-deflection curves for slab S1 after being subjected to CAF 246 loading

Fig. $\quad 5.23 \quad$ Crack pattern after failure of slab S1 after testing under static load tocollapse

Fig. $\quad 5.24 \quad$ Views of punching shear failure of slab S2 tested under VAF loading tocollapse

Fig. $\quad 5.25$ Static load-deflection curves of the slab S2 with A-joint after every 100,000 cycles of VAF loading

Fig. $\quad 5.26$ Degradation of the precast FDDPs with A-joint details under CAF and 250 VAF loading

Fig. $\quad 5.27 \quad$ Construction of the precast FDDP with C-shape joint for slabs S3 and S4 251

Fig. $\quad 5.28 \quad$ Close-up view of forming the C-joint using Styrofoam 252

Fig. $\quad 5.29 \quad$ Longitudinal cross-section in the precast FDDP with C-joint showing 252 details of GFRP arrangement

Fig. $\quad 5.30 \quad$ View of slab S3 with precast FDDP segments placed side by side normal 253 the steel girders

Fig. $\quad 5.31 \quad$ Photo of the closure strip for the C-joint before casting 253

Fig. $\quad 5.32 \quad$ View of the test setup for fatigue loading for slabs S3 and S4 254

Fig. $\quad 5.33 \quad$ View of the test setup for the static load test for slab S3 with C-joint 254

Fig. $\quad 5.34 \quad$ Monotonic load-deflection curves for slab S3 during CAF loading and 255 static load test to-collapse 
Fig. $\quad 5.35 \quad$ Crack pattern after failure of slab S3 with C-joint tested under CAF 256 loading followed by static load test to-collapse

Fig. $\quad 5.36 \quad$ Views of punching shear failure of slab S4 tested under VAF loading to- 257 collapse

Fig. $\quad 5.37 \quad$ Monotonic load-deflection curves for slab S4 tested after each 100,000 258 cycles of VAF loading

Fig. $\quad 5.38 \quad$ Degradation of the precast FDDPs subjected to CAF and VAF loading 260

Fig. $\quad 5.39$ Construction of the precast FDDP with zigzag-shape joint for slabs S5 261 and S6

Fig. $\quad 5.40 \quad$ Longitudinal cross-section for the precast FDDP with zigzag joint $\quad 261$

Fig. $\quad 5.41 \quad$ Placing of reinforcement for the precast FDDP with zigzag joint 262

Fig. $\quad 5.42 \quad$ Close-up view for the reinforcement through the zigzag connection $\quad 262$

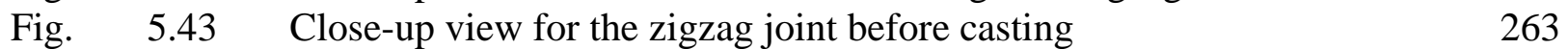

Fig. $\quad 5.44 \quad$ View of the test setup for fatigue loading for slabs S5 and S6 263

Fig. $\quad 5.45 \quad$ View of the test setup for the monotonic loading for slab S5 with Z- $\quad 264$ shape joint

Fig. $\quad 5.46 \quad$ Monotonic load-deflection history for slab S5 under CAF loading 265

Fig. $\quad 5.47$ Crack pattern after failure of slab S5 under CAF loading, followed by 266

static load test to-collapse

Fig. $\quad 5.48 \quad$ Views of punching shear failure of slab S6 tested under VAF loading 267

Fig. $\quad 5.49 \quad$ Monotonic load-deflection history for slab S6 at every 100,000 cycles of 268 VAF loading

Fig. $\quad 5.50 \quad$ Degradation of the precast FDDP with Z-shape joint under CAF and 269 VAF loading

Fig. $\quad 5.51 \quad$ Degradation of the precast FDDPs under CAF and VAF loading $\quad 271$

Fig. $\quad 5.52 \quad$ The P-N fatigue models for the precast FDDPs on log-graphs 273

$\begin{array}{llll}\text { Fig. } & 5.53 & \text { P-N curves for the ribbed-surface GFRP-reinforced precast FDDPs } & 274\end{array}$

Fig. $\quad 5.54 \quad$ Views of punching shear failure in tested slabs after slicing them at 276 wheel load location

Fig. $\quad 5.55 \quad$ Schematic diagrams of the punching shear crack patterns for the tested 277 slabs

Fig. $\quad 5.56 \quad$ Schematic diagram for the critical section of the punching shear $\quad 278$

Fig. A5.1 d-N curve for deflection of the A-Jointed precast FDDP 286

Fig. A5.2 d-N curve for deflection of the C-Jointed precast FDDP 286

Fig. $\quad$ A5.3 d-N curve for deflection of the Z-Jointed precast FDDP 287

Fig. $\quad$ A5.4 ACZ jointed precast FDDPs - deflection versus number of cycles 287

Fig. A5.5 The A-Precast FDDP's concrete, longitudinal bar and transverse bar 288 strain versus load cycles

Fig. A5.6 The C-Precast FDDP's concrete, longitudinal bar and transverse bar 288 strain versus load cycles

Fig. $\quad$ A5.7 The Z-Precast FDDP's concrete, longitudinal bar and transverse bar 289 strain versus load cycles 


\section{Abbreviations}

AASHTO American Association of State Highway and Transportation Officials

$\mathrm{ABC}$

$\mathrm{ACI}$

ACTT

AFGC

AR

ASR

ASTM

BMD

CAF

CAN

CBM

CDOT

CFD

CHBDC

CIP

CMA

ComBar

$\mathrm{CP}$

CSA

DBT

DOT

FCM

FDDP

FEA

FES

FHWA

Fig

Figs

FLS

FRP

GFRP

GU

$\mathrm{HCC}$

$\mathrm{HCF}$

$\mathrm{HM}$

HPC

HRWR

HSC

HSS

LCF

LES

LM

LRFD
Accelerated Bridge Construction

American Concrete Institute

Accelerated Construction Technology Transfer

French Association of Civil Engineering

Abrasion Resistance

Alkali-Silica Reactivity

American Society for Testing and Materials

Bending Moment Diagram

Constant Amplitude Fatigue

Canada

Canada Building Materials

Connecticut Department of Transportation

Cumulative Fatigue Damage

Canadian Highway Bridge Design Code

Cast-in-Place

Compressive Membrane Action

Composite Bar

Chloride Penetration

Canadian Standard Association

Deck Bult-Tee

Department of Transportation

Federation of Canadian Municipalities

Full Depth Deck Panel

Finite Element Analysis

Free-End Slip

Federal Highway Administration

Figure

Figures

Fatigue Limit State

Fiber Reinforced Polymer

Glass Fiber Reinforced Polymer

General Use Cement

Hydraulic cement concrete

High Cycle Fatigue

High Modulus

High Performance Concrete

High Range Water Reducer

High Strength Concrete

Hollow Structural Steel

Low Cycle Fatigue

Load-End Slip

Low Modulus

Load and Resistance Factor Design 


$\begin{array}{ll}\text { MCJ } & \text { Monolithic Concrete Joint } \\ \text { MF } & \text { Modification Factor } \\ \text { MOE } & \text { Modulus of Elasticity } \\ \text { MRC } & \text { Moment Resistance Connection } \\ \text { MTO } & \text { Ministry Transportation of Ontario } \\ \text { NCHRP } & \text { National Cooperative Highway Research Program } \\ \text { NDOR } & \text { Nebraska Department of Roads } \\ \text { NSC } & \text { Normal Strength Concrete } \\ \text { NU } & \text { Nebraska University Deck } \\ \text { OPC } & \text { Ordinary Portland Cement } \\ \text { PBES } & \text { Prefabricated Bridge Elements and Systems } \\ \text { PCI } & \text { Prestressed/Precast Concrete Institute } \\ \text { PCINER } & \text { Precast/Prestressed Concrete Institute New England Region } \\ \text { POT } & \text { Potentiometer } \\ \text { PPC } & \text { Partial-Depth Precast } \\ \text { RBR } & \text { Rapid Bridge Replacement } \\ \text { RCCAO } & \text { Residential and Civil Construction Alliance of Ontario } \\ \text { RULD } & \text { Road-User Liquidated Damage } \\ \text { SCM } & \text { Self-Consolidated Concrete } \\ \text { SETRA } & \text { Road and Motorway Technical Studies Department } \\ \text { SHRP } & \text { Strategic Highway Research Program } \\ \text { SLS } & \text { Serviceability Limit State } \\ \text { TAC } & \text { Transportation Association of Canada } \\ \text { TYP } & \text { Typical } \\ \text { UDOT } & \text { Utah Department of Transportation } \\ \text { UHPC } & \text { Ultra-High Performance Concrete } \\ \text { UHPFRC } & \text { Ultra-High Performance Fiber Reinforced Concrete } \\ \text { ULS } & \text { Ultimate Limit State } \\ \text { USA } & \text { United States of America } \\ \text { VAF } & \text { Variable Amplitude Fatigue }\end{array}$




\section{Chapter I \\ Introduction}

\subsection{General}

Bridges are one of the key elements in surface infrastructures and transportation networks. Canada invests billions of dollars every year to repair, upgrade and expand its public infrastructures. The Federation of Canadian Municipalities (FCM) added the importance of the gridlock costs and the loss in productivity due to the growth of road-users and bridge construction. Accelerated bridge construction (ABC) addresses the dual needs of fast construction and long service life. Also, rapid bridge replacement allows minimum disruption to traffic for the replacement of the deteriorated bridge deck. Prefabricated bridge elements and systems offers rapid onsite installation, reducing the site access footprint and the environmental impact on construction. In $\mathrm{ABC}$, structural components are built off-site or near the site of the bridge and then transported to the construction site.

Prefabricated bridge systems have five element types, namely: deck elements (precast deck panels), barriers and guardrails, pier elements (caps, columns, and footings), abutments and wall elements (wing walls and back walls), and miscellaneous elements (including approach slabs and overlap systems). In an ideal environment, construction materials are durable. However, discrepancies in design or construction, lack of proper maintenance and aging result in less-thanideal conditions and degradation of construction materials. One of the major issues with service life of bridges is the corrosion of reinforcing steel bars used in bridge decks and barriers when they are exposed to atmospheric conditions, de-icing, moisture and oxygen. Corrosion of steel bars is an electrochemical reaction that occurs when iron atoms loose electrons in the presence of water and oxygen, leading to loss of bond between steel and concrete, concrete spalling and reduction in member load carrying capacity. To address this issue, bridge owners employed several techniques such as: (i) applying protective coating, (ii) the use of high performance coating systems that can provide a prolonged service life, (iii) the use of well-placed and highlyimpermeable high-performance concrete (HPC) with deeply embedded reinforcing bars to

prevent chloride build-up, and (iii) applying regular maintenance before the corrosion propagation phase takes place. 
In November 2007, The Residential and Civil Construction Alliance of Ontario (RCCAO, 2007) released a report on the state of Ontario bridges, entitled "Ontario's Bridges: Bridging the Gap." The study noted that many of Ontario's bridges were built in the 1950s and 1960s, and "it is expected that most bridges will require costly rehabilitation or replacement after 50 years of life." Bridges built prior to the 1970s did not use air-entrained concrete and coated reinforcing steel bars to protect from the effects of freeze-thaw cycles and the application of winter de-icing salt. This leads to corrosion-induced degradation in bridge decks and barriers. Accordingly, bridge decks, railings and barrier walls are all likely candidates for expensive replacement on the majority of these older bridges. The RCCAO report stated some recommendations to be made to promote the public's safety and the sustainability of Ontario's bridges. One of these recommendations includes promoting bridge engineering designs that improve the life expectancy and reduce maintenance costs of bridges. This can be achieved by using fibre reinforced polymer (FRP) bars. The RCCAO report also recommended seeking accelerated delivery methods to address the mounting infrastructure repair and construction backlog. This can be achieved by utilizing prefabricated bridge elements and connection technology to accelerate bridge replacement.

One of the prefabricated systems to accelerate bridge construction involves full-depth, full width, precast concrete deck slab placed transversally over steel or concrete girders where grout pockets, shown in Fig. 1.1, are provided to accommodate clusters of shear connectors welded to steel girders or embedded in concrete girders. In this system, transverse joints, as shown in Fig. 1.2(a), are required to connect adjacent panels in the negative moment region. Also, these transverse joints exist at the negative moment region, at which many serviceability problems such as cracking and water leakage were reported (Porter et al., 2011; Issa et al., 2006). As such, questions arise as how to connect full-depth deck panels at the negative moment region to prevent water leakage and to contribute to ultimate and fatigue limit state designs of the composite girders. Figure 1.2(b) shows a photo of assembled transverse precast deck panels in Passe-à-Fontaine Bridge while Figs. 1.2(c) and 1.2(d) show photos of transverse closure strip in deck and barrier wall in Hwy 401/Mull Road Underpass in Ontario. These bridges were built using epoxy-coated steel bars, dictating wide closure strips in the order of 300 to $400 \mathrm{~mm}$ width and filled with non-shrink grout. However, to reduce closure strip width, high strength materials such as UHPFRC can be used with increased durability. 
Other prefabricated bridge system is made of deck bulb-tee girders as shown in Fig. 1.3. In this system, the concrete deck slab is cast with the prestressed girder in a controlled environment at the fabrication facility and then shipped to the bridge site. This system requires a closure strip to be poured on site between the precast girders to make it continuous for live load distribution. Figure 1.4 depicts an example of bulb-tee girder system used in Moose Creek bridge in Ontario. As it can be observed, deck slab thickness was taken as $250 \mathrm{~mm}$ with the use of epoxy-coated bars. However, this slab thickness can be reduced to $200 \mathrm{~mm}$ with use of GFRP bars since they provide tensile strength more than double the yield strength or reinforcing steel. Also, GFRP bars, as non-corrosive materials, require reduced concrete cover. Figure 1.4(b) shows that the closure strip between precast girders in Moose Creek bridge was of $400 \mathrm{~mm}$ width and filled with $35 \mathrm{MPa}$ non-shrink grout. However, the current research proposes the use of UHPFRC of $150 \mathrm{MPa}$ as filling material in the closure strip so that its width can be significantly reduced to be around $200 \mathrm{~mm}$.

\subsection{The Problem}

Research on the materials between precast bridge components has emerged in the last decade, including non-shrink cementitous grouts, ultra-high performance concrete, epoxy grout, magnesium ammonium phosphate grout and post-tensioning cable grout (among them: NCHRP Report 584, 2008; Li et al., 2010a, 2010b; NCHRP Report 10-71, 2011). More recently, the concept of using the properties of UHPFRC to redesign the connections between prefabricated bridge components has been recognized in North America (among them: Graybeal, 2010, Khalafalla, 2014). Field-cast UHPFRC connections between prefabricated bridge components have now been implemented in few bridges in Canada and U.S. UHPFRC whose mechanical and durability properties far exceed those of conventional concretes present the opportunity to significantly enhance the performance of these field-applied connections, thus facilitating the wider use of prefabricated bridge deck systems. Of particular interest here, UHPFRC can exhibit exceptional bond when cast against previously cast concrete and can significantly shorten the development length of embedded discrete GFRP bars. Based on literature survey, the minimum development length of GFRP bars produced by different manufacturers, with either straight or 
headed-end, embedded in UHPFRC is as yet unavailable. As such, Phase I of the research addresses this gap in research data.

Because $A B C$ is a relatively new technology, the Canadian Highway Bridge Design Code, (CHBDC, 2014), and AASHTO-LRFD Bridge Design Specifications (AASHTO, 2012) do not provide guidance to design prefabricated concrete girder/deck joints made with GFRP bars. Also, there is no enough information available in the literature to design such joints, nor is there test data available to give confidence when designing such joints. Moreover, the literature survey showed limited number of experiments conducted on bridge deck slabs to examine their fatigue and ultimate load carrying capacities under wheel loads. It is important to develop effective precast deck joints to provide continuity of reinforcement in the closure strips so that load sharing between girders is not compromised. In addition, the use of high-performance concrete (HPC) in forming the precast concrete bridge deck panels, following the recent US DOTs' trends (Culmo, 2011, UDOT, 2010), in lieu of the conventional concretes would increase strength, durability and long-term stability, especially its material cost in slightly higher than for conventional concrete. It should be noted that bridges in Canada built with precast panels use 35 MPa normal strength concrete (NSC) while a few US DOTs use high-performance concrete (HPC) with 70-100 MPa compressive strength to cast the deck panels. As such, phase II of this research involves developing closure strip details incorporating GFRP bars and UHPFRC, with the precast deck panels made either from NSC or HPC.

In case of slab-on-girder bridges, deck slab design follows two approaches based on the span-todepth ratio as well as edge restraint conditions. AASHTO-LRFD design specifications consider the design of deck slabs as a continuous strip, of $1000 \mathrm{~mm}$ width, supported freely over the main bridge beams. In this case, the load carrying capacity of the slab is based on its bending moment capacity. However, CHBDC specifies an empirical design method of the deck slabs based on punching shear capacity provided that (i) the main beam spacing-to-slab depth ratio is less than 18; (ii) the slab free edges normal to the main beams are stiffened by composite end beams and increase in slab thickness per Clause 8.18.6; and (iii) the deck slab acts compositely with the supporting beams. As an alternative to the empirical method, CHBDC specifies the flexural design method if the above-mentioned conditions are not met to promote punching shear failure. Phase II in this research addresses the flexural behavior of the deck slabs when designed to 
perform in flexure, while Phase III in this research addresses the punching shear behavior of the restrained deck slabs.

In addition to studying the closure strip performance under wheel load in the positive moment region, questions arise as how to connect full-depth deck panels at the negative moment region to prevent water leakage and to contribute to ultimate and fatigue limit state designs of the composite girders. A review of bridge project inventory in USA (PCI, 2011) revealed critical factors contributing to reduced performance of precast bridge deck panels. Those factors include debonding and water leakage through panel-to-panel transverse joints that lead to severe corrosion due to lack of post-tensioning in the longitudinal direction, inadequate material in the joint, inadequate configuration of the joint, and inadequate surface preparation of the joint. In phases II and III of this research, a zigzag-shape joint was proposed to be used in the negative moment region to assist in limiting crack width for serviceability limit state design. However, this proposed joint was examined only in this research to resist eccentric CHBDC wheel loading. Further research will be conducted to examine this joint in a composite girder system to carry the global girder moment in such a way concrete crack width is controlled.

In conventional bridge construction, expansion joints are used at bridge support locations to accommodate for movement, shrinkage and temperature variations. These joints create shortterm and long-term problems, caused by leaking, debris accumulation, corrosion, and concrete spalling, that causes severe strength reductions. Therefore, there was a need to eliminate the expansion and develop new techniques using advanced construction materials and methods. Two types of jointless bridges can be used to face such challenge, namely: (i) fully integral abutment bridges and (ii) semi-integral abutment bridges which is considered a floating type structure. Figure 1.5 shows typical elevation for an integrated abutment bridge in the traffic direction. Integral abutment has no joints and is constructed with single row of piles. Design issues for the integral abutment bridges consider translation due to abutment movement and rotation of abutment that depends on the height, beam depth, connection details, span length and backfill compaction. The cost-effective intelligent bidding for accelerated bridge construction of integral abutment bridges used " $\mathrm{A}+\mathrm{Bx}$ " bidding, that also referred to as cost-plus time bidding, where " $\mathrm{A}$ " is the dollar amount for the contract, " $\mathrm{B}$ " is the number of days to complete and " $\mathrm{x}$ " is the road-user liquidated damage (RULD). 
The use of precast FDDPs, shown in Figs. 1.1 and 1.2(a), in acceleration bridge construction of the integral abutment bridge, shown in Fig. 1.5, required the use of the transverse joint details for the panel-to-panel connection in the positive moment region. The positive moment region is identified in the schematic diagram in Fig. 1.6 as the girder length of $0.6 \mathrm{~L}$ in each span of a typical two-equal-span integral abutment bridge, where L is the girder span. On the other hand, the negative moment region is identified as the girder length of $0.25 \mathrm{~L}$ on each side of the interior supports (i.e. at pier locations) and at $0.15 \mathrm{~L}$ at the integral abutment locations. The joint located into the negative moment region will be subjected to tensile stresses resulting from composite girder section analysis shown in Fig. 1.7. In the calculations of the negative resisting moment for the composite girder section shown in Fig. 1.7 with Class 1 or 2 steel I-shape section, the full-plasticity stress distribution at failure is of intensity $\varphi_{s} F_{y}$ where $\varphi_{s}$ is the structural steel resistance factor and $\mathrm{F}_{\mathrm{y}}$ is the yield stress of structural steel. Considering concrete deck ineffective in tension, the force in the GFRP reinforcement embedded in the deck will balance the forces in the steel section as a result of the presence of shear studs, ensuring full shear interaction between the steel top flange and the concrete deck slab. The force in the GFRP reinforcement is considered as $\Phi_{\text {frp }} . A_{\text {frp }} . F_{\text {frp }}$ where $\Phi_{\text {frp }}$ is the GFRP resistance factor, $A_{\text {frp }}$ is the cross-section area of the GFRP bars within the effective slab width and $F_{\text {frp }}$ is the flexural stress of GFRP bars at failure. In continuous GFRP bar reinforcement, $F_{\text {frp }}$ is taken the design tensile strength of GFRP bars as specified by the manufacturer. However, with the presence of the UHPFRC-filled transverse joint at the negative moment region, GFRP bars projecting in the joint will overlap over significant portion of the joint width. Thus, $\mathrm{F}_{\text {frp }}$ will depend on the actual force in the bar at the time it pulled out of the jointed-filled concrete. To ensure that GFRP bars are utilized in design at their full capacity, their development length should be calculated at the time the bars rupture under tension. Since research data on the development length of GFRP bars embedded in UHPFRC is as yet unavailable. Phase I would address this research gap in the literature.

CHBDC Clause 10.11.5.3.1 specifies the calculations of the resisting moment, $\mathrm{Mr}$, in the negative moment region per equation 1.1 when (i) shear connectors are provided between the deck slab and the steel girder ensuring full-shear interaction, (ii) slab reinforcement is continuous over the interior supports and (iii) the girders are cross-braced against lateral torsional buckling failure. $M_{r}$ is to be designed for full plastic stress distribution in the structural steel and 
reinforcement, as shown in Figure 1.7. To ensure full tension capacity of GFRP bars in equation 1.1, the minimum embedment length of the bar into the UHPFRC-filled joints resulting from phase I of the research should be used.

$$
M_{r}=T_{f r p, t o p} e_{r, t o p}+T_{f r p, b o t t o m} e_{r, b o t t o m}+T_{s} e_{s}
$$

Where $M_{r}$ is the resisting moment of the composite section around the compression force; $T_{f r p, t o p}$ and $T_{f r p, b o t t o m}$ are the tension force in the top and bottom rebar, respectively; $T_{s}$ is the tension force in the girder above the neutral axis; $e_{r, t o p}, e_{r, b o t t o m}$ and $e_{s}$ are the arm distances from the forces to the compression force.

\subsection{Objectives of Research}

The objective of this research is to develop and study closure strips between adjacent precast full-depth concrete bridge deck panels (FDDPs) incorporating GFRP bars as reinforcement in deck slabs and UHPFRC as filling-material in the joints. The specific objectives of this research can be listed as follow:

1- Conduct experimental parametric study on the pullout capacity of GFRP bars embedded in UHPFRC with ultimate goal to determine the minimum GFRP bar development length to ensure full capacity of the bar when conducting section analysis.

2- Investigate experimentally the mechanical behavior and ultimate flexural load carrying capacity under monotonic loading of precast FDDPs incorporating three developed jointed precast FDDPs.

3- Investigate experimentally the mechanical behavior and punching shear capacity of restrained jointed FDDPs under fatigue loading with ultimate goal to develop prediction model for fatigue life of UHPFRC-filled jointed deck slabs.

4- Examine whether GFRP-reinforcement ratio for internally-restrained deck slabs recommended by CHBDC Section 16 is adequate for ultimate, serviceability and fatigue limit state designs of jointed FDDPs. 


\subsection{Scope of Research}

The experimental program investigates the structural behavior of the jointed precast FDDPs Under monotonic and fatigue loading. This research has three phases as follows.

Phase I investigated the pullout mechanism of GFRP bars embedded into UHPFRC. Four sets of GFRP bar configurations with bar ends embedded into UHPFRC blocks. The key parameters considered in this experimental study included (i) bar location within the block (i.e. central or eccentric), (ii) type of bar end (i.e. straight- or headed-end), (iii) bar diameter, and (iv) bar embedment length. Results from this phase let to determine the minimum development length into UHPFRC to ensure full capacity of the bars based on their tensile strength.

Phase II included the development and study of closure strip details incorporating UHPFRC as joint-filling materials and GFRP bars projecting into the joint. Three joints of width $200 \mathrm{~mm}$ between precast FDDPs were developed, namely: angle-shape joint (A-joint), $\mathrm{C}$-shape joint (Cshape), and zigzag-shape joint (Z-joint), with 175-mm projecting length of GFRP bars into the joint. Two series of $2500 \times 600 \times 200 \mathrm{~mm}$ one-way slabs were cast to investigate the flexural strength of the jointed precast slabs compared to cast-in-place slabs. Two types of concrete were used to fabricate the precast FDDPs, namely: normal concrete (NSC) and high-performance concrete (HPC). The applied wheel load was located such that the joint is subjected to pure moment in one specimen and combined moment and shear in another identical specimen. Correlation between experimental results and available design equations for moment and shear capacities, as well as CHBDC and AASHTO-LRFD applied factored design moments, was performed.

Phase III included testing three pairs of 3700x2500x200 mm laterally-restrained precast FDDPs incorporating the three developed joint details in the transverse direction of the girders. Each pair of specimens was tested under $600 \times 250 \mathrm{~mm}$ wheel loading located beside the closure strip, considering (i) constant amplitude fatigue (CAF) loading up to 4 million cycles followed by increasing static loading to-collapse, and (ii) incremental variable amplitude fatigue (VAF) loading to-collapse. Correlation between the experimental ultimate loads and available factored design moments bridge codes was performed. A new prediction model for fatigue life of the 
GFRP-reinforced, UHPFRC-filled jointed deck slabs was developed. Conclusion regarding the adequacy of the GFRP-reinforcement ratio for internally-restrained deck slabs recommended by CHBDC Section 16 for ultimate, serviceability and fatigue limit state designs of jointed FDDPs was drawn.

\subsection{Contents and Arrangements of the Thesis}

This thesis consists of six chapters. Chapter I presents the introduction, rationale for research, research objective and scope of work. Chapter II presents the research program to investigate the pullout capacity of GFRP bars embedded in UHPFRC blocks. Chapters III and IV present the development of transverse joints for full-depth precast deck panel reinforced with GFRP bars using normal concrete and high-performance concrete, respectively. Chapter V presents fatigue evaluation of full-scale restrained precast FDDPs with transverse joint details as well as their

ultimate load carrying capacities. Chapter VI presents the summary of findings, conclusions and recommendations for the future research.

\section{References}

AASHTO, (2012). LRFD Bridge design specifications. 2nd Ed. American Association of State Highway and Transportation Officials. Washington. D. C. USA.

CHBDC. 2006. Canadian Highway Bridge Design Code, CAN/CSA-S6-06. Canadian Standard Association, Toronto, Ontario, Canada.

Culmo, M. 2011. Accelerated Bridge Construction - Experience in Design, Fabrication and Erection of Prefabricated Bridge Elements and Systems. Report No. FHWA-HIF-12-013, Federal Highway Admin., 347 pages.

Graybeal, B. 2010. Behavior of Field-Cast Ultra-High Performance Concrete Bridge Deck Connections under Cyclic and Static Structural Loading. Federal Highway Administration, Report No. FHWA-HRT-11-023, 106 pp.

Issa, M., Salas, J., Shabila, H., and Alrousan, R. 2006. Composite Behavior of Precast Concrete Full-Depth Panels and Prestressed Girders. PCI Journal, 132-145.

Khalafalla, I. 2014. Development of Sustainable Concrete Bridge Deck Slab Systems using Corrosion-resistant GFRP bars. Ph.D. Thesis, Civil Engineering Department, Ryerson 
University, Toronto, Ontario, Canada.

Li, L., Ma, J., and Oesterle, R. 2010a. Improved Longitudinal Joint Details in Decked For Accelerated Bridge Construction: Concept Development. ASCE Journal of Bridge Engineering, V. 15, No. 3, May-June 2010, p. 327-336.

Li, L., Ma, J., and Oesterle, R. 2010b. Improved Longitudinal Joint Details in Decked For Accelerated Bridge Construction: Fatigue Evaluation. ASCE Journal of Bridge Engineering, V. 15, No. 5, September-October 2010, p. 511-522.

Hossain, I., and Lam, C. 2006. Guidelines for Prefabricated Bridge. Highway Standard Branch, Ministry of Transportation of Ontario, 128 pages.

NCHRP. 2008. Full-Depth Precast Concrete Bridge Deck Panel Systems. NCHRP Report 584, Transportation Research Board, Washington DC.

NCHRP. 2011. Summary of Cast-In-Place Concrete Connections for Precast Deck Systems. Research Results Digest 355, Transportation Research Board, pp. 1-33.

PCI. 2011. State-of-the-art Report on Full-Depth Precast Concrete Bridge Deck Panels. Prepared by PCI Committee on Bridges and PCI Bridge Producers Committee. Precast/prestressed Concrete Institute, PCI, 141 Pages.

Porter S.D., Julander J. L., Halling M.W., Barr P. J., Boyle H., Xing S. 2011. Flexural Testing of Precast Bridge Deck Panel Connections. Journal of Bridge Engineering, Vol. 16, No. 3.

RCCAO. 2007. Ontario's Bridges Bridging the Gap. Reported prepared by MMM Group for Infrastructure Investment Coalition (IIC) and Residential and Civil Construction Alliance of Ontario (RCCAO).

UDOT. 2010. Full Depth Precast Concrete Deck Panel Manual. Utah Department of Transportation, 24 pages. 


\section{Figures}

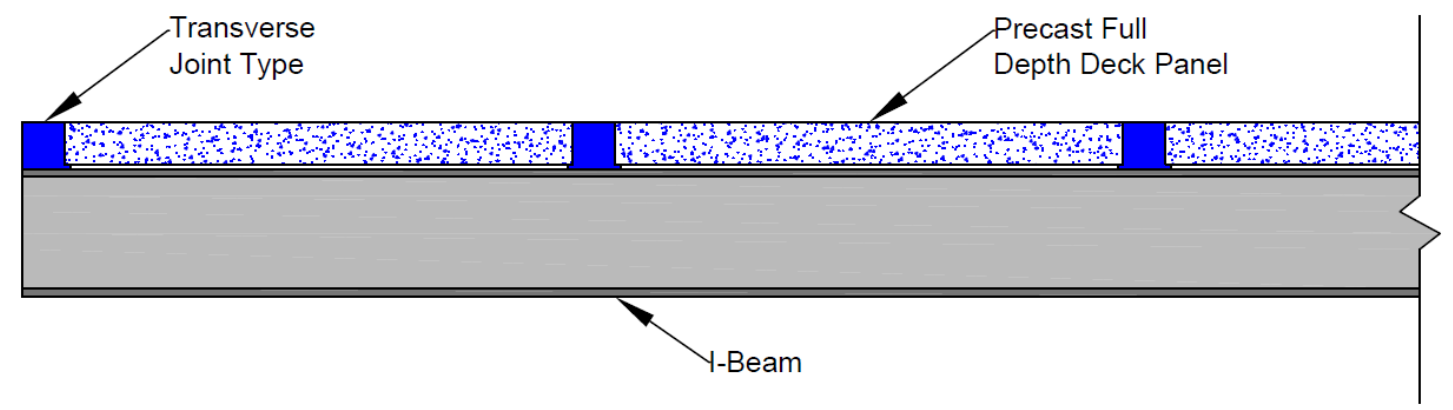

Figure 1.1 Precast full-depth deck panels (FDDPs) placed transversally over girders 


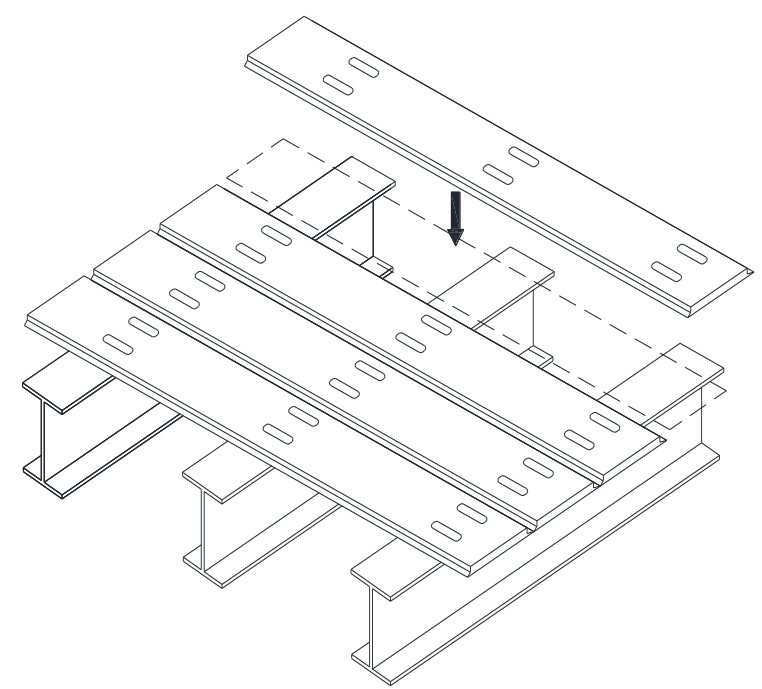

a) Isometric view

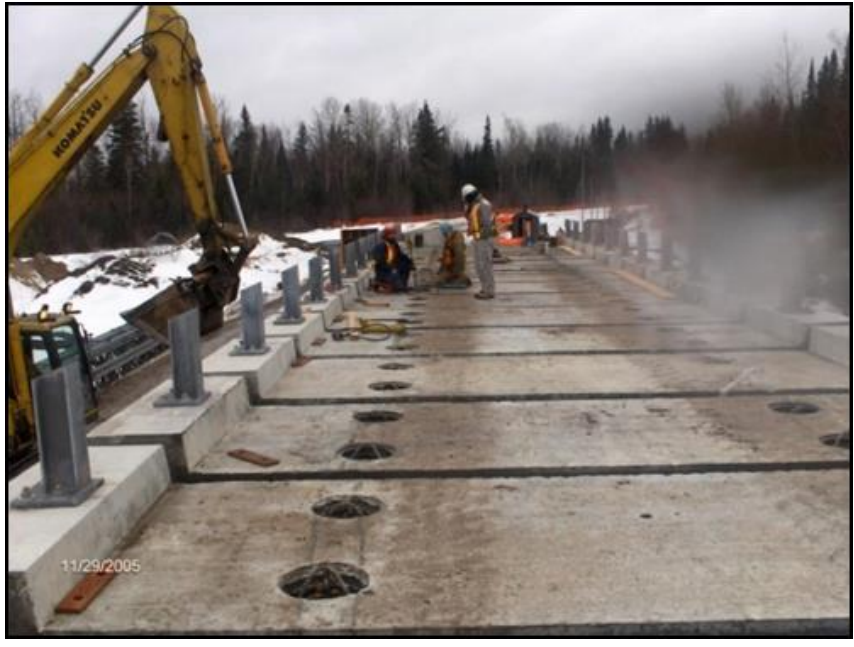

b) Photo of assembled transverse precast deck panels in Passe-à-Fontaine Bridge (source: Hossain and Lam, 2006)

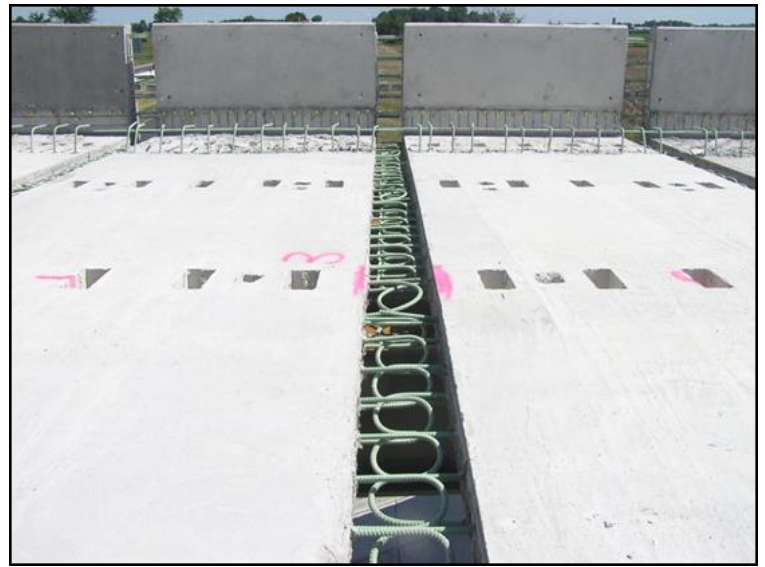

d) Transverse closure Strip in deck and barrier in Hwy 401/Mull Road Underpass (source: Hossain and Lam, 2006)

Figure 1.2 Views of full-depth, full width, deck panels (FDDPs) placed transversally on girders 


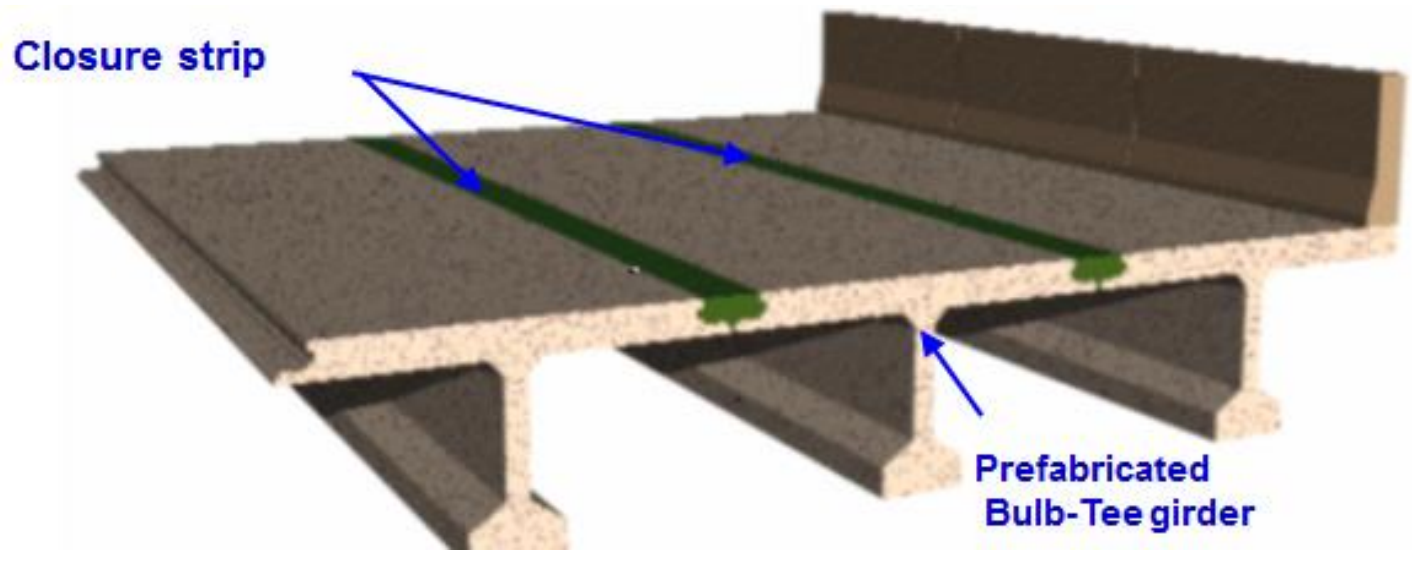

Figure 1.3. Schematic view of precast bulb-tee girders with closure strips oriented in girder direction 


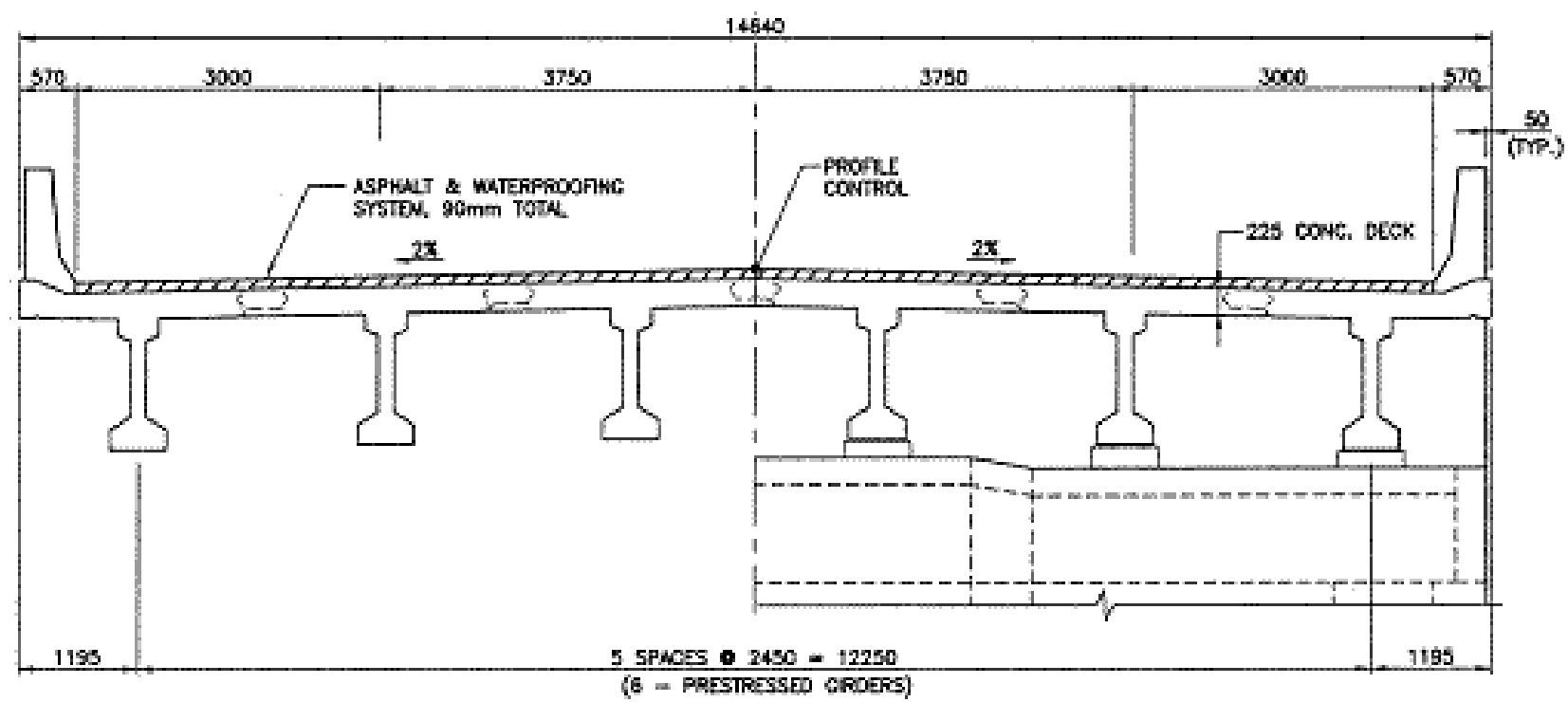

a) Bridge cross-section of Moose Creek Bridge
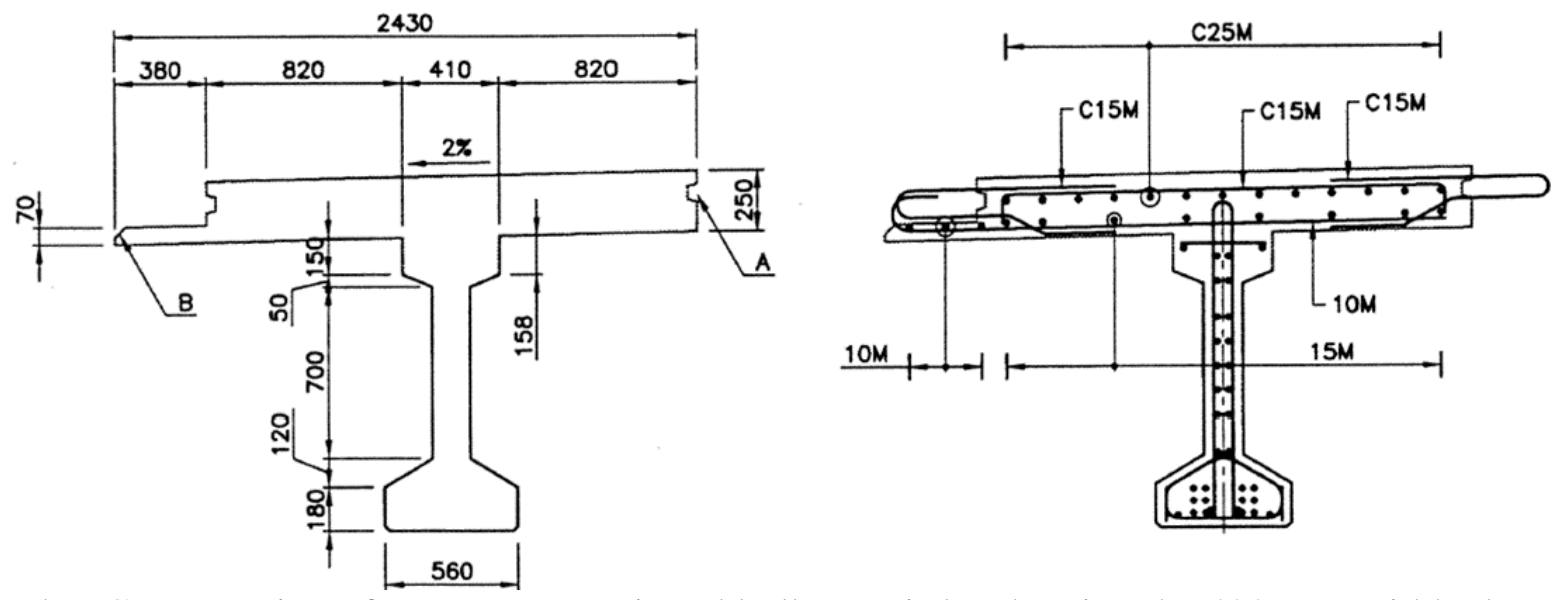

b) Cross-section of precast pretensioned bulb-tee girder showing the 400-mm width closure strip and projecting epoxy-coated steel bars into the joint

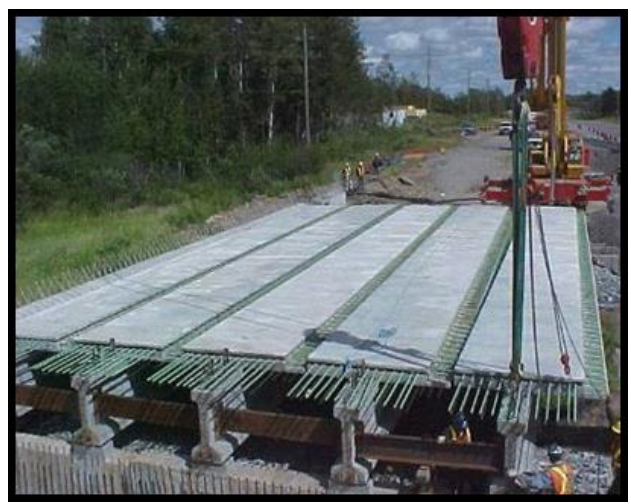

c) Photo of precast girders assembled

Figure 1.4. Prefabricated bulb-tee bridge girder system in Moose Creek Bridge (source: Hossain and Lam, 2006) 


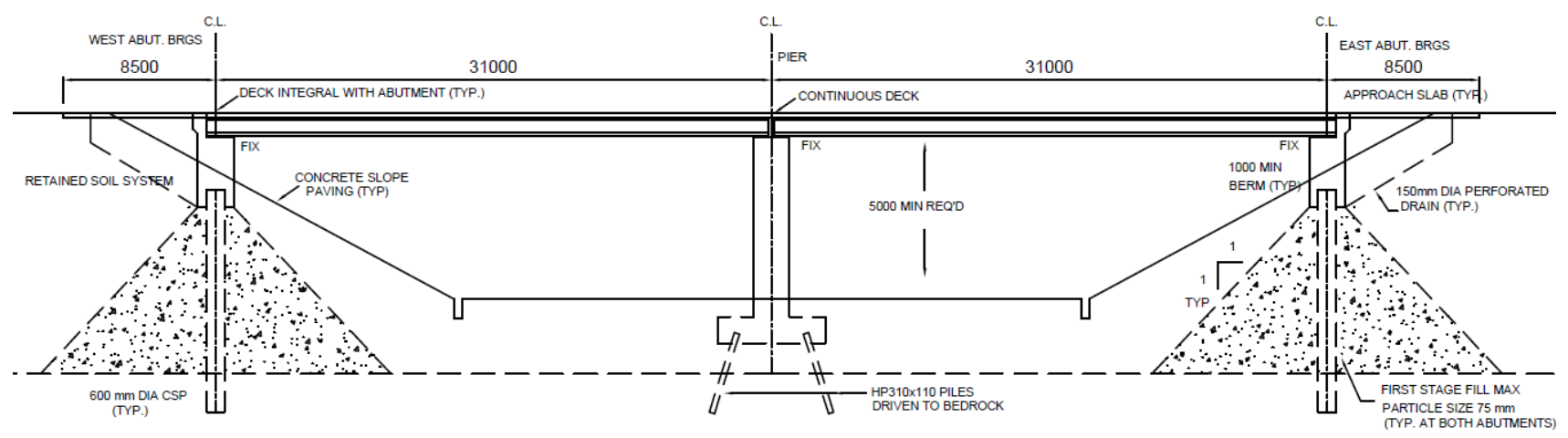

Figure 1.5. Typical elevation for an integral abutment bridge in the longitudinal direction

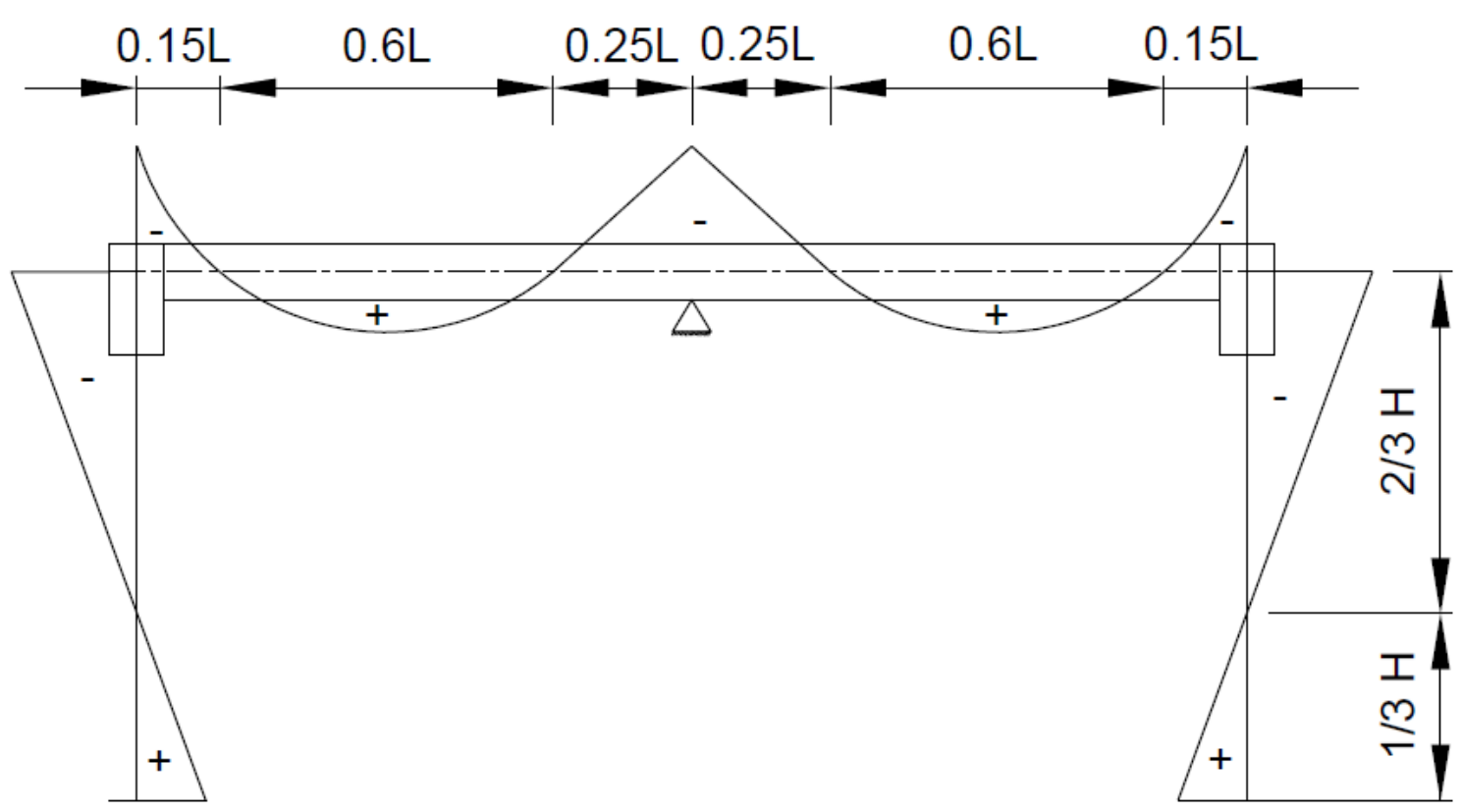

Figure 1.6. Typical bending moment diagram in the longitudinal direction of an integral abutment bridge 


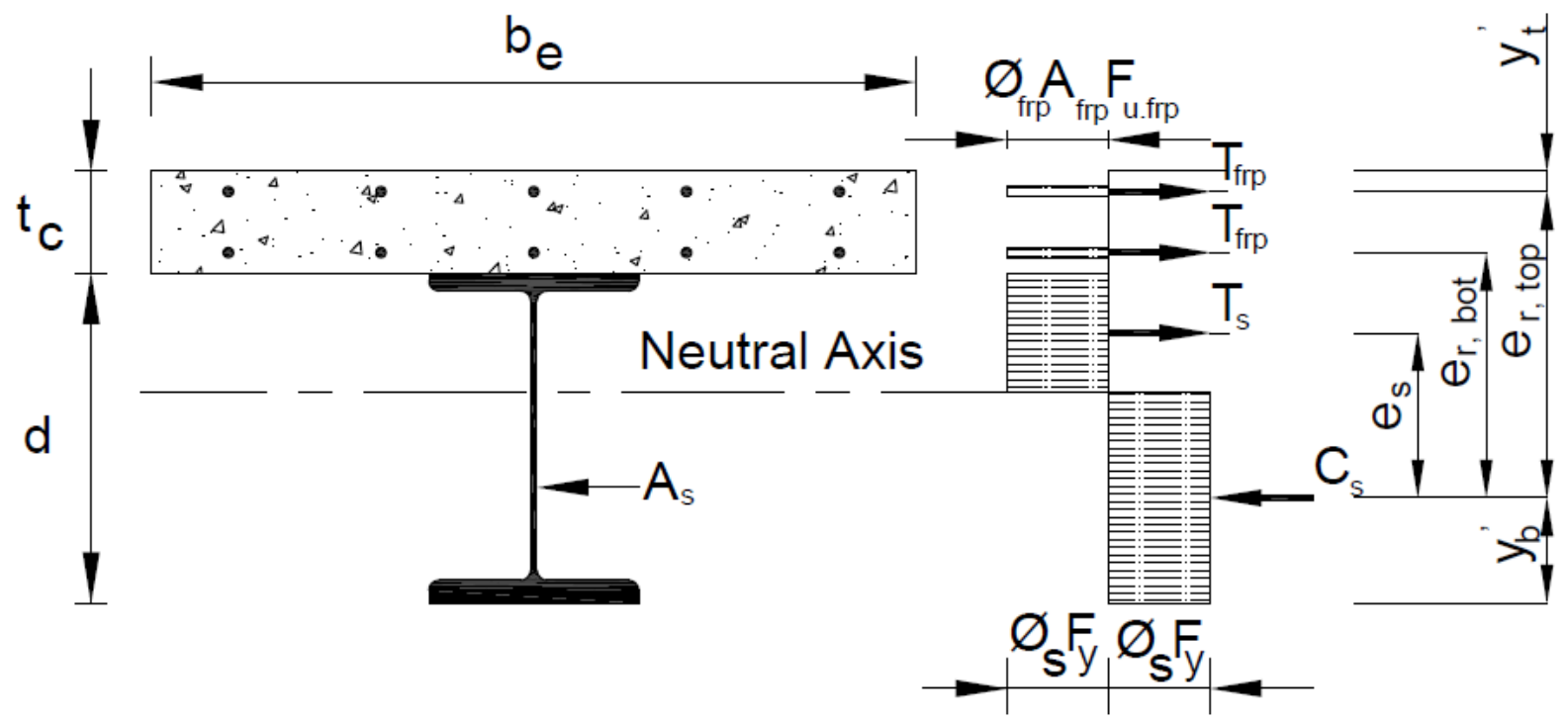

Figure 1.7. Cross-section analysis in the negative moment regions showing stress and force distribution for fully-plastic composite girder design 


\section{Chapter II}

\section{Bond Strength of High-Modulus Glass Fiber Reinforced Polymer Bars Embedded into Unconfined Ultra-high Performance Concrete}

\subsection{General}

High-modulus (HM) ribbed-surface and sand-coated glass fiber reinforced polymer (GFRP) bars have recently been used in concrete bridge decks and barriers to avoid corrosion of steel reinforcement resulting from the use of de-icing salts in winter times in Canada. Recently, prefabricated full-depth deck panels (FDDPs), made of normal strength concrete or high performance concrete and reinforced with GFRP bars, are used in Canada in accelerated bridge construction. FDDPs are connected through panel-to-panel and panel-to-girder joints. These joints are filled with cementitious materials as ultra-high performance fiber-reinforced concrete (UHPFRC). This chapter presents the experimental program to investigate the bond strength of GFRP bars embedded into unconfined UHPFRC using pull-out testing, leading to the proper GFRP bar development length required to determine the width of the closure strip between connected slabs. The longitudinal GFRP/UHPFRC interface is influenced by (i) the development length-to-nominal diameter of the bar ratio, (ii) the concrete cover-to-bar diameter ratio and (iii) the development length-to-embedment depth ratio due to lugs or headed-end and (iv) concrete compressive strength. GFRP bars embedded into UHPFRC would rely less on the friction and adhesion of the interface, and more on the bearing of the lugs against the concrete. These bearing forces act at an angle to the axis of the bar, causing radial outward forces. Pullout failure of the GFRP/UHPFRC interface leads to shearing of the lugs and bar slippage from the headed-end. Adequate bond strength between the GFRP/UHPFRC interfaces is necessary for design of jointed FDDPs. Therefore, accurate predictions of development length and bond strength of straight-end as well as headed-end GFRP bars, without passing through the high localized stresses due to flexure, are essential for safe design. The results from this research can be used further to analyse the joint between the precast slabs to determine their resisting moment given 
the fact that GFRP tensile strength can be determined based on the pullout capacity of the projecting bar from the precast slab and embedded into the UHPFRC filling the closure strip.

\subsection{Background of Research}

The concept of the accelerated bridge construction (ABC) and rapid bridge replacement (RBR) introduces the prefabricated bridge elements and systems (PBES). Bridge elements are manufactured in plant, transported, installed on-site with cast-in-place joints using high early strength concrete in order to open the bridge for traffic. Precast FDDPs are connected through panel-to-panel and panel-to-girder joints. In this research, the closure strip is filled with UHPFRC and GFRP are used to reinforce the FDDPs and the closure strips. Bond strength of GFRP bar embedded into UHPFRC needs experimental investigation to determine the reasonable width for the panel-to-panel joints. The interaction between GFRP bars and concrete is influenced by (i) bond strength between the two materials that prevents slip of the bar relative to concrete, (ii) concrete mix design that provides structural member with high concrete capacity design load, and (iii) similar rates of thermal expansion for the concrete and GFRP bars under weather conditions.

UHPFRC is made by mixing ordinary Portland cement, supplementary cementitious materials such as Silica Fume, very fine aggregate such as ground quartz, steel fiber reinforcement, admixtures such as the high range water reducer (HRWR), and water (Graybeal, 2006, 2007). UHPFRC is a self-consolidated concrete with high fluidity and deformation capability that levels itself without vibration. Its strength increases with age and curing, with accelerated compressive strength of $100 \mathrm{MPa}$ at 96 hours and $140 \mathrm{MPa}$ at 28 days.

Fiber reinforced polymer (FRP) bars have high tensile strength-to-weight ratio compared to steel reinforcement (i.e. the weight of the FRP bar is approx. 25\% of weight for the same bar diameter of steel reinforcement). FRP bars are characterized by being corrosion resistant, leading to increase in bridge service life (durability) as a result of significant reduction in maintenance cost. FRP bar is composed of fibres and resin. Fibres are made of carbon, glass, or aramid to provide strength, stiffness. While the resin binds the fibers together. FRP is anisotropic material that provides high strength in the direction of the fibers, and low shear strength perpendicular to the axes of the bar. FRP bar exhibits linear elastic behavior until failure with no yielding point as for 
steel reinforcement, thus design should account for the lack of ductility. The external surface of the FRP bar may be ribbed-surface, sand-coated surface, deformed, or helical to achieve the desired bonding to the surrounding concrete. GFRP bars manufactured with straight end, headedend or with $180^{\circ}$ hook. GFRP bars are classified into low modulus (LM) and high modulus (HM) based on the value of their modulus of elasticity. Figure 2.1 shows views of the ribbed-surface and sand-coated GFRP bars considered in this research.

The common mechanisms for bond strength between reinforcing bars and concrete depends on several parameters including: (i) reinforcement properties, surface condition, modulus of elasticity, and Poisson's ratio; (ii) concrete properties including compression and tensile strength, modulus of elasticity and Passion's ratio; (iii) confinement of concrete; (iv) type of loading and (v) time dependent effect (ACI 440R, 1996). The common bond failure for the GFRP bars embedded into concrete can be one of the following: (i) concrete splitting; (ii) bar pullout; (iii) bar fracture ; (iv) interlaminar shear strength failure; (v) peel-off surface failure; and (vi) bar slippage from the headed bar, as shown in Figure 2.2. Bond strength means the capacity to transfer the force in the reinforcement to the surrounding concrete. The interacting force may arise from: (i) chemical adhesion and friction at the surface area of the bar to the concrete; and (ii) the bearing of the ribs or lugs that act at an angle to the axis of the bar, causing radial outward forces counterpartyed with the surrounding concrete (ACI Committee 408, 2003). Structural members are subjected to axial load and or flexural loads. Bond strength due to tension loading fails under pullout of the bar, progressive splitting of concrete, or the combined failure mode of bar pullout and concrete splitting. Flexural bond (i.e. localized interaction) generates high localized stress due to the rate of change of longitudinal tensile force along the span adjacent to the flexural cracks, and is proportional in magnitude to the shear, $\mathrm{dM} /$ $(\operatorname{arm}) \mathrm{dz}=\mathrm{V} /(\mathrm{arm})$. Thus, the localized stress condition doesn't directly correlate to the development-length-related strength of the member. Equation 2.1 determines the horizontal force equilibrium over a segment $D D^{\prime}$ of the reinforcing bar noted $d z$ as that depends on the magnitude of $\left(\left(T_{D}-T_{D^{\prime}}\right) / d z\right)$.

(2.1) $\quad u_{s} \pi d_{b} d z+u_{b} A_{b r} d z=T_{D}-T_{D^{\prime}}$ 
Where $u_{s}$ is the localized surface stress over nominal contact area between the steel bar and the concrete; $d_{b}$ is the diameter of the single bar; $u_{b}$ is the localized bearing stress over the area $A_{b r}$ per unit length between the lugs and the concrete (Wang and Salmon, 2002).

ACI 318 defined the development length concept as the attainable average bond stress over the length of embedment of the reinforcement (ACI 318-15, 2015). The development length is a function of (i) the embedment length-to-nominal diameter of the bar ratio $\left(l_{e} / d_{b}\right)$; (ii) the embedment length-to-concrete cover ratio $\left(l_{e} / c_{\min }\right)$ or concrete cover to bar diameter ratio $\left(c_{\min } / d_{b}\right)$; (iii) the embedment length-to-depth ratio $\left(l_{e} / h_{e f}\right)$ due to lugs or headed-end or the $180^{\circ}$ hook; and (iv) concrete compressive strength $\left(f_{c}^{\prime}\right)$ for confined and unconfined sections. One step to find the development length is to determine the bond behavior and develop the empirical bond constant $(1 / k)$. The nonlinearity of bond between reinforcement and concrete has been investigated by several researchers. It was found that GFRP bar has nonlinear distribution of bond stresses along the embedment length similar to the uncoated steel rebar (Benmokrane, et al., 1996). To find empirical constants for bond-slip relationships, researchers obtained experimentally data on peak load, concrete peak load, load-end slip and free-end slip (Hanus, et al., 2000). FRP bar diameter attributes such lower bond strength to three factors (Achillides \& Pilakoutas, 2004):

- Larger diameter bars require longer embedment lengths in order to reach the same normal bond stress. As greater embedment lengths reduce the average bond strength.

- Poisson's effect can allow a slight reduction in bar diameter as a result of longitudinal stress. This reduction increases with bar diameter leading to reduced frictional/mechanical locking stresses.

- Shear stiffness of FRP bars depends mainly on the shear stiffness of the resin and the shear strength at the resin-fibre interface. When a FRP bar is pulled in tension, some differential movement between the core and surface fibres takes place which results in a non-uniform distribution of normal stresses through the bar cross section (shear lag phenomenon),

Researchers investigated experimentally the behavior of sand-coated low modulus (LM) and high modulus (HM) GFRP bars among them Hossain, et al. (2012) using high strength concrete. 
Khederzadeh and Sennah (2013) conducted similar research but using normal strength concrete and sand-coated GFRP bars with straight, hooked and headed ends. Headed-end and straightend, ribbed-surface, GFRP bars were tested after embedding them into high-strength concrete (Islam, et al., 2015). This research showed that bond stresses of headed-end bars were enhanced by $135 \%$ and $59 \%$ compared to the straight bars with embedment length of $6 \mathrm{~d}_{\mathrm{b}}$ for the 12 and 16 $\mathrm{mm}$ diameters, respectively. Other researchers conducted similar tests but with GFRP bars embedded in normal-strength, steel-reinforced, slabs where the confinement contributed to higher bond strength (Tropynina, 2012).

Design recommendations for bond of GFRP bars to concrete were performed by several researchers. Direct pullout tests were carried out on cantilever beams and pull-out specimens. The basic development length of FRP bars was written in form of Equation 2.2, where $f_{y f}$ is the effective yield strength of the FRP bar in MPa, $\mathrm{A}_{\mathrm{b}}$ is the bar nominal cross-sectional area and the compressive strength of concrete $f_{c}^{\prime}$ does not exceed 69 MPa (Faza \& GangaRao, 1990).

$$
l_{d}=0.028 \frac{A_{b} f_{y f}}{\sqrt{f_{c}^{\prime}}}
$$

The basic development lengths for straight and hooked GFRP bars were introduced into Equations 2.3 and 2.4 based on experimental study using direct pullout and beam tests. The 0.022 coefficient in Equation 2.3 ensures that all development lengths calculated will be larger than the measured ones. The 152 coefficient in Equation 2.4 was modified after the ACI Basic coefficient of 100 that simulates the steel bar response. The modification factor (MF) herein introduces the effect of the bar locations and valued by 1 to 1.25 (Ehsani, et al., 1996).

$$
\begin{aligned}
& l_{d b}=M F \times 0.022\left(A_{b} f_{y} / \sqrt{f_{c}^{\prime}}\right) \\
& L_{h b}=M F \times 152\left(d_{b} / \sqrt{f_{c}^{\prime}}\right)
\end{aligned}
$$

A more recent study investigated bond performance of GFRP bars embedded in UHPFRC and HSC (Mak, 2011). Such study was extended by conducting reliability analysis to determine a resistance factor of 0.5 for bond of HM GFRP bars in UHPFRC (Banson, 2013). Researchers suggested that Equation 2.5 be used to find the development length of low modulus GFRP that has elastic modulus of 49.4 or $47.8 \mathrm{MPa}$ with $f_{f r p, u}$ of $1132 \mathrm{MPa}$, embedded into UHPFRC with 
$f_{c}^{\prime}$ of $201.8 \mathrm{MPa}$. The $15.9 \mathrm{~mm}$ diameter GFRP bar will result in a development length of 373 $\mathrm{mm}\left(23 d_{b}\right)$ before bar reached pullout failure (Yoo, et al., 2015).

$$
l_{d, p u l l o u t}=\frac{d_{b} f_{f r p, u}}{3.4 \sqrt{f_{c}^{\prime}}}
$$

Other researchers (Firas, et al., 2011) tested control specimen of $8 \mathrm{~mm}$ diameter deformed steel bar embedded $40 \mathrm{~mm}$ into UHPFRC of $170 \mathrm{MPa}$. The test showed that bar bond strength of 50 MPa and slip of $0.038 \mathrm{~mm}$, while for the smooth CFRP bar with same bar diameter and embedment length, the experimental bond strength was $22.05 \mathrm{MPa}$ at slip of $0.14 \mathrm{~mm}$. Research found that bond strength decreases with the increase of the embedment length or with the increase of bar diameter

New York State Department of Transportation performed pullout test on steel reinforcing bar embedded in $400 \mathrm{~mm}$ diameter UHPFRC cylinders, where \# 4, \#5 and \#6 bars were embedded at 75, 100 and $125 \mathrm{~mm}$ into UHPFRC (Graybeal and March 2011). Results showed that all bars were fractured before bond failure. Pullout tests were also conducted on \# 4 (12 mm diameter) bar considering different concrete cover and embedment length in UHPFRC. Results indicated that increasing concrete cover and embedment length resulted into increase in bond strength (Fehling, et al., 2012). Other researchers (Swenty and Graybeal, 2012) pulled out a \#4 steel bar embedded into $152 \mathrm{~mm}$ UHPFRC cube with $76 \mathrm{~mm}$ of unbonded length, followed by $76 \mathrm{~mm}$ of bond length along the centerline of the cube. Results showed bar yield before ultimate pullout or bar rupture before bond failure

Yuan and Graybeal (Yuan and Graybeal, 2014) conducted 200 direct pullout tests on steel bars embedded into UHPFRC of $150 \mathrm{MPa}$ compressive strength. They found out that bar development length into UHPFRC can be significantly reduced compared to that for normal concrete. Also, they concluded that the non-contact lap splice exhibits higher bond strength than contact lap splice as long as the bar clear spacing is bigger than $l_{s} \tan (\theta)$ where $1_{\mathrm{s}}$ is splice length and $\theta$ is angle between the diagonal crack and testing bar. Moreover, they concluded that bar splice is recommended to be at $75 \%$ of the embedment length. 
Saleem et al. (Saleem, et al., 2012) conducted pullout tests on \#10 and \#22 high-strength steel bars embedment into UHPC. They recommended bar development lengths of 12 and 18 times the bar diameter for \#10 and \#22 bars, respectively.

\subsection{Experimental Program}

The objective of this experimental research is to investigate the direct pullout load for the ribbedsurface and sand-coated straight and headed-end HM GFRP bars with different embedment lengths into UHPFRC. The key parameters considered in this study were: (i) embedment lengthto-nominal diameter of the bar ratio $\left(l_{e} / d_{b}\right)$; (ii) concrete cover-to-bar diameter ratio $\left(c_{\min } / d_{b}\right)$; (iii) type of bar end anchorage (i.e. straight end or headed end); and (iv) concrete compressive strength $\left(f_{c}^{\prime}\right)$. The data collected during each test included the applied pullout load, and the slip at the loaded and free ends. The data generated form the pullout tests were used to determine the GFRP bar bond strength as well as minimum embedment length to ensure that the bar reaches it tensile strength before bond failure.

\subsubsection{Description of Specimens}

To perform pullout tests, two sizes of concrete block cross-section were used, namely: 200x200 $\mathrm{mm}$ for straight bars and 300x300 mm for headed-end bars. The depth of the concrete block in direction of the bar depends on the bar embedment length as depicted in Fig. 2.3. Three types of concrete cover were considered in this study, namely: (i) centric bar as shown in Figs. 2.3.a and 2.3.b, and (ii) eccentric bar with concrete cover of 40 and $60 \mathrm{~mm}$ as depicted in Figs. 2.3.c and 2.3.d. In all specimens, $1500 \mathrm{~mm}$ length GFRP bars were used. The first $50 \mathrm{~mm}$ from the embedded length of all tested bars into the concrete blocks was unbonded as depicted in Fig. 2.3. For straight-end bars, different embedment length of $4 \mathrm{~d}_{b}, 6 \mathrm{~d}_{\mathrm{b}}$ and $8 \mathrm{~d}_{\mathrm{b}}$ were considered. However, for headed-end bars, the bonded length of the bar was taken as the length of the anchorage head of $100 \mathrm{~mm}$. Table 2.1 summarizes the test matrix. It should be noted that both Table 2.1 and Figs. 2.3.b and 2.3.d show a bonded length, $\mathrm{L}_{\mathrm{e} 2}$, for the straight portion of the bar in addition to the head-end length. However, only one specimen was tested in this configuration to examine the effect of added bonded length of the straight portion to the anchorage head (i.e. specimens with 20M GFRP bar with $100 \mathrm{~mm}$ anchorage head and $80 \mathrm{~mm}$ straight bonded length. This specimen showed higher pullout force than that for the anchorage head only. So, it was decided to conduct 
pullout tests on headed-end bars with $\mathrm{L}_{\mathrm{e} 2}$ equal zero due to the limited capacity of the test setup. It should be noted that for each pull out test configuration, 4 identical specimens were tested for better representation of test data.

\subsubsection{Material Properties}

The ultra-high performance fiber reinforced concrete (UHPFRC) considered in this research had a nominal compressive strength $140 \mathrm{MPa}$ after 28 days. Tables 2.2 and 2.3 show the concrete mix design and mechanical properties of UHPFRC, respectively. The cementitious materials-fine aggregate-water ratio is 1.33: 1.74: 0.20. Figure 2.4 shows the compressive strength of UHPFRC versus the curing age, $\mathrm{t}\left(f_{C, U H P F R C}^{\prime}=74.865 t^{0.191}\right)$.

Two types of GFRP bars were used in this study, namely: sand-coated and ribbed-surface bars. The ribbed-surface profile of these bars, shown in Fig. 2.1.a, ensure optimal bond between concrete and the bar. These bars have tensile strength of $1188 \mathrm{MPa}$, compared to $400 \mathrm{MPa}$ yield strength of the currently used reinforcing steel bars (Schoeck Canada Inc, 2011). Their modulus of elasticity and strain at failure are $64 \mathrm{GPa}$ and $2.61 \%$, respectively. Table 2.4 summarizes the mechanical properties of the ribbed-surface bars. Until recently, the installation of GFRP bars was often hampered by the fact that bent bars have to be produced in the factory since GFRP bars cannot be bent at the site. Also, bent GFRP bars are much weaker than straight bars, due to the redirection and associated rearrangement of the fibres in the bend. As a result, number of bent GFRP bars is increased and even doubled at such locations where bar bents are required. The use of headed-end GFRP bars is intended to eliminate the unnecessary and expensive use of bar bends. This headed end is made of a thermo-setting polymeric concrete with a compressive strength far greater than that of normal grade concrete. It is cast onto the end of the straight bar and hardened at elevated temperatures. The concrete mix contains an alkali resistant Vinyl Ester resin, the same material used in the straight bars, and a mixture of fine aggregates. The maximum outer diameter of the end heads is 2.5 times the diameter of the bar. The head of the $16 \mathrm{~mm}$ bar is approximately $100 \mathrm{~mm}$ long. It begins with a wide disk which transfers a large portion of the load from the bar into the concrete. Beyond this disk, the head tapers in five steps

to the outer diameter of the blank bar. This geometry ensures optimal anchorage forces and minimal transverse splitting action in the vicinity of the head. Figures 2.1.b and 2.1c shows views the ribbed surface GFRP bar with headed end. In this research, 15M ribbed-surface GFRP 
bars with straight as well as headed ends were tested. However, only straight-ended 20M bars were tested.

The sand-coated surface profile of GFRP bars, shown in Fig 2.1a. ensure optimal bond between concrete and the bar. 15M (\#5) high-modulus (HM) GFRP bars of specified tensile strength of $1184 \mathrm{MPa}$, modulus of elasticity of $62.6 \pm 2.5 \mathrm{GPa}$ and strain at rupture of $1.89 \%$, as listed in the manufacturers catalogue, were used in this research. Also, 20M (\#6) high-modulus (HM) GFRP bars of specified tensile strength of $1105 \mathrm{MPa}$, modulus of elasticity of $64.6 \pm 2.5 \mathrm{GPa}$ and strain at rupture of $1.71 \%$ were considered in this research. Table 2.5 summarizes the mechanical properties of these sand-coated bars. GFRP bents are produced to allow for anchorage in concrete. However, GFRP bents are much weaker than straight bars, due to the redirection and associated rearrangement of the fibres in the bent. As a result, the number of bent GFRP bars is increased and even doubled at such locations where bar bents are required. The use of headedend GFRP bars is proposed in this research to allow for anchorage in concrete at lower cost that the bent bars. This GFRP bar headed end, shown in Figs. 2.1.b and 2.1.c, is made of a thermosetting polymeric material cast onto the end of the straight bar and hardened at elevated temperatures. Before casting the head, the bar end that was left smooth with no sand-coating was machined to provide intermittent circular ribs to increase bond between the bar the head. The maximum outer diameter of the head at its end is 2.5 times the diameter of the bar. The head of $15 \mathrm{M}$ bar is approximately $100 \mathrm{~mm}$ long. It begins with a wide disk which transfers a large portion of the load from the bar into the concrete. Beyond this disk, the head tapers in five steps to the outer diameter of the blank bar. This geometry ensures optimal anchorage forces and minimal transverse splitting action in the vicinity of the head.

\subsubsection{Test Setup and Instrumentation}

Static loading was applied along the $1500 \mathrm{~mm}$ long HM GFRP bars in gradual increments until failure occurs. The applied load was measured by a load cell, while the slip between the GFRP bar and concrete was measured through two potentiometers (POTs) placed to record the loaded end-slip (LES) and free-end slip (FES). Figure 2.5.a depicts a schematic diagram of the test setup and location of POTs. While Fig. 2.5.b showed a view of the test setup. The GFRP bar was loaded by gripping the bars in conventional steel wedge friction grips, considering that the bar 
should not fail under transverse pressure (Castro and Carino, 1998) before it reaches to its ultimate tensile strength or pullout failure, whichever comes first.

\subsection{Experimental Results for Ribbed-Surface GFRP Bars}

Experimental results from pullout tests on 15M and 20M ribbed-surface GFRP bars embedded into unconfined UHPFRC showed that all bars failed in pullout failure mode. The pullout failure mode was found to be due to (i) interlaminar shear strength failure between the GFRP bar and its surface, also slippage from the headed-end and (ii) shear-off failure of ribbed-surface. Figure 2.6 shows typical failure for the $15 \mathrm{M}$ ribbed-surface GFRP bar with embedment length of $4 \mathrm{~d}_{\mathrm{b}}$. The straight bar appeared to be slipped off concrete with shearing off of the lugs. When slicing the concrete block as shown in Fig. 2.6.e, the bar ribs appeared to be sheared. Figures 2.7 and 2.8 show the concrete cracks for the $20 \mathrm{M}$ ribbed-surface GFRP bars with embedment lengths of $4 \mathrm{~d}_{\mathrm{b}}$ and $6 \mathrm{~d}_{\mathrm{b}}$. One may observe that while the primary failure of the bars were pullout from concrete, signs of splitting cracks appeared. Figure 2.9 shows the bar slippage failure for the $15 \mathrm{M}$ ribbedsurface bar with headed-end. Figure 2.9.d shows view of the two sides of the concrete block after slicing it through bar location. Figure 2.9.e shows close-up view of the bar slipped from its head at failure.

Table 2.6 summaries the pullout test results for the 15M ribbed-surface GFRP bars with central bar location. The specimen designation shown in the first column of this table show the parameters involved in each test. The first symbol "C" represents the concrete block and bar located centrally. The first number " 16 " represents the bar size, while the net figure " $4 \mathrm{~d}$ " , " $6 \mathrm{~d}$ " or " $8 \mathrm{~d}$ " represents the embedment length as a function of the bar diameter. The last symbol "S1 through S5" represents each identical sample of the 5 samples in a group. As for the headed-end bar, the set of identical samples in a group is identified as $\mathrm{H} 1$ through $\mathrm{H} 5$. It should be noted that for headed bars, the total development length is taken as the anchorage head length of $100 \mathrm{~mm}$ plus the length of the straight portion of the bonded length of the bar before the head, denoted as $\mathrm{L}_{\mathrm{e} 2}$ in Fig. 2.3. In the last group of specimens in Table 2.6, $\mathrm{L}_{\mathrm{e} 2}$ is taken as zero, thus the third symbol is the specimen designation is taken as " $0 \mathrm{~d}$ ".

Results in Table 2.6 show that the $15 \mathrm{M}$ straight bar with centrically-loaded specimens and with $l_{e} / d_{b}$ series equal to 4,6 and 8 exhibits average pullout load, $P_{u l t}$, of $94.2,59.8$ and $74.6 \mathrm{kN}$, 
respectively. The corresponding average critical load-end slip (LES) was found to be 14.028, 17.446 and $25.574 \mathrm{~mm}$ while the critical values for the free-end slip (FES) was found to be $0.228,0.232$ and $0.356 \mathrm{~mm}$ for the same series arrangement, respectively. The $15 \mathrm{M}$ headed-end bar with centrically-loaded specimen recorded pullout load, LES and FES as of $122.2 \mathrm{kN}, 14.8$ $\mathrm{mm}$ and $0.714 \mathrm{~mm}$, respectively. One may observe that the pullout capacity of the $100-\mathrm{mm}$ length headed-end bar of $122.2 \mathrm{kN}$ is far greater that the pullout capacity of similar straight-end bar of $59.80 \mathrm{kN}$ for $96 \mathrm{~mm}$ embedment length and $74.60 \mathrm{kN}$ for $128 \mathrm{~mm}$ embedment length.

Results in Table 2.7 show that the 15M straight bar with $40 \mathrm{~mm}$ eccentrically-loaded specimens. The specimen designation shown in the first column of the table is identical to those in Table 2.6 for centrally-loaded specimens except that the first two symbols "E40" represent the eccentric bar with $40 \mathrm{~mm}$ concrete cover. One may observe that the eccentrically loaded specimens with $l_{e} / d_{b}$ series equal to 4,6 and 8 exhibit average pullout load, $P_{u l t}$, of 97, 72.6 and $85.2 \mathrm{kN}$, respectively. The corresponding average critical load-end slip was found to be 16.00, 23.432 and $23.486 \mathrm{~mm}$ while the critical values for the free-end slip was found to be $0.416,0.36 \mathrm{~mm}$ and $0.486 \mathrm{~mm}$ for the same series arrangement, respectively. The $15 \mathrm{M}$ headed-end bar recorded pullout load, LES, FES of $122 \mathrm{kN}, 17.028 \mathrm{~mm}$ and $0.48 \mathrm{~mm}$ for $l_{e} / d_{b}$ equal 6.25 , respectively.

Results in Table 2.8 show that the $15 \mathrm{M}$ straight bars with $60 \mathrm{~mm}$ concrete cover in an eccentrically-loaded specimens with $\mathrm{l}_{\mathrm{e}} / \mathrm{d}_{\mathrm{b}}$ series equal to 4,6 and 8 exhibit average pullout load, $\mathrm{P}_{\mathrm{ult}}$, of 51.8, 64.4 and $81.2 \mathrm{kN}$, respectively. The corresponding average critical load-end slip was found to be $17.928,16.542$ and $26.428 \mathrm{~mm}$ while the critical values for the free-end slip was found to be $0.238,0.282$ and $0.856 \mathrm{~mm}$ for $\mathrm{l}_{\mathrm{e}} / \mathrm{d}_{\mathrm{b}}$ series equal to 4,6 and 8 , respectively.

Table 2.9 shows a summary of pullout test results for the 20M GFRP bars in a centrically-loaded specimen. The $20 \mathrm{M}$ straight bars with $\mathrm{l}_{\mathrm{e}} / \mathrm{d}_{\mathrm{b}}$ series equal to 4,6 and 8 exhibit average pullout load, $\mathrm{P}_{\mathrm{ult}}$, of 59.4, 115.4 and $127 \mathrm{kN}$, respectively. The corresponding average critical load-end slip was found to be $15.492,18.966$ and $27.614 \mathrm{~mm}$ while the critical values for the free-end slip was found to be $0.29,0.784$ and $0.274 \mathrm{~mm}$ for the same series arrangement, respectively.

Table 2.10 summarizes the pullout test results for the $20 \mathrm{M}$ ribbed-surface bars with $40 \mathrm{~mm}$ eccentric bar location. The $20 \mathrm{M}$ straight bar with $40 \mathrm{~mm}$ eccentric loaded specimens and with 
$\mathrm{l}_{\mathrm{e}} / \mathrm{d}_{\mathrm{b}}$ series equal to 8 exhibits average pullout load, LES and FES as $117.4 \mathrm{kN}, 23.278 \mathrm{~mm}$ and $0.296 \mathrm{~mm}$, respectively, with signs of hair cracks on the concrete block.

Figures 2.10, 2.11, 2.12 and 2.13 depict the relationship of the pullout load, normalized bond stress, combined bond coefficients, and bar stress ratio versus the development length-to-bar diameter, $\mathrm{l}_{\mathrm{e}} / \mathrm{d}_{\mathrm{b}}$, respectively. It should be noted that the normalized bond strength is taken as the ratio between the bond strength and square root of the compressive strength of UHPFRC $\left(\mathrm{u} / \sqrt{f_{c}^{\prime}}\right)$. In this research the normalized concrete compressive strength is considered to account for its variation, $\sqrt{f_{c}^{\prime}}$. One may observe that the pullout load versus the embedment length-tonominal bar diameter ratio $\left(l_{e} / d_{b}\right)$ was found to be quadratic polynomial of the second degree, with parabola opens upwards. The vertex of the parabola, also called the turning point of such curve, is located at the mid points of the $l_{e} / d_{b}$ series of 4,6 , and 8 considered in this study. Unlike the polynomial behaviour of the $15 \mathrm{M}$ bars, the $20 \mathrm{M}$ bars follows downward parabola with turning point at $\mathrm{l}_{\mathrm{e}} / \mathrm{d}_{\mathrm{b}}$ equals to 6 with minor hair cracks observed into the concrete blocks at failure. Figures 2.14.a and 2.14.b depict the comparison between the predicted and experimentally measured embedment-to-diameter ratio based on Equation 2.12, and the pullout load based on Equation 2.21.

Tables 2.11 summarizes the experimental data and the developed polynomial modeling of the pullout load as a function of the actual displacement of the bar. Table 2.12 shows the values of the bond factor, $\mathrm{B}$, and the bar stress ratio, $\varphi$, for ribbed-surface bars of different configurations. Tables 2.13, 2.14, 2.15 and 2.16 summarize the pullout load, the normalized bond stress, the concrete and reinforcement location factor (Bond factor) and the reinforcement location factor (stress factor) as a function of $\mathrm{l}_{\mathrm{e}} / \mathrm{d}_{\mathrm{b}}$, respectively. Finally, Table 2.17 shows the ACI pullout basic coefficients for the ribbed-surface bars that will be discussed in the analytical section.

\subsection{Experimental Results for Sand-Coated GFRP bars}

Experimental results from pullout tests on 15M and 20M sand-coated GFRP bars embedded into unconfined UHPFRC showed that all bars failed in pullout failure mode. Figure 2.15 shows views of the pullout failure of the $15 \mathrm{M}$ sand-coated bar due to interlaminar shear failure of the sand coating as well as shearing-off failure of the sand coating from bar surface. Figure 2.16 
shows similar failure mode for the $15 \mathrm{M}$ sand-coated bars with 40 and $60 \mathrm{~mm}$ concrete cover. In addition, Fig. 2.15.e shows peeling of the sand-coating at the bar end. Figure 2.17 depicts the pullout failure of the $20 \mathrm{M}$ sand-coated bar as a result of interlaminar shear failure of the sand coating and shearing-off the sand-coating form the bar surface. Similar reasons for failure appeared in blocks with 20M sand-coated GFRP bar with 40 and $60 \mathrm{~mm}$ concrete are depicted in Fig. 2.18. Figure 2.19 shows typical failure of the 15M sand-coated, headed-end, GFRP bar with different concrete cover due to slippage of the bar from the anchorage head as a result of shear failure along the grooves at the interface between the bar and the anchorage inner surface. Figure 2.20. Typical failure of the 20M sand-coated, headed-end, GFRP bar with different concrete covers (i.e. central bar and bar with 40 and $60 \mathrm{~mm}$ concrete covers). After failure, each of these blocks were sliced at the bar head location to show the relative movement between the bar and the head. It can be observed that the primary reason of bar pullout is the slippage of the bar from the anchorage head as a result of shear failure along the grooves at the interface between the bar and the anchorage inner surface. Signed of splitting cracks appeared, as depicted in Fig. 2.20.a for central bar and Fig. 2.20.f for bar with $40 \mathrm{~mm}$ concrete cover. However, this was not the primary case of bar pullout.

Table 2.19 summaries the pullout test results for the 15M sand-coated GFRP bars with central bar location. The specimen designation shown in the first column of this table show the parameters involved in each test. The first symbol " $\mathrm{C}$ " represents the concrete block and bar located centrally. The first number " 16 " represents the bar size, while the net figure " $4 \mathrm{~d}$ " , " $6 \mathrm{~d}$ " or " $8 \mathrm{~d}$ " represents the embedment length as a function of the bar diameter. The last symbol "S1 through S5" represents each identical sample of the 5 samples in a group. As for the headed-end bar, the set of identical samples in a group is identified as H1 through H5. It should be noted that for headed bars, the total development length is taken as the anchorage head length of $100 \mathrm{~mm}$ plus the length of the straight portion of the bonded length of the bar before the head, denoted as $\mathrm{L}_{\mathrm{e} 2}$ in Fig. 2.3. In the last group of specimens in Table 2.18, $\mathrm{L}_{\mathrm{e} 2}$ is taken as zero, thus the third symbol is the specimen designation is taken as " $0 \mathrm{~d}$ ".

Results in Table 2.18 show that the $15 \mathrm{M}$ straight bar with centrically-loaded specimens and with $l_{e} / d_{b}$ series equal to 4,6 and 8 exhibits average pullout load, $P_{u l t}$, of $68.3,53.9$ and $108.8 \mathrm{kN}$, respectively. The corresponding average critical load-end slip (LES) was found to be 13.656, 
12.74 and $16.298 \mathrm{~mm}$ while the critical values for the free-end slip (FES) was found to be 0.834 , 0.33 and $0.76 \mathrm{~mm}$ for the same series arrangement, respectively. The $15 \mathrm{M}$ headed-end bar with centrically-loaded specimen recorded pullout load, LES and FES as of $116.6 \mathrm{kN}, 19.4 \mathrm{~mm}$ and $0.0 \mathrm{~mm}$, respectively. One may observe that the pullout capacity of the $100-\mathrm{mm}$ length headedend bar of $116.6 \mathrm{kN}$ is far greater that the pullout capacity of similar straight-end bar of $53.9 \mathrm{kN}$ for $96 \mathrm{~mm}$ embedment length and $108.8 \mathrm{kN}$ for $128 \mathrm{~mm}$ embedment length.

Results in Table 2.19 show that the $15 \mathrm{M}$ straight bar with $40 \mathrm{~mm}$ eccentrically-loaded specimens. The specimen designation shown in the first column of the table is identical to those in Table 2.18 for centrally-loaded specimens except that the first two symbols "E40" represent the eccentric bar with $40 \mathrm{~mm}$ concrete cover. One may observe that the eccentrically loaded specimens with $l_{e} / d_{b}$ series equal to 4,6 and 8 exhibit average pullout load, $P_{u l t}$, of 43.7, 73.25 and $149.15 \mathrm{kN}$, respectively. The corresponding average critical load-end slip was found to be 20.95, 12.22 and $20.74 \mathrm{~mm}$ while the critical values for the free-end slip was found to be 0.192 , $1.283 \mathrm{~mm}$ and $0.744 \mathrm{~mm}$ for the same series arrangement, respectively. The $15 \mathrm{M}$ headed-end bar with $40 \mathrm{~mm}$ concrete cover recorded pullout load, LES, FES of $140 \mathrm{kN}, 26.4 \mathrm{~mm}$ and $0.2 \mathrm{~mm}$ for $l_{e} / d_{b}$ equal 6.25 , respectively.

Results in Table 2.20 show that the $15 \mathrm{M}$ straight bars with $60 \mathrm{~mm}$ concrete cover in an eccentrically-loaded specimens with $l_{e} / d_{b}$ series equal to 4,6 and 8 exhibit average pullout load, $\mathrm{P}_{\text {ult }}$, of 40.9, 67.45 and $149.4 \mathrm{kN}$, respectively. The corresponding average critical load-end slip was found to be $20.49,26.09$ and $24.65 \mathrm{~mm}$ while the critical values for the free-end slip was found to be $0.352,0.706$ and $0.318 \mathrm{~mm}$ for $\mathrm{l}_{\mathrm{e}} / \mathrm{d}_{\mathrm{b}}$ series equal to 4,6 and 8 , respectively. The 15M headed-end bar with $60 \mathrm{~mm}$ concrete cover recorded pullout load, LES, FES of 142.05 $\mathrm{kN}, 28.6 \mathrm{~mm}$ and $1.23 \mathrm{~mm}$ for $l_{e} / d_{b}$ equal 6.25 , respectively.

Table 2.21 shows a summary of pullout test results for the 20M sand-coated GFRP bars in a centrically-loaded specimen. The $20 \mathrm{M}$ straight bars with $\mathrm{l}_{\mathrm{e}} / \mathrm{d}_{\mathrm{b}}$ series equal to 6 and 8 exhibit average pullout load, $\mathrm{P}_{\mathrm{ult}}$, of 77.7 and $85.25 \mathrm{kN}$, respectively. The corresponding average critical load-end slip was found to be 16.28 and $27.62 \mathrm{~mm}$ while the critical values for the free-end slip was found to be 0.226 and $0.772 \mathrm{~mm}$ for the same series arrangement, respectively. The $20 \mathrm{M}$ headed-end bar with centrally-loaded concrete block recorded pullout load, LES, FES of 218.35 $\mathrm{kN}, 27.192 \mathrm{~mm}$ and $0.744 \mathrm{~mm}$ for $l_{e} / d_{b}$ equal 5 , respectively. 
Table 2.22 shows a summary of pullout test results for the 20M sand-coated GFRP bars in an eccentrically-loaded specimen with $40 \mathrm{~mm}$ concrete cover to the bar. The 20M straight bars with $\mathrm{l}_{\mathrm{e}} / \mathrm{d}_{\mathrm{b}}$ series equal to 4, 6 and 8 exhibit average pullout load, $\mathrm{P}_{\mathrm{ult}}$, of 54.05, 68.05 and $165.25 \mathrm{kN}$, respectively. The corresponding average critical load-end slip was found to be $24.53,21.31$ and $26.08 \mathrm{~mm}$ while the critical values for the free-end slip was found to be $0.73,0.396$ and 0.826 $\mathrm{mm}$ for the same series arrangement, respectively. The $20 \mathrm{M}$ headed-end bar with $40 \mathrm{~mm}$ concrete cover in an eccentrically-loaded concrete block recorded pullout load, LES, FES of 247 $\mathrm{kN}, 27 \mathrm{~mm}$ and $0.0 \mathrm{~mm}$ for $l_{e} / d_{b}$ equal 5, respectively.

Table 2.23 summarizes the pullout test results for the $20 \mathrm{M}$ sand-coated bars with $60 \mathrm{~mm}$ eccentric bar location. The 20M straight bar with $60 \mathrm{~mm}$ eccentric loaded specimens and with $\mathrm{l}_{\mathrm{e}} / \mathrm{d}_{\mathrm{b}}$ series equal to 4 exhibits average pullout load, LES and FES as $71.85 \mathrm{kN}, 33.34 \mathrm{~mm}$ and $1.062 \mathrm{~mm}$, respectively, with signs of hair cracks on the concrete block.

Figures 2.21, 2.22, 2.23 and 2.24 depict the relationship of the pullout load, normalized bond stress, combined bond coefficients, and bar stress ratio versus the development length-to-bar diameter, $\mathrm{l}_{\mathrm{e}} / \mathrm{d}_{\mathrm{b}}$, respectively. It should be noted that the normalized bond strength is taken as the ratio between the bond strength and square root of the compressive strength of UHPFRC $\left(\mathrm{u} / \sqrt{f_{c}^{\prime}}\right)$. In this research the normalized concrete compressive strength is considered to account for its variation, $\sqrt{f_{c}^{\prime}}$. One may observe that the pullout load versus the embedment length-tonominal bar diameter ratio $\left(l_{e} / d_{b}\right)$ was found to be quadratic polynomial of the second degree, with parabola opens upwards. The vertex of the parabola, also called the turning point of such curve, is located at the mid points of the $l_{e} / d_{b}$ series of 4,6 , and 8 considered in this study. Unlike the polynomial behaviour of the $15 \mathrm{M}$ bars, the $20 \mathrm{M}$ bars follows downward parabola with turning point at $\mathrm{l}_{\mathrm{e}} / \mathrm{d}_{\mathrm{b}}$ equals to 6 with minor hair cracks observed into the concrete blocks at failure. Figures 2.25.a and 2.25.b depict the comparison of the predicted and experimentally measured embedment-to-diameter ratio based on Equation 2.12, and the pullout load based on Equation 2.21.

Table 2.24 summarizes the experimental data and the developed polynomial modeling of the pullout load as a function of the actual displacement of the bar. Table 2.25 shows the values of the bond factor, $\mathrm{B}$, and the bar stress ratio, $\varphi$, for sand-coated bars of different configurations. 
Tables 2.26, 2.27, 2.28 and 2.29 summarize the pullout load, the normalized bond stress, the concrete and reinforcement location factor (Bond factor) and the reinforcement location factor (stress factor) as a function of $\mathrm{l}_{\mathrm{e}} / \mathrm{d}_{\mathrm{b}}$, respectively. Finally, Table 2.30 shows the ACI pullout basic coefficients for the ribbed-surface bars that will be discussed in the analytical section.

\subsection{Analytical Study of GFRP Bar Bond Strength and Development Length}

Data from the pullout tests were analyzed to develop design guidelines for anchorage of straight and headed-end GFRP bars with ribbed-surface or sand-coated surface. The pullout load values, load-end slip (LES) and free-end slip (FES) were measured at each monotonic static loading level. Bond stress, $u$, is defined as the shear force per unit surface area of the rebar as per Equation 2.6.

$$
u=\frac{P_{u l t}}{\pi d_{b} l_{e}}
$$

Where $P_{u l t}$ is the ultimate applied tensile force, $d_{b}$ is the bar diameter, $l_{e}$ is the embedment length. Due to the unbonded length and low modulus of elasticity, the elastic elongation of the unbonded length portion the GFRP bar should be deducted from the measured slip values. Equation 2.7 derivew the actual slip, $\delta_{\text {Actual }}$, as the difference between the slip at the load end, $\delta_{L E S}$, and the slip at the free end, $\delta_{F E S}$. The value of the loaded-end slip is greater than the freeended slip, i.e. $\delta_{L E S} \gg \delta_{F E S}$, due to the absence of the concrete flexural cracks in pullout blocks that generates higher ultimate bond stress and greater slip at the FES.

$$
\delta_{\text {Actual }}=\delta_{L E S}-\delta_{F E S}
$$

Concrete cover has significant effect on the bond failure of the GFRP bars. The concrete cover, $c_{\min }=d_{b}$, causes side concrete cracks and/or spitting cracks, while with the increase of concrete cover, $c_{\min } \geq 2 d_{b}$, pullout failure or bar rupture is dominant. Due to the change of the embedment length, it was necessary to calculate the ratio the experimental bar stress to the ultimate tensile strength of the GFRP bar, $\varphi$, per Equations 2.8 and 2.9. Equation 2.10 is proposed to predict the experimental bond factor, $k$. Equation 2.10 is rearranged into Equation 2.11 to determine the experimental embedment (development) length, $l_{e}$, this is finally presented in Equation 2.12. Figs 2.14.a and 2.25.a depict the comparisons for the predicted and 
experimentally measured embedment length for the ribbed-surface and sand-coated GFRP bars respectively.

$$
\begin{aligned}
& f_{\text {frp,experiment }}=4 \frac{P_{u l t}}{\pi d_{b}^{2}} \\
& \varphi=\frac{f_{\text {frp,experimental }}}{f_{\text {frp,ultimate }}}
\end{aligned}
$$

$$
k=c_{\min } \frac{l_{e}}{d_{b}} \frac{\sqrt{f_{c}^{\prime}}}{\varphi f_{\text {frp, ultimate }}}
$$

$k . \varphi=\frac{l_{e}}{d_{b}} c_{\min } \frac{\sqrt{f_{c}^{\prime}}}{f_{\text {frp }, \text { ultimate }}}$

$$
l_{e}=\frac{k}{c_{\min }} d_{b} \frac{\varphi f_{\text {frp }, \text { ultimate }}}{\sqrt{f_{c}^{\prime}}}
$$

The Nonlinear Regression analysis of the experimental data was performed for the polynomial equation of the second degree in the form shown in Equations 2.13 and 2.15, to obtain its coefficients $a_{i}$ per Equation 2.15. The reliability of the predicted coefficients is determined by the coefficient of determination, $R^{2}$, as shown in Equations 2.16 through 2.19.

$$
\begin{aligned}
& f(x)=Y=\sum_{i=0}^{n} a_{i} X^{i} \text { where } i<n \\
& f(x)=Y=a_{0}+a_{1} X+a_{2} X^{2} \\
& {\left[\begin{array}{ccc}
n & \sum_{i=1}^{n} X_{i} & \sum_{i=1}^{n} X_{i}^{2} \\
\sum_{i=1}^{n} X_{i} & \sum_{i=1}^{n} X_{i}^{2} & \sum_{i=1}^{n} X_{i}^{3} \\
\sum_{i=1}^{n} X_{i}^{2} & \sum_{i=1}^{n} X_{i}^{3} & \sum_{i=1}^{n} X_{i}^{4}
\end{array}\right]\left[\begin{array}{l}
a_{0} \\
a_{1} \\
a_{2}
\end{array}\right]=\left[\begin{array}{c}
\sum_{i=1}^{n} Y_{i} \\
\sum_{i=1}^{n} X_{i} Y_{i} \\
\sum_{i=1}^{n} X_{i}^{2} Y_{i}
\end{array}\right]}
\end{aligned}
$$

(2.16) Mean:

$$
\bar{y}_{\text {observed }}=\frac{1}{n} \sum_{i=1}^{n} y_{i, \text { observed }}
$$

(2.17) Sum of Squared Errors:

$$
S S E=\min \sum_{i=1}^{n}\left[f\left(x_{i}\right)_{\text {predicted }}-y_{i, \text { observed }}\right]^{2}
$$

(2.18) Total Sum of Squares:

$$
S S T=\sum_{i=1}^{n}\left(y_{i, \text { observed }}-\bar{y}_{\text {observed }}\right)^{2}
$$

(2.19) Coefficient of determination: $R^{2}=1-\frac{S S E}{S S T} ; 0.0<R^{2}<1.0$ 
The load-slip $\left(P \sim \delta_{\text {act }}\right)$ relationship can be determined by Equation 2.20. The load versus the embedment length-to-nominal diameter of the bar ratio $\left(P \sim l_{e} / d_{b}\right)$ can be expressed by quadratic polynomial of second degree per Equation 2.21.

$$
\begin{aligned}
& P=a_{2} \delta_{a c t}^{2}+a_{1} \delta_{a c t} \\
& P=a_{2}\left(\frac{l_{e}}{d_{b}}\right)^{2}+a_{1}\left(\frac{l_{e}}{d_{b}}\right)+a_{0}
\end{aligned}
$$

Normalized bond stress that account for the difference of the compressive strength and the embedment length-to-nominal bar diameter ratio $\left(u / \sqrt{f_{c}^{\prime}} \sim l_{e} / d_{b}\right)$ can be expressed by quadratic polynomial of second degree per Equation 2.22.

$$
u / \sqrt{f_{c}^{\prime}}=a_{2}\left(\frac{l_{e}}{d_{b}}\right)^{2}+a_{1}\left(\frac{l_{e}}{d_{b}}\right)+a_{0}
$$

The bond factor, B, considers the combination of the bond factor, $k$ and bar stress ratio, $\varphi$. The first degree of linear polynomial equation can be expressed as shown in Equation 2.23.

$$
B=k . \varphi=a\left(\frac{l_{e}}{d_{b}}\right)
$$

The bar stress ratio versus the embedment length-to-nominal diameter of the bar ratio $\left(\varphi \sim l_{e} / d_{b}\right)$, can be expressed by quadratic polynomial of the second degree per Equation 2.24.

$$
\varphi=a_{2}\left(\frac{l_{e}}{d_{b}}\right)^{2}+a_{1}\left(\frac{l_{e}}{d_{b}}\right)+a_{0}
$$

The ACI approximate method is used to determine the embedment length through Equations 2.25 through 2.28. Equation 2.27 is used to determine the experimental factor, $k$. The ACI assumed that the factor $k_{d, \text { larger value }}>k_{\text {d,experimental, }}$, where this coefficient ensures that it will be larger than all measured embedment lengths and yields conservative development length, $l_{d}$. The modification factor (MF $=1.0$ to 1.25 ) is introduced to represent the effect of the bar location with respect to minimum concrete cover, resulting into increase of the development length up to $125 \%$ (Ehsani et al. 1996).

$$
u \pi d_{b} l_{e}=A_{b} f_{\text {frp,ultimate }}
$$




$$
l_{e}=k_{\text {exp }}\left(A_{b} f_{\text {frp,ultimate }}\right) / \sqrt{f_{c}^{\prime}}
$$

$$
\begin{aligned}
& k_{\text {exp }}=\sqrt{f_{c}^{\prime}} /\left(u \pi d_{b}\right) \quad \text { where } \quad u=\frac{1}{k_{d}} \frac{\sqrt{f_{c}^{\prime}}}{\pi d} \\
& l_{d}=M F \times k_{d}\left(A_{b} f_{\text {frp }, \text { ultimate }}\right) / \sqrt{f_{c}^{\prime}} \\
& k_{d}=\frac{l_{d} \sqrt{f_{c}^{\prime}}}{A_{b} f_{f r p, u}}
\end{aligned}
$$

The CSA A23.3 states that the development length for steel hooked bars or steel headed-end bars in tension should be obtained by multiplying the basic hook / headed development length, $l_{h b}$, by appropriate modification factors (MF) for steel hooked anchorage; that is

$$
l_{h b}=M F \times 100 \frac{d_{b}}{\sqrt{f_{c}^{\prime}}}
$$

The CSA A23.3 Cl.12.5.1 recommended that $l_{h b}$ should not be less than $8 d_{b}$ or $150 \mathrm{~mm}$, whichever is greater (CSA A23.3) for reinforcing steel bars only.

\subsubsection{Analytical Results for Ribbed-Surface GFRP Bars}

The longitudinal HM GFRP/UHPFRC interface is highly influenced by the embedment lengthto-nominal bar diameter ratio $\left(l_{e} / d_{b}\right)$, when the minimum concrete cover is greater than 2 times the bar diameter $\left(c_{\min } \geq 2 d_{b}\right)$. The proposed Equation 2.12 used to determine the embedment length, $l_{e}$, that have highest coefficient of determination close to 1. Equation 2.9 is calculated first to determine the bar stress ratio, $\varphi$, followed by Equation 2.10 to determine the $k$ factor, then substitute the values into Equation 2.12, the concrete cover, $c_{\min }$, is left for designer prediction and / or further parametric studies. For example, $l_{e}=64 \mathrm{~mm}$, when $B=k . \varphi=4.29$ $d_{b}=16 \mathrm{~mm}, \sqrt{f_{c}^{\prime}}=13.84 \mathrm{MPa}, f_{\text {frp,ultimate }}=1188 \mathrm{MPa}$, and $c_{\min }=92 \mathrm{~mm}$. Thus, the proposed equations simulate the experimental behavior with excellent coefficient of determination.

The ACI basic development factor for the straight-ended ribbed-surface HM GFRP bar, $k_{d}$, equals to 0.00743 and 0.00594 for the $15 \mathrm{M}$ and $20 \mathrm{M}$ bars, respectively, as shown in Table 2.31 . 
These $k_{d}$ factors for the same bar arrangement are equivalent to $34 \%$ and $31 \%$ of the bar maximum ultimate strength of $1188 \mathrm{MPa}$ that yields to $403.86 \mathrm{MPa}$ and $373.69 \mathrm{MPa}$ for the 15M and $20 \mathrm{M}$ bars, respectively. Thus, the development length is recommended to be taken as 150 $\mathrm{mm}$ and $190 \mathrm{~mm}$ for the $15 \mathrm{M}$ and $20 \mathrm{M}$ bars, respectively, when embedded into UHPFRC with nominal compressive strength of $140 \mathrm{MPa}$, to ensure that the bar tensile strength will reach first before bar bond failure as depicted in Equations 2.31 through 2.33. The calculations for the $l_{d}$ resulted into minimum $l_{d}$ of 9.5 times the bar diameter for the $15 \mathrm{M}$ and $20 \mathrm{M}$ straight bars.

$$
\begin{aligned}
& l_{d}=M F \times k_{d}\left(A_{b} f_{\text {frp, ultimate }}\right) / \sqrt{f_{c}^{\prime}} \\
& l_{d}=1.0 \times 0.00743(201 \times 1188) / \sqrt{140}=150 \mathrm{~mm} \\
& l_{d}=1.0 \times 0.00594(314 \times 1188) / \sqrt{140}=190 \mathrm{~mm}
\end{aligned}
$$

The ACI basic development factor for the headed-end HM GFRP bar, $k_{h b}$, equals to 0.00547 for the $15 \mathrm{M}$ bar. The $k_{h b}$ factor is equivalent to the $51 \%$ of the bar ultimate tensile strength of 1188 $\mathrm{MPa}$ that yields to $607 \mathrm{MPa}$ before bar slips from the anchorage head. Thus the basic development length for the 15M headed-end bar embedded into UHPFRC with compressive strength of $140 \mathrm{MPa}$ results into $l_{h b}$ equals to $111 \mathrm{~mm}$ as shown in Equation 2.34. The calculations for the $l_{h b}$ resulted into minimum development length of 7 times the bar diameter.

$$
\begin{aligned}
& l_{h b}=0.00547 \frac{A_{b} f_{f r p, u}}{\sqrt{f_{c}^{\prime}}}=82 \frac{d_{b}}{\sqrt{f_{c}^{\prime}}}=111 \mathrm{~mm} \\
& l_{h b}=82 \frac{d_{b}}{\sqrt{f_{c}^{\prime}}}
\end{aligned}
$$

\subsubsection{Analytical Results for Sand-Coated GFRP Bars}

The ACI basic development factor for the straight-ended sand-coated HM GFRP bar, $k_{b}$, equals to 0.00752 and 0.0066 for the $15 \mathrm{M}$ and $20 \mathrm{M}$ bars, respectively, as shown in Table 2.31 . The $k_{b}$ factor is equivalent to $63 \%$ and $48 \%$ for the same arrangement of bars that yields to $743 \mathrm{MPa}$ and $526 \mathrm{MPa}$ for the $15 \mathrm{M}$ and $20 \mathrm{M}$ bars, respectively. Thus, the development length is taken as 150 and $176 \mathrm{~mm}$ for the $15 \mathrm{M}$ and $20 \mathrm{M}$ bars, respectively, when embedded into UHPFRC with 
nominal compressive strength of $140 \mathrm{MPa}$, to ensure that the bar tensile strength will reach first before bar bond failure as depicted in Equations 2.36 through 2.38. The calculations for the development length resulted in minimum $l_{d}$ of 9.5 times for both of the $15 \mathrm{M}$ and $20 \mathrm{M}$ straight bars.

$$
\begin{aligned}
& l_{d}=M F \times k_{d}\left(A_{b} f_{\text {frp }, \text { ultimate }}\right) / \sqrt{f_{c}^{\prime}} \\
& l_{d}=1.0 \times 0.00752(198 \times 1184) / \sqrt{140}=150 \mathrm{~mm} \\
& l_{d}=1.0 \times 0.0066(285 \times 1105) / \sqrt{140}=176 \mathrm{~mm}
\end{aligned}
$$

The ACI basic development factor for the headed-end HM GFRP bar, $k_{h b}$, equals to 0.00552 for the $15 \mathrm{M}$ bars and has a tensile stress of $49 \%$ of the ultimate tensile strength of $1184 \mathrm{MPa}$ that yields to $580 \mathrm{MPa}$ before bar slips from the anchorage head. Thus, the basic development length for the $15 \mathrm{M}$ headed-end bar embedded into UHPFRC with compressive strength of $140 \mathrm{MPa}$ results in $l_{h b}$ equals to $110 \mathrm{~mm}$ as presented in Equations 2.39 and 2.40 .

$$
\begin{aligned}
& l_{h b}=0.00552 \frac{A_{b} f_{f r p, u}}{\sqrt{f_{c}^{\prime}}}=0.00552 \frac{\pi d_{b}}{4} f_{f r p, u} \frac{d_{b}}{\sqrt{f_{c}^{\prime}}}=82 \frac{d_{b}}{\sqrt{f_{c}^{\prime}}}=110 \mathrm{~mm} \\
& l_{h b}=82 \frac{d_{b}}{\sqrt{f_{c}^{\prime}}}
\end{aligned}
$$

The ACI basic development factor for the headed-end HM GFRP bar, $k_{d}$, equals to 0.00428 for $20 \mathrm{M}$ bars, and has tensile stress at bond failure of $63 \%$ of the ultimate tensile strength of 1105 MPa that yields to $695 \mathrm{MPa}$ before bar slips from the anchorage head. Thus, the basic development for the 20M headed-end bar embedded into UHPFRC with compressive strength of $140 \mathrm{MPa}$ results in $l_{h b}$ equals to $114 \mathrm{~mm}$ per equation 2.42 .

$$
\begin{aligned}
& l_{h b}=0.00428 \frac{A_{b} f_{f r p, u}}{\sqrt{f_{c}^{\prime}}}=71 \frac{d_{b}}{\sqrt{f_{c}^{\prime}}}=114 \mathrm{~mm} \\
& l_{h b}=71 \frac{d_{b}}{\sqrt{f_{c}^{\prime}}}
\end{aligned}
$$


Equation 2.43 can be used to represent the development length $l_{h b}$ for the headed-end bars by defining the $k_{h b}$ to be 82 and 71 for the $15 \mathrm{M}$ and $20 \mathrm{M}$ headed-end bars. These calculations resulted into minimum development length not less than 7 times and 6 times the bar diameter for the $15 \mathrm{M}$ and $20 \mathrm{M}$ headed-end bars, respectively. Table 2.31 summarizes the findings with respect to the proposed development length of studies GFRP bar types when embedded into UHPFRC.

\subsubsection{Development of Modification Factor for Bond Strength Based on Concrete Cover}

The modification factor (MF) is the relationship between the relative normalized bond strength $u / \sqrt{f_{c}^{\prime}}$ obtained from the pullout tests based on bar locations within the UHPFRC blocks (i.e. different concrete cover, $\left.c_{\min } / d_{b}\right)$. MF should be multiplied by the development length $\left(l_{d}\right)$ calculated from previous equations to account for bar location.

The straight 15M ribbed-surface HM GFRP bar has normalized bond stress of 2.12, 1.16, 2.18 at minimum concrete cover of 92, 60 and $40 \mathrm{~mm}$, respectively, as shown in Table 2.12. The modification factor is found to be 1 to 1.03 . The straight 20M ribbed-surface HM GFRP has normalized bond stress of 1.11 and 0.84 at minimum concrete cover of 90 and $40 \mathrm{~mm}$, respectively, where the MF is found to be 1.31. The headed-end 15M ribbed-surface HM GFRP bar has normalized bond stress of 1.87 and 1.86 for the minimum of concrete cover of 142 and $40 \mathrm{~mm}$, respectively, where the MF is found to be 1 .

The straight 15M sand-coated HM GFRP bar has normalized bond stress of 1.64, 1.69 and 1.69 at minimum concrete cover of 92,60 , and $40 \mathrm{~mm}$, respectively, as shown in Table 2.25 . The modification fact is found to be 1.0,1.026 and 1.027 for the same concrete cover arrangement. The straight 20M sand-coated HM GFRP bar has normalized bond stress of 0.80 and 1.26 for minimum concrete cover of 90 and $40 \mathrm{~mm}$, respectively. The MF was found to be 1.00 and 1.58 for the same arrangement of the concrete covers. The headed end 15M sand-coated HM GFRP bars has normalized bond stress of 1.79, 2.17 and 2.15 for the minimum concrete cover of 142, 60 and $40 \mathrm{~mm}$. The MF was found to be 1.0, 1.21 and 1.20, respectively. The headed-end 20M sand-coated HM GFRP bars has normalized bond stress of 2.58 and 2.75 and minimum concrete cover 140 and $40 \mathrm{~mm}$. The MF was found to be 1.0 and 1.065 , respectively. 
In summary, the Modification Factor (MF) is affected by the bar/concrete interface, bar diameter and bar location with respect to the concrete cover. The $\mathrm{MF}$ for the $15 \mathrm{M}$ and $20 \mathrm{M}$ straight ribbed-surface bar has a range of 1 to 1.03 and 1 to 1.31 for two bar sizes, respectively. The MF for the $15 \mathrm{M}$ ribbed-surface headed-end equals to 1.0 . As for the $15 \mathrm{M}$ and $20 \mathrm{M}$ sand-coated bars have range of MF as 1 to 1.027 and 1 to 1.58 for the same bar types, respectively. The $15 \mathrm{M}$ and 20M sand-coated headed-end bars have range of MF as 1 to 1.21 and 1 to 1.065 for the same bar types, respectively. Beam analysis should be conducted to adjust these values which were taken in the range of 1 to 1.25 on previous research (Ehsani, et al., 1996).

\subsection{Recommendations for Minimum GFRP Development Length}

Table 2.31 provides the design recommendations for the ribbed-surface and sand-coated HM GFRP bars embedded into UHPFRC. The development length of the straight bars should not be less than $9.5 d_{b}$ or $150 \mathrm{~mm}$, whichever is greater. The development length of the headed-end bar should not be less than $7 \mathrm{~d}_{\mathrm{b}}$ or $111 \mathrm{~mm}$, whichever is greater. Table 2.31 shows the ACI basic coefficient, $\mathrm{k}$, for future use by code writers.

\subsection{Conclusions}

Based on the experimental observations and theoretical analysis of the pullout test results, the following conclusions were drawn:

1. The allowable slips at loaded-end and free-end of the HM GFRP bars should be limited with acceptable range as bar fails suddenly due to interlaminar shear strength (ILSS) failure of the GFRP bar, with shear failure of the ribbed-surface or sand coated surface for the straight bars and bar slippage from the anchor headed bars.

2. The ACI basic coefficients were able to predict reliable development lengths.

3. For straight HM GFRP bars embedded into UHPFRC, it is recommended to use minimum development length of 9.5 times the bar diameter or $152 \mathrm{~mm}$, whichever is greater, to ensure bar reaching its tensile strength before anchorage failure.

4. For headed-end HM GFRP bars embedded into UHPFRC, it is recommended to use minimum development length of 7 times the bar diameter or $111 \mathrm{~mm}$, whichever is greater, to ensure bar reaching its tensile strength before anchorage failure. 
5. The developed equations for the embedment length and pullout load given into Equations 2.12 and 2.21 respectively were able to predict the experimental values with $\mathrm{R}^{2}$ equals to $100 \%$.

\section{References}

Achillides, Z., and Pilakoutas, K. 2004. Bond Behavior of Fiber Reinforced Polymer Bars under Direct Pullout Conditions. ASCE Journal for Composites in Construction, 8(2): 173-181.

ACI 440R. 1996. State of the Art Report: FRP Reinforcement, 440R-32, Detroit, MI: American Concrete Institute.

ACI Committee 408. 2003. Bond and Development of Straight Reinforcing Bars in Tension, ACI 408R-03, USA: American Concrete Institute.

ACI 318-15. 2015. Building Code Requirements for Reinforced Concrete, Detroit, MI: American Concrete Institute.

Banson, A. K. 2013. Precast Bridge Deck Joins using FRP and Ultra High Perfromance Concrete. Master's Thesis, Western University, London, Ontario, Canada.

Benmokrane, B., Tighiouart, B., and Chaallal, O. 1996. Bond Strength and Load Distribution of Composite GFRP Reinforcing Bars in Concrete. ACI Materials Journal, pp. 246-253.

Castro, P. F., and Carino, N. J. 1998. Tensile and Nondestructive Testing of FRP Bars. Journal of Composite for Construction, 2(1): 17-27.

CSA A23.2. 2004. Design of Concrete Structures, CSA A23.3-04. Canadian Standard Association, Mississauga, ON, Canada.

Ehsani, M. R., Saadatmanesh, H., and Tao, S. 1996. Design Recommendations for Bond of GFRP Rebars to Concrete. Journal of Structural Engineering, 122(3): 247-254.

Faza, S. S., and GangaRao, H. V. S. 1990. Bending and Bond Behavior of Concrete Beams Reinforced with Plastic Rebars. Transportation Research Record, 1290, pp. 185-193. 
Fehling, E., Lorenz, P., and Leutbecher, T. 2012. Experimental Investigations on Anchorage of Rebars in UHPC. Kassel,Germany, Proceedings of Hipermat 2012 3rd International Symposium on UHPC and Nanotechnology for High Performance Construction Materials.

Firas, S. A., Gilles, F., and Robert, L. R. 2011. Bond Between Carbon Fibre-reinforced Polymer (CFRP) Bars and Ultra-High Performance Fibre Reinforced Concrete (UHPFRC): Experimental study. Construction and Building Materials, 25: 479-485.

Graybeal, B. A. 2006. Material Property Characterization of Ultra-High Performance Concrete. Publication No. FHWA-HRT-06-103., Mclean, VA.: U.S. Department of Transportation. Federal Highway Administration.

Graybeal, B. A. 2007. Compressive Behaviour of Ultra-High-Performance-Fibre-Reinforced Concrete. ACI Materials Journal , 104(2): 146-152.

Graybeal, B., March 2011. Ultra-High Performance Concrete, FHWA-HRT-11-038, Federal Highway Administration: U.S. Department of Transportation,.

Hanus, J., Shield, C. \& French, C. W., 2000. Development Length of GFRP Reinforcement in Concrete Bridge Decks, Minneapolis, MN: University of Minnesota.

Hossain, K. M., Ametrano, D., and Lachemi, M. 2012. Bond Strength of Standard and High Modulus GFRP Bars in High Strength Concrete. Journal of Materials in Civil Engineering, 26(3): 449-456.

Islam, S., Afefy, H. M., Sennah, K., and Azimi, H. 2015. Bond Characteristics of Straight- and Headed-End, Ribbed-surface, GFRP Bars Embedded in High-Strength Concrete. Construction and Building Materials, 83: 283-298.

Khederzadeh, H. R., and Sennah, K. 2013. Pullout Strength of Pre-installed GFRP Bars in Concrete. Proceedings of the the 2013 CSCE Annual General Conference, Canadian Society for Civil Engineering, Montreal, Quebec, pp. 1-10.

Lafarge Canada Inc., 2009. Ductal Joint Fill JS1000. [Online]

Available at: www.ductal.com/JS1000_2009.pdf 
[Accessed 15 September 2011].

Mak, C. 2011. Experimental and Theoretical Investigation of Glass Fibre Reinforced Polymer Tension Lap Splices in Ultra High Performance Concrete, M.A.Sc. Thesis, Civil Engineering Department, Ryerson University, Toronto, Ontario, Canada.

Saleem , M. A., Mirmiran, A., Xia, J., and Mackie, K. 2013. Development Length of HighStrength Steel Rebar in Ultra-High Performance Concrete. Journal of Materials in Civil Engineering, 25: 991-998.

Schoeck Canada Inc. 2011. ComBar Technical Information. [Online] Available at: www.schoeck-canada.com [Accessed Dec 2011].

Swenty, M., and Graybeal, B.. 2012. Influence of Differential Deflection on Staged Construction Deck-Level Connections, Report No. FHWAHRT-12-057, s.l.: National Technical Information Service Accession No. PB2012-111528.

Tropynina, E. 2012. Structural Design Issues on GFRP-Reinforced Concrete Bridge Barriers, M.A.Sc. Thesis, Civil Engineering Department, Ryerson University, Toronto, Ontario, Canada.

VROD - Pultrall, 2013. Pultral V-Rod Standard. [Online]

Available at: www.pultrall.com/dbfiles/Document/722201382006AMDOCen40.pdf

[Accessed 15 September 2011].

Wang, C.-K., and Salmon, C. G. 2002. Reinforced Concrete Design, Sixth Edition. USA: John Wiley \& Sons, Inc.

Yoo, D.-Y., Kwon, K.-Y., Park, J.-J., and Yoon, Y.-S. 2015. Local Bond-Slip Response of GFRP Rebar in Ultra-High Performance Fiber-Reinforced Concrete. Composite Structures, 120: 53-64.

Yuan, J., and Graybeal, B. 2014. Bond Behavior of Reinforcing Steel in Ultra-High Performance Concrete, FHWA Publication No. FHWA-HRT-14-090, McLean, VA: Office of Infrastructure Research and Development. 
Table 2.1. Pullout test matrix

\begin{tabular}{ccccccccc}
\hline \multirow{2}{*}{ Bar Location } & $\mathrm{d}_{\mathrm{b}}$, & \multicolumn{3}{c}{$\mathrm{L}_{\mathrm{e}}{ }^{*}, \mathrm{~mm}$} & \multicolumn{2}{c}{$\mathrm{L}_{\mathrm{e} 2}{ }^{*}, \mathrm{~mm}$} & Head length, & Remarks \\
& $\mathrm{mm}$ & $4 \mathrm{~d}_{\mathrm{b}}$ & $6 \mathrm{~d}_{\mathrm{b}}$ & $8 \mathrm{~d}_{\mathrm{b}}$ & 0 & $4 \mathrm{~d}_{\mathrm{b}}$ & $\mathrm{mm}$ & \\
\hline Centric & 16 & 64 & 96 & 128 & 0 & 64 & 100 & 5 specimens \\
& 20 & 80 & 120 & 160 & 0 & 80 & 100 & 5 specimens \\
\hline Eccentric with 40 & 16 & 64 & 96 & 128 & 0 & 64 & 100 & 5 specimens \\
mm concrete cover & 20 & 80 & 120 & 160 & 0 & 80 & 100 & 5 specimens \\
\hline $\begin{array}{c}\text { Eccentric with 60 } \\
\text { mm concrete cover }\end{array}$ & 16 & 64 & 96 & 128 & 0 & 64 & 100 & 5 specimens \\
\hline * See Figure 3 for definitions of $\mathrm{L}_{\mathrm{e} 1}$ and $\mathrm{L}_{\mathrm{e} 2}$ & & & & & & \\
\hline
\end{tabular}

Table 2.2 Proportion of UHPFRC (Ductal) constituent materials

\begin{tabular}{ccc}
\hline Constituent materials & Percentage by weight (\%) & Weight relative to cement \\
\hline Portland Cement & 28.6 & 1.00 \\
Silica fume & 9.3 & 0.33 \\
Ground quartz & 8.5 & 0.30 \\
Fine sand & 41.1 & 1.44 \\
Steel Fiber Reinforcement & 6.4 & 0.22 \\
(High Range Water Reducer, HRWR) & 0.5 & 0.02 \\
Water & 5.6 & 0.20 \\
\hline Total & 100 & \\
\hline
\end{tabular}

Note: Data from http://www.lafargnorthamerica.com (May, 2008).

Table 2.3. UHPFRC (Ductal JS1000) physical properties (Lafarge Canada Inc., 2009)

\begin{tabular}{lccc}
\hline & \multicolumn{2}{c}{ Characteristic values for design } & Design value \\
\cline { 2 - 3 } & Mean & Standard Deviation & \\
\cline { 2 - 3 } & $\mathrm{MPa}$ & $\mathrm{MPa}$ & $\mathrm{MPa}$ \\
\hline Compression & 140 & 5 & 100 \\
Flexural & 30 & 1 & -- \\
Direct tension & 8 & $2 \mathrm{GPa}$ & $45 \mathrm{GPa}$ \\
\hline Young's modulus & $50 \mathrm{GPa}$ & & \\
\hline
\end{tabular}


Table 2.4. Material properties 15M and 20M straight ribbed-surface GFRP bar (Schoeck Canada, 2013)

\begin{tabular}{lccccccc}
\hline $\begin{array}{c}\text { Nominal } \\
\text { bar size }\end{array}$ & $\begin{array}{c}\text { Tensile } \\
\text { strength, } \\
\mathrm{MPa}\end{array}$ & $\begin{array}{c}\text { Strain, } \\
\%\end{array}$ & $\begin{array}{c}\text { Poisson's } \\
\text { ratio }\end{array}$ & $\begin{array}{c}\text { Gross cross- } \\
\text { section including } \\
\text { ribs, } \mathrm{mm}^{2}\end{array}$ & $\begin{array}{c}\text { Nominal } \\
\text { cross- } \\
\text { section, } \mathrm{mm}^{2}\end{array}$ & $\begin{array}{c}\text { Weight } \\
\mathrm{g} / \mathrm{m}\end{array}$ & $\begin{array}{c}\text { Nominal tensile } \\
\text { modulus, GPa }\end{array}$ \\
\hline 16 & 1188 & 2.61 & $0.20-0.22$ & 254.47 & 201.06 & 0.53 & 64 \\
20 & 1188 & 2.61 & $0.20-0.22$ & 380.13 & 314.16 & & 64 \\
\hline
\end{tabular}

Table 2.5. Mechanical properties 15M and 20M straight sand-coated GFRP bars (VROD Pultrall, 2013)

\begin{tabular}{cccccccc}
\hline $\begin{array}{c}\text { Bar } \\
\text { size }\end{array}$ & $\begin{array}{c}\text { Metric } \\
\text { size }\end{array}$ & $\begin{array}{c}\text { Cross- } \\
\text { sectional } \\
\text { area, } \\
\mathrm{mm}^{2}\end{array}$ & $\begin{array}{c}\text { Glass content } \\
\% \text { volume } \\
(\% \text { weight })\end{array}$ & $\begin{array}{c}\text { Nominal } \\
\text { bond } \\
\text { strength, } \\
\mathrm{MPa}\end{array}$ & $\begin{array}{c}\text { Tensile } \\
\text { strain, } \%\end{array}$ & $\begin{array}{c}\text { Nominal } \\
\text { tensile } \\
\text { modulus, } \\
\mathrm{MPa}\end{array}$ & $\begin{array}{c}\text { Guaranteed } \\
\text { tensile } \\
\text { strength, } \\
\mathrm{MPa}\end{array}$ \\
\hline$\# 5$ & $15 \mathrm{M}$ & 198 & $65(83)$ & 14 & 1.89 & $62.6 \pm 2.5$ & 1184 \\
\hline$\# 6$ & $20 \mathrm{M}$ & 285 & $65(83)$ & 14 & 1.71 & $64.7 \pm 2.5$ & 1105 \\
\hline
\end{tabular}

Note: Poisson's ratio $=0.25$ 
Table 2.6. Pullout test results for 15M ribbed-surface GFRP bars with centric bar location

\begin{tabular}{|c|c|c|c|c|c|c|c|c|}
\hline Specimen & $d_{b}$ & $\mathrm{~mm}$ & $\begin{array}{c}\text { Concrete } \\
\text { cover } \\
\mathrm{mm} \\
\end{array}$ & $\mathrm{MPa}$ & $\begin{array}{r}P_{\text {ult }} \\
\mathrm{kN} \\
\end{array}$ & $\begin{array}{r}\text { LES } \\
\mathrm{mm} \\
\end{array}$ & $\begin{array}{l}\text { FES } \\
\mathrm{mm} \\
\end{array}$ & $\begin{array}{l}\text { Failure } \\
\text { mode }\end{array}$ \\
\hline C16.4d.S1 & 16 & 64 & 92 & 191.60 & 95.00 & 13.18 & 0.23 & Pullout \\
\hline C16.4d.S2 & 16 & 64 & 92 & & 98.00 & 14.01 & 0.24 & Pullout \\
\hline C16.4d.S3 & 16 & 64 & 92 & & 104.00 & 13.02 & 0.25 & Pullout \\
\hline C16.4d.S4 & 16 & 64 & 92 & & 88.00 & 18.00 & 0.21 & Pullout \\
\hline C16.4d.S5 & 16 & 64 & 92 & & 86.00 & 11.93 & 0.21 & Pullout \\
\hline Mean & & & & & 94.20 & 14.03 & 0.23 & \\
\hline C16.6d.S1 & 16 & 96 & 92 & 191.86 & 62.00 & 16.22 & 0.26 & Pullout \\
\hline C16.6d.S2 & 16 & 96 & 92 & & 61.00 & 16.46 & 0.28 & Pullout \\
\hline C16.6d.S3 & 16 & 96 & 92 & & 53.00 & 14.42 & 0.25 & Pullout \\
\hline C16.6d.S4 & 16 & 96 & 92 & & 54.00 & 22.14 & 0.16 & Pullout \\
\hline C16.6d.S5 & 16 & 96 & 92 & & 69.00 & 17.99 & 0.21 & Pullout \\
\hline Mean & & & & & 59.80 & 17.45 & 0.23 & \\
\hline C16.8d.S1 & 16 & 128 & 92 & 191.86 & 84.00 & 25.81 & 0.53 & Pullout \\
\hline C16.8d.S2 & 16 & 128 & 92 & & 77.00 & 28.58 & 0.23 & Pullout \\
\hline C16.8d.S3 & 16 & 128 & 92 & & 73.00 & 30.52 & 0.40 & Pullout \\
\hline C16.8d.S4 & 16 & 128 & 92 & & 76.00 & 23.49 & 0.41 & Pullout \\
\hline C16.8d.S5 & 16 & 128 & 92 & & 63.00 & 19.47 & 0.21 & Pullout \\
\hline Mean & & & & & 74.60 & 25.57 & 0.36 & \\
\hline C16.0d.H1 & 16 & 100 & 142 & 168.53 & 115.00 & 18.00 & 0.28 & Pullout \\
\hline C16.0d.H2 & 16 & 100 & 142 & & 125.00 & 14.00 & 0.30 & Pullout \\
\hline C16.0d.H3 & 16 & 100 & 142 & & 123.00 & 14.00 & 1.30 & Pullout \\
\hline C16.0d.H4 & 16 & 100 & 142 & & 133.00 & 12.00 & 1.41 & Pullout \\
\hline C16.0d.H5 & 16 & 100 & 142 & & 115.00 & 16.00 & 0.28 & Pullout \\
\hline Mean & & & & & 122.20 & 14.80 & 0.714 & \\
\hline
\end{tabular}

Notes: $\mathrm{d}_{\mathrm{b}}=$ nominal bar diameter; $\mathrm{l}_{\mathrm{e}}=$ bar embedment length; $\mathrm{P}_{\mathrm{ult}}=$ pullout load; $\mathrm{LES}=$ loaded-end slip; FES $=$ free-end slip. 
Table 2.7. Pullout test results for 15M ribbed-surface GFRP bars with $40 \mathrm{~mm}$ eccentric bar location

\begin{tabular}{|c|c|c|c|c|c|c|c|c|}
\hline Specimen & $d_{b}$ & $\begin{array}{c}l_{e} \\
\mathrm{~mm}\end{array}$ & $\begin{array}{c}\text { Concrete } \\
\text { cover } \\
\mathrm{mm}\end{array}$ & $\begin{array}{c}f_{c}^{\prime} \\
\mathrm{MPa}\end{array}$ & $\begin{array}{l}P_{\text {ult }} \\
\mathrm{kN}\end{array}$ & LES & FES & $\begin{array}{c}\text { Failure } \\
\text { mode }\end{array}$ \\
\hline E40-16.4d.S1 & 16 & 64 & 40 & 191.86 & 93.00 & 12.68 & 0.37 & Pullout \\
\hline E40-16.4d.S2 & 16 & 64 & 40 & & 101.00 & 13.67 & 0.35 & Pullout \\
\hline E40-16.4d.S3 & 16 & 64 & 40 & & 95.00 & 22.05 & 1.01 & Pullout \\
\hline E40-16.4d.S4 & 16 & 64 & 40 & & 93.00 & 16.27 & 0.18 & Pullout \\
\hline E40-16.4d.S5 & 16 & 64 & 40 & & 103.00 & 15.33 & 0.17 & Pullout \\
\hline Mean & & & & & 97.00 & 16.00 & 0.42 & \\
\hline E40-16.6d.S1 & 16 & 96 & 40 & 191.60 & 74.00 & 24.94 & 0.38 & Pullout \\
\hline E40-16.6d.S2 & 16 & 96 & 40 & & 88.00 & 28.74 & 0.45 & Pullout \\
\hline E40-16.6d.S3 & 16 & 96 & 40 & & 75.00 & 25.17 & 0.38 & Pullout \\
\hline E40-16.6d.S4 & 16 & 96 & 40 & & 68.00 & 22.21 & 0.35 & Pullout \\
\hline E40-16.6d.S5 & 16 & 96 & 40 & & 58.00 & 16.10 & 0.24 & Pullout \\
\hline Mean & & & & & 72.60 & 23.43 & 0.36 & \\
\hline E40-16.8d.S1 & 16 & 128 & 40 & 191.86 & 109.00 & 26.43 & 0.66 & Pullout \\
\hline E40-16.8d.S2 & 16 & 128 & 40 & & 89.00 & 26.19 & 0.72 & Pullout \\
\hline E40-16.8d.S3 & 16 & 128 & 40 & & 73.00 & 22.29 & 0.33 & Pullout \\
\hline E40-16.8d.S4 & 16 & 128 & 40 & & 75.00 & 23.24 & 0.24 & Pullout \\
\hline E40-16.8d.S5 & 16 & 128 & 40 & & 80.00 & 19.28 & 0.48 & Pullout \\
\hline Mean & & & & & 85.20 & 23.49 & 0.49 & \\
\hline E40-16.0d.H1 & 16 & 100 & 40 & 170.77 & 131.00 & 18.00 & 0.51 & Pullout \\
\hline E40-16.0d.H2 & 16 & 100 & 40 & & 132.00 & 18.14 & 0.51 & Pullout \\
\hline E40-16.0d.H3 & 16 & 100 & 40 & & 114.00 & 16.00 & 0.44 & Pullout \\
\hline E40-16.0d.H4 & 16 & 100 & 40 & & 119.00 & 17.00 & 0.48 & Pullout \\
\hline E40-16.0d.H5 & 16 & 100 & 40 & & 114.00 & 16.00 & 0.46 & Pullout \\
\hline Mean & & & & & 122.00 & 17.03 & 0.48 & \\
\hline
\end{tabular}

Notes: $\mathrm{d}_{\mathrm{b}}=$ nominal bar diameter; $\mathrm{l}_{\mathrm{e}}=$ bar embedment length; $\mathrm{P}_{\mathrm{ult}}=$ pullout load; $\mathrm{LES}=$ loaded-end slip; FES = free-end slip. 
Table 2.8. Pullout test results for 15M ribbed-surface GFRP bars with $60 \mathrm{~mm}$ eccentric bar location

\begin{tabular}{|c|c|c|c|c|c|c|c|c|}
\hline Specimen & $\begin{array}{c}d_{b} \\
\mathrm{Mm}\end{array}$ & $\mathrm{mm}$ & $\begin{array}{c}\text { Concrete } \\
\text { cover } \\
\mathrm{mm}\end{array}$ & $\mathrm{MPa}$ & $\begin{array}{l}P_{\text {ult }} \\
\mathrm{kN}\end{array}$ & $\begin{array}{l}\text { LES } \\
\text { mm }\end{array}$ & $\begin{array}{l}\text { FES } \\
\mathrm{mm}\end{array}$ & $\begin{array}{l}\text { Failure } \\
\text { mode }\end{array}$ \\
\hline E60-16.4d.S1 & 16 & 64 & 60 & 191.60 & 45.00 & 18.38 & 0.26 & Pullout \\
\hline E60-16.4d.S2 & 16 & 64 & 60 & & 49.00 & 19.60 & 0.19 & Pullout \\
\hline E60-16.4d.S3 & 16 & 64 & 60 & & 55.00 & 16.16 & 0.24 & Pullout \\
\hline E60-16.4d.S4 & 16 & 64 & 60 & & 46.00 & 16.53 & 0.22 & Pullout \\
\hline E60-16.4d.S5 & 16 & 64 & 60 & & 64.00 & 18.97 & 0.28 & Pullout \\
\hline Mean & & & & & 51.80 & 17.93 & 0.24 & \\
\hline E60-16.6d.S1 & 16 & 96 & 60 & 191.60 & 83.00 & 19.60 & 0.37 & Pullout \\
\hline E60-16.6d.S2 & 16 & 96 & 60 & & 53.00 & 19.00 & 0.24 & Pullout \\
\hline E60-16.6d.S3 & 16 & 96 & 60 & & 60.00 & 19.00 & 0.27 & Pullout \\
\hline E60-16.6d.S4 & 16 & 96 & 60 & & 55.00 & 16.00 & 0.25 & Pullout \\
\hline E60-16.6d.S5 & 16 & 96 & 60 & & 71.00 & 9.11 & 0.28 & Pullout \\
\hline Mean & & & & & 64.40 & 16.54 & 0.28 & \\
\hline E60-16.8d.S1 & 16 & 128 & 60 & 192.39 & 83.00 & 26.24 & 0.33 & Pullout \\
\hline E60-16.8d.S2 & 16 & 128 & 60 & & 78.00 & 28.88 & 0.55 & Pullout \\
\hline E60-16.8d.S3 & 16 & 128 & 60 & & 86.00 & 25.25 & 0.38 & Pullout \\
\hline E60-16.8d.S4 & 16 & 128 & 60 & & 84.00 & 24.88 & 0.37 & Pullout \\
\hline E60-16.8d.S5 & 16 & 128 & 60 & & 75.00 & 26.89 & 2.65 & Pullout \\
\hline Mean & & & & & 81.20 & 26.43 & 0.86 & \\
\hline
\end{tabular}

Notes: $\mathrm{d}_{\mathrm{b}}=$ nominal bar diameter; $\mathrm{l}_{\mathrm{e}}=$ bar embedment length; $\mathrm{P}_{\mathrm{ult}}=$ pullout load; $\mathrm{LES}=$ loaded-end slip; FES $=$ free-end slip. 
Table 2.9. Pullout test results for 20M ribbed-surface GFRP bars with centric bar location

\begin{tabular}{lcccccccc}
\hline Specimen & $d_{b}$ & $l_{e}$ & $\begin{array}{c}\text { Concrete } \\
\text { cover }\end{array}$ & $f_{c}^{\prime}$ & $P_{\text {ult }}$ & LES & FES & $\begin{array}{c}\text { Failure } \\
\text { mode }\end{array}$ \\
\hline C20.4d.S1 & 20 & 80 & 90 & 191.86 & 66.00 & 17.86 & 0.31 & Pullout \\
C20.4d.S2 & 20 & 80 & 90 & & 58.00 & 15.69 & 0.27 & Pullout \\
C20.4d.S3 & 20 & 80 & 90 & & 45.00 & 12.18 & 0.21 & Pullout \\
C20.4d.S4 & 20 & 80 & 90 & & 63.00 & 16.91 & 0.29 & Pullout \\
C20.4d.S5 & 20 & 80 & 90 & & 65.00 & 14.82 & 0.37 & Pullout \\
Mean & & & & & $\mathbf{5 9 . 4 0}$ & $\mathbf{1 5 . 4 9}$ & $\mathbf{0 . 2 9}$ & \\
C20.6d.S1 & 20 & 120 & 90 & 191.06 & 128.00 & 20.21 & 0.50 & Pullout \\
C20.6d.S2 & 20 & 120 & 90 & & 133.00 & 21.44 & 0.52 & Pullout \\
C20.6d.S3 & 20 & 120 & 90 & & 123.00 & 19.83 & 0.48 & Pullout \\
C20.6d.S4 & 20 & 120 & 90 & & 97.00 & 15.64 & 2.04 & Pullout \\
C20.6d.S5 & 20 & 120 & 90 & & 96.00 & 17.71 & 0.38 & Pullout \\
Mean & & & & & $\mathbf{1 1 5 . 4 0}$ & $\mathbf{1 8 . 9 7}$ & $\mathbf{0 . 7 8}$ & \\
C20.8d.S1 & 20 & 160 & 90 & 191.60 & 133.00 & 26.38 & 0.25 & Pullout \\
C20.8d.S2 & 20 & 160 & 90 & & 133.00 & 26.38 & 0.25 & Pullout \\
C20.8d.S3 & 20 & 160 & 90 & & 147.00 & 29.15 & 0.27 & Pullout \\
C20.8d.S4 & 20 & 160 & 90 & & 109.00 & 33.85 & 0.39 & Pullout \\
C20.8d.S5 & 20 & 160 & 90 & & 113.00 & 22.31 & 0.21 & Pullout \\
Mean & & & & & $\mathbf{1 2 7 . 0 0}$ & $\mathbf{2 7 . 6 1}$ & $\mathbf{0 . 2 7}$ & \\
\hline
\end{tabular}

Notes: $\mathrm{d}_{\mathrm{b}}=$ nominal bar diameter; $\mathrm{l}_{\mathrm{e}}=$ bar embedment length; $\mathrm{P}_{\mathrm{ult}}=$ pullout load; $\mathrm{LES}=$ loaded-end slip; FES = free-end slip. 
Table 2.10. Pullout test results for 20M ribbed-surface GFRP bars with $40 \mathrm{~mm}$ eccentric bar location

\begin{tabular}{|c|c|c|c|c|c|c|c|c|}
\hline Specimen & $\begin{array}{l}d_{b} \\
\mathrm{~mm}\end{array}$ & $\begin{array}{c}l_{e} \\
\mathrm{~mm}\end{array}$ & $\begin{array}{c}\text { Concrete } \\
\text { cover } \\
\mathrm{mm}\end{array}$ & $\begin{array}{c}f_{c}^{\prime} \\
\mathrm{MPa}\end{array}$ & $\begin{array}{l}P_{\text {ult }} \\
\\
\mathrm{kN}\end{array}$ & $\begin{array}{r}\text { LES } \\
\mathrm{mm}\end{array}$ & $\begin{array}{l}\text { FES } \\
\mathrm{mm}\end{array}$ & $\begin{array}{l}\text { Failure } \\
\text { mode }\end{array}$ \\
\hline E40-20.8d.S1 & 20 & 160 & 40 & 191.86 & 119.00 & 20.35 & 0.61 & Pullout \\
\hline E40-20.8d.S2 & 20 & 160 & 40 & & 117.00 & 27.22 & 0.33 & Pullout \\
\hline E40-20.8d.S3 & 20 & 160 & 40 & & 112.00 & 22.91 & 0.22 & Pullout \\
\hline E40-20.8d.S4 & 20 & 160 & 40 & & 108.00 & 22.38 & 0.12 & Pullout \\
\hline E40-20.8d.S5 & 20 & 160 & 40 & & 131.00 & 23.53 & 0.20 & Pullout \\
\hline Mean & & & & & 117.40 & 23.28 & 0.30 & \\
\hline
\end{tabular}

Notes: $\mathrm{d}_{\mathrm{b}}=$ nominal bar diameter; $\mathrm{l}_{\mathrm{e}}=$ bar embedment length; $\mathrm{P}_{\mathrm{ult}}=$ pullout load; $\mathrm{LES}=$ loaded-end slip; FES $=$ free-end slip. 
Table 2.11. Pullout load - slip modeling for ribbed-surface GFRP bars

\begin{tabular}{|c|c|c|c|c|c|c|}
\hline Specimen & $P$ & $\delta_{L E S}$ & $\delta_{F E S}$ & $\delta_{A C T}$ & $P-\delta_{A C T}$ Equation & $R^{2}$ \\
\hline C16.4d.S & 94.2 & 14.028 & 0.228 & 13.8 & $P=0.272 \delta_{a c t}^{2}+3.06 \delta_{a c t}$ & 0.99 \\
\hline C16.6d.S & 59.8 & $\mathbf{1 7 . 4 4 6}$ & 0.232 & 17.21 & $P=0.142 \delta_{a c t}^{2}+1.032 \delta_{a c t}$ & 1.00 \\
\hline C16.8d.S & 74.6 & 25.574 & 0.356 & 25.218 & $P=0.106 \delta_{a c t}^{2}+0.275 \delta_{a c t}$ & 0.99 \\
\hline C16.0d.H & 122.2 & 14.8 & 0.714 & 14.086 & $P=0.487 \delta_{a c t}^{2}+1.788 \delta_{a c t}$ & 0.99 \\
\hline C20.4d.S & 59.4 & 15.492 & 0.29 & 15.202 & $P=0.1529 \delta_{a c t}^{2}+1.5824 \delta_{a c t}$ & 0.99 \\
\hline C20.6d.S & 115.4 & 18.966 & 0.784 & 18.182 & $P=0.1626 \delta_{a c t}^{2}+3.391 \delta_{a c t}$ & 1.00 \\
\hline C20.8d.S & 127.00 & 27.614 & 0.274 & 27.34 & $P=0.21 \delta_{a c t}^{2}-1.2 \delta_{a c t}$ & 0.98 \\
\hline E40-16.4d.S & 97 & 16 & 0.416 & 15.584 & $P=0.148 \delta_{a c t}^{2}+3.92 \delta_{a c t}$ & 1.00 \\
\hline E40-16.6d.S & 72.6 & 23.432 & 0.36 & 23.792 & $P=0.101 \delta_{a c t}^{2}+0.64 \delta_{a c t}$ & 0.99 \\
\hline E40-16.8d.S & 85.2 & 23.486 & 0.486 & 23.00 & $P=0.168 \delta_{a c t}^{2}-0.16 \delta_{a c t}$ & 0.99 \\
\hline E40-16.0d.H & 122 & $\mathbf{1 7 . 0 2 8}$ & 0.48 & 16.548 & $P=0.374 \delta_{a c t}^{2}+1.189 \delta_{a c t}$ & 1.00 \\
\hline E40-20.8d.S & 117.4 & 23.278 & 0.296 & 22.982 & $P=0.114 \delta_{a c t}^{2}+2.44 \delta_{a c t}$ & 0.99 \\
\hline E60-16.4d.S & 51.8 & $\mathbf{1 7 . 9 2 8}$ & 0.238 & 17.69 & $P=0 . .1255 \delta_{a c t}^{2}+0.706 \delta_{a c t}$ & 0.99 \\
\hline E60-16.6d.S & 64.4 & 16.542 & 0.282 & 16.26 & $P=-0.056 \delta_{a c t}^{2}+4.874 \delta_{a c t}$ & 1.00 \\
\hline E60-16.8d.S & 81.2 & 26.428 & 0.856 & 25.572 & $P=0.0976 \delta_{a c t}^{2}+0.666 \delta_{a c t}$ & 0.99 \\
\hline
\end{tabular}

Notes: $P=$ pullout load; $\delta_{L E S}=$ displacement at the load-end slip; $\delta_{F E S}=$ displacement at the free-end slip; $\delta_{A C T}=$ actual displacement; $R^{2}=$ coefficient of determination 
Table 2.12. Bond stress and experimental coefficients for ribbed-surface GFRP bars

\begin{tabular}{|c|c|c|c|c|c|c|}
\hline Specimen & $l_{e} / c_{\min }$ & $l_{e} / d_{b}$ & $u, \mathrm{MPa}$ & $u$ & $\boldsymbol{B}=k \cdot \varphi$ & $\varphi$ \\
\hline C16.4d.S & 0.69 & 4 & 29.28 & $\begin{array}{l}\sqrt{f_{c}} \\
2.11\end{array}$ & 4.29 & 0.39 \\
\hline C16.6d.S & 1.04 & 6 & 12.39 & 0.89 & 6.43 & 0.25 \\
\hline C16.8d.S & 1.39 & 8 & 11.59 & 0.84 & 8.58 & 0.31 \\
\hline C16.0d.H & 0.70 & 6.25 & 24.31 & 1.87 & 9.70 & 0.51 \\
\hline C20.4d.S & 0.89 & 4 & 11.81 & 0.85 & 4.20 & 0.16 \\
\hline C20.6d.S & 1.33 & 6 & 15.30 & 1.11 & 6.28 & 0.31 \\
\hline C20.8d.S & 1.78 & 8 & 12.63 & 0.91 & 8.39 & 0.34 \\
\hline E40-16.4d.S & 1.60 & 4 & 30.15 & 2.18 & 1.87 & 0.41 \\
\hline E40-16.6d.S & 2.40 & 6 & 15.04 & 1.09 & 2.80 & 0.30 \\
\hline E40-16.8d.S & 3.20 & 8 & 13.24 & 0.96 & 3.73 & 0.36 \\
\hline E40-16.0d.H & 2.50 & 6.25 & 24.27 & 1.86 & 2.75 & 0.51 \\
\hline E40-20.8d.S & 4.00 & 8 & 11.68 & 0.84 & 3.73 & 0.31 \\
\hline E60-16.4d.S & 1.07 & 4 & 16.10 & 1.16 & 2.80 & 0.22 \\
\hline E60-16.6d.S & 1.60 & 6 & 13.35 & 0.96 & 4.19 & 0.27 \\
\hline E60-16.8d.S & 2.13 & 8 & 12.62 & 0.91 & 5.60 & 0.34 \\
\hline
\end{tabular}

Notes: $l_{e}=$ embedment length; $c_{\min }=$ minimum concrete cover; $d_{b}=$ bar diameter; $f_{c}^{\prime}=$ compressive strength of concrete; $u=$ bond stress; $k=$ bond empirical constant; $\varphi=$ bar stress ratio to its ultimate stress. 
Table 2.13. Pullout load versus the embedment length equations for ribbed-surface GFRP bars

\begin{tabular}{llc}
\hline Group & Equation $P$ versus $\frac{l_{e}}{d_{b}}$ & $\boldsymbol{R}^{2}$ \\
\hline C20.S & $P==-5.55\left(\frac{l_{e}}{d_{b}}\right)^{2}+83.5\left(\frac{l_{e}}{d_{b}}\right)-185.8$ & 1 \\
\hline C16.S & $P==6.15\left(\frac{l_{e}}{d_{b}}\right)^{2}-78.7\left(\frac{l_{e}}{d_{b}}\right)+310.6$ & 1 \\
\hline E40-16.S & $P==4.625\left(\frac{l_{e}}{d_{b}}\right)^{2}-58.45\left(\frac{l_{e}}{d_{b}}\right)+256.8$ & 1 \\
\hline E60-16.S & $P==0.525\left(\frac{l_{e}}{d_{b}}\right)^{2}+1.05\left(\frac{l_{e}}{d_{b}}\right)+39.2$ & 1
\end{tabular}

Notes: $P=$ pullout load; $l_{e}=$ embedment length; $d_{b}=$ bar diameter; $R^{2}=$ coefficient of determination.

Table 2.14. Bond stress versus the embedment length equations for ribbed-surface GFRP bars

\begin{tabular}{llc}
\hline Group & Equation $\frac{\boldsymbol{u}}{\sqrt{\boldsymbol{f}_{c}^{\prime}}} \operatorname{versus} \frac{l_{e}}{\boldsymbol{d}_{\boldsymbol{b}}}$ & $\boldsymbol{R}^{\mathbf{2}}$ \\
\hline C20.S & $\frac{u}{\sqrt{f_{c}^{\prime}}}=-0.056\left(\frac{l_{e}}{d_{b}}\right)^{2}+0.687\left(\frac{l_{e}}{d_{b}}\right)-0.999$ & 1 \\
\hline C16.S & $\frac{u}{\sqrt{f_{c}^{\prime}}}=0.1453\left(\frac{l_{e}}{d_{b}}\right)^{2}-2.0625\left(\frac{l_{e}}{d_{b}}\right)+8.041$ & 1 \\
\hline E40-16.S & $\frac{u}{\sqrt{f_{c}^{\prime}}}=0.1199\left(\frac{l_{e}}{d_{b}}\right)^{2}-1.7438\left(\frac{l_{e}}{d_{b}}\right)+7.234$ & 1 \\
\hline E60-16.S & $\frac{u}{\sqrt{f_{c}^{\prime}}}=0.0181\left(\frac{l_{e}}{d_{b}}\right)^{2}-2.808\left(\frac{l_{e}}{d_{b}}\right)+1.996$ & 1 \\
\hline
\end{tabular}

Notes: $u=$ bond stress; $f_{c}^{\prime}=$ compressive strength of concrete; $l_{e}=$ embedment length; $d_{b}=$ bar diameter; $R^{2}=$ coefficient of determination. 
Table 2.15. Concrete and reinforcement location factor (bond factor) for ribbed-surface GFRP bars

\begin{tabular}{lcc}
\hline Group & Equation $B=k . \varphi$ versus $\frac{l_{e}}{d_{b}}$ & $\boldsymbol{R}^{2}$ \\
\hline C20.S & $B=1.0483\left(\frac{l_{e}}{d_{b}}\right)$ & 1 \\
\hline C16.S & $B=1.0726\left(\frac{l_{e}}{d_{b}}\right)$ & 1 \\
\hline E40-16.S & $B=0.4663\left(\frac{l_{e}}{d_{b}}\right)$ & 1 \\
\hline E60-16.S & $B=0.6999\left(\frac{l_{e}}{d_{b}}\right)$ & 1 \\
\hline
\end{tabular}

Notes: $k=$ bond empirical constant; $\varphi=$ bar stress ratio to its ultimate stress; $l_{e}=$ embedment length; $d_{b}=$ bar diameter; $\mathrm{B}=$ compound empirical constant; $R^{2}=$ coefficient of determination

Table 2.16. Reinforcement location factor for ribbed-surface GFRP bars

\begin{tabular}{lcc}
\hline Group & Equation $\varphi$ versus $\frac{l_{e}}{d_{b}}$ & $\boldsymbol{R}^{2}$ \\
\hline C20.S & $\varphi=0.0149\left(\frac{l_{e}}{d_{b}}\right)^{2}+0.2237\left(\frac{l_{e}}{d_{b}}\right)-0.4978$ & 1 \\
\hline C16.S & $\varphi=0.0257\left(\frac{l_{e}}{d_{b}}\right)^{2}-0.3295\left(\frac{l_{e}}{d_{b}}\right)+1.3003$ & 1 \\
\hline E40-16.S & $\varphi=0.0194\left(\frac{l_{e}}{d_{b}}\right)^{2}-0.2447\left(\frac{l_{e}}{d_{b}}\right)+1.0751$ & 1 \\
\hline E60-16.S & $\varphi=0.0022\left(\frac{l_{e}}{d_{b}}\right)^{2}+0.0044\left(\frac{l_{e}}{d_{b}}\right)+0.1641$ & 1 \\
\hline
\end{tabular}

Notes: $\varphi=$ bar stress ratio to its ultimate stress; $l_{e}=$ embedment length; $d_{b}=$ bar diameter; $R^{2}=$ coefficient of determination. 
Table 2.17. ACI basic factor $k_{\text {exp }}$ for ribbed-surface GFRP bars

\begin{tabular}{|c|c|c|c|c|}
\hline Loading location & Bar type* & $\begin{array}{c}\text { Bar diameter, } \\
\mathrm{d}_{\mathrm{b}} \\
\end{array}$ & $l_{e} / d_{b} * *$ & $k_{\exp } * * *$ \\
\hline \multirow[t]{4}{*}{ Centric } & $\mathrm{S}$ & 16 & 4.00 & 0.00371 \\
\hline & $\mathrm{S}$ & 16 & 6.00 & 0.00557 \\
\hline & $\mathrm{S}$ & 16 & 8.00 & 0.00742 \\
\hline & $\mathrm{H}$ & 16 & 6.26 & 0.00543 \\
\hline \multirow[t]{4}{*}{ Eccentric at $40 \mathrm{~mm}$ concrete cover } & $\mathrm{S}$ & 16 & 4.00 & 0.00371 \\
\hline & $S$ & 16 & 6.00 & 0.00556 \\
\hline & $\mathrm{S}$ & 16 & 8.00 & 0.00742 \\
\hline & $\mathrm{H}$ & 16 & 6.25 & 0.00547 \\
\hline \multirow[t]{3}{*}{ Eccentric at $60 \mathrm{~mm}$ concrete cover } & $\mathrm{S}$ & 16 & 4.00 & 0.00371 \\
\hline & $\mathrm{S}$ & 16 & 6.00 & 0.00556 \\
\hline & $\mathrm{S}$ & 16 & 8.00 & 0.00743 \\
\hline \multirow[t]{3}{*}{ Centric } & $\mathrm{S}$ & 20 & 4.00 & 0.00297 \\
\hline & $\mathrm{S}$ & 20 & 6.00 & 0.0044 \\
\hline & $\mathrm{S}$ & 20 & 8.00 & 0.00593 \\
\hline Eccentric at $40 \mathrm{~mm}$ concrete cover & $\mathrm{S}$ & 20 & 8.00 & 0.00594 \\
\hline
\end{tabular}


Table 2.18. Pullout test results for 15M sand-coated GFRP bars with centric bar location

\begin{tabular}{|c|c|c|c|c|c|c|c|c|}
\hline Specimen & $\begin{array}{c}d_{b} \\
\mathrm{~mm}\end{array}$ & $\begin{array}{c}l_{e} \\
\mathrm{~mm} \\
\end{array}$ & $\begin{array}{c}\text { Concrete } \\
\text { cover } \\
\mathrm{mm} \\
\end{array}$ & $\begin{array}{c}f_{c}^{\prime} \\
\mathrm{MPa} \\
\end{array}$ & $\begin{array}{l}P_{u l t} \\
\mathrm{kN} \\
\end{array}$ & $\mathrm{mm}$ & $\begin{array}{l}\text { FES } \\
\mathrm{mm} \\
\end{array}$ & $\begin{array}{c}\text { Failure } \\
\text { mode }\end{array}$ \\
\hline C16.4d.S1 & 16 & 64 & 92 & 167.13 & 67.50 & 13.80 & 0.4 & Pullout \\
\hline C16.4d.S2 & 16 & 64 & 92 & 167.13 & 80.00 & 20.34 & 2.09 & Pullout \\
\hline C16.4d.S3 & 16 & 64 & 92 & 167.13 & 59.25 & 10.87 & 0.79 & Pullout \\
\hline C16.4d.S4 & 16 & 64 & 92 & 167.13 & 58.75 & 12.45 & 0.63 & Pullout \\
\hline C16.4d.S5 & 16 & 64 & 92 & 167.13 & 76.00 & 10.82 & 0.26 & Pullout \\
\hline Mean & & & & 167.13 & 68.3 & 13.66 & 0.83 & \\
\hline C16.6d.S1 & 16 & 96 & 92 & 168.07 & 55.50 & 20.17 & 0.45 & Pullout \\
\hline C16.6d.S2 & 16 & 96 & 92 & 168.07 & 56.50 & 0.51 & 0.35 & Pullout \\
\hline C16.6d.S3 & 16 & 96 & 92 & 168.07 & 43.75 & 6.98 & 0.35 & Pullout \\
\hline C16.6d.S4 & 16 & 96 & 92 & 168.07 & 56.50 & 15.01 & 0.2 & Pullout \\
\hline C16.6d.S5 & 16 & 96 & 92 & 168.07 & 57.25 & 21.03 & 0.3 & Pullout \\
\hline Mean & & & & 168.07 & 53.90 & 12.74 & 0.33 & \\
\hline C16.8d.S1 & 16 & 128 & 92 & 168.99 & 99.00 & 14.78 & 1.05 & Pullout \\
\hline C16.8d.S2 & 16 & 128 & 92 & 168.99 & 104.25 & 16.64 & 0.33 & Pullout \\
\hline C16.8d.S3 & 16 & 128 & 92 & 168.99 & 126.75 & 17.74 & 1.75 & Pullout \\
\hline C16.8d.S4 & 16 & 128 & 92 & 168.99 & 112.00 & 16.97 & 0.32 & Pullout \\
\hline C16.8d.S5 & 16 & 128 & 92 & 168.99 & 102.00 & 15.36 & 0.35 & Pullout \\
\hline Mean & & & & 168.99 & 108.80 & 16.30 & 0.76 & \\
\hline C16.0d.H1 & 16 & 100 & 142 & 167.61 & 114.00 & 24.00 & 0 & Pullout \\
\hline C16.0d.H2 & 16 & 100 & 142 & 167.61 & 111.00 & 21.00 & 0 & Pullout \\
\hline C16.0d.H3 & 16 & 100 & 142 & 167.61 & 125.00 & 18.00 & 0 & Pullout \\
\hline C16.0d.H4 & 16 & 100 & 142 & 167.61 & 134.00 & 15.00 & 0 & Pullout \\
\hline C16.0d.H5 & 16 & 100 & 142 & 167.61 & 99.00 & 19.00 & 0 & Pullout \\
\hline Mean & & & & 167.61 & 116.60 & 19.40 & 0 & \\
\hline
\end{tabular}

Notes: $d_{b}=$ nominal bar diameter; $1_{e}=$ bar embedment length; $P_{\text {ult }}=$ pullout load; $L E S=$ loaded-end slip; FES $=$ free-end slip. 
Table 2.19. Pullout test results for 15M sand-coated GFRP bars with $40 \mathrm{~mm}$ of eccentric bar location

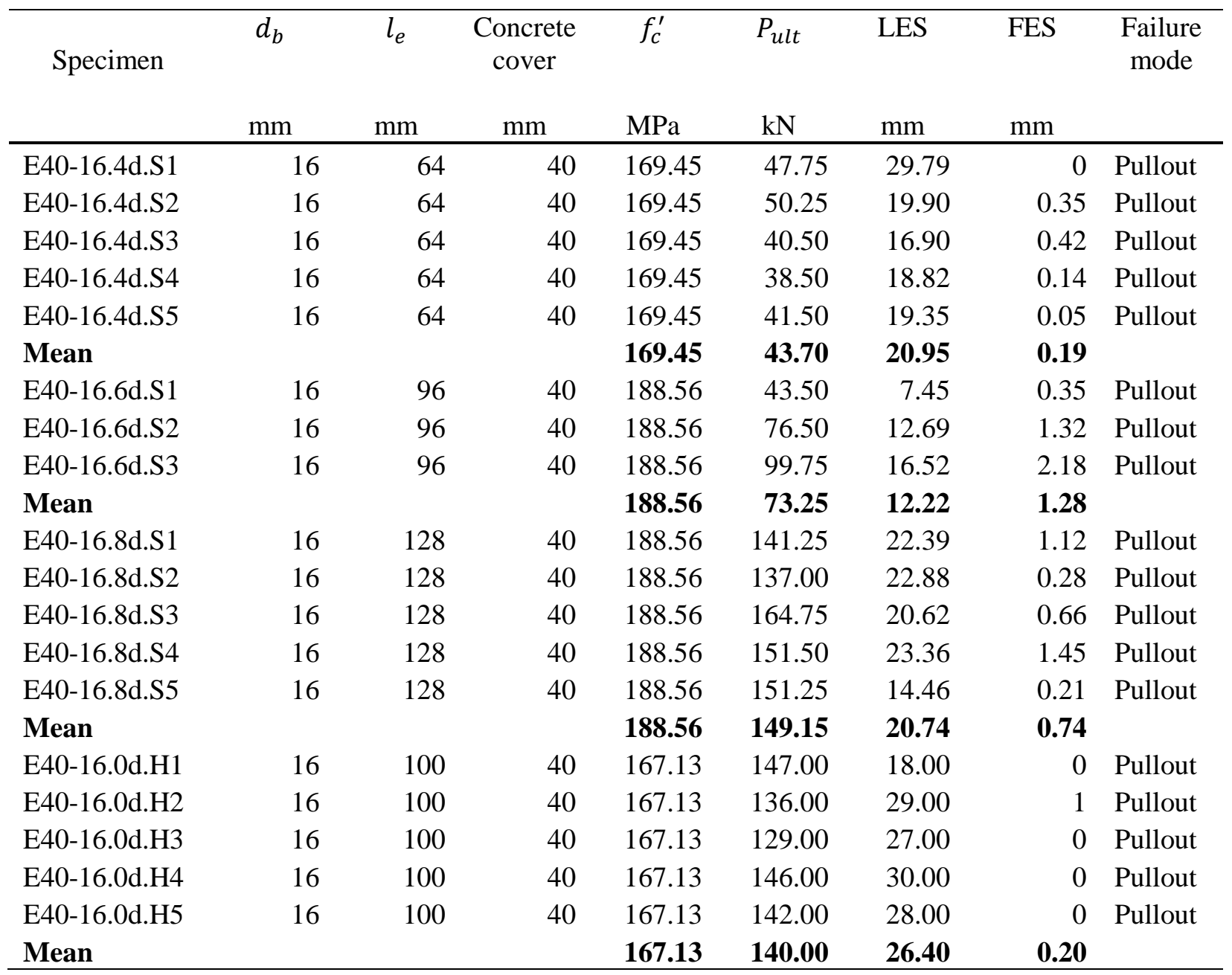

Notes: $\mathrm{d}_{\mathrm{b}}=$ nominal bar diameter; $\mathrm{l}_{\mathrm{e}}=$ bar embedment length; $\mathrm{P}_{\mathrm{ult}}=$ pullout load; $\mathrm{LES}=$ loaded-end slip; FES = free-end slip. 
Table 2.20. Pullout test results for 15M sand-coated GFRP bars with $60 \mathrm{~mm}$ eccentric bar location

\begin{tabular}{|c|c|c|c|c|c|c|c|c|}
\hline Specimen & $d_{b}$ & $l_{e}$ & $\begin{array}{c}\text { Concrete } \\
\text { cover }\end{array}$ & $f_{c}^{\prime}$ & $P_{u l t}$ & LES & FES & $\begin{array}{c}\text { Failure } \\
\text { mode }\end{array}$ \\
\hline & $\mathrm{mm}$ & $\mathrm{mm}$ & $\mathrm{mm}$ & $\mathrm{MPa}$ & $\mathrm{kN}$ & $\mathrm{mm}$ & $\mathrm{mm}$ & \\
\hline E60-16.4d.S1 & 16 & 64 & 60 & 168.54 & 45.75 & 27.31 & 0.88 & Pullout \\
\hline E60-16.4d.S2 & 16 & 64 & 60 & 168.54 & 40.50 & 20.55 & 0.19 & Pullout \\
\hline E60-16.4d.S3 & 16 & 64 & 60 & 168.54 & 36.50 & 18.37 & 0.32 & Pullout \\
\hline E60-16.4d.S4 & 16 & 64 & 60 & 168.54 & 41.25 & 18.62 & 0.27 & Pullout \\
\hline E60-16.4d.S5 & 16 & 64 & 60 & 168.54 & 40.50 & 17.60 & 0.1 & Pullout \\
\hline Mean & & & & 168.54 & 40.90 & 20.49 & 0.35 & \\
\hline E60-16.6d.S1 & 16 & 96 & 60 & 168.54 & 65.25 & 25.87 & 0.57 & Pullout \\
\hline E60-16.6d.S2 & 16 & 96 & 60 & 168.54 & 66.50 & 28.36 & 0.52 & Pullout \\
\hline E60-16.6d.S3 & 16 & 96 & 60 & 168.54 & 68.50 & 28.03 & 0.73 & Pullout \\
\hline E60-16.6d.S4 & 16 & 96 & 60 & 168.54 & 69.25 & 21.47 & 0.92 & Pullout \\
\hline E60-16.6d.S5 & 16 & 96 & 60 & 168.54 & 67.75 & 26.73 & 0.79 & Pullout \\
\hline Mean & & & & 168.54 & 67.45 & 26.09 & 0.71 & \\
\hline E60-16.8d.S1 & 16 & 128 & 60 & 189.69 & 151.75 & 27.85 & 0.18 & Pullout \\
\hline E60-16.8d.S2 & 16 & 128 & 60 & 189.69 & 145.25 & 28.91 & 0.98 & Pullout \\
\hline E60-16.8d.S3 & 16 & 128 & 60 & 189.69 & 162.25 & 22.60 & 0.19 & Pullout \\
\hline E60-16.8d.S4 & 16 & 128 & 60 & 189.69 & 142.00 & 23.60 & 0.23 & Pullout \\
\hline E60-16.8d.S5 & 16 & 128 & 60 & 189.69 & 145.75 & 20.29 & 0.01 & Pullout \\
\hline Mean & & & & 189.69 & 149.4 & 24.65 & 0.32 & \\
\hline E60-16.0d.H1 & 16 & 100 & 60 & 169.45 & 161.00 & 45.86 & 3.96 & Pullout \\
\hline E60-16.0d.H2 & 16 & 100 & 60 & 169.45 & 140.00 & 22.79 & 0.32 & Pullout \\
\hline E60-16.0d.H3 & 16 & 100 & 60 & 169.45 & 127.75 & 20.96 & 0.03 & Pullout \\
\hline E60-16.0d.H4 & 16 & 100 & 60 & 169.45 & 151.00 & 30.27 & 0.60 & Pullout \\
\hline E60-16.0d.H5 & 16 & 100 & 60 & 169.45 & 130.50 & 23.41 & 1.25 & Pullout \\
\hline Mean & & & & 169.45 & 142.05 & 28.66 & 1.23 & \\
\hline
\end{tabular}

Notes: $d_{b}=$ nominal bar diameter; $1_{e}=$ bar embedment length; $P_{\text {ult }}=$ pullout load; $L E S=$ loaded-end slip; FES = free-end slip. 
Table 2.21. Pullout test results for 20M sand-coated GFRP bars with centric bar location

\begin{tabular}{lccccccrcc}
\hline \multicolumn{1}{c}{ Specimen } & $d_{b}$ & $l_{e}$ & $\begin{array}{c}\text { Concrete } \\
\text { cover }\end{array}$ & $f_{c}^{\prime}$ & $P_{u l t}$ & LES & FES & $\begin{array}{c}\text { Failure } \\
\text { mode }\end{array}$ \\
& $\mathrm{mm}$ & $\mathrm{mm}$ & $\mathrm{mm}$ & $\mathrm{MPa}$ & \multicolumn{1}{c}{$\mathrm{kN}$} & $\mathrm{mm}$ & $\mathrm{mm}$ & \\
\hline C20.6d.S1 & 20 & 120 & 90 & 166.65 & 71.25 & 1.28 & 0.01 & Pullout \\
C20.6d.S2 & 20 & 120 & 90 & 166.65 & 68.50 & 20.22 & 0.12 & Pullout \\
C20.6d.S3 & 20 & 120 & 90 & 166.65 & 85.25 & 15.79 & 0.43 & Pullout \\
C20.6d.S4 & 20 & 120 & 90 & 166.65 & 84.25 & 24.56 & 0.24 & Pullout \\
C20.6d.S5 & 20 & 120 & 90 & 166.65 & 79.25 & 19.55 & 0.33 & Pullout \\
Mean & & & & $\mathbf{1 6 6 . 6 5}$ & $\mathbf{7 7 . 7 0}$ & $\mathbf{1 6 . 2 8}$ & $\mathbf{0 . 2 3}$ & \\
C20.8d.S1 & 20 & 160 & 90 & 166.65 & 80.75 & 24.72 & 0.13 & Pullout \\
C20.8d.S2 & 20 & 160 & 90 & 166.65 & 99.25 & 29.17 & 1.82 & Pullout \\
C20.8d.S3 & 20 & 160 & 90 & 166.65 & 89.75 & 29.63 & 0.03 & Pullout \\
C20.8d.S4 & 20 & 160 & 90 & 166.65 & 84.50 & 25.07 & 0.18 & Pullout \\
C20.8d.S5 & 20 & 160 & 90 & 166.65 & 72.00 & 29.51 & 1.7 & Pullout \\
Mean & & & & $\mathbf{1 6 6 . 6 5}$ & $\mathbf{8 5 . 2 5}$ & $\mathbf{2 7 . 6 2}$ & $\mathbf{0 . 7 7}$ & \\
C20.0d.H1 & 20 & 100 & 140 & 181.77 & 222.75 & 34.88 & 0.2 & Pullout \\
C20.0d.H2 & 20 & 100 & 140 & 181.77 & 226.25 & 24.23 & 0.83 & Pullout \\
C20.0d.H3 & 20 & 100 & 140 & 181.77 & 200.50 & 23.57 & 0.28 & Pullout \\
C20.0d.H4 & 20 & 100 & 140 & 181.77 & 247.50 & 29.10 & 1.99 & Pullout \\
C20.0d.H5 & 20 & 100 & 140 & 181.77 & 194.75 & 24.18 & 0.42 & Pullout \\
Mean & & & & $\mathbf{1 8 1 . 7 7}$ & $\mathbf{2 1 8 . 3 5}$ & $\mathbf{2 7 . 1 9}$ & $\mathbf{0 . 7 4}$ & \\
\hline Nes: d & & & & &
\end{tabular}

Notes: $\mathrm{d}_{\mathrm{b}}=$ nominal bar diameter; $\mathrm{l}_{\mathrm{e}}=$ bar embedment length; $\mathrm{P}_{\mathrm{ult}}=$ pullout load; $\mathrm{LES}=$ loaded-end slip; FES $=$ free-end slip. 
Table 2.22. Pullout test results for 20M sand-coated GFRP bars with $40 \mathrm{~mm}$ eccentric bar location

\begin{tabular}{|c|c|c|c|c|c|c|c|c|}
\hline Specimen & $d_{b}$ & $l_{e}$ & $\begin{array}{c}\text { Concrete } \\
\text { cover }\end{array}$ & $f_{c}^{\prime}$ & $P_{\text {ult }}$ & LES & FES & $\begin{array}{c}\text { Failure } \\
\text { mode }\end{array}$ \\
\hline & $\mathrm{mm}$ & $\mathrm{mm}$ & $\mathrm{mm}$ & $\mathrm{MPa}$ & $\mathrm{kN}$ & $\mathrm{mm}$ & $\mathrm{mm}$ & \\
\hline E40-20.4d.S1 & 20 & 80 & 40 & 168.54 & 58.00 & 29.75 & 0.36 & Pullout \\
\hline E40-20.4d.S2 & 20 & 80 & 40 & 168.54 & 57.00 & 27.19 & 0.95 & Pullout \\
\hline E40-20.4d.S3 & 20 & 80 & 40 & 168.54 & 47.75 & 26.10 & 1.16 & Pullout \\
\hline E40-20.4d.S4 & 20 & 80 & 40 & 168.54 & 53.00 & 22.74 & 0.57 & Pullout \\
\hline E40-20.4d.S5 & 20 & 80 & 40 & 168.54 & 54.5 & 16.88 & 0.61 & Pullout \\
\hline Mean & & & & 168.54 & 54.05 & 24.53 & 0.73 & \\
\hline E40-20.6d.S1 & 20 & 120 & 40 & 166.65 & 69.75 & 17.03 & 0.37 & Pullout \\
\hline E40-20.6d.S2 & 20 & 120 & 40 & 166.65 & 74.75 & 24.49 & 0.20 & Pullout \\
\hline E40-20.6d.S3 & 20 & 120 & 40 & 166.65 & 68.50 & 21.74 & 0.24 & Pullout \\
\hline E40-20.6d.S4 & 20 & 120 & 40 & 166.65 & 58.00 & 19.36 & 0.74 & Pullout \\
\hline E40-20.6d.S5 & 20 & 120 & 40 & 166.65 & 69.25 & 23.91 & 0.43 & Pullout \\
\hline Mean & & & & 166.65 & 68.05 & 21.31 & 0.40 & \\
\hline E40-20.8d.S1 & 20 & 160 & 40 & 168.99 & 160.50 & 21.69 & 0.46 & Pullout \\
\hline E40-20.8d.S2 & 20 & 160 & 40 & 168.99 & 137.00 & 18.89 & 0.64 & Pullout \\
\hline E40-20.8d.S3 & 20 & 160 & 40 & 168.99 & 154.00 & 42.4 & 0.31 & Pullout \\
\hline E40-20.8d.S4 & 20 & 160 & 40 & 168.99 & 180.00 & 24.62 & 2.13 & Pullout \\
\hline E40-20.8d.S5 & 20 & 160 & 40 & 168.99 & 194.75 & 22.8 & 0.59 & Pullout \\
\hline Mean & & & & 168.99 & 165.25 & 26.08 & 0.83 & \\
\hline E40-20.0d.H1 & 20 & 100 & 40 & 168.07 & 225.25 & 39.38 & 0.31 & Pullout \\
\hline E40-20.0d.H2 & 20 & 100 & 40 & 168.07 & 233.25 & 29.23 & 0.60 & Pullout \\
\hline E40-20.0d.H3 & 20 & 100 & 40 & 168.07 & 211.50 & 25.06 & 0.25 & Pullout \\
\hline E40-20.0d.H4 & 20 & 100 & 40 & 168.07 & 214.25 & 32.78 & 0.20 & Pullout \\
\hline E40-20.0d.H5 & 20 & 100 & 40 & 168.07 & 234.00 & 32.06 & 0.26 & Pullout \\
\hline Mean & & & & 168.07 & 223.65 & 31.70 & 0.32 & \\
\hline E40-20.4d.H1 & 20 & 180 & 40 & 168.07 & 247.00 & 27.00 & 0.00 & $*$ \\
\hline
\end{tabular}


Table 2.23. Pullout test results for 20M sand-coated GFRP bars with $60 \mathrm{~mm}$ eccentric bar location

\begin{tabular}{|c|c|c|c|c|c|c|c|c|}
\hline Specimen & $d_{b}$ & $l_{e}$ & $\begin{array}{l}\text { Concrete } \\
\text { cover }\end{array}$ & $f_{c}^{\prime}$ & $P_{u l t}$ & LES & FES & $\begin{array}{c}\text { Failure } \\
\text { mode }\end{array}$ \\
\hline & $\mathrm{mm}$ & $\mathrm{mm}$ & $\mathrm{mm}$ & $\mathrm{MPa}$ & $\mathrm{kN}$ & $\mathrm{mm}$ & $\mathrm{mm}$ & \\
\hline E60-20.4d.S1 & 20 & 80 & 60 & 168.07 & 79.75 & 11.77 & 0.26 & Pullout \\
\hline E60-20.4d.S2 & 20 & 80 & 60 & 168.07 & 72.75 & 14.44 & 0.45 & Pullout \\
\hline E60-20.4d.S3 & 20 & 80 & 60 & 168.07 & 68.75 & 52.38 & 1.15 & Pullout \\
\hline E60-20.4d.S4 & 20 & 80 & 60 & 168.07 & 72.5 & 44 & 2.41 & Pullout \\
\hline E60-20.4d.S5 & 20 & 80 & 60 & 168.07 & 65.5 & 44.12 & 1.04 & Pullout \\
\hline Mean & & & & 168.07 & 71.85 & 33.34 & 1.06 & \\
\hline
\end{tabular}


Table 2.24. Pullout load - slip modeling for sand-coated GFRP bars

\begin{tabular}{|c|c|c|c|c|c|c|}
\hline Specimen & $P$ & $\delta_{L E S}$ & $\delta_{F E S}$ & $\delta_{A C T}$ & $P-\delta_{A C T}$ Equation & $R^{2}$ \\
\hline C16.4d.S & 68.3 & 13.656 & .834 & 12.822 & $P=0.14 \delta_{a c t}^{2}+3.53 \delta_{a c t}$ & 0.99 \\
\hline C16.6d.S & 53.9 & 12.74 & $\mathbf{0 . 3 3}$ & 12.41 & $P=0.275 \delta_{a c t}^{2}+0.926 \delta_{a c t}$ & 0.99 \\
\hline C16.8d.S & 108.8 & 16.298 & 0.76 & 15.538 & $P=0.333 \delta_{a c t}^{2}+1.83 \delta_{a c t}$ & 1.00 \\
\hline C16.0d.H & 116.6 & 19.4 & 0.00 & 19.4 & $P=0.1864 \delta_{a c t}^{2}+2.391 \delta_{a c t}$ & 0.99 \\
\hline C20.6d.S & 77.7 & 16.28 & 0.226 & 16.054 & $P=0.202 \delta_{a c t}^{2}+1.598 \delta_{a c t}$ & 1.00 \\
\hline C20.8d.S & 85.25 & 27.62 & 0.772 & 26.848 & $P=0.144 \delta_{a c t}^{2}-0.693 \delta_{a c t}$ & 0.99 \\
\hline C20.0d.H & 218.35 & 27.192 & 0.744 & 26.448 & $P=0.085 \delta_{a c t}^{2}+5.993 \delta_{a c t}$ & 0.99 \\
\hline E40-16.4d.S & 43.7 & 20.952 & 0.192 & 20.76 & $P=0.076 \delta_{a c t}^{2}+0.5255 \delta_{a c t}$ & 0.99 \\
\hline E40-16.6d.S & 73.25 & 12.22 & 1.283 & 10.937 & $P=0.341 \delta_{a c t}^{2}+2.965 \delta_{a c t}$ & 0.99 \\
\hline E40-16.8d.S & 149.15 & 20.742 & 0.744 & 19.998 & $P=0.3175 \delta_{a c t}^{2}+1.102 \delta_{a c t}$ & 0.99 \\
\hline E40-16.0d.H & 140 & 26.4 & $\mathbf{0 . 2}$ & 26.2 & $P=0.15246 \delta_{a c t}^{2}+1.349 \delta_{a c t}$ & 0.99 \\
\hline E40-20.4d.S & 54.05 & 24.532 & 0.73 & 23.802 & $P=0.1766 \delta_{a c t}^{2}-1.933 \delta_{a c t}$ & 0.99 \\
\hline E40-20.6d.S & 68.05 & 21.306 & 0.396 & 20.91 & $P=0.1414 \delta_{a c t}^{2}+0.297 \delta_{a c t}$ & 0.99 \\
\hline E40-20.8d.S & 165.25 & 26.08 & 0.826 & 25.254 & $P=0.2394 \delta_{a c t}^{2}+0.4958 \delta_{a c t}$ & 0.99 \\
\hline E40-20.0d.H & 223.65 & 31.65 & 0.324 & 31.326 & $P=0.1244 \delta_{a c t}^{2}+3.24 \delta_{a c t}$ & 0.99 \\
\hline E40-20.4d.H & 247 & 27 & 0 & 27 & $P=.15174 \delta_{a c t}^{2}+5.051 \delta_{a c t}$ & 0.99 \\
\hline E60-16.4d.S & 40.9 & 20.49 & 0.352 & 20.138 & $P=0.143 \delta_{a c t}^{2}-0.855 \delta_{a c t}$ & 0.99 \\
\hline E60-16.6d.S & 67.45 & 26.092 & 0.706 & 25.386 & $P=0.1709 \delta_{a c t}^{2}-1.682 \delta_{a c t}$ & 0.99 \\
\hline E60-16.8d.S & 149.4 & 24.65 & 0.318 & 24.332 & $P=0.1563 \delta_{a c t}^{2}+2.336 \delta_{a c t}$ & 0.99 \\
\hline E60-16.0d.H & 142.05 & 28.658 & 1.232 & 27.426 & $P=0.1617 \delta_{a c t}^{2}+0.744 \delta_{a c t}$ & 0.99 \\
\hline E60-20.4d.S & 71.85 & 33.342 & 1.062 & 32.28 & $P=0.0571 \delta_{a c t}^{2}+0.38 \delta_{a c t}$ & 0.99 \\
\hline
\end{tabular}

Notes: $P=$ ultimate pullout load; $\delta_{L E S}=$ displacement at the load-end slip; $\delta_{F E S}=$ displacement at the free-end slip; $\delta_{A C T}=$ actual displacement; $R^{2}=$ coefficient of determination 
Table 2.25. Bond stress and experimental coefficients for sand-coated GFRP bars

\begin{tabular}{|c|c|c|c|c|c|}
\hline Specimen & $\frac{l_{e}}{d_{b}}$ & $u$ & $\frac{u}{\sqrt{f_{c}^{\prime}}}$ & $\boldsymbol{B}=k \cdot \varphi$ & $\varphi$ \\
\hline C16.4d.S & 4 & 21.23 & 1.64 & 4.018 & 0.29 \\
\hline C16.6d.S & 6 & 11.17 & 0.86 & 6.044 & 0.23 \\
\hline C16.8d.S & 8 & 16.91 & 1.30 & 8.081 & 0.46 \\
\hline C16.0d.H & 6.25 & 23.20 & 1.79 & 9.704 & 0.49 \\
\hline C20.6d.S & 6 & 10.31 & 0.80 & 6.309 & 0.22 \\
\hline C20.8d.S & 8 & 8.48 & 0.66 & 8.411 & 0.25 \\
\hline C20.0d.H & 5 & 34.75 & 2.58 & 8.541 & 0.63 \\
\hline E40-16.4d.S & 4 & 13.58 & 1.04 & 1.759 & 0.18 \\
\hline E40-16.6d.S & 6 & 9.11 & 0.66 & 2.783 & 0.18 \\
\hline E40-16.8d.S & 8 & 23.18 & 1.69 & 3.711 & 0.63 \\
\hline E40-16.0d.H & 6.25 & 27.85 & 2.15 & 2.730 & 0.59 \\
\hline E40-20.4d.S & 4 & 10.75 & 0.83 & 1.880 & 0.16 \\
\hline E40-20.6d.S & 6 & 9.02 & 0.70 & 2.804 & 0.12 \\
\hline E40-20.8d.S & 8 & 16.44 & 1.26 & 3.765 & 0.48 \\
\hline E40-20.0d.H & 5 & 35.60 & 2.75 & 2.346 & 0.64 \\
\hline E40-20.4d.H & 9 & 21.84 & 1.68 & 4.224 & 0.71 \\
\hline E60-16.4d.S & 4 & 12.71 & 0.98 & 2.632 & 0.17 \\
\hline E60-16.6d.S & 6 & 13.98 & 1.08 & 3.947 & 0.28 \\
\hline E60-16.8d.S & 8 & 23.22 & 1.69 & 5.584 & 0.63 \\
\hline E60-16.0d.H & 6.25 & 28.26 & 2.17 & 4.123 & 0.60 \\
\hline E60-20.4d.S & 4 & 14.29 & 0.28 & 2.816 & 1.10 \\
\hline
\end{tabular}

Notes: $l_{e}=$ embedment length; $c_{\min }=$ minimum concrete cover; $d_{b}=$ bar diameter; $f_{c}^{\prime}=$ compressive strength of concrete; $u=$ bond stress; $k=$ bond empirical constant; $\varphi=$ bar stress ratio to its ultimate stress. 
Table 2.26. Pullout load versus the embedment length equations for sand-coated GFRP bars

\begin{tabular}{lcc}
\hline Group & Equation $P$ versus $\frac{l_{e}}{d_{b}}$ & $\boldsymbol{R}^{2}$ \\
\hline C16.S & $P=8.6625\left(\frac{l_{e}}{d_{b}}\right)^{2}-93.825\left(\frac{l_{e}}{d_{b}}\right)+305$ & 1 \\
\hline E40-16.S & $P=5.7938\left(\frac{l_{e}}{d_{b}}\right)^{2}-43.163\left(\frac{l_{e}}{d_{b}}\right)+123.65$ & 1 \\
\hline E60-16.S & $P=6.925\left(\frac{l_{e}}{d_{b}}\right)^{2}-55.975\left(\frac{l_{e}}{d_{b}}\right)+154$ & 1 \\
\hline C20.S & $P=3.775\left(\frac{l_{e}}{d_{b}}\right)+55.05$ & 1 \\
\hline E40-20.S & $P=10.4\left(\frac{l_{e}}{d_{b}}\right)^{2}-97\left(\frac{l_{e}}{d_{b}}\right)+275.65$ & 1 \\
\hline
\end{tabular}

Notes: $P=$ ultimate pullout load; $l_{e}=$ embedment length; $d_{b}=$ bar diameter; $R^{2}=$ coefficient of determination.

Table 2.27. Bond stress versus the embedment length equations for sand-coated GFRP bars

\begin{tabular}{llc}
\hline Group & \multicolumn{1}{c}{ Equation $\frac{u}{\sqrt{f_{c}^{\prime}}}$ versus $\frac{l_{e}}{\boldsymbol{d}_{b}}$} & $\boldsymbol{R}^{2}$ \\
\hline C16.S & $\frac{u}{\sqrt{f_{c}^{\prime}}}=0.1525\left(\frac{l_{e}}{d_{b}}\right)^{2}-1.9152\left(\frac{l_{e}}{d_{b}}\right)+6.8633$ & 1 \\
\hline E40-16.S & $\frac{u}{\sqrt{f_{c}^{\prime}}}=0.1756\left(\frac{l_{e}}{d_{b}}\right)^{2}-1.9466\left(\frac{l_{e}}{d_{b}}\right)-6.0196$ & 1 \\
\hline E60-16.S & $\frac{u}{\sqrt{f_{c}^{\prime}}}=0.064\left(\frac{l_{e}}{d_{b}}\right)^{2}-0.5912\left(\frac{l_{e}}{d_{b}}\right)+2.3203$ & 1 \\
\hline C20.S & $\frac{u}{\sqrt{f_{c}^{\prime}}}=-0.0707\left(\frac{l_{e}}{d_{b}}\right)+1.2225$ & 1 \\
\hline E40-20.S & $\frac{u}{\sqrt{f_{c}^{\prime}}}=0.0868\left(\frac{l_{e}}{d_{b}}\right)^{2}-0.9327\left(\frac{l_{e}}{d_{b}}\right)+3.17$ & 1 \\
\hline
\end{tabular}

Notes: $u=$ bond stress; $f_{c}^{\prime}=$ compressive strength of concrete; $l_{e}=$ embedment length; $d_{b}=$ bar diameter; $R^{2}=$ coefficient of determination. 
Table 2.28. Concrete and reinforcement location factors for sand-coated GFRP bars

\begin{tabular}{lcc}
\hline Group & Equation $B=k . \varphi$ versus $\frac{l_{e}}{d_{b}}$ & $\boldsymbol{R}^{2}$ \\
\hline C16.S & $B=0.10085\left(\frac{l_{e}}{d_{b}}\right)$ & 0.99 \\
\hline E40-16.S & $B=0.04606\left(\frac{l_{e}}{d_{b}}\right)$ & 0.99 \\
\hline E60-16.S & $B=0.68\left(\frac{l_{e}}{d_{b}}\right)$ & 0.99 \\
\hline C20.S & $B=0.1052\left(\frac{l_{e}}{d_{b}}\right)-6 \times 10^{-14}$ & 1 \\
\hline E40-20.S & $B=0.04695\left(\frac{l_{e}}{d_{b}}\right)$ & 0.99 \\
\hline
\end{tabular}

Notes: $k=$ bond empirical constant; $\varphi=$ bar stress ratio to its ultimate stress; $l_{e}=$ embedment length; $d_{b}=$ bar diameter; $\mathrm{B}=$ compound empirical constant; $R^{2}=$ coefficient of determination

Table 2.29. Reinforcement location factor for sand-coated GFRP bars

\begin{tabular}{lcc}
\hline Group & Equation $\varphi$ versus $\frac{l_{e}}{d_{b}}$ & $\boldsymbol{R}^{2}$ \\
\hline C16.S & $\varphi=0.0364\left(\frac{l_{e}}{d_{b}}\right)^{2}-0.3941\left(\frac{l_{e}}{d_{b}}\right)+1.2812$ & 1 \\
\hline E40-16.S & $\varphi=0.0551\left(\frac{l_{e}}{d_{b}}\right)^{2}-0.5506\left(\frac{l_{e}}{d_{b}}\right)+1.504$ & 1 \\
\hline E60-16.S & $\varphi=0.0291\left(\frac{l_{e}}{d_{b}}\right)^{2}-0.2351\left(\frac{l_{e}}{d_{b}}\right)+0.6469$ & 1 \\
\hline C20.S & $\varphi=0.0109\left(\frac{l_{e}}{d_{b}}\right)+0.1586$ & 1 \\
\hline E40-20.S & $\varphi=0.03\left(\frac{l_{e}}{d_{b}}\right)^{2}-0.2794\left(\frac{l_{e}}{d_{b}}\right)+0.794$ & 1 \\
\hline
\end{tabular}

Notes: $\varphi=$ bar stress ratio to its ultimate stress; $l_{e}=$ embedment length; $d_{b}=$ bar diameter; $R^{2}=$ coefficient of determination 
Table 2.30. ACI basic factor $k_{\text {exp }}$ for the sand-coated HM GFRP bars

\begin{tabular}{|c|c|c|c|c|}
\hline Loading location & Bar type* & Bar diameter, $d_{b}$ & $l_{e} / d_{b} * *$ & $k_{\exp } * * *$ \\
\hline \multirow{4}{*}{ Centric } & $\mathrm{S}$ & 16 & 4 & 0.00353 \\
\hline & $\mathrm{S}$ & 16 & 6 & 0.00531 \\
\hline & $\mathrm{S}$ & 16 & 8 & 0.0071 \\
\hline & $\mathrm{H}$ & 16 & 6.26 & 0.00552 \\
\hline \multirow{4}{*}{ Eccentric at $40 \mathrm{~mm}$ concrete cover } & $\mathrm{S}$ & 16 & 4 & 0.0035 \\
\hline & $\mathrm{S}$ & 16 & 6 & 0.00562 \\
\hline & $\mathrm{S}$ & 16 & 8 & 0.0075 \\
\hline & $\mathrm{H}$ & 16 & 6.25 & 0.00551 \\
\hline \multirow{4}{*}{ Eccentric at $60 \mathrm{~mm}$ concrete cover } & $\mathrm{S}$ & 16 & 4 & 0.00354 \\
\hline & $\mathrm{S}$ & 16 & 6 & 0.00532 \\
\hline & $\mathrm{S}$ & 16 & 8 & 0.00752 \\
\hline & $\mathrm{H}$ & 16 & 6.25 & 0.0055 \\
\hline \multirow{3}{*}{ Centric } & $\mathrm{S}$ & 20 & 6 & 0.00492 \\
\hline & S & 20 & 8 & 0.00656 \\
\hline & $\mathrm{H}$ & 20 & 5 & 0.00428 \\
\hline \multirow{4}{*}{ Eccentric at $40 \mathrm{~mm}$ concrete cover } & S & 20 & 4 & 0.0033 \\
\hline & $\mathrm{S}$ & 20 & 6 & 0.00492 \\
\hline & $\mathrm{S}$ & 20 & 8 & 0.0066 \\
\hline & $\mathrm{H}$ & 20 & 5 & 0.00412 \\
\hline Eccentric at $60 \mathrm{~mm}$ concrete cover & $\mathrm{S}$ & 20 & 4 & 0.00329 \\
\hline
\end{tabular}

$* \mathrm{~S}=$ bar with straight end; $\mathrm{H}=$ bar with headed end

$* * l_{e}=$ embedment length; $d_{b}=$ bar diameter

$* * * k_{\text {exp }}=$ experimental basic factor

Table 2.31. Bond factors, $\mathrm{k}$, and development length, $1_{\mathrm{d}}$, of GFRP bars embedded into UHPFRC

\begin{tabular}{ccccccc}
\hline Type & Size & $\begin{array}{c}\text { Bar } \\
\text { diameter, } \\
\text { mm }\end{array}$ & $\begin{array}{c}f_{\text {frp }, u}, \\
\mathrm{MPa}\end{array}$ & $k$ & \multicolumn{2}{c}{$f_{c}^{\prime}=140 \mathrm{MPa}$} \\
\hline & & \multicolumn{5}{c}{ Ribbed-Surface GFRP Bar } \\
\hline Straight & $15 \mathrm{M}$ & 16 & 1188 & 0.00743 & 150 & -- \\
\hline Straight & $20 \mathrm{M}$ & 20 & 1188 & 0.00594 & 190 & -- \\
\hline Headed & $15 \mathrm{M}$ & 16 & 1188 & 82 & -- & 111 \\
\hline & & \multicolumn{5}{c}{ Sand-Coated GFRP Bar } \\
\hline Straight & $15 \mathrm{M}$ & 15.9 & 1184 & 0.00752 & 150 & -- \\
\hline Straight & $20 \mathrm{M}$ & 19.0 & 1105 & 0.0066 & 176 & -- \\
\hline Headed & $15 \mathrm{M}$ & 15.9 & 1184 & 82 & -- & 110 \\
\hline Headed & $20 \mathrm{M}$ & 19.0 & 1105 & 71 & -- & 114 \\
\hline
\end{tabular}

Note: $l_{d}$ should not be less than $9.5 d_{b}$ or $150 \mathrm{~mm}$, whichever is greater

And $l_{h b}$ should not be less than $7 d_{b}$ or $111 \mathrm{~mm}$, whichever is greater 


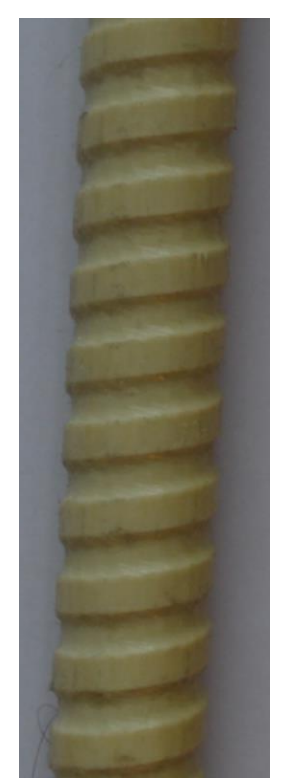

a) Ribbed-surface bar

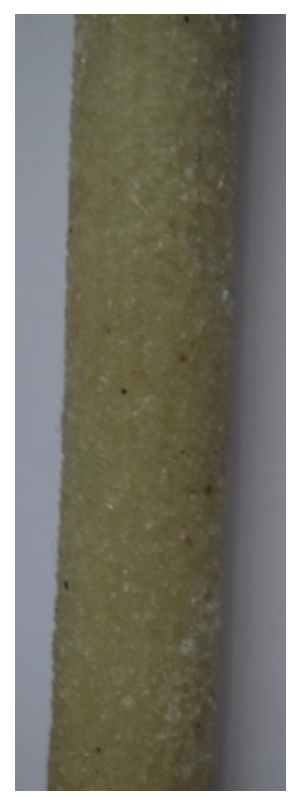

a) Sand-coated bar

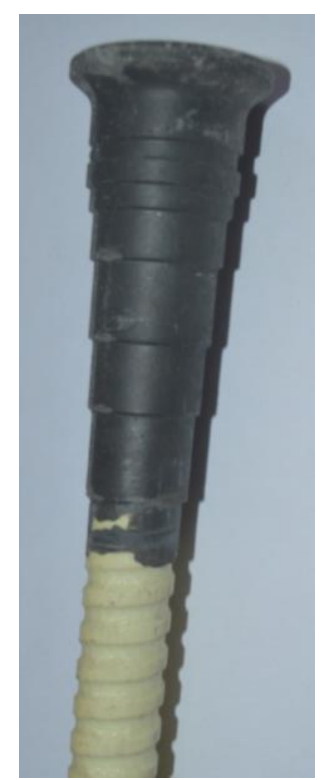

b) Headed-end bar

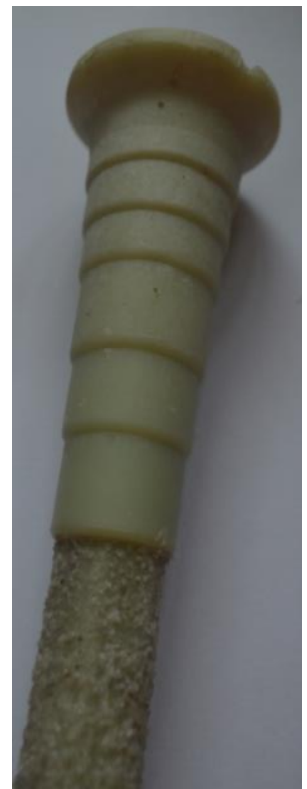

b) Headed-end bar

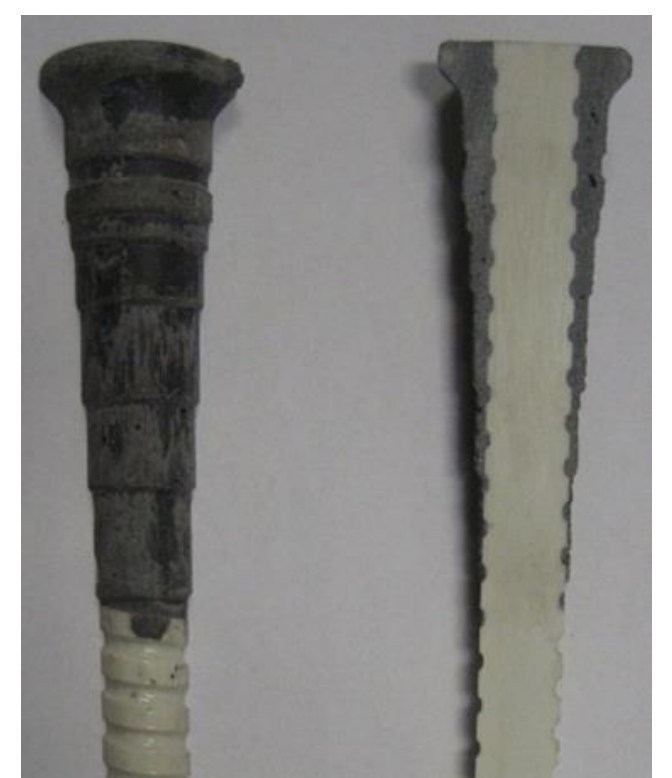

c) Sliced head-end bar showing ribs interacting with bar head material

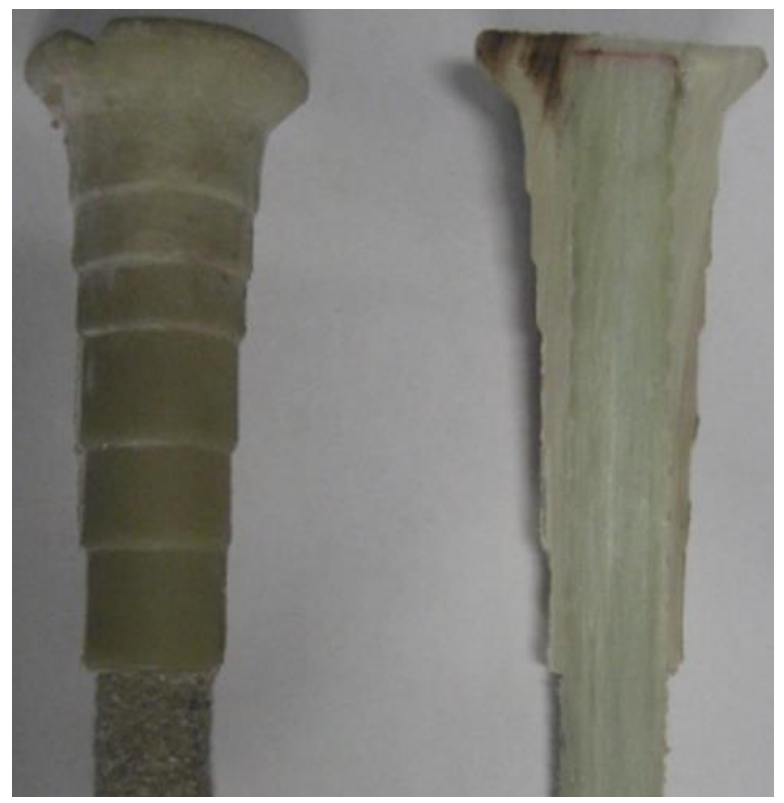

c) Sliced head-end, sand-coated, bar showing the grooves on bar surface filled will head material

Figure 2.1. Ribbed-surface and sand-coated GFRP bars considered in this study 

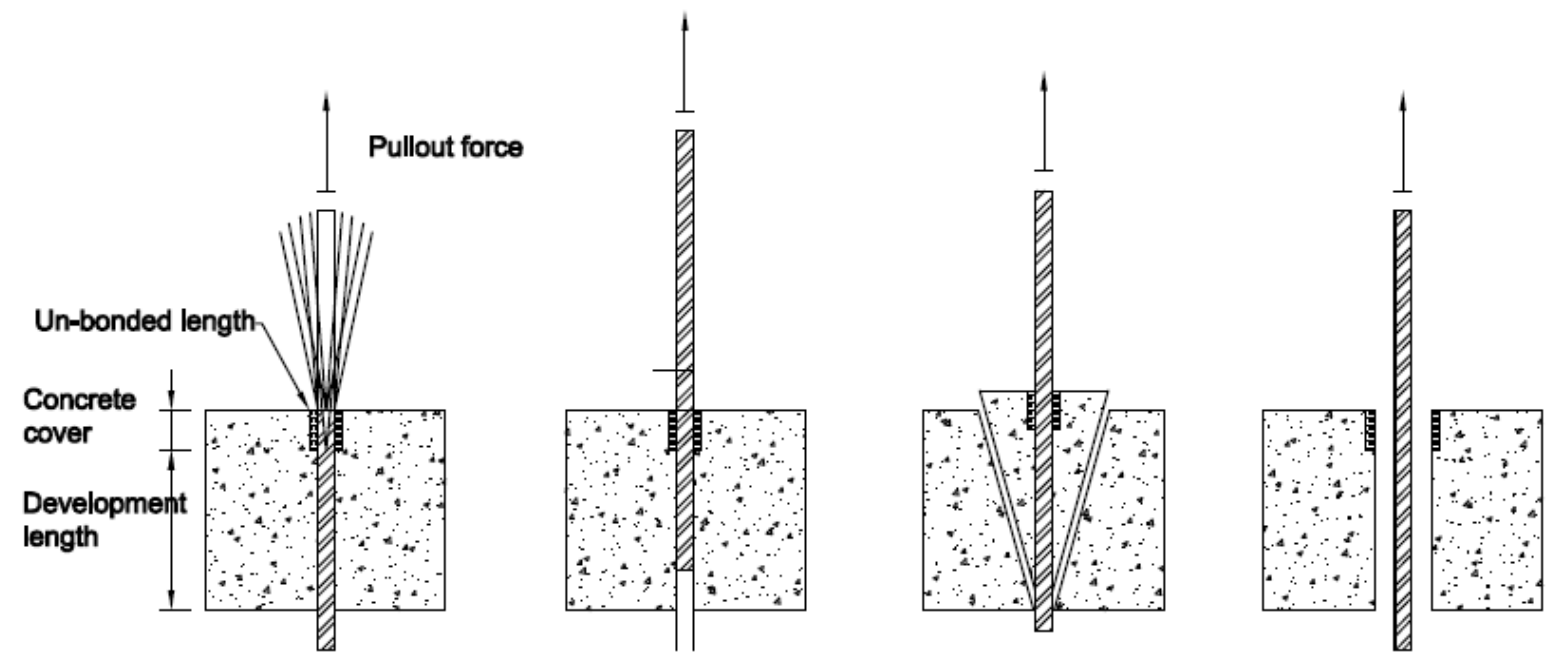
a) Bar rupture
b) Bar pullout
c) Concrete breakout
d) Concrete Splitting

Figure 2.2. Possible failure modes of GFRP bar embedded in concrete

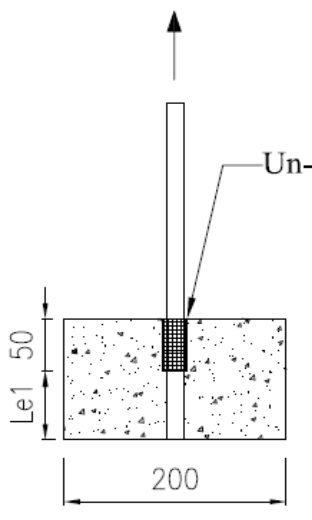

a) Straight bar

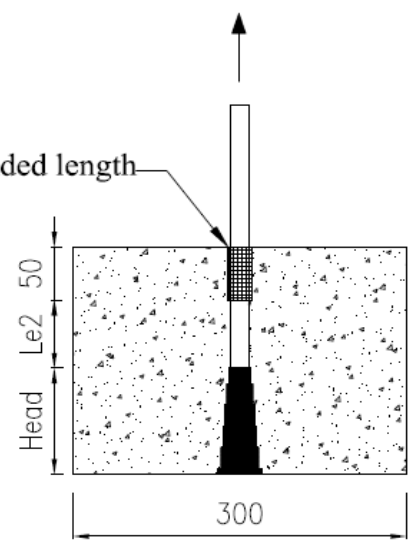

b) Headed bar

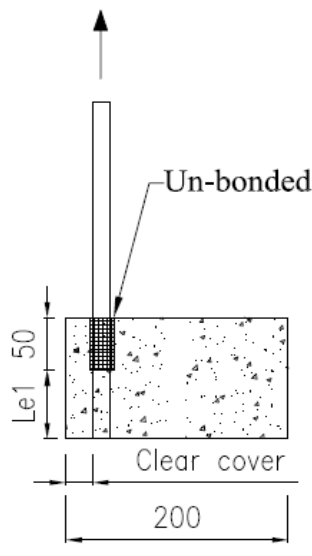

a) Straight bar

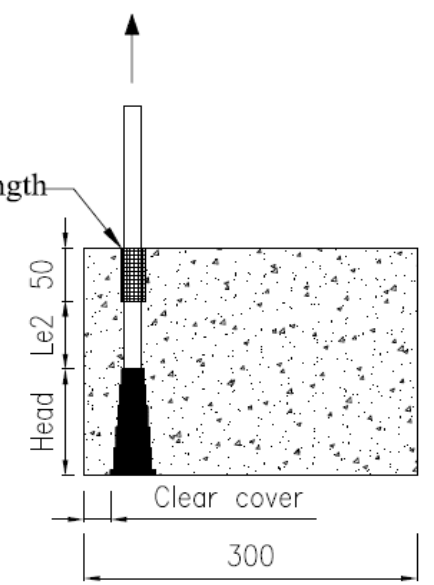

b) Headed bar

Figure 2.3. Concentric and eccentric cube specimens 


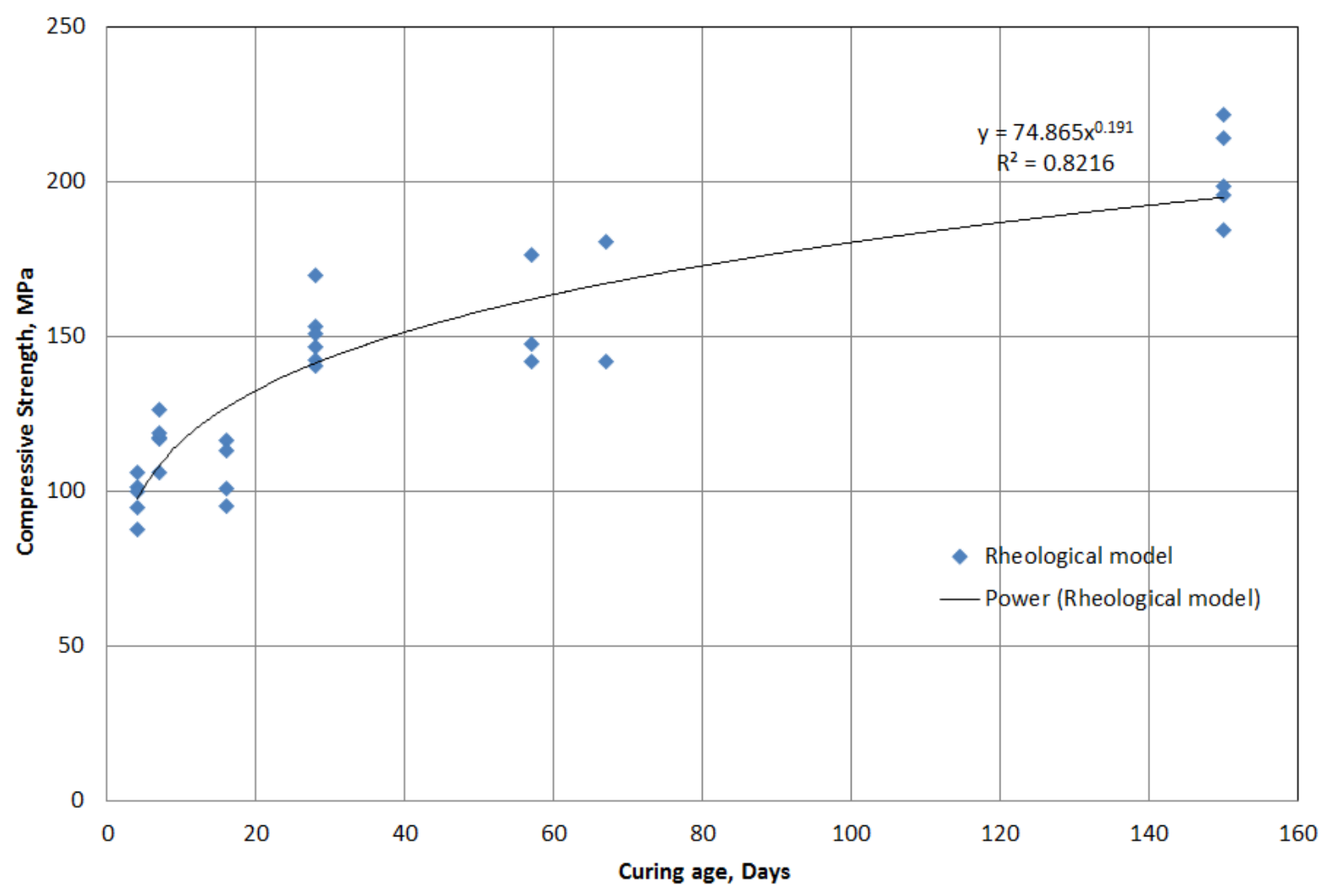

Figure 2.4. Rheological model for the UHPC (Ductal JS1000) 


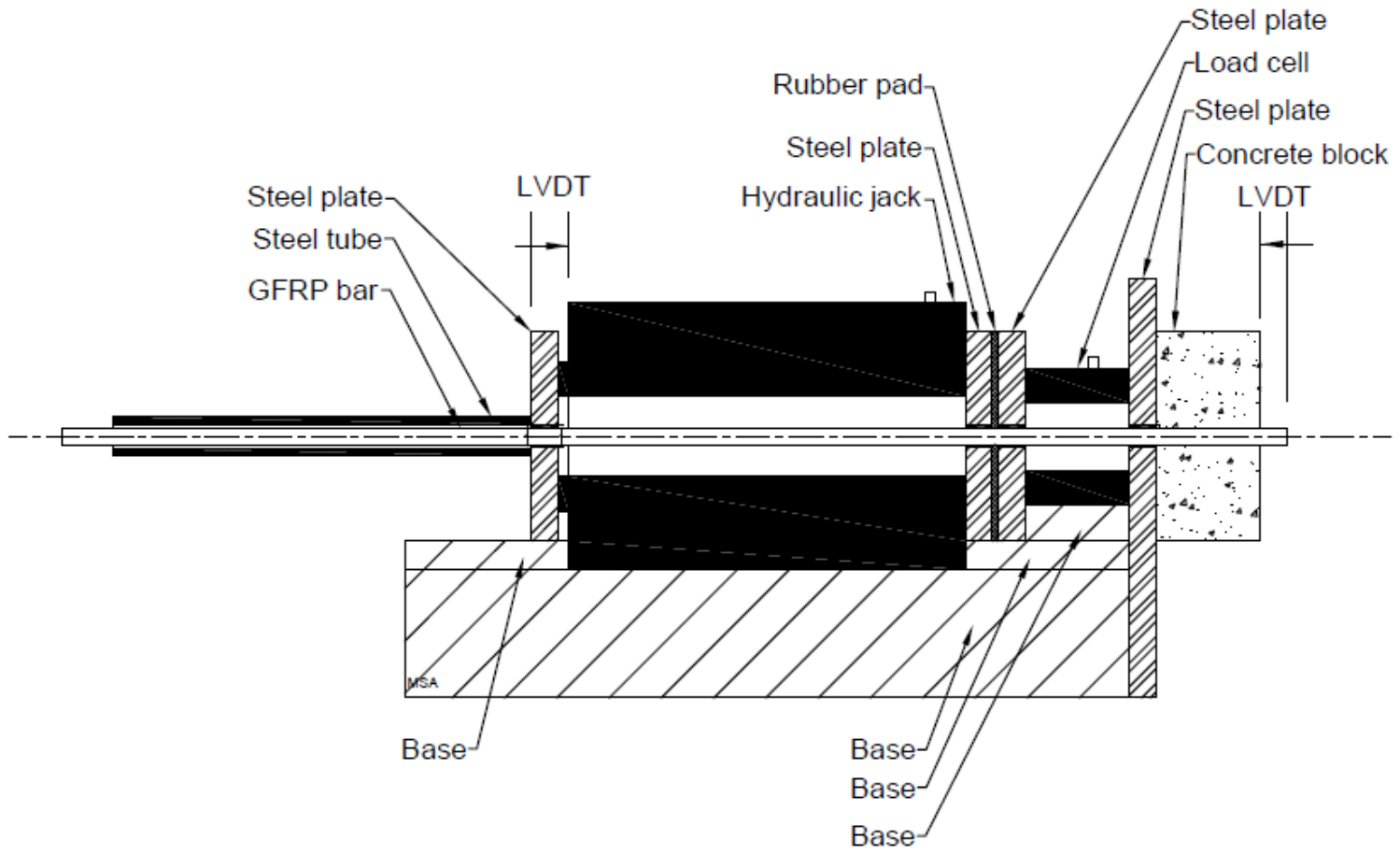

a. Detailed test setup

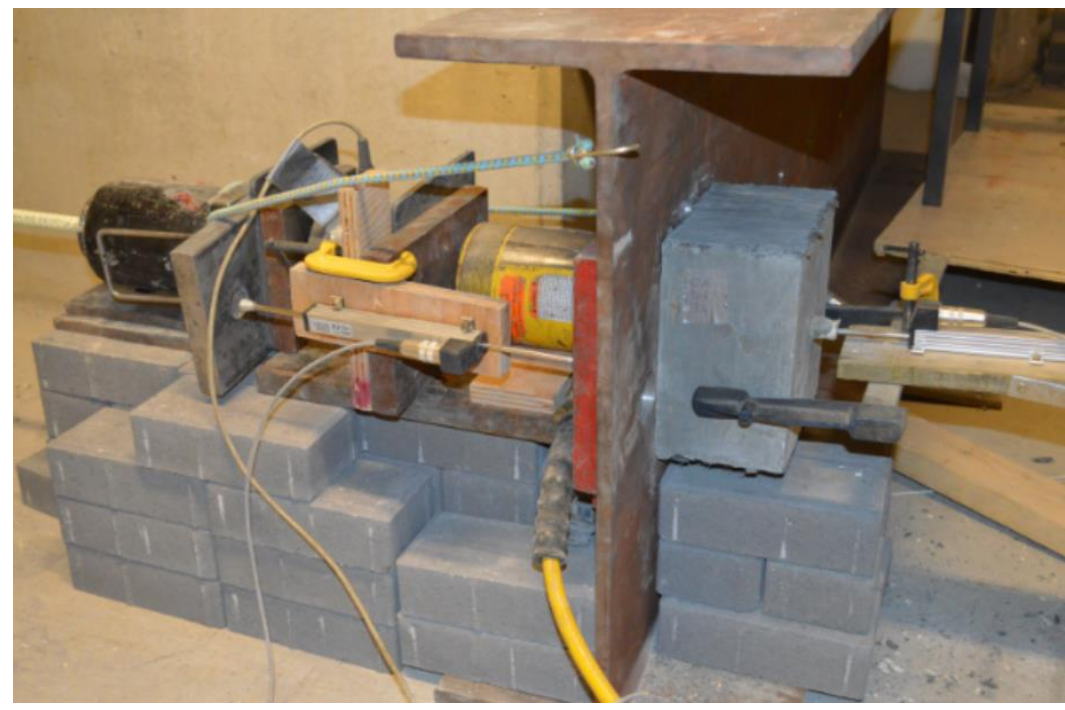

b. View of a typical test setup

Figure 2.5. Pullout test setup

(LES: loaded-end slip; FES: free-end slip)

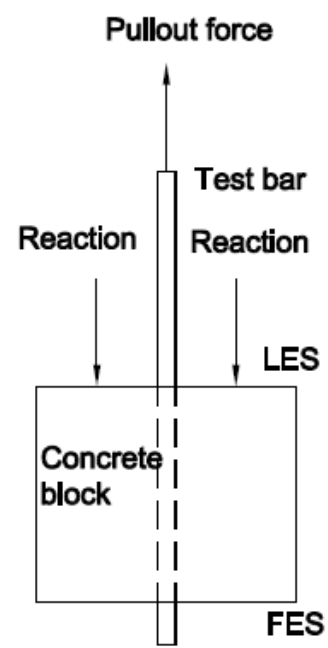

c. Cube forces and slip measurement locations 


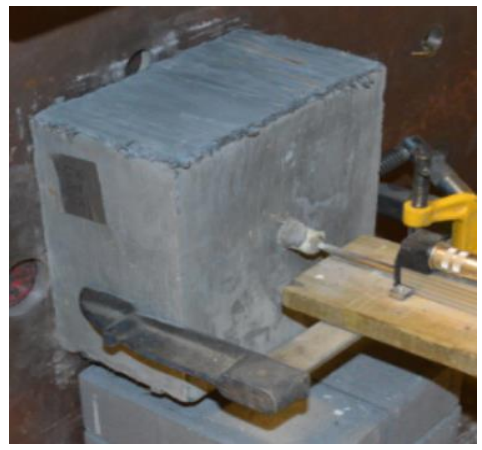

a) Test setup

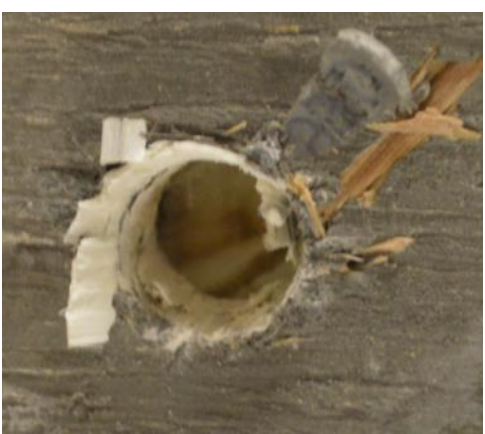

d) Close-up view of bar slip at free end

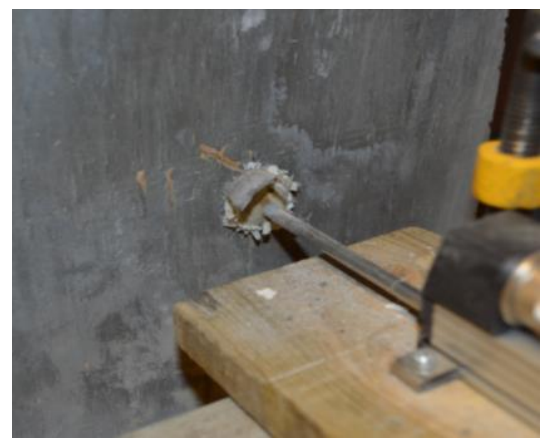

b) Block view at bar end slip

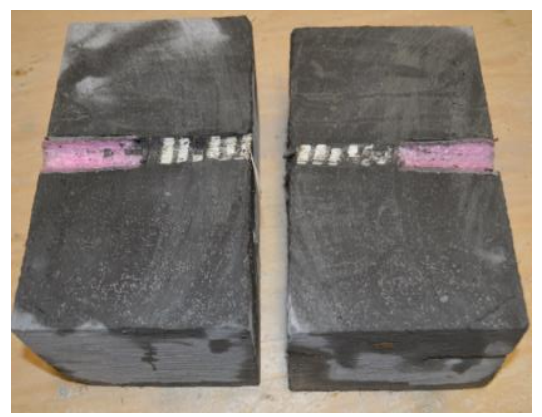

e) Section cut in the UHPC cube at bar location

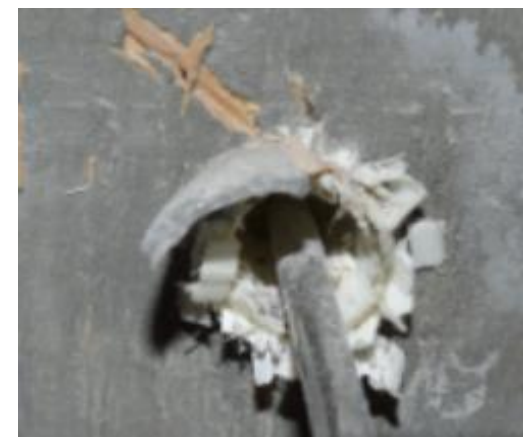

c) View of sheared lugs

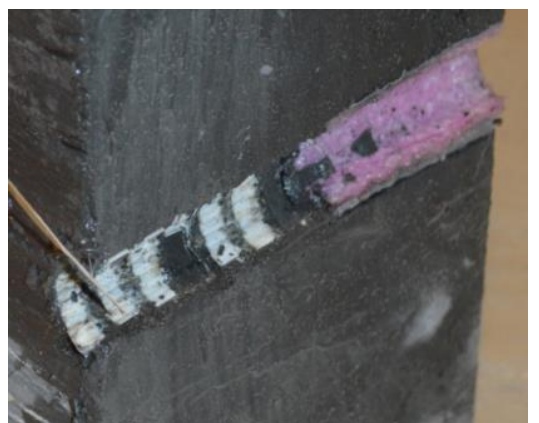

f) Close-up view of sheared ribs

Figure 2.6. Typical failure of the $15 \mathrm{M}$ ribbed-surface bar with embedment length equal 4 times the diameter showing bar slip and sheared lugs 


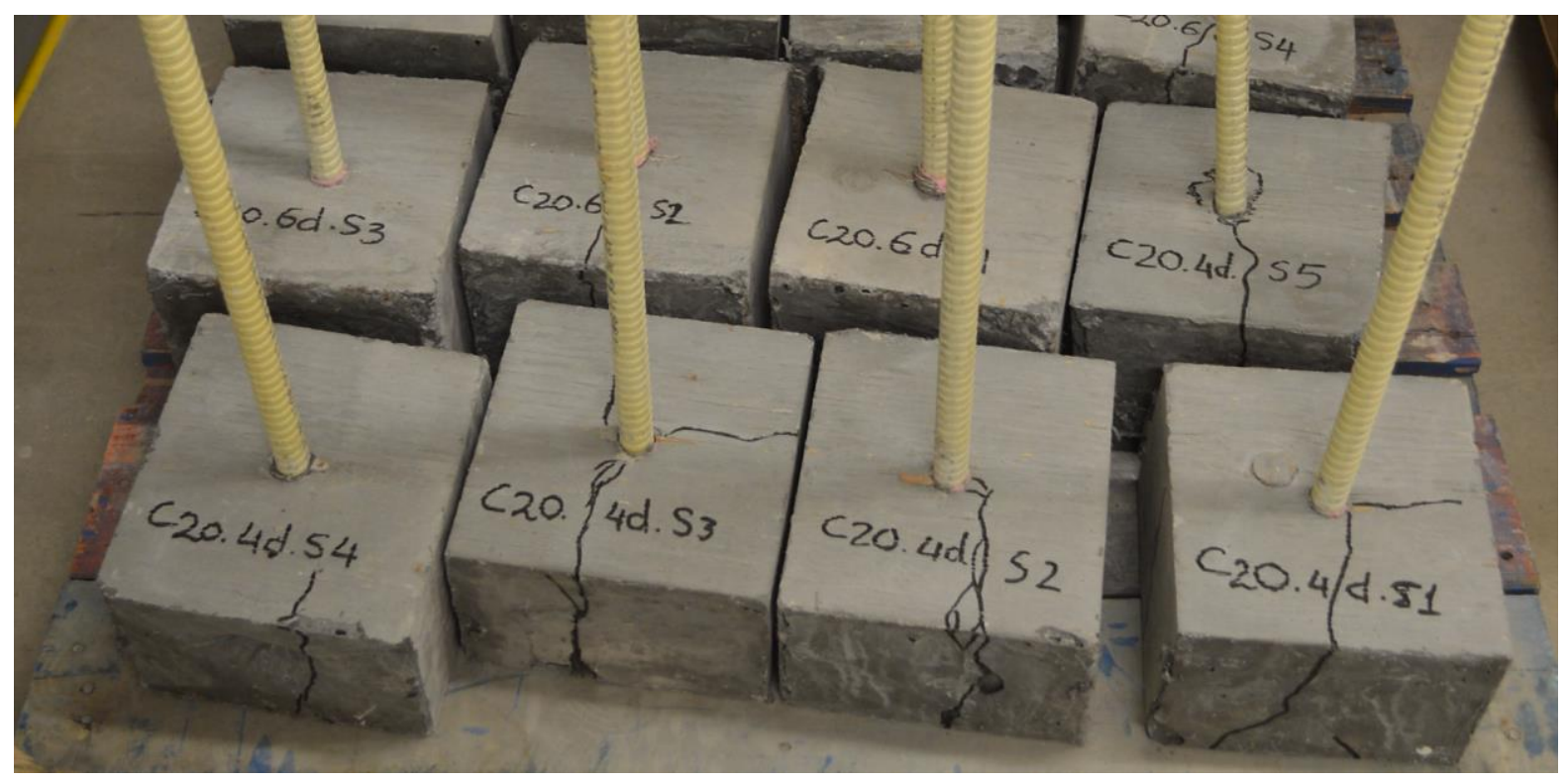

Figure 2.7. Views of splitting cracks after pullout test for the 20M ribbed-surface GFRP bars with embedment length of 4 and 6 times the bar diameters 


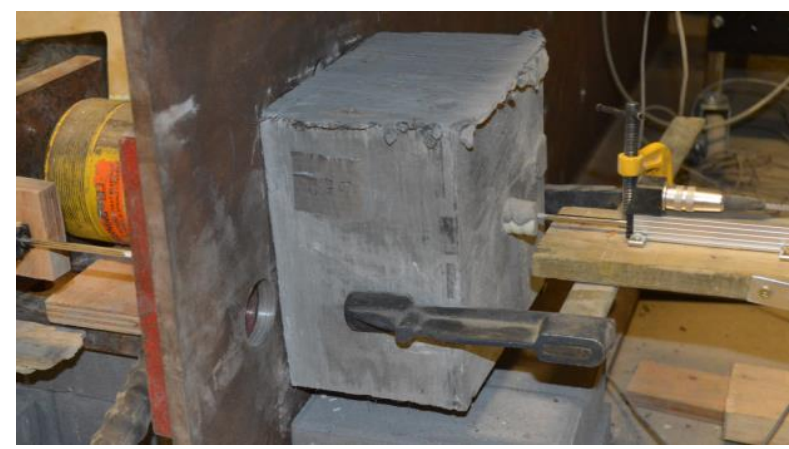

a) Test setup

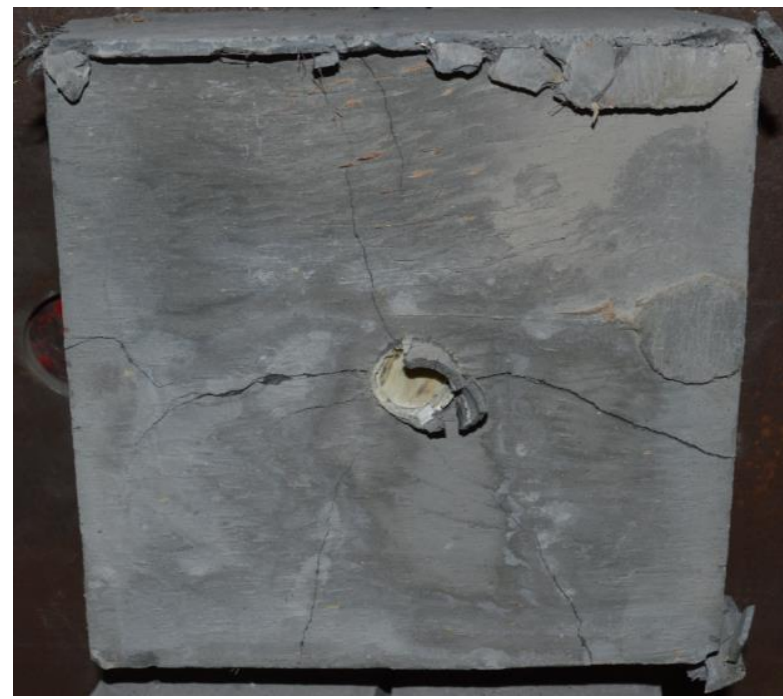

c) Splitting Cracks at free end of the UHPC block after bar pullout

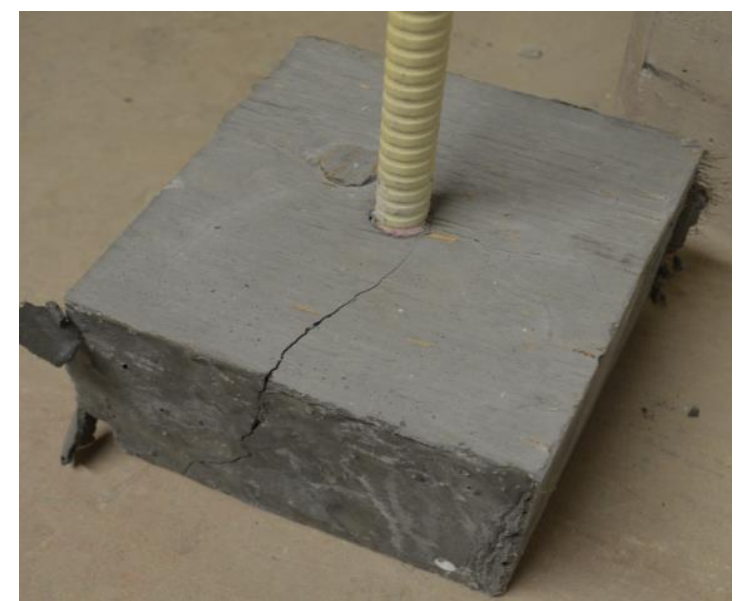

b) Concrete crack at top and side of the UHPC block after bar pullout

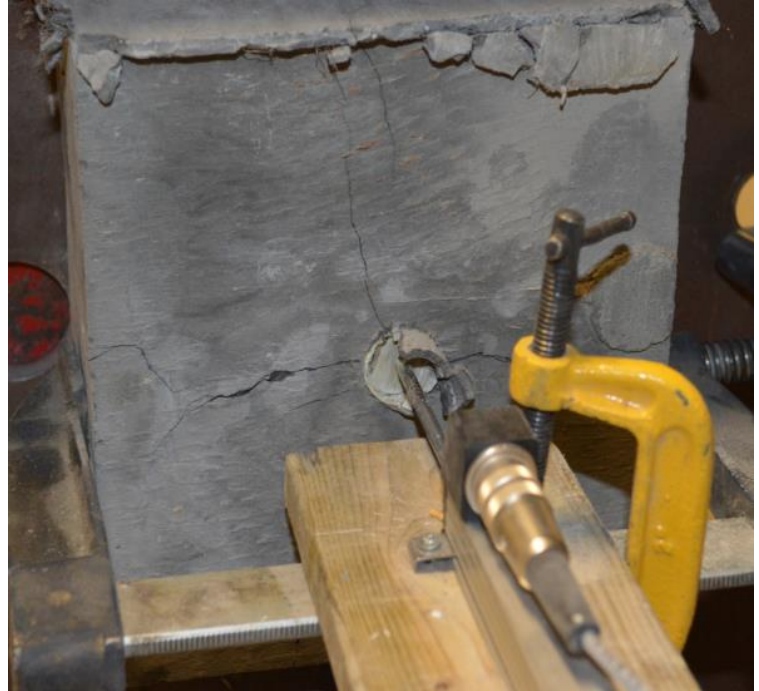

d) Close-up view of the bar pullout and splitting cracks

Figure 2.8. Typical pullout failure of the 20M ribbed surface GFRP bar with embedment length of 4 times the bar diameter 


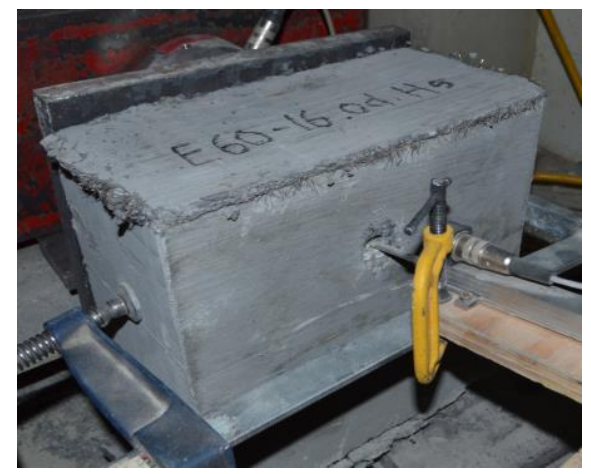

a) Test setup

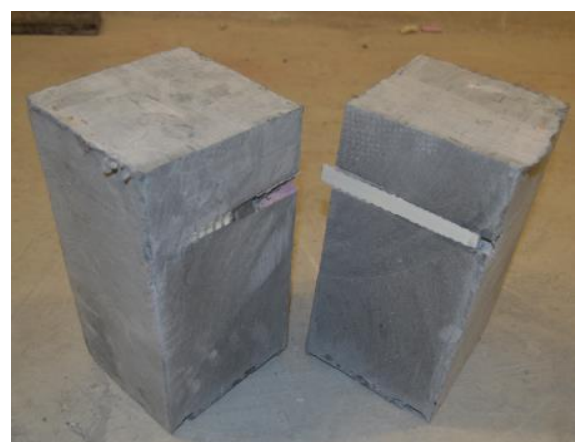

d) Section cut at bar location

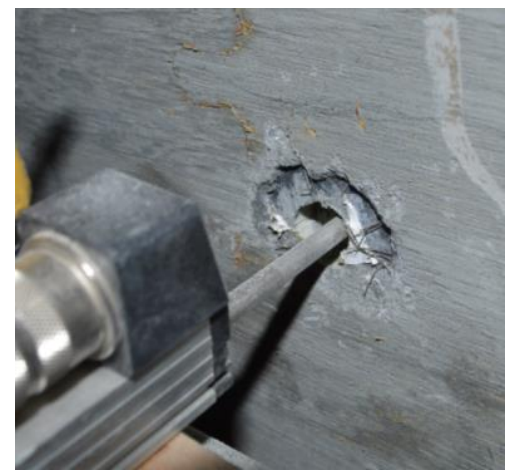

b) Bar slippage

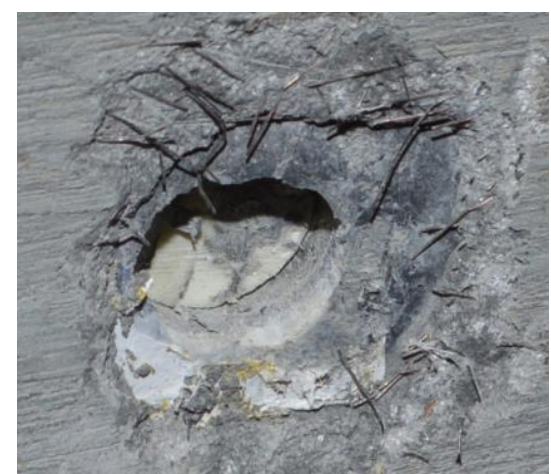

c) Close-up view of bar slip at its end

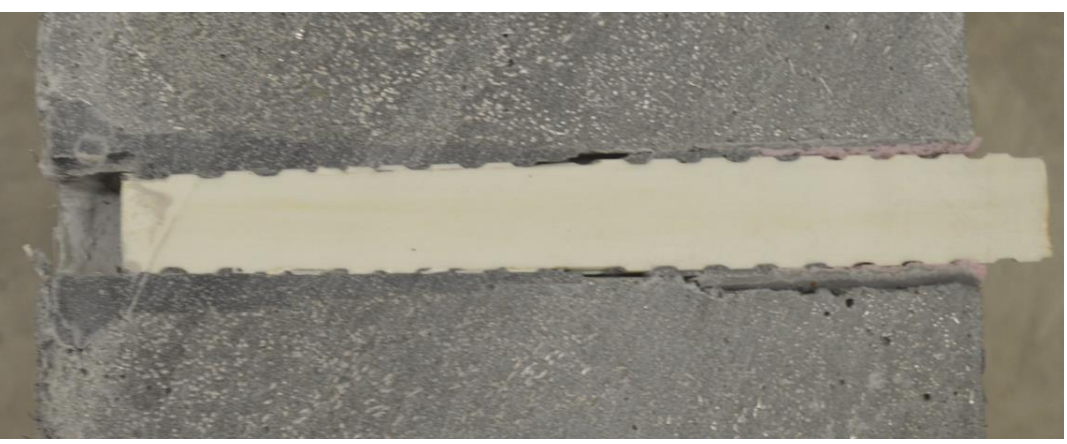

e) Close-up view for the bar slippage from its black head

Figure 2.9. Typical bar slippage failure from its anchorage head for the $15 \mathrm{M}$ ribbed surface GFRP bar embedded in UHPC block with $60 \mathrm{~mm}$ concrete cover 


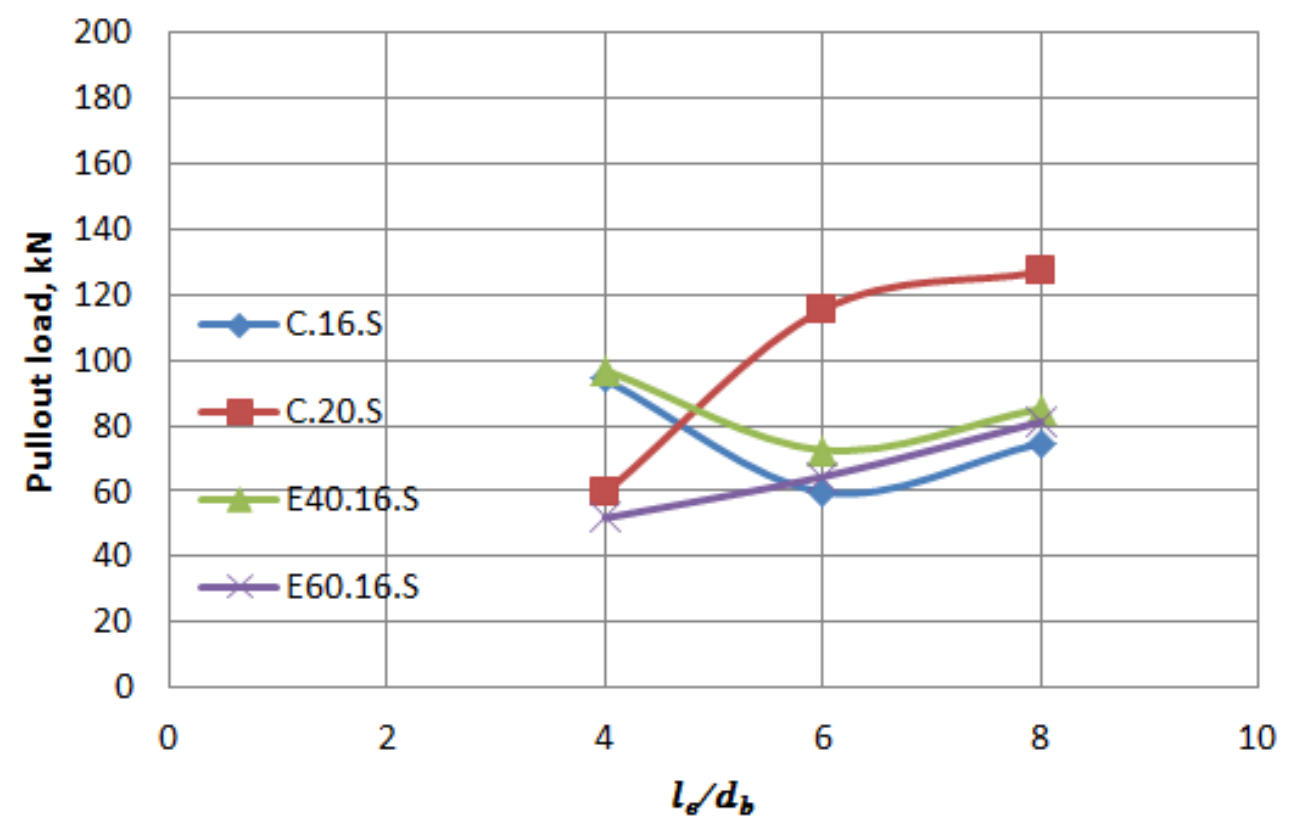

Figure 2.10. Experimental pullout load for the ribbed-surface GFRP bars embedded in UHPC cube specimens

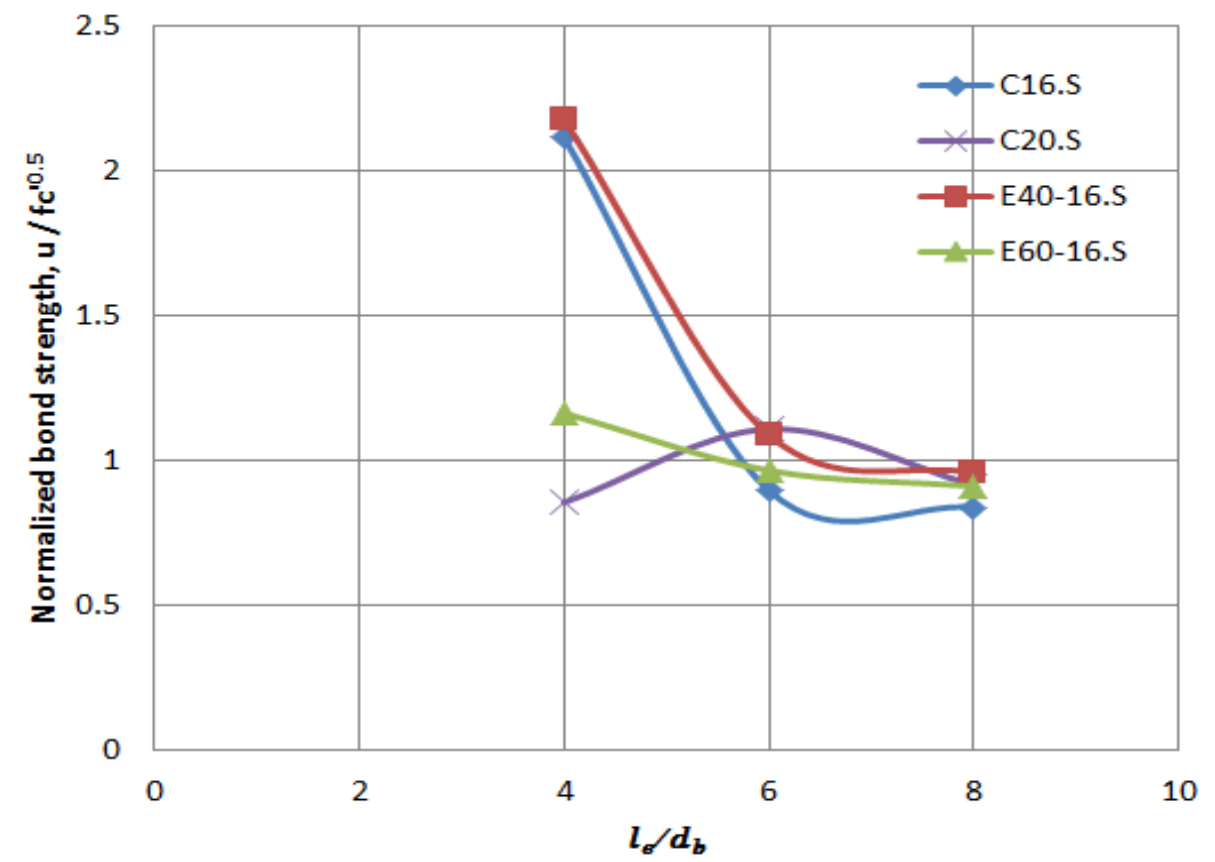

Figure 2.11. Normalized bond stress, $\mu$, for the ribbed-surface GFRP bars embedded in UHPC cube specimens 


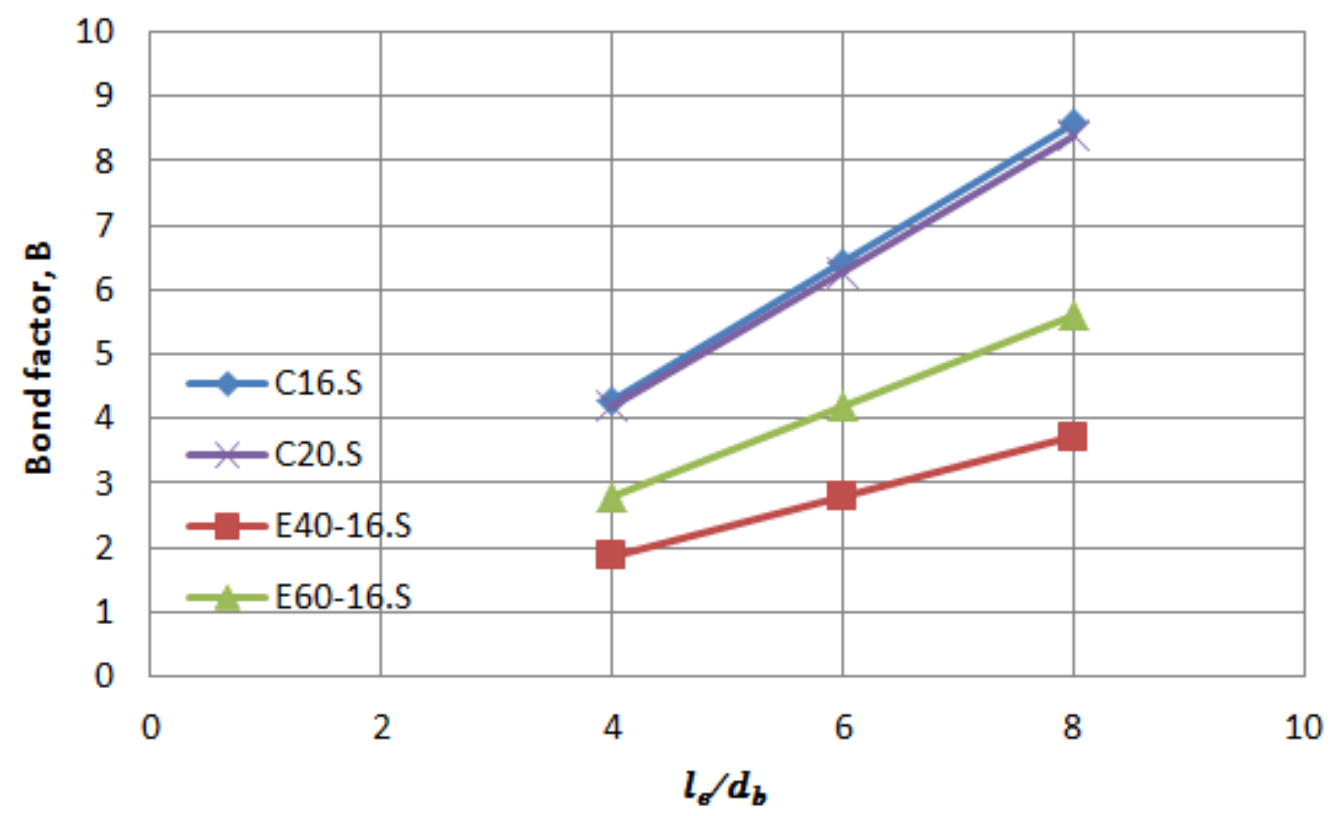

Figure 2.12. Combined bond factor, B, for the ribbed-surface GFRP bars

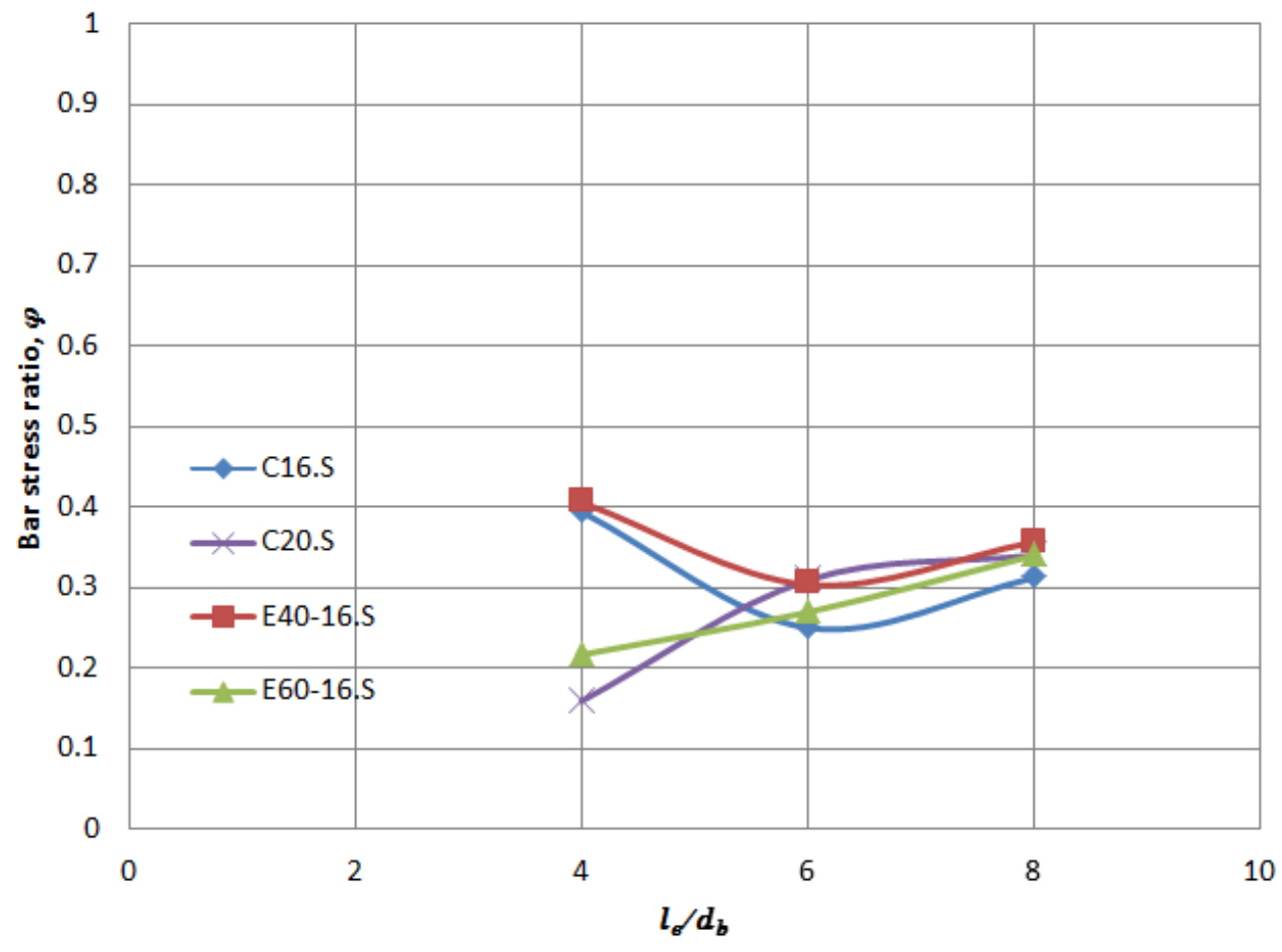

Figure 2.13. Bar stress ratio, $\varphi$, for the ribbed-surface GFRP bars 


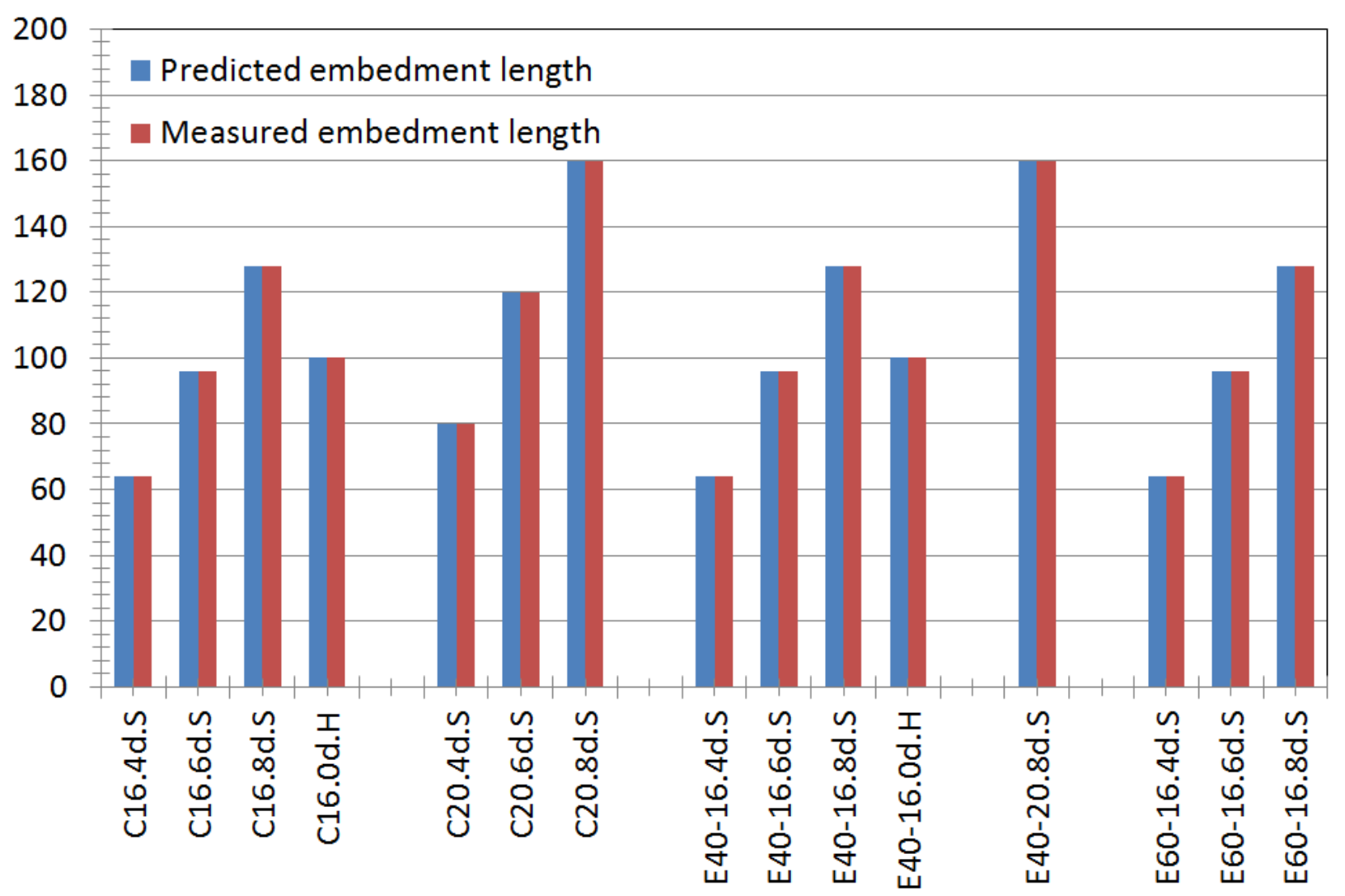

a) Comparison between predicted and experimentally measured embedment length for the ribbedsurface GFRP bar, based on Equation 2.12 


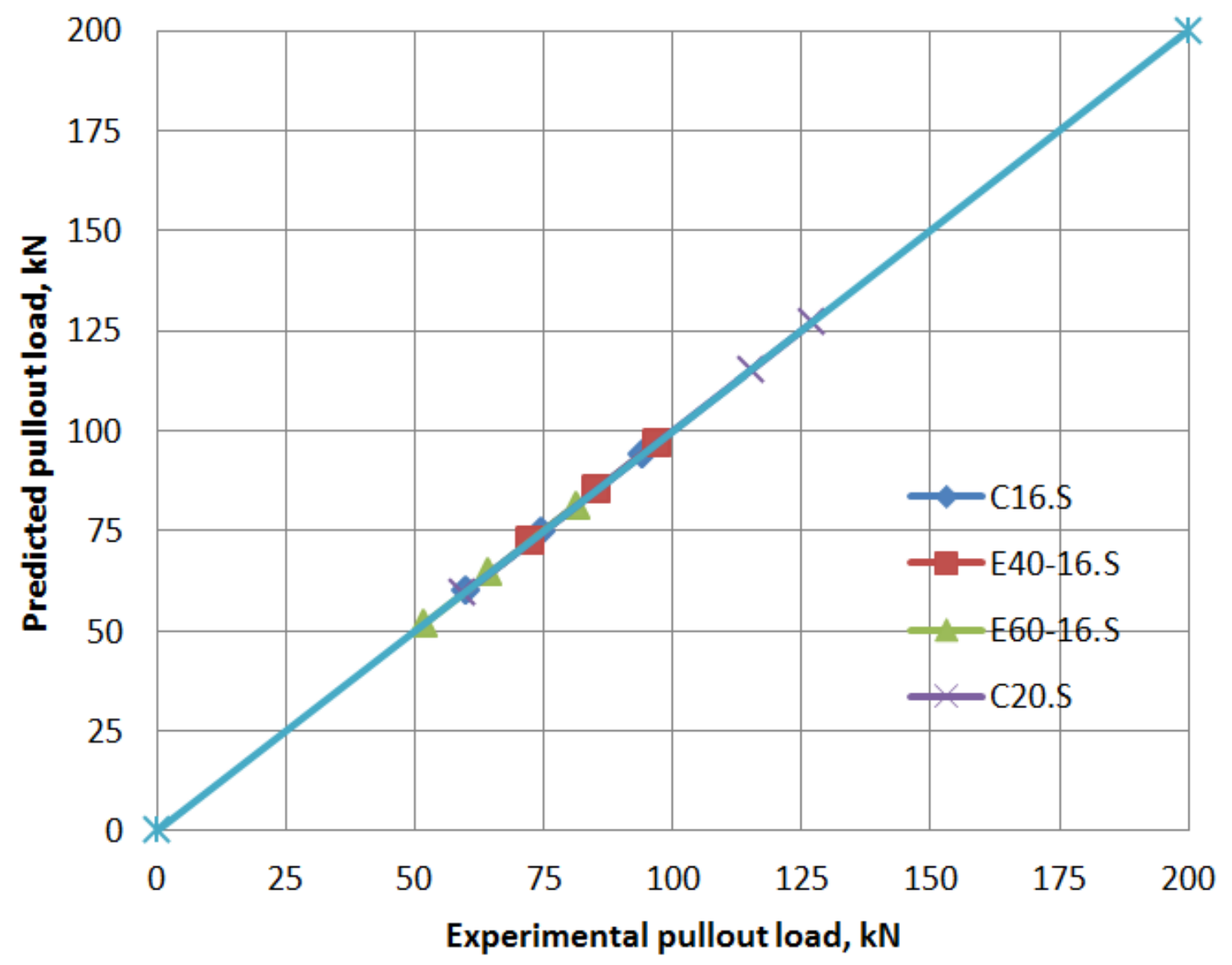

b) Comparison between predicted and experimentally pullout load for the ribbed-surface GFRP bar, based on Equation 2.21 and Table 2.13.

Figure 2.14. Comparisons between predicted and experimental embedment length and pullout-load for the ribbed-surface GFRP bar respectively 


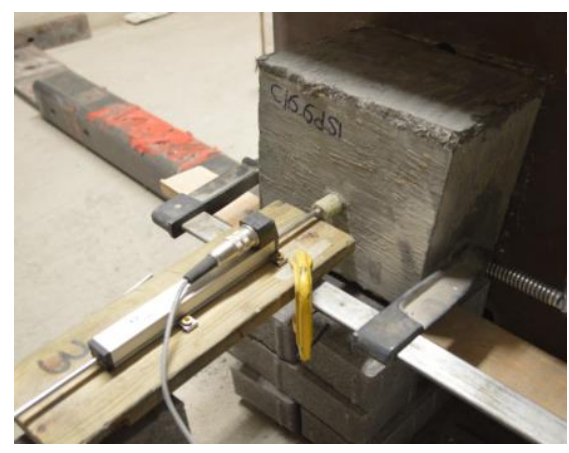

a. Bar projecting length at the free end before test

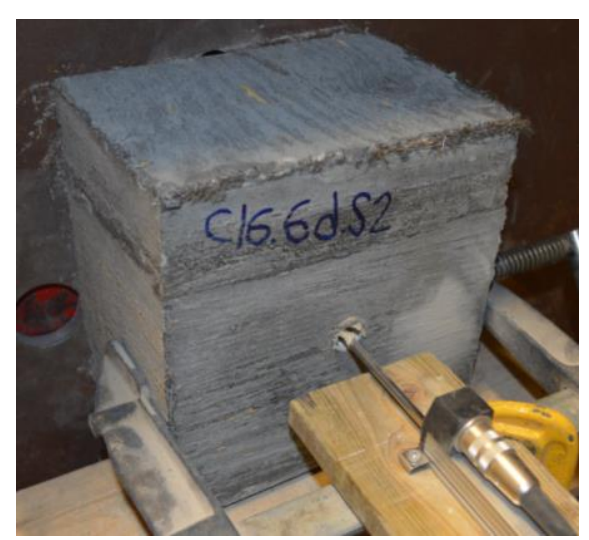

d. Block view at failure shown projecting bar end slipped inside the block at failure

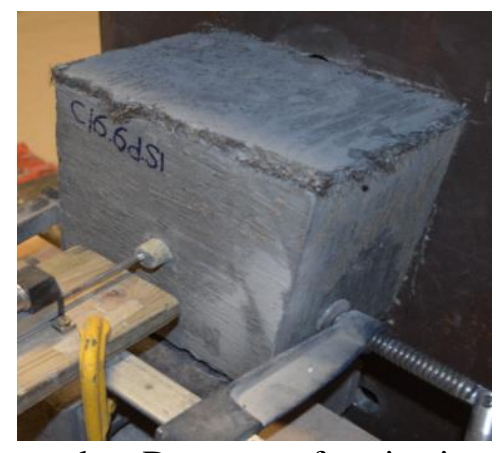

b. Decrease of projecting bar length during test

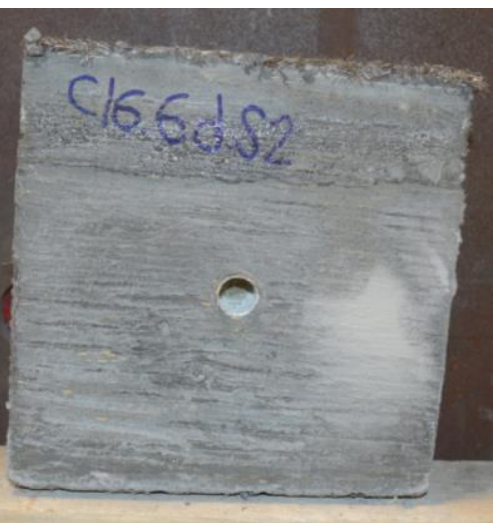

e. View of the block free face showing the bar end went deeply in the hole

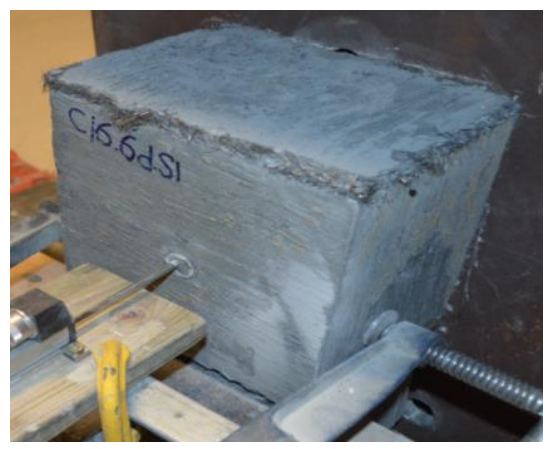

c. Projecting bar end became flush with the block free-end face during test

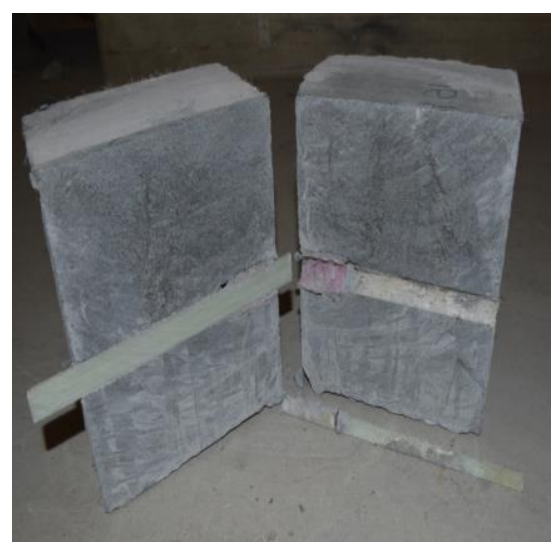

f. Section cut at bar location

Figure 2.15. Typical failure of the 15M sand-coated GFRP bar due to: (i) interlaminar shear failure of sand coating and (ii) shear-off failure of sand coating from bar surface 


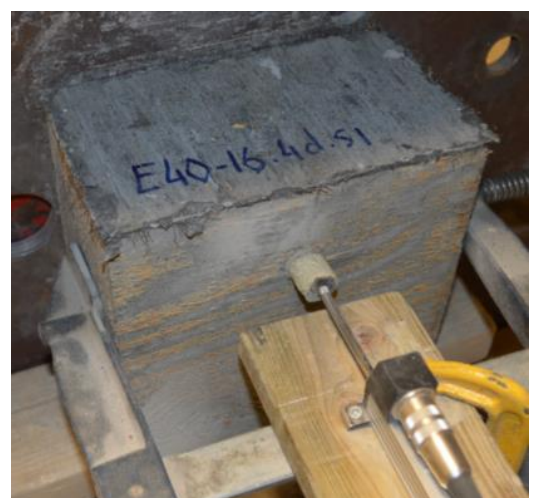

a. Bar projecting length, with $40 \mathrm{~mm}$ concrete cover, at the free end before test

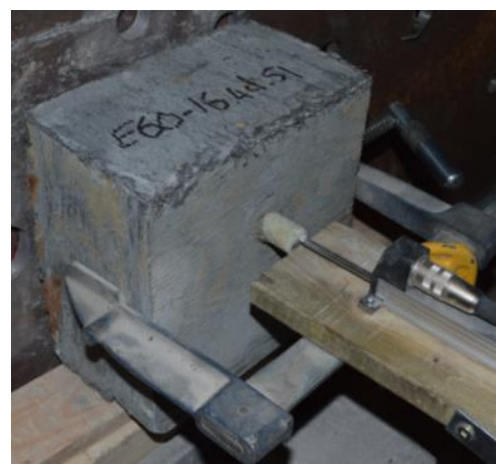

d. Bar projecting length, with $60 \mathrm{~mm}$ concrete cover, at the free end before test

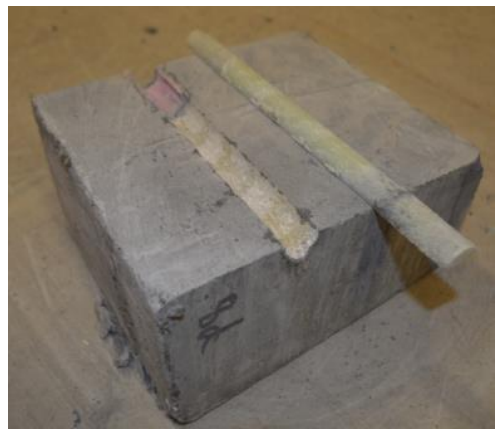

g. Close-up view of section cut

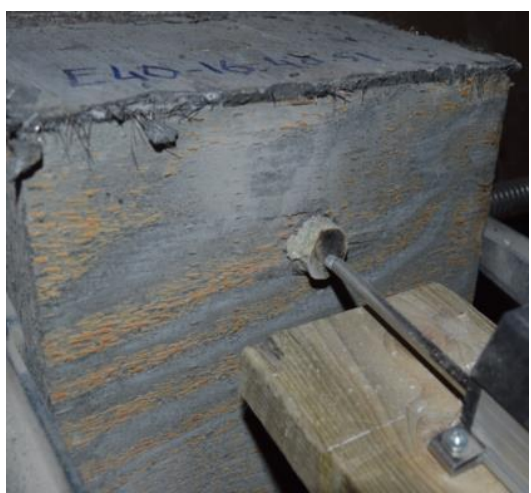

b. Decrease of projecting bar length during test

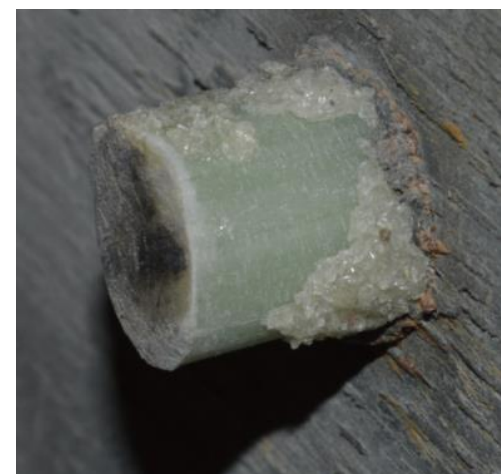

e. Peeling-off failure of the sand coating at failure
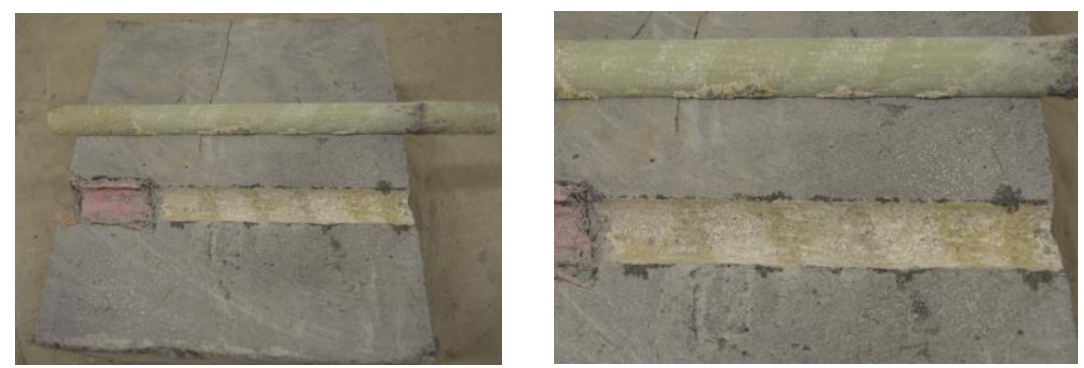

h. Interlaminar shear failure of the coating and shear-off of sand coating from bar surface

Figure 2.16. Typical failure of the $15 \mathrm{M}$ sand-coated GFRP bar with 40 and $60 \mathrm{~mm}$ concrete cover due to: (i) interlaminar shear failure of sand coating and (ii) shear-off failure of sand coating from bar surface 


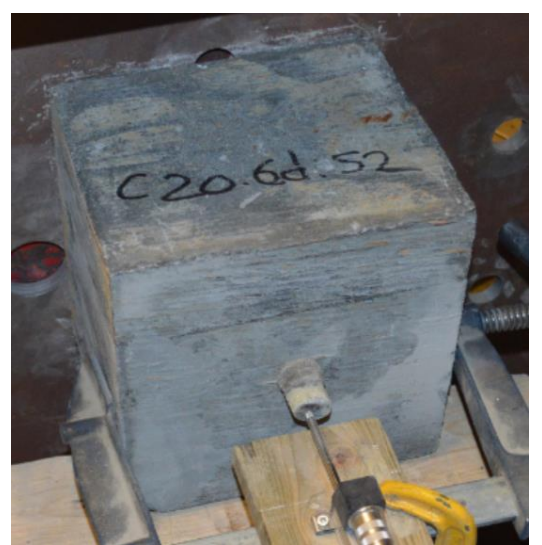

a. Bar projecting length at the free end before test

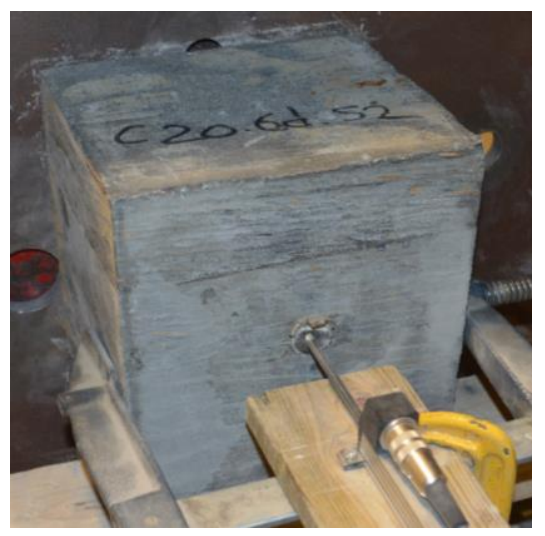

b. Decrease of projecting bar length during test

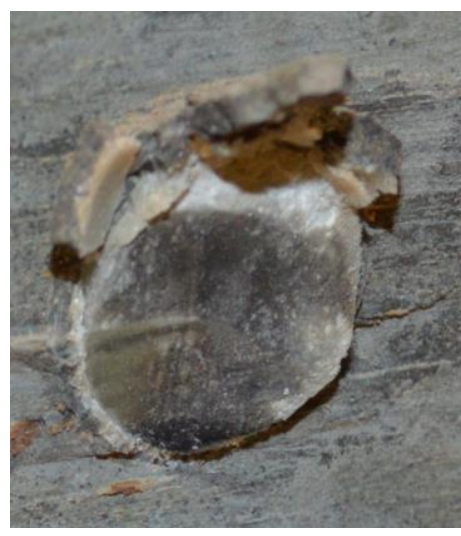

c. Bar free end after pullout failure

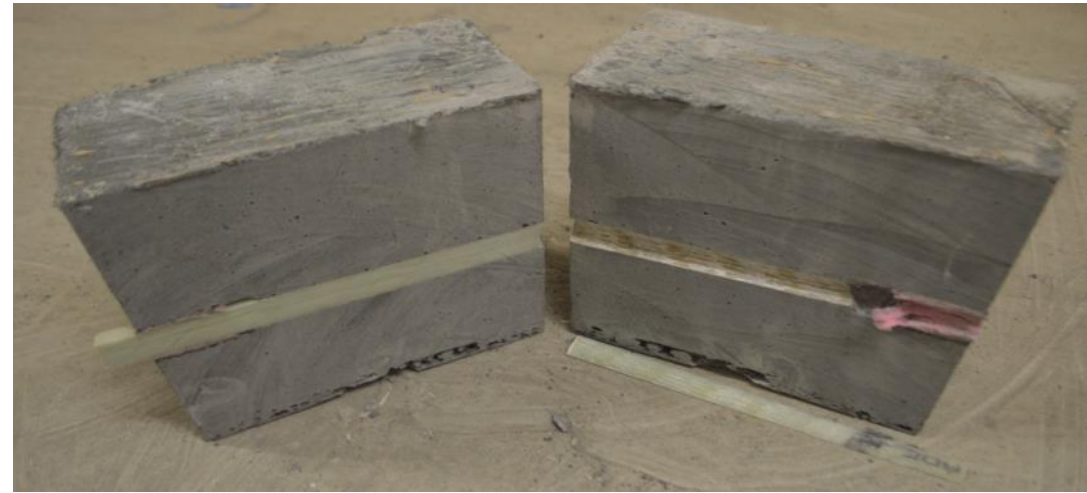

d. Interlaminar shear failure of the sand coating shown after slicing the block at bar location

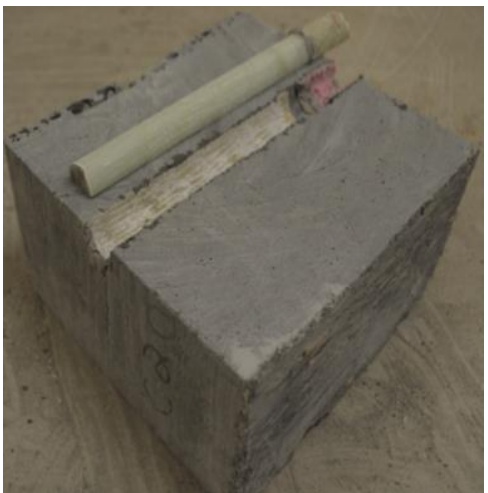

e. Close up view of section cut showing shearing-off of coating from bar surface

Figure 2.17. Typical failure of the 20M sand-coated GFRP bar due to: (i) interlaminar shear failure of sand coating and (ii) shear-off failure of sand coating from bar surface 


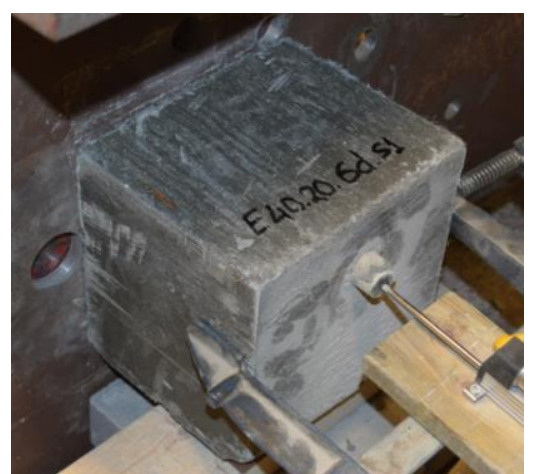

a. Bar projecting length at the free end before test

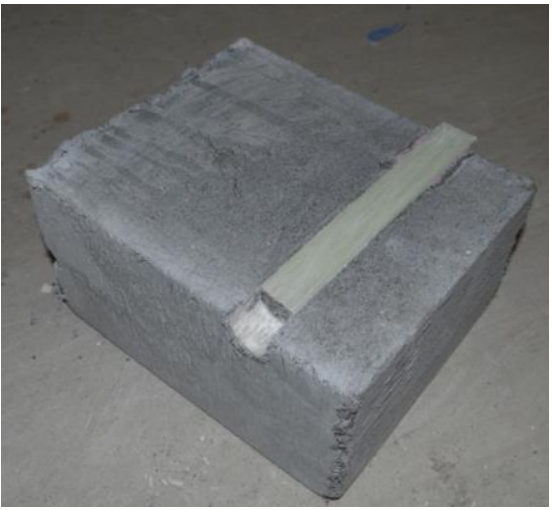

d. Close up view of section cut showing bar slip length at the free end

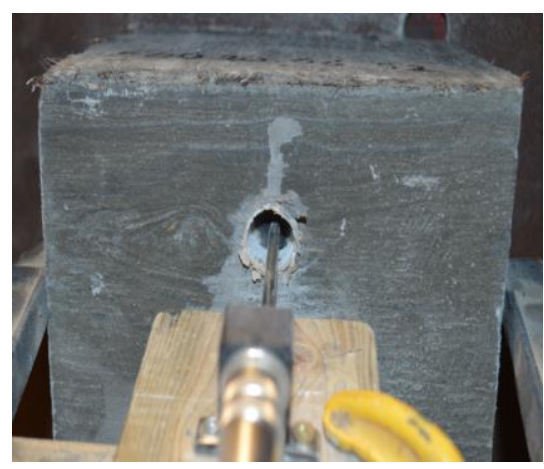

b. Bar projecting length disappeared inside the hole after test

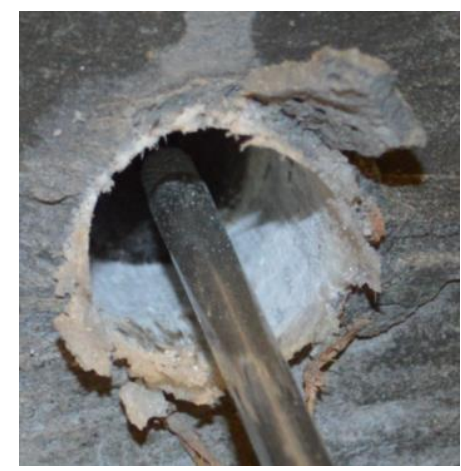

c. Close-up view of the concrete hole at block free end sand-coating left behind along the perimeter of the hole

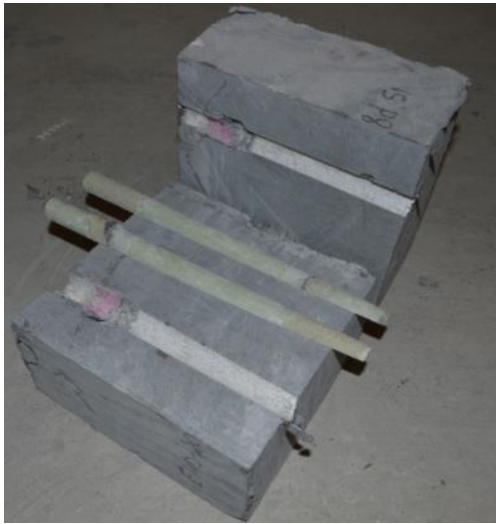

e. View of interlaminar shear failure of the sand coating along with shearing-off of the coating from bar surface

Figure 2.18. Typical failure of the 20M sand-coated GFRP bar with 40 and $60 \mathrm{~mm}$ concrete cover due to: (i) interlaminar shear failure of sand coating and (ii) shear-off failure of sand coating from bar surface 


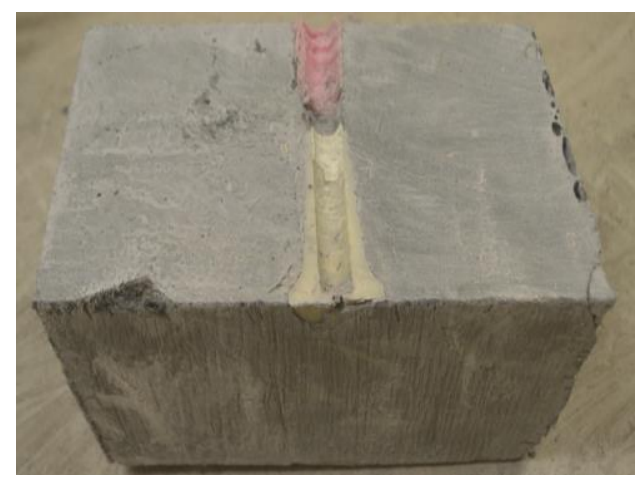

a. Sliced block at centered bar head

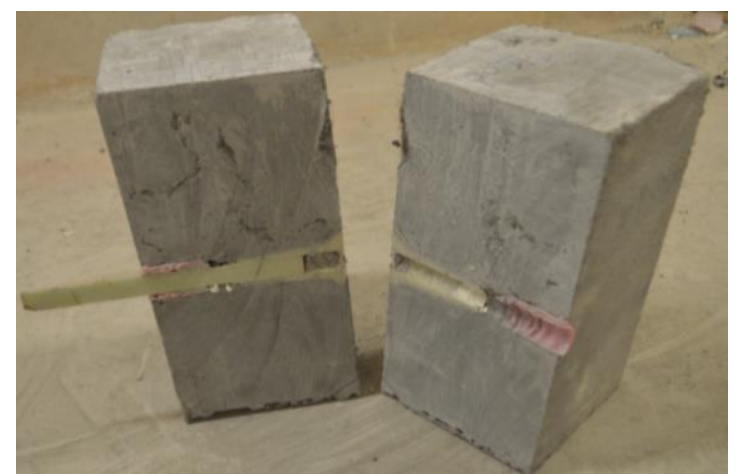

b. Centric bar slippage from the anchorage head

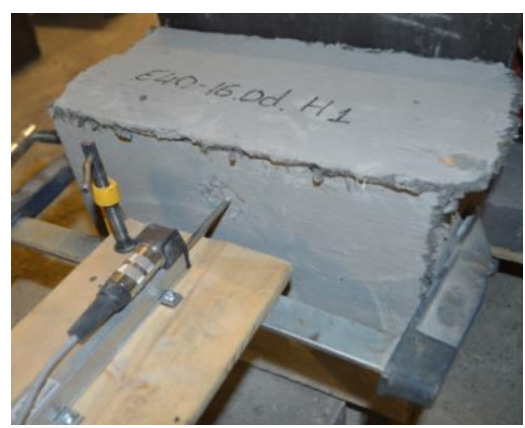

c. View of front and back face of block for GFRP headed bar with $40 \mathrm{~mm}$ concrete cover

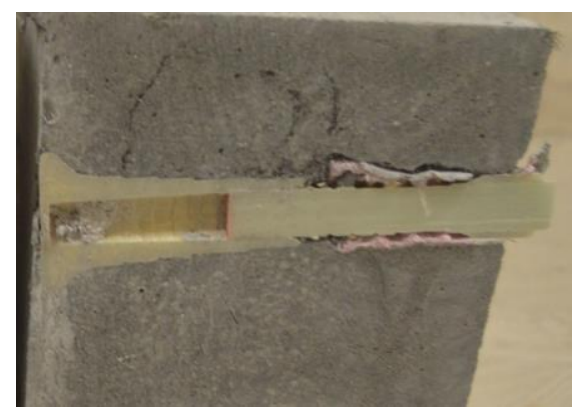

e. View of the GFRP bar with $60 \mathrm{~mm}$ concrete cover showing bar slippage from anchorage head

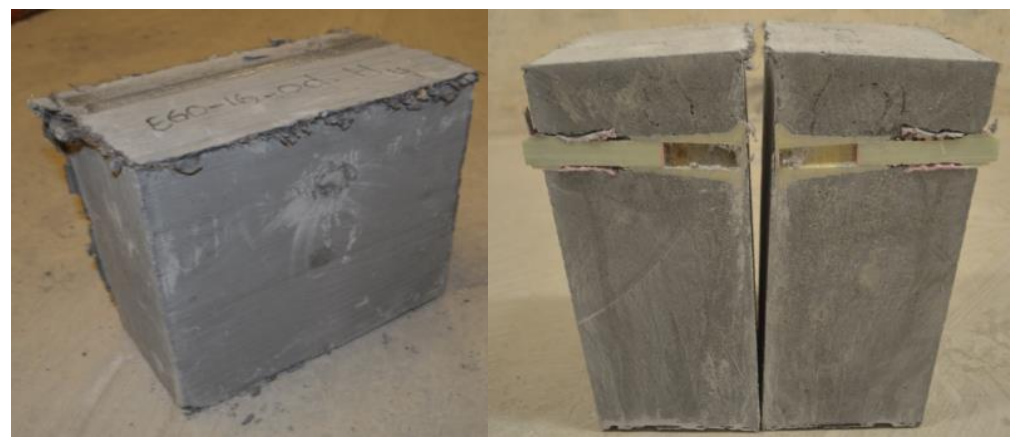

d. Bar pull-through failure and slippage from anchorage head

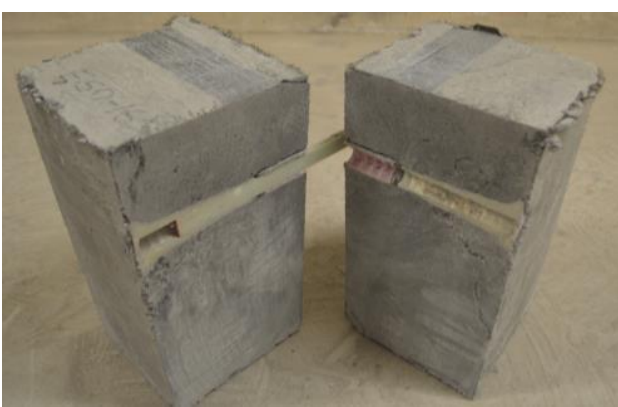

f. Section cut for headed bar with $60 \mathrm{~mm}$ concrete cover showing bar slippage from head and pull-though failure

Figure 2.19. Typical failure of the 15M sand-coated, headed-end, GFRP bar with different concrete cover due to slippage of the bar from the anchorage head as a result of shear failure along the grooves at the interface between the bar and the anchorage inner surface 


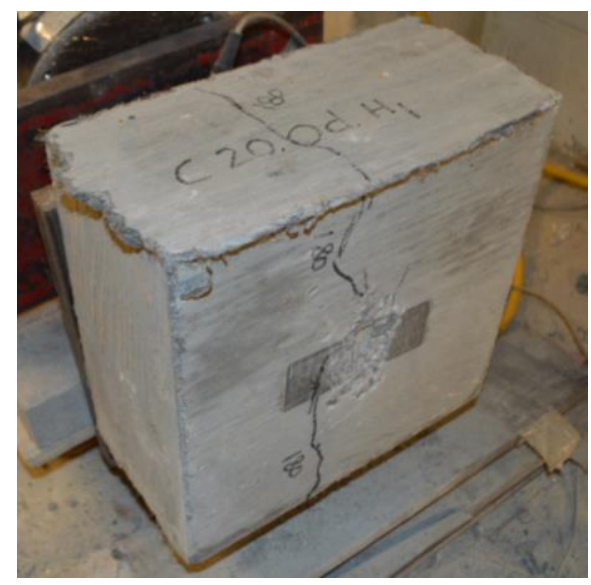

a. Block with centric headedend bar showing splitting cracks

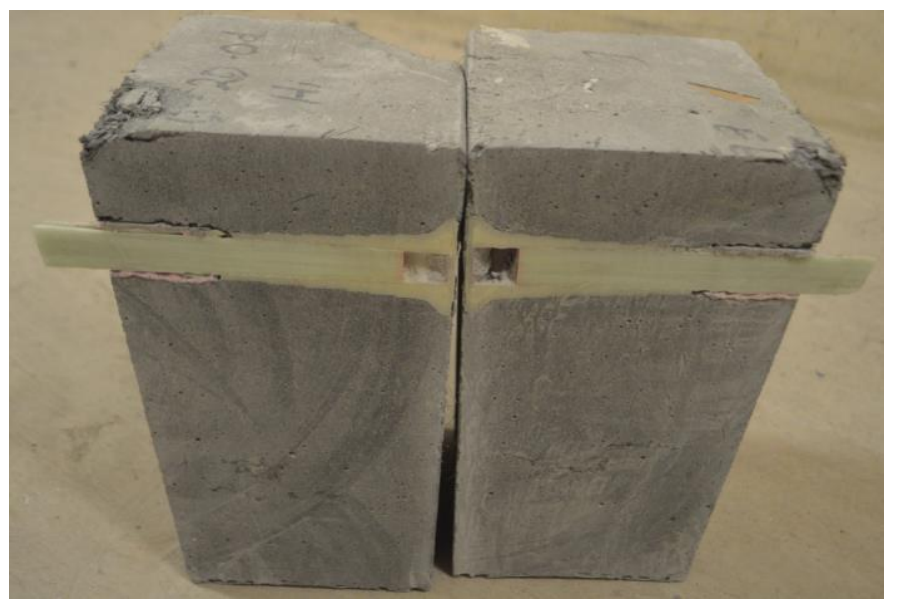

b. Section cut in the block with GFRP headed-end bar at 40 $\mathrm{mm}$ concrete cover showing bar slippage from head

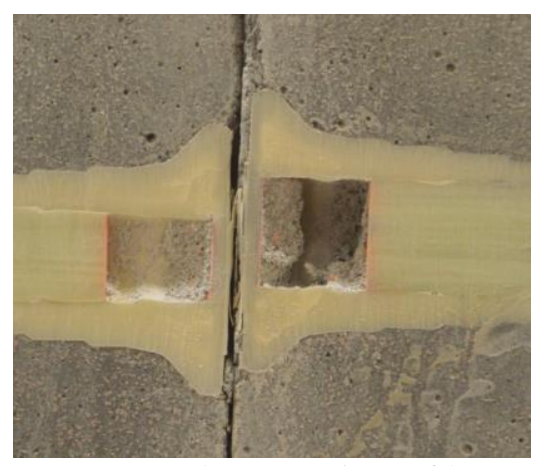

c. Close-up view of bar slippage from head

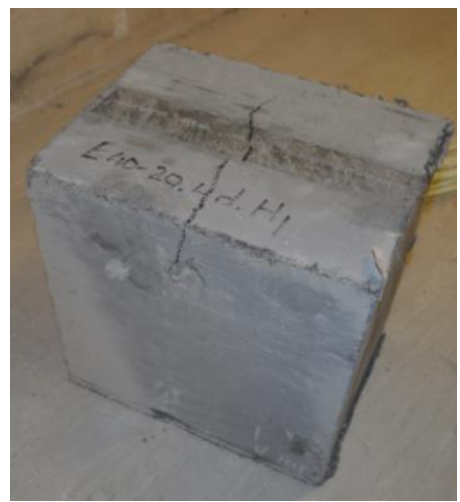

f. Block with headed-end bar at $40 \mathrm{~mm}$ concrete cover showing side crack

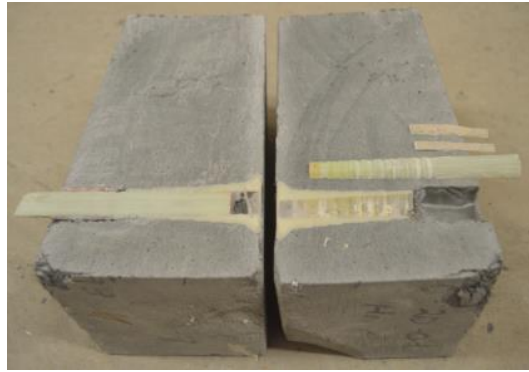

d. Section cut in the block with GFRP bar head at 40 mm concrete cover

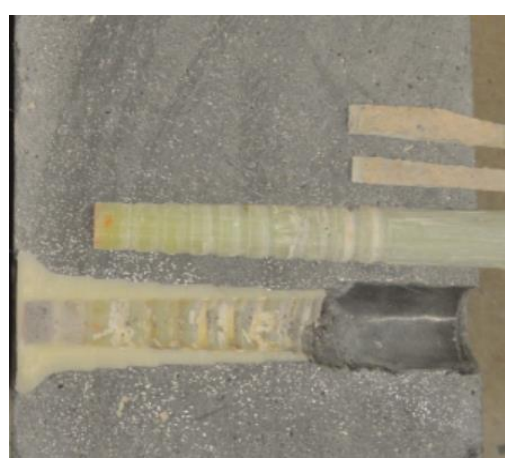

e. Close-up of shearing the grooves along the bar embeded length in the head

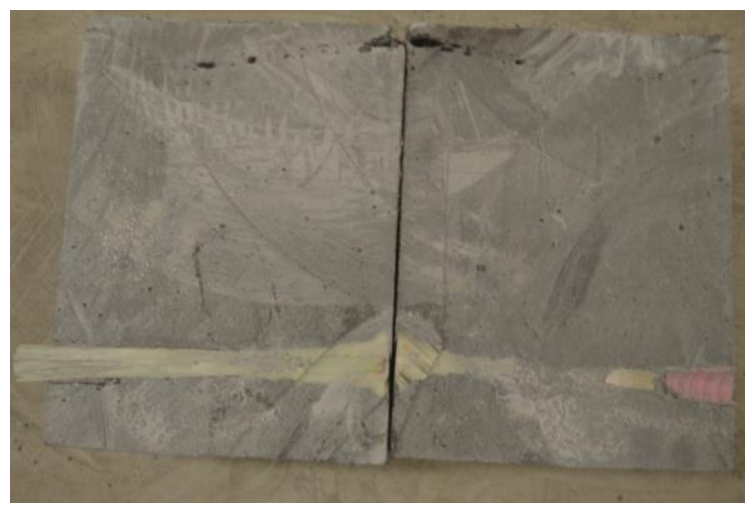

g. Section cut headed-end bar with $40 \mathrm{~mm}$ concrete cover

Figure 2.20. Typical failure of the 20M sand-coated, headed-end, GFRP bar with different concrete cover due to slippage of the bar from the anchorage head as a result of shear failure along the grooves at the interface between the bar and the anchorage inner surface 


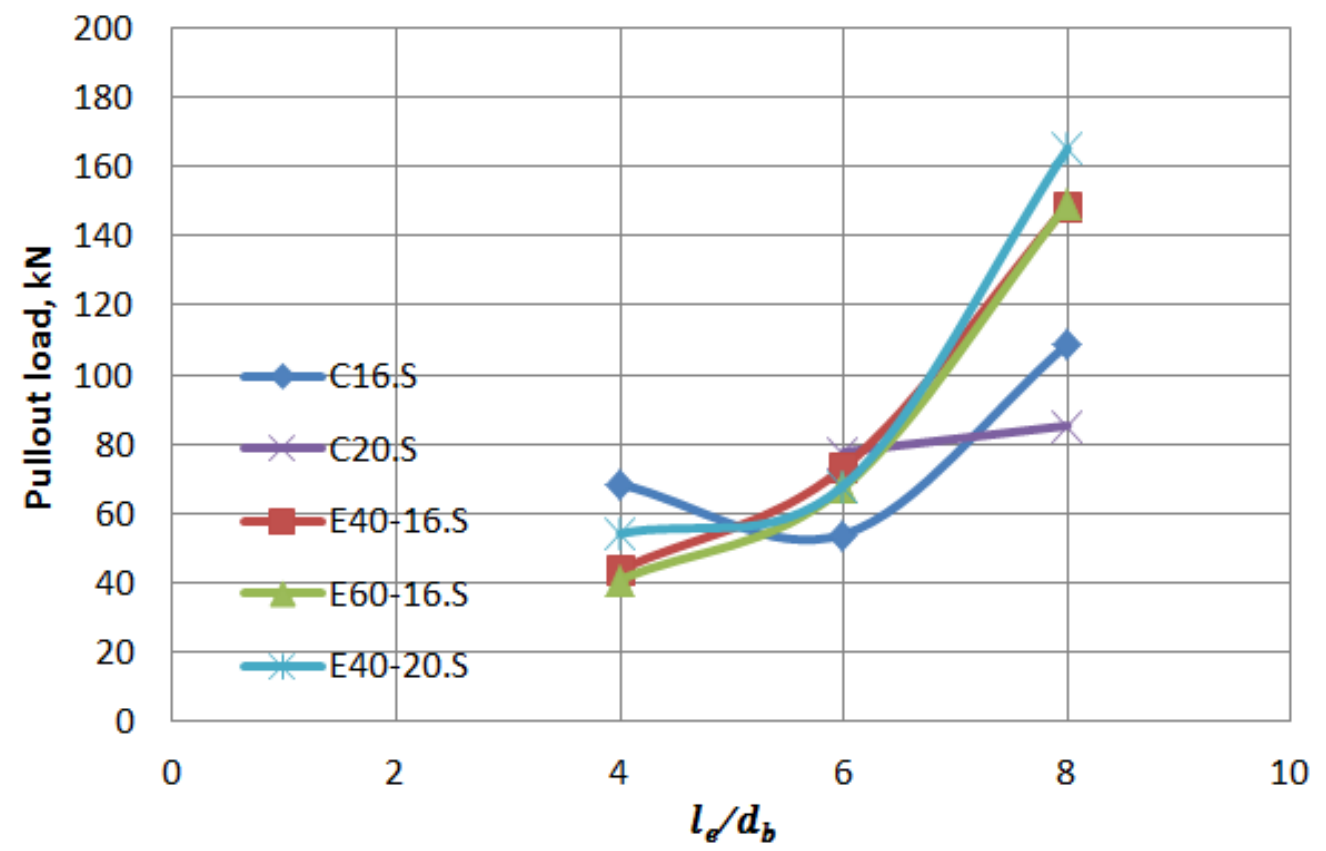

Figure 2.21. Experimental pullout load for the sand-coated GFRP bars embedded in UHPC cube specimens

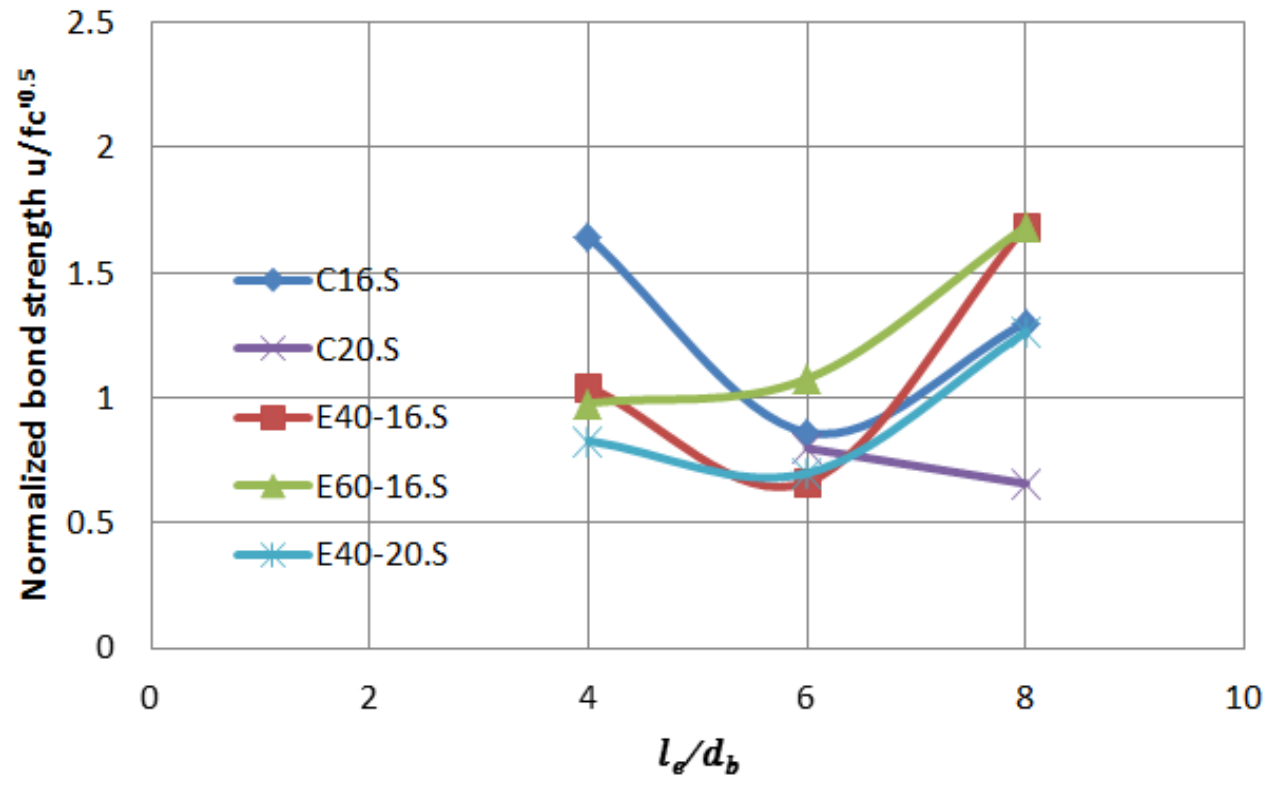

Figure 2.22. Normalized bond stress, $\mu$, for the sand-coated GFRP bars embedded in UHPC cube specimens 


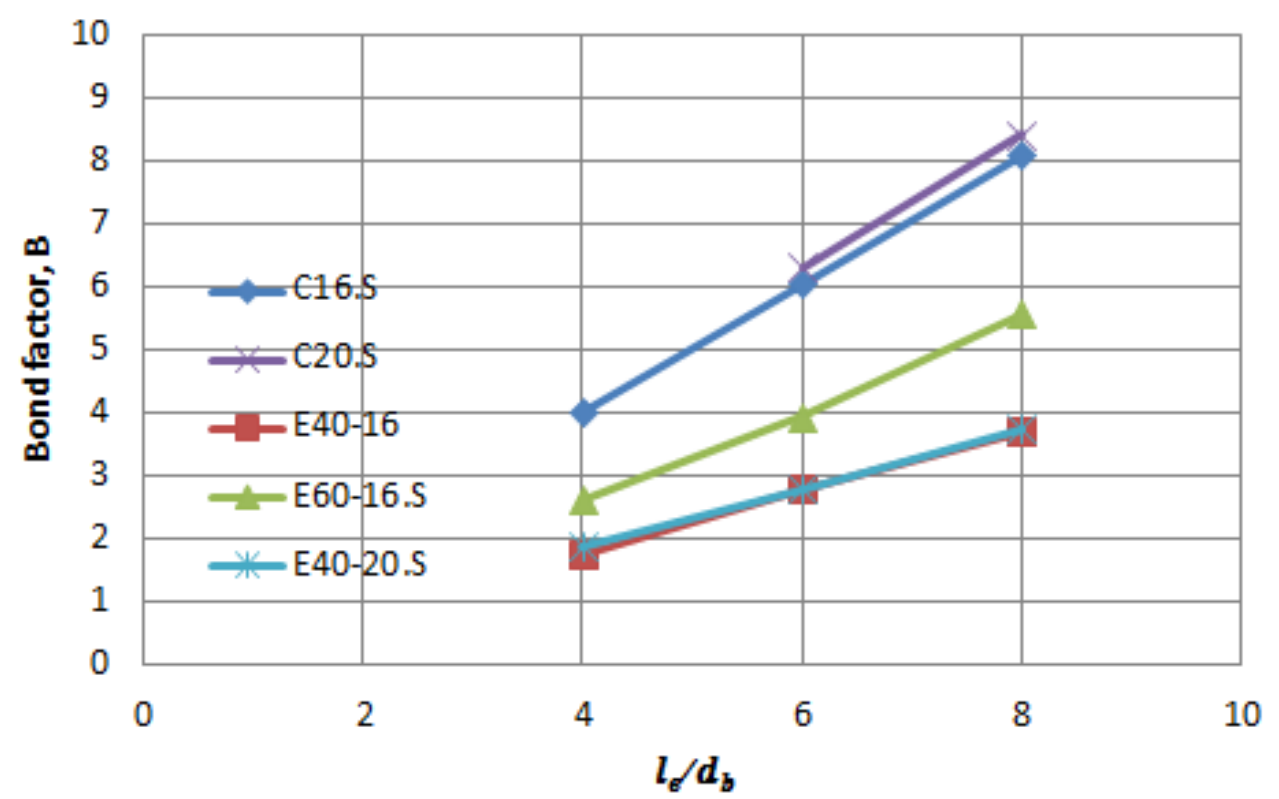

Figure 2.23. Combined bond factor, B, for the sand-coated GFRP bars

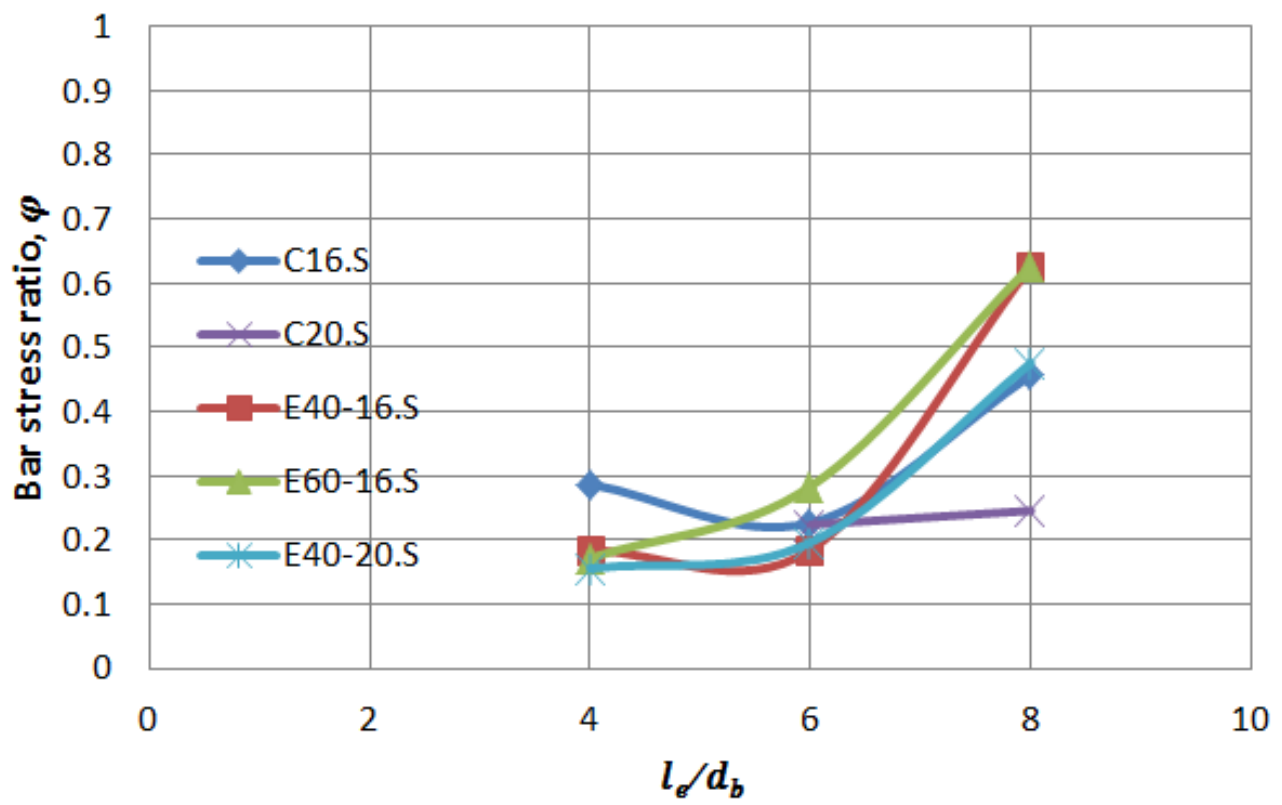

Figure 2.24. Bar stress ratio, $\varphi$, for the sand-coated GFRP bars 


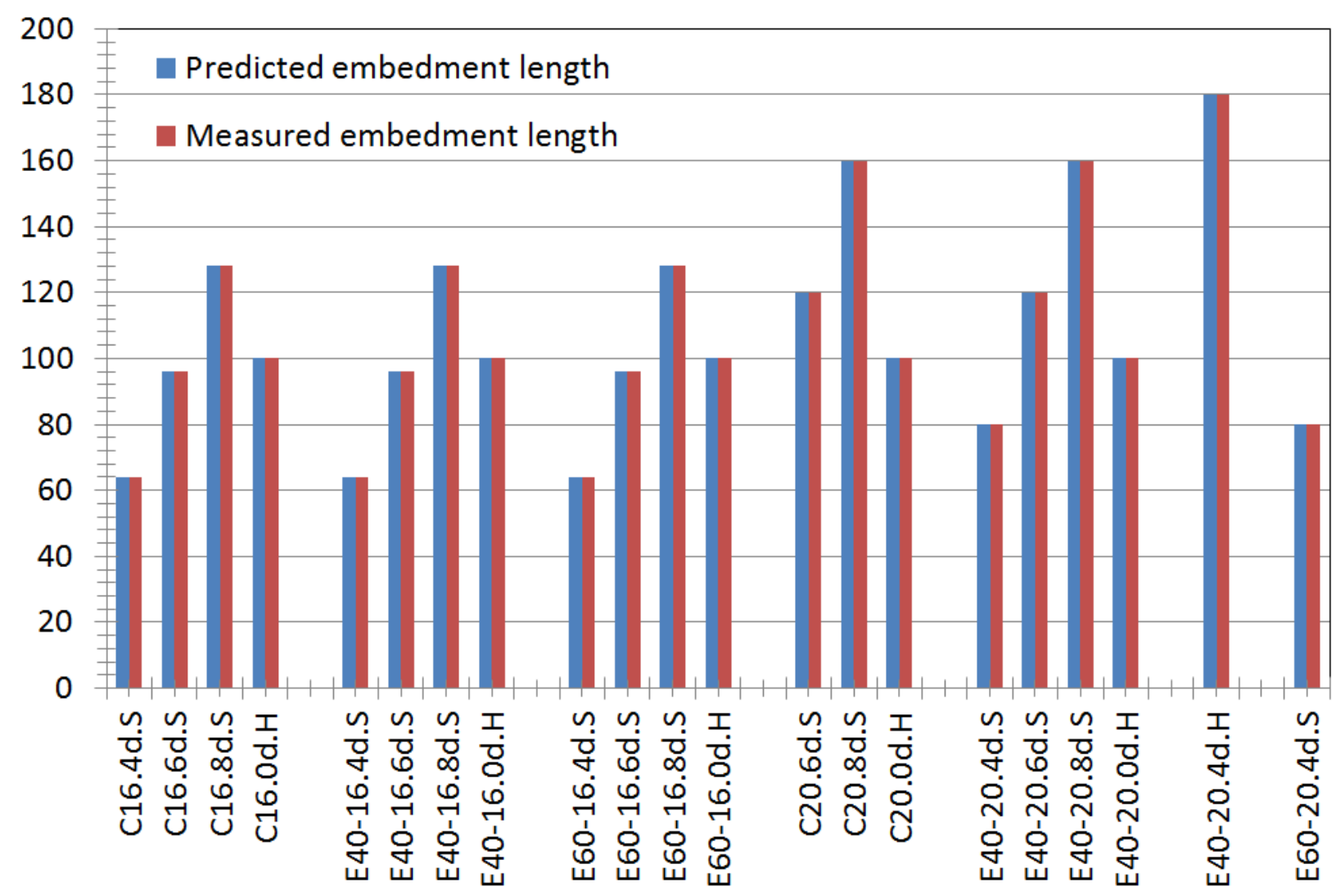

c) Comparison between predicted and experimentally measured embedment length for the sandcoated GFRP bar based on Equation 2.12 


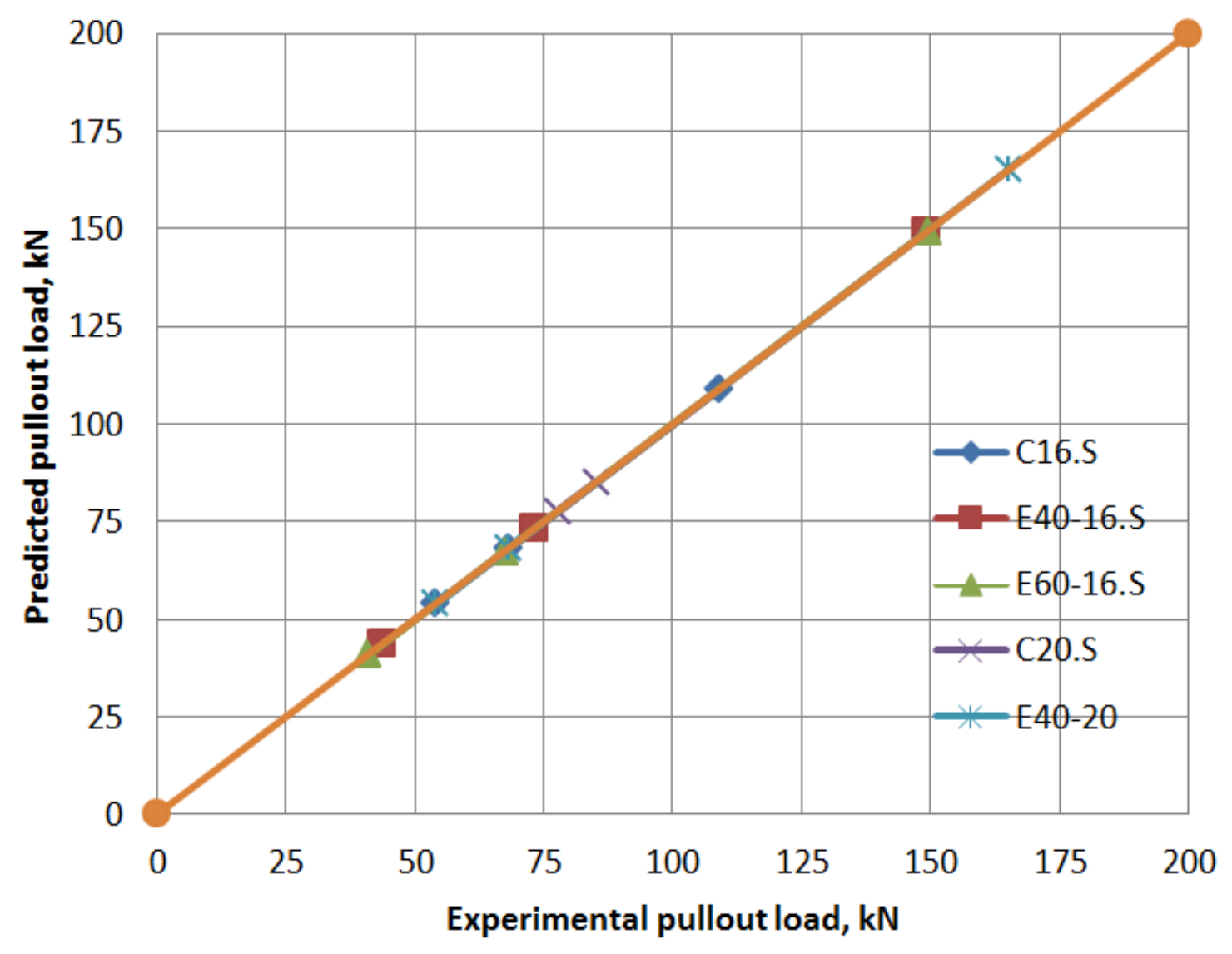

d) Comparison between predicted and experimentally pullout load for the sand-coated GFRP bar, based on Equation 2.21 and Table

Figure 2.25. Comparisons between predicted and experimental embedment length and pullout-load for the sand-coated GFRP bar respectively 


\section{Chapter III}

\section{Structural Behavior of NSC Full-Depth Deck Panels}

\section{with Closure Strips Reinforced with Glass Fiber Reinforced Polymer Bars and Filled with Ultra-High Performance Fiber Reinforced Concrete}

\subsection{General}

Corrosion of reinforcing steel in bridge decks decreases life expectancy of bridge superstructures, leading to costly and frequent maintenance or replacement. The use of noncorrosive glass fiber reinforced polymer (GFRP) bars as internal reinforcement is a viable option for the replacement of deteriorated concrete bridge deck slabs due to steel bar corrosion. Precast full depth deck panels (FDDPs) have recently used in new accelerated bridge construction $(\mathrm{ABC})$ or for the rapid bridge replacement (RBR) of existing deteriorated bridge decks. FDDPs are produced off-site, quickly assembled on-site, reduce construction time, minimize lane closure and are considered as a good solution to minimize traffic disruption (Clumo, 2011). FDDPs are placed side by side, as shown in Fig. 3.1, then closure strips between them are filled with bonding material. FDDP closure strips should take the advantage of high quality concrete such as ultra-high performance concrete (UHPFRC) and non-corrosive reinforcement such as GFRP bars for enhanced strength and durability. Although the Canadian Highway Bridge Design Code, CHBDC, (CHBDC, 2014) allows the use of GFRP-reinforced FDDPs in bridge construction, there is no code provision on the joint details between such precast systems. The behaviour of the FDDP monolithic concrete joints, also known as moment resisting joints, accounts for the state of bond of the projected longitudinal bars anchored through the cast-field joints. UHPFRC is a relatively new class of cementitious matrix with steel fiber content that has high compressive strength (in order of $140 \mathrm{MPa}$ ) and relatively large tensile strength (in order of $8 \mathrm{MPa}$ ), with strain hardening behavior in tension that ensure crack opening remain very small (Russell and Graybeal, 2013). The use of UHPFRC as a filling material of the closure strip between connected 
FDDPs have numerous benefits, including reduction of joint size, improving durability, speed of construction and prolonging usage life. The proposed research investigates the use of ribbedsurface GFRP bars in prefabricated full-depth precast bridge deck panels to accelerate bridge construction. In this research, three joints between precast panels were developed using straight GFRP bars embedded in a closure strip filled with UHPFRC. Normal strength concrete was used to cast the prefabricated panels. Two control cast-in-place slabs reinforced with steel and GFRP bars, respectively, were cast to form the baseline structural performance of the developed jointed panels. Concentric and eccentric wheel loading, with respect to the centre of the joint, were applied at the joint to expose it to pure bending and combined bending and shear, respectively. Results were compared to the wheel load provided into the Canadian Highway Bridge Design Code.

\subsection{Research Background}

\subsubsection{Bridge Deck Slabs and Accelerated Bridge Construction}

The use of prefabricated elements and systems in accelerating construction of new bridges and replacement of deteriorated ones has recently been the subject of much attention and interest amongst bridge jurisdictions. Prefabricated elements and systems can be quickly assembled, reduce the impact on the environment in the vicinity of the site, minimize the delays and lane closure time and inconvenience to the traveling public, saving time and tax payers money. In 2011, Federal Highway Administration published the most comprehensive "State-of-Practice" document on accelerated bridge construction that includes experience in design, fabrication and erection of prefabricated bridge elements and systems (Culmo, 2011). Other reports in ABC were published in 2012 (SHRP2, 2012a, 2012b). These reports covered similar ABC projects for the superstructure deck elements including: (a) partial-depth precast concrete deck panels, (b) precast full-depth deck panels (FDDP) with and without longitudinal post-tensioning, (c) steel grid (open of filled with concrete), (d) orthotropic deck, (e) adjacent deck bulb tee beams (f) adjacent double tee beams, (g) adjacent inverted tee beams (h) adjacent box beams (i) modular beams with decks, and (j) full-width beam span with deck.

Recent surveys of the state of practice of full-depth, full-width panel-girder system and joints were conducted elsewhere (Roddenberry, 2012; NCHRP, 2011; Badie and Tadros, 2008; Hieber 
and Wacker, 2005; Versace and Ramirez, 2004). Connections suitable for simple and continuous spans for composite design were developed using different means including headed reinforcing steel bars, steel couples and post-tensioning the precast panels. Although deck panel posttensioning puts the joint in compression and secure it against leakage, it increases the cost of the deck system and delay deck construction. PCI (2011) and UDOT (2010) Published a guide for full-depth deck panels for accelerated bridge deck replacement using deck panels connected to girders using stud clusters at a specified AASHTO-LRFD spacing of $600 \mathrm{~mm}$ and connected with each other using lateral post-tensioning. The guide specifies skewed deck panels placed on girders along the skew of the bridge for skew angles less than $15^{\circ}$, and rectangular panels placed orthogonal to the supporting girders for skew angles more than $15^{\circ}$.

The Technical committee of the Precast/Prestressed Concrete Institute of New England Region introduced the "New England Region System" and recommended the typical thickness of the panel to be $200 \mathrm{~mm}$, with composite action achieved through square tapered pockets. This system does not require prestressing of the deck panels (PCINER Technical Committee, 2002). Later, the Connecticut Department of Transportation (CDOT) developed the connection details for such panels (Price, 2010; Versace, 2003). Tadros and Baishya (1998) developed full-depth precast, prestressed concrete bridge deck with pre-tensioning in the transverse direction and posttensioning in the longitudinal direction with threaded bars. The system incorporated shear blockouts and female-to-female transverse connection. The panel-to-girder was composed of welded headless studs and welded threaded studs with nuts. Hanna and Tadros (2010) developed the second generation of this deck system using high performance concrete (HPC). The width of the panel ranged from 2.4 to $3.65 \mathrm{~m}$ along the roadway. The length of the panel was typically equal to the full width of the bridge. These precast FDDPs have (i) shear pockets or block-outs to accommodate the shear connectors, (ii) transverse joint between the precast FDDPs and (iii) longitudinal post-tensioning to tie the FDDPs together. Porter et al. (2011) tested precast deck panels with few connections types, namely: welded connection, post-tensioned connection and curved bolt connection, to determine their cracking and ultimate flexural strengths. Badie et al. (2006) developed panel-to-panel connection to eliminate the use of the post-tensioning. The female-to-female connection system uses hidden HSS tubes cast in the panels to provide confinement for full develop mild reinforcement between the adjacent FDDPs. 
Few authors dealt with full-depth panel joint with concrete girders (among them: Price, 2010; Issa et al., 2006). Also, few authors (among them: Afefy et al., 2015; Sennah et al., 2015; Shah et al., 2007 and 2006; He et al., 2013; Ma et al., 2012, Li et al., 2010) dealt with panel-to-panel longitudinal joints in bulb-tee girders. While others (among them: Porter, et al., 2012; Issa et al., 2007, 2006, 2003; Markowiski, 2005) dealt with panel-to-panel transverse joints, all using reinforcing steel bars in the form or straight bars, headed-end bars and U-bars. Other researchers extended this research by using ultra-high performance concrete ((UHPFRC) as filling materials in the closure strip to reduce joint width with using steel bars to reinforce the deck slab (Lee and Lee, 2015, 2014; Hwang and Park, 2014; Graybeal, 2010). Thompson et al. (2006a and 2006b) investigated the anchorage behavior of headed steel bars in noncontact lap splices, both experimentally and using the strut-and-tie model. Results demonstrated that headed reinforcement can significantly reduce the required lap length of the sliced reinforcement. Liu (2011) conducted experimental tests to-collapse on precast full-depth deck panels made of normal-weight and light-weight concrete and reinforced with GFRP bars to evaluate the shear capacity, deck deflection and moment of inertia. No joints between the panels were studied in Liu's research. Khalafalla (2014) developed and tested experimentally under static and fatigue loading, joint details between flanges of precast bulb-tee girders incorporating non-corrosive GFRP bars with headed ends in precast girder panels and UHPFRC as filling material in the closure strips.

Very few researchers (Chim and Chang, 2003; Ryu et al., 2003) investigated experimentally and theoretically cracking behavior and girder ultimate strength at the transverse joint of full-depth deck panels acting compositely with the girder at the negative moment region with the use of longitudinal post-tensioning. Due to insufficient data on the tension capacity of the transverse joint at the negative moment region, bridge designers used to neglect the precast deck-girder composite action, leading to expensive design. Zhu et al. (2012) proposed continuous transverse U-bar joint details, incorporating projecting reinforced steel bars from the jointed panels which can provide negative moment continuity in multi-span bridges, no pretensioning was used. Based on ultimate and fatigue test results, the developed transverse U-bar joint detail proved promising to provide continuity of deck panels to carry tensile forces when acting composedly with the girders. Chapman (2010) investigated similar U-bar joint details for transverse joint under tension for the negative moment region as well as for longitudinal joint under flexure for the 
positive moment region. Ong and Paramasivam (2006) proposed a strut-and-tie model to calculate the ultimate tensile load of precast concrete joints with two or one loop connections subjected to static uniaxial tensile loading. Results showed that the predictions using the proposed model correlated well with the experimental data.

Few authors dealt with finite-element modeling of full-depth panel-girder joint (among them: Julander, 2009; Sullivan and Roberts-Wollmann, 2008). Niroumand (2009) investigated, using 3D FEA modeling, the resistance mechanism of the simple-made-continuous connections in skew and non-skew steel girder bridges using accelerated type of construction, followed by experimental verification of full-scale model of the composite girder with the constructed precast deck panel joint, using reinforcing steel bars, through testing to-collapse.

Literature survey revealed that PCI Report along with other ABC manuals produced by US DOTs specify the use of high-performance concrete (HPC) with 70 to $100 \mathrm{MPa}$ compressive strength in precast deck panels to provide sufficient strength and durability. Such US reports showed that skewed bridges are becoming increasingly common. With the use of full-depth precast panels in skew bridges, questions arise regarding the performance of transverse connections between panels at bridge obtuse corners, at which high shear force and torsional moments exist. However, the performance of the transverse panel-to-panel joints in bridge systems are still not knows at ultimate and fatigue limit states with the use of UHPFRC and GFRP bars in the closure strip for straight bridges. In addition, at transverse joints that exist at the negative moment region, many serviceability problems such as cracking and water leakage were reported (Porter et al., 2011; Issa et al., 1995). As such, questions arise as how to connect full-depth deck panels at the negative moment region to prevent water leakage and to contribute to ultimate and fatigue limit state designs of the composite girders. A review of bridge project inventory in USA (PCI, 2011) revealed critical factors contributing to reduced performance of precast bridge deck panels:

1- Debonding and leakage through the panel-to-panel joints, either at the positive or negative moment regions that lead to severe corrosion due to lack of post-tensioning in the longitudinal direction, inadequate material in the joint, inadequate configuration of the joint, and inadequate surface preparation of the joint. 
2- $\quad$ Loss of full composite action between precast slabs and the supporting system due to lack of haunch, inadequate design and distribution of shear connectors, and inadequate materials in the shear pockets.

\subsubsection{Background on Ultra-High Performance Fibre-Reinforced Concrete (UHPFRC)}

The term UHPFRC refers to a class of advanced cementitious composite materials. Although the general concepts which lead to the advanced performance characteristics of UHPFRC are well known, the commercial availability of UHPFRC and the development of locally-sources UHPFRC mixes has been limited in North America. The availability of UHPFRC has developed differently in other parts of world, most notably Europe, where multiple pre-bagged and locallysources UHPFRCs are available. Ductal product, supplied by Lafarge, is currently considered the only commercial product widely available in North America in the quantities necessary for large scale infrastructure applications and remains a proprietary product with considerable cost premium. Typical UHPFRC composition and material properties are available elsewhere (Graybeal, 2010 and 2006). While a state-of-the-art report in UHPFRC for the bridge community was produced by Russell and Graybeal (2013). The concept of using the advanced properties of UHPFRC to significantly modify the design of connections between precast concrete components is not new. In fact, research and deployments in this area date back to at least 1995. At that time, a commercially available UHPFRC was used as a closure pour material in the connection of slab elements in a building being constructed at Aalborg University.

A few years later, a second project at the same university resulted in the use of field-cast UHPFRC connections both between slab elements and between slabs and columns. In support and association with these two projects, a series of research projects were completed to assess the bonding performance between the UHPFRC and straight lengths of mild steel reinforcement (Aarup et al., 2000; Hansen and Jensen, 1999; Nielsen et al., 1996; Aarup and Jensen, 1998). Additional research, focused specifically on field-cast UHPFRC connections for precast bridge deck panels, was completed at Chalmers University of Sweden (Harryson, 2000 and 1999; Broo and Broo, 1997). Saleem et al. (2012) conducted tested on cube and beam samples to determine the developed length of steel bars in UHPFRC, while Ametrano (2011) conducted similar bond tests but for glass fiber polymer bars. 
Research on the materials between precast bridge components has emerged in the last decade, including non-shrink cementitous grouts, ultra-high performance concrete, epoxy grout, magnesium ammonium phosphate grout and post-tensioning cable grout (among them: NCHRP, 2008; Li et al., 2010; NCHRP, 2011). More recently, the concept of using the properties of UHPFRC to redesign the connections between prefabricated bridge components has been recognized in North America (among them: Graybeal, 2010). Field-cast UHPFRC connections between prefabricated bridge components have now been implemented in few bridges in Canada and U.S. However, research is need to develop guidelines for the design of transverse joints between precast deck panels for bridges at the positive moment region, incorporating GFRP bars and UHPFRC.

\subsection{New Connection Details}

The prefabricated bridge system shown in Fig. 3.1 incorporates precast concrete panels placed side-by-side to form the bridge deck. In this system, the concrete deck slab is cast in a controlled environment at the fabrication facilities and then transported to the construction site. One of the main issues inherent in these prefabricated systems is the presence of cold joints created by the closure pours and their potential impact on the overall deck system behavior. In addition, it is important to develop effective connection details between the prefabricated elements to provide continuity of reinforcement in the closure strips so that load sharing between girders is not compromised. This research GFRP bars as main reinforcement in the precast panels, projecting in the closure strip with straight ends. FRPs, as non-corrodible materials, are considered as excellent alternative to reinforcing steel bars in bridge decks to overcome steel corrosion-related problems. Since it is less expensive than carbon and aramid FRPs, GFRP bars are more attractive for bridge deck applications. GFRP bars used in this study have a tensile strength of $1188 \mathrm{MPa}$, compared to $400 \mathrm{MPa}$ yield strength of reinforcing steel bars. The special "ribbed" surface profile of these bars, shown in Fig. 2.1.c, ensure optimal bond between the concrete and the bar. The favorable GFRP characteristics include (i) high-strength-to-weight ratio; (ii) resistant to corrosion; (iii) freeze and thaw resistance; and (iv) high chemical resistant (Schoeck, 2013). Table 3.2 summarizes the rest of the mechanical properties of GFRP bars considered in this study. 
The common practice in bridge design in Ontario is to use $225 \mathrm{~mm}$ thick cast-in-place deck slab in bridges. This slab thickness incorporates $60 \pm 10 \mathrm{~mm}$ top concrete cover as recommended by CHBDC for reinforcing steel bars for protection against possible corrosion. However, with the use of non-corrosive FRP bars, the deck slab thickness can be reduced by $35 \mathrm{~mm}$ as specified in CHBDC with the use of FRP reinforcement. This change makes the FRP-reinforced deck slab thickness $200 \mathrm{~mm}$, thus reducing the material of the deck slab by about $12 \%$. As a result, it was decided to conduct this research using $200 \mathrm{~mm}$ thick slabs in this study. A vertical shear key, shown in Fig. 3.2.a, was used on each side of the joint to improve the shear resistance between the jointed parts.

Three details for the joints between precast panels were proposed incorporating GFRP bars as depicted in Fig. 3.2. The first proposed joint has a 200-mm wide closure strip as shown in Fig. 3.2.a. In this joint, both the top and bottom GFRP bars in the precast slab project into the joint with a 175-mm anchorage length. In this joint, the precast panel has projected slab of $90 \mathrm{~mm}$ length at the bottom of the joint to hold UHPFRC within the closure strip during casting. A foam-type packing rod is inserted in the $20-\mathrm{mm}$ gap between the two projected slabs at the bottom of the closure strip to avoid material leakage. This joint is called "Angle-shape" joint or "A-joint" in this research.

The second proposed joint has a 200-mm wide closure strip as shown in Fig. 3.2.b. This joint is identical to the first proposed joint but without the $90-\mathrm{mm}$ projecting slab. Both the top and bottom GFRP bars in the precast slab project into the joint with a $175-\mathrm{mm}$ anchorage length. It is assumed that temporary form work will be used to close the bottom of the closure strip to hold UHPFRC materials before hardening. This joint is called "C-shape" joint or " $\mathrm{C}$-joint" in this research. The third proposed joint has a 100-mm wide closure strip with a staggered $100 \mathrm{~mm}$ wide trapezoidal-shaped (zigzagged-shaped) interlock between the precast slabs as shown in the plan of Fig. 3.2.c. In this connection, the top and bottom GFRP bars of the precast slab project into the joint with a $175 \mathrm{~mm}$ anchorage length. Temporary form work will be required to hold the cast UHPFRC into the closure strip during concrete placement. This joint is called "Zigzagshape" joint or "Z-joint" in this research. 


\subsection{Experimental Program}

The experimental program investigated two types of loading that can be applied at the closure strip region, namely concentric and eccentric truck wheel loading. Figure 3.3-a shows a schematic diagram of the jointed slab with truck wheel load located over the joint creating pure bending with UHPFRC. However, Fig. 3.3.b shows the wheel load located at the edge of joint creating combined shear and bending on the UHPFRC. As such, the test setup considered in this study includes (i) pure flexural tests, and (ii) combined flexural-shear tests. The experimental program included seven full-scale deck slab specimens 2500 -mm long and 200-mm thick. A $600-\mathrm{mm}$ slab width is considered for a slab strip. The $600-\mathrm{mm}$ width is assumed oriented in the direction normal to the traffic, while the slab span is oriented in the direction of traffic. It should be noted that the selection of this slab size and details is considered for the sake of correlating test results for the jointed precast slab with identical cast-in-place slabs reinforced by either steel bars or GFRP bars. In fact, the results from this research can be applied to the joint between bulb-tee girders since the objective of this testing is to qualify the jointed precast slab to be "as good as" the cast-in-place slab of similar geometry, concrete type and reinforcement sizes but with different reinforcement material.

In this study, the wheel load is distributed only over a $600 \mathrm{~mm}$ width of the slab and over a length of $250 \mathrm{~mm}$ in the direction of slab span. The span of the slab was taken as $2000 \mathrm{~mm}$ with a slab total length of $2500 \mathrm{~mm}$ to accommodate proper bars anchorage beyond the supporting points. Table 3.1 summarizes the name coding for the tested slabs considered in this study. The first deck slab specimen, S1, is formed of cast-in-place concrete reinforced with steel bars of 400-MPa yield strength and 200-GPa modulus of elasticity. In this research, 3-20M reinforcing steel bars spaced at $200 \mathrm{~mm}$ on centre were used as bottom and top layers representing the CHBDC-specified minimum steel reinforcement of $1.0 \%$ of the concrete area. Transverse 10M steel bars were used at the top and bottom layers at $300 \mathrm{~mm}$ spacing. Figure 3.4.a shows layout of reinforcement in slab S1, while Fig. 3.6.a shows view of the steel reinforcement in slab S1. The second deck slab strip, S2, is similar to cast-in-place slab S1 but with 3-20M straight GFRP bars spaced at $200 \mathrm{~mm}$ on centre, as bottom tension reinforcement. Transverse reinforcement of 20M GFRP bars was used at the top and bottom layers at $300 \mathrm{~mm}$ on centre spacing. Fig. 3.5.b shows view of the GFRP reinforcement in slab S2. The third deck slab, S3, is similar to slab S2 
but uses the precast deck C-joint shown in Fig. 3.2.b. The amount and spacing of GFRP bars in the precast flanges were the same as those for the cast-in-place deck slab S2. Figure 3.5.b shows a view of the GFRP bar layout in S3 slab formwork, while Fig. 2.6.a shows a view of straight GFRP bars projecting into the closure strip of slab S3 before casting the UHPFRC. It should be noted that slabs S1 to S3 were loaded with the wheel load located concentrically over the joint as depicted in Fig. 3.3.a. The fourth deck slab, S4, is identical to the joined precast slab S3 and had the joint eccentrically loaded as depicted in Fig. 3.3.b.

The fifth slab S5 incorporates the trapezoidal-shaped (zigzagged-shaped) interlocking closure strip between precast panels shown in Fig. 3.2.c. The slab size and GFRP reinforcement in slab S5 is identical to those for slabs S3 and S4. Figure 3.5.c shows a view of the GFRP bar layout in S5 slab formwork, while Figure 3.6.b shows a view of the straight GFRP bars projecting into the closure strip of slab S5 before casting the UHPFRC. The sixth and the seventh deck slabs, S6 and S7, respectively, are similar to the joined precast slabs S3 and S4 but with the closure strip details shown in Fig. 3.2.a. Figure 3.5.d shows view of the GFRP bar layout in S6 slab formwork, while Figure 3.6.c shows view of the straight GFRP bars projecting into the closure strip of slab S6 before casting the UHPFRC. The only difference between slabs S6 and S7 is that the former was loaded with concentric loading while the latter was loaded with eccentric loading.

Table 3.1 summarizes the main variables of the tested slabs. Concrete having a specified 28-day compressive strength of $35 \mathrm{MPa}$ was used for both cast-in-place and precast deck slabs. Standard cylinders of 150-mm diameter and 300-mm height were cast concurrently with the casting of the deck slabs. An average of three cylinders were cast and stored close to the test samples to ensure the same curing conditions after casting. The tested cylinder had a concrete compressive strength of $45 \mathrm{MPa}$. Ultra-High-Performance Fiber Reinforced Concrete (UHPFRC) of a specified compressive concrete strength of $140 \mathrm{MPa}$ was used to fill the closure strip. Table 3.2 shows typical UHPFRC composition, while Table 3.4 shows the characteristic values of UHPFRC Type JS1000 considered in this study and available elsewhere (Lafarge, 2009). During pouring UHPFRC into the precast deck slab closure strips, standard cylinders of 100-mm diameter and 200-mm height were cast and kept close to the test samples. Table 3.5 summarizes the average strength of cylinders tested on the day of slab testing. 
The structural response during the static loading of the specimens was captured through the use of electronic instrumentation. Potentiometers and Linear variable differential transducers (LVDTs) were used to measure deflections under the loading point of the slab specimens as depicted in Fig. 3.4. Concrete strain gauges were installed along the depth of the UHPFRC joint at mid-span as depicted in Fig. 3.4. Also, strain gauges were installed on the bottom steel and GFRP bars at the mid-span point in the cast-in-place slabs S1 and S2, respectively. While they were installed in the bottom GFRP bars at the interface between the precast slab and the UHPFRC but embedded in the precast slab S3 through S7. Figures 3.4 shows locations of these gauges in the tested slabs.

A static patch load, simulating CHBDC truck wheel load was applied to examine the structural behavior and ultimate load carrying capacity of the proposed connection details as compared to the control cast-in-place slabs S1 and S2 reinforced with steel and GFRP bars, respectively. Slabs S1, S2, S3 and S6 were tested under a 250x600 mm single patch load at the center of their clear span. Figures 3.7.a and 3.8.a show the locations of the wheel load at the mid-span for slabs S1 and S2, respectively. While Figure 3.9.a shows the wheel load applied concentrically over the joint for slab S3. This patch load is equivalent to the foot print of CHBDC wheel load of 87.5 $\mathrm{kN}$. The slab ends were simply-supported over roller support at one end and hinged support at the other end. Slabs S4, S5, and S7 were tested under a 250x600 mm single patch load located at the edge of the joint as shown in Figs. 3.10-a, 3.11-a and 3.13-a, respectively.

To conduct static load tests to-collapse, the jacking load was applied in increments to allow for visual inspection of the specimen and to mark cracks. The available data acquisition system was used to capture readings from sensors as well as the load cell located between the jacking piston and the top of the deck slab. After every load increment, the start of tension cracks and crack propagations were monitored. It should be noted that the incremental loading technique was used during testing on which the specimen was loaded to $10 \mathrm{kN}$, followed by load release. The specimen was loaded then to $20 \mathrm{kN}$, followed by load release. These incremental loading steps were repeated with a total load increase of $10 \mathrm{kN}$ in each step until the specimen failed. The following section discusses the structural behavior of the test specimens. ASTM E529-04 (2011), Standard Guide for Conduction Flexural Tests on Beams and Girders for Building Construction, specifies the monotonic load to be applied and removed in equal increments at constant rate as possible. 
Under repeated load (incremental load-to-collapse) at levels that cause significant yielding, there is the potential for the slab deck to fail due to incremental accumulation of vertical deflection at loads less than the inelastic monotonic limit load. This shakedown load converges to stable elastic state under repeated loading. The shakedown load lies between the load at which the first hinge forms and the inelastic limit point that accounts on: (i) load-deformation (vertical direction); and (ii) load-slip (horizontal direction).

\subsection{Test Results}

\subsubsection{Behavior of the Tested Slabs}

This section discusses the structural behavior of the tested specimens in the form of crack pattern, slab deflection and monotonic ultimate load carrying capacity. Table 3.5 summarizes the tested slab designations (S1 through S7), concrete strengths and modes of failure. The second column of the table shows the slab configurations through three different letters. The first letter "C" for slabs S1 and S2 stands for cast-in-place slab while the first letter "J" for slabs S3 through S7 stands for jointed precast slabs. The second parts for slabs S1 and S2 as "ST" and "GFRP" stand for steel reinforcement and GFRP reinforcement, respectively. The second letter in jointed slabs S3 through S7 stand for the joint type per Fig. 3.2 (C stands for C-shape joint; Z stands for zigzag joint; A stands for Angle-shape joint). The last letter in slab configurations stands for the location of the wheel load ( $\mathrm{C}$ stands for centric loading and $\mathrm{E}$ stands for eccentric loading).

Figure 3.7 shows the crack pattern at failure at the side of the slab for the cast-in-place slab S1 (C.ST.C) reinforced with steel bars. It was observed that the first visual flexural crack appeared at the bottom of the slab at the mid-span location at a load of $18.94 \mathrm{kN}$. Other flexural cracks appeared within the quarter points of the span and propagated towards the top surface of the slab with increase in load till failure. Crushing of concrete at the top surface of the slab at the midspan location was observed at the maximum jacking load of $163.11 \mathrm{kN}$ and slab deflection of $34.05 \mathrm{~mm}$, leading to pure flexural failure. Figure 3.8 shows the crack pattern at failure of castin-place slab S2 (C.GFRP.C) reinforced with GFRP bars. It was observed that the first visible flexural crack appeared at a load of $18.79 \mathrm{kN}$. Other flexural cracks appeared at higher load increments and spread over a length greater than that for slab S1 (C.ST.C). The slab failed mainly due to large flexural-shear crack with concrete crushing at the top surface of slab at an ultimate load of $137.22 \mathrm{kN}$ and deflection of $38.95 \mathrm{~mm}$. By comparing the ultimate load capacity 
of slabs S1 (C.ST.C) and slab S2 (C.GFRP.C), it can be observed that the GFRP-reinforced slab exhibited a flexural strength about $15 \%$ less than that for a similar slab reinforced with steel bars and deflection at failure of about $26 \%$ more than that for steel-reinforced slab. This increase in deflection in the GFRP-reinforced slab may be attributed to the significant reduction in the modulus of elasticity of the GFRP bars compared to that for steel bars.

Figures 3.9-b and 3.9-c show the crack pattern at failure of precast slab S3 (J.C.C) with 200-mm wide closure strip and projecting GFRP bars into the UHPFRC-filled closure strip. It was observed that the first hairline cracks were formed at the cold joint between the precast concrete and the closure strip at a load of $18.05 \mathrm{kN}$. These fine cracks started to widen gradually with increase in applied load. Several flexural cracks as well as flexural-shear cracks appeared in the precast slab closer to the closure strip, with a wide flexural-shear crack at one side of the cold joint appearing at slab failure. Failure of slab S3 (J.C.C) was at $132.19 \mathrm{kN}$ ultimate load and $31.29 \mathrm{~mm}$ deflection. By comparing the ultimate load capacity of cast-in-place slab S2 (C.GFRP.C) and the jointed slab S3 (J.C.C), it can be observed that the GFRP-reinforced jointed slab exhibited a flexural strength about $4 \%$ less than that for a similar cast-in-place slab. This slight decrease in slab flexural strength would be acceptable given the presence of the joint at the maximum moment location with short splice length of GFRP bars. It should be noted that very light flexural crack appeared in the UHPFRC at the slab mid-span, with no visible cracks appeared in the UHPFRC material at the free sides of the slab as depicted in Figures 3.9-b and 3.9-c. As such, it can be concluded that the jointed slab S3 (J.C.C) is "as good as" the cast-inplace GFRP-reinforced concrete slab S2 (C.GFRP.C).

Figures 3.10-b and 3.10-c show the crack pattern at failure of precast GFRP-reinforced slab S4 ( J.C.E) with eccentric truck wheel loading at the joint. It was noticed that the first hairline cracks were formed at the cold joint between the precast concrete and the closure strip at a load of 18.64 $\mathrm{kN}$. These fine cracks started to widen gradually with increase in applied load. Few flexural cracks appeared in the precast slab closer to the closure strip. Close to failure, a wide diagonalshear crack, as depicted in Figure 3.10-b, propagated along the slab between the support and the applied load location, leading to slab failure. Failure of slab S4 (J.C.E) occurred at $151.87 \mathrm{kN}$ ultimate load and $39.98 \mathrm{~mm}$ deflection. Comparing jointed slabs S3 (J.C.C) with concentric loading and slab S4 (J.C.E), it can be observed that the slab ultimate load slightly increased (i.e. 
by $16 \%$ ) as a result of using eccentric loading rather than concentric loading at the closure strip, while the slab ultimate deflection decreased by about $24 \%$. This may be attributed to the fact that the shear span of the precast slab with eccentric loading decreases, leading to a higher load carrying capacity.

Figure 3.11-b and 3.11-c shows the crack pattern at failure of precast slab S5 (J.Z.E) with 100 $\mathrm{mm}$ wide closure strip and staggered 100-mm wide trapezoidal-shaped interlock, and projected GFRP bars into the joint. It was noticed that the first hairline cracks were formed at the cold joint between the precast concrete and the closure strip at a load of $17.16 \mathrm{kN}$. These fine cracks started to widen gradually with increase in applied load. Few flexural cracks appeared in the precast slab closer to the closure strip, while no cracks appeared in UHPFRC strip as shown in Figure 3.11-c. Prior to failure, a wide flexural-shear crack appeared on one both side of the cold joint at the quarter point of the precast slab as depicted in Figure 3.11-b that led to failure. Failure of slab S5 (J.Z.E) occurred at $135.30 \mathrm{kN}$ ultimate load and $30.28 \mathrm{~mm}$ deflection.

Figures 3.12-b and 3.12-c shows the crack pattern at failure of the precast slab S6 (J.A.C) with 200-mm wide closure strip and projected bottom slab segment. It was observed that the first hairline cracks were formed at the cold joint between the precast concrete and the closure strip and propagated towards the top of the slab with load increase as depicted in the close-up in Figure 3.11-c at a load of about $16.42 \mathrm{kN}$. These cracks widened with increase in the applied load as depicted in Figure 3.10-c, leading to concrete crushing at the top surface of the slab with the sudden flexural shear crack at one side of the A-Joint. Other flexural crack appeared in the UHPFRC as depicted in Figure 3.10-b. The slab failed at $121.24 \mathrm{kN}$ ultimate load and $35.16 \mathrm{~mm}$ deflection. It can be noted that slab S6 has a flexural capacity less than those for the steelreinforced slab S1 (C.ST.C) and the GFRP-reinforced slab S2 (C.GFRP.C) by about 25\% and $12 \%$, respectively. Figures 3.13-b and 3.13-c show the crack pattern at failure for precast slab S7 (J.A.E) which was loaded with eccentric load as depicted in Fig. 3.13.a. It was observed that first hair crack load was at $19.90 \mathrm{kN}$. The failure occurred at ultimate load of $130.71 \mathrm{kN}$ and 34.49 $\mathrm{mm}$ deflection and the failure mode was combined flexure and shear. One may observe that slab S7 (J.A.E) with eccentric loading exhibited 7.8\% load carrying capacity greater than that for the identical slab S6 (J.A.C) with concentric loading. 
Figure 3.14 depicts the incremental load-deflection history of all tested specimens. Although the load-deflection history presents a measure of the change in slab flexural stiffness due to cracking, and yielding of steel in case of steel-reinforced slab, with increase in applied load, the given data can be used further to determine slab ductility under wheel load. Such analysis as well as forthcoming testing of these slabs under fatigue loading is expected to provide engineers with clear understanding of the best joint between precast slab for enhanced strength, fatigue service life and durability. Table 3.6 summarizes the test results in the form of cracking and ultimate loads, ultimate deflections and ultimate strains in concrete and bars. Results in Fig. 3.14 for each slab and the combined results for all slabs show that incremental loading on the tested slabs per ASTM E529-04 test method did not cause significant yielding, of failure due to incremental accumulation of vertical deflection at loads less than the inelastic monotonic limit load.

\subsubsection{Analysis of Test Data for Flexural and Shear Resistance}

The objective of this analysis is to determine the resisting moment of the concrete and UHPFRC sections by manual calculations and correlate them to the experimental ultimate moment. Figure 3.16 depicts the structural analysis of the tested slabs so that the reaction forces and moments can be obtained through the following equations.

$$
\begin{array}{ll}
R_{1}=V_{1}(\max . \text { when } \mathrm{a}<\mathrm{c}) & =\frac{w b}{2 L}(2 c+b) \\
R_{2}=V_{2}(\max . \text { when } \mathrm{a}>\mathrm{c}) & =\frac{w b}{2 L}(2 a+b) \\
V_{x}(\text { when } \mathrm{x}>\mathrm{a} \text { and }<(\mathrm{a}+\mathrm{b})) & =R_{1}-w(x-a) \\
M_{\max }\left(\text { at } x=a+\frac{R_{1}}{w}\right) & =R_{1}\left(a+\frac{R_{1}}{2 w}\right)
\end{array}
$$

The resisting bending moment of the reinforced concrete slab of width $600 \mathrm{~mm}$ was calculated based on the procedure available elsewhere (ISIS, 2007) for singly-reinforced section and based on the rectangular section analysis shown in Fig. 3.17 and the following set of equations. Also, the theoretical shear capacity of the tested slabs was obtained using Equation 3.12 (ISIS, 2007).

$$
\rho_{f r p}=\frac{A_{f r p}}{b d}
$$




$$
\epsilon_{f r p u}=\frac{f_{f r p u}}{E_{f r p}} \mathrm{e}
$$

$$
\rho_{f r p b}=\frac{A_{f r p}}{b d}=\alpha_{1} \beta_{1} \frac{\phi_{c}}{\phi_{f r p}} \frac{f_{c}^{\prime}}{f_{f r p u}}\left(\frac{\epsilon_{c u}}{\epsilon_{c u}+\epsilon_{f r p u}}\right)
$$$$
\rho_{\text {frp }}=0.011636>\rho_{\text {frpb }}=0.00655
$$$$
f_{f r p}=0.5 E_{f r p} \epsilon_{c u}\left[\sqrt{1+\frac{4 \alpha_{1} \beta_{1} \phi_{c} f_{c}^{\prime}}{\rho_{f r p} \phi_{f r p} E_{f r p} \epsilon_{c u}}}-1\right]
$$

$$
\beta_{1} c=a=\frac{\phi_{f r p} A_{f r p} f_{f r p}}{\alpha_{1} \phi_{c} f_{c}^{\prime} b}
$$

$$
M_{r}=\phi_{f r p} A_{f r p} f_{f r p}\left[d-\frac{a}{2}\right]
$$

In section analysis, the resistance factors for concrete, $\phi_{c}$, and GFRP reinforcing bars, $\phi_{f r p}$, were taken as 0.75 and 0.55 , respectively (CHBDC, 2014). Using the above-mentioned equations, the bottom HM GFRP reinforcement ratio, $\rho_{f r p}$, and the balanced HM GFRP reinforcement ratio, $\rho_{f r p b}$, were found to be $1.16 \%$ and $0.52 \%$, respectively. As such, the slab section would fail under concrete compression failure mode. Section analysis showed that the tensile stress in HM GFRP reinforcement at compression failure of the section was $f_{f r p}=$ 785.32 MPa which is far below the tensile strength of the bars. The stress block depth, a, was found to be $25.69 \mathrm{~mm}$. Thus, the calculated resisting moment, $M_{r}$, was found to be 49.73 kN.m/0.6-m from Equation [3.11], or $82.88 \mathrm{kN} . \mathrm{m} / \mathrm{m}$. Equation 3.12 provided the shear resistance of the slab as $76.84 \mathrm{kN} / \mathrm{m}$.

The generalized internal stress distribution for the flexural behavior of UHPFRC rectangular section can be simplified as shown in Fig. 3.18 (Garcia, 2007). The compressive force is approximated through a triangular stress distribution, where the compressive force, $\mathrm{C}$, acts at one-third the neutral axis depth below the extreme compression fiber of the rectangular section. A uniform tensile stress of $f_{t}$ acts along the section depth from the neutral axis to the extreme 
fibers in tension. The tensile force from the HM GFRP bars is expressed in the same manner as that for reinforced concrete section (Garcia, 2007).

$$
\begin{aligned}
& C=\frac{1}{2} \phi_{c} f_{c}^{\prime} a b \\
& T_{c}=\phi_{c} f_{t}(h-a) b \\
& T_{f r p}=\phi_{f r p} f_{f r p u} A_{f r p}
\end{aligned}
$$

Calculations showed that the depth of the stress block was calculated from Equation 3.15 to be $35.23 \mathrm{~mm}$ when the compression stress, $f_{c}^{\prime}$, and tension stress, $f_{t}$, of UHPFRC are 140 and 8 $\mathrm{MPa}$ respectively, and the ultimate tensile strength of the bar, $f_{\text {frpu }}$, is $1188 \mathrm{MPa}$. The resistance factor of concrete and HM GFRP considered in these calculations were equal to 0.75 and 0.55 , respectively (CHBDC, 2014).

$$
a=\frac{\phi_{f r p} A_{f r p} f_{f r p u}+\phi_{c} f_{t} h b}{b\left(0.5 \phi_{c} f_{c}^{\prime}+\phi_{c} f_{t}\right)}
$$

The calculated resisting moment of the UHPFRC section was then obtained from Equation 3.16 as $137.29 \mathrm{kN} . \mathrm{m} / 0.6-\mathrm{m}$ or $228.81 \mathrm{kN} . \mathrm{m} / \mathrm{m}$.

$$
\begin{aligned}
& M_{n}=T_{f r p}\left(d-\frac{a}{3}\right)+T_{c}\left(\frac{h}{2}+\frac{a}{6}\right) \\
& M_{n}=\phi_{\text {frp }} f_{f r p} A_{f r p}\left(d-\frac{a}{3}\right)+\phi_{c} f_{t}(h-c) b\left(\frac{h}{2}+\frac{a}{6}\right)
\end{aligned}
$$

Table 3.7 shows summary of experimental data as well as theoretical bending and shear resistance of the tested slabs. The ratio between the experimental resisting moment and theoretical resisting moment, $\beta$, was calculated using equation Equation 3.17. Results show shows the ratio between the experimental resisting moment and theoretical moment for the precast slabs as 1.29, 1.25, 1.36, 1.18, 1.14 and 1.17 for slabs S2, S3, S4, S5, S6 and S7, respectively. So, Bridge Design Engineers can use the traditional procedure for section analysis mentioned earlier to determine the resisting moment of the slabs made of normal concrete as the ratio $\beta$ is more than 1 for all slabs. However, designers may consider the durability effect on the GFRP bars in the tested slabs of 0.75 as specified in CHBDC chapter 16 . In this case, the ratio $\beta$ may be $0.97,0.93,1.02,0.88,0.86$ and 0.88 for slabs S2, S3, S4, S5, S6 and S7, respectively. As 
such, Designers may consider the resisting moment of the reinforced concrete section as the revised ratio $\beta$, taking into account durability effects of GFRP bars, times the resisting moment calculated based on Equation 3.11. On the other hand, Designers should not rely on the moment resistance of UHPFRC section, of $228.81 \mathrm{kN} . \mathrm{m} / \mathrm{m}$, since it is far greater than the experimental resisting moment as well as the theoretical resisting moment of the normal concrete section.

$$
\beta=M_{r, \text { experimental }} / M_{r, \text { theoretical }}
$$

As for shear, the last column in Table 3.7 depicts the ratio between the experimental resisting shear force and the theoretical value. Results show shows the ratio between the experimental resisting shear force and theoretical value for the precast slabs as 1.49, 1.43, 2.02, 1.87, 1.31 and 1.74 for slabs S2, S3, S4, S5, S6 and S7, respectively. However, designers may consider the durability effect on the GFRP bars in the tested slabs of 0.75 as specified in CHBDC chapter 16. In this case, the ratio $\beta$ may be $1.12,1.08,1.51,1.40,0.99$ and 1.30 for slabs S2, S3, S4, S5, S6 and S7, respectively. In this case, designers may use Equation 3.12 to determine the shear resistance of the precast slab made of normal concrete without correction factors.

Table 3.8 provides the coefficients for the third-degree polynomial equation that used to model the moment-deflection behavior from starting of the load to the ultimate to-collapse loading. Polynomial equations show excellent coefficient of determination $\mathrm{R}^{2}$ close to 1 .

\subsubsection{Moment-Strain Relationship and Associated Bond Stress for GFRP Bars in UHPFRC}

Figures 3.19 to 3.25 depict the moment-strain relationships for the bottom and top HM GFRP bars and for concrete at mid-span for cast-in-place concrete and at the edge of the applied load at the mid-span for the jointed slabs. General purpose, 10-mm, strain gages were placed at the midlength of the bar for the cast-in-place control slabs reinforced with steel bars or HM GFRP bars. However, for jointed slabs, the 10-mm strain gages were attached to the HM GFRP bars embedded into the precast FDDP but just at the interface between the precast slab and the joint. Foil strain gauges of $60 \mathrm{~mm}$ length were placed on the top surface of the concrete and along the depth of the mid-point of the control slabs or along the depth of the joint to measure concrete strains. Figure 3.4 shows locations of sensors used in this research. Table 3.9 record three readings for the micro-strain gages glued to the top surface of the concrete slab, to the top 
reinforcement, and to the bottom reinforcement, respectively. These values were recorded at the failure load. This strain data was considered for further analysis to determine the bottom bar tensile force as shown in Table 3.10. By multiplying the tensile force by the bar cross-sectional area, the actual bar stresses at slab failure was recorded as 537, 662, 620, 508, 473, and $420 \mathrm{MPa}$ for slabs S2, S3, S4, S5, S6 and S7, respectively. This values were far below the tensile strength of the GFRP bars of $1188 \mathrm{MPa}$, as expected. To determine the actual bond stress $(\tau)$ developed in the GFRP bar embedded into UHPFRC in the jointed slabs, the following equations were used and the results were presented in Table 3.10.

$$
\begin{aligned}
\frac{1}{k} & =\frac{T}{L_{d} \sqrt{f_{c}^{\prime}}}=\frac{A_{f r p} E_{f r p} \epsilon_{f r p}}{L_{d} \sqrt{f_{c}^{\prime}}} \\
\tau & =\frac{\sqrt{f_{c}^{\prime}}}{\pi k d}
\end{aligned}
$$

Results in Table 3.10 show that the actual bond stress in GFRP bars embedded into UHPFRC in the tested slabs were found to be $18.92,17.73,14.53,13.52$ and $12.03 \mathrm{MPa}$ for jointed slabs S3, S4, S5, S6 and S7, respectively. These values are generally lower than those obtained from direct pullout tests on GFRP bars embedded in UHPFRC blocks presented in Chapter II of this Thesis.

\subsection{Conclusions}

This research presented the mechanical behavior of precast full-depth deck panels (FDDPs) made of normal-strength concrete (NSC) and reinforced with straight-ended ribbed-surface HM GFRP bars. For FDDPs constructed using NSC, the following conclusions were drawn:

1- The ultimate load capacity of GFRP-reinforced cast-in-place slab was about $15 \%$ less than the capacity of a similar slab reinforced with steel bars in accordance with the CHBDC and having the same cross-sectional area as the GFRP bars.

2- GFRP-reinforced jointed slab with a $200 \mathrm{~mm}$ wide joint width with a zigzag-shape exhibited a flexural strength that was about $1.4 \%$ less than that for a similar cast-in-place slab. 
3- Comparing jointed precast slabs with different applied load patterns, it was observed that the ultimate load of the jointed slab with a vertical shear key increased by $15 \%$ as a result of using eccentric loading rather than the concentric loading at the closure strip.

4- The precast slab with a $200-\mathrm{mm}$ wide closure strip and projected bottom slab segment proved to have flexural capacity less than those for the steel-reinforced cast-in-place slab and the GFRP-reinforced cast-in-place slab by $25 \%$ and $12 \%$, respectively.

5- In case, of the effect of load location on this slab, it was observed that the eccentric wheel loading, when compared to the concentric wheel loading, increased the load carrying capacity by $7.8 \%$. As such, concentric loading will be used in further tests to examine the fatigue life of the successful control joints under simulated vehicular wheel loading in case of bulb-tee girder system. However, in case of FDDPs placed transversally over the girders, eccentric loading may be the proper choice given the distribution of wheel loads within the NSC panel is more critical than that when the wheel load is located over the UHPFRC-filled closure strip.

6- The failure mode in all tested one-way slab specimens was either pure flexural of combined flexural and shear in the NSC panel rather than (i) in the UHPFRC-filled joint or (ii) bond failure of GFRP bars embedded into the joint. This conclusion would allow Bridge Design Engineers design the precast jointed NSC-built deck slabs using the oneway slab action specified in AASHTO-LRFD Specification or the flexural design method specified in CHBDC. In this case, Bridge Design Engineers will adopt one of the developed UHPFRC-filled joint configurations without design and consider the design of the precast slab under factored applied wheel load specified in the code per meter width to prevent pure flexure failure or combined shear-flexural failure outside the joint.

7- Considering the ratios between the experimental moment and the theoretical moment resistance of the tested slabs made normal-strength concrete, $\beta$, as $0.97,0.93,1.02,0.88$, 0.86 and 0.88 for slabs S2, S3, S4, S5, S6 and S7, respectively, designers may consider the resisting moment of the reinforced concrete section as the ratio $\beta$ times the resisting moment calculated based on traditional procedure available in CHBDC. This conclusion was reached by applying a general durability factor of 0.75 to the test results of the tested slabs made of GFRP bars as specified in CHBDC. However, test results showed that the shear resistance of the precast slab made of normal concrete calculated using ISIS shear 
equation is conservative even with the application of the 0.75 durability factor to the experimental shear resistance.

\section{References}

Aarup, B., and Jensen, B. 1998. Bond Properties of High Strength Fiber Reinforced Concrete. Bond and Development of Reinforcement, ACI Publication SP-180, pp. 459-472.

Aarup, B., Karlsen, J., and Lindström, G. 2000. Fiber Reinforced High Performance Concrete for In-Situ Cast Joints. Proceedings, PCI/FHWA/FIB International Symposium on High Performance Concrete, Orlando, Florida, 9 pp.

Afefy, H., Sennah, K., Tu, S., Ismail, M., and Kianoush, R. 2015. Experimental Study on the Ultimate Capacity of Deck Joints in Prefabricated Concrete Bulb-Tee Bridge Girders. Journal of Bridge Structures, Design, Assessment and Construction, 11 (2015) 55-71.

Ametrano, D. 2011. Bond Characteristics of Glass Fibre Reinforced Polymer Bars Embedded in High Performance and Ultra-High Performance Concrete. M.A.SC. Thesis, Civil Engineering Department, Ryerson University, Toronto, Ontario, Canada.

ASTM. 2011. Standard Guide for Conduction Flexural Tests on Beams and Girders for Building Construction, ASTM E529-04. American Society for Testing and Materials Standard Practice, Philadelphia, PA, USA.

Badie, S., and Tadros, M. 2008. NCHRP Report 584: Full-Depth Precast Concrete Bridge Deck Panel Systems. Transportation Research Board, Washington, D.C.

Badie, S., Tadros, M., and Girgis, A. 2006. Full-Depth Precast-Concrete Bridge Deck Panel Systems, Final Report NCHRP-12-65, s.1.: National Cooperative Highway Research Program.

Broo, H., and Broo, M. 1997. Fog av högpresterande fiberbetong i prefabricerad brobaneplatta," examensarbete 97:2, Chalmers Tekniske Högskola, avd. för Betongbyggnad. (in Swedish, Joint of High Performance Fiber Reinforced Concrete in Precast Bridge Slabs).

Garcia, H. 2007. Analysis of an Ultra-High Performance Concrete Two-Way Ribbed Bridge Deck Slab, FHWA-HRT-07-056. Federal Highway Adminstration, McLean, VA.

Chapman, C. 2010. Behavior of Precast Bridge Deck Joints with Small Bend Diameter U-Bars. Master's Thesis, University of Tennessee, Konxville.

CHBDC. 2014. Canadian Highway Bridge Design Code, CAN/CSA-S6-06. Canadian Standard Association, Toronto, Ontario, Canada. 
Chim, C., and Chang, S. 2003. Cracking of Continuous Composite Beams with Precast Decks. Journal of Constructional Steel Research, 59: 201-204.

Culmo, M. 2011. Accelerated Bridge Construction - Experience in Design, Fabrication and Erection of Prefabricated Bridge Elements and Systems. Report No. FHWA-HIF-12-013, Federal Highway Admin., 347 pages.

Graybeal, B. 2006. Material Property Characterization of Ultra-High Performance Concrete. Federal Highway Administration, Report No. FHWA-HRT-06-103, 186 pp.

Graybeal, B. 2010. Behavior of Field-Cast Ultra-High Performance Concrete Bridge Deck Connections under Cyclic and Static Structural Loading. Report No. FHWA-HRT-11-023, Federal Highway Administration, 116 pages.

Hanna, K., Morcous, G. and Tadros, M. 2010. Second Generation Precast Deck Panel (NUDECK) System, University of Nebraska-Lincoln, Omaha, Nebraska.

Hansen, L., and Jensen, B. 1999. A New Building System Using Joints of Ultra High-Strength Fibre Reinforced Concrete, Innovation in Concrete Structures: Design and Construction, Dundee, pp. 543-552.

Harryson, P. 1999. Böjprovning av fog i högpresterande fiberbetong för prefabricerade brobaneplattor, statisk belastning," rapport 99:1, Chalmers Tekniske Högskola, avd. för Betonbyggnad. (in Swedish, Bending Test of Joint in High Performance Fiber Reinforced Concrete for Precast Bridge Slabs, Static Loading).

Harryson, P. 2000. Utmattningprovning av fog i högpresterande fiberbetong för prefabricerade brobaneplattor, rapport 00:2, Chalmers Tekniske Högskola, avd. för Betongbyggnad. (in Swedish, Fatigue Test of Joint in High Performance Fiber Reinforced Concrete for Precast Bridge Slabs).

He, Z., Ma, Z., Chapman, C., and Liu, Z. 2013. Longitudinal Joints with Accelerated Construction Features in Decked Bulb-Tee Girder Bridges: Strut-and-Tie Model and Design Guidelines. ASCE Journal of Bridge Engineering, 18(5): 372-379.

Hieber, D., and Wacker, J. 2005. State-of-the-Art Report on Precast Concrete Systems for Rapid Construction of Bridges. Report No. WA-RD 594.1, Washington State Department of Transportation, 112 pages.

Hwang, H., and Park, S. 2014. A study on the Flexural Behavior of Lap-Splices Cast-in-Place Joints under Static Loading in Ultra-High Performance Concrete Bridge Deck Slabs. Canadian 
Journal for Civil Engineering, 41: 615-623.

ISIS Canada Research Network. 2007. Reinforcing Concrete Structures with Fibre Reinforced Polymers, Design Manual No. 3. ISIS Canada, Intelligent Sensing for Innovative Structures, University of Manitoba, Winnipeg, Manitoba, Canada.

Issa, M., Anderson, R., Domagalski, T., Asfour, S., Islam, M. 2007. Full-Scale Testing of Prefabricated Full-Depth Precast Concrete Bridge Deck Panel System. ACI Structural Journal, 104(3): 324-332.

Issa, M., Idriss, A., Kaspar, I., and Khayyat, S. 1995. Full depth precast and Precast, Prestressed Concrete Bridge Deck Panels. PCI Journal, pp. 59-69.

Issa, M., Ribeiro, C. Abdalla, H., Islam, S., Issa, M. 2003. Performance of Transverse Joint Grout Materials in Full-Depth Precast Concrete Bridge Deck Systems. PCI Journal, 48(4): 92-103.

Issa, M., Salas, J., Shabila, H., and Alrousan, R. 2006. Composite Behavior of Precast Concrete Full-Depth Panels and Prestressed Girders. PCI Journal, 132-145.

Julander, J. 2009. Finite Element Modeling of Full-Depth Precast Concrete Transverse Bridge Deck Connections. Master's Thesis, Civil and Environmental Engineering, Utah State University, Utah.

Khalafalla, I. 2013. Development of sustainable concrete bridge deck slab systems using corrosionresistant GFRP bars, Ph.D. Thesis, Ryerson University, Toronto, Ontario, Canada.

Lee, J., and Lee S. 2015. Flexural Behavior of Ultra-High Performance Fibre-Reinforced Concrete Moment Connection for Precast Concrete Decks. ACI Structural Journal, 112(4): 451-638.

Lee, J., and Lee S. 2014. Evaluation of Flange-to-Flange Connection of Precast Concrete Deck using UHPFRC. PCI and National Bridge Conference, pp. 1-12.

Li, L., Ma, Z., Griffey, M., Oesterle, R. 2011. Improved Longitudinal Joint Details in Decked Bulb Tees for Accelerated Bridge Construction: Concept Development, Journal of Bridge Engineering, 15(3): 327-336.

Li, L., Ma, J., and Oesterle, R. 2010. Improved Longitudinal Joint Details in Decked For Accelerated Bridge Construction: Fatigue Evaluation. ASCE Journal of Bridge Engineering, V. 15, No. 5, September-October 2010, p. 511-522.

Liu, R. 2011. Precast Concrete Bridge Deck Panels Reinforced with Glass Fibre Reinforced Polymer Bars. Ph.D. Dissertation, Department of Civil and Environmental Engineering, University of Utah. 
Ma, Z., Cao, Q., Chapman, C., Burdette, E., and French, C. 2012. Longitudinal Joint Details with Tight Bend Diameter U-Bars. ACI Structural Journal, 109(6): 815- 824.

Markowski. S. 2005. Experimental and Analytical Study of Full-Depth Precast/Prestressed Concrete Deck Panels for Highway Bridges. M.SC. Thesis, Department of Civil Engineering, University of Wisconsin-Madison, Wisconsin.

NCHRP. 2008. Full-Depth Precast Concrete Bridge Deck Panel Systems. NCHRP Report 584, Transportation Research Board, Washington DC.

NCHRP. 2011. Summary of Cast-In-Place Concrete Connections for Precast Deck Systems. Research Results Digest 355, Transportation Research Board, pp. 1-33.

Nielsen, C., Olesen, J. and Aarup, B. 1996. Effects of Fibres on the Bond Strength of High Strength Concrete. BHP96 Fourth International Symposium on Utilization of High-Strength/HighPerformance Concrete, Paris, France.

Niroumand, S. 2009. Resistance mechanism of simple-made-continuous connections in skew and non-skew steel girder bridges using conventional and accelerated types of construction. Ph.D. Dissertation, University of Nebraska - Lincoln, Nebraska, USA.

Ong, K., Hao, j., and Paramasivam, P. 2006. A strut-and-Tie Model for Ultimate Loads for Precast Concrete Joints with Loop Connections in Tension. Journal of Construction and Building Materials, 20: 169-176.

PCI. 2011. State-of-the-art Report on Full-Depth Precast Concrete Bridge Deck Panels. Prepared by PCI Committee on Bridges and PCI Bridge Producers Committee. Precast/prestressed Concrete Institute, PCI, 141 Pages.

PCINER Technical Committee, 2002. Full Depth Precast Concrete Deck Slabs, Report No. PCINER-02-FDPCDS, New England Region: Precast/Prestressed Concrete Institute New England Region.

Porter, S., Julander, J., Halling, M., Barr, P., Boyle, H., and Xing, S. 2011. Flexural Testing of Precast Bridge Deck Panel Connections. ASCE Journal of Bridge Engineering, 16(3): 422430.

Porter, S., Julander, J., Halling, M. and Barr, P. 2012. Shear Testing of Precast Bridge Deck Panel Transverse Connections. ASCE Journal of Performance of Constructed Facilities, 26(2): 462-468.

Price, S. 2010. Innovative Connection Details for Full-Depth Precast Bridge Deck Panels for Use on 
Prestresssed Concrete Bridges. Ph.D. Dissertation, Purdue University, Indiana.

Roddenberry, M. 2012. Prefabricated/Precast Bridge Elements and Systems (PBES) for Off-System Bridges. Final Report submitted to Florida Department of Transportation and prepared by Florida A\&M University, Florida, 85 pages.

Russell, H. G. and Graybeal, B. A. 2013. Ultra-High Performance Concrete: A State-of-the-Art Report for the Bridge Community, McLean, VA: Office of Infrastructure Research \& Development, Federal Highway Administration.

Ryu, H., Chang, S., and Shim, C. 2003. An Estimation of Ultimate Strength of Continuous Composite Box-Girder with Precast Decks. Journal of Korean Society of Civil Engineers, 23(2A): 355-362.

Saleem, M., Mirmairan, A., Xia, J., and Mackie, K. 2012. Development Length of High-Strength Steel Rebar in Ultra-High Performance Concrete. ASCE Journal of Materials in Civil Engineering, online.

Schoeck Canada Inc. 2011. ComBar Technical Information. [Online] Available at: www.schoeck-canada.com [Accessed Dec 2011].

Sennah, K., and Afefy, H. 2015. Development and Study of Deck Joints in Prefabricated Concrete Bulb-Tee Bridge Girders: Conceptual Design. Journal of Bridge Structures, Design, Assessment and Construction, 11 (2015) 33-53.

Shah, B., Sennah, K., Kianoush, R., Tu, S., and Lam, C. 2007. Experimental Study on Prefabricated Concrete Bridge Girder-to-Girder Intermittent-Bolted Connection Systems. ASCE journal of Bridge Engineering, 12(5): 570-584.

Shah, B., Sennah, K., Kianoush, K., Tu, S., and Lam. C. 2006. Flange-to-Flange Moment Connections for Precast Concrete Deck Bulb-Tee Bridge Girders. Journal of Prestressed Concrete Institute, PCI, 51(6): 86-107.

SHRP2. 2012a. Innovative Bridge Deigns for Rapid Renewal: ABC Toolkit. Report prepared for the Strategic Highway Research Program of the Transportation Research Board, HNTB Corp, 308 pages.

SHRP2. 2012b. Innovative Bridge Deigns for Rapid Renewal: Final Report. Report prepared for the Strategic Highway Research Program of the Transportation Research Board, HNTB Corp, 873 pages.

Sullivan, S., and Roberts-Wollmann, C. 2008. Experimental and Analytical Investigation of Full- 
Depth Precast Deck Panels on Prestressed I-Girders, Report No. FWHA/VTRC-09-CR4, Federal Highway Admin., 84 pages.

Tadros, M. K. and Baishya, M. C. 1998. Rapid Replacement of Bridge Decks, NCHRP Report 407, Transportation Research Board, Washington, D.C.

Thompson, M., Jirsa, J., and Breen, J. 2006a. Behavior and Capacity of Headed Reinforcement. ACI Structural Journal, 103(4): 522-530.

Thompson, M., Jirsa, J., and Breen, J. 2006b. Lap Splices Anchored by Headed Bars. ACI Structural Journal, 103(2): 271-279.

UDOT. 2010. Full Depth Precast Concrete Deck Panel Manual. Utah Department of Transportation, 24 pages.

Versace, J. D. 2003. Implementation of Full-Depth Precast Deck Panels. Master's Thesis, Purdue University, West Lafayette, IN.

Versace, J. D., and J. A. 2004. Ramirez. Implementation of Full-Width Bridge Deck Panels: A Synthesis Study. Publication FHWA/IN/JTRP-2003/24. Joint Transportation Research Program, Indiana Department of Transportation and Purdue University, West Lafayette, Indiana.

Zhu, P., Ma, Z., Cao, Q., and French, C. 2012. Fatigue Evaluation of Transverse U-Bar Joint Details for Accelerated Bridge Construction. Journal of Bridge Engineering, 17:191-200. 
Tables

Table 3.1. Name coding

\begin{tabular}{|c|c|c|c|c|c|}
\hline \multicolumn{5}{|c|}{ Name coding } & \multirow[t]{2}{*}{ Description } \\
\hline Slab type & \multicolumn{3}{|c|}{ Connection type } & Loading case & \\
\hline \multirow[t]{2}{*}{ C: Control Slab } & \multicolumn{3}{|c|}{ ST: Steel bars - no joint } & \multirow{5}{*}{$\begin{array}{c}\text { C: Centric loading } \\
\text { E: Eccentric loading }\end{array}$} & \multirow{2}{*}{ Cast-in-place } \\
\hline & \multicolumn{3}{|c|}{ GFRP: GFRP bars - no joint } & & \\
\hline \multirow[t]{3}{*}{ J: Joint slab } & \multicolumn{3}{|c|}{ A: Angle shape joint with GFRP bars } & & \multirow{3}{*}{$\begin{array}{l}\text { Precast } \\
\text { FDDP }\end{array}$} \\
\hline & \multirow{2}{*}{\multicolumn{3}{|c|}{$\begin{array}{l}\text { C: C-Shape joint with GFRP bars } \\
\text { Z: Zigzag shape joint with GFRP bars }\end{array}$}} & & \\
\hline & & & & & \\
\hline \multicolumn{6}{|c|}{ Example: C.ST.C means Control slab with steel reinforcement bars with applied centric loading } \\
\hline \multicolumn{6}{|c|}{ Table 3.2. Properties of GFRP bars considered in slab testing (Schoeck Canada, 2013) } \\
\hline Bar & $\begin{array}{l}\text { Designated } \\
\text { diameter }\end{array}$ & $\begin{array}{l}\text { Core diameter } \\
\quad(\mathrm{mm})\end{array}$ & $\begin{array}{c}\text { Exterior } \\
\text { diameter } \\
(\mathrm{mm})\end{array}$ & $\begin{array}{c}\text { Cross- } \\
\text { sectional-area } \\
\left(\mathrm{mm}^{2}\right)\end{array}$ & $\begin{array}{c}\text { Specific } \\
\text { weight }(\mathrm{kg} / \mathrm{m})\end{array}$ \\
\hline$\varnothing 12$ & $12 \mathrm{M}$ & 12 & 13.5 & 113 & 0.30 \\
\hline$\varnothing 20$ & $20 \mathrm{M}$ & 20 & 22 & 314 & 0.80 \\
\hline
\end{tabular}

Table 3.2. Typical UHPFRC composition (Graybeal, 2006)

\begin{tabular}{lll}
\hline Material & Size & Percentage by weight \\
\hline Portland Cement & $15 \mu \mathrm{m}$ & $27-38$ \\
\hline Fine sand & $150-600 \mu \mathrm{m}$ & $39-41$ \\
\hline Silica fume & $0.2 \mu \mathrm{m}$ & $8-9$ \\
\hline Ground Quartz & $10 \mu \mathrm{m}$ & $0-8$ \\
\hline Steel fibers & $0.2 \mathrm{~mm} \times 12.7 \mathrm{~mm}$ & $5-8$ \\
\hline Water & - & $5-8$ \\
\hline High Range Water Reducer (HRWR) & - & $0.5-1.0$ \\
\hline
\end{tabular}

Table 3.4. Characteristic values of UHPFRC type JS1000 for the design of joint (Lafarge Canada Inc., 2009)

\begin{tabular}{lccc}
\hline Mechanical properties & \multicolumn{2}{c}{ Test data } & \multirow{2}{*}{ Design values } \\
\cline { 2 - 3 } & Mean & Standard deviation & \\
\hline Compression & $140 \mathrm{MPa}$ & $10 \mathrm{MPa}$ & $100 \mathrm{MPa}$ \\
\hline Flexural & $30 \mathrm{MPa}$ & $5 \mathrm{MPa}$ & $27 \mathrm{MPa}$ \\
\hline Direct tension & $8 \mathrm{MPa}$ & $1 \mathrm{MPa}$ & $5 \mathrm{MPa}$ \\
\hline Young's modulus & $50 \mathrm{GPa}$ & $2 \mathrm{GPa}$ & $45 \mathrm{GPa}$ \\
\hline
\end{tabular}


Table 3.5. Summary of slab configurations and test results

\begin{tabular}{|c|c|c|c|c|c|c|c|}
\hline \multirow{2}{*}{$\begin{array}{c}\text { Slab } \\
\text { number }\end{array}$} & \multirow{2}{*}{ Slab type } & \multirow{2}{*}{ Reinforcement } & \multirow{2}{*}{ Slab type } & \multirow{2}{*}{$\begin{array}{c}\text { Wheel } \\
\text { load } \\
\text { location }\end{array}$} & \multicolumn{2}{|c|}{$\mathrm{f}_{\mathrm{c}}^{\prime}(\mathrm{MPa})$} & \multirow{2}{*}{$\begin{array}{c}\text { Failure } \\
\text { mode }\end{array}$} \\
\hline & & & & & Concrete & UHPFRC & \\
\hline S1 & C.ST.C & Steel bars & Cast-in-place & Concentric & 45 & - & Flexural \\
\hline S2 & C.GFRP.C & GFRP bars & Cast-in-place & Concentric & 45 & - & $\begin{array}{c}\text { Flexural- } \\
\text { shear }\end{array}$ \\
\hline S3 & J.C.C & GFRP bars & $\begin{array}{l}\text { Precast with } 200 \\
\text { mm closure strip } \\
\text { filled with } \\
\text { UHPFRC }\end{array}$ & Concentric & 45 & 141.11 & $\begin{array}{c}\text { Flexural- } \\
\text { shear }\end{array}$ \\
\hline S4 & J.C.E & GFRP bars & $\begin{array}{c}\text { Precast with } 200 \\
\text { mm closure strip } \\
\text { filled with } \\
\text { UHPFRC }\end{array}$ & Eccentric & 45 & 166.58 & $\begin{array}{c}\text { Flexural- } \\
\text { shear }\end{array}$ \\
\hline S5 & J.Z.E & GFRP bars & $\begin{array}{c}\text { Precast with } \\
\text { zigzag-shaped } \\
\text { closure strip filled } \\
\text { with UHPFRC }\end{array}$ & Eccentric & 45 & 166.58 & $\begin{array}{c}\text { Flexural- } \\
\text { shear }\end{array}$ \\
\hline S6 & J.A.C & GFRP bars & $\begin{array}{l}\text { Precast with } 200 \\
\text { mm closure strip } \\
\text { with bottom } \\
\text { projecting slab and } \\
\text { filled with } \\
\text { UHPFRC }\end{array}$ & Concentric & 45 & 141.11 & $\begin{array}{c}\text { Flexural- } \\
\text { shear }\end{array}$ \\
\hline S7 & J.A.E & GFRP bars & $\begin{array}{l}\text { Precast with } 200 \\
\text { mm closure strip } \\
\text { with bottom } \\
\text { projecting slab and } \\
\text { filled with } \\
\text { UHPFRC }\end{array}$ & Eccentric & 45 & 141.11 & $\begin{array}{c}\text { Flexural- } \\
\text { shear }\end{array}$ \\
\hline
\end{tabular}

Note: $\mathrm{f}_{\mathrm{c}}^{\prime}=$ the compressive strength of the concrete 
Table 3.6. Test results

\begin{tabular}{clccccc}
\hline Serial & $\begin{array}{c}\text { Specimen } \\
\text { name }\end{array}$ & $\begin{array}{c}\text { Cracking } \\
\text { load }\end{array}$ & $\begin{array}{c}\text { Ultimate } \\
\text { load }\end{array}$ & $\begin{array}{c}\text { Ultimate } \\
\text { deflection }\end{array}$ & $\begin{array}{c}\text { Max. side } \\
\text { concrete strain } \\
\text { x } 10^{-6}\end{array}$ & $\begin{array}{c}\text { Max. bar } \\
\text { strain } \\
\text { x 10 }\end{array}$ \\
\hline S1 & C.ST.C & 18.94 & 163.11 & 34.05 & 1949 & 11637 \\
\hline S2 & C.GFRP.C & 18.79 & 137.22 & 38.95 & 2664 & 9953 \\
\hline S3 & J.C.C & 18.05 & 132.19 & 31.29 & 2088 & 11240 \\
\hline S4 & J.C.E & 18.64 & 151.87 & 39.98 & 1449 & 9729 \\
\hline S5 & J.Z.E & 17.16 & 135.30 & 30.28 & 1250 & 8194 \\
\hline S6 & J.A.C & 16.42 & 121.24 & 35.16 & 2808 & 8173 \\
\hline S7 & J.A.E & 19.90 & 130.71 & 34.49 & 2233 & 6833 \\
\hline
\end{tabular}


Table 3.7. Shear and bending moment values

\begin{tabular}{|c|c|c|c|c|c|c|c|c|c|c|c|c|c|c|c|}
\hline \multirow[t]{2}{*}{ Specimen } & \multirow{2}{*}{$\frac{\text { Load }}{\mathrm{kN}}$} & \multirow{2}{*}{$\frac{\mathrm{w}}{\mathrm{kN} / \mathrm{b}}$} & \multirow{2}{*}{$\mathrm{a}$} & \multirow{2}{*}{$\mathrm{c}$} & \multirow{2}{*}{$\frac{\mathrm{R}_{1}}{\mathrm{kN}}$} & \multirow{2}{*}{$\frac{\mathrm{R}_{2}}{\mathrm{kN}}$} & \multirow{2}{*}{$\frac{0.75 \mathrm{R}}{\mathrm{kN} / \mathrm{m}}$} & \multicolumn{4}{|c|}{ Experimental resisting moment } & \multicolumn{2}{|c|}{$\begin{array}{c}\text { Theoretical } \\
\text { resisting } \\
\text { moment }\end{array}$} & \multicolumn{2}{|c|}{$\begin{array}{l}\text { Theoretical } \\
\text { resisting shear }\end{array}$} \\
\hline & & & & & & & & $\begin{array}{c}\mathrm{M}_{\mathrm{r} \text {.precast }} \\
\mathrm{kN} . \mathrm{m} / 0.6 \mathrm{~m}\end{array}$ & $\begin{array}{l}\mathrm{M}_{\mathrm{r} \text {.precast }} \\
\mathrm{kN} . \mathrm{m} / \mathrm{m}\end{array}$ & $\begin{array}{c}0.75 \mathrm{M}_{\mathrm{r} \text {.precast }} \\
\mathrm{kN} \cdot \mathrm{m} / \mathrm{m}\end{array}$ & $\begin{array}{l}\mathrm{M}_{\mathrm{r} . \mathrm{prec} \text { ast }} \\
\mathrm{kN} \cdot \mathrm{m} / \mathrm{m}\end{array}$ & $\frac{\mathrm{M}_{\mathrm{r} . \text { precast }}}{\mathrm{Mr}}$ & $\begin{array}{l}\mathrm{M}_{\mathrm{r} . \mathrm{UHPFRC}} \\
\mathrm{kN} \cdot \mathrm{m} / \mathrm{m}\end{array}$ & $\begin{array}{l}\mathrm{Vr}, \text { ISIS } \\
\mathrm{kN} / \mathrm{m}\end{array}$ & $\begin{array}{l}\text { Vexp. } \\
\mathrm{Vr}_{\text {, IsIS }}\end{array}$ \\
\hline S1 (C.ST.C) & 163.11 & 652.44 & 0.875 & 0.875 & 81.555 & 81.555 & 101.94 & 76.45 & 127.42 & -- & -- & -- & -- & -- & -- \\
\hline S2 (C.GFRP.C) & 137.22 & 548.88 & 0.875 & 0.875 & 68.61 & 68.61 & 85.77 & 64.32 & 107.20 & 80.40 & 82.88 & 0.97 & -- & 76.84 & 1.12 \\
\hline S3 (J.C.C) & 132.19 & 528.76 & 0.875 & 0.875 & 66.10 & 66.10 & 82.63 & 61.96 & 103.27 & 77.45 & 82.88 & 0.93 & 228.81 & 76.84 & 1.08 \\
\hline S4 (J.C.E) & 151.87 & 607.48 & 0.650 & 1.100 & 93.02 & 58.85 & 116.28 & 67.58 & 112.64 & 84.48 & 82.88 & 1.02 & 228.81 & 76.84 & 1.51 \\
\hline S5 (J.Z.E) & 135.20 & 540.80 & 0.600 & 1.150 & 86.19 & 49.01 & 107.73 & 58.58 & 97.63 & 73.22 & 82.88 & 0.88 & 228.81 & 76.84 & 1.40 \\
\hline S6 (J.A.C) & 121.24 & 484.96 & 0.875 & 0.875 & 60.62 & 60.62 & 75.78 & 56.83 & 94.71 & 71.03 & 82.88 & 0.86 & 228.81 & 76.84 & 0.99 \\
\hline S7 (J.A.E) & 130.71 & 522.84 & 0.650 & 1.100 & 80.06 & 50.65 & 100.08 & 58.16 & 96.94 & 72.71 & 82.88 & 0.88 & 228.81 & 76.84 & 1.30 \\
\hline
\end{tabular}

Notes: See Fig. 3.16 for definitions of symbols

$\mathrm{w}=$ uniform distributed load, $\mathrm{L}=$ span length equal $2 \mathrm{~m} ; \mathrm{a}, \mathrm{b}, \mathrm{c}=$ slab dimension where $\mathrm{b}=0.25 \mathrm{~m} ; \mathrm{R}_{1}$ and $\mathrm{R}_{2}=$ support reactions; $M_{r}=$ resisting moment.

$\mathrm{V}_{\text {exp }}$ in $\mathrm{kN} / \mathrm{m}=\left(\right.$ greater of $\mathrm{R}_{1}$ and $\left.\mathrm{R}_{2}\right) / 0.6 \mathrm{~m}$

The 0.75 is durability factor to be multiplied by the experimental moment and shear capacity 
Table 3.8. Experimental parameters for the moment-deflection equation

\begin{tabular}{llccccc}
\hline \multirow{2}{*}{$\begin{array}{l}\text { Specimen } \\
\text { number }\end{array}$} & Specimen & \multicolumn{5}{c}{ Polynomial parameters* } \\
\cline { 3 - 6 } S1 & C.ST.C & $a_{3}$ & $a_{2}$ & $a_{1}$ & $a_{0}$ & $R^{2}$ \\
\hline S2 & C.GFRP.C & 0.003 & -0.23 & 6.64 & 3.25 & 0.9979 \\
\hline S3 & J.C.C. & & -0.04 & 2.54 & 3.30 & 0.9944 \\
\hline S4 & J.C.E. & & -0.12 & 4.50 & 2.33 & 0.9873 \\
\hline S5 & J.Z.E. & & -0.03 & 2.73 & 2.73 & 0.9970 \\
\hline S6 & J.A.C. & & -0.04 & 2.89 & 3.84 & 0.9928 \\
\hline S7 & J.A.E. & & -0.03 & 2.68 & 4.78 & 0.9879 \\
\hline
\end{tabular}

$*$ Moment-deflection equation: $M=a_{3} \delta^{3}+a_{2} \delta^{2}+a_{1} \delta^{1}+a_{o} ; R^{2}$ is the coefficient of determination

Table 3.9. Maximum micro-strain readings for the tested slabs

\begin{tabular}{|c|c|c|c|c|c|c|c|}
\hline \multirow{2}{*}{$\begin{array}{l}\text { Specimen } \\
\text { number }\end{array}$} & \multirow[t]{2}{*}{ Slab name } & \multirow[t]{2}{*}{ Location } & \multirow{2}{*}{$\begin{array}{l}\text { Depth } \\
(\mathrm{mm})\end{array}$} & \multicolumn{4}{|c|}{ Micro-strain at ultimate load } \\
\hline & & & & 1 & 2 & 3 & Average \\
\hline \multirow{3}{*}{ S1 } & \multirow{3}{*}{ C.ST.C } & Concrete & 0 & 1949 & 1698 & - & 1823.5 \\
\hline & & Top bar & 65 & 11423 & 15182 & - & 13303 \\
\hline & & Bottom bar & 135 & 11637 & 7934 & 8119 & 9230 \\
\hline \multirow{3}{*}{ S2 } & \multirow{3}{*}{ C.GFRP.C } & Concrete & 0 & 2434 & 2664 & - & 2549 \\
\hline & & Top bar & 65 & 1320 & 2843 & - & 2082 \\
\hline & & Bottom bar & 135 & 7669 & 7563 & 9953 & 8395 \\
\hline \multirow{3}{*}{ S3 } & \multirow{3}{*}{ J.C.C } & Concrete & 0 & 2088 & 1212 & - & 1650 \\
\hline & & Top bar & 65 & 2400 & 3592 & - & 2996 \\
\hline & & Bottom bar & 135 & 11240 & 10003 & 9791 & 10344 \\
\hline \multirow{3}{*}{ S4 } & \multirow{3}{*}{ J.C.E } & Concrete & 0 & 912 & 885 & 1449 & 1082 \\
\hline & & Top bar & 65 & 1851 & 922 & - & 1387 \\
\hline & & Bottom bar & 135 & 9641 & 9711 & 9729 & 9694 \\
\hline \multirow{3}{*}{ S5 } & \multirow{3}{*}{ J.Z.E } & Concrete & 0 & 686 & 909 & 1250 & 948 \\
\hline & & Top bar & 65 & 982 & 1020 & - & 1001 \\
\hline & & Bottom bar & 135 & 8006 & 7664 & 8194 & 7954.7 \\
\hline \multirow{3}{*}{ S6 } & \multirow{3}{*}{ J.A.C } & Concrete & 0 & 2808 & - & - & 2808 \\
\hline & & Top bar & 65 & 2709 & 1406 & - & 2058 \\
\hline & & Bottom bar & 135 & 6618 & 8173 & - & 7396 \\
\hline \multirow{3}{*}{ S7 } & \multirow{3}{*}{ J.A.E } & Concrete & 0 & 833 & 2233 & - & 1528 \\
\hline & & Top bar & 65 & 1846 & 2645 & 3403 & 2631 \\
\hline & & Bottom bar & 135 & 6833 & 6590 & 6309 & 6577 \\
\hline
\end{tabular}


Table 3.10. Calculated pullout force of bottom GFRP bars embedded in the UHPFRC-filled joint based on experimental data

\begin{tabular}{|c|c|c|c|c|c|c|c|c|c|c|c|}
\hline & Name & $\begin{array}{c}\mathrm{A} \\
\left(\mathrm{mm}^{2}\right)\end{array}$ & $\begin{array}{c}\mathrm{E} \\
(\mathrm{MPa})\end{array}$ & $\begin{array}{c}\epsilon \\
\left(\mathrm{x} 10^{-6}\right)\end{array}$ & $\begin{array}{c}\mathrm{T} \\
(\mathrm{kN})\end{array}$ & $\begin{array}{c}\mathrm{L}_{\mathrm{d}} \\
(\mathrm{mm})\end{array}$ & $\begin{array}{c}\mathrm{d} \\
(\mathrm{mm})\end{array}$ & $\begin{array}{c}\mathrm{f}_{\mathrm{c}}{ }^{\prime} \\
(\mathrm{MPa})\end{array}$ & $k$ & $1 / \mathrm{k}$ & $\begin{array}{c}\tau \\
(\mathrm{MPa})\end{array}$ \\
\hline \multirow{4}{*}{ S1 } & & 300 & 200,000 & 11637 & 698.22 & & 20 & 45.00 & -- & -- & -- \\
\hline & C.ST.C & & & 7934 & 476.04 & & & & & & \\
\hline & & & & 8119 & 487.14 & & & & & & \\
\hline & Average & & & 9230 & $\mathbf{5 5 3 . 8 0}$ & & & & & & \\
\hline \multirow{4}{*}{ S2 } & & 314 & 64,000 & 7669 & 154.12 & & 20 & 45.00 & -- & -- & -- \\
\hline & C.GFRP.C & & & 7563 & 151.99 & & & & & & \\
\hline & & & & 9953 & 200.02 & & & & & & \\
\hline & Average & & & 8395 & 168.71 & & & & & & \\
\hline \multirow{4}{*}{ S3 } & & 314 & 64,000 & 11240 & 225.88 & 175 & 20 & 141.11 & 0.00999 & 100.00 & 18.92 \\
\hline & J.C.C & & & 10003 & 201.02 & & & & & & \\
\hline & & & & 9791 & 196.76 & & & & & & \\
\hline & Average & & & 10344.67 & 207.87 & & & & & & \\
\hline \multirow{4}{*}{ S4 } & & 314 & 64,000 & 9641 & 193.75 & 175 & 20 & 166.58 & 0.0115 & 86.25 & 17.73 \\
\hline & J.C.E & & & 9711 & 195.15 & & & & & & \\
\hline & & & & 9729 & 195.51 & & & & & & \\
\hline & Average & & & 9693.667 & 194.80 & & & & & & \\
\hline \multirow{4}{*}{ S5 } & & 314 & 64,000 & 8006 & 160.89 & 175 & 20 & 166.58 & 0.0141 & 70.72 & 14.53 \\
\hline & J.Z.E & & & 7644 & 153.61 & & & & & & \\
\hline & & & & 8194 & 164.67 & & & & & & \\
\hline & Average & & & 7954.7 & 159.72 & & & & & & \\
\hline \multirow{3}{*}{ S6 } & $\mathrm{I} A C$ & 314 & 64,000 & 6618 & 132.99 & 175 & 20 & 141.11 & 0.0139 & 71.49 & 13.52 \\
\hline & & & & 8173 & 164.24 & & & & & & \\
\hline & Average & & & 7395.5 & 148.62 & & & & & & \\
\hline \multirow{4}{*}{ S7 } & & 314 & 64,000 & 6833 & 137.32 & 175 & 20 & 141.11 & 0.01572 & 63.58 & 12.03 \\
\hline & J.A.E & & & 6590 & 132.43 & & & & & & \\
\hline & & & & 6309 & 126.79 & & & & & & \\
\hline & Average & & & 6577.33 & 132.18 & & & & & & \\
\hline
\end{tabular}

Note: $T=E A . \epsilon, \quad 1 / k=T /\left(l_{d} \sqrt{f_{c}^{\prime}}\right), \tau=\sqrt{f_{c}^{\prime}} / \pi k d$

$\mathrm{A}=$ cross-sectional area of the bar; $\mathrm{E}=$ modulus of elasticity; $\epsilon=$ bar strain; $\mathrm{T}=$ pullout load; $\mathrm{L}_{\mathrm{d}}$ $=$ development length; $\mathrm{d}=$ bar diameter; $\mathrm{f}_{\mathrm{c}}{ }^{\prime}=$ compressive strength of concrete; $k=$ bond basic factor; $\tau=$ bond stress. 
Figures

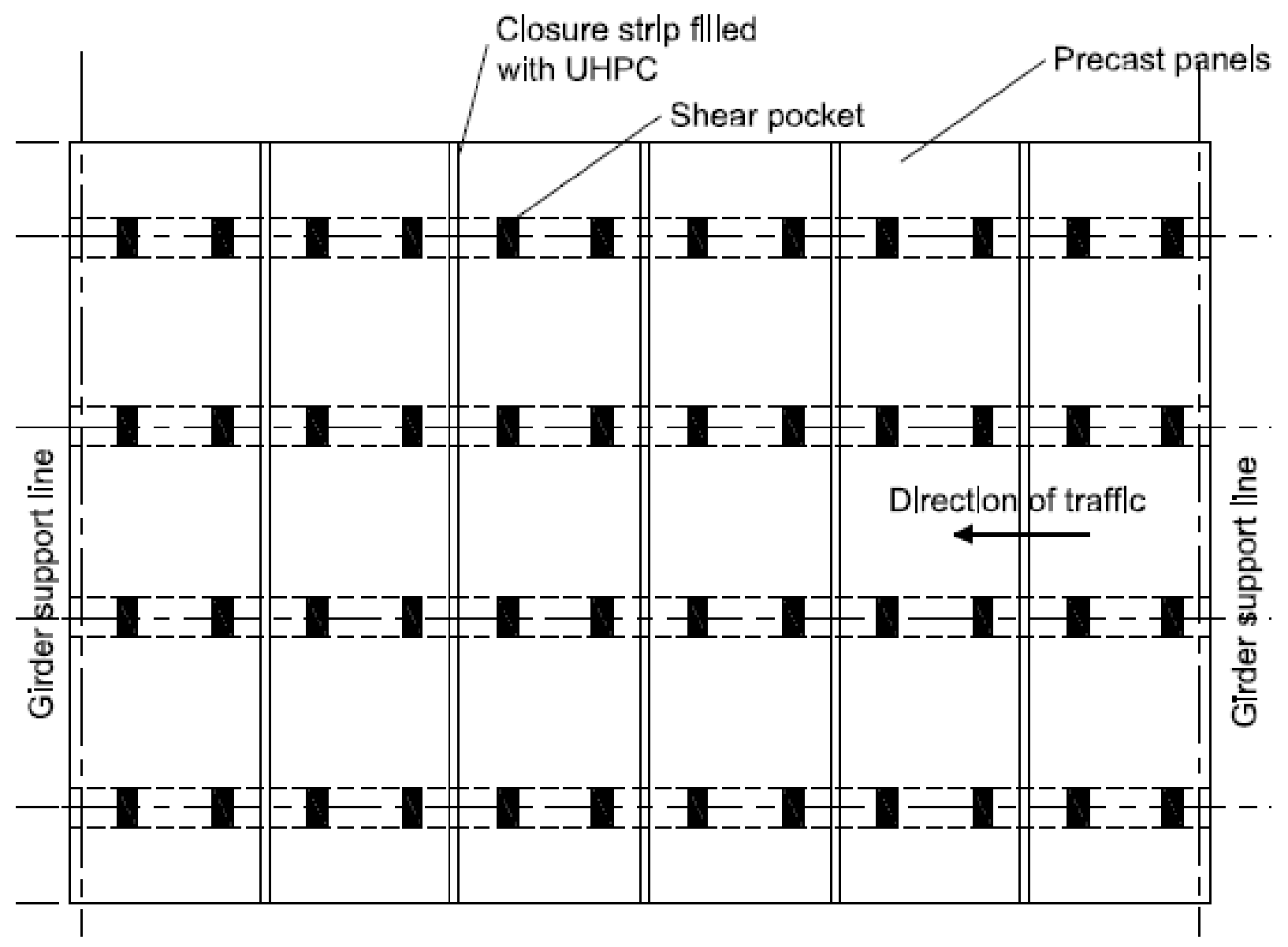

Figure 3.1. Plan view of a slab-on-girder bridge with full-depth precast deck panels 


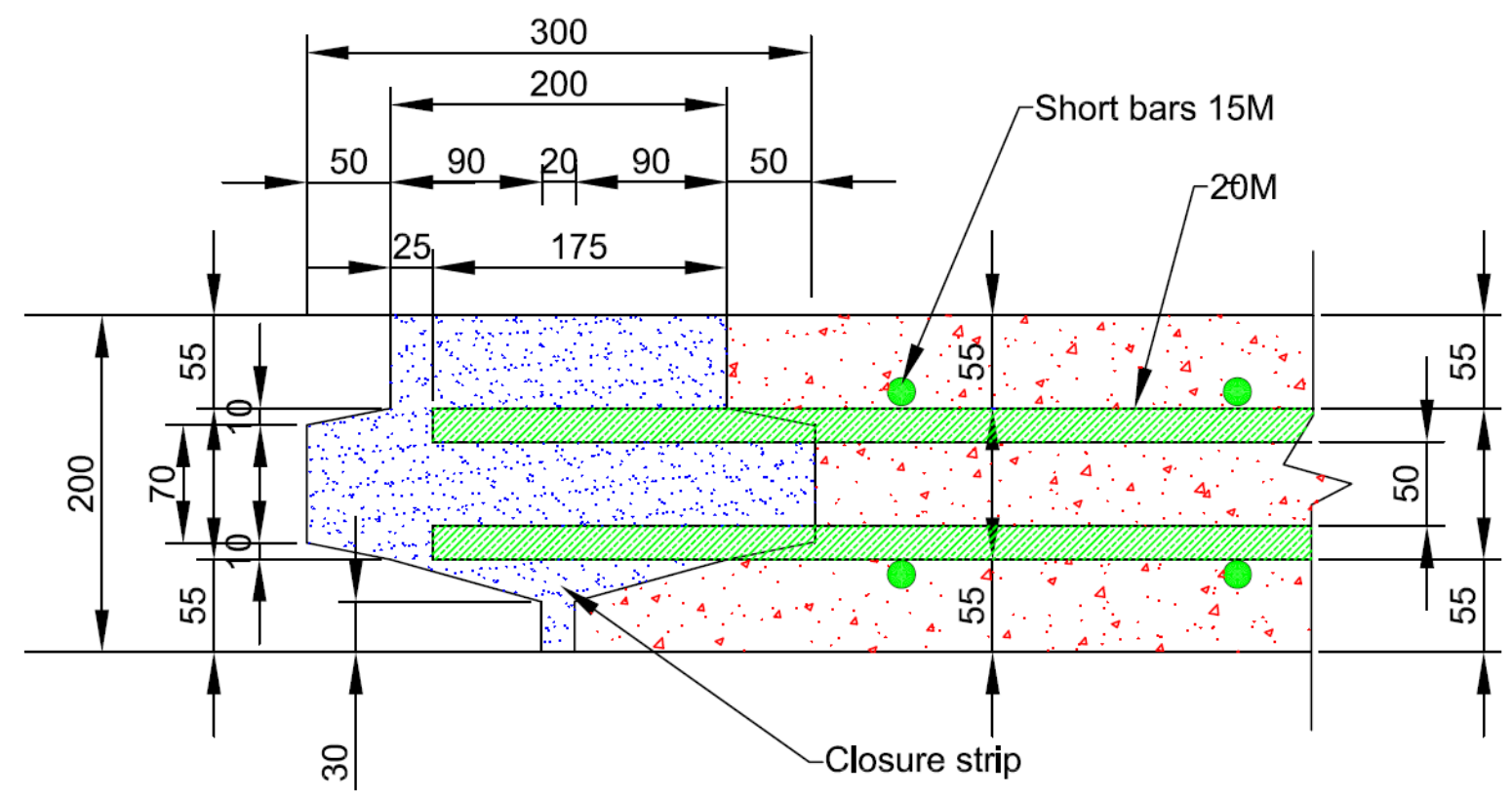

a) Angle-shape joint with pojecting bottom slab (Joint A)

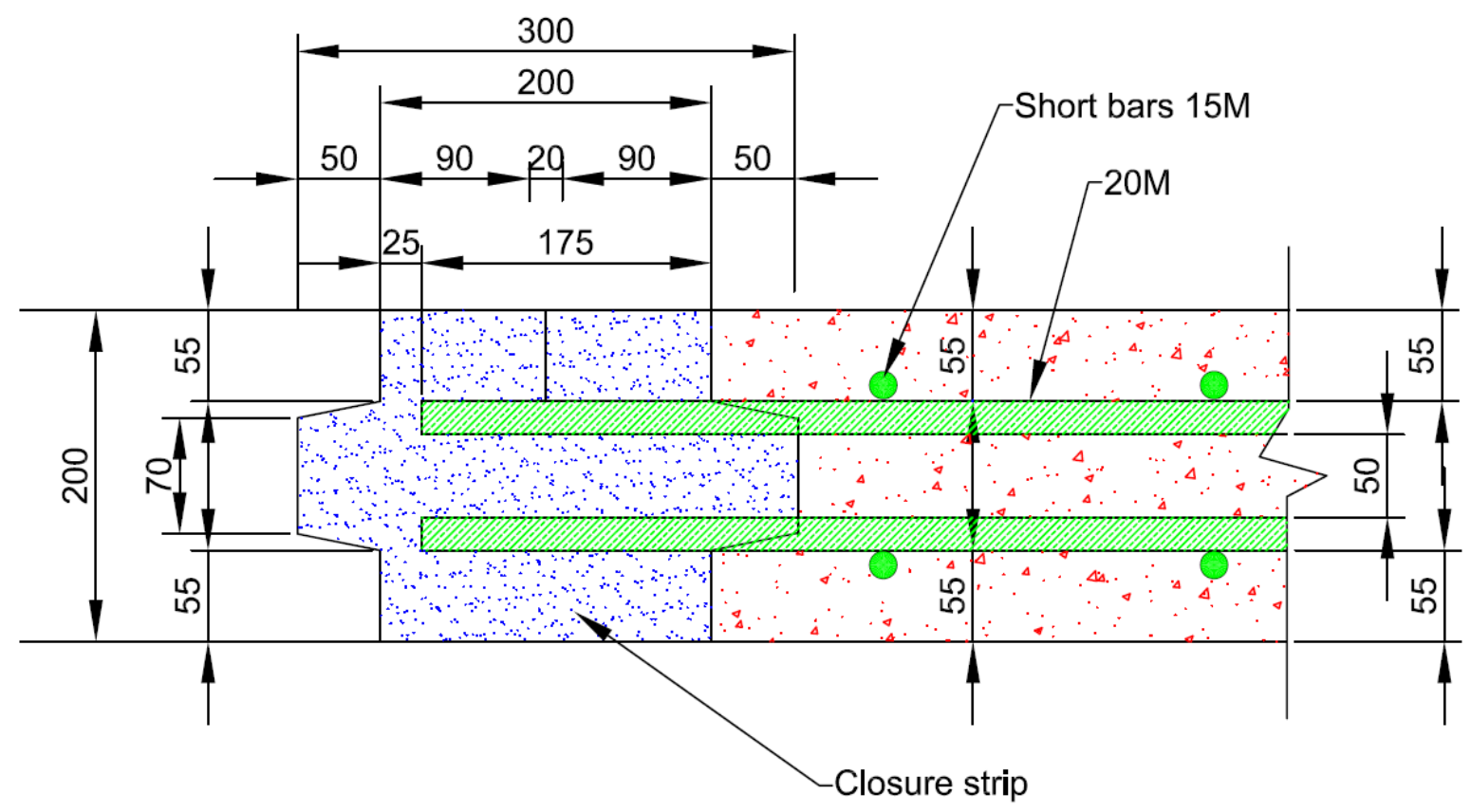

b) C-Shape joint with vertical shear key (Joint C) 


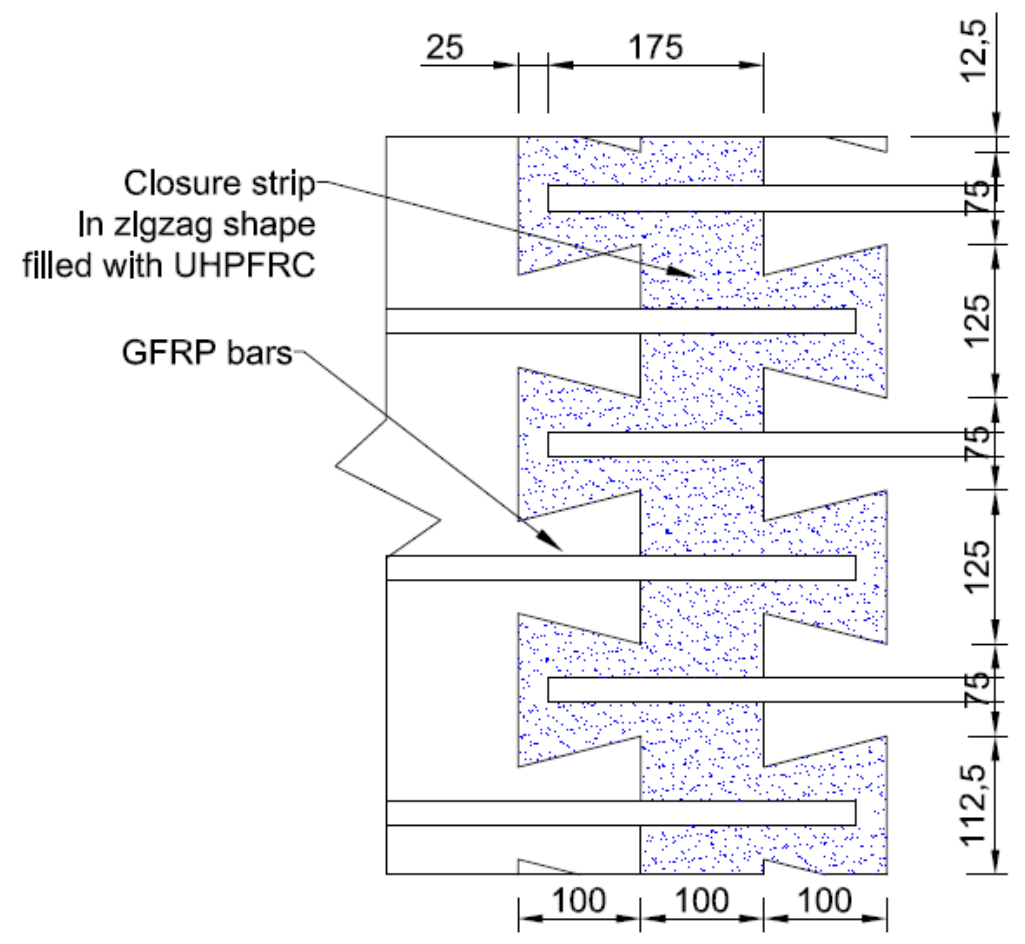

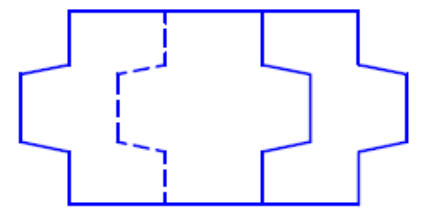

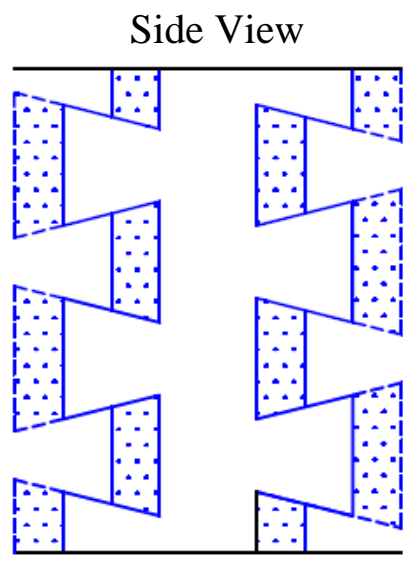

Top View

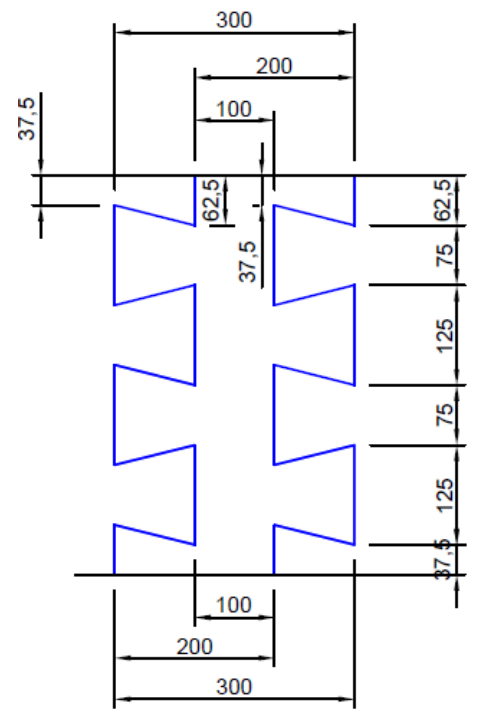

Top \& Bottom Layers

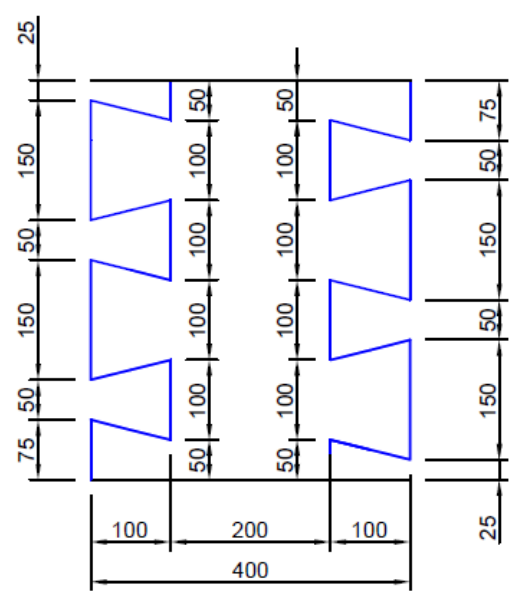

Middle Layers

c) Zigzag-shape joint with interlocking horizontal and vertical shear key (joint Z)

Figure 3.2. Schematic diagrams of the three proposed joints between precast deck panels 


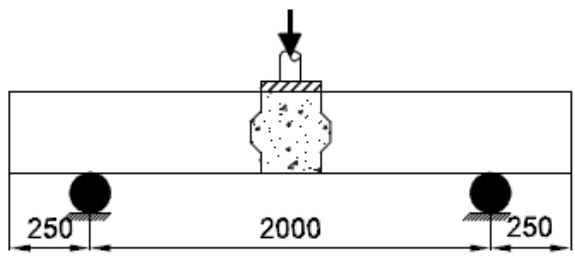

a) Simply-supported slab with centric load (C)

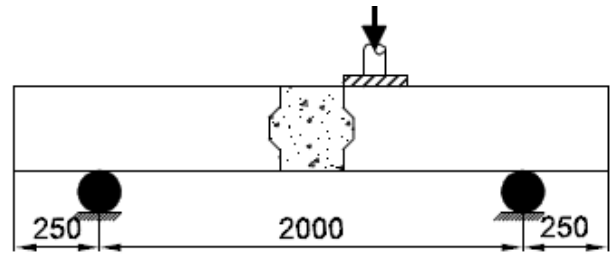

b) Simply-supported slab with eccentric load (E)

Figure 3.3. Schematic diagram showing location of simulated wheel load at the joint between precast deck panels

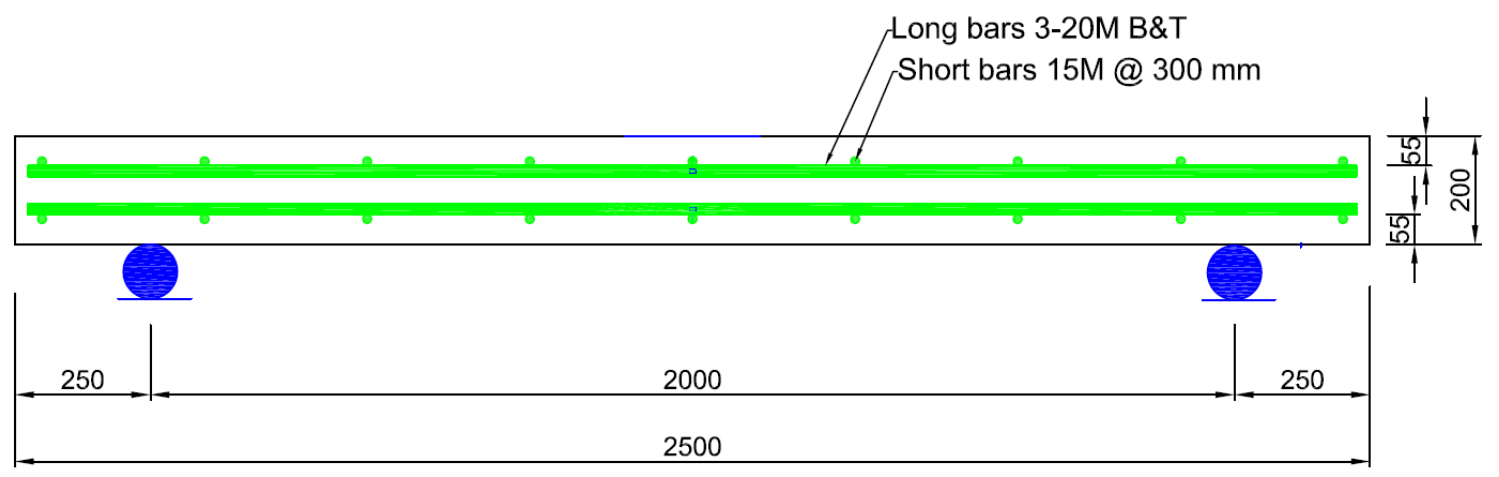

a) Longitudinal section for the cast-in-place deck slab S 2 (C.GFRP.C)

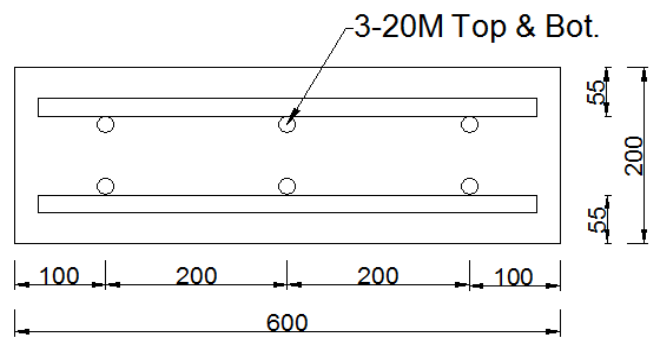

b) Cross-section for the cast-in-place deck slab S 2 (C.GFRP.C) 


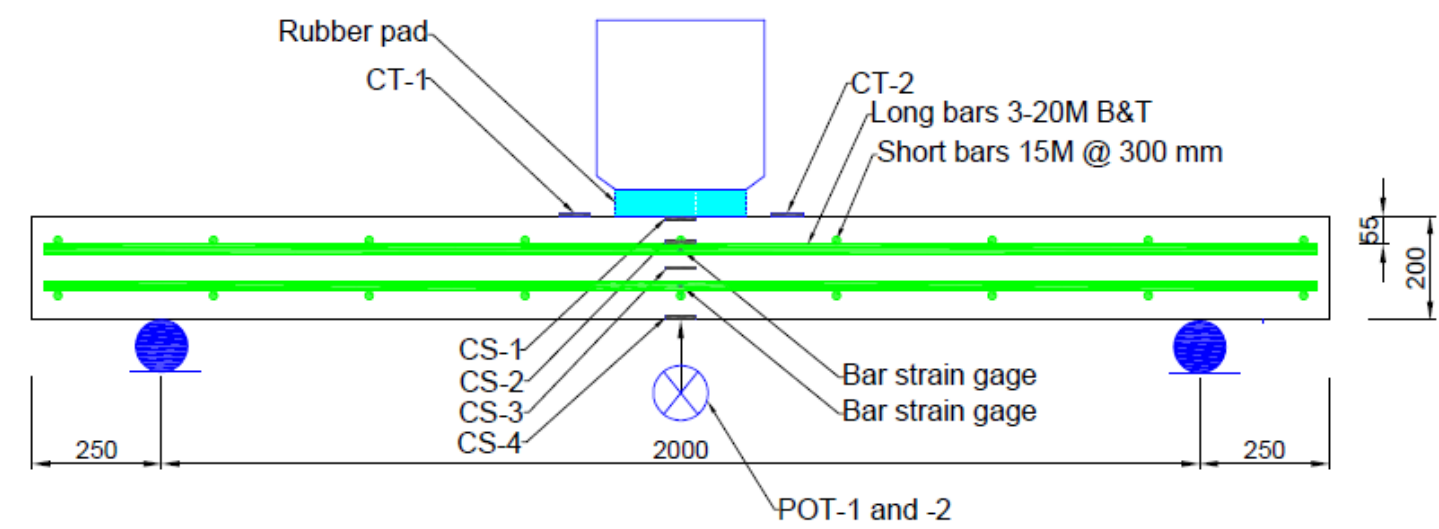

c) Locations of POTs and strain gages in cast-in-place slabs

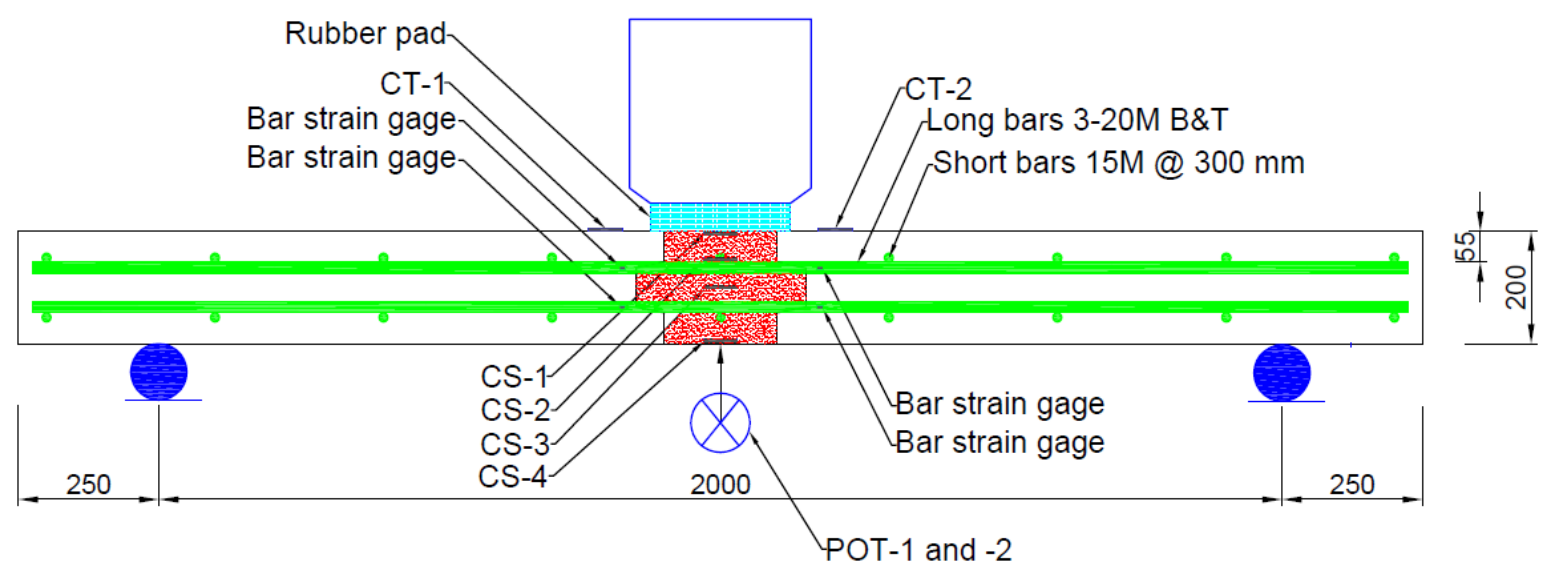

d) Locations of POTs and strain gages in precast FDDP under centric loading

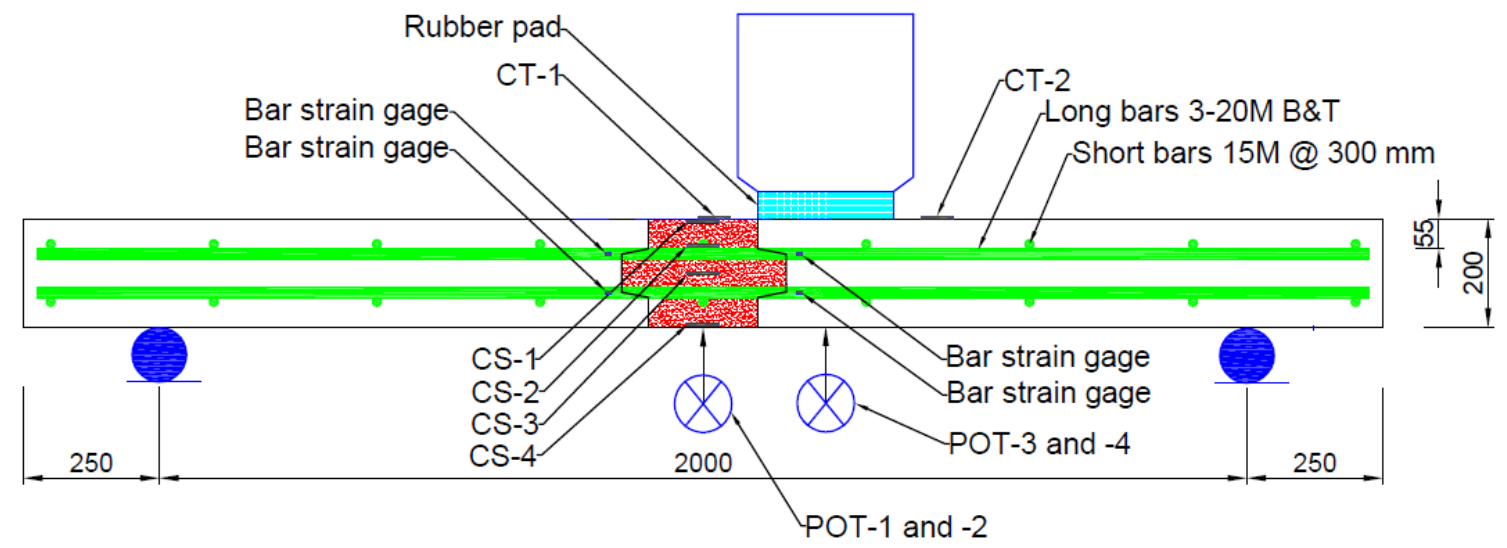

e) Locations of POTs and strain gages in precast FDDP under eccentric loading

Figure 3.4. Reinforcement details and locations of strain gauges and potentiometers (POTs) 


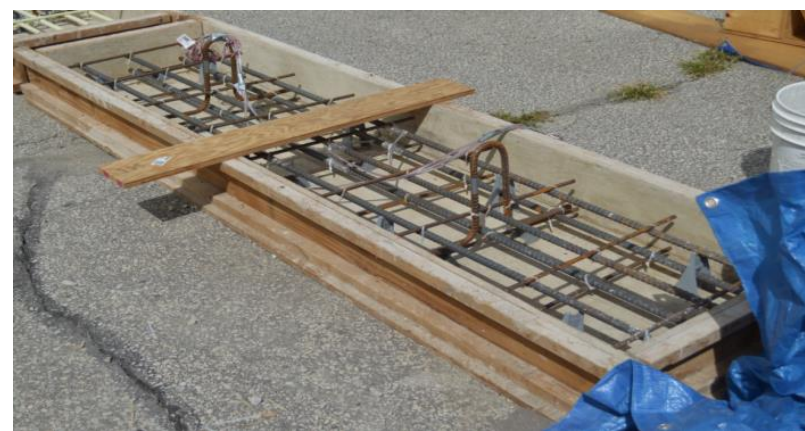

a) Slab S1 (C.ST.C)

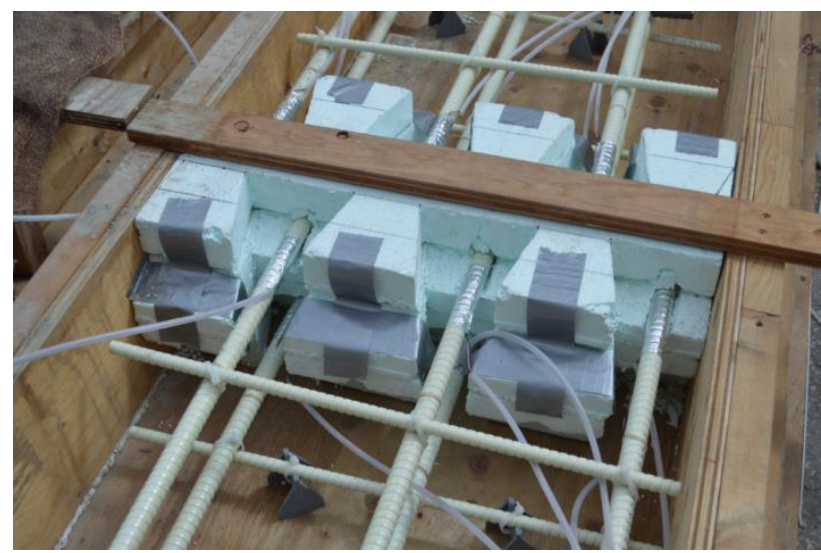

c) Slab S5 (J.Z.E)

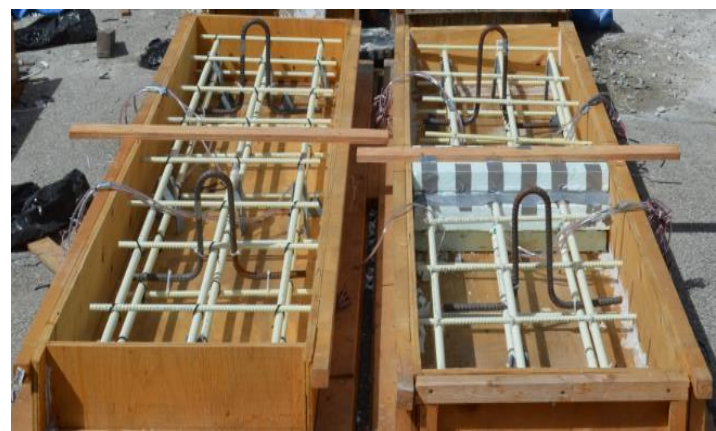

b) Slabs S2 (C.GFRP.C) and S3 (J.C.C)

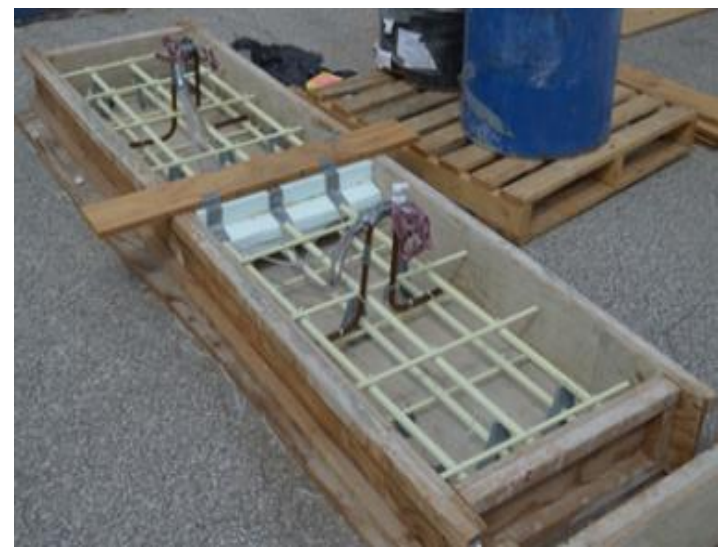

d) Slab S6 (J.A.C)

Figure 3.5. Views of reinforcement layout and form work for slab specimens

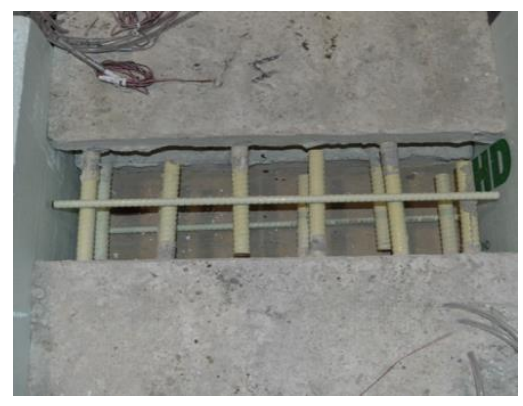

a) Closure strip for slab S3

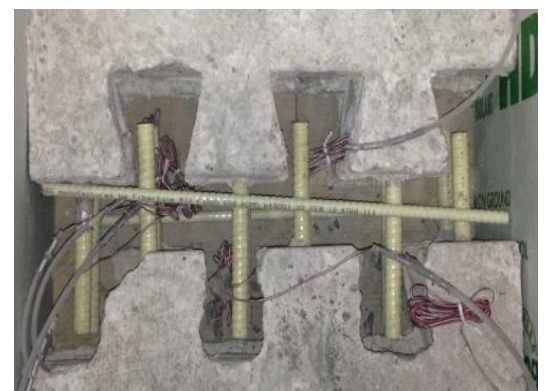

b) Closure strip for slab S5

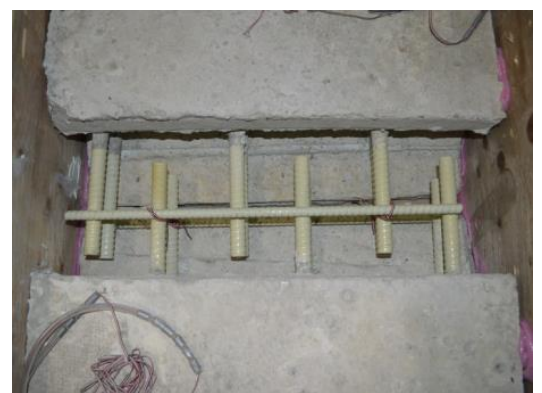

c) Closure strip for slab S6

Figure 3.6. Plan views of closure strips in jointed slabs 


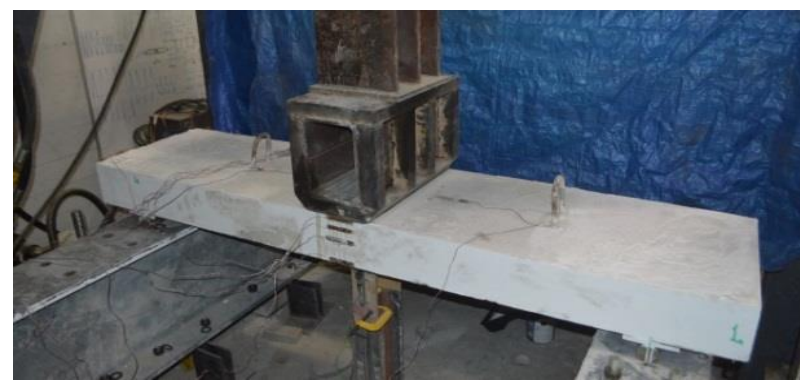

a) Test setup

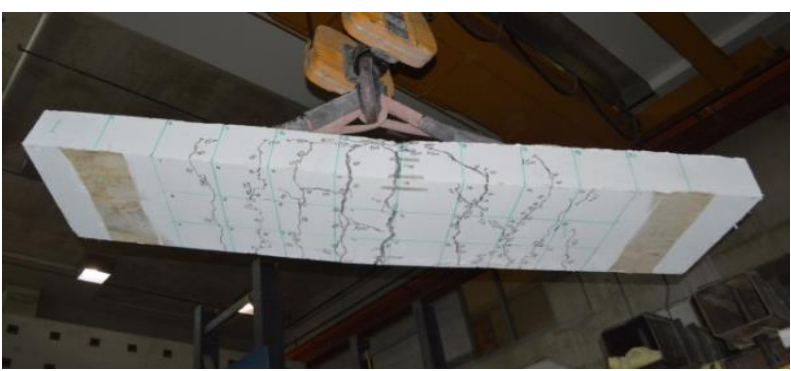

b) crack pattern after failure

Figure 3.7. Test setup and crack pattern at failure for cast-in-place specimen S1 (C.ST.C) reinforced with steel bars

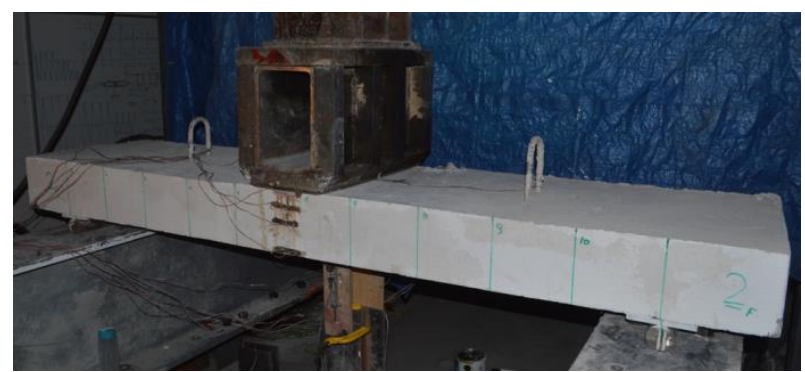

a) Test setup

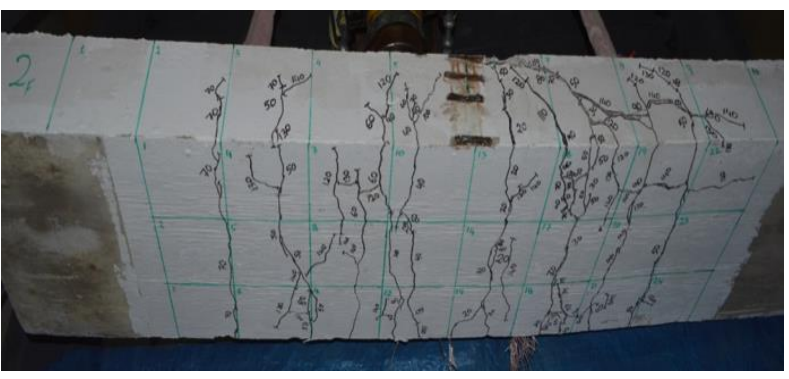

b) crack pattern after failure

Figure 3.8. Test setup and crack pattern at failure of cast-in-place specimen S2 (C.GFRP.C) reinforced with GFRP bars 


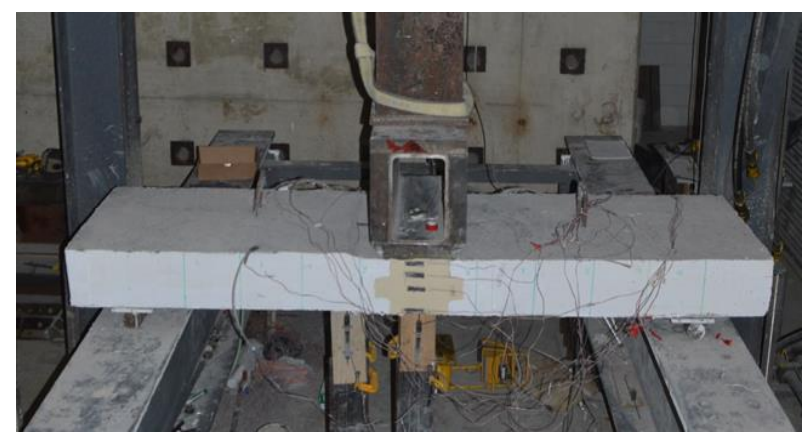

a) Test Setup

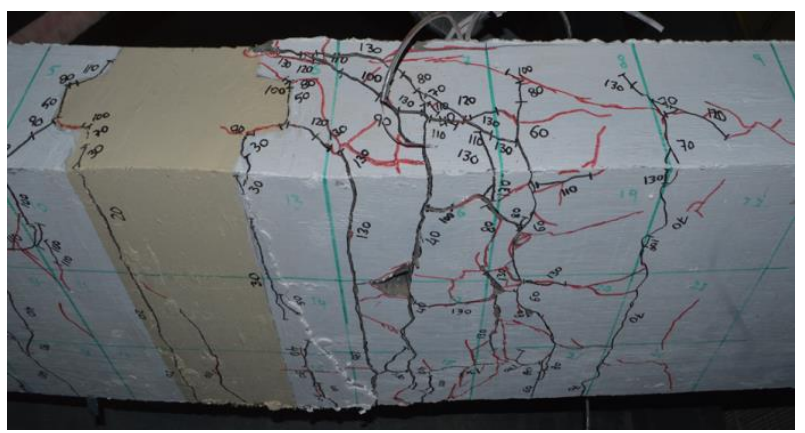

b) Crack pattern at joint region

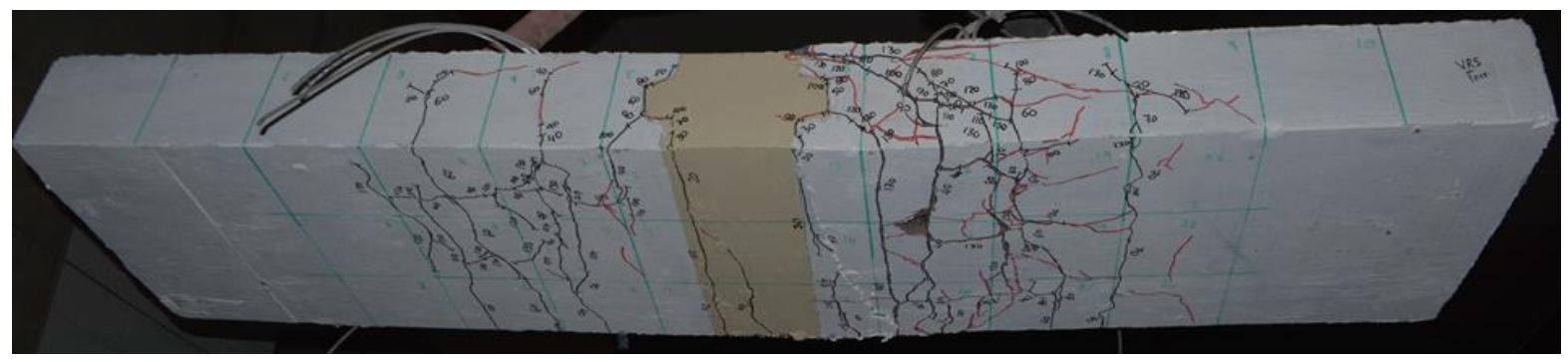

c) Crack pattern along the specimen length

Figure 3.9. Test setup and crack pattern at failure of jointed slab S3 (J.C.C) and centric loading over the joint

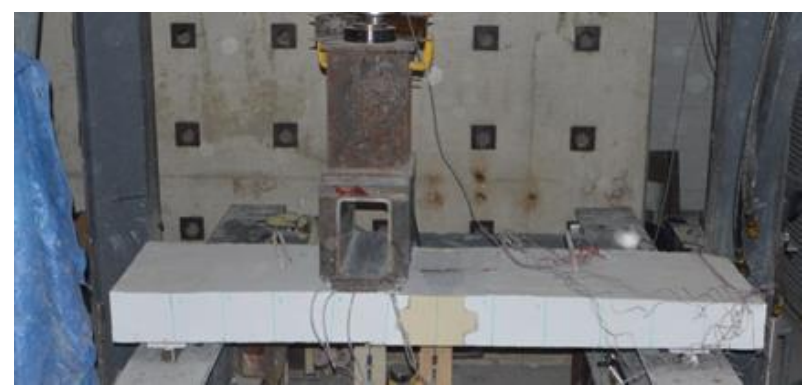

a) Test Setup

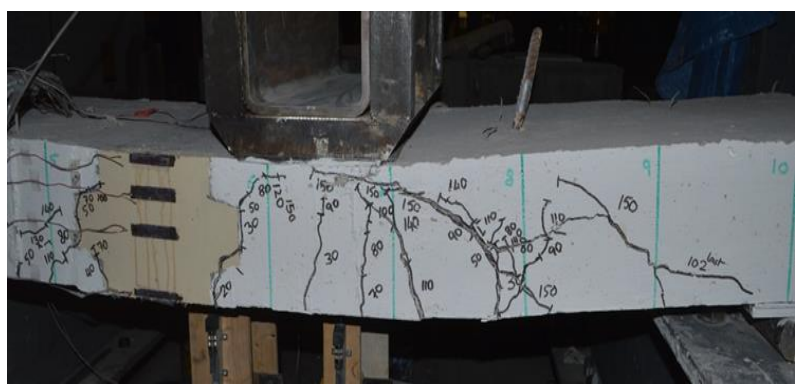

b) Close-up view of flexural-shear crack in precast slab

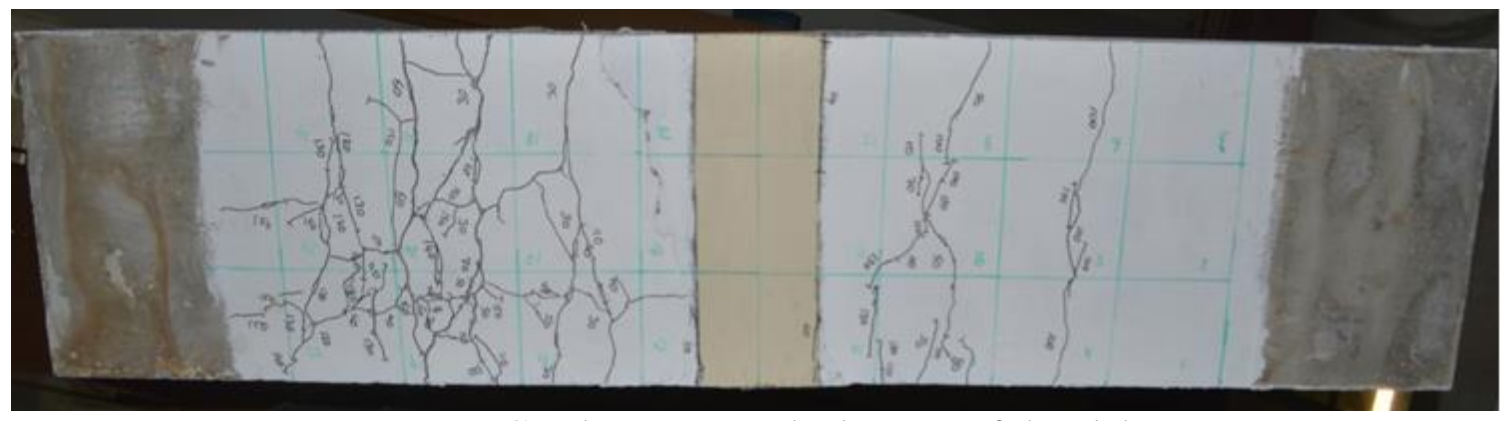

c) Crack pattern at the bottom of the slab

Figure 3.10. Test setup and crack pattern at failure of jointed slab S4 (J.C.E) with eccentric loading at the joint 


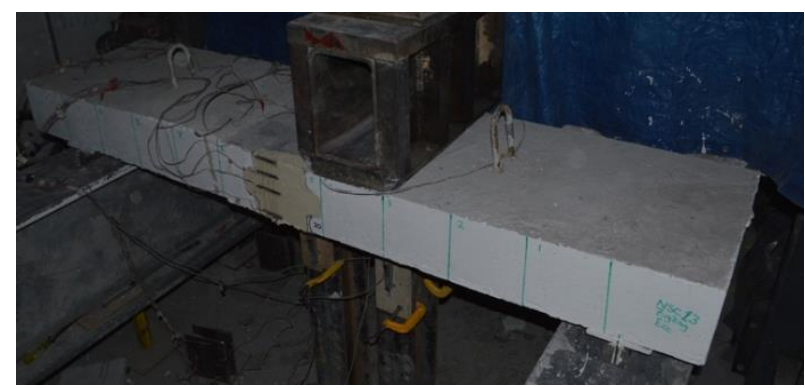

a) Test Setup

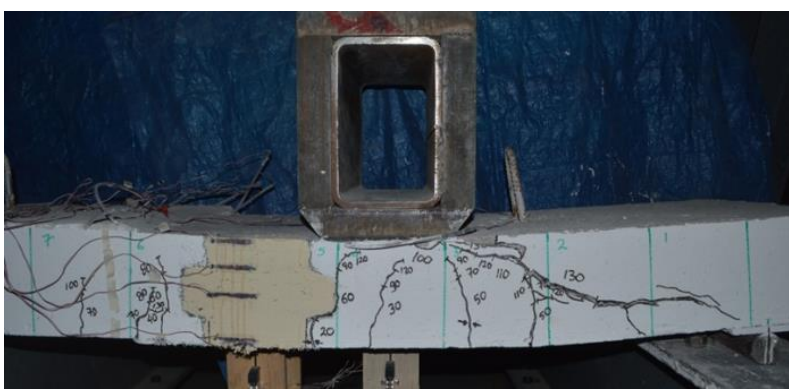

b) Close-up view of flexural-shear crack in precast slab

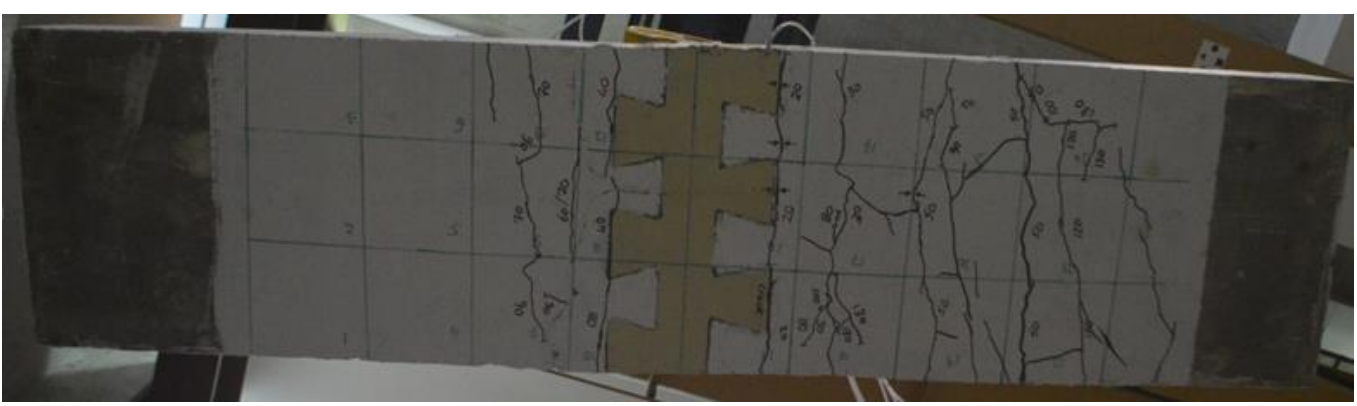

c) Crack pattern at the bottom of the slab

Figure 3.11. Test setup and crack pattern at failure of slab S5 (J.Z.E) with zigzag-shape joint under eccentric loading

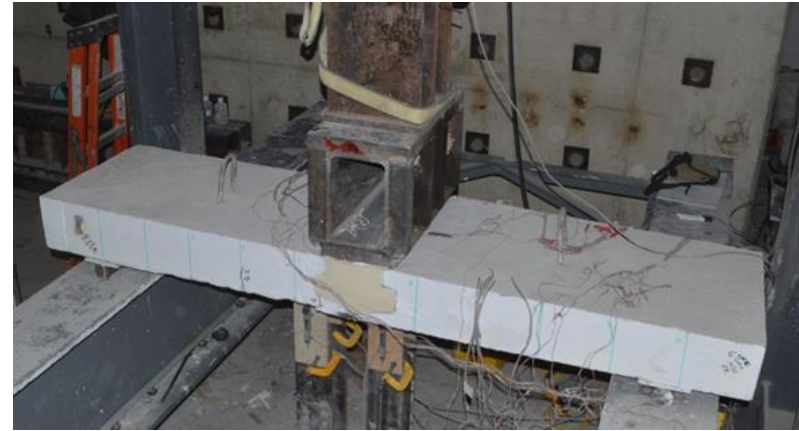

a) Test Setup

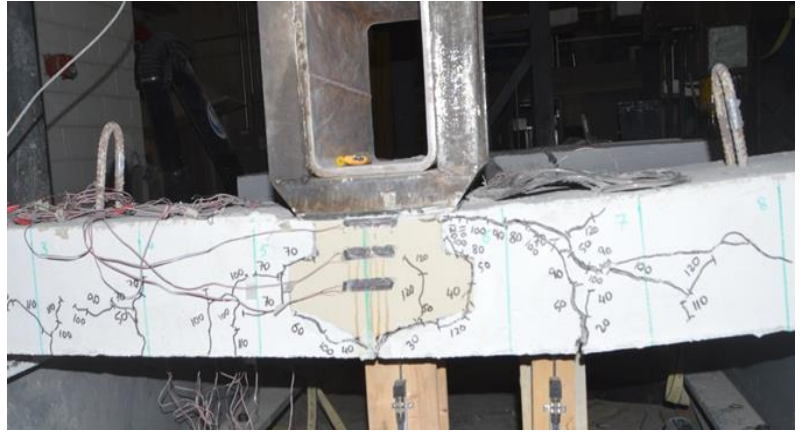

b) Crack pattern at the joint region

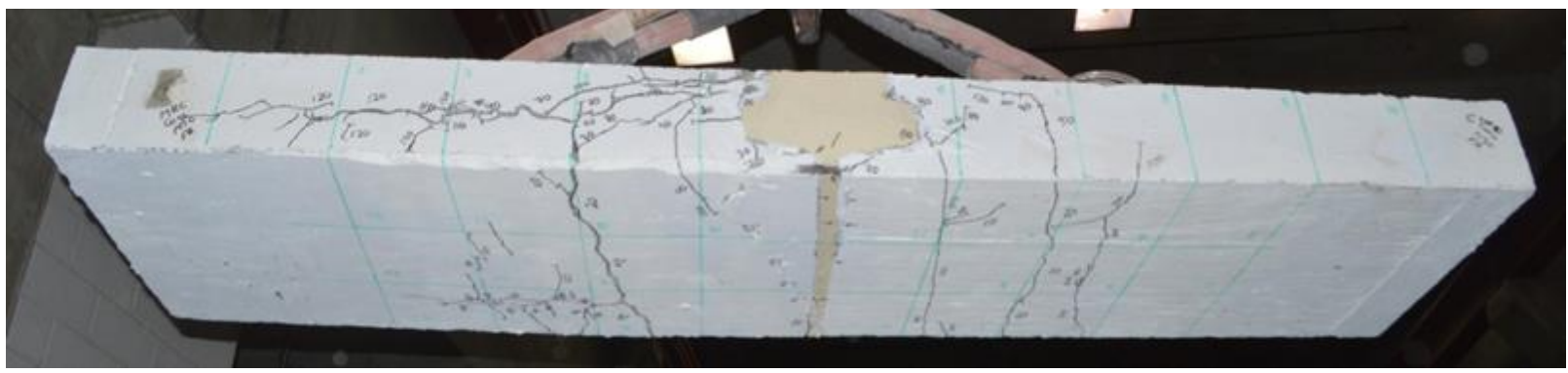

c) Crack pattern at the bottom of the slab

Figure 3.12. Test setup and crack pattern at failure of slab S6 (J.A.C) with angle-shape joint under centric loading 


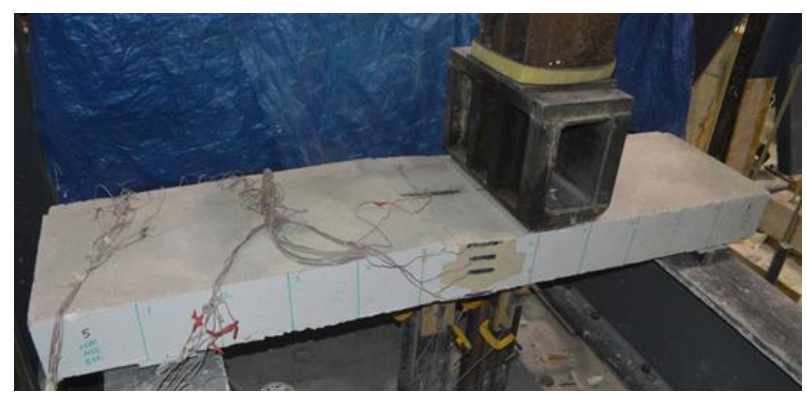

a) Test Setup

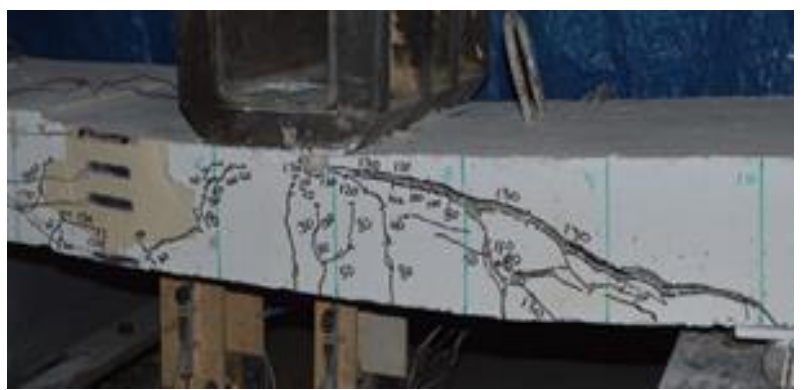

b) Crack pattern at the joint region showing major flexural-shear crack

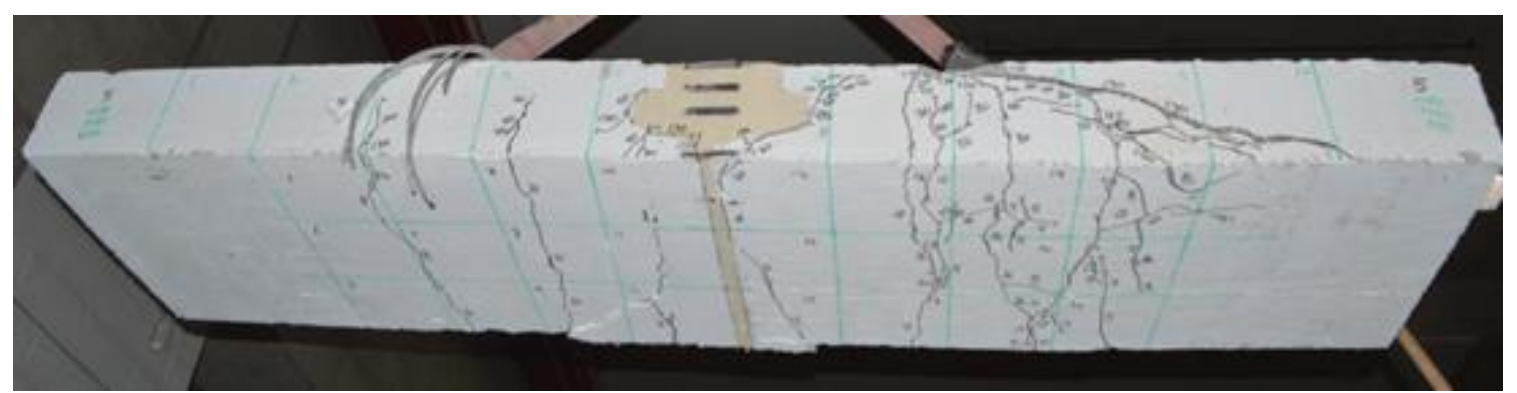

c) Crack pattern at the bottom of the slab

Figure 3.13. Test setup and crack pattern at failure of slab S6 (J.A.E) with angle-shape joint under eccentric loading 


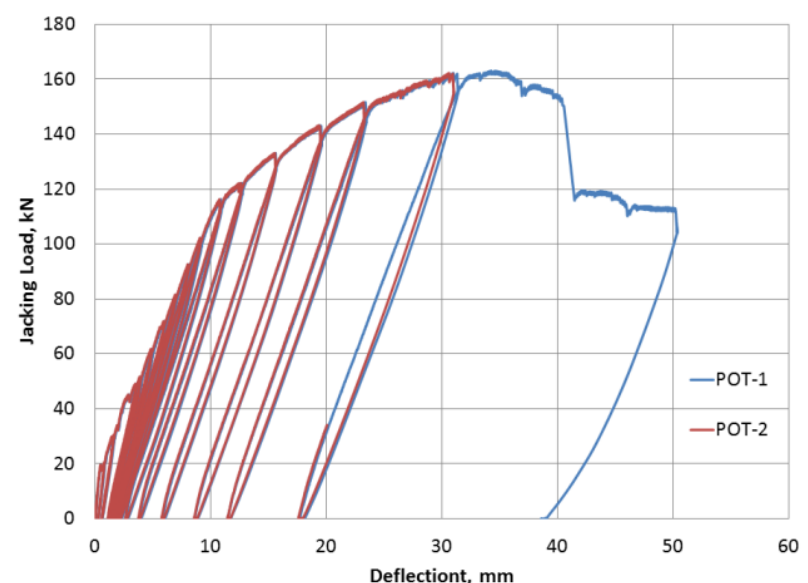

a) Slab S1 (C.ST.C)

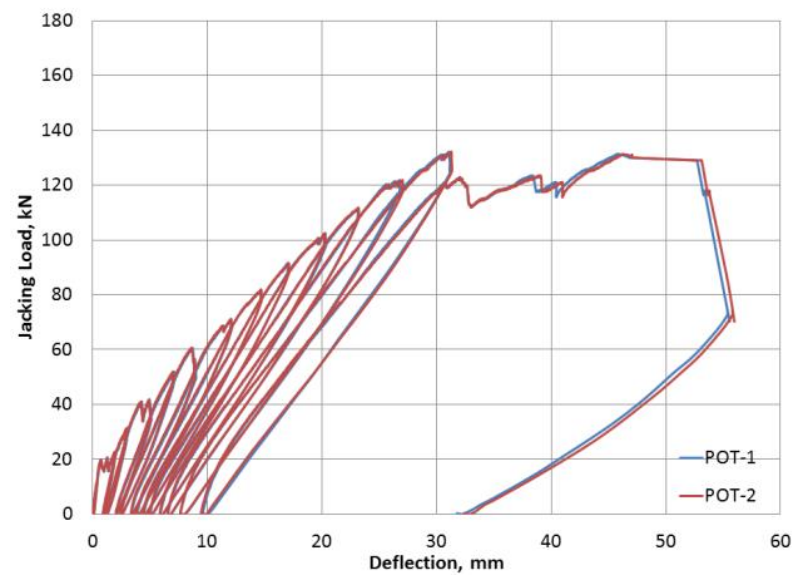

c) Slab S3 (J.C.C)

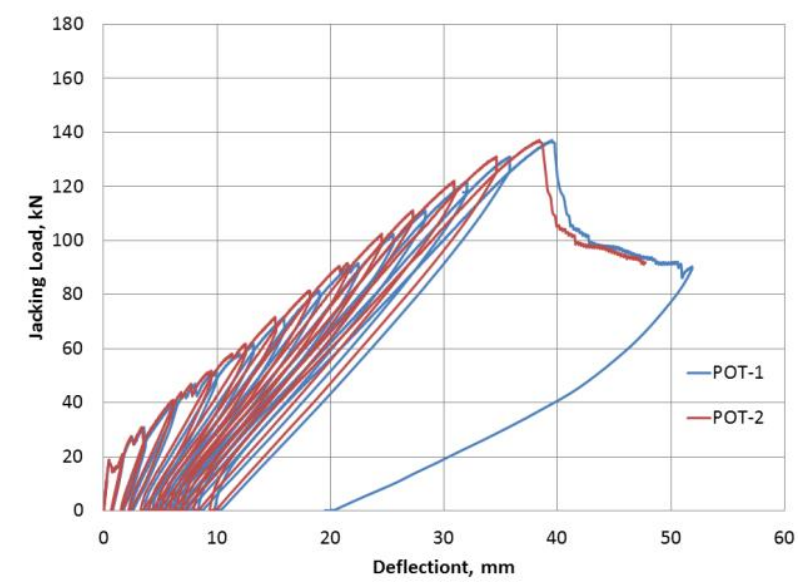

b) Slab S2 (C.GFRP.C)

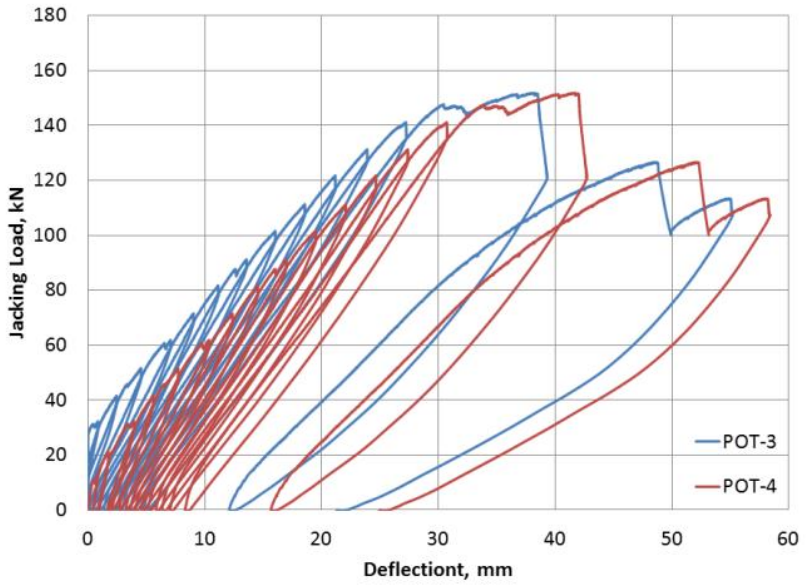

d) Slab S4 (J.C.E)

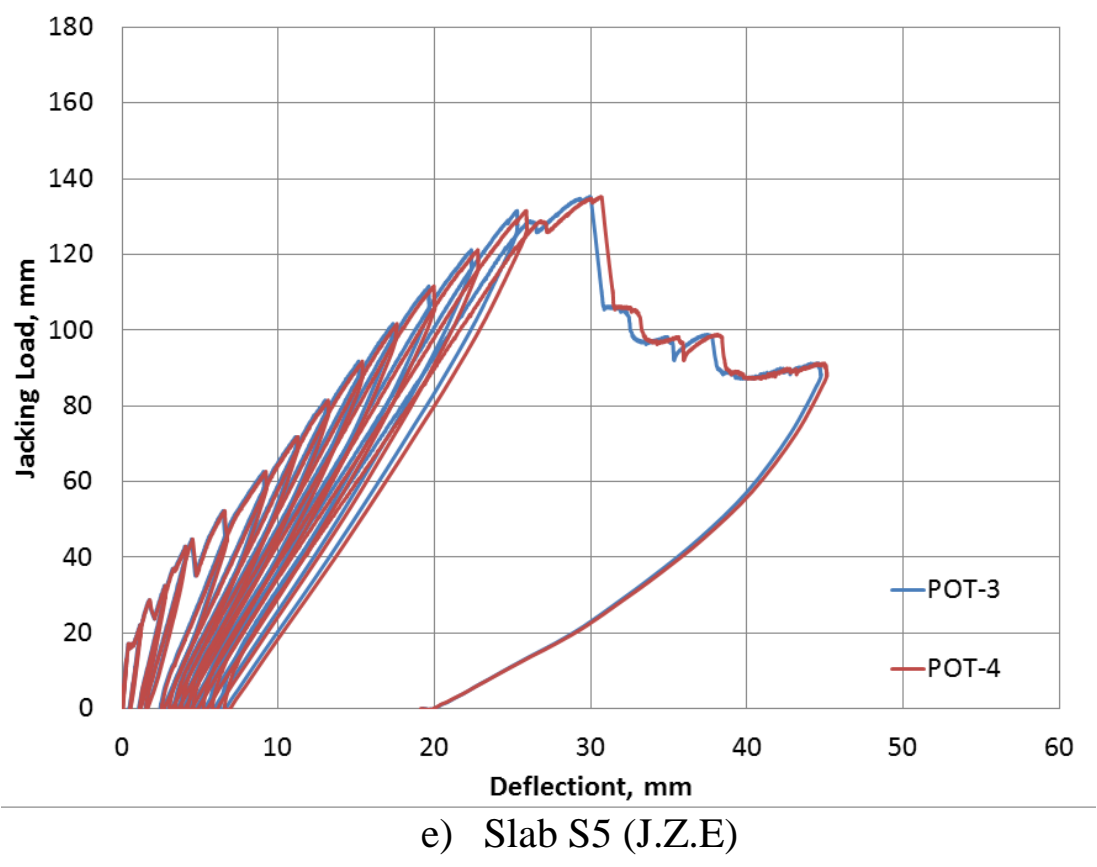




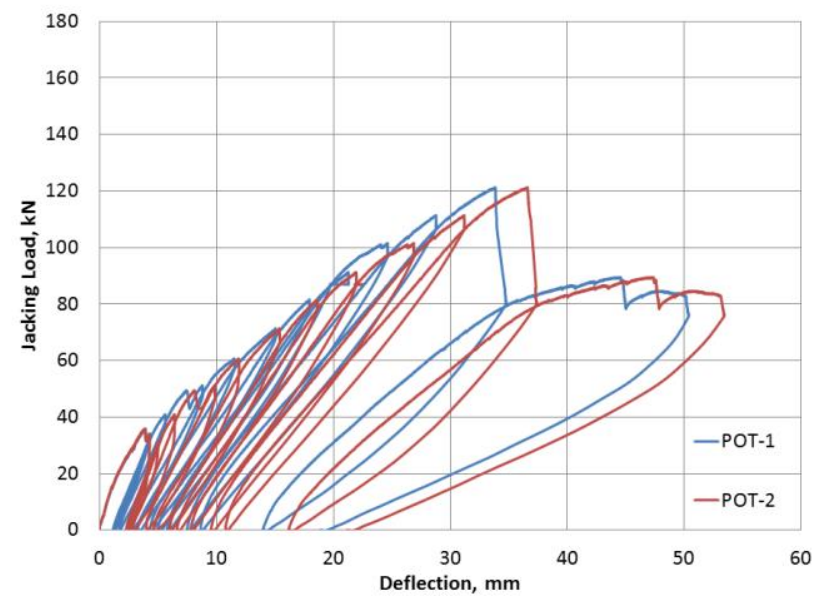

f) Slab S6 (J.A.C)

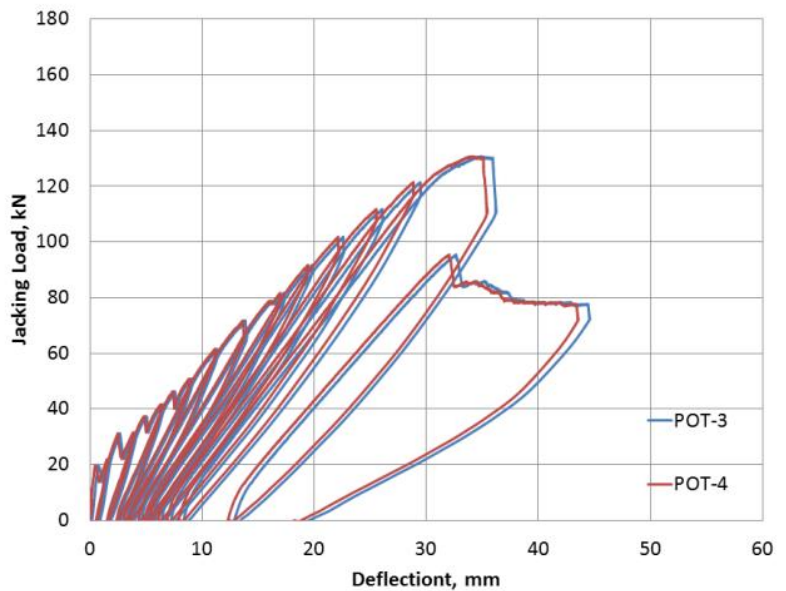

g) Slab S7 (J.A.E)

Figure 3.14. Load-deflection relationships obtained at mid-span of the slab specimens

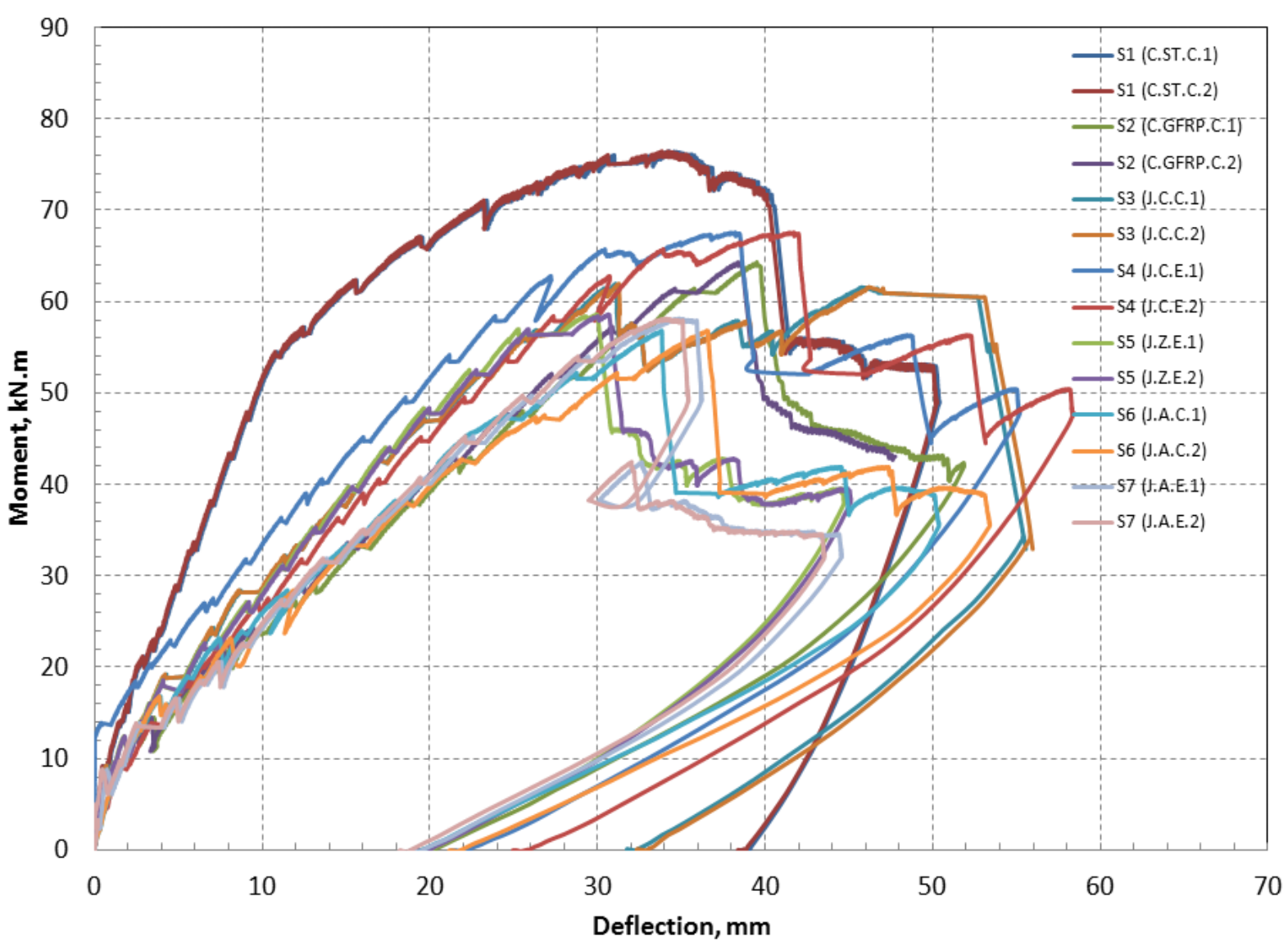

Figure 3. 15. Applied moment-deflection curves for the tested specimens 

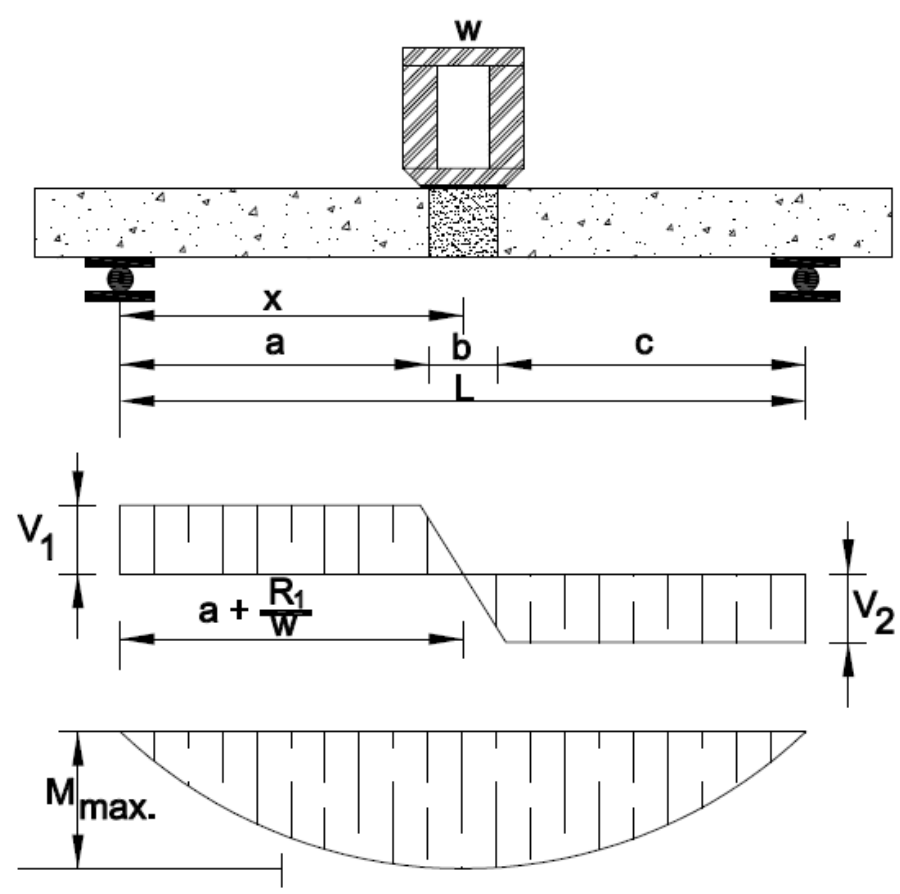

a) Analysis of slab with centric patch loading
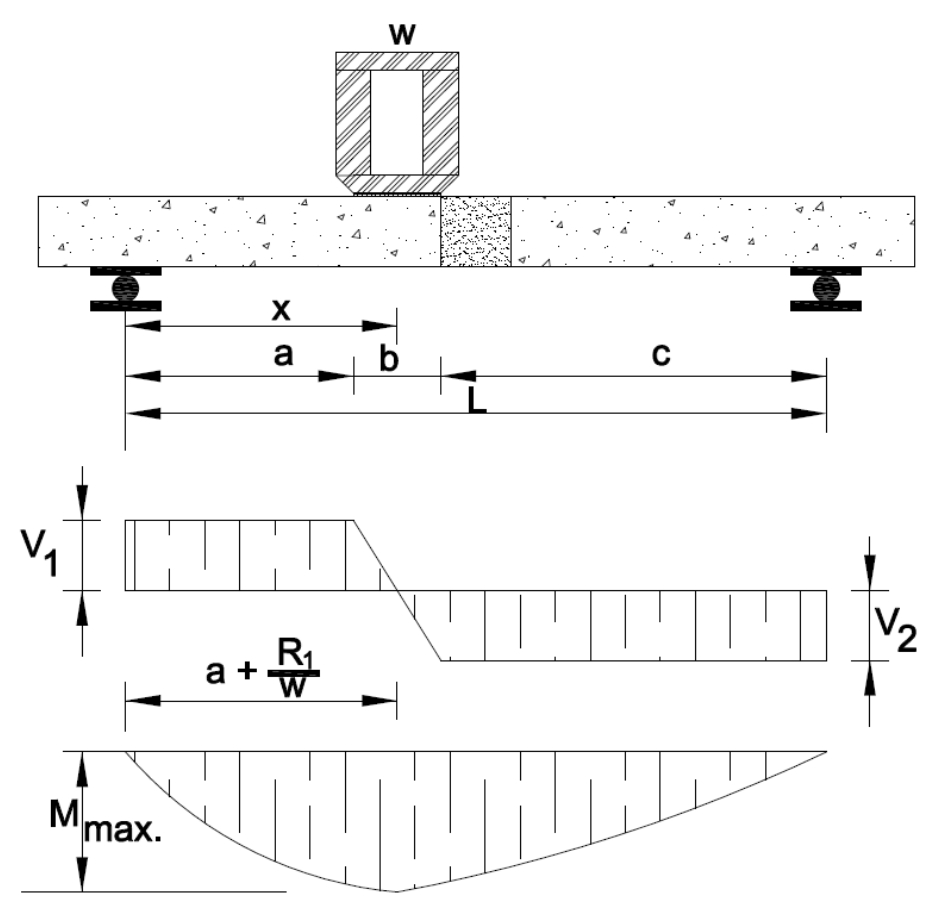

b) Analysis of slab with eccentric patch loading

Figure 3.16. Structural analysis of the tested slabs 


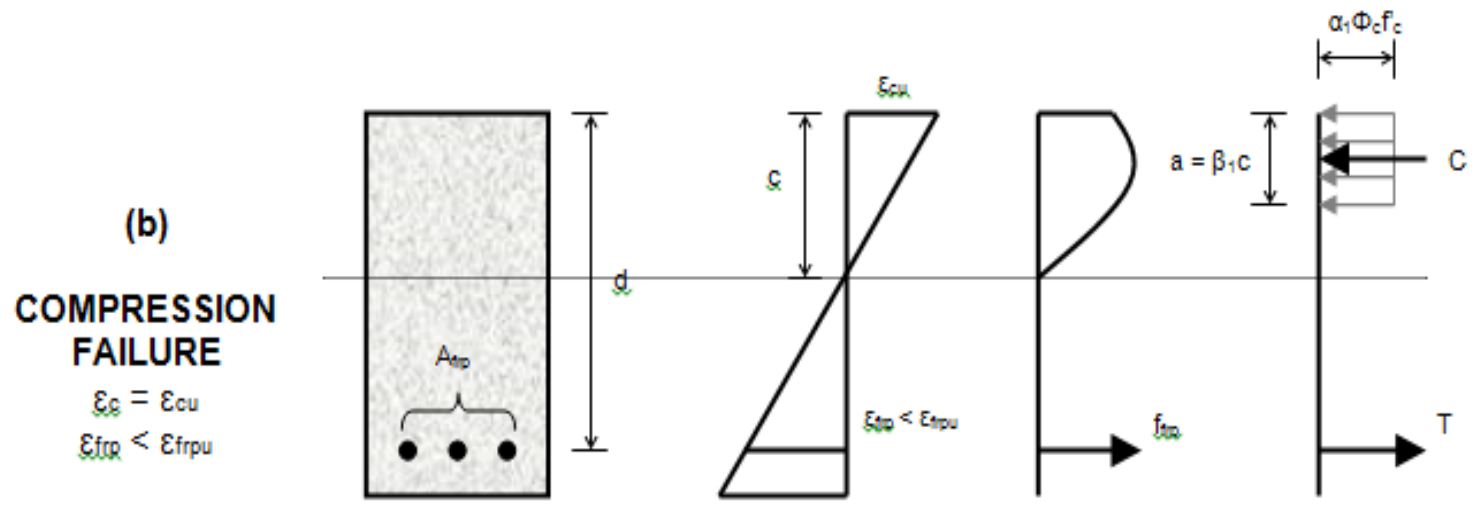

Figure 3. 17. Whitney rectangular stress distribution for flexural design

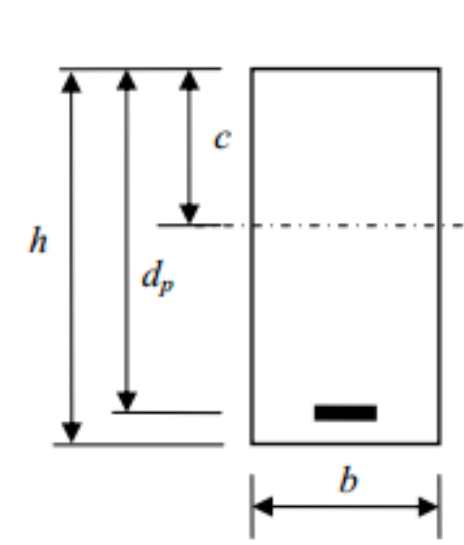

(a)

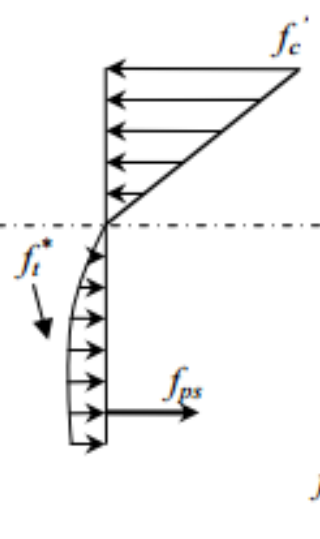

(b) (c) (d)

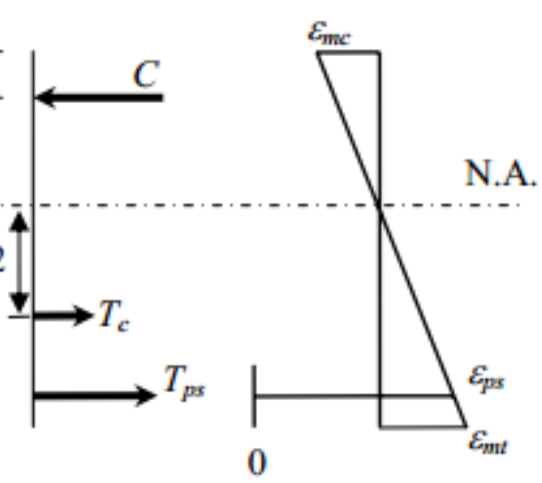

(e)

Figure 3.18. UHPFRC internal stress behavior 


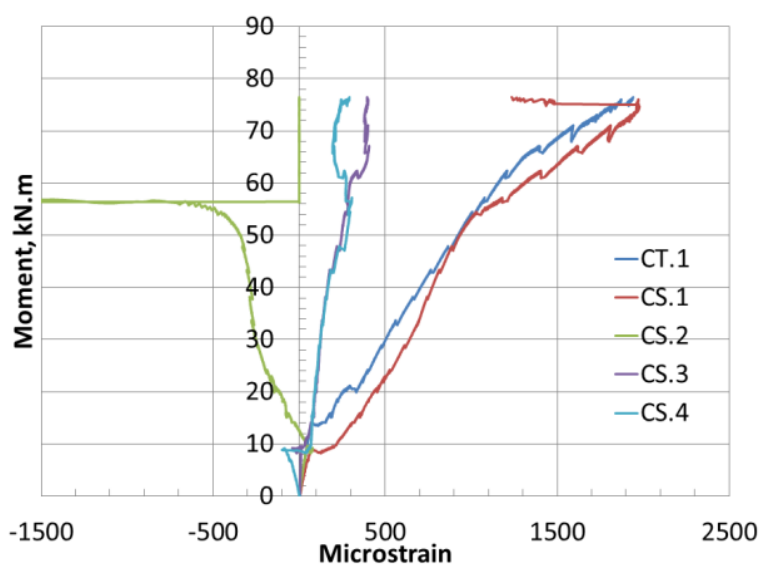

a) Moment-concrete strain

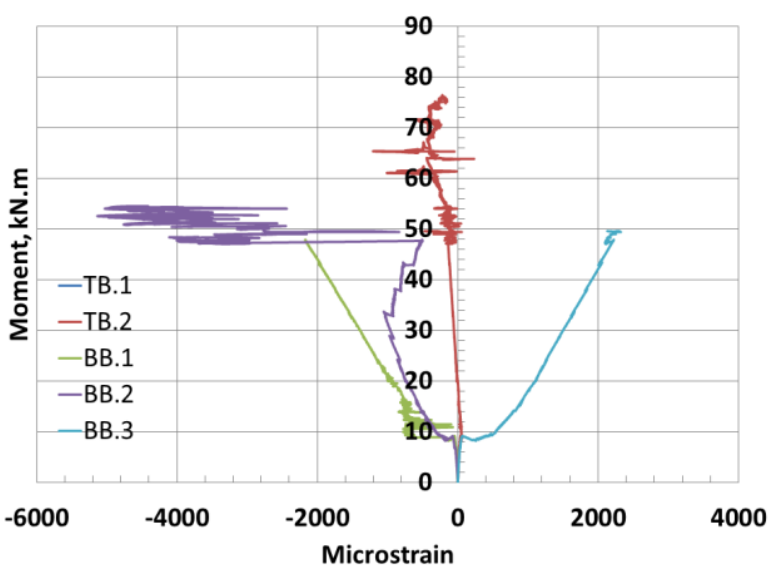

b) Moment-bar strain

Figure 3. 19. Control CIP steel slab S1 (C.ST.C) under centric load

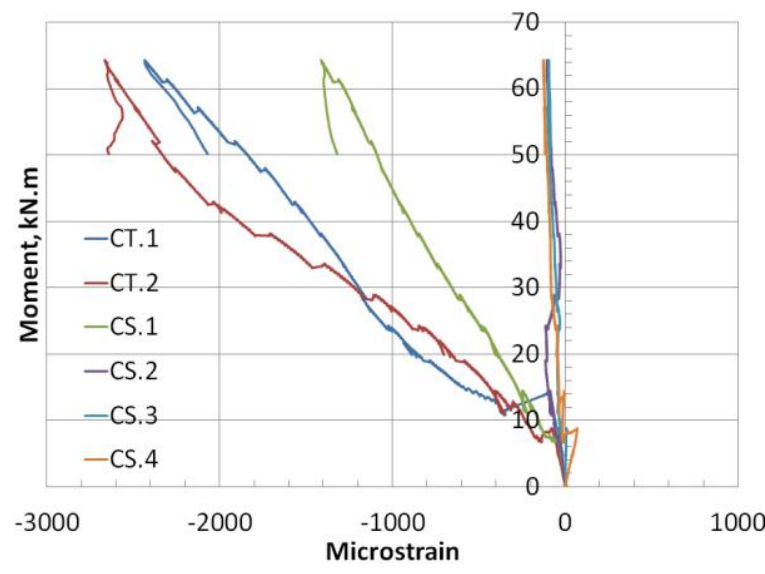

a) Moment-concrete strain

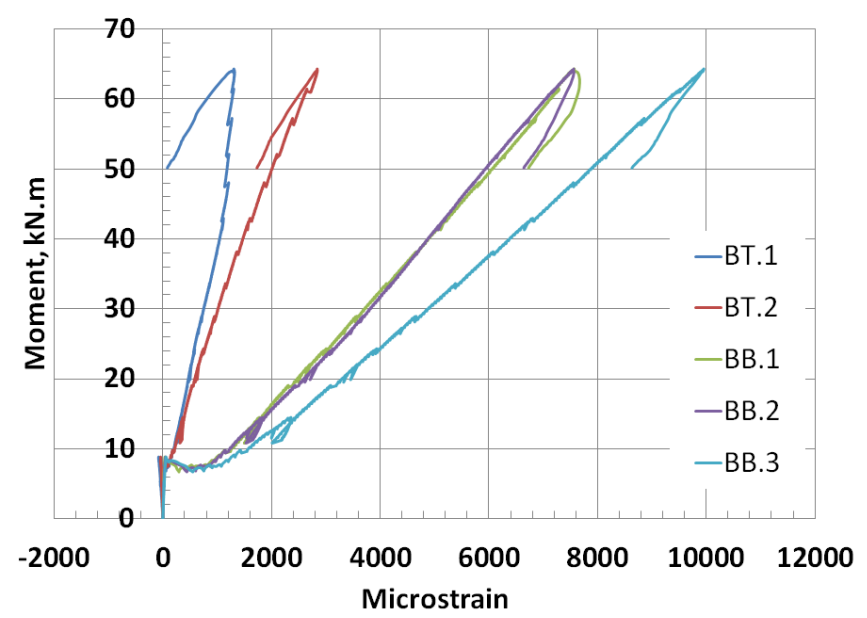

b) Moment-bar strain

Figure 3.20. Control CIP GFRP slab S2 (C.GFRP.C) under centric load

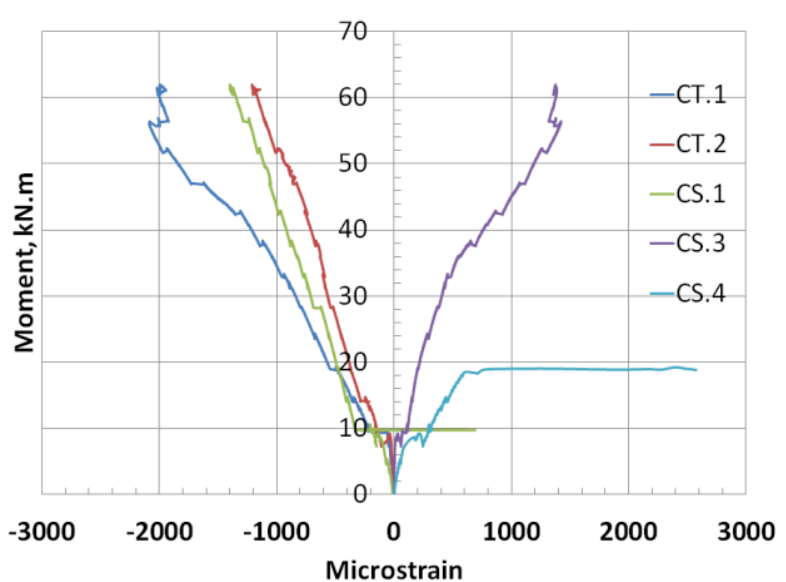

a) Moment-concrete strain

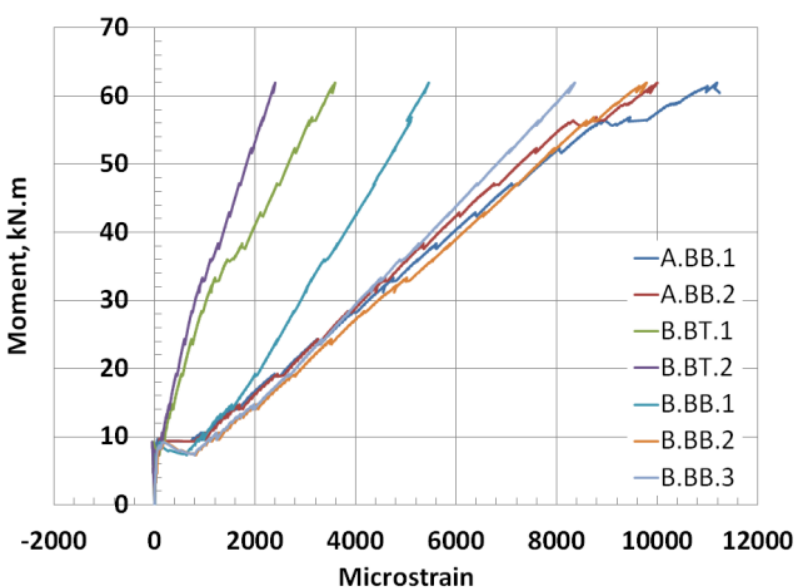

b) Moment-bar strain

Figure 3.21. Jointed GFRP-reinforced FDDP S3 (JCC) under centric load 


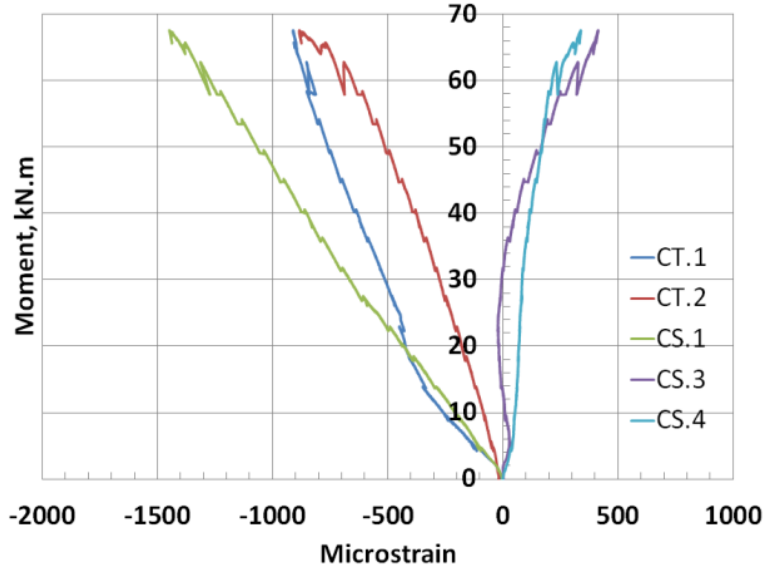

a) Moment-concrete strain

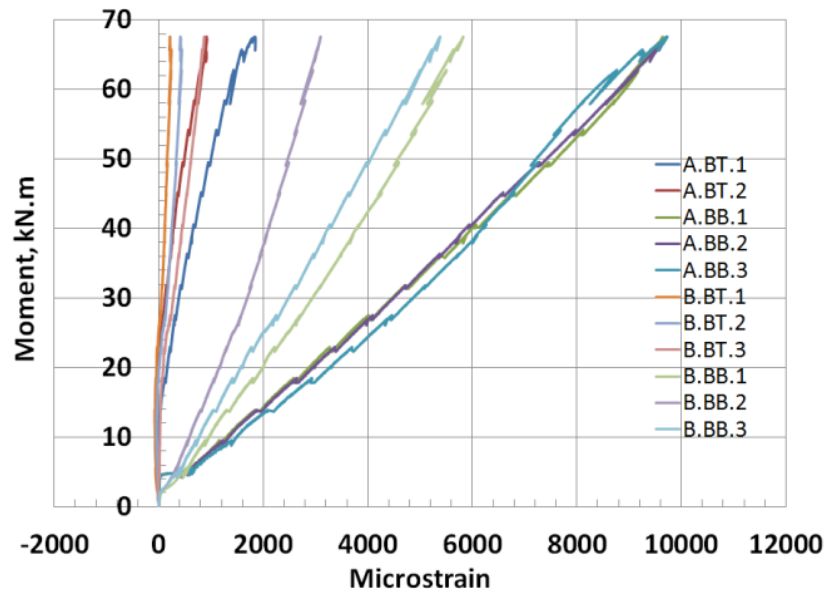

b) Moment-bar strain

Figure 3.22. Jointed GFRP-reinforced FDDP S4 (JCE) under eccentric load

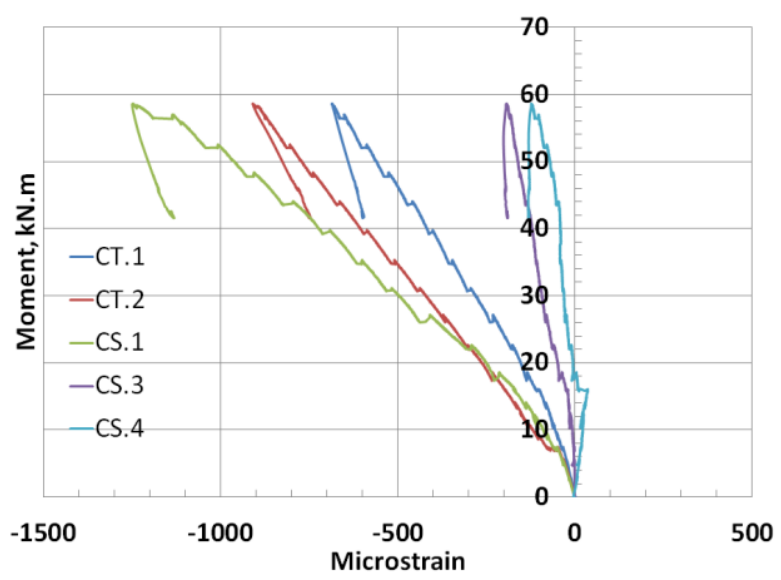

a) Moment-concrete strain

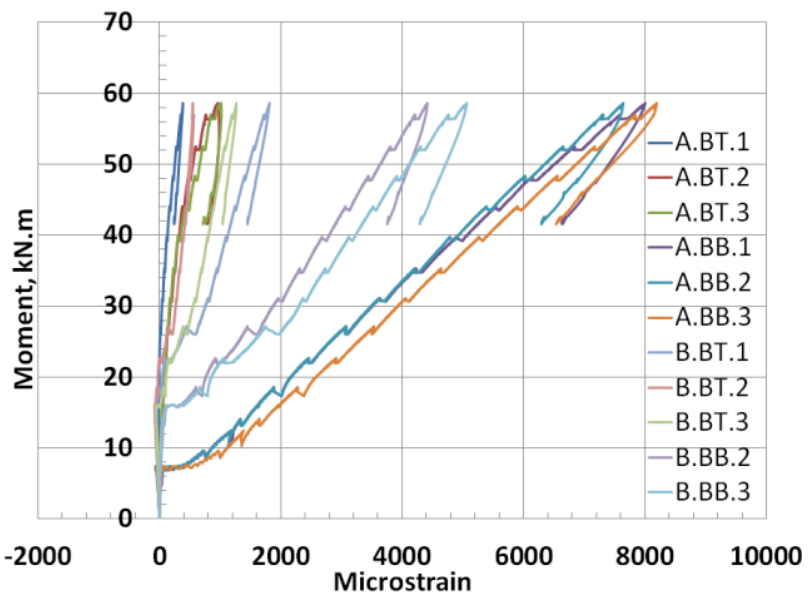

b) Moment-bar strain

Figure 3.23. Jointed GFRP-reinforced FDDP S5 (JZE) under eccentric load

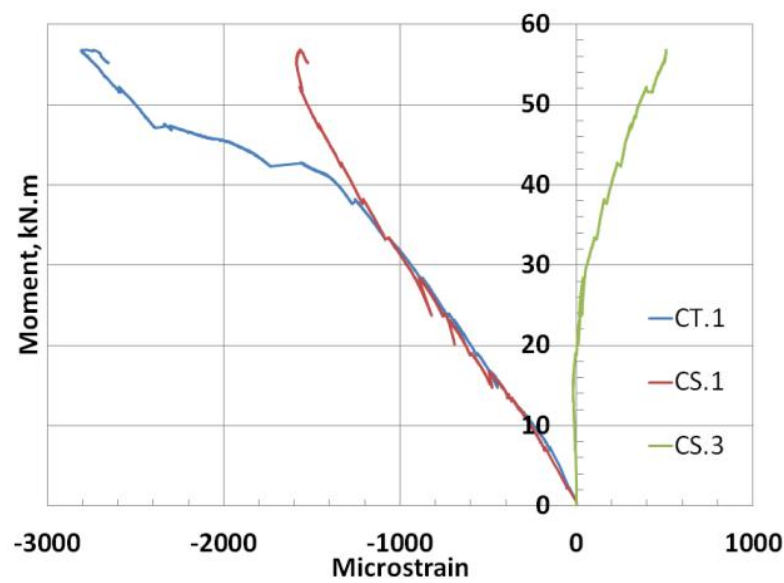

a) Moment-concrete strain

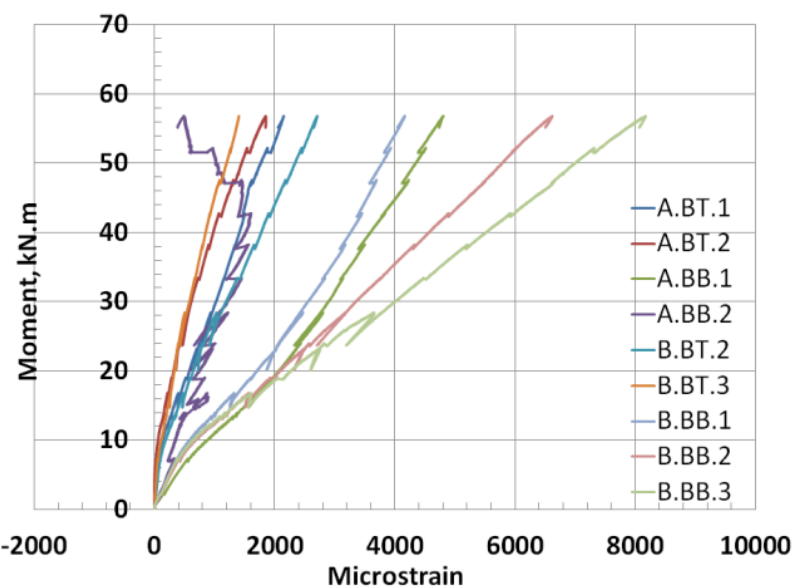

b) Moment-bar strain

Figure 3.24. Jointed GFRP-reinforced FDDP S6 (JAC) under centric load 


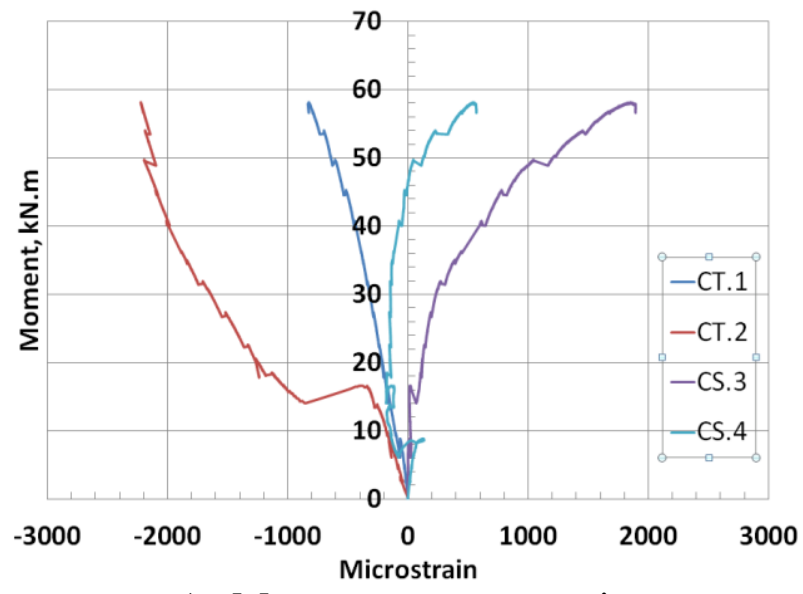

a) Moment-concrete strain

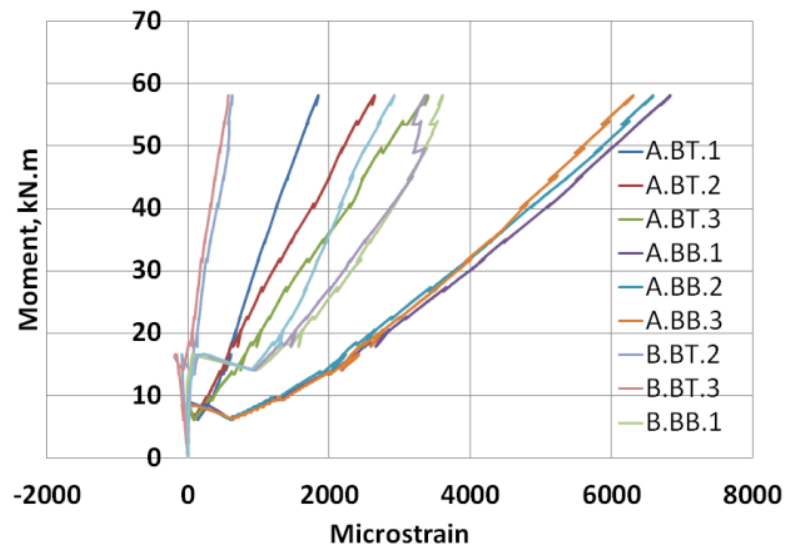

b) Moment-bar strain

Figure 3.25. Jointed GFRP-reinforced FDDP S7 (JAE) under eccentric load 


\section{Appendix: Design Data}

Moment capacity of a rectangular slab with tension reinforcement (Compression Failure)

Data:

Concrete compressive strength, $f_{c}^{\prime}=45 \mathrm{MPa}$

Elastic modulus, $\mathrm{E}$ for the GFRP bar $=64 \mathrm{GPa}$

Ultimate tensile strength, $f_{f r p}=1188 \mathrm{MPa}$

Area of $20 \mathrm{M}$ rebar is $314.16 \mathrm{~mm}^{2}$

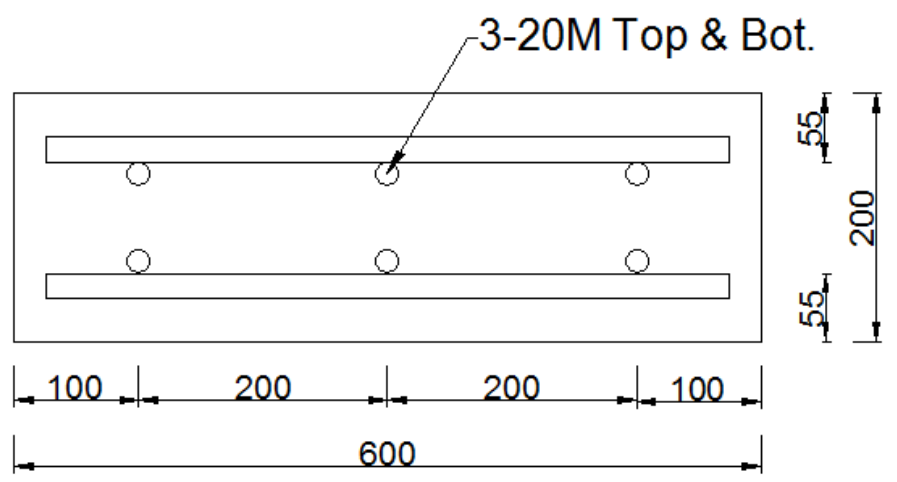

Figure B.1. Slab cross-section

1 Determine the concrete cover and the effective depth of the section

$2.5 d_{b}=2.5 \times 20=50 \mathrm{~mm}$

The effective depth, $d$, is calculated from

$d=h-$ cover $-\frac{d_{b}}{2}=200-50-20 / 2=140 \mathrm{~mm}$

However $\mathrm{d}=200-55-20 / 2=135 \mathrm{~mm}$

2 Calculate the FRP reinforcement ratio

$$
\rho_{\text {frp }}=\frac{A_{f r p}}{b d}=(3 \times 314.16) /(600 \times 135)=0.011636=1.1636 \%
$$

3 Calculate the balanced FRP reinforcement ratio

$\rho_{f r p b}=\frac{A_{f r p}}{b d}=\alpha_{1} \beta_{1} \frac{\phi_{c}}{\phi_{f r p}} \frac{f_{c}^{\prime}}{f_{f r p u}}\left(\frac{\epsilon_{c u}}{\epsilon_{c u}+\epsilon_{f r p u}}\right)$

Where

$\alpha_{1}=0.85-0.0015 f_{c}^{\prime} \geq 0.67 ;=0.85-0.0015(45)=0.7825$

$\beta_{1}=0.97-0.0025 f_{c}^{\prime} \geq 0.67 ;=0.97-0.0025(45)=0.8575$

$\epsilon_{\text {frpu }}=\frac{f_{\text {frpu }}}{E_{\text {frp }}}=1188 /\left(64 \times 10^{3}\right)=0.0186$

$\phi_{c}=0.75$ for concrete

$\phi_{\text {frp }}=0.55$ for Glass FRP 


$$
\rho_{f r p b}=0.7825 \times 0.8575 \times \frac{0.75}{0.55} \times \frac{45}{1188}\left(\frac{0.0035}{0.0035+0.0186}\right)=0.0055
$$

4 Check if the section will fail by tension failure or compression failure

$$
\rho_{\text {frp }}=0.011636>\rho_{\text {frpb }}=0.0055
$$

Therefore, we have COMPRESSION FAILURE, and the distribution is as follows:

5 Determine the tensile stress in the FRP reinforcement at compressive failure of the section

$$
\begin{aligned}
f_{f r p}= & 0.5 E_{f r p} \epsilon_{c u}\left[\sqrt{1+\frac{4 \alpha_{1} \beta_{1} \phi_{c} f_{c}^{\prime}}{\rho_{\text {frp }} \phi_{f r p} E_{f r p} \epsilon_{c u}}}-1\right] \\
& =0.5 \times 64 \times 10^{3} \times 0.0035\left[\sqrt{1+\frac{4 \times 0.7825 \times 0.8575 \times 0.75 \times 45}{0.011636 \times 0.55 \times 64 \times 10^{3} \times 0.0035}}-1\right] \\
& =785.32 \mathrm{MPa}
\end{aligned}
$$

6 Determine the stress block depth, a

$$
\begin{aligned}
\beta_{1} c= & a=\frac{\phi_{f r p} A_{f r p} f_{f r p}}{\alpha_{1} \phi_{c} f_{c}^{\prime} b} \\
& =\frac{0.55 \times 3 \times 314.16 \times 785.32}{0.7825 \times 0.75 \times 45 \times 600}=25.69 \mathrm{~mm}
\end{aligned}
$$

7 Determine the flexural capacity

$$
\begin{aligned}
M_{r}= & \phi_{\text {frp }} A_{\text {frp }} f_{\text {frp }}\left[d-\frac{a}{2}\right] \\
& =0.55 \times 3 \times 314.16 \times 785.32[135-25.69 / 2] \\
& =49727054.86 \mathrm{~N} . \mathrm{mm}=49.73 \times 10^{6} \mathrm{~N} . \mathrm{mm} \\
& =\underline{\mathbf{4 9 . 7 3} \text { kN.m }}
\end{aligned}
$$

Thus, the moment capacity of the section is $48.48 \mathrm{kNm}$.

Finally, we must check that the minimum flexural capacity requirements are satisfied

$$
M_{r} \geq 1.5 M_{c r}
$$

The cracking moment is determined by

$$
M_{c r}=\frac{f_{r} I_{t}}{y_{t}}
$$

Where $f_{r}=0.6 \sqrt{f_{c}^{\prime}}$

$I_{t}=$ transformed section moment of inertia

$y_{t}=$ distance from N.A. to extreme tension fiber

$$
\begin{aligned}
& f_{r}=0.6 \sqrt{45}=4.025 \\
& I_{t}=\frac{b h^{3}}{12}+b h\left(\bar{y}-y_{t}\right)^{2}+(n-1) A_{f r p}(d-\bar{y})^{2} \\
& \bar{y}=\frac{(b h) \frac{h}{2}+(n-1) A_{f r p} d}{A_{t r}} \\
& A_{t r}=b h+(n-1) A_{f r p} \\
& n=\frac{E_{f r p}}{E_{c}}
\end{aligned}
$$




$$
\begin{aligned}
& E_{c}=\left(3300 \sqrt{f_{c}^{\prime}}+6900\right)=3300 \sqrt{45}+6900=29037 \mathrm{MPa} \\
& E_{f r p}=64 \times 10^{3} \mathrm{MPa} \\
& n=\frac{64000}{29037}=2.20 \\
& A_{t r}=600 \times 200+(2.20-1) \times 3 \times 314.16=121130.98 \mathrm{~mm}^{2} \\
& \bar{y}=\frac{(600 \times 200) \frac{200}{2}+(2.20-1) \times 3 \times 314.16 \times 135}{121130.98}=100.33 \mathrm{~mm} \\
& y_{t}=\frac{h}{2}=200 / 2=100 \mathrm{~mm} \\
& I_{t}=\frac{600 \times 200^{3}}{12}+600 \times 200(100.33-100)^{2}+(2.20-1) \times 3 \times 314.16(135- \\
& 100.33)^{2} \quad=401372511.2 \mathrm{~mm}^{4}=0.401 \times 10^{9} \mathrm{~mm}^{4} \\
& M_{c r}=\frac{f_{r} I_{t}}{y_{t}}=\frac{4.025 \times 0.401 \times 10^{9}}{100}=16140250 \mathrm{~N} \cdot \mathrm{mm}=16.14 \times 10^{6} \mathrm{~N} . \mathrm{mm}=\underline{\mathbf{1 6 . 1 4} \mathbf{k N} . \mathbf{m}} \\
& \text { Thus we have } \\
& M_{r}=49.73 \mathrm{kNm} \geq 1.5 M_{c r}=1.5 \times 16.14=24.21 \mathrm{kNm} \rightarrow \mathrm{OK}
\end{aligned}
$$

This, the beam has satisfactory capacity to avoid failure upon cracking.

\section{Flexural capacity of UHPFRC beam}

The flexural strength of singly GFRP reinforced beam. The moment is taken about the compressive force resultant of the UHPFRC (Garcia, 2007).

To determine the stress block of the UHPFRC beam

$$
\begin{gathered}
c=\frac{\phi_{\text {frp }} A_{\text {frp }} f_{\text {frpu }}+\phi_{c} f_{t} h b}{b\left(0.5 \phi_{c} f_{c}+\phi_{c} f_{t}\right)} \\
c=\frac{0.55 \times 3 \times 314 \times 1188+0.75 \times 8 \times 200 \times 600}{600 \times 0.75(0.5 \times 140+8)} \\
\mathrm{c}=38.05 \mathrm{~mm} \\
M_{n}=T_{\text {frp }}\left(d-\frac{c}{3}\right)+T_{c}\left(\frac{h}{2}+\frac{c}{6}\right) \\
M=0.55 \times 1188 \times 3 \times 314\left(135-\frac{38.05}{3}\right)+0.75 \times 8 \times 600(200-38.05)\left(\frac{200}{2}+\frac{38.05}{6}\right) \\
\mathrm{M}=137.286 \mathrm{kN} . \mathrm{m}
\end{gathered}
$$


Shear resistance provided by concrete (ISIS Canada Research Network's Design Manual, 2007)

$$
\begin{aligned}
V_{c}= & 0.2 \lambda \phi_{c} \sqrt{f_{c}^{\prime}} b_{w} d \sqrt{\frac{E_{f r p}}{E_{s}}} \\
= & 0.2 \times 1 \times 0.75 \sqrt{45} \times 1000 \times 135 \sqrt{\frac{64}{200}}=76.84 \mathrm{kN}
\end{aligned}
$$

Where

$V_{c} \quad$ factors shear resistance attributed to concrete, $\mathrm{N}$

$\lambda \quad$ modification factor for density of concrete

$\phi_{c} \quad$ resistance factor for concrete

$b_{w} \quad$ minimum effective web width within depth d, mm

$d$ distance from the extreme compression surface to the centroid of the reinforcement, $\mathrm{mm}$

$E_{f r p} \quad$ modulus of elasticity of flexural FRP reinforcement, MPa

$E_{S} \quad$ modulus of elasticity of steel taken as $200 \times 10^{3} \mathrm{MPa}$ 


\section{Chapter IV}

\section{Structural Behavior of HPC Full-Depth Deck Panels}

\section{with Closure Strips Reinforced with Glass Fiber Reinforced Polymer Bars and Filled with Ultra-High Performance Fiber Reinforced Concrete}

\subsection{General}

Corrosion of reinforcing steel in bridge decks decreases life expectancy of bridge superstructure, leading to costly and frequent maintenance or replacement. The use of high-modulus glass fiber reinforced polymer (HM GFRP) bars as internal reinforcement is a viable option for the replacement of deteriorated concrete bridge deck slabs due to steel bar corrosion. The proposed research investigates the use of ribbed-surface HM GFRP bars in cast-in-place bridge deck slabs as well as precast bridge deck slab of prefabricated full-depth deck panels to accelerate bridge construction. In this research, three joints between precast panels were developed using straight HM GFRP bars embedded in a closure strip filled with ultra-high performance concrete (UHPFRC). High-performance concrete (HPC) of $70-\mathrm{MPa}$ compressive strength was used to cast the prefabricated panels. Two control cast-in-place slabs reinforced with steel bars and HM GFRP bars, respectively, were casted to form the baseline of the structural performance of the developed jointed panels. Concentric and eccentric wheel loading were applied at the joint to expose it to pure bending and combined bending and shear, respectively. Table 4.1 summarizes the parameters considered to form the text matrix in this research. Results were presented in the form of crack pattern at failure, ultimate strength and deflection and strain data. Comparison between the experimental resisting moment and shear with those obtained from design codes were conducted.

\subsection{Research Background on Accelerated Bridge Construction}

One of the prefabricated bridge system used to accelerate bridge construction is the full-depth, full width, precast concrete deck slab with transverse joint placed over steel or concrete girders. 
In this system shown in Fig. 4.1, grout pockets are provided to accommodate clusters of shear connectors welded to steel girders or embedded in concrete girders. Literature survey revealed that PCI Report (2011) along with other ABC manuals produced by US DOTs specify the use of high-performance concrete (HPC) with 70 to $100 \mathrm{MPa}$ compressive strength in precast deck panels to provide sufficient strength and durability. With the use of UHPFRC and GFRP bars in the closure strip, the performance of the transverse panel-to-panel joints in bridge system shown in Fig. 4.1 needs to be investigated at ultimate and fatigue limit states.

Recent surveys of the state of practice of full-depth, full-width panel-girder system and joints were conducted elsewhere (among them: NCHRP, 2011; Badie and Tadros, 2008; Hieber and Wacker, 2005). Connections suitable for simple and continuous spans for composite design were developed using different means including headed reinforcing steel bars, steel couplers and posttensioning the precast panels. Although deck panel post-tensioning puts the joint in compression and secure it against leakage, it increases the cost of the deck system and delays deck construction. A few authors (among them: Li et al. 2011; Graybeal, 2010) have dealt with panelto-panel longitudinal joints in bulb-tee girders. While others authors have (among them: Graybeal, 2010; Issa et al. 2006) dealt with panel-to-panel transverse joints, all using steel reinforcing bars. None of these transverse joints were tested using GFRP bars. Zhu et al. (2012) proposed continuous transverse U-bar joint details, incorporating projecting reinforced steel bars from the jointed panels which can provide negative moment continuity in multi-span bridges, however no pretensioning was used. Based on ultimate and fatigue test results, the developed transverse U-bar joint detail appeared to provide continuity of the reinforcement in the deck panels to carry tensile forces while acting compositely with the girders.

One of the prefabricated bridge elements and systems (PBES) for the accelerate bridge construction (ABC) is the precast full-depth deck panel (FDDP) (Culmo, 2011). Precast FDDP is placed transversally over steel or concrete girders, as shown in Fig. 4.1. Two types of connections are considered with this construction method, namely: (i) transverse panel-to-panel connections and (ii) panel-to-girder connections. The composite action of the panel-to-girder connection and for the superstructure is achieved throughout the clustering of the shear connectors that are welded to steel girders or embedded to concrete girders. Detailed literature 
review pertained to accelerated bridge construction and ultra-high performance concrete is presented in Chapter III.

In 1993, The Federal Highway Administration (FHWA) introduced the high performance concrete (HPC) designation for the construction of bridge use with eight performance characteristics; four for concrete durability and four for its strength. The definition consisted of four durability characteristics that include freeze-thaw resistance, scaling resistance, abrasion resistance and chloride penetration, while the four strength characteristics include compressive strength, modulus of elasticity, drying shrinkage and creep (Russell, 2013). High-strength concrete (HSC) allows the use of longer span lengths, wider girder spacing, shallow girders, or their combination resulting into economical structures. Inspection for constructed HPC reinforced concrete bridge deck depicts cracks from none to more than expected (Russell, 2004). ACI defined HPC as the concrete that has compressive strength of $55.16 \mathrm{MPa}$ or greater (ACI 318, 2010). AASHTO-LRFD Bridge Construction Specification (AASHTO, 2010) included two classes of HPC designated as P (HPC) for the prestressed with strength greater than 41.37 MPa and A (HPC) for the cast-in-place (CIP) construction with strength less than or equal to 41.37 MPa. HPC is composed from supplementary cementitious materials (SCM) that consist of cement, fly ash, silica fume and slag cement. Water/cement ratio is used to represent the weight of water to the binding materials. In 1987, the Strategic Highway Research Program (Zia et al, 1991) proposed the mechanical behavior of HPC to be: (i) maximum w/cm ratio of 0.35 ; (ii) minimum durability factor of $80 \%$ as determined by ASTM C666 Method A; and (iii) minimum compressive strength of $21 \mathrm{MPa}$ within 4 hours after placement, $35 \mathrm{MPa}$ within 24 hours and 70 MPa within 28 days.

In 2006, FHWA proposed new revision for eleven characteristics and three grades of performances of HPC (Russell, 2013). The eleven performance characteristics includes: freezethaw (F/T) durability, scaling resistance (SR), abrasion resistance (AR), chloride penetration (CP), alkali-silica reactivity (ASR), sulfate resistance (SR), flowability, strength; elasticity, drying shrinkage, and creep. Supplementary cementitious materials (SCM) limited the use of Type III cement in only precast concrete members. Fly ash of Class C, F or N Pozzolan is used with upper limits of $15 \%$ to $30 \%$ of the total cementitious materials. Silica fume is restricted to an upper limit between $7 \%$ to $10 \%$ and lower limit of $5 \%$ to $7 \%$ of the total cementitious 
materials. The use of slag cement has upper limit of $30 \%$ to $50 \%$ of the total cementitious materials. The aggregate for concrete bridge decks is taken as normal weight aggregate conforming to AASHTO Spec, M6 and M80, or the lightweight aggregate conforming to AASHTO M195, or the combination of them. AASHTO Specs. M6 and M43 contain the grade spec. for the coarse normal weight aggregates. Chemical admixtures conforming to AASHTO M194 or ASTM C494 through its seven types of admixtures (A through G) are permitted.

\subsection{New Connection Details}

The common practice in Canada for precast concrete bridges is to use concrete with $35 \mathrm{MPa}$ compressive strength. However, given the trend in US DOTs of using high-performance concrete (HPC), it was decided to use $70 \mathrm{MPa}$ compressive strength to cast the prefabricated deck panels for enhanced durability and impact resistance.

The prefabricated bridge system shown in Fig. 4.1 incorporates precast concrete panels placed side-by-side to form the bridge deck. In this system, the concrete deck slab is cast in a controlled environment at the fabrication facilities and then transported to the construction site. One of the main issues inherent in these prefabricated systems is the presence of cold joints created by the closure pours and their potential impact on the overall deck system behavior. In addition, it is important to develop effective connection details between the prefabricated elements to provide continuity of reinforcement in the closure strips so that load sharing between girders is not compromised. This research GFRP bars as main reinforcement in the precast panels, projecting in the closure strip with straight ends. FRPs, as non-corrodible materials, are considered as excellent alternative to reinforcing steel bars in bridge decks to overcome steel corrosion-related problems. GFRP bars used in this study have a tensile strength of $1188 \mathrm{MPa}$, compared to 400 MPa yield strength of reinforcing steel bars. The special "ribbed" surface profile of these bars, shown in Fig. 2.1.c, ensure optimal bond between the concrete and the bar. The favorable GFRP characteristics include (i) high-strength-to-weight ratio; (ii) resistant to corrosion; (iii) freeze and thaw resistance; and (iv) high chemical resistant (Schoeck, 2013). Table 4.2 summarizes the rest of the mechanical properties of GFRP bars considered in this study.

The common practice in bridge design in Ontario is to use $225 \mathrm{~mm}$ thick cast-in-place deck slab in bridges. This slab thickness incorporates $60 \pm 10 \mathrm{~mm}$ top concrete cover as recommended by 
CHBDC for reinforcing steel bars for protection against possible corrosion. However, with the use of non-corrosive FRP bars, the deck slab thickness can be reduced by $35 \mathrm{~mm}$ as specified in CHBDC with the use of FRP reinforcement. This change makes the FRP-reinforced deck slab thickness $200 \mathrm{~mm}$, thus reducing the material of the deck slab by about $12 \%$. As a result, it was decided to conduct this research using $200 \mathrm{~mm}$ thick slabs in this study. A vertical shear key, shown in Fig. 4.2.a, was used on each side of the joint to improve the shear resistance between the jointed parts.

Three details for the joints between precast panels were proposed incorporating GFRP bars as depicted in Fig. 4.2. The first proposed joint has a 200-mm wide closure strip as shown in Fig. 4.2.a. In this joint, both the top and bottom GFRP bars in the precast slab project into the joint with a 175-mm anchorage length. In this joint, the precast panel has projected slab of $90 \mathrm{~mm}$ length at the bottom of the joint to hold UHPFRC within the closure strip during casting. A foam-type packing rod is inserted in the $20-\mathrm{mm}$ gap between the two projected slabs at the bottom of the closure strip to avoid material leakage. This joint is called "Angle-shape" joint or "A-joint" in this research.

The second proposed joint has a 200-mm wide closure strip as shown in Fig. 4.2.b. This joint is identical to the first proposed joint but without the $90-\mathrm{mm}$ projecting slab. Both the top and bottom GFRP bars in the precast slab project into the joint with a $175-\mathrm{mm}$ anchorage length. It is assumed that temporary form work will be used to close the bottom of the closure strip to hold UHPFRC materials before hardening. This joint is called " $\mathrm{C}$-shape" joint or " $\mathrm{C}$-joint" in this research.

The third proposed joint has a 100-mm wide closure strip with a staggered $100 \mathrm{~mm}$ wide trapezoidal-shaped (zigzagged-shaped) interlock between the precast slabs as shown in the plan of Fig. 4.2.c. In this connection, the top and bottom GFRP bars of the precast slab project into the joint with a $175 \mathrm{~mm}$ anchorage length. Temporary form work will be required to hold the cast UHPFRC into the closure strip during concrete placement. This joint is called "Zigzagshape" joint or "Z-joint" in this research. It should be noted that this joint with zigzag shape and vertical shear key at joint edges would be a good choice for the closure strip a the negative moment region since it will provide continuity through bar splice along with interlocking of the zigzagged UHPFRC closure strip with the zigzagged edges of the precast panel. The use of 70 
$\mathrm{MPa}$ concrete along with this joint in the vicinity of the piers would allow for enhanced continuity that increase moment resistance of the composite slab-girder section at ultimate and fatigue limit state designs.

\subsection{Experimental Program}

The experimental program investigated two types of loading that can be applied at the closure strip region, namely concentric and eccentric truck wheel loading. Figure 4.3-a shows a schematic diagram of the jointed slab with truck wheel load located over the joint creating pure bending with UHPFRC. However, Fig. 4.3.b shows the wheel load located at the edge of joint creating combined shear and bending on the UHPFRC. As such, the test setup considered in this study includes (i) pure flexural tests, and (ii) combined flexural-shear tests. The experimental program included seven full-scale deck slab specimens 2500-mm long and 200-mm thick. A 600-mm slab width is considered for a slab strip. The 600-mm width is assumed oriented in the direction normal to the traffic, while the slab span is oriented in the direction of traffic. It should be noted that the selection of this slab size and details is considered for the sake of correlating test results for the jointed precast slab with identical cast-in-place slabs reinforced by either steel bars or GFRP bars. In fact, the results from this research can be applied to the joint between bulb-tee girders since the objective of this testing is to qualify the jointed precast slab to be "as good as" the cast-in-place slab of similar geometry, concrete type and reinforcement sizes but with different reinforcement material.

In this study, the span of the slab was taken as $2000 \mathrm{~mm}$ with a slab total length of $2500 \mathrm{~mm}$ to accommodate proper bars anchorage beyond the supporting points. The wheel load is distributed only over a $600 \mathrm{~mm}$ width of the slab and over a length of $250 \mathrm{~mm}$ in the direction of slab span. Table 4.1 summarizes the name coding for the tested slabs considered in this study. The first deck slab specimen, S1, is formed of cast-in-place concrete reinforced with steel bars of 400MPa yield strength and 200-GPa modulus of elasticity. In this research, 3-20M reinforcing steel bars spaced at $200 \mathrm{~mm}$ on centre were used as bottom and top layers representing the CHBDCspecified minimum steel reinforcement of $1.0 \%$ of the concrete area. Transverse $10 \mathrm{M}$ steel bars

were used at the top and bottom layers at $300 \mathrm{~mm}$ spacing. Figure 4.4.a shows layout of reinforcement in slab S1, while Fig. 4.6.a shows view of the steel reinforcement in slab S1. The second deck slab strip, S2, is similar to cast-in-place slab S1 but with 3-20M straight GFRP bars 
spaced at $200 \mathrm{~mm}$ on centre, as bottom tension reinforcement. Transverse reinforcement of $20 \mathrm{M}$ GFRP bars was used at the top and bottom layers at $300 \mathrm{~mm}$ on centre spacing. Fig. 4.5.b shows view of the GFRP reinforcement in slab S2. The third deck slab, S3, is similar to slab S2 but uses the precast deck C-joint shown in Fig. 4.2.b. The amount and spacing of GFRP bars in the precast flanges were the same as those for the cast-in-place deck slab S2. Figure 4.5.b shows a view of the GFRP bar layout in S3 slab formwork, while Fig. 4.6.a shows a view of straight GFRP bars projecting into the closure strip of slab S3 before casting the UHPFRC. It should be noted that slabs S1 to S3 were loaded with the wheel load located concentrically over the joint as depicted in Fig. 4.3.a. The fourth deck slab, S4, is identical to the joined precast slab S3 and had the joint eccentrically loaded as depicted in Fig. 4.3.b.

The fifth slab S5 incorporates the trapezoidal-shaped (zigzagged-shaped) interlocking closure strip between precast panels shown in Fig. 4.2.c. The slab size and GFRP reinforcement in slab $\mathrm{S} 5$ is identical to those for slabs S3 and S4. Figure 4.5.c shows a view of the GFRP bar layout in S5 slab formwork, while Figure 4.6.b shows a view of the straight GFRP bars projecting into the closure strip of slab S5 before casting the UHPFRC. The sixth and the seventh deck slabs, S6 and S7, respectively, are similar to the joined precast slabs S3 and S4 but with the closure strip details shown in Fig. 4.2.a. Figure 4.5.d shows view of the GFRP bar layout in S6 slab formwork, while Figure 4.6.c shows view of the straight GFRP bars projecting into the closure strip of slab S6 before casting the UHPFRC. The only difference between slabs S6 and S7 is that the former was loaded with concentric loading while the latter was loaded with eccentric loading.

Concrete having a specified 28-day compressive strength of $70 \mathrm{MPa}$ was used for both cast-inplace and precast deck slabs. Standard cylinders of 150-mm diameter and 300-mm height were cast concurrently with the casting of the deck slabs. An average of three cylinders were cast and stored close to the test samples to ensure the same curing conditions after casting. The tested cylinder had an average concrete compressive strength of $76.94 \mathrm{MPa}$ (the average of 77.75, 79.65, 71.23 and 79.11 MPa). Ultra-High-Performance Fiber Reinforced Concrete (UHPFRC) of a specified compressive concrete strength of $140 \mathrm{MPa}$ was used to fill the closure strip. Table 4.2 shows typical UHPFRC composition, while Table 4.4 shows the characteristic values of UHPFRC Type JS1000 considered in this study and available elsewhere (Lafarge, 2009). During pouring UHPFRC into the precast deck slab closure strips, standard cylinders of 100-mm 
diameter and 200-mm height were cast and kept close to the test samples. Table 4.5 summarizes the average strength of cylinders tested on the day of slab testing.

The behavior of the specimens under static loading to-collapse was captured through the use of electronic instrumentation. Potentiometers and Linear variable differential transducers (LVDTs) were used to measure deflections under the loading point of the slab specimens as depicted in Fig. 4.4. Concrete strain gauges were installed along the depth of the UHPFRC joint at mid-span as depicted in Fig. 4.4. Also, strain gauges were installed on the bottom steel and GFRP bars at the mid-span point in the cast-in-place slabs S1 and S2, respectively. While they were installed in the bottom GFRP bars at the interface between the precast slab and the UHPFRC but embedded in the precast slab S3 through S7. Figures 4.4 shows locations of these gauges in the tested slabs.

CHBDC truck wheel load was applied to examine the structural behavior and ultimate load carrying capacity of the proposed connection details as compared to the control cast-in-place slabs S1 and S2 reinforced with steel and GFRP bars, respectively. Slabs S1, S2, S3 and S6 were tested under a 250x600 mm single patch load at the center of their clear span. Figures 4.7.a and 4.8.a show the locations of the wheel load at the mid-span for slabs S1 and S2, respectively. While Figure 4.9.a shows the wheel load applied concentrically over the joint for slab S3. The slab ends were simply-supported over roller support at one end and hinged support at the other end. Slabs S4, S5, and S7 were tested under a 250x600 mm single patch load located at the edge of the joint as shown in Figs. 4.10-a, 4.11-a and 4.13-a, respectively.

\section{ASTM E529-04 (2011), Standard Guide for Conduction Flexural Tests on Beams and Girders} for Building Construction, specifies the monotonic load to be applied and removed in equal increments at constant rate as possible. Under repeated load (incremental load-to-collapse) at levels that cause significant yielding, there is the potential for the slab deck to fail due to incremental accumulation of vertical deflection at loads less than the inelastic monotonic limit load. So, to conduct static load tests to-collapse, the jacking load was applied in increments to allow for visual inspection of the specimen and to mark cracks. The available data acquisition system was used to capture readings from sensors as well as the load cell located between the jacking piston and the top of the deck slab. After every load increment, the start of tension cracks and crack propagations were monitored. It should be noted that the incremental loading 
technique was used during testing on which the specimen was loaded to $10 \mathrm{kN}$, followed by load release. The specimen was loaded then to $20 \mathrm{kN}$, followed by load release. These incremental loading steps were repeated with a total load increase of $10 \mathrm{kN}$ in each step until the specimen failed. The following section discusses the structural behavior of the test specimens.

\subsection{Test Results}

\subsubsection{Behavior of the Tested Slabs}

This section discusses the structural behavior of the tested specimens in the form of crack pattern, slab deflection and ultimate load carrying capacity. Figure 4.7.b shows the crack pattern at failure at the side of the slab for the cast-in-place slab S1 (C.ST.C) with reinforcing steel bars. It was observed that the first visual flexural crack appeared at the bottom of the slab at the midspan location at a load of $28 \mathrm{kN}$. Other flexural cracks appeared within the quarter points of the span and propagated towards the top surface of the slab with increase in load till failure. Crushing of concrete at the top surface of the slab at the mid-span location was observed at the maximum jacking load of $169.47 \mathrm{kN}$ and slab deflection of $29.81 \mathrm{~mm}$, leading to pure flexural flexure. Figure 4.8.b shows the crack pattern at failure of cast-in-place slab S2 (C.GFRP.C) with HM GFRP bars. It was observed that the first visible flexural crack appeared at a load of $28 \mathrm{kN}$. Other flexural crack appeared at higher load increments and spread over a length greater than that for slab S1. The slab failed mainly due to large flexural cracks with concrete crushing at the top surface of slab at an ultimate load of $161.34 \mathrm{kN}$ and deflection of $42.24 \mathrm{~mm}$. By comparing the ultimate load capacity of slabs S1 (C.ST.C) and S2 (C.GFRP.C), it can be observed that the HM GFRP-reinforced slab exhibited a flexural strength about $4.8 \%$ less than that for a similar slab reinforced with steel bars and deflection at failure of about $42 \%$ more than that for steelreinforced slab. This increase in deflection in the HM GFRP-reinforced slab may be attributed to the significant reduction in the modulus of elasticity of the HM GFRP bars compared to that for steel bars.

Figures 4.9-b and 4.9-c show the crack pattern at failure of precast slab S3 (J.C.C) with 200-mm wide closure strip and projecting straight GFRP bars into the UHPFRC-filled closure strip. It was observed that the first hairline cracks were formed at the cold joint between the precast concrete and the closure strip. These fine cracks started to widen gradually with increase in applied load. 
Several flexural, as well as flexural-shear, cracks appeared in the precast slab closer to the closure strip, with a wide flexural crack at both sides of the cold joint. Failure of slab S3 (J.C.C) was at $148.39-\mathrm{kN}$ ultimate load and 37.84-mm deflection. By comparing the ultimate load capacity of slabs S2 (C.GFRP.C) and S3 (J.C.C), it can be observed that the HM GFRPreinforced jointed slab exhibited a flexural strength about $8 \%$ less than that for a similar cast-inplace slab. This slight decrease in slab flexural strength would be acceptable given the presence of the joint at the maximum moment location with short splice length of HM GFRP bars. It should be noted that very light flexural crack appeared in the UHPFRC at the slab mid-span, with no visible cracks appeared in the UHPFRC material at the free sides of the slab as depicted in Figs. 4.9-b and 4.9-c.

Figures 4.10-b and 4.10-c show the crack pattern at failure of precast HM GFRP-reinforced slab S4 (J.C.E) with eccentric truck wheel loading at the joint. It was noticed that the first hairline cracks were formed at the cold joint between the precast concrete and the closure strip at a load of $30 \mathrm{kN}$. These fine cracks started to widen gradually with increase in applied load. Few flexural cracks appeared in the precast slab closer to the closure strip. Close to failure, a wide diagonal-shear crack, as depicted in Figure 4.10-b, propagated along the slab between the support and the applied load location, leading to slab failure. Failure of slab S4 (J.C.E) occurred at $152.53-\mathrm{kN}$ ultimate load and 22.12-mm deflection. Comparing slabs S3 (J.C.C) and S4 (J.C.E), it can be observed that the slab ultimate load slightly increased (i.e. by 3\%) as a result of using eccentric loading rather than concentric loading at the closure strip, while the slab ultimate deflection decreased by about $41 \%$. This may be attributed to the fact that the shear span of the precast slab with eccentric loading decreases, leading to a higher load carrying capacity.

Figure 4.11-b and 4.11-c shows the crack pattern at failure of precast slab S5 (J.Z.E) with 100$\mathrm{mm}$ wide closure strip and staggered 100-mm wide trapezoidal-shaped interlock, and projected HM GFRP bars into the joint. It was noticed that the first hairline cracks were formed at the cold joint between the precast concrete and the closure strip at a load of $15 \mathrm{kN}$. These fine cracks started to widen gradually with increase in applied load. Few flexural cracks appeared in the precast slab closer to the closure strip as shown in Figure 4.11-c. Prior to failure, a wide diagonal flexural crack appeared on one both side of the cold joint at the quarter point of the precast slab as depicted in Figure 4.11-b that led to failure. Failure of slab S5 (J.Z.E) occurred at 162.52-kN 
ultimate load and 26.89-mm deflection. No cracks appeared in UHPFRC at the bottom or the sides of the joint. As such, it can be concluded that slab S5 is as good as the steel-reinforced slab S1 (C.ST.C) (i.e. 4\% difference) and HM GFRP-reinforced slab (i.e. 0.7\% difference) with respect to ultimate strength.

Figures 4.12.b and 4.12.c show the crack pattern at failure of the precast slab S6 (J.A.C) with 200-mm closure strip and projected bottom slab segment under concentric loading, while Figs. 4.13.b and 4.13.c show the crack pattern for slab S7 (J.A.E) with eccentric loading. It was observed that the first hairline cracks were formed at the cold joint between the precast concrete and the closure strip and propagated towards the top of the slab with load increase as depicted in the close-up in Figure 4.13-c at a load of about $30 \mathrm{kN}$. These cracks widened with increase in the applied load as depicted in Figure 4.13-d, leading to concrete crushing at the top surface of the slab. Other flexural cracks appeared between the joint and the quarter point of the precast slab as depicted in Figure 4.13-b. The S3 (J.C.C) slab failed at $132.64 \mathrm{kN}$ ultimate load and $34.1 \mathrm{~mm}$ deflection while the S4 (J.C.E) failed at $111.33-\mathrm{kN}$ ultimate load and 24.26-mm deflection. It can be noted that both slabs S3 (J.C.C) and S4 (J.C.E) has a flexural capacity less than those for the steel-reinforced slab S1 (C.ST.C) and the HM GFRP-reinforced slab S2 (C.GFRP.C) by 21.73\% and $17.8 \%$ for the S3 (J.C.C) slab and $34 \%$ and $31 \%$ for the S4 (J.C.E) slab, respectively.

Figure 4.14 depicts the incremental load-deflection history of all tested specimens, and Fig. 4.15 depicts the combined load-deflection curves for all testes slabs. Although the load-deflection history presents a measure of the change in slab flexural stiffness due to cracking, and yielding of steel in case of steel-reinforced slab, with increase in applied load, the given data can be used further to determine slab ductility under wheel load. Such analysis as well as forthcoming testing of these slabs under fatigue loading is expected to provide engineers with clear picture on the best joint between precast slab for enhanced strength, fatigue service life and durability.

\subsubsection{Analysis of Test Data for Flexural and Shear Resistance}

The objective of this analysis is to determine the resisting moment of the concrete and UHPFRC sections by manual calculations and correlate them to the experimental ultimate moment. Figure 4.17 depicts the structural analysis of the tested slabs so that the reaction forces and moments can be obtained through the following equations. 


$$
R_{1}=V_{1}(\max . \text { when } \mathrm{a}<\mathrm{c})
$$$$
=\frac{w b}{2 L}(2 c+b)
$$

$$
R_{2}=V_{2}(\max . \text { when } \mathrm{a}>\mathrm{c})
$$$$
=\frac{w b}{2 L}(2 a+b)
$$

$$
V_{x}(\text { when } \mathrm{x}>\mathrm{a} \text { and }<(\mathrm{a}+\mathrm{b}))
$$$$
=R_{1}-w(x-a)
$$

$$
M_{\max }\left(\text { at } x=a+\frac{R_{1}}{w}\right)
$$$$
=R_{1}\left(a+\frac{R_{1}}{2 w}\right)
$$

The resisting bending moment of the reinforced concrete slab of width $600 \mathrm{~mm}$ was calculated based on the procedure available elsewhere (ISIS, 2007) for singly-reinforced section and based on the rectangular section analysis shown in Fig. 4.18 and the following set of equations. Also, the theoretical shear capacity of the tested slabs was obtained using Equation 4.12 (ISIS, 2007).

$$
\begin{aligned}
& \rho_{f r p}=\frac{A_{f r p}}{b d} \\
& \epsilon_{f r p u}=\frac{f_{f r p u}}{E_{f r p}} \mathrm{e}
\end{aligned}
$$

$$
\rho_{f r p b}=\frac{A_{f r p}}{b d}=\alpha_{1} \beta_{1} \frac{\phi_{c}}{\phi_{f r p}} \frac{f_{c}^{\prime}}{f_{f r p u}}\left(\frac{\epsilon_{c u}}{\epsilon_{c u}+\epsilon_{f r p u}}\right)
$$

$$
\rho_{\text {frp }}=0.011636>\rho_{\text {frpb }}=0.00655
$$

$$
f_{f r p}=0.5 E_{f r p} \epsilon_{c u}\left[\sqrt{1+\frac{4 \alpha_{1} \beta_{1} \phi_{c} f_{c}^{\prime}}{\rho_{f r p} \phi_{f r p} E_{f r p} \epsilon_{c u}}}-1\right]
$$

$$
\beta_{1} c=a=\frac{\phi_{f r p} A_{f r p} f_{f r p}}{\alpha_{1} \phi_{c} f_{c}^{\prime} b}
$$

$$
M_{r}=\phi_{f r p} A_{f r p} f_{f r p}\left[d-\frac{a}{2}\right]
$$

$$
V_{c}=0.2 \lambda \phi_{c} \sqrt{f_{c}^{\prime}} b_{w} d \sqrt{\frac{E_{f r p}}{E_{s}}}
$$

In section analysis, the resistance factors for concrete, $\phi_{c}$, and GFRP reinforcing bars, $\phi_{f r p}$, were taken as 0.75 and 0.55 , respectively (CHBDC, 2014). Using the above-mentioned equations, the bottom HM GFRP reinforcement ratio, $\rho_{f r p}$, and the balanced HM GFRP reinforcement ratio, $\rho_{f r p b}$, were found to be $1.16 \%$ and $0.76 \%$, respectively. As such, the slab 
section would fail under concrete compression failure mode. Section analysis showed that the tensile stress in HM GFRP reinforcement at compression failure of the section was $f_{\text {frp }}=968$ MPa which is far below the tensile strength of the bars. The stress block depth, a, was found to be $19.73 \mathrm{~mm}$. Thus, the calculated resisting moment, $M_{r}$, was found to be $62.79 \mathrm{kN} . \mathrm{m} / 0.6-\mathrm{m}$ from Equation (4.11), or $104.65 \mathrm{kN} . \mathrm{m} / \mathrm{m}$. Equation 4.12 provided the shear resistance of the slab as $100.48 \mathrm{kN} / \mathrm{m}$.

The generalized internal stress distribution for the flexural behavior of UHPFRC rectangular section can be simplified as shown in Fig. 4.19 (Garcia, 2007). The compressive force is approximated through a triangular stress distribution, where the compressive force, $\mathrm{C}$, acts at one-third the neutral axis depth below the extreme compression fiber of the rectangular section. A uniform tensile stress of $f_{t}$ acts along the section depth from the neutral axis to the extreme fibers in tension. The tensile force from the HM GFRP bars is expressed in the same manner as that for reinforced concrete section (Garcia, 2007).

$$
\begin{aligned}
& C=\frac{1}{2} \phi_{c} f_{c}^{\prime} a b \\
& T_{c}=\phi_{c} f_{t}(h-a) b \\
& T_{f r p}=\phi_{f r p} f_{f r p u} A_{f r p}
\end{aligned}
$$

Calculations showed that the depth of the stress block was calculated from Equation 3.15 to be $38.05 \mathrm{~mm}$ when the compression stress, $f_{c}^{\prime}$, and tension stress, $f_{t}$, of UHPFRC are 140 and 8 $\mathrm{MPa}$ respectively, and the ultimate tensile strength of the bar, $f_{\text {frpu }}$, is $1188 \mathrm{MPa}$. The resistance factor of concrete and HM GFRP considered in these calculations were equal to 0.75 and 0.55 , respectively (CHBDC, 2014).

$$
a=\frac{\phi_{f r p} A_{f r p} f_{f r p u}+\phi_{c} f_{t} h b}{b\left(0.5 \phi_{c} f_{c}^{\prime}+\phi_{c} f_{t}\right)}
$$

The calculated resisting moment of the UHPFRC section was then obtained from Equation 4.16 as $137.29 \mathrm{kN} . \mathrm{m} / 0.6-\mathrm{m}$ or $228.82 \mathrm{kN} . \mathrm{m} / \mathrm{m}$.

$$
M_{n}=T_{f r p}\left(d-\frac{a}{3}\right)+T_{c}\left(\frac{h}{2}+\frac{a}{6}\right)
$$




$$
M_{n}=\phi_{f r p} f_{f r p} A_{f r p}\left(d-\frac{a}{3}\right)+\phi_{c} f_{t}(h-c) b\left(\frac{h}{2}+\frac{a}{6}\right)
$$

Table 4.7 shows summary of experimental data as well as theoretical bending and shear resistance of the tested slabs. The ratio between the experimental resisting moment and theoretical resisting moment, $\beta$, was calculated using Equation 4.17. Results show shows the ratio between the experimental resisting moment and theoretical moment for the precast slabs as 1.18, 1.11, 1.08, 1.12, 0.99 and 0.79 for slabs S2, S3, S4, S5, S6 and S7, respectively. So, Bridge Design Engineers can use the traditional procedure for section analysis mentioned earlier to determine the resisting moment of the slabs made of normal concrete as the ratio $\beta$ is more than 1 for all slabs. However, designers may consider the durability effect on the GFRP bars in the tested slabs of 0.75 as specified in CHBDC chapter 16 . In this case, the ratio $\beta$ may be 0.89 , 0.83, 0.81, 0.84, 0.75 and 0.59 for slabs S2, S3, S4, S5, S6 and S7, respectively. As such, Designers may consider the resisting moment of the reinforced concrete section as the revised ratio $\beta$, taking into account durability effects of GFRP bars, times the resisting moment calculated based on Equation 4.11. On the other hand, Designers should not rely on the moment resistance of UHPFRC section, of $228.81 \mathrm{kN} . \mathrm{m} / \mathrm{m}$, since it is far greater than the experimental resisting moment as well as the theoretical resisting moment of the normal concrete section.

$$
\beta=M_{r, \text { experimental }} / M_{r, \text { theoretical }}
$$

As for shear, the last column in Table 4.7 depicts the ratio between the experimental resisting shear force and the theoretical value. Results show shows the ratio between the experimental resisting shear force and theoretical value for the precast slabs as 1.34, 1.23, 1.55, 1.72, 1.1 and 1.13 for slabs S2, S3, S4, S5, S6 and S7, respectively. However, designers may consider the durability effect on the GFRP bars in the tested slabs of 0.75 as specified in CHBDC chapter 16. In this case, the ratio $\beta$ may be $1.01,0.92,1.16,1.29$ and 0.83 for slabs S2, S3, S4, S5, S6 and S7, respectively. In this case, designers may use Equation 4.12 to determine the shear resistance of the precast slab made of normal concrete with a reduction factor equal to the ration $\beta$ if it is less than 1. Table 4.8 provides the coefficients for the third-degree polynomial equation that used to model the moment-deflection behavior from starting of the load to the ultimate to-collapse loading. Polynomial equations show excellent coefficient of determination $\mathrm{R}^{2}$ close to 1 . 


\subsubsection{Moment-Strain Relationship and Associated Bond Stress for GFRP Bars in UHPFRC}

Figures 4.20 to 4.26 depict the moment-strain relationships for the bottom and top HM GFRP bars and for concrete at mid-span for cast-in-place concrete and at the edge of the applied load at the mid-span for the jointed slabs. General purpose, $10-\mathrm{mm}$, strain gages were placed at the midlength of the bar for the cast-in-place control slabs reinforced with steel bars or HM GFRP bars. However, for jointed slabs, the 10-mm strain gages were attached to the HM GFRP bars embedded into the precast FDDP but just at the interface between the precast slab and the joint. Foil strain gauges of $60 \mathrm{~mm}$ length were placed on the top surface of the concrete and along the depth of the mid-point of the control slabs or along the depth of the joint to measure concrete strains. Figure 4.4 shows locations of sensors used in this research. Table 4.9 records three readings for the micro-strain gages glued to the top surface of the concrete slab, to the top reinforcement, and to the bottom reinforcement, respectively. These values were recorded at the failure load. This strain data was considered for further analysis to determine the bottom bar tensile force as shown in Table 4.10. By multiplying the tensile force by the bar cross-sectional area, the actual bar stresses at slab failure was recorded as 377, 699, 450, 392, 426 and 382 MPa for slabs S2, S3, S4, S5, S6 and S7, respectively. This values were far below the tensile strength of the GFRP bars of $1188 \mathrm{MPa}$, as expected. To determine the actual bond stress $(\tau)$ developed in the GFRP bar embedded into UHPFRC in the jointed slabs, the following equations were used and the results were presented in Table 4.10.

$$
\begin{aligned}
\frac{1}{k} & =\frac{T}{L_{d} \sqrt{f_{c}^{\prime}}}=\frac{A_{f r p} E_{f r p} \epsilon_{f r p}}{L_{d} \sqrt{f_{c}^{\prime}}} \\
\tau & =\frac{\sqrt{f_{c}^{\prime}}}{\pi k d}
\end{aligned}
$$

Results in Table 3.10 show that the actual bond stress in GFRP bars embedded into UHPFRC in the tested slabs were found to be 19.98, 12.88, 11.19, 12.16 and 10.92 MPa for jointed slabs S3, S4, S5, S6 and S7, respectively. These values are generally lower than those obtained from direct pullout tests on GFRP bars embedded in UHPFRC blocks presented in Chapter II of this Thesis. 


\subsection{Conclusions}

This research presented the mechanically behavior of precast full-depth deck panels (FDDPs) made of high-performance concrete (HPC) and reinforced with straight-ended ribbed-surface HM GFRP bars. For FDDPs constructed using HPC, the following conclusions were drawn:

1. The ultimate load capacity of GFRP-reinforced cast-in-place slab was about 5\% greater than the capacity of a similar slab reinforced with steel bars in accordance with the CHBDC and having the same cross-sectional area as the GFRP bars.

2. The GFRP-reinforced jointed slab with a $200 \mathrm{~mm}$ wide joint width with vertical shear keys exhibited a flexural strength about $8 \%$ less than that for a similar cast-in-place slab.

3. Comparing jointed precast slabs with different applied load patterns, it was observed that the slab ultimate load slightly increased (i.e. by 3\%) as a result of using eccentric loading rather than concentric loading at the closure strip, while the slab ultimate deflection decreased by about $41 \%$.

4. The precast slab with a zigzag-shaped joint appeared to be as good as the steelreinforced slab (i.e. $4 \%$ difference) and GFRP-reinforced slab (i.e. $0.7 \%$ difference) with respect to ultimate strength.

5. The precast slab with a $200-\mathrm{mm}$ wide closure strip and projected bottom slab segment proved to have flexural capacity less than those for the steel-reinforced cast-in-place slab and the GFRP-reinforced cast-in-place slab 34\% and 31\%, respectively.

6. The failure mode in all tested one-way slab specimens was either pure flexural of combined flexural and shear in the HPC panel rather than (i) in the UHPFRC-filled joint or (ii) bond failure of GFRP bars embedded into the joint. This conclusion would allow Bridge Design Engineers design the precast jointed NSC-built deck slabs using the one-way slab action specified in AASHTO-LRFD Specification or the flexural design method specified in CHBDC. In this case, Bridge Design Engineers will adopt one of the developed UHPFRC-filled joint configurations without design and consider the design of the precast slab under factored applied wheel load specified in the code per meter width to prevent pure flexure failure or combined shear-flexural failure outside the joint. 
7. Considering the ratios between the experimental moment and the theoretical moment resistance of the tested slabs made normal-strength concrete, $\beta$, as $0.89,0.83,0.81$, 0.84, 0.75 and 0.59 for slabs S2, S3, S4, S5, S6 and S7, respectively, designers may consider the resisting moment of the reinforced concrete section as the ratio $\beta$ times the resisting moment calculated based on traditional procedure available in CHBDC. This conclusion was reached by applying a general durability factor of 0.75 to the test results of the tested slabs made of GFRP bars as specified in CHBDC. In case of shear design, the ratio $\beta$ may be taken as $1.01,0.92,1.16,1.29$ and 0.83 for slabs S2, S3, S4, S5, S6 and S7, respectively. In this case, designers may use ISIS shear equation to determine the shear resistance of the precast slab made of highperformance concrete with a reduction factor equal to the ratio $\beta$ if it is less than 1 .

\section{References}

ACI. 2004. Guide Test Methods for Fiber-Reinforced Polymers (FRPs) for Reinforcing or Strengthening Concrete Structures, ACI 440.3R-04, American Concrete Institute, USA.

ACI COMMITTEE 363. 2010. Report on High-Strength Concrete. American Concrete Institute, USA.

AASHTO. 2016. AASHTO-LRFD Bridge Design Specification. The American Association of State Highway and Transportation Officials.

ASTM. 2011. Standard Guide for Conduction Flexural Tests on Beams and Girders for Building Construction, ASTM E529-04. American Society for Testing and Materials Standard Practice, Philadelphia, PA, USA.

Badie, S., and Tadros, M. 2008. Full-Depth Precast Concrete Bridge Deck Panel Systems. NCHRP Report 584, Transportation Research Board, Washington, D.C.

CHBDC. 2014. Canadian Highway Bridge Design Code, CAN/CSA-S6-14. Canadian Standards Association, Toronto, Ontario, Canada.

Culmo, M. P. 2011. Accelerated Bridge Construction - Experience in Design, Fabrication and Erection of Prefabricated Bridge Elements and Systems. Report FHWA-HIF-12-013, Federal Highway Administration, McLean, VA, USA. 
Garcia, H. M. 2007. Analysis of an Ultra-High Performance Concrete Two-Way Ribbed Bridge Deck Slab. Report FHWA-HRT-07-056, Federal Highway Administration, McLean, VA, USA.

Graybeal, B. 2010. Behavior of Field-Cast Ultra-High Performance Concrete Bridge Deck Connections under Cyclic and Static Structural Loading. Federal Highway Administration, Report No. FHWA-HRT-11-023, 106 pp.

Hieber, D., and Wacker, J. 2005. State-of-the-Art Report on Precast Concrete Systems for Rapid Construction of Bridges. Report No. WA-RD 594.1, Washignton State Department of Transportation, 112 pages.

ISIS. 2007. Reinforcing Concrete Structures with Fibre Reinforced Polymers, Design Manual No. 3. The Canadian Network of Centres of Excellence on Intelligent Sensing for Innovative Structures, ISIS, University of Manitoba, Winnipeg, Manitoba, Canada.

Issa, M., Salas, J., Shabila, H., and Alrousan, R. 2006. Composite Behavior of Precast Concrete Full-Depth Panels and Prestressed Girders. PCI Journal, 132-145.

Lafarge Canada Inc., 2009. Ductal Joint Fill JS1000. [Online], Available at: www.ductal.com/JS1000_2009.pdf, [Accessed 15 September 2011].

Li, L., Ma, Z., Griffey, M., Oesterle, R. 2011. Improved Longitudinal Joint Details in Decked Bulb Tees for Accelerated Bridge Construction: Concept Development, Journal of Bridge Engineering, 15(3): 327-336.

NCHRP. 2011. Summary of Cast-In-Place Concrete Connections for Precast Deck Systems. Research Results Digest 355, Transportation Research Board, pp. 1-33.

PCI. 2011. PCI State-of-the-Art Report on Full-Depth Precast Concrete Bridge Deck Panels (SOA01-1911), PCI Committee on Bridges and the PCI Bridge Producers Committee, Precast/Prestressed Concrete Institute. Chicago, IL.

Russell, H. G. 2013. NCHRP Synthesis 441: High Performance Concrete Specifications and Practices for Bridges. Transportation Research Board, Washington, D.C. 
Schoeck. 2013. Technical Information Schoeck ComBAR. [Online] Available at: http://www.schoeck.ca/upload/documents/flashbook/en_ca/combar/technical_information_sch oeck_combar_13-05-14_1513/index.html (Accessed on April 23, 2015).

Zia, P., Lemin, M. L., and Ahmad, S.H. 1991. High Performance Concrete, A State-of-the-Art Report No. SHRP-C/FR-91-103. Strategic Highway Research Program, National Research Council, Washington, D.C.

Zhu, P., Ma, Z., Cao, Q., and French, C. 2012. Fatigue Evaluation of Transverse U-Bar Joint Details for Accelerated Bridge Construction. Journal of Bridge Engineering, 17:191-200. 


\section{Tables}

Table 4.1. Name coding

\begin{tabular}{|c|c|c|c|}
\hline \multicolumn{3}{|c|}{ Name coding } & \multirow[t]{2}{*}{ Description } \\
\hline Slab Type & Connection Type & Loading case & \\
\hline \multirow[t]{2}{*}{ C: Control Slab } & ST: Steel bars - no joint & \multirow{5}{*}{$\begin{array}{c}\text { C: Centric loading } \\
\text { E: Eccentric loading }\end{array}$} & \multirow{2}{*}{ Cast-in-place } \\
\hline & GFRP: GFRP Bars - no joint & & \\
\hline \multirow[t]{3}{*}{ J: Joint Slab } & A: Angle shape joint with GFRP bars & & \multirow{3}{*}{ Precast deck } \\
\hline & C: C-Shape joint with GFRP bars & & \\
\hline & Z: Zigzag shape joint with GFRP bars & & \\
\hline
\end{tabular}

Table 4.2. Properties of GFRP bars considered in slab testing (Schoeck Canada, 2013)

\begin{tabular}{cccccc}
\hline Bar & $\begin{array}{c}\text { Designated } \\
\text { diameter }\end{array}$ & $\begin{array}{c}\text { Core diameter } \\
(\mathrm{mm})\end{array}$ & $\begin{array}{c}\text { Exterior } \\
\text { diameter } \\
(\mathrm{mm})\end{array}$ & $\begin{array}{c}\text { Cross- } \\
\text { sectional-area } \\
\left(\mathrm{mm}^{2}\right)\end{array}$ & $\begin{array}{c}\text { Specific } \\
\text { weight }(\mathrm{kg} / \mathrm{m})\end{array}$ \\
\hline$\varnothing 12$ & $12 \mathrm{M}$ & 12 & 13.5 & 113 & 0.30 \\
\hline$\varnothing 20$ & $20 \mathrm{M}$ & 20 & 22 & 314 & 0.80 \\
\hline
\end{tabular}

Table 4.3. Typical UHPFRC composition (Graybeal, 2006)

\begin{tabular}{lll}
\hline Material & Size & Percentage by weight \\
\hline Portland Cement & $15 \mu \mathrm{m}$ & $27-38$ \\
\hline Fine sand & $150-600 \mu \mathrm{m}$ & $39-41$ \\
\hline Silica fume & $0.2 \mu \mathrm{m}$ & $8-9$ \\
\hline Ground Quartz & $10 \mu \mathrm{m}$ & $0-8$ \\
\hline Steel fibers & $0.2 \mathrm{~mm} \mathrm{x} \mathrm{12.7} \mathrm{mm}$ & $5-8$ \\
\hline Water & - & $5-8$ \\
\hline High Range Water Reducer (HRWR) & - & $0.5-1.0$ \\
\hline
\end{tabular}

Table 4.4. Characteristic values of UHPFRC type JS1000 for the design of joint (Lafarge Canada Inc., 2009)

\begin{tabular}{lccc}
\hline Mechanical properties & \multicolumn{2}{c}{ Test data } & \multirow{2}{*}{ Design values } \\
\cline { 2 - 3 } & Mean & Standard deviation & \\
\hline Compression & $140 \mathrm{MPa}$ & $10 \mathrm{MPa}$ & $100 \mathrm{MPa}$ \\
\hline Flexural & $30 \mathrm{MPa}$ & $5 \mathrm{MPa}$ & $27 \mathrm{MPa}$ \\
\hline Direct tension & $8 \mathrm{MPa}$ & $1 \mathrm{MPa}$ & $5 \mathrm{MPa}$ \\
\hline Young's modulus & $50 \mathrm{GPa}$ & $2 \mathrm{GPa}$ & $45 \mathrm{GPa}$ \\
\hline
\end{tabular}


Table 4.5. Summary of slab configurations and test results

\begin{tabular}{|c|c|c|c|c|c|c|c|}
\hline \multirow[t]{2}{*}{$\begin{array}{c}\text { Slab } \\
\text { number }\end{array}$} & \multirow{2}{*}{ Slab } & \multirow{2}{*}{ Reinforcement } & \multirow{2}{*}{ Slab type } & \multirow{2}{*}{$\begin{array}{c}\text { Wheel } \\
\text { load } \\
\text { location }\end{array}$} & \multicolumn{2}{|c|}{$\mathrm{f}_{\mathrm{c}}^{\prime} \mathrm{MPa}$} & \multirow[t]{2}{*}{$\begin{array}{c}\text { Failure } \\
\text { mode }\end{array}$} \\
\hline & & & & & Concrete & UHPFRC & \\
\hline S1 & C.ST.C & Steel bars & Cast-in-place & Concentric & 76.94 & - & Flexural \\
\hline S2 & C.GFRP.C & GFRP bars & Cast-in-place & Concentric & 76.94 & - & Flexural \\
\hline S3 & J.C.C & GFRP bars & $\begin{array}{l}\text { Precast with } 200 \mathrm{~mm} \\
\text { closure strip filled } \\
\text { with UHPFRC }\end{array}$ & Concentric & 76.94 & 141.11 & Flexural \\
\hline S4 & J.C.E & GFRP bars & $\begin{array}{l}\text { Precast with } 200 \mathrm{~mm} \\
\text { closure strip filled } \\
\text { with UHPFRC }\end{array}$ & Eccentric & 76.94 & 141.11 & $\begin{array}{c}\text { Flexural- } \\
\text { shear }\end{array}$ \\
\hline S5 & J.Z.E & GFRP bars & $\begin{array}{l}\text { Precast with zigzag- } \\
\text { shaped closure strip } \\
\text { filled with UHPFRC }\end{array}$ & Eccentric & 76.94 & 166.58 & $\begin{array}{c}\text { Flexural- } \\
\text { shear }\end{array}$ \\
\hline S6 & J.A.C & GFRP bars & $\begin{array}{l}\text { Precast with } 200 \mathrm{~mm} \\
\text { closure strip and } \\
\text { bottom projecting slab } \\
\text { and filled with } \\
\text { UHPFRC }\end{array}$ & Concentric & 76.94 & 166.58 & $\begin{array}{c}\text { Flexural- } \\
\text { Shear }\end{array}$ \\
\hline S7 & J.A.E & GFRP bars & $\begin{array}{l}\text { Precast with } 200 \mathrm{~mm} \\
\text { closure strip and } \\
\text { bottom projecting slab } \\
\text { and filled with } \\
\text { UHPFRC }\end{array}$ & Eccentric & 76.94 & 141.11 & Flexural \\
\hline
\end{tabular}


Table 4.6. Test results

\begin{tabular}{|c|c|c|c|c|c|c|}
\hline Serial & $\begin{array}{l}\text { Specimen } \\
\text { name }\end{array}$ & $\begin{array}{c}\text { Cracking } \\
\text { load }\end{array}$ & $\begin{array}{c}\text { Ultimate } \\
\text { load }\end{array}$ & $\begin{array}{c}\begin{array}{c}\text { Ultimate } \\
\text { deflection }\end{array} \\
\mathrm{mm}\end{array}$ & $\begin{array}{c}\text { Max concrete } \\
\text { strain } \times 10^{-6}\end{array}$ & $\begin{array}{c}\text { Max. bar } \\
\text { strain } \times 10^{-6}\end{array}$ \\
\hline 1 & C.ST.C & 27.15 & 169.47 & 29.81 & 3006 & 11819 \\
\hline 2 & C.GFRP.C & 26.93 & 161.34 & 42.24 & 2531 & 7744 \\
\hline 3 & J.C.C & 31.07 & 148.39 & 37.84 & 1574 & 12156 \\
\hline 4 & J.C.E & 39.06 & 152.53 & 22.12 & 1283 & 7356 \\
\hline 5 & J.Z.E & 30.48 & 162.52 & 26.89 & 1076 & 6572 \\
\hline 6 & J.A.C & 31.66 & 132.64 & 34.31 & 2566 & 6756 \\
\hline 7 & J.A.E & 29.29 & 111.33 & 24.26 & 1745 & 6464 \\
\hline
\end{tabular}


Table 4.7. Shear and bending moment values

\begin{tabular}{|c|c|c|c|c|c|c|c|c|c|c|c|c|c|c|c|}
\hline \multirow[t]{2}{*}{ Specimen } & \multirow{2}{*}{$\frac{\text { Load }}{\mathrm{kN}}$} & \multirow{2}{*}{$\frac{\mathrm{w}}{\mathrm{kN} / \mathrm{b}}$} & \multirow{2}{*}{$\frac{\mathrm{a}}{\mathrm{m}}$} & \multirow{2}{*}{$\frac{\mathrm{c}}{\mathrm{m}}$} & \multirow{2}{*}{$\frac{\mathrm{R}_{1}}{\mathrm{kN}}$} & \multirow{2}{*}{$\frac{\mathrm{R}_{2}}{\mathrm{kN}}$} & \multirow{2}{*}{$\frac{0.75 \mathrm{R}}{\mathrm{kN} / \mathrm{m}}$} & \multicolumn{3}{|c|}{ Experimental resisting moment } & \multicolumn{3}{|c|}{$\begin{array}{c}\text { Theoretical resisting } \\
\text { moment }\end{array}$} & \multicolumn{2}{|c|}{$\begin{array}{l}\text { Theoretical } \\
\text { resisting shear }\end{array}$} \\
\hline & & & & & & & & $\begin{array}{c}\mathrm{M}_{\mathrm{r} . \text { precast }} \\
\mathrm{kN} . \mathrm{m} / 0.6 \mathrm{~m}\end{array}$ & $\begin{array}{l}\mathrm{M}_{\mathrm{r} . \text { precast }} \\
\mathrm{kN} . \mathrm{m} / \mathrm{m}\end{array}$ & $\begin{array}{c}0.75 \mathrm{M}_{\mathrm{r} \text {.precast }} \\
\mathrm{kN} \cdot \mathrm{m} / \mathrm{m}\end{array}$ & $\begin{array}{l}\mathrm{M}_{\mathrm{r} \text {.precast }} \\
\mathrm{kN} . \mathrm{m} / \mathrm{m}\end{array}$ & $\frac{\mathrm{M}_{\mathrm{r.precast}}}{\mathrm{Mr}}$ & $\begin{array}{l}\mathrm{M}_{\mathrm{r} . \mathrm{UHPFRC}} \\
\mathrm{kN} \cdot \mathrm{m} / \mathrm{m}\end{array}$ & $\begin{array}{l}\mathrm{Vr}, \mathrm{ISIS} \\
\mathrm{kN} / \mathrm{m}\end{array}$ & $\frac{\text { Vexp. }}{\mathrm{Vr}_{\text {, IsIS }}}$ \\
\hline S1 (C.ST.C) & 169.47 & 677.88 & 0.875 & 0.875 & 84.735 & 84.735 & 65.80 & 79.44 & 132.40 & 99.30 & -- & -- & -- & -- & -- \\
\hline S2 (C.GFRP.C) & 161.34 & 645.36 & 0.875 & 0.875 & 80.670 & 80.670 & 60.50 & 75.63 & 126.05 & 94.54 & 104.65 & 0.90 & -- & 100.48 & 0.60 \\
\hline S3 (J.C.C) & 148.39 & 593.56 & 0.875 & 0.875 & 74.195 & 74.195 & 55.65 & 69.56 & 115.93 & 86.95 & 104.65 & 0.83 & 228.82 & 100.48 & 0.55 \\
\hline S4 (J.C.E) & 152.53 & 610.12 & 0.650 & 1.100 & 93.420 & 59.110 & 44.44 & 67.88 & 113.13 & 84.85 & 104.65 & 0.81 & 228.82 & 100.48 & 0.44 \\
\hline S5 (J.Z.E) & 162.52 & 650.08 & 0.600 & 1.150 & 103.61 & 58.914 & 44.19 & 70.42 & 117.37 & 88.03 & 104.65 & 0.84 & 228.82 & 100.48 & 0.44 \\
\hline S6 (J.A.C) & 132.64 & 530.56 & 0.875 & 0.875 & 66.32 & 66.320 & 49.74 & 62.18 & 103.63 & 77.72 & 104.65 & 0.74 & 228.82 & 100.48 & 0.49 \\
\hline S7 (J.A.E) & 111.33 & 445.32 & 0.650 & 1.100 & 68.190 & 43.140 & 32.36 & 49.54 & 82.57 & 61.93 & 104.65 & 0.59 & 228.82 & 100.48 & 0.32 \\
\hline
\end{tabular}

Notes: See Fig. 4.17 for definitions of symbols

$\mathrm{w}=$ uniform distributed load, $\mathrm{L}=$ span length equal $2 \mathrm{~m} ; \mathrm{a}, \mathrm{b}, \mathrm{c}=$ slab dimension where $\mathrm{b}=0.25 \mathrm{~m} ; \mathrm{R}_{1}$ and $\mathrm{R}_{2}=$ support reactions; $M_{r}=$ resisting moment.

$\mathrm{V}_{\text {exp }}$ in $\mathrm{kN} / \mathrm{m}=\left(\right.$ greater of $\mathrm{R}_{1}$ and $\left.\mathrm{R}_{2}\right) / 0.6 \mathrm{~m}$

The 0.75 is durability factor to be multiplied by the experimental moment and shear capacity 
Table 4.8. Experimental parameters for the moment-deflection

\begin{tabular}{lccccc}
\hline Specimen & \multicolumn{5}{c}{ Polynomial Parameters* } \\
\hline & $a_{4}$ & $a_{3}$ & $a_{2}$ & $a_{1}$ & $R^{2}$ \\
\hline S1 (C.ST.C) & -0.00008 & 0.0075 & -0.3253 & 7.7159 & 0.9948 \\
\hline S2 (C.GFRP.C) & -0.0001 & 0.0099 & -0.3112 & 5.3276 & 0.9944 \\
\hline S3 (J.C.C.) & -0.0001 & 0.0109 & -0.3279 & 5.5057 & 0.9873 \\
\hline S4 (J.C.E.) & -0.0012 & 0.0583 & -0.962 & 8.7267 & 0.9970 \\
\hline S5 (J.Z.E.) & -0.0005 & 0.0284 & -0.5866 & 7.0541 & 0.9928 \\
\hline S6 (J.A.C.) & -0.0002 & 0.0143 & -0.3557 & 5.3364 & 0.9879 \\
\hline S7 (J.A.E.) & & & -0.0697 & 3.6536 & 0.9963 \\
\hline
\end{tabular}

* Moment-Deflection Equation: $M=a_{3} \delta^{4}+a_{3} \delta^{3}+a_{2} \delta^{2}+a_{1} \delta^{1}+a_{o} ; \mathbf{R}^{2}$ is the coefficient of determination

Table 4.9. Maximum micro-strain readings for the tested slabs

\begin{tabular}{llccccc}
\hline Slab Name & Location & Depth & \multicolumn{3}{c}{ Micro-Strain } \\
\hline & & $\mathrm{mm}$ & 1 & 2 & 3 & Average \\
\hline S1 (C.ST.C) & Concrete & 0 & 1678 & 1989 & 3006 & 2224 \\
\hline & Top Bar & 65 & 9300 & & & 9300 \\
\hline & Bottom Bar & 135 & 7935 & 11819 & & 9877 \\
\hline S2 (C.GFRP.C) & Concrete & 0 & 2531 & 2462 & & 2496.5 \\
\hline & Top Bar & 65 & 1965 & 4039 & & 3002 \\
\hline & Bottom Bar & 135 & 3260 & 6684 & 7744 & 5896 \\
\hline S3 (J.C.C) & Concrete & 0 & 1574 & 909 & 1314 & 1265.7 \\
\hline & Top Bar & 65 & 1579 & 1085 & 1669 & 1444 \\
\hline & Bottom Bar & 135 & 12156 & 10731 & 9886 & 10924.3 \\
\hline S4 (J.C.E) & Concrete & 0 & 998 & 1100 & 1283 & 1127 \\
\hline & Top Bar & 65 & 2712 & 2358 & 1783 & 2284 \\
\hline & Bottom Bar & 135 & 7356 & 6730 & & 7043 \\
\hline S5 (J.Z.E) & Concrete & 0 & 1076 & 857 & & 966.5 \\
\hline & Top Bar & 65 & 1750 & 1678 & 1741 & 1723 \\
\hline & Bottom Bar & 135 & 5326 & 6466 & 6572 & 6121.33 \\
\hline S6 (J.A.C) & Concrete & 0 & 2566 & 2242 & & 2404 \\
\hline & Top Bar & 65 & 1198 & 1107 & 1477 & 1260.67 \\
\hline & Bottom Bar & 135 & 6756 & 6703 & 6487 & 6648.7 \\
\hline S7 (J.A.E) & Concrete & 0 & 1745 & 1443 & & 1594 \\
\hline & Top Bar & 65 & 2384 & 3506 & 3522 & 3137.3 \\
\hline & Bottom Bar & 135 & 6464 & 5554 & 5901 & 5973 \\
\hline
\end{tabular}


Table 4.10. Pullout force from the bottom reinforcement

\begin{tabular}{|c|c|c|c|c|c|c|c|c|c|c|c|}
\hline & Name & $\begin{array}{c}\mathrm{A}, \\
\mathrm{mm} 2\end{array}$ & $\mathrm{E}, \mathrm{MPa}$ & $\epsilon \times 10^{-6}$ & $\mathrm{~T}, \mathrm{kN}$ & $\begin{array}{l}\mathrm{L}_{\mathrm{d}} \\
\mathrm{mm}\end{array}$ & $\begin{array}{c}\mathrm{d}, \\
\mathrm{mm}\end{array}$ & $\begin{array}{l}\mathrm{f}_{\mathrm{c}}{ }^{\prime}, \\
\mathrm{MPa}\end{array}$ & $1 / \mathrm{k}$ & $\mathrm{k}$ & $\begin{array}{c}\tau, \\
\mathrm{MPa}\end{array}$ \\
\hline \multirow{3}{*}{ S1 } & C.ST.C & 300 & 200,000 & 7935 & 476.10 & & & & & & \\
\hline & & & & 11819 & 709.14 & & 20 & 45 & -- & -- & -- \\
\hline & Average & & & 9877 & 592.62 & & & & & & \\
\hline \multirow{4}{*}{$\mathrm{S} 2$} & C.GFRP.C & 314 & 64,000 & 3260 & 65.51 & & & & & & \\
\hline & & & & 6684 & 134.32 & & 20 & 45 & & & \\
\hline & & & & 7744 & 155.62 & & & & -- & -- & -- \\
\hline & Average & & & 5896 & 118.49 & & & & & & \\
\hline \multirow{4}{*}{ S3 } & J.C.C & 314 & 64,000 & 12156 & 244.29 & & & & & & \\
\hline & & & & 10731 & 215.65 & 175 & 20 & 141.11 & 0.00947 & 105.61 & 19.98 \\
\hline & & & & 9886 & 198.67 & & & & & & \\
\hline & Average & & & 10924 & 219.54 & & & & & & \\
\hline \multirow{3}{*}{ S4 } & J.C.E & 314 & 64,000 & 7356 & 147.83 & & & & & & \\
\hline & & & & 6730 & 135.25 & 175 & 20 & 141.11 & 0.01469 & 68.08 & 12.88 \\
\hline & Average & & & 7043 & 141.54 & & & & & & \\
\hline \multirow{4}{*}{ S5 } & J.Z.E & 314 & 64,000 & 5326 & 107.03 & & & & & & \\
\hline & & & & 6466 & 129.94 & 175 & 20 & 166.58 & 0.01836 & 54.46 & 11.19 \\
\hline & & & & 6572 & 132.07 & & & & & & \\
\hline & Average & & & 6121 & 123.01 & & & & & & \\
\hline \multirow{4}{*}{ S6 } & J.A.C & 314 & 64,000 & 6756 & 135.77 & & & & & & \\
\hline & & & & 6703 & 134.70 & 175 & 20 & 166.58 & 0.01690 & 59.16 & 12.16 \\
\hline & & & & 6487 & 130.36 & & & & & & \\
\hline & Average & & & 6648.7 & 133.61 & & & & & & \\
\hline \multirow{4}{*}{ S7 } & J.A.E & 314 & 64,000 & 6464 & 129.90 & & & & & & \\
\hline & & & & 5554 & 111.61 & 175 & 20 & 141.11 & 0.017312 & 57.74 & 10.92 \\
\hline & & & & 5901 & 118.59 & & & & & & \\
\hline & Average & & & 5973 & 120.03 & & & & & & \\
\hline
\end{tabular}

Note: $T=E A . \epsilon, \quad 1 / k=T /\left(l_{d} \sqrt{f_{c}^{\prime}}\right), \tau=\sqrt{f_{c}^{\prime}} / \pi k d$

$\mathrm{A}=$ cross-sectional area of the bar; $\mathrm{E}=$ modulus of elasticity; $\epsilon=$ bar strain; $\mathrm{T}=$ pullout load; $\mathrm{L}_{\mathrm{d}}$ $=$ development length; $\mathrm{d}=$ bar diameter; $\mathrm{f}_{\mathrm{c}}{ }^{\prime}=$ compressive strength of concrete; $k=$ bond basic factor; $\tau=$ bond stress. 
Figures

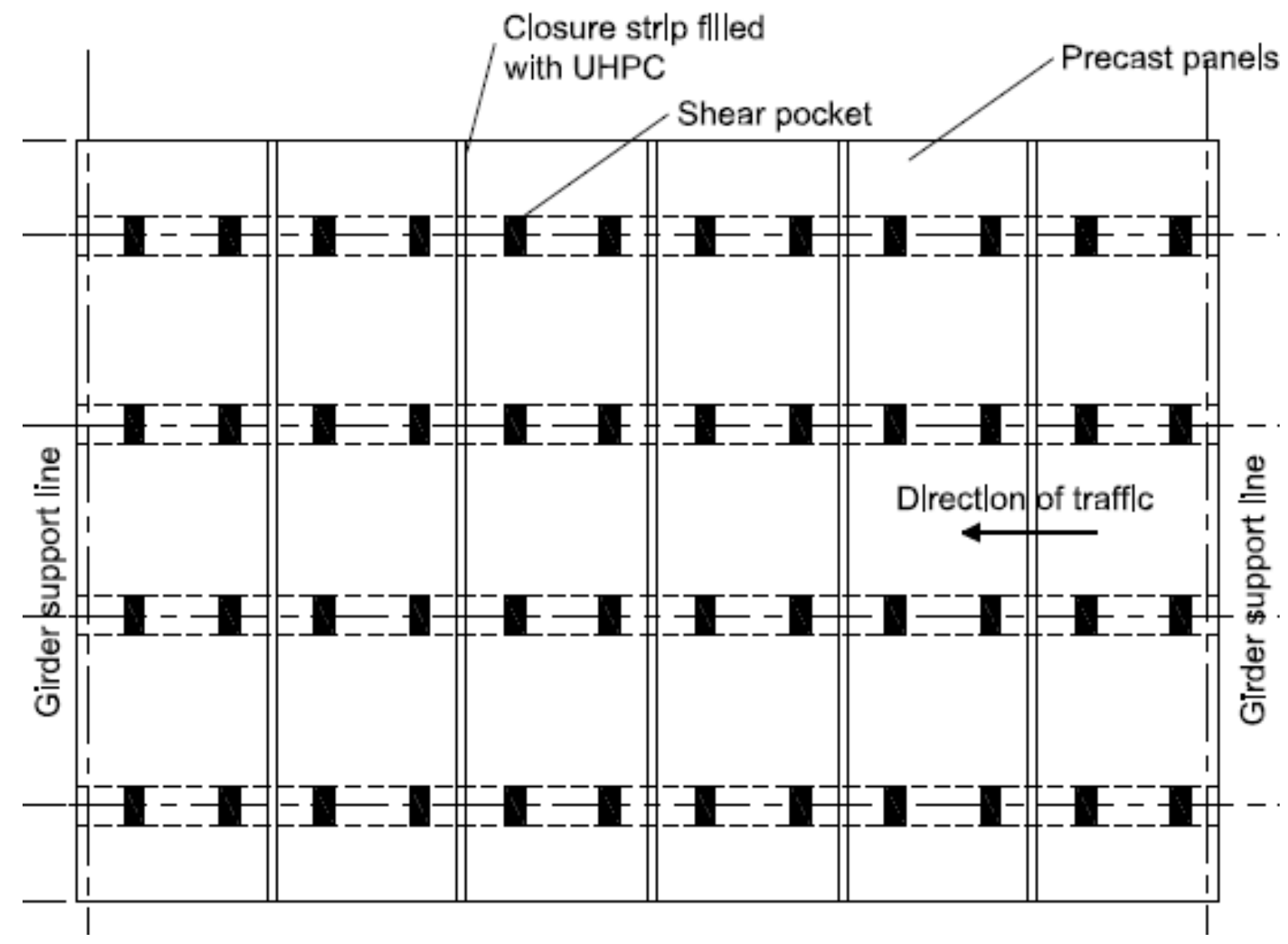

Figure 4.1. Plan view of a slab-on-girder bridge with full depth precast deck panels 


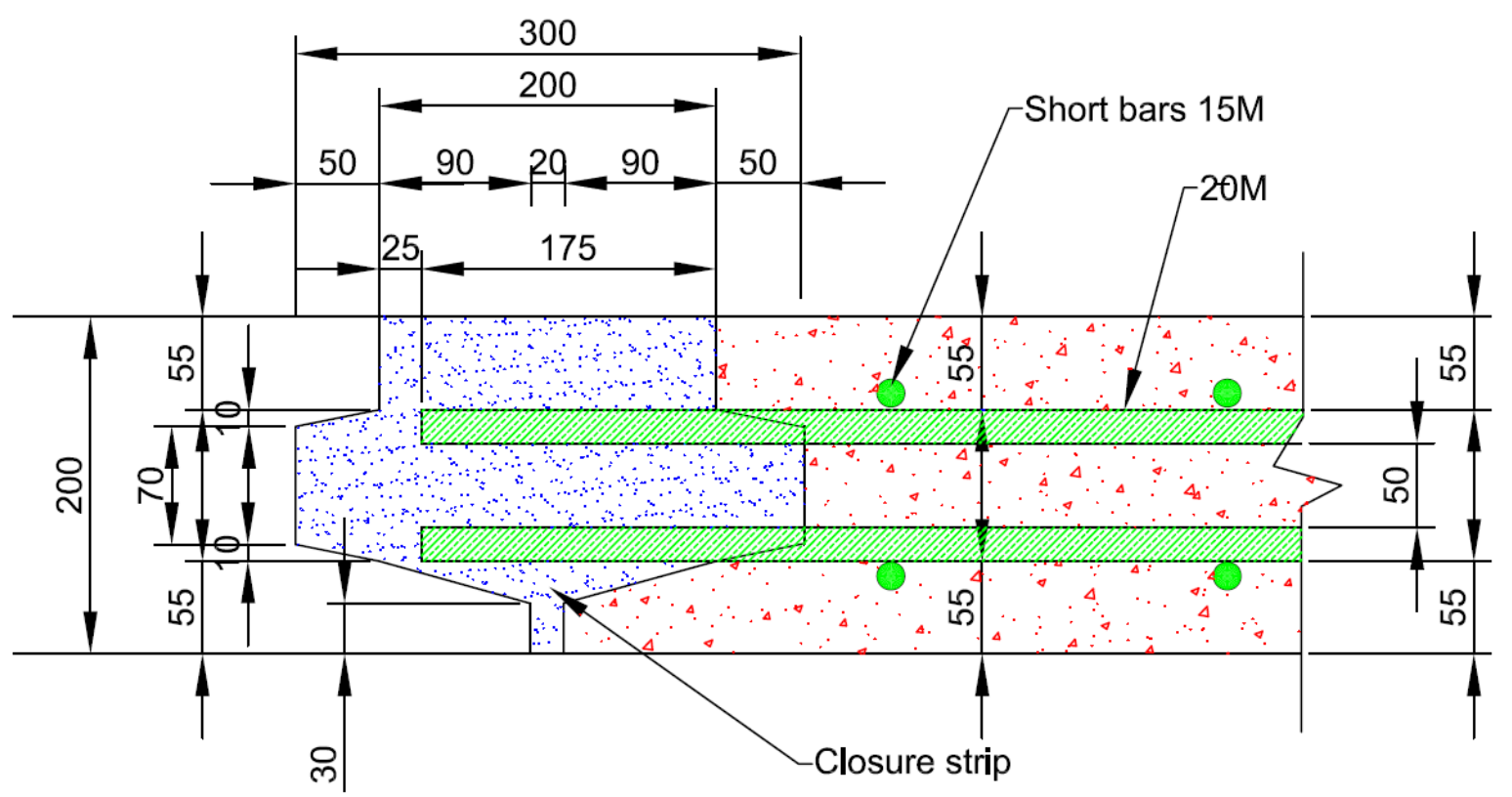

a) Angle-shape joint with pojecting bottom slab (Joint A)

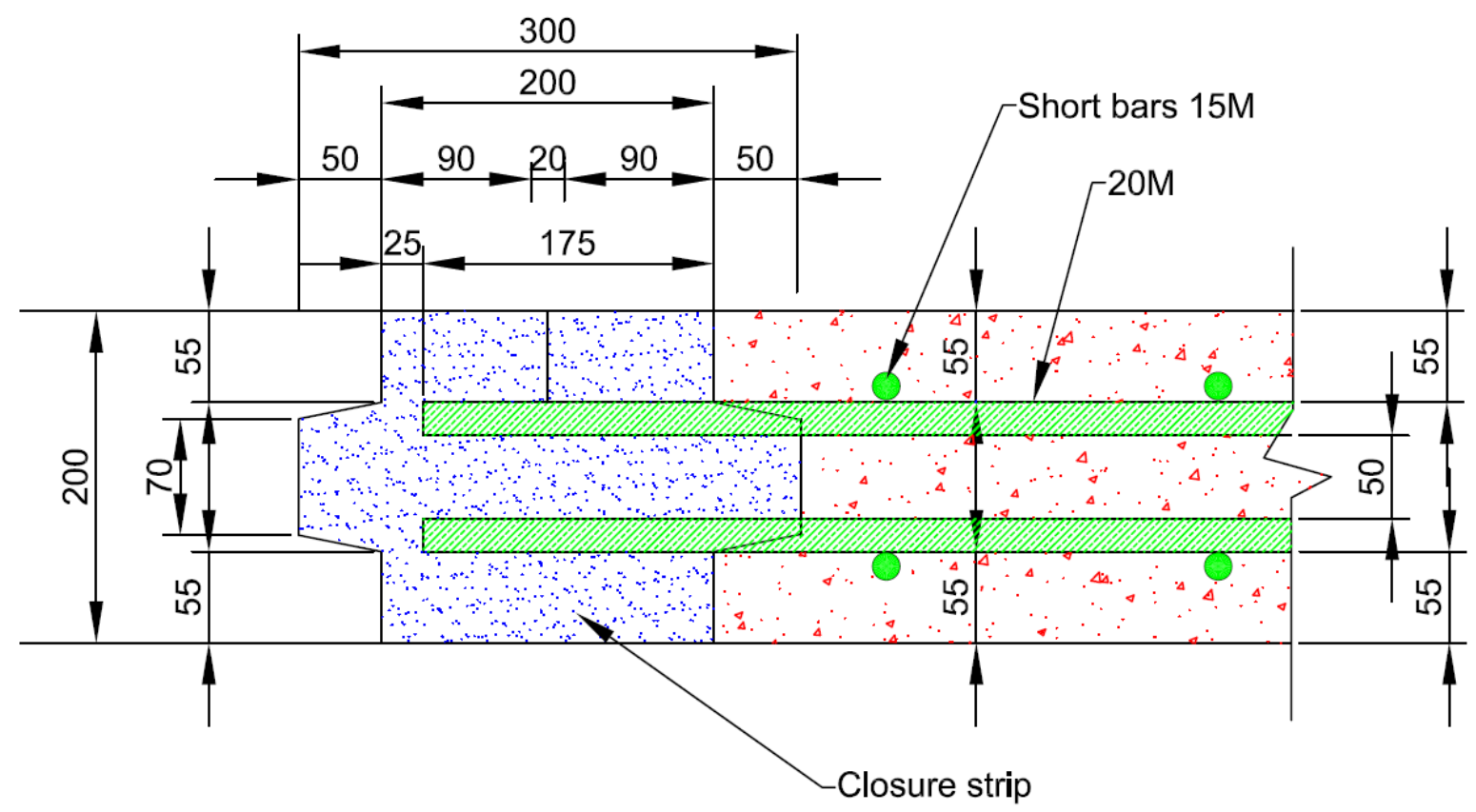

b) C-Shape joint with vertical shear key (Joint C) 

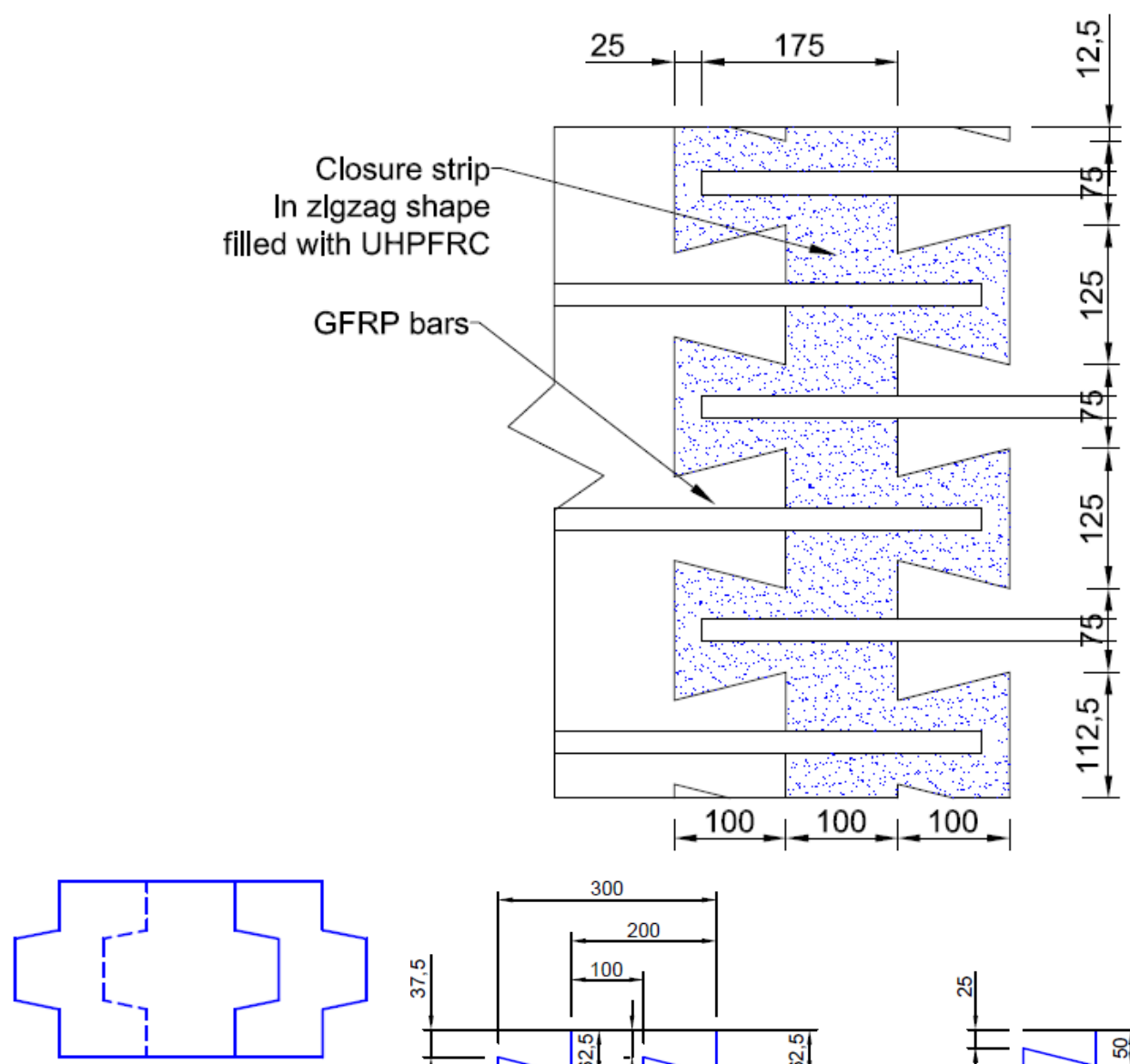

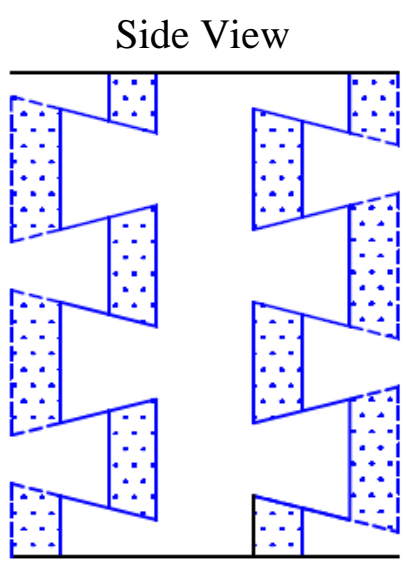

Top View

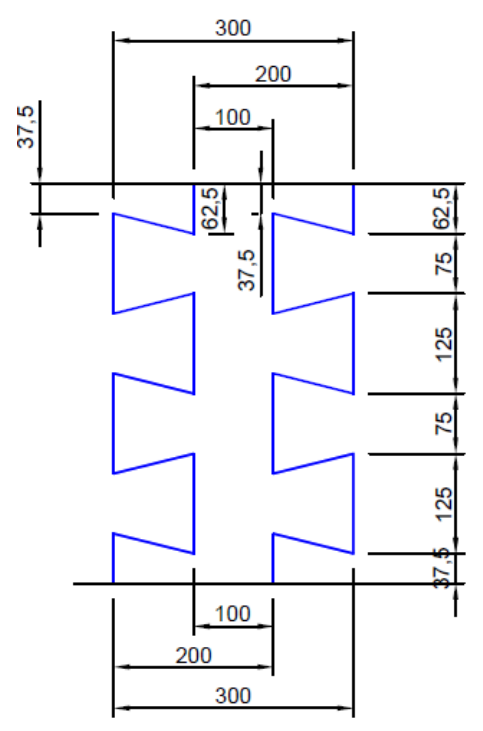

Top \& Bottom Layers

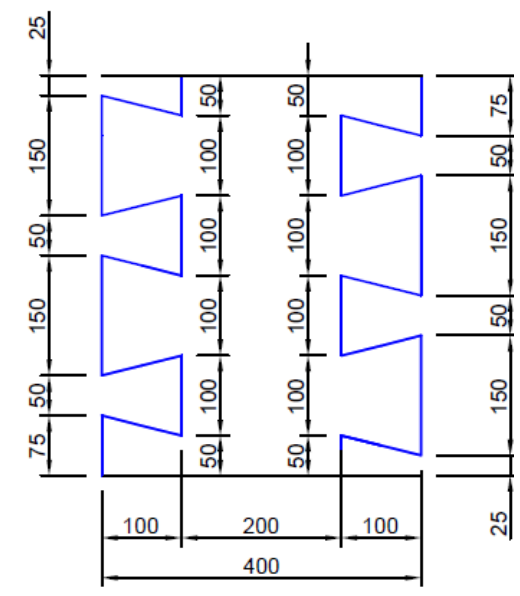

Middle Layers

c) Zigzag-shape joint with interlocking horizontal and vertical shear key (joint Z)

Figure 4.2. Schematic diagrams of the three proposed joints between precast deck panels 


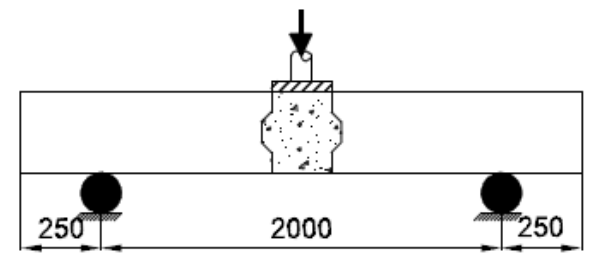

a) Simple beam with center load (C)

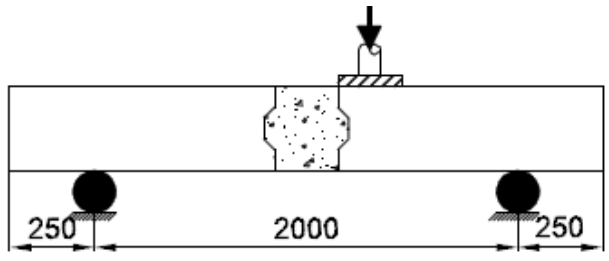

b) Simple beam with eccentric load (E)

Figure 4.3. Schematic diagram showing location of simulated wheel load at the joint between precast deck panels

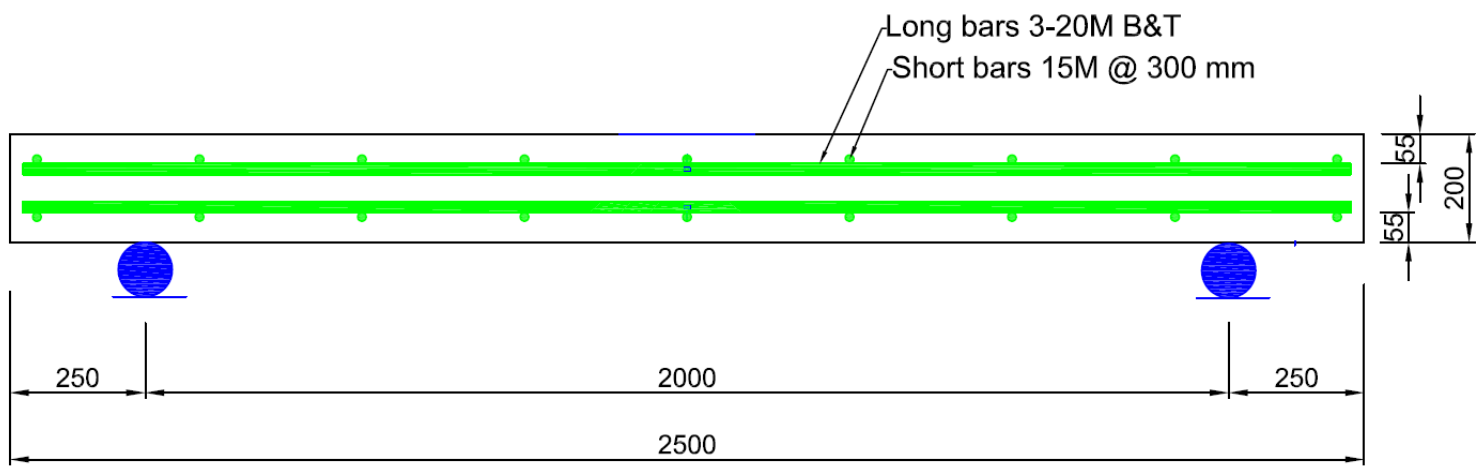

a) Longitudinal section for the cast-in-place deck slab S 2 (C.GFRP.C)

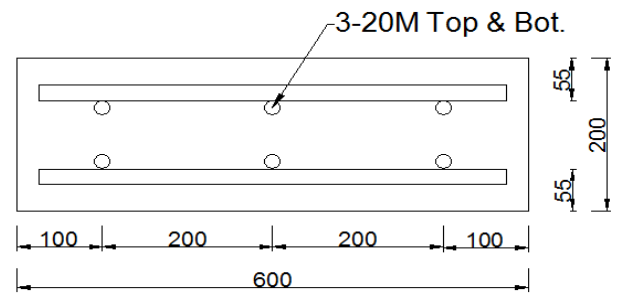

b) Cross-section for the cast-in-place deck slab S 2 (C.GFRP.C) 


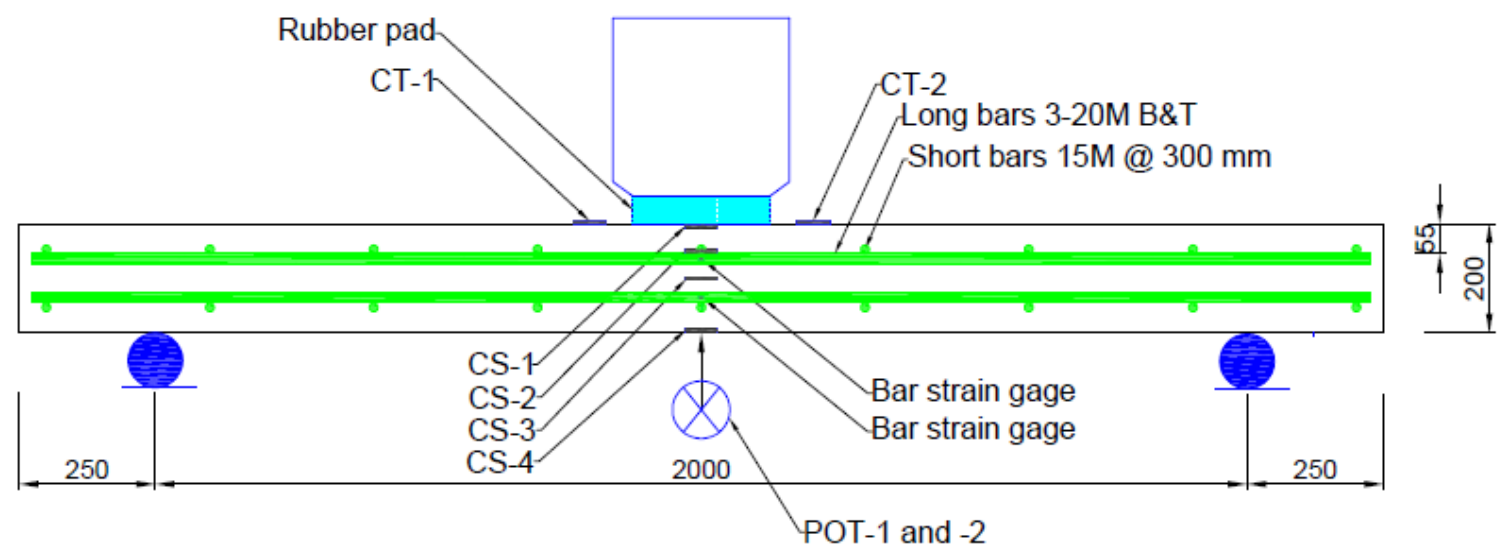

c) Locations of POTs and strain gages in cast-in-place slabs

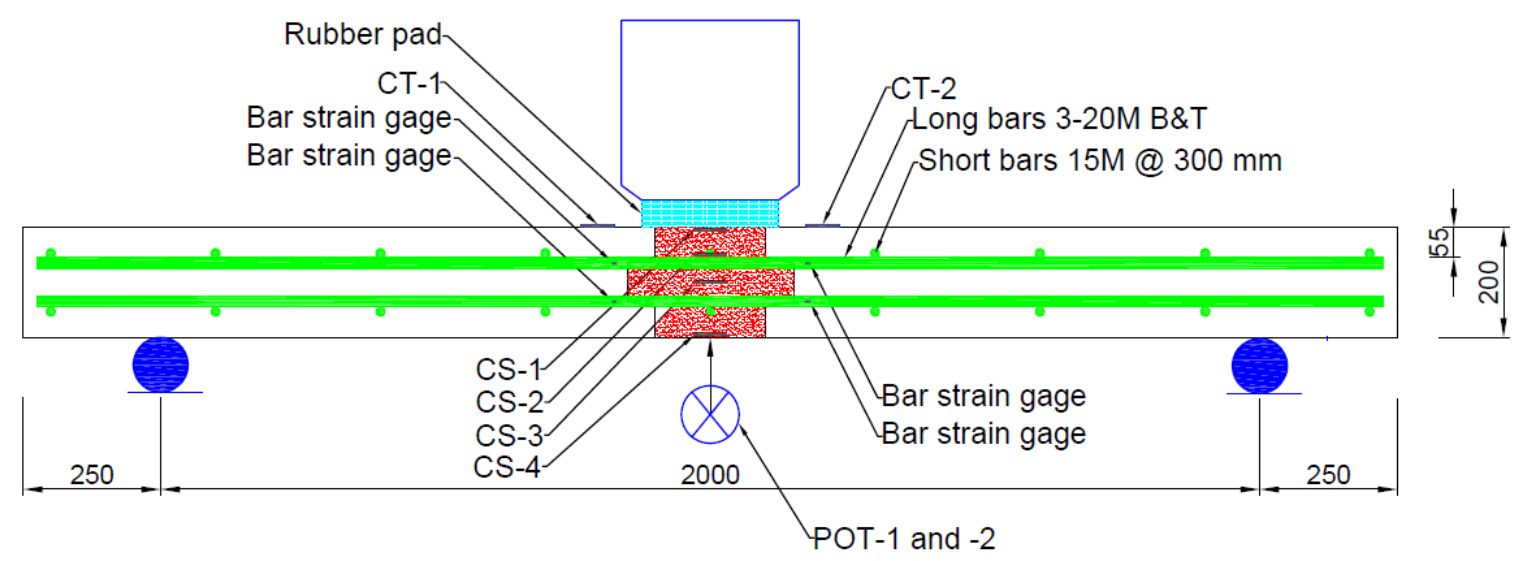

d) Locations of POTs and strain gages in precast FDDP under centric loading

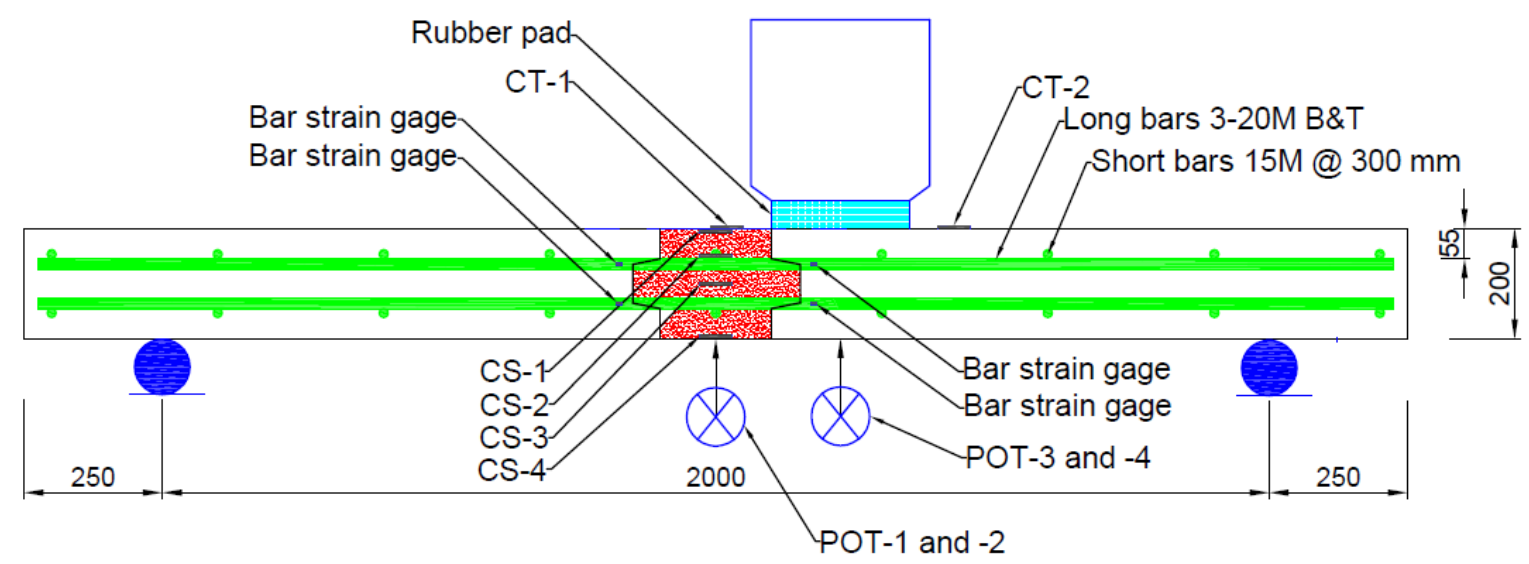

e) Locations of POTs and strain gages in precast FDDP under eccentric loading

Figure 4.4. Reinforcement details and locations of strain gauges and potentiometers (POTs) 


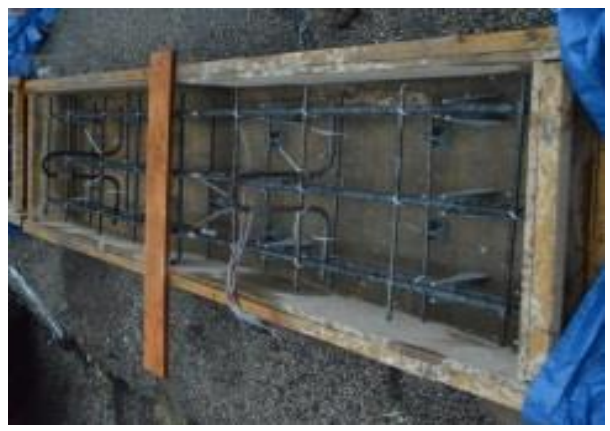

a) Slab C.ST.C

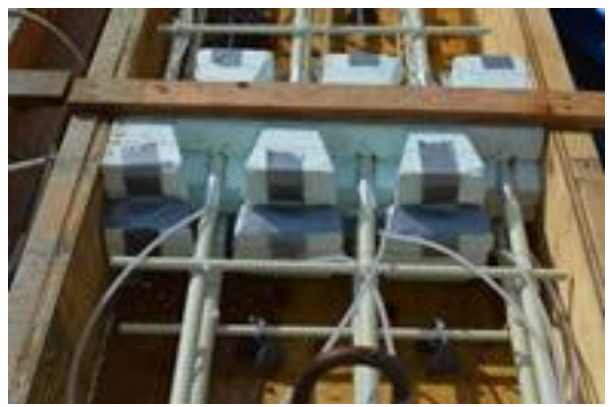

c) Slab J.Z.E

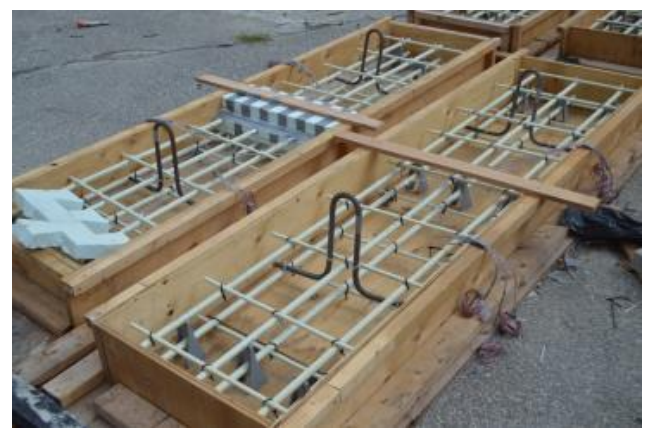

b) Slab J.C.C and C.GFRP.C

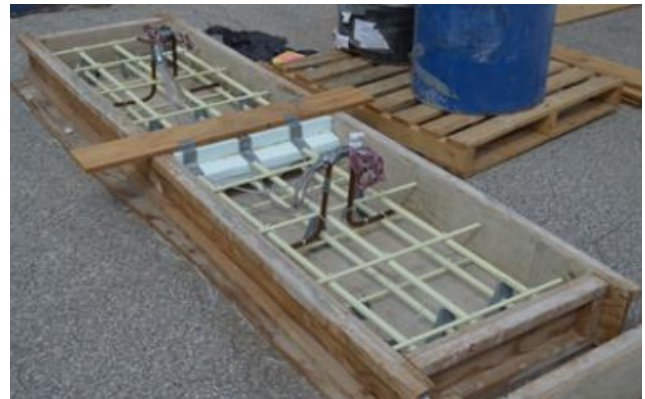

d) Slab J.A.C and J.A.E

Figure 4.5. Views of reinforcement layout and formwork for slab specimens

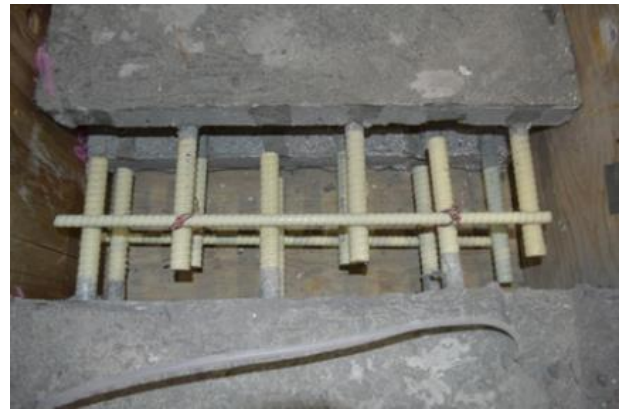

a) Closure strip for J.C.C and J.C.E

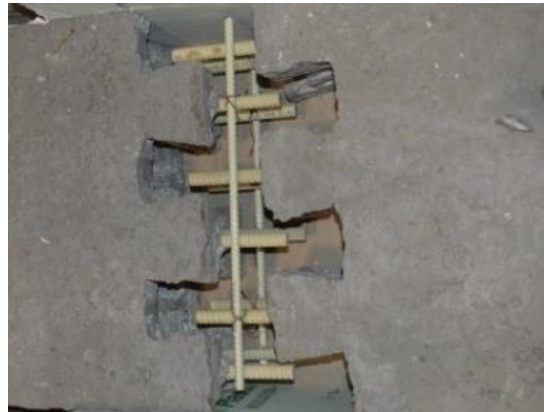

b) Closure strip for slab J.Z.E

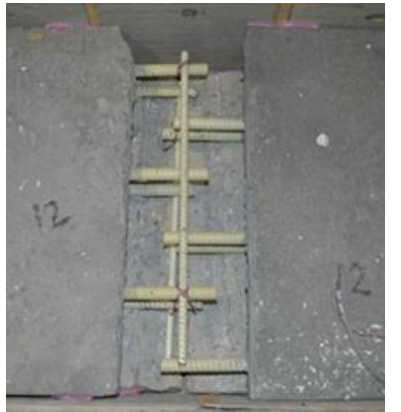

c) Closure strip for J.A.C and J.A.E

Figure 4. 6. Plan views of closure strip in jointed slabs 


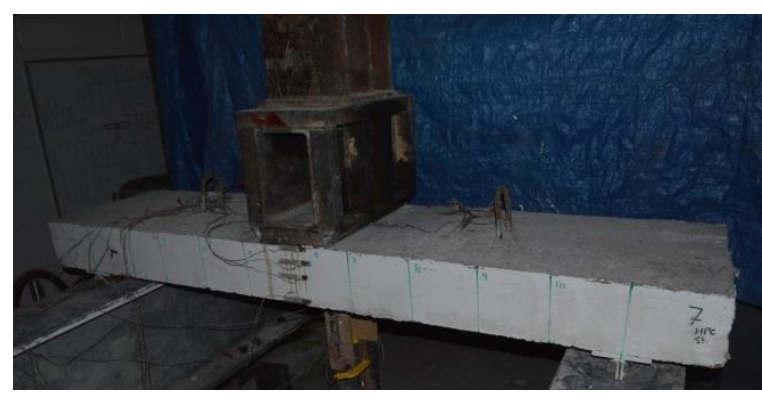

a) Test Setup

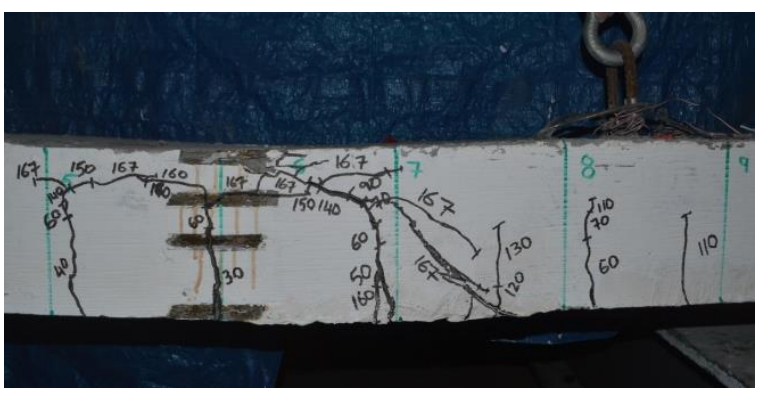

b) Crack pattern at the mid-span region

Figure 4.7. Test setup and crack pattern at failure for cast-in-place specimen S1 (C.ST.C) reinforced with steel bars

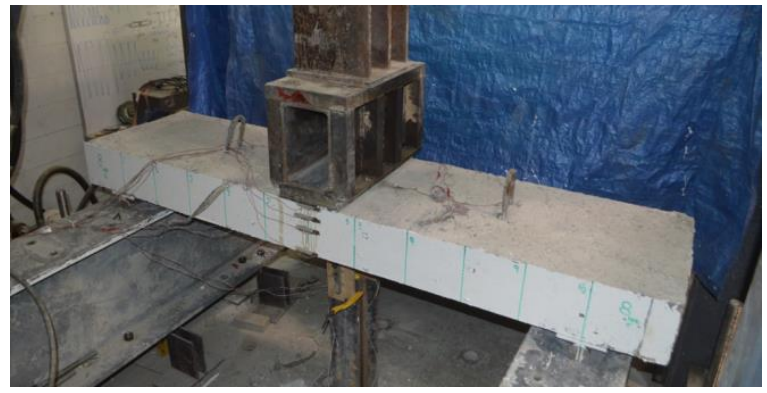

a) Test Setup

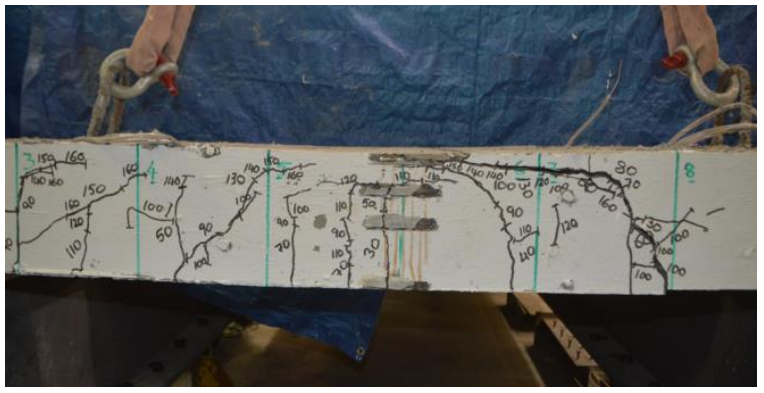

b) Crack pattern at the mid-span region

Figure 4.8. Test setup and crack pattern at failure of cast-in-place specimen S2 (C.GFRP.C) reinforced with GFRP bars 


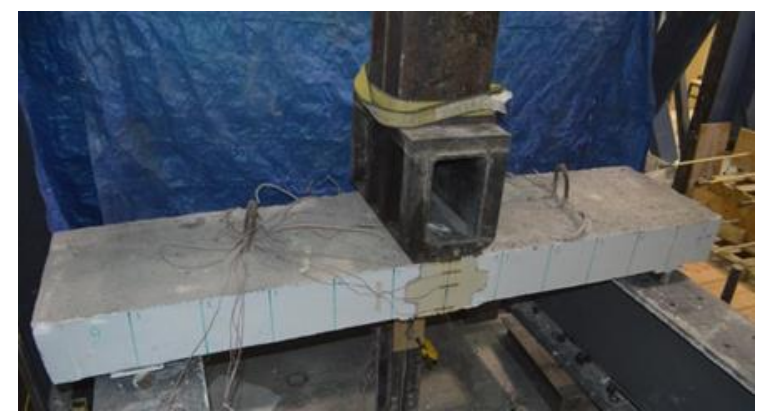

a) Test Setup

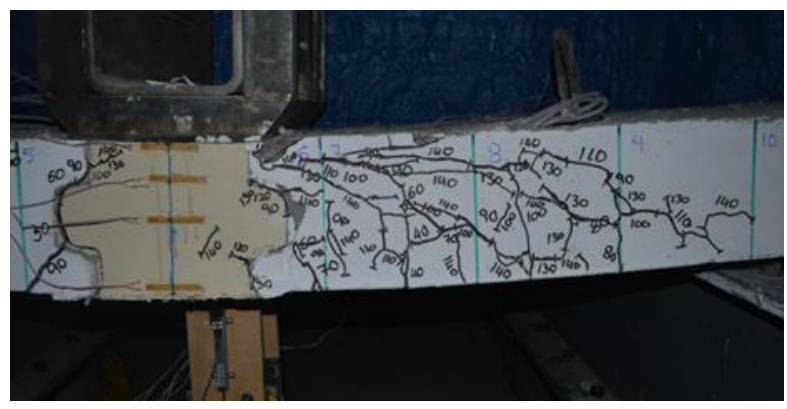

b) Crack pattern at joint region

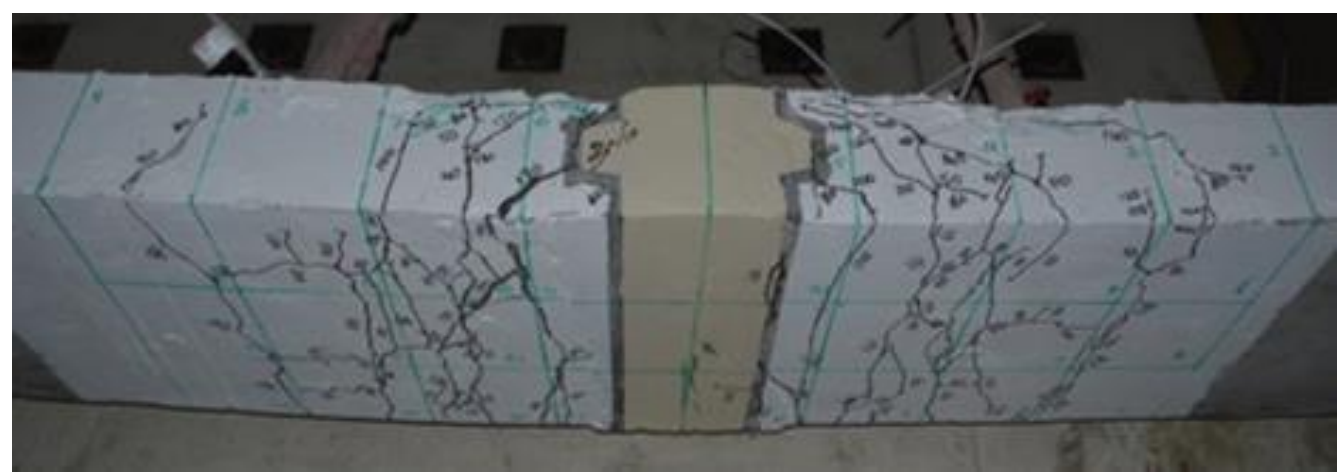

c) Crack pattern at the bottom and the side the FDDP

Figure 4.9. Test setup and crack pattern at failure of jointed slab S3 (J.C.C) and centric loading over the joint 


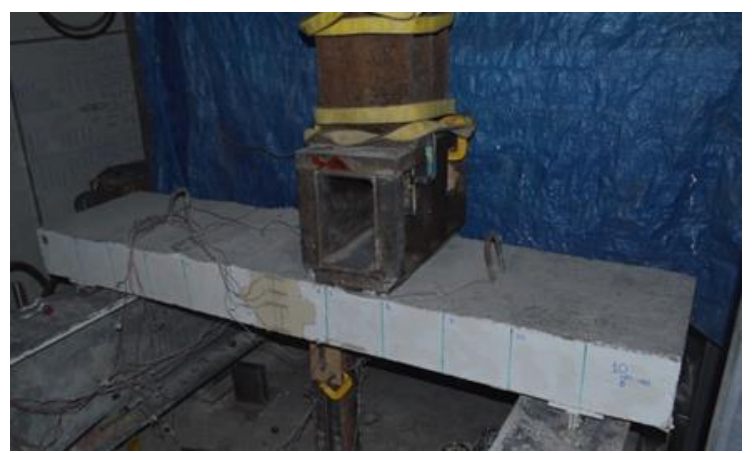

a) Test setup

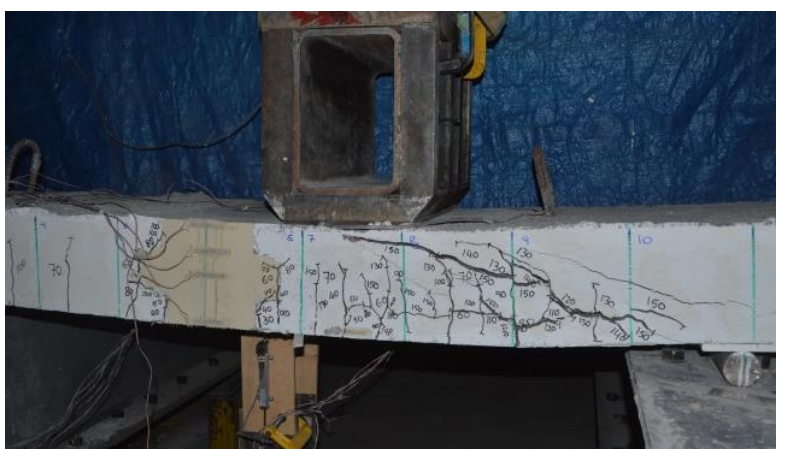

b) Crack pattern at the joint region

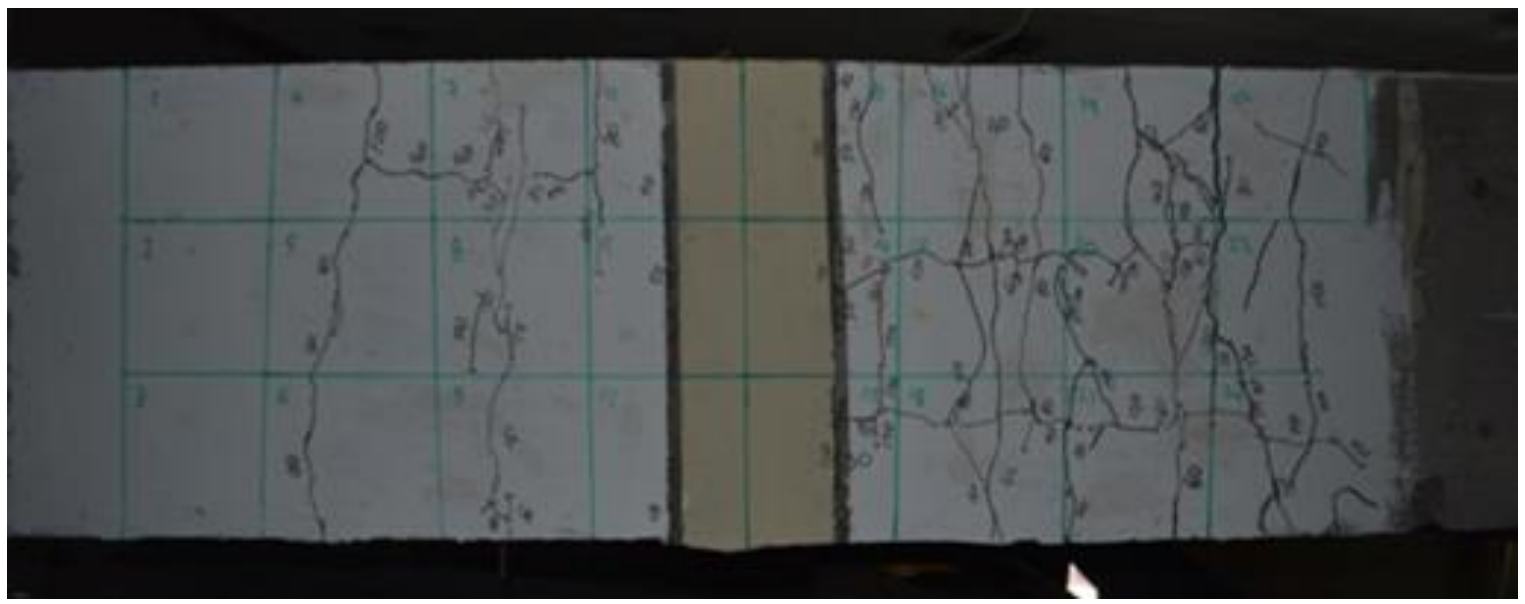

c) Crack pattern at the bottom of the FDDP

Figure 4.10. Test setup and crack pattern at failure of jointed slab S4 (J.C.E) with eccentric loading at the joint 


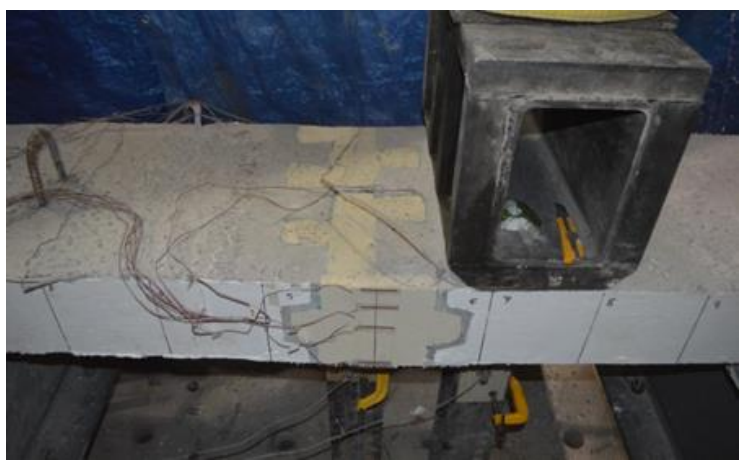

a) Test setup

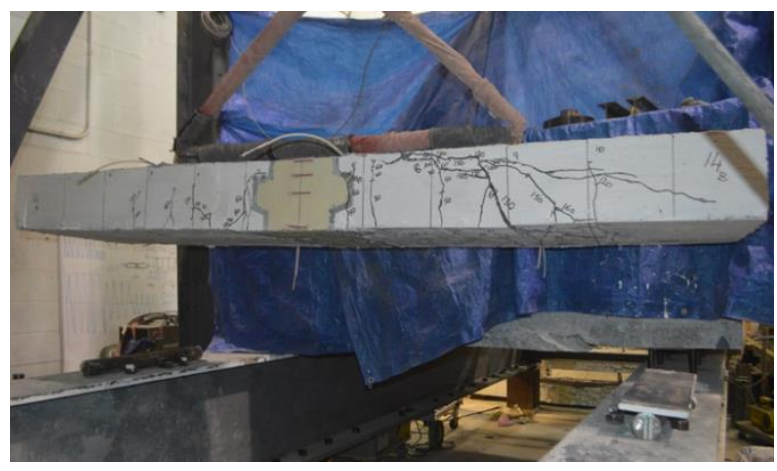

b) Crack pattern at the joint region

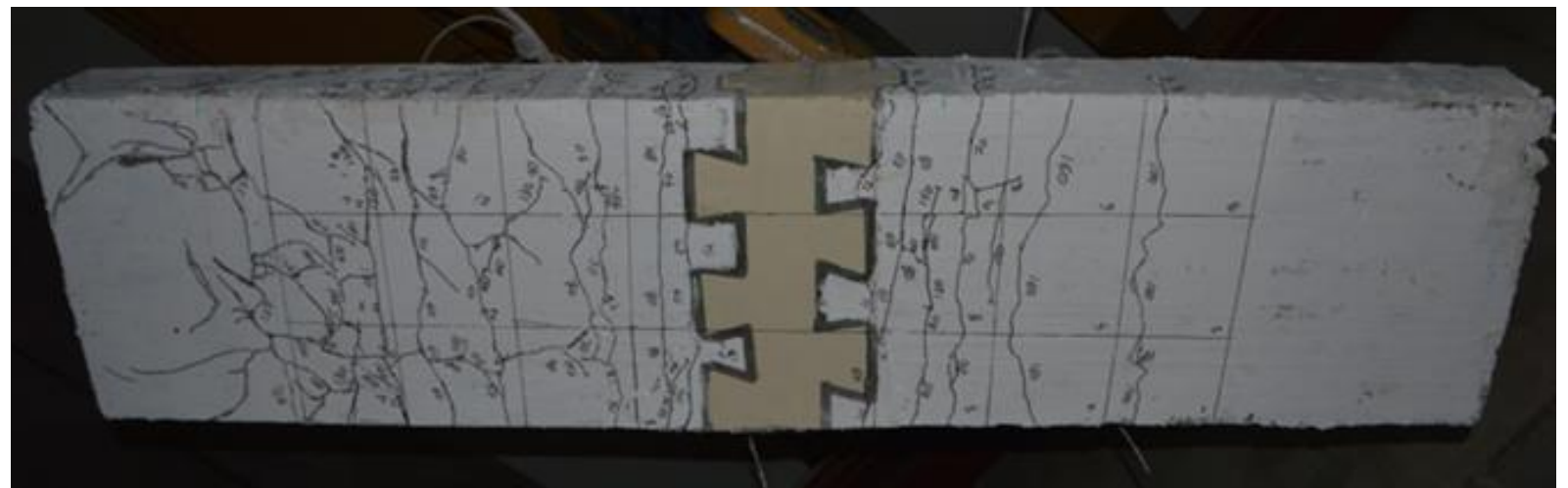

c) Crack pattern at the bottom of the FDDP

Figure 4.11. Test setup and crack pattern at failure of slab S5 (J.Z.E) with zigzag-shape joint under eccentric loading 


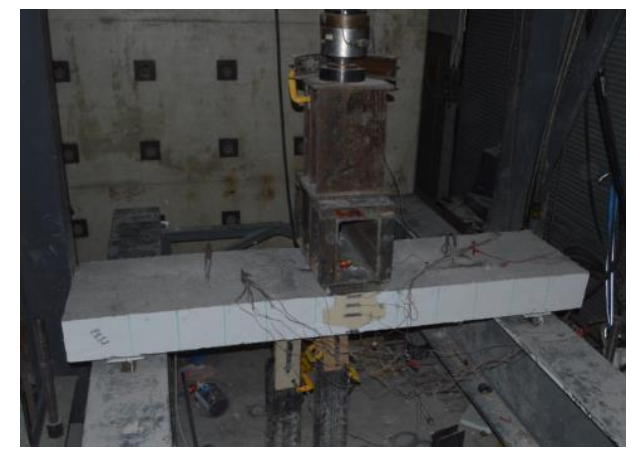

a) Test setup

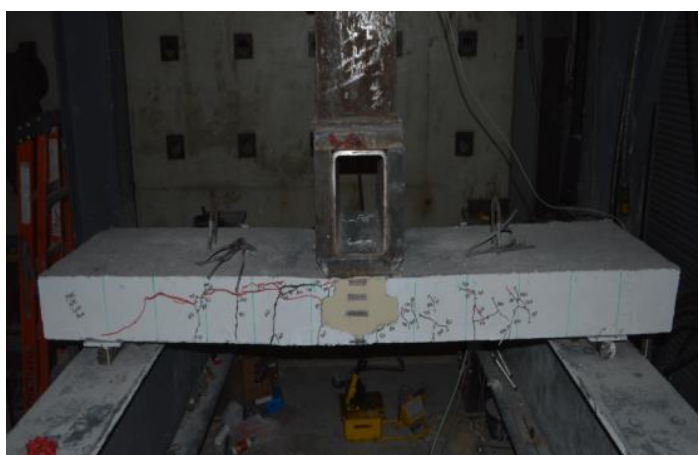

b) Crack pattern at the joint region

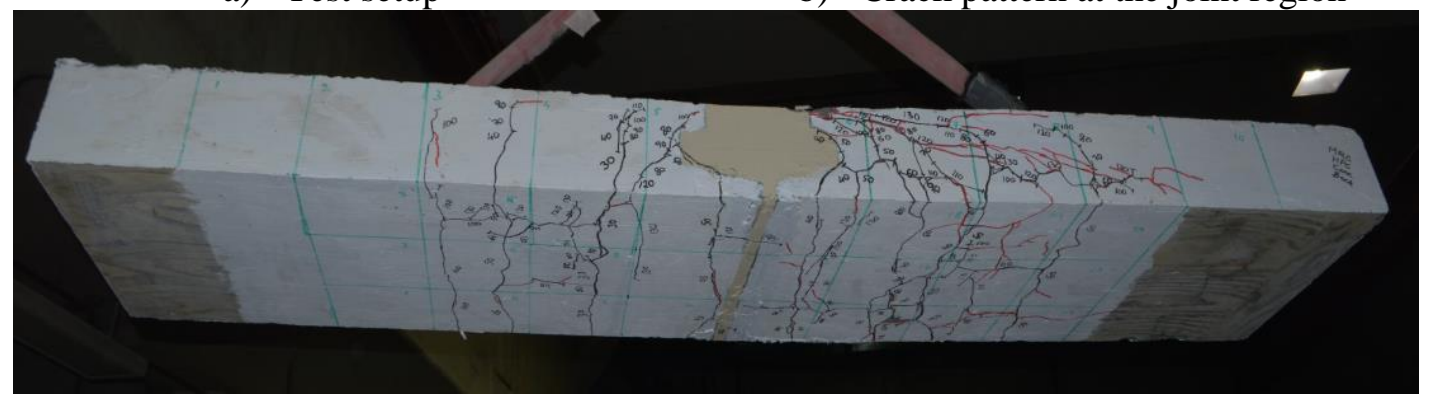

c) Crack pattern at the bottom of the FDDP

Figure 4.12. Test setup and crack pattern at failure of slab S6 (J.A.C) with angle-shape joint under centric loading

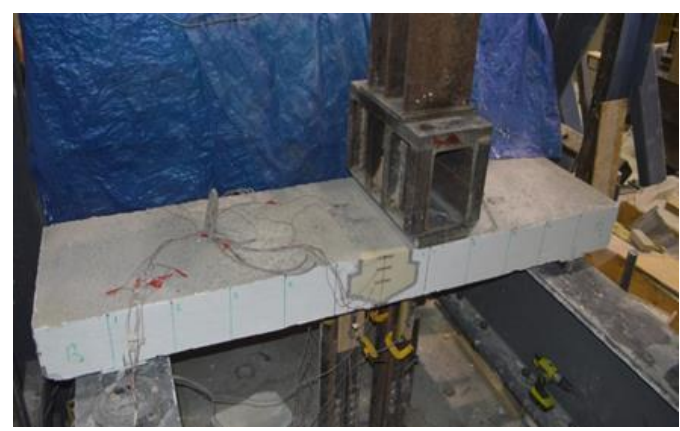

a) Test setup

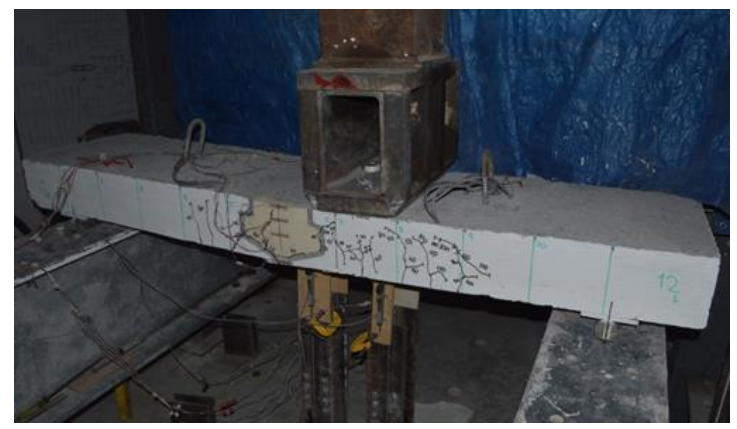

b) Crack pattern at the joint region

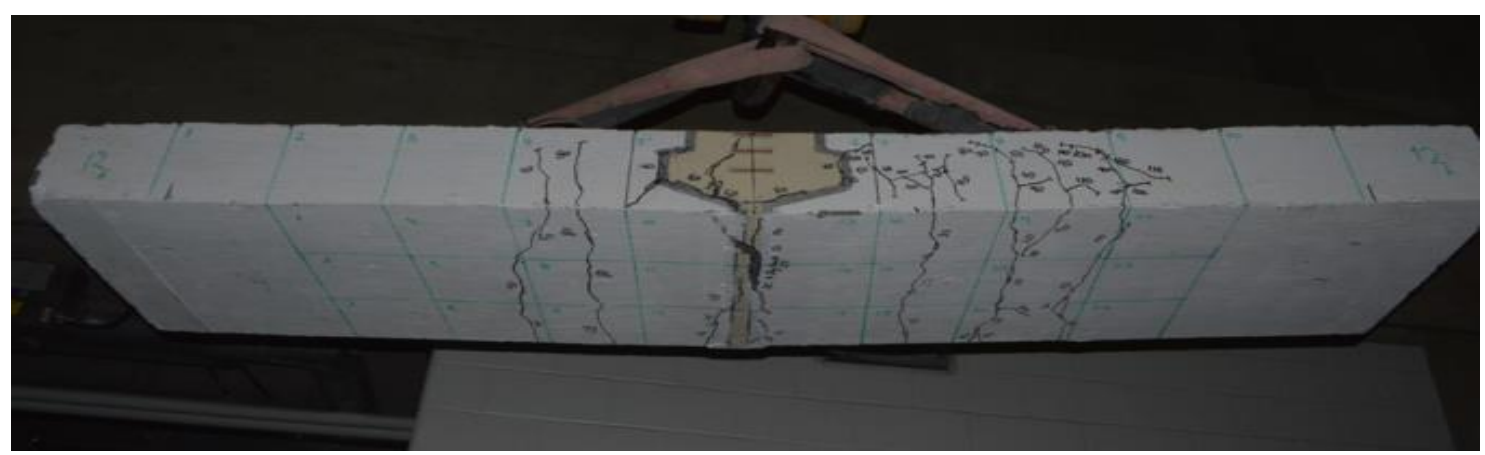

c) Crack pattern at the bottom of the FDDP

Figure 4.13. Test setup and crack pattern at failure of slab S6 (J.A.E) with angle-shape joint under eccentric loading 


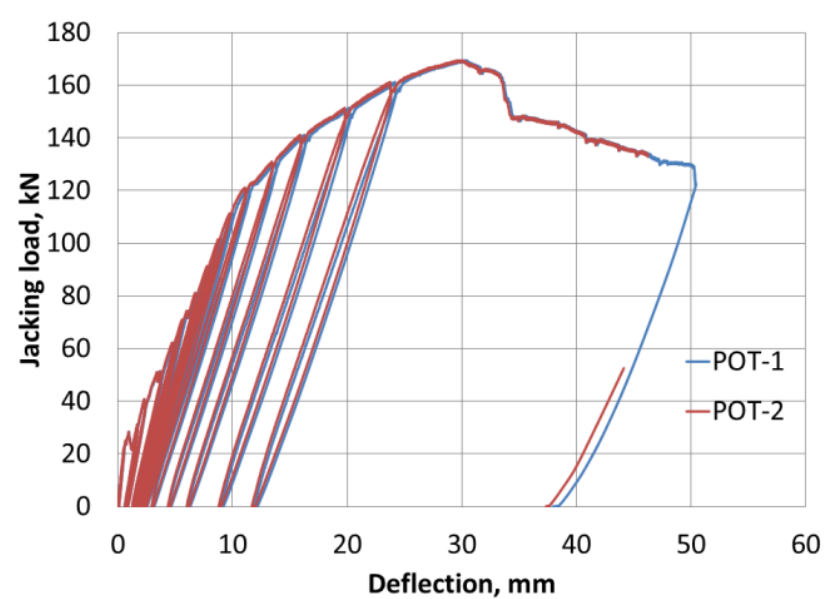

\section{a) Slab S1 (C.ST.C)}

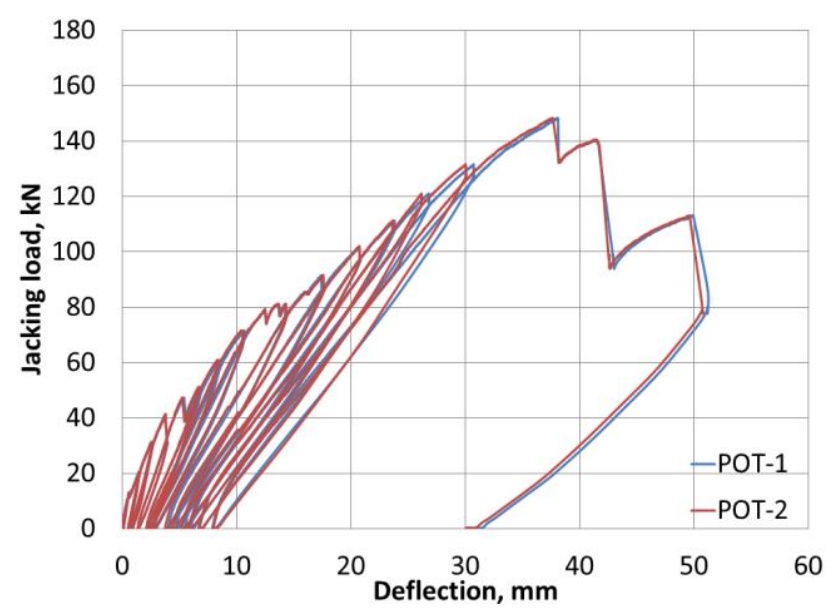

c) Slab S3 (J.C.C)

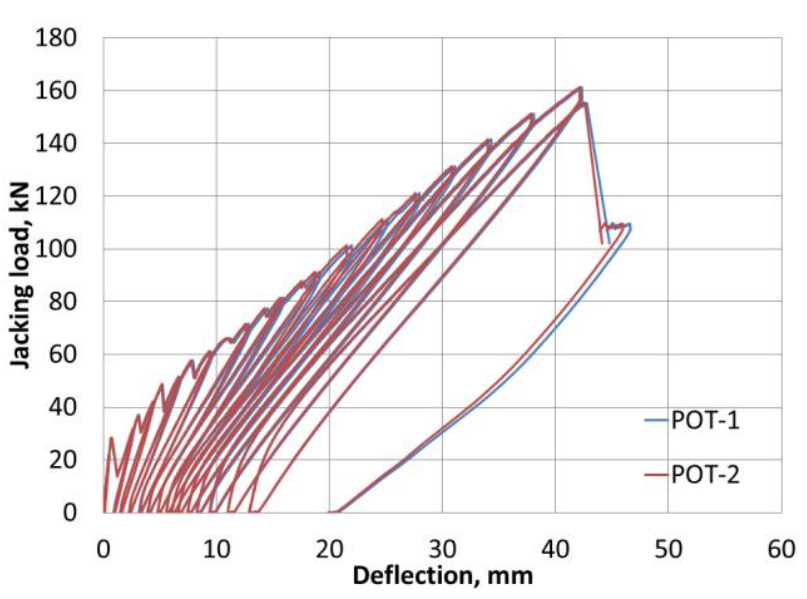

b) Slab S2 (C.GFRP.C)

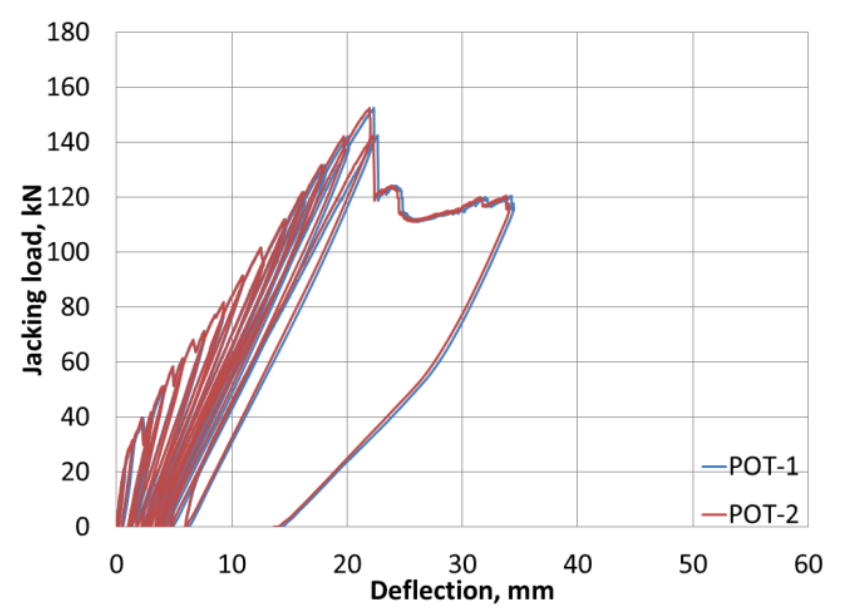

d) Slab S4 (J.C.E)

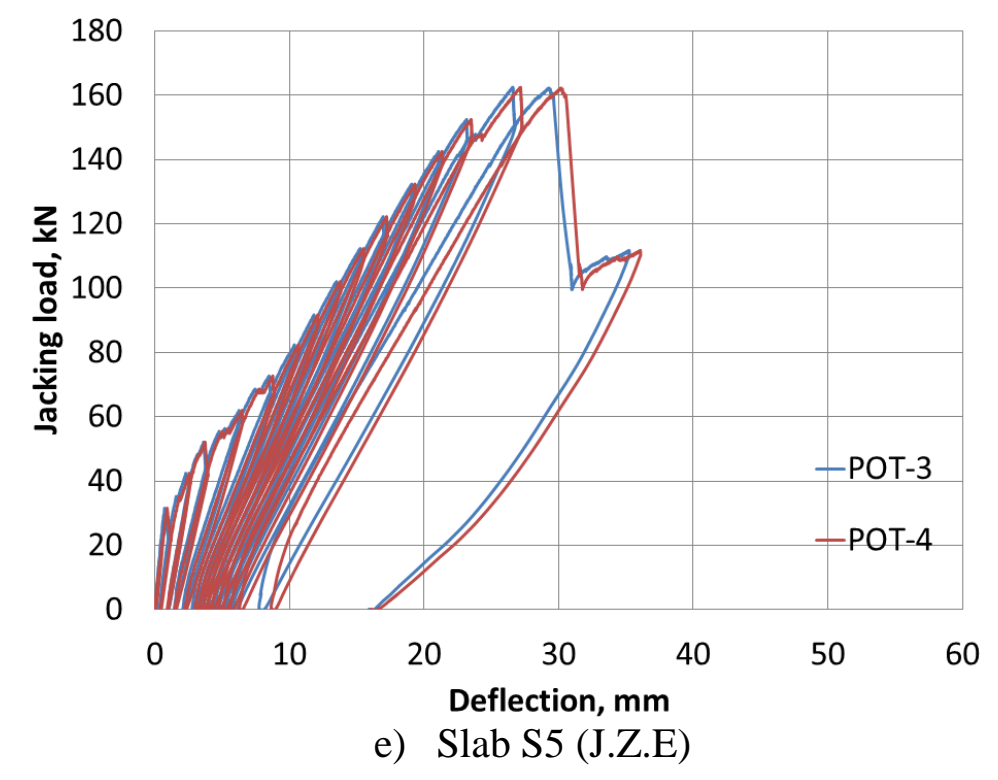




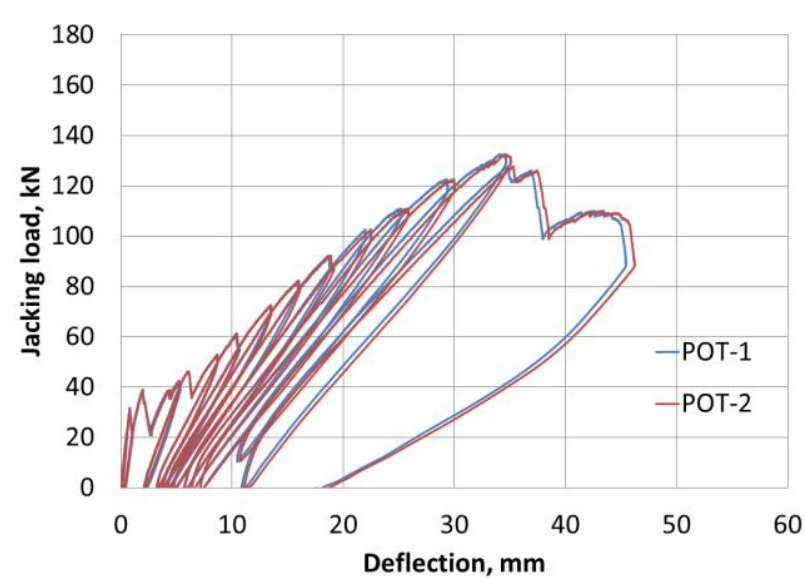

f) Slab S6 (J.A.C)

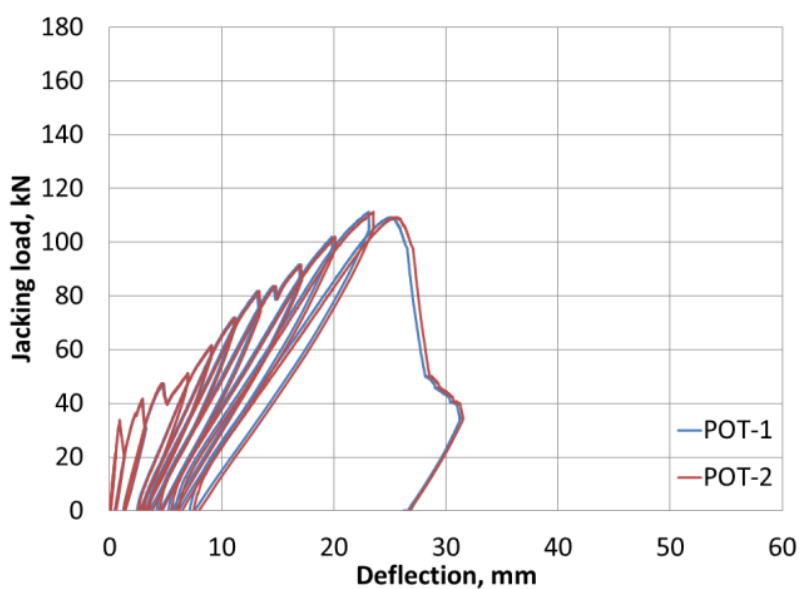

g) Slab S7 (J.A.E)

Figure 4.14. Load-deflection relationships obtained at mid-span of slab specimens 


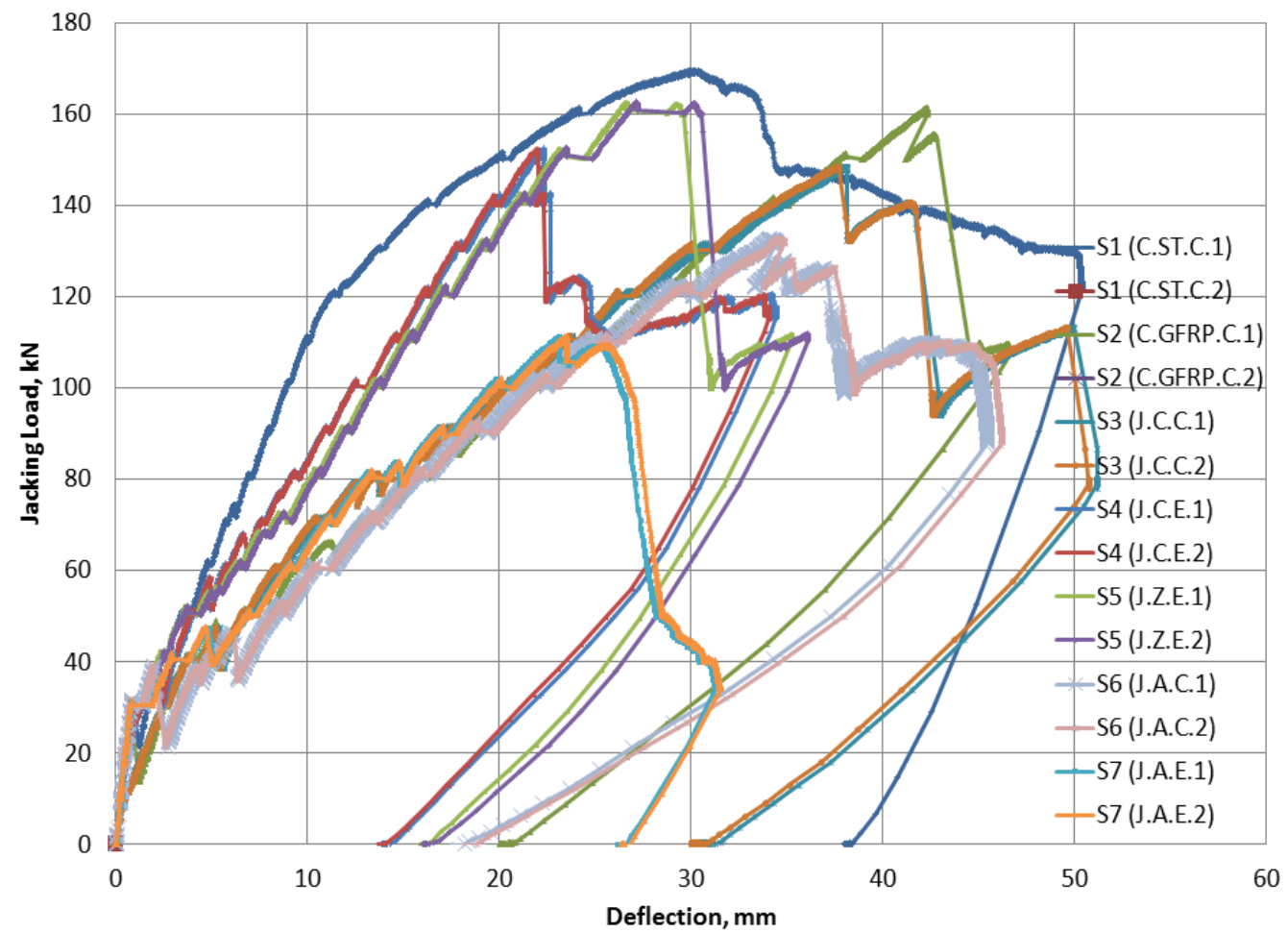

Figure 4.15. Load-deflection curves for the tested specimens

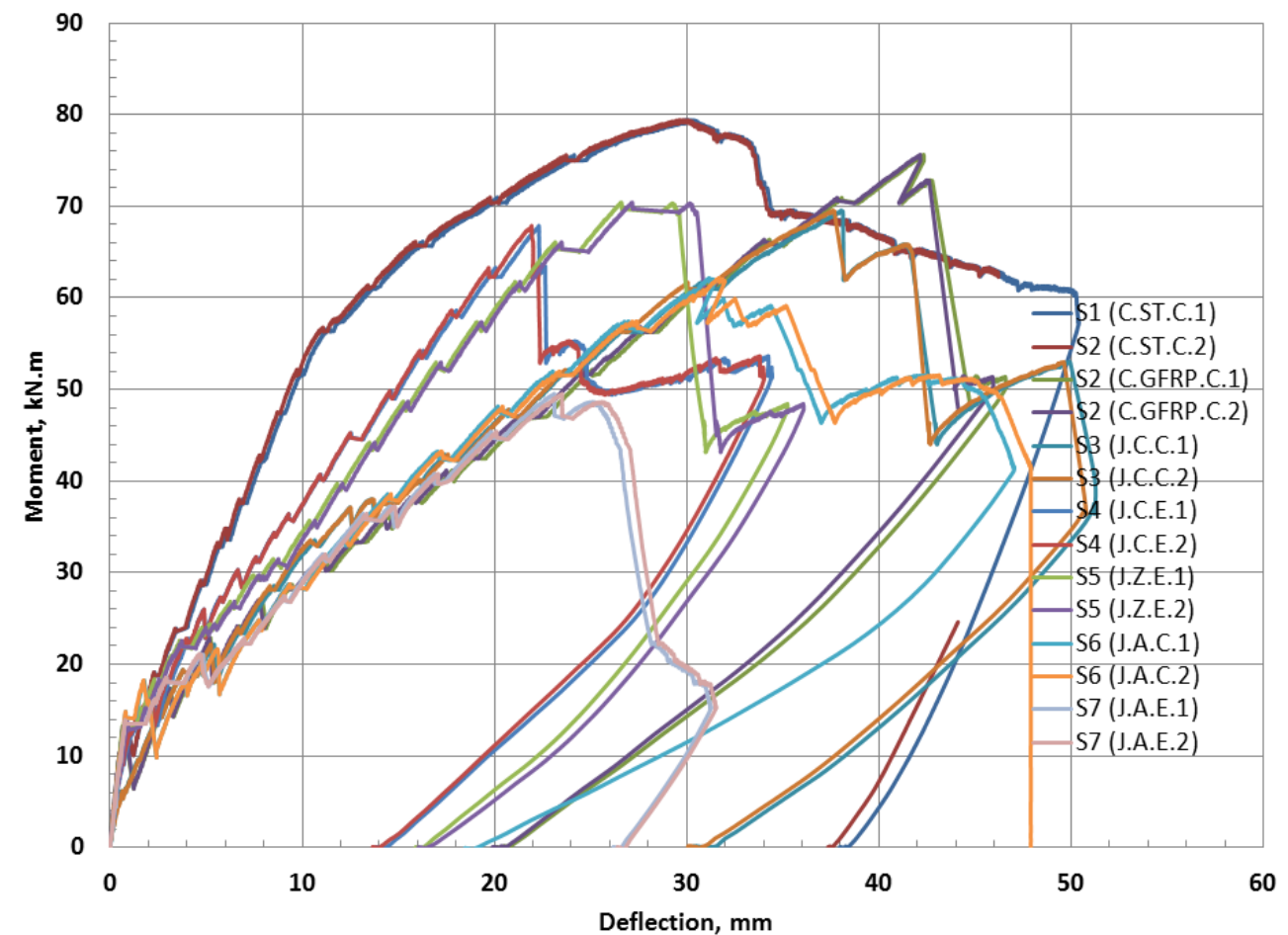

Figure 4.16. Moment-deflection curves for the tested specimens 

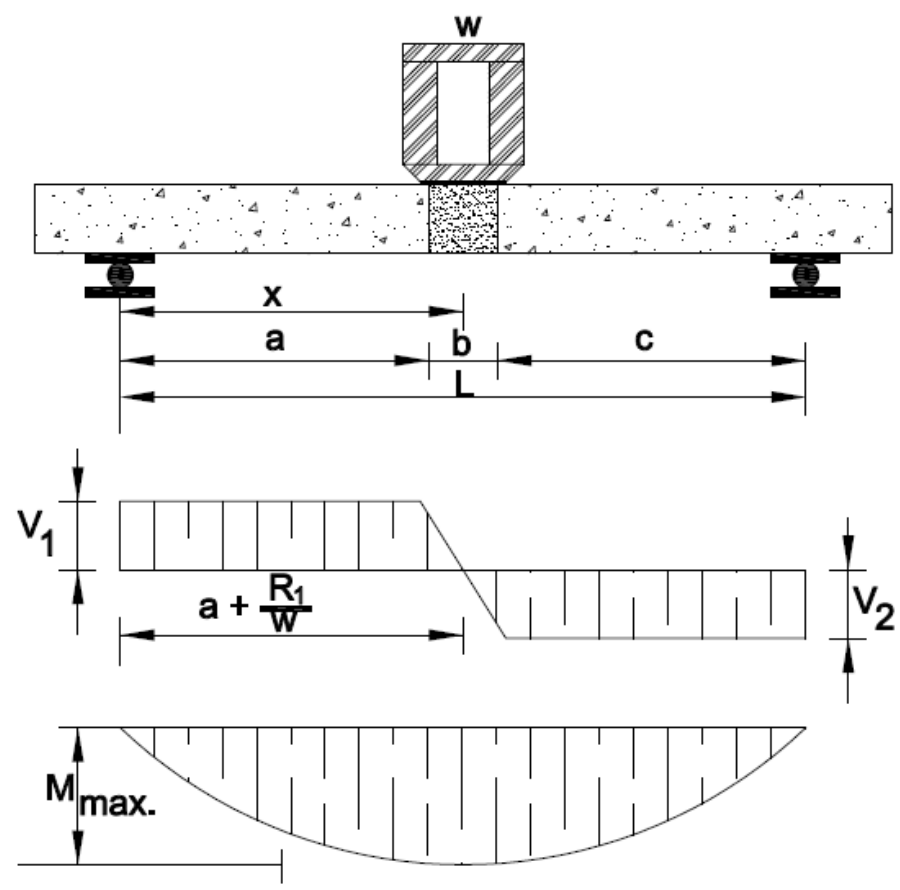

a) Analysis of slab with centric patch loading
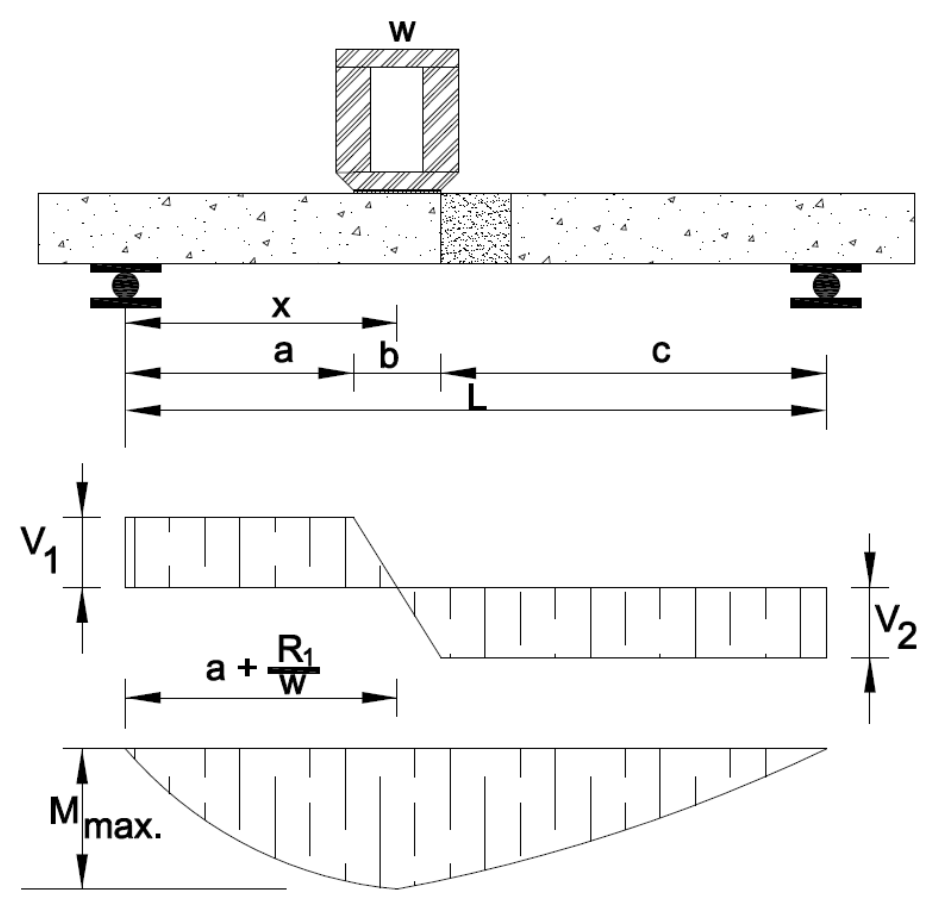

b) Analysis of slab with eccentric patch loading

Figure 4.17. Structural analysis of the tested slabs 


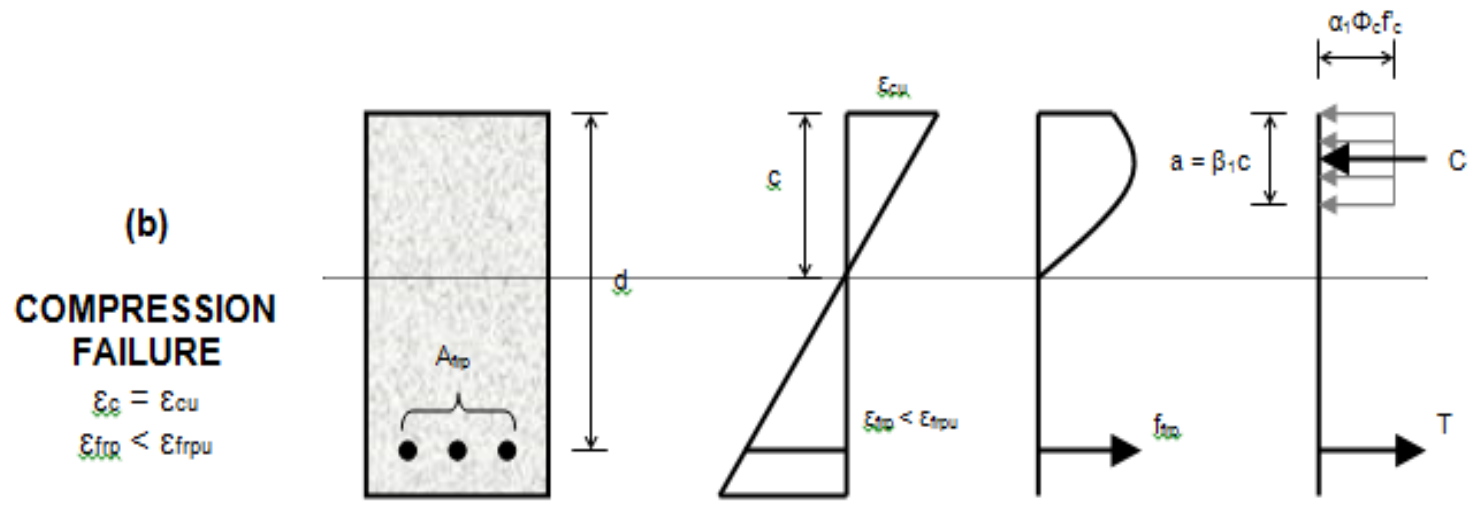

Figure 4. 18. Whitney rectangular stress distribution for flexural design

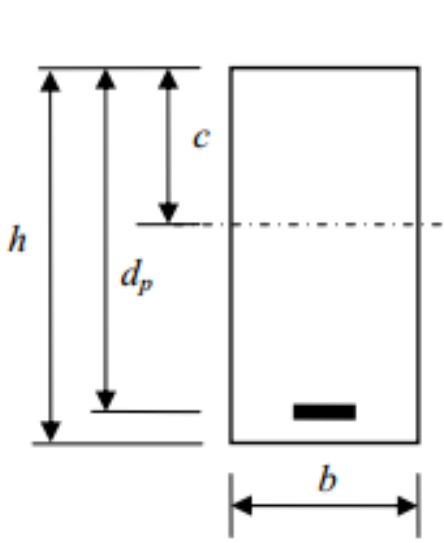

(a)

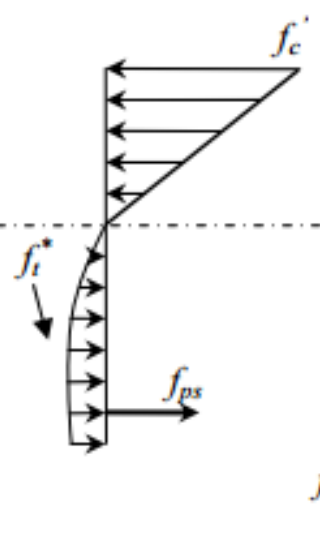

(b) (c) (d)

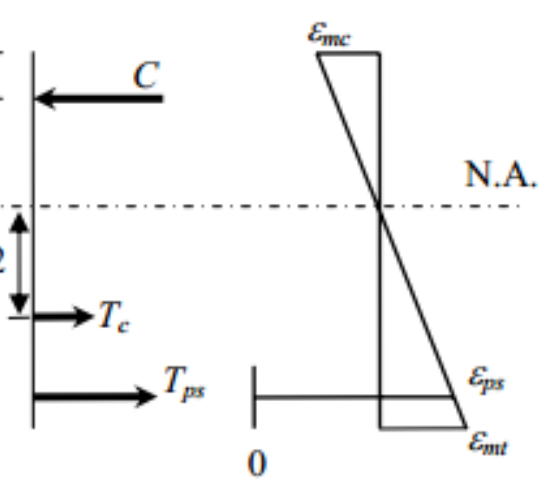

(e)

Figure 4.19. UHPFRC internal stress behavior 


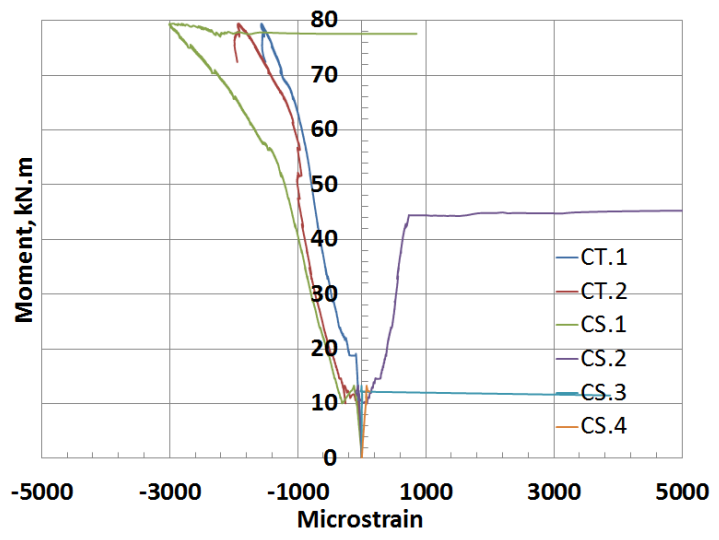

Moment-concrete strain

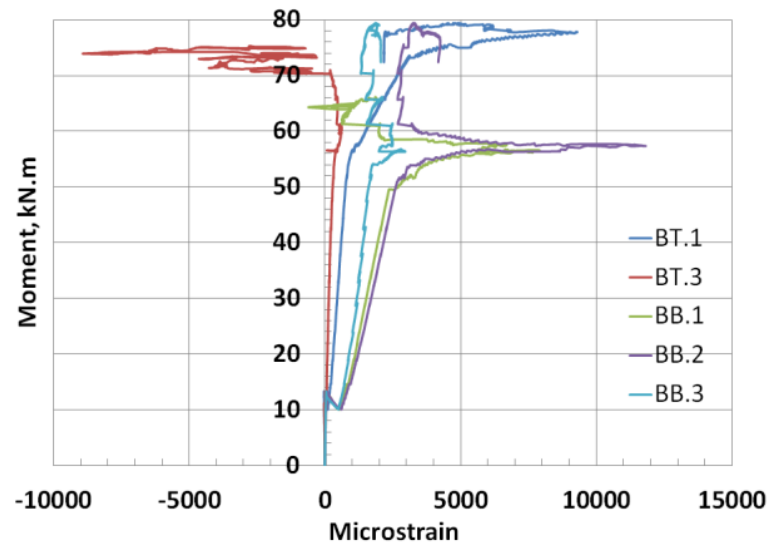

Moment-bar strian

Figure 4.20. Control CIP steel slab S1 (C.ST.C) under centric load

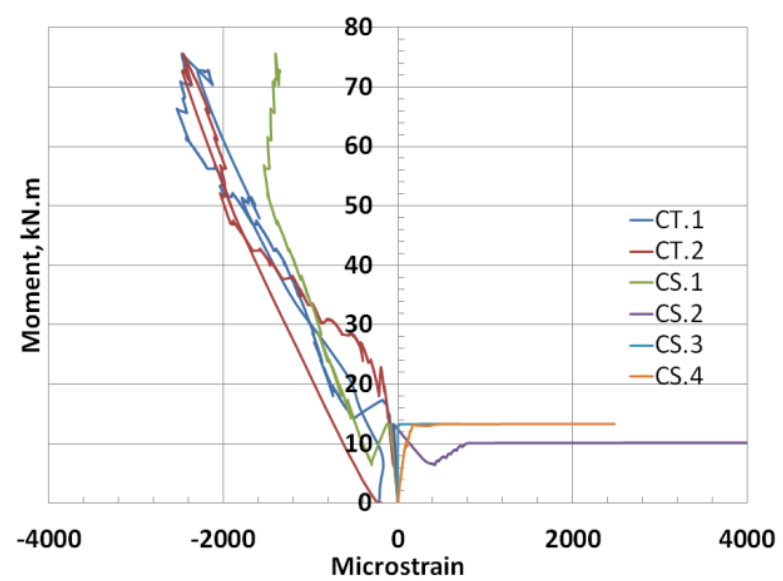

Moment-concrete strain

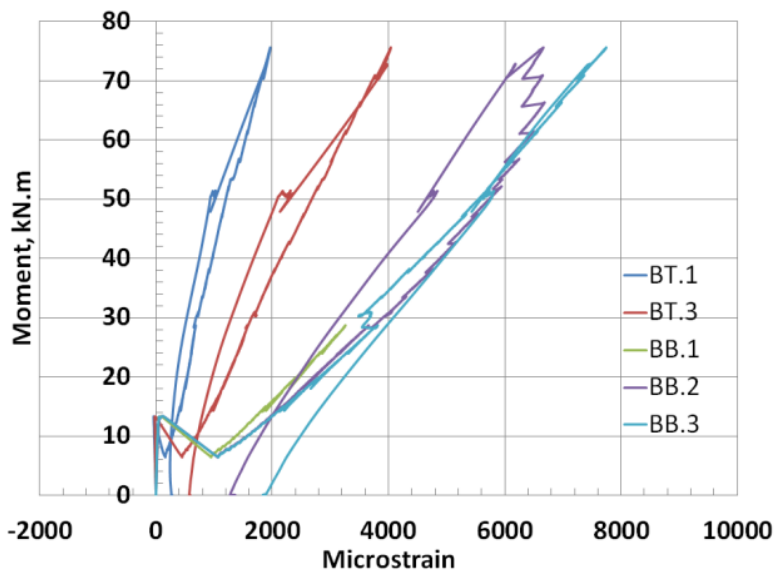

Moment-bar strain

Figure 4. 21. Control CIP GFRP slab S2 (C.GFRP.C) under centric load

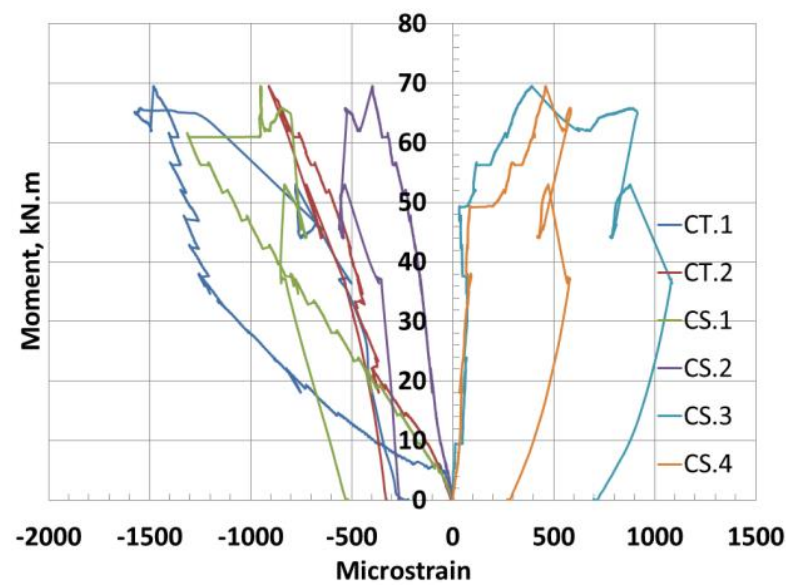

Moment-concrete strain

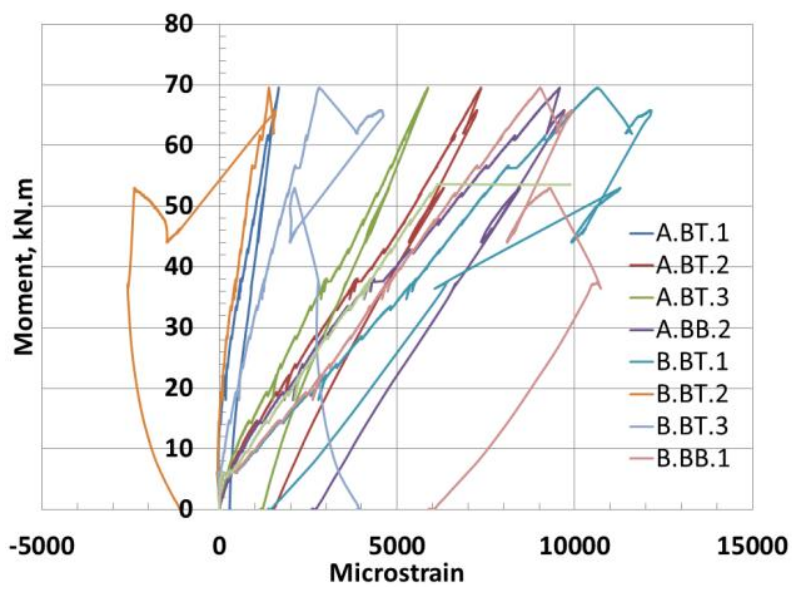

Moment-bar strain

Figure 4.22. Jointed GFRP-reinforced FDDP S3 (JCC) under centric load 


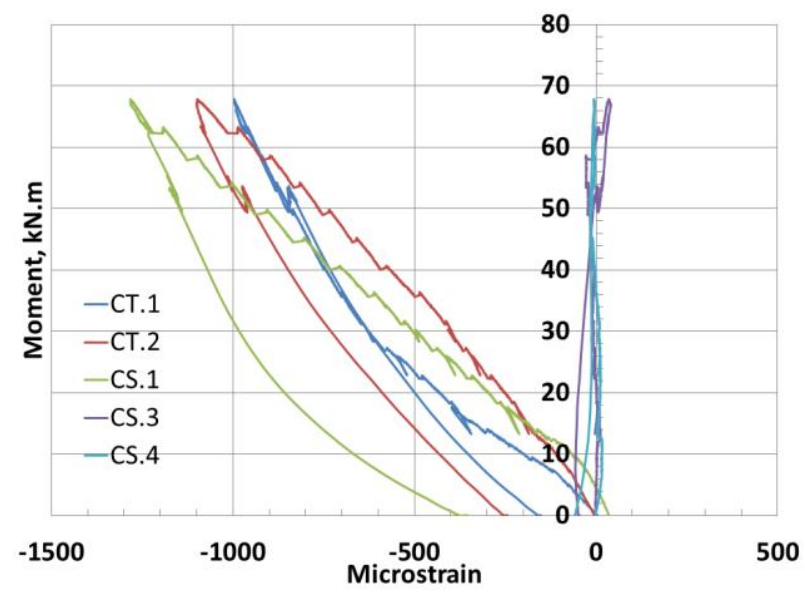

Moment-concrete strain

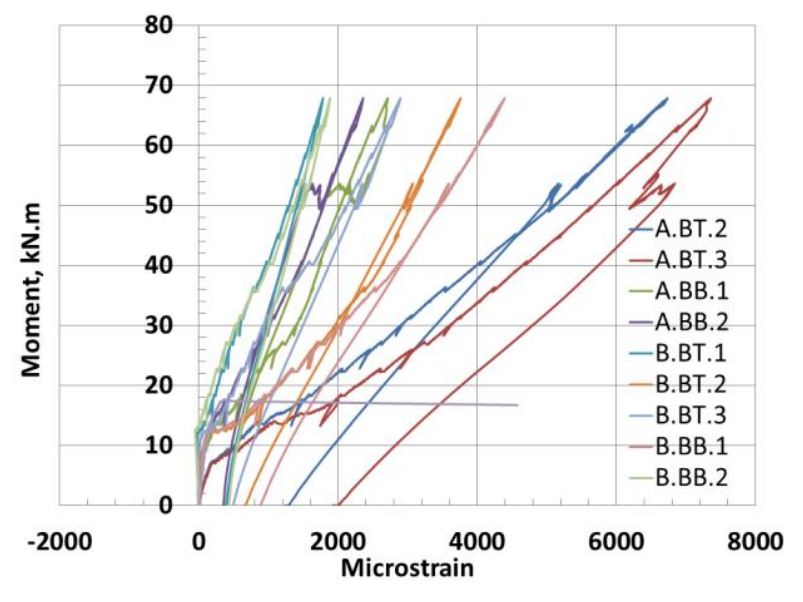

Moment-bar strain

Figure 4.23. Jointed GFRP-reinforced FDDP S4 (JCE) under eccentric load

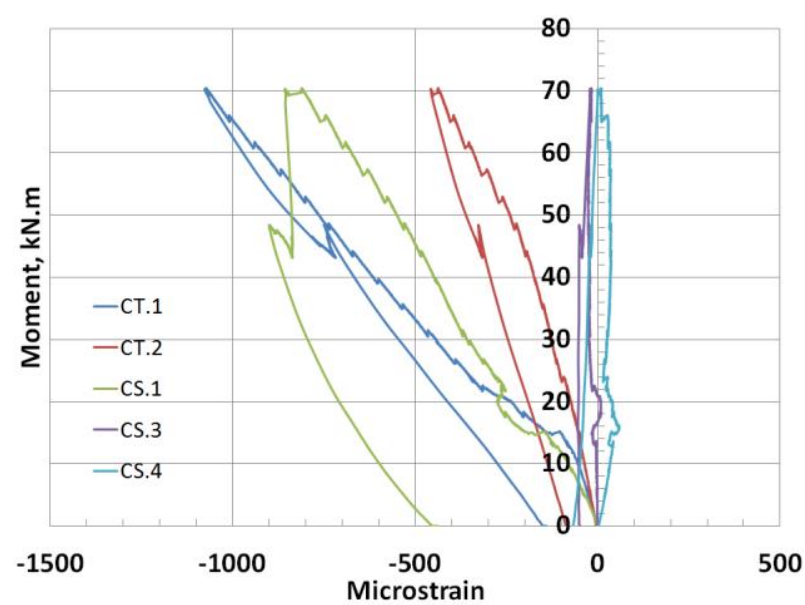

Moment-concrete strain

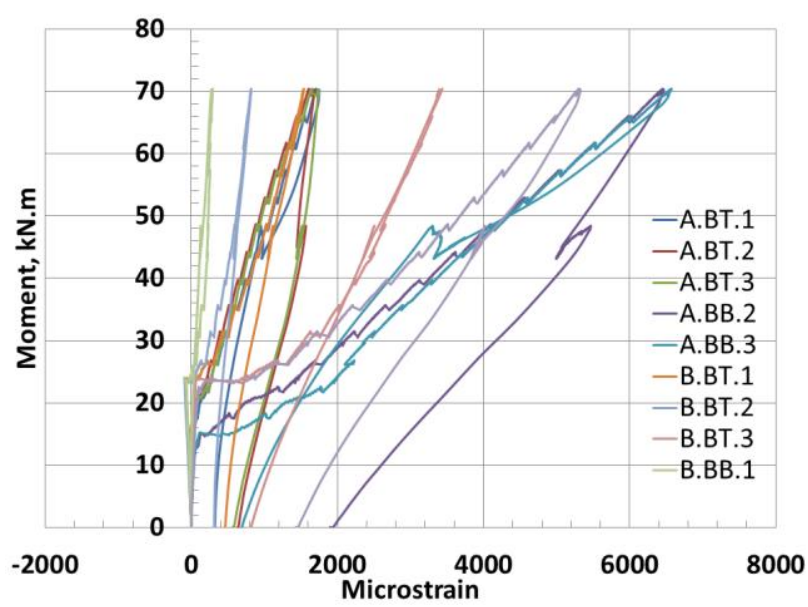

Moment-bar strain

Figure 4.24. Jointed GFRP-reinforced FDDP S5 (JZE) under eccentric load

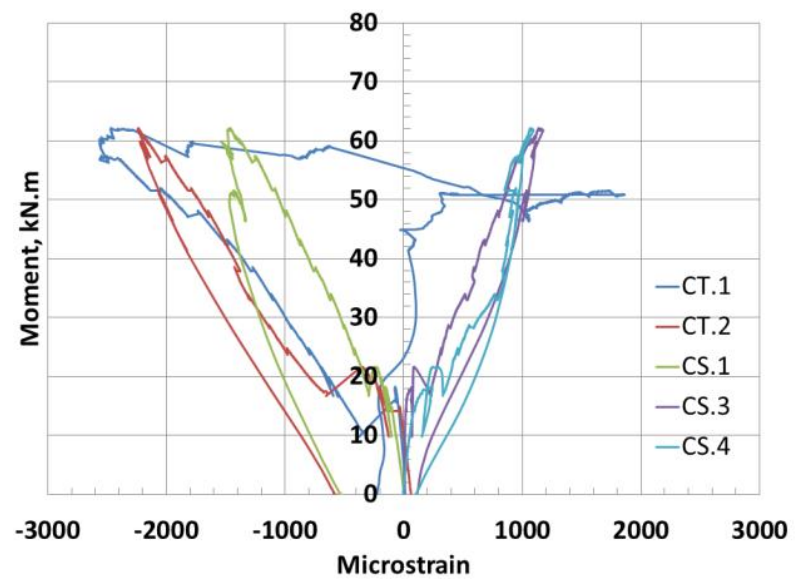

Moment-concrete strain

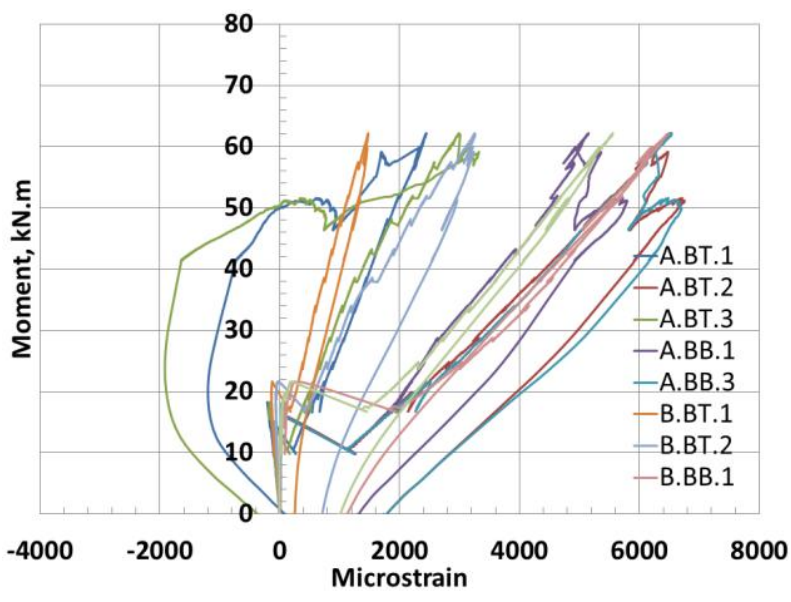

Moment-bar strian

Figure 4.25. Jointed GFRP-reinforced FDDP S6 (JAC) under centric load 


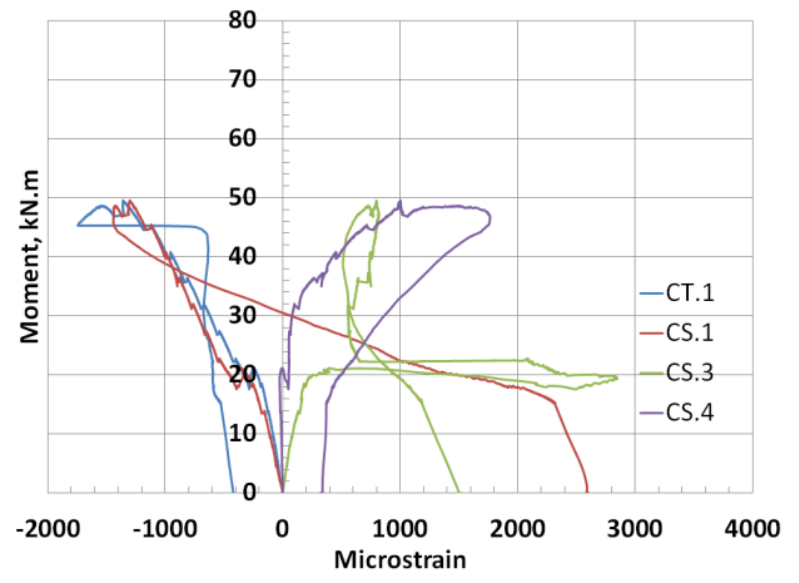

Moment-concrete strain

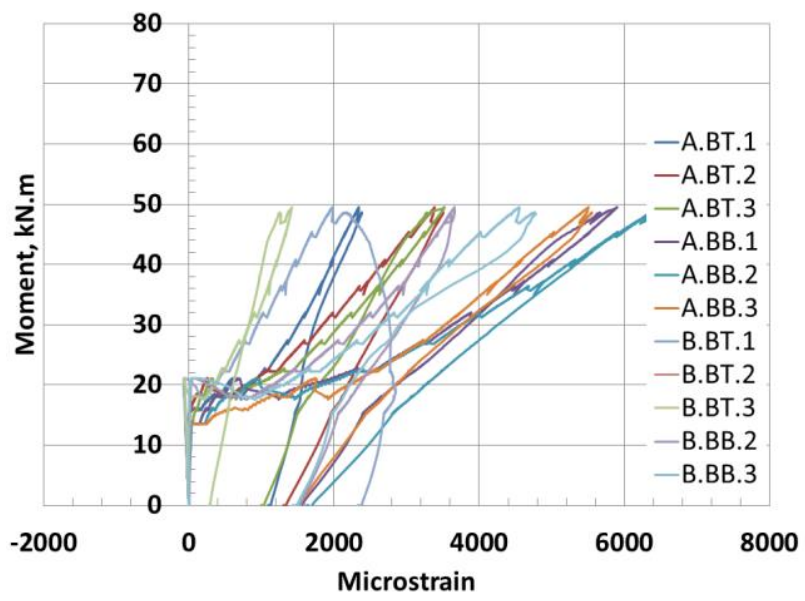

Moment-bar strain

Figure 4.26. Jointed GFRP-reinforced FDDP S7 (JAE) under eccentric load 


\section{Appendix: Design Data}

Data:

Concrete compressive strength, $f_{c}^{\prime}=76.94 \mathrm{MPa}$

Elastic modulus for the GFRP bar, $\mathrm{E}=64 \mathrm{GPa}$

Ultimate tensile strength, $f_{\text {frp }}=1188 \mathrm{MPa}$

Area of $20 \mathrm{M}$ rebar is $314.16 \mathrm{~mm}^{2}$ for nominal area

and $380.13 \mathrm{~mm}^{2}$ for the area + the ribbed part

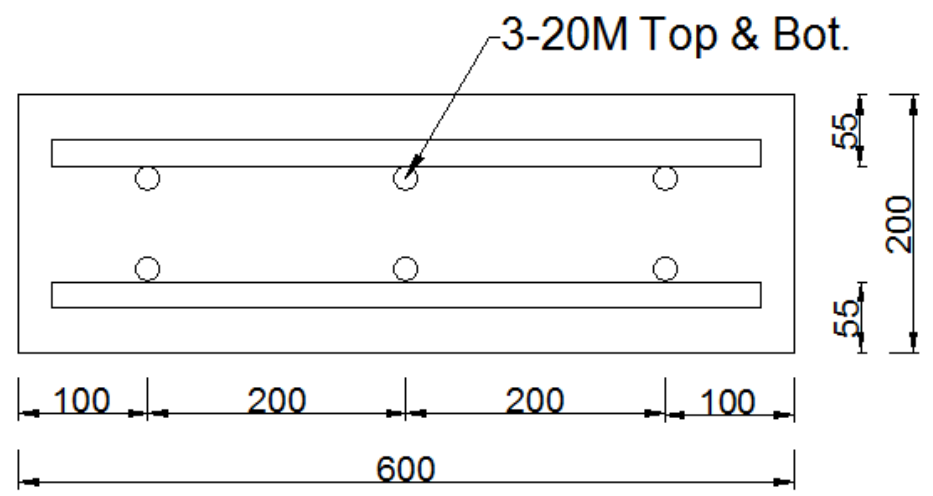

Figure C.1. Beam's cross section

1 Determine the concrete cover and the effective depth of the section

$2.5 d_{b}=2.5 \times 20=50 \mathrm{~mm}$

The effective depth, $d$, is calculated from

$d=h-$ cover $-\frac{d_{b}}{2}=200-50-20 / 2=140 \mathrm{~mm}$

However $\mathrm{d}=200-55-20 / 2=135 \mathrm{~mm}$

2 Calculate the FRP reinforcement ratio

$$
\rho_{f r p}=\frac{A_{f r p}}{b d}=(3 \times 314.16) /(600 \times 135)=0.011636=1.1636 \%
$$

3 Calculate the balanced FRP reinforcement ratio

$$
\rho_{\text {frpb }}=\frac{A_{f r p}}{b d}=\alpha_{1} \beta_{1} \frac{\phi_{c}}{\phi_{f r p}} \frac{f_{c}^{\prime}}{f_{f r p u}}\left(\frac{\epsilon_{c u}}{\epsilon_{c u}+\epsilon_{f r p u}}\right)
$$

Where

$$
\begin{aligned}
& \alpha_{1}=0.85-0.0015 f_{c}^{\prime} \geq 0.67 ;=0.85-0.0015(76.94)=0.7346 \\
& \beta_{1}=0.97-0.0025 f_{c}^{\prime} \geq 0.67 ;=0.97-0.0025(76.94)=0.7777 \\
& \epsilon_{f r p u}=\frac{f_{f r p u}}{E_{\text {frp }}}=1188 /\left(64 \times 10^{3}\right)=0.0186 \\
& \phi_{c}=0.75 \\
& \phi_{\text {frp }}=0.55 \text { for Glass FRP } \\
& \rho_{\text {frpb }}=0.7346 \times 0.7777 \times \frac{0.75}{0.55} \times \frac{76.94}{1188}\left(\frac{0.0035}{0.0035+0.0186}\right)=0.00799
\end{aligned}
$$


4 Check if the section will fail by tension failure or compression failure

$\rho_{\text {frp }}=0.011636>\rho_{\text {frpb }}=0.00799$

Therefore, we have COMPRESSION FAILURE, and the distribution is as follows:

5 Determine the tensile stress in the FRP reinforcement at compressive failure of the section

$$
\begin{aligned}
f_{\text {frp }}= & 0.5 E_{\text {frp }} \epsilon_{c u}\left[\sqrt{1+\frac{4 \alpha_{1} \beta_{1} \phi_{c} f_{c}^{\prime}}{\rho_{f r p} \phi_{f r p} E_{f r p} \epsilon_{c u}}}-1\right] \\
& =0.5 \times 64 \times 10^{3} \times 0.0035\left[\sqrt{1+\frac{4 \times 0.7346 \times 0.7777 \times 0.75 \times 76.94}{0.011636 \times 0.55 \times 64 \times 10^{3} \times 0.0035}}-1\right] \\
& =968 \mathrm{MPa}
\end{aligned}
$$

6 Determine the stress block depth, a

$$
\begin{aligned}
\beta_{1} c=a & =\frac{\phi_{f r p} A_{f r p} f_{f r p}}{\alpha_{1} \phi_{c} f_{c}^{\prime} b} \\
& =\frac{0.55 \times 3 \times 314.16 \times 968}{0.7346 \times 0.75 \times 76.94 \times 600}=19.73 \mathrm{~mm}
\end{aligned}
$$

7 Determine the flexural capacity

$$
\begin{aligned}
M_{r}= & \phi_{\text {frp }} A_{\text {frp }} f_{\text {frp }}\left[d-\frac{a}{2}\right] \\
& =0.55 \times 3 \times 314.16 \times 968[135-19.73 / 2] \\
& =62789783.81 \mathrm{~N} . \mathrm{mm}=62.79 \times 10^{6} \mathrm{~N} . \mathrm{mm} \\
& =\underline{\mathbf{6 2 . 7 9} \mathbf{~ k N . m}}
\end{aligned}
$$

Thus, the moment capacity of the section is $62.79 \mathrm{kN} . \mathrm{m}$.

Finally, we must check that the minimum flexural capacity requirements are satisfied

$M_{r} \geq 1.5 M_{c r}$

The cracking moment is determined by

$M_{c r}=\frac{f_{r} I_{t}}{y_{t}}$

Where $f_{r}=0.6 \sqrt{f_{c}^{\prime}}$

$I_{t}=$ transformed section moment of inertia

$y_{t}=$ distance from N.A. to extreme tension fiber

$f_{r}=0.6 \sqrt{76.94}=5.26$

$I_{t}=\frac{b h^{3}}{12}+b h\left(\bar{y}-y_{t}\right)^{2}+(n-1) A_{f r p}(d-\bar{y})^{2}$

$\bar{y}=\frac{(b h) \frac{h}{2}+(n-1) A_{f r p} d}{A_{t r}}$

$A_{t r}=b h+(n-1) A_{f r p}$

$n=\frac{E_{\text {frp }}}{E_{c}}$

$E_{c}=\left(3300 \sqrt{f_{c}^{\prime}}+6900\right)=3300 \sqrt{45}+6900=29037 \mathrm{MPa}$

$E_{\text {frp }}=60 \times 10^{3} \mathrm{MPa}$

$n=\frac{64000}{29037}=2.2$

$A_{t r}=600 \times 200+(2.2-1) \times 3 \times 314.16=121130.98 \mathrm{~mm}^{2}$

$\bar{y}=\frac{(600 \times 200) \frac{200}{2}+(2.2-1) \times 3 \times 314.16 \times 135}{121130.98}=100.22 \mathrm{~mm}$ 


$$
\begin{aligned}
& \begin{array}{l}
y_{t}=\frac{h}{2}=200 / 2=100 \mathrm{~mm} \\
I_{t}=\frac{600 \times 200^{3}}{12}+600 \times 200(100.22-100)^{2}+(2.20-1) \times 3 \times 314.16(135-100.22)^{2} \\
=401259884.4 \mathrm{~mm}^{4}=0.401 \times 10^{9} \mathrm{~mm}^{4} \\
M_{c r}=\frac{f_{r} I_{t}}{y_{t}}=\frac{5.26 \times 0.401 \times 10^{9}}{100}=21092600 \mathrm{~N} . \mathrm{mm}=21.09 \times 10^{6} \mathrm{~N} . \mathrm{mm}=\underline{\mathbf{2 1 . 0 9} \mathbf{k N} . \mathbf{m}}
\end{array}
\end{aligned}
$$

Thus we have

$M_{r}=62.79 \mathrm{kNm} \geq 1.5 M_{c r}=1.5 x 21.09=31.635 \mathrm{kNm} \rightarrow \mathrm{OK}$

This, the beam has satisfactory capacity to avoid failure upon cracking.

Note: serviceability requirements for cracking and deflection should be investigated.

$$
1 \mathrm{MPa}=1 \mathrm{~N} / \mathrm{mm}^{2}
$$

Shear resistance provided by concrete section of $600-\mathrm{mm}$ wide

$$
\begin{aligned}
V_{c}=0.2 \lambda \phi_{c} \sqrt{f_{c}^{\prime}} b_{w} d \sqrt{\frac{E_{f r p}}{E_{s}}} \\
=0.2 \times 1 \times 0.75 \sqrt{76.94} \times 600 \times 135 \sqrt{\frac{64}{200}}=60.29 \mathrm{kN}
\end{aligned}
$$

\section{Flexural capacity of UHPFRC beam}

The flexural strength of singly-reinforced GFRP reinforced beam. The moment is taken about the compressive force resultant of the UHPFRC.

To determine the stress block of the UHPFRC beam

$$
\begin{gathered}
c=\frac{\phi_{\text {frp }} A_{\text {frp }} f_{\text {frpu }}+\phi_{c} f_{t} h b}{b\left(0.5 \phi_{c} f_{c}+\phi_{c} f_{t}\right)} \\
c=\frac{0.55 \times 3 \times 314 \times 1188+0.75 \times 8 \times 200 \times 600}{600 \times 0.75(0.5 \times 140+8)} \\
\mathrm{c}=38.05 \mathrm{~mm} \\
M=0.55 \times 1188 \times 3 \times 314\left(135-\frac{38.05}{3}\right)+0.75 \times 8 \times 600(200-38.05)\left(\frac{200}{2}+\frac{38.05}{6}\right) \\
M=137.29 \mathrm{kN} . \mathrm{m}
\end{gathered}
$$




\section{Chapter VI}

\section{Fatigue and Ultimate Strength Evaluation of GFRP- Reinforced Full-Depth Precast Deck Panels with UHPFRC-Filled Transverse Closure Strips}

\subsection{General}

This research investigates the structural response of full depth precast deck panels (FDDPs) constructed with new construction materials and connection details. FDDP is one element of the prefabricated bridge element and systems (PBES) that allows for quick unshored assembly of the bridge deck on-site as part of the accelerated bridge construction (ABC) technology. FDDP is constructed with normal strength concrete (NSC) and reinforced with high modulus (HM) glass fiber reinforced polymer (GFRP) ribbed bars. The panel-to-girder V-shape connections adopt the use of the shear pockets to accommodate the clustering of the shear connectors. In this research, the transverse panel-to-panel connection was developed, forming three different female-tofemale joint shapes with (i) 175-mm projected GFRP bars from the FDDP into the closure strip, (ii) female vertical shear key and (iii) joint-filled with cementitious materials. The ultra-high performance fiber reinforced concrete (UHPFRC) was used to joint-fill the 200-mm transverse joint between adjacent precast panels and the shear pockets connecting the panels to the supporting girders to ensure full shear interaction. Two actual-size FDDP specimens for each type of the three developed joints were erected to perform fatigue tests under the foot print of the Canadian Highway Bridge Design Code (CHBDC) truck wheel loading. The FDDP had 200-mm thickness, 2500-mm width and 2400-mm length in the direction of traffic and rest over braced steel twin-girders. Two types of fatigue tests were performed, namely: (i) incremental variable amplitude fatigue (VAF) loading and (ii) constant amplitude fatigue (CAF) loading, followed by monotonically loaded the slab ultimate-to-collapse. It was observed that fatigue test results showed that the ultimate capacity of the slab under VAF loading or after 4 million cycles of CAF 
exceeds the factored design wheel load specified into the CHBDC. Also, punching shear failure mode was dominant in all the tested FDDP specimens.

\subsection{Research Background}

Precast full depth deck panels (FDDPs) have recently used in new accelerated bridge construction $(\mathrm{ABC})$ or for the rapid bridge replacement $(\mathrm{RBR})$ of existing deteriorated bridge decks. FDDPs are produced off-site, quickly assembled on-site, reduce construction time, minimize lane closure and are considered as a good solution to minimize traffic disruption (Clumo, 2011). FDDPs are placed side by side as shown in Fig. 5.1, then the closure strips between them are filled with bonding material. FDDP closure strips should take the advantage of high quality concrete and non-corrosive reinforcement as glass fiber reinforced polymer (GFRP) bars for enhanced strength and durability. Although the Canadian Highway Bridge Design Code, CHBDC (CSA, 2014) allows the use of GFRP-reinforced FDDPs in bridge construction, there is no code provision on the joint details between such precast systems. The behaviour of the FDDP monolithic concrete joint, also known as moment resisting connection (MRC), accounts for the state of bond of the projected longitudinal bars anchored through the cast-field joints. Ultra-high performance fiber reinforced concrete (UHPFRC) is a relatively new class of cementitious matrix with steel fiber content that has high compressive strength (in order of $140 \mathrm{MPa}$ at 28 days) and relatively large tensile strength (in order of $8 \mathrm{MPa}$ ), with strain hardening behavior in tension that ensure crack opening remain very small (Russell and Graybeal, 2013). The use of UHPFRC as a filling material of the closure strip between connected FDDPs have numerous benefits, including reduction of joint size, improving durability, speed of construction and prolonging usage life.

GFRP reinforcement is a composite material made of polymer matrix reinforced with fibers. It has high strength-to-weight ratio, is free of corrosion and lasts longer. The mechanical properties of the GFRP depend on different several factors such as fiber quality, orientation, volumetric ratio, and adhesion to the matrix and the manufacturing process such as pultrusion (ACI, 2003). The stress-strain relationship for the GFRP in tension is linear up to failure. Fibers near to the outer surface are stressed more than those at the center of the bar (Faza, 1991). The tensile strength of GFRP bars ranges from 483 to $1600 \mathrm{MPa}$ based on the manufacturer, while steel bars have tensile strength between 483 to $690 \mathrm{MPa}$. GFRP bars has no yield stress values, while steel 
bars have yield strength ranging from 276 to $517 \mathrm{MPa}$. The elastic modulus of the GFRP bars ranges from 35 to $65 \mathrm{GPa}$, while steel bars have modulus of elasticity of $200 \mathrm{GPa}$. GFRP bars have no yield strain values while its weight is about 0.14 to $0.25 \%$ that of steel bars. The rupture strain percentage of the GFRP bars ranges from 1.2 to $3.1 \%$ based on the manufacturer while for steel bars have rupture strain ranging from 6.0 to $12.0 \%$. GFRP bar has relatively weak interlaminar shear strength that is governed by the weak matrix. The shear strength of the sandcoated GFRP is $184 \mathrm{MPa}$ (Pultrall, 2013). The bond force in GFRP bar is transferred to the concrete by adhesion, friction and mechanical interlock provided that adequate concrete cover is available. Time-dependent behavior of the GFRP bars can be listed as: (i) creep and relaxation, and (ii) fatigue of GFRP bars. Creep of GFRP bar is characterized by the initial elastic response followed by non-linear creep response until it fails at the endurance time. Environmental conditions such as high temperature, high alkalinity wet and dry cycles and freeze-thaw cycles shorten the creep time.

AASHT-LRFD design specifications (AASHTO, 2012) considers the design of the deck slab as a continuous strip of $1000 \mathrm{~mm}$ width resting freely over the bridge beams. The load carrying capacity of such slab which is based on the bending moment capacity makes such design to be over conservative (Perdikaris and Beim, 1988). CHBDC specifies two different design methods for the slab-on-girder type, namely: (i) the flexural design method and (ii) the empirical method that accounts for the arching action of the laterally restrained slabs. The laterally-restrained concrete bridge slab deck fails in punching shear failure mode due to the effect of the concentrated wheel load causing the arching or compressive membrane action. Laterallyrestrained precast FDDP under arching action should exhibit higher load carrying capacity than those of one-way precast FDDP failed under pure flexural loading or combined flexural shear failure. Thus, it was deemed necessary to investigate the behavior of the laterally-restrained precast FDDP under the effect of truck wheel load. The flexural design method specified an equation to determine the factored applied moment in the deck slab as a function of the heaviest wheel load in CHBDC truck as well as girder spacing. The positive and negative moment for the simply supported slab is reduced by coefficient for positive and negative moment for the continuous two-way slab resting over three or more stiff supports (Thorburn, 1998). CHBDC adopts the empirical design method (Clause 8.18.4.1) for uniform thickness slab bounded by exterior supporting beams and met the following conditions: 
a) The deck slab is composite with the supporting beams, which are parallel to each other, and the lines of supports for the beams are also parallel to each other;

b) The ratio of the spacing of the supporting beams to the thickness of the slab is less or equal to 18.0. The spacing of the supporting beams used in calculating this ratio is taken parallel to the direction of the transverse reinforcement;

c) The spacing of the supporting beams does not exceed $4.0 \mathrm{~m}$ and the slab extends sufficiently beyond the external beams to provide full development length for the bottom transverse reinforcement; and

d) Longitudinal reinforcement in the deck slab in the negative moment regions of continuous composite beams is provided for in accordance with Clause 8.19.4 and Section 10, if applicable.

CHBDC added clause 8.18.4.4 for the use of the empirical method to the full-depth precast panels provided that:

a) The panels cover the full width of the bridge;

b) The depth of the panels is not less than $190 \mathrm{~mm}$;

c) At their transverse joints, the panels are joined together by grouted shear keys and are longitudinally post-tensioned with a minimum effective prestress of $1.7 \mathrm{MPa}$;

d) The ducts for longitudinal post-tensioning are located at the mid-depth of the panels, and openings (also known as blockouts) are provided at the joints to accommodate splices for tendons;

e) The blockouts are provided in the panels at locations where the panels are to be connected to the beams for composite action;

f) Initially, the panels are supported on the beams by means of temporary levelling devices, with the blockouts for connections to beams for composite action and the gap between the panels and beams being filled with grout after completion of post-tensioning; and

g) The grout used in the shear keys has a minimum strength of 35 MPa at $24 \mathrm{~h}$.

CHBDC provides provision for the edge stiffening for the deck slabs to meet the following specification: 
The transverse free edges of all deck slabs shall be stiffened by composite edge beams and shall be proportioned for the effects of wheel loads. Where the unsupported length of an edge stiffening beam, $S_{e}$, is less than or equal to $5 \mathrm{~m}$ and the slab is designed in accordance with Clause 8.18.4, the details as shown in any one of the diagrams of Figure 8.8 may be considered satisfactory.

Furthermore, precast FDDP needs further analysis for the transverse negative moment due to expected loads from the deck slab overhang and the barrier walls. Deflection and vibration play an important role on the serviceability of bridges. AASHTO-LRFD Bridge Design Specification specifies bridge serviceability deflection limits at L/800 for vehicular bridges and L/1000 for pedestrian bridges as optional criteria where $\mathrm{L}$ is the span of the structural element.

According to the empirical method specified in CHBDC Clause 8.18.4, an isotropic steel reinforcement of $0.3 \%$ ratio is specified in all directions for the bottom and top layers. This approach resulted in $15 \mathrm{M}$ steel bars spaced at $300 \mathrm{~mm}$. CHBDC Section 16 permits the use of GFRP bars in bridge deck slabs. Clause 16.8.18 allows the use of either the flexural or the empirical method for the internally-restrained cast-in-place deck slab. CHBDC included the following conditions of applicability of such methods: (i) the deck shall contain two orthogonal assemblies of the GFRP bars with clear distance between the top and bottom transverse bars of not less than $55 \mathrm{~mm}$, and the diameter for the bars not be less than $15 \mathrm{~mm}$. For the transverse GFRP bars in the bottom assembly, CHBDC specifies the minimum cross-section of GFRP bars to be $500 \mathrm{~d}_{s} / \mathrm{E}_{\mathrm{FRP}}$ in the $\mathrm{mm}^{2} / \mathrm{mm}$ where $\mathrm{d}_{\mathrm{s}}$ is the distance from the compression side of the slab to the centre of the tension reinforcement and $\mathrm{E}_{\mathrm{FRP}}$ is the modulus of elasticity of GFRP bars. This reinforcement ratio was specified to have the same axial stiffness as the average between the minimum and the recommended steel reinforcement ratio allowed by CHBDC Commentary Clause C.16.8.8.1. This approach results in using 16M HM GFRP bars spaced at $150 \mathrm{~mm}$ in the bottom transverse direction with a reinforcement ratio of about $0.90 \%$. Also, CHBDC specifies a minimum ratio of $0.35 \%$ for the longitudinal bars in bottom assembly and both the transverse and longitudinal bars in the top assembly. Moreover, CHBDC refers to the distribution reinforcement to the main reinforcement to be a minimum of $120 /(\mathrm{S})^{0.5}$, up to a maximum of $67 \%$, as a percentage of the main reinforcement, if the main reinforcement is perpendicular to traffic, where $\mathrm{S}$ herein is considered the spacing of the supporting beams in meters. Thus, the 
longitudinal bottom reinforcement for the slab would be M16 HM GFRP bars spaced at $225 \mathrm{~mm}$ with a reinforcement ratio of $0.60 \%$. For the top reinforcement layers, M12 GFRP bars at 200 mm spacing can be used.

Few authors Conducted fatigue tests on steel-reinforced deck slabs (among them: Edalatmanesh and Newhook, 2013a and 2013b; Hwang et al., 2010; Klowak et al., 2007; Sonoda and Horikawa, 1982). Few authors tested two-way concrete bridge decks reinforced with GFRP bars under concentrated wheel loads which failed in punching shear failure mode (Hassan et al., 2013, Dulude et al., 2013; Hassan et al., 2000a and 2000b; El-Ghandour et al., 2003; El-Ghandour et al., 1999; Braimah et. A., 1998). Others (among them: Benmokrane et al., 2006; El-Salakawy et al., 2005; Hacobson et al., 2005; El-Gamal, 2005; Khanna et al., 2000; Matthys and Taerwe, 2000; Banthia et al., 1995; Mufti et al., 1993) tested one-way bridge deck slabs supported over girders and reinforced with GFRP bars and using carbon fibre fabric (among them: Ahmad et al., 1993). Correlation between experimental data and available equations for punching shear capacity was conducted. Few authors tested cast-in-place deck slabs reinforced with GFRP bars under fatigue loading (among them: You et al., 2015; Khalafalla 2014; El-Ragaby et al., 2007; Kumar and GangaRao, 1992) and using CFRP grid (among them: Rahman et al. 2000). Few authors conducted static and fatigue load tests on jointed slabs reinforced with steel bars and supported over girders (among them: Zhu et al., 2012; Graybeal 2010). Others conduced similar tests but on FRP-reinforced jointed deck slabs (among them: Khalafalla, 2014; Hwang and Park, 2014; Gar et al., 2014). Hassan et al. (2000a) concluded that the presence of the top FRP reinforcement in bridge deck slab has negligible effect on the punching shear capacity. Research on fatigue performance on bridge deck slabs showed negligible deterioration of the loaded slabs

Simply supported RC slabs reinforced with FRP grids with different depths of 120 or $150 \mathrm{~mm}$ and different reinforcement ratios. The study resulted in the increase of the punching failure load with the increase of the reinforcement ratio, and enhance of the crack behavior, ultimate load, and stiffness of the fully cracked state (Matthys and Taerwe, 2000). Simply supported square RC slabs reinforced with GFRP and CFRP were loaded with and without shear reinforcement. Slabs failed under the centered concentrated load, and the presence of the shear reinforcement was observed to be inefficient due to the brittleness of the concrete (El-Ghandour et al., 2003 and 1999). It worth mentioning that the fiber reinforced concrete (FRC) deck without internal 
reinforcement supported over restrained girders by transverse steel straps exhibit the necessary internal arching system to carry the wheel load (Mufti et al, 1993). Restrained longitudinal jointed precast bridge decks, also known as deck bulb-tee (DBT), were constructed with 3500mm long, 2500-mm width including the joint width, and 200-mm thickness resting over twin steel girders were cast and tested under fatigue loading as well as static loading to collapse (Khalafalla, 2014). The wheel footprint placed on the center of the slab and loaded with constant amplitude fatigue (CAF) loading for 4 million cycles at $4 \mathrm{~Hz}$ followed by static loading tocollapse, while other slabs were loaded under variable amplitude fatigue (VAF) loading tocollapse at $2 \mathrm{~Hz}$ and less. All restrained slabs failed in punching shear failure mode at a wheel load greater than 3 times the specified CHBDC factored wheel loading.

Traditionally bridges are designed using static loads that include the dynamic load allowance (DLA) due to passing trucks at the ultimate, serviceability and fatigue limit states. It is important to examine the structural behaviour of the jointed precast FDDPs under different fatigue loading conditions which lead to progressive, internal and permanent structural changes in the materials. After crack initiation and propagation, failure is caused by the deterioration of the bond between coarse aggregate, reinforcing bars and the binding matrix. Two types of fatigue loading are considered in testing, namely: constant amplitude fatigue loading (CAF) and variable amplitude fatigue loading (VAF). Fatigue loading is known to reduce the life span for the bridge deck (Karunananda et al., 2010). Constant amplitude fatigue (CAF) is a classical method for fatigue analysis of the material to obtain the three fatigue resistance components for a structure, namely: stress-life (S-N) known as Wöhler curve, strain-life $(\varepsilon-N)$ and fatigue crack growth (FCG). CAF limit is the safe stress level under elastic deformation for design that can take a very large number of cycles, longer than one-million cycles. The Average Daily Truck Traffic (ADTT) of 100 trucks per lane over 25 years produce number of cycles exceeding the CAF limit of two million cycles. Variable amplitude fatigue (VAF) limit investigates the effect of periodic overloading cycles. VAF is based on the same concepts with addition of cycle counting and damage summation due to increasing step loading. However, the resulting stresses are high enough for plastic deformation to occur within the number of cycles very much less than onemillion cycles. 
Few researchers studied fatigue strength of the reinforced concrete slabs under fixed-point pulsating and repetitive moving loads. Experimental tests showed that moving loads produces more fatigue deterioration than the pulsating load, and the influence of the transverse reinforcement enhance the fatigue performance (Sonoda and Horikawa, 1982). Reduced-scale model deck slabs were tested under both pulsating and moving loads, although the moving load produced more fatigue deterioration, but all slabs failed in punching (Perdikaris and Beim, 1988). Under fatigue loading, plain concrete exhibits increasing strain at the beginning forming initial cracks followed by steady state for longer time before it crushes. Typical fatigue damage mechanisms for the FRP bars subjected to fatigue includes matrix cracking, fiber-matrix debonding, void growth and fiber breakage. FRP bar-concrete under fatigue loading may result in abrasion of the bar surface due to shear lag (Adimi et al., 2000; Katz, 2000). Sand-coated GFRP-reinforced slab decks with transverse post-tensioning were constructed and subjected to cyclic loading at the center of the slab with load range of $169.1 \mathrm{kN}$ and frequency of $1 \mathrm{~Hz}$. Monotonic tests were performed every 100,000 cycles up to 4 million cycles at the load of 178 $\mathrm{kN}$ to measure the deterioration. The results shows that the transverse post-tensioning enhance the deck performance and limit the crack growth (Kumar and GangaRao, 1992). A 52.08-m twoequal-span bridge was constructed in Quebec, Canada, named Cookshire-Eaton Bridge, with one span reinforced with steel bars and another span reinforced with GFRP bars (El-Salakawy et al., 2005). The bridge passed the service performance test using calibrated CHBDC truck after one year. Field test results showed no signs of cracks and deflection was under the allowable limits. In Vermont, USA, Morristown Bridge was reinforced with GFRP bars. Field test results revealed the good performance of the GFRP bars (Benmokrane et al., 2006). Full scale deck slabs reinforced with GFRP bars were tested under pulsating concentrated loading up to failure, showing their superior performance (El-Ragaby et al., 2007; Klowak et al., 2007).

The use of the precast FDDPs in bridge construction started in United States in early 1960s, with the purpose to shorten the deck construction time in areas with high traffic volumes. The deckgirder system was primarily non-composite, and the panel-to-panel connections exhibit partial failures. By 1974, FDDPs were made composite with the superstructure by extending the steel shear stud into the deck. The spacing of the shear pockets ranged from $457 \mathrm{~mm}$ to $610 \mathrm{~mm}$ and the number of studs per pocket ranged from 4 to 12. Two sizes of steel studs are typically used, namely: $19 \mathrm{~mm}$ and $22 \mathrm{~mm}$. FDDP were supported on girders and secured to it using shear studs 
embedded in the shear pockets that are normally filled with non-shrink grout to eliminate stress concentrations in the panels (Badie and Tadors, 2008). The transverse panel-to-panel connection is provided with shear keys to protect adjacent panels from relative vertical movement due to traffic load. This type of joint has two types of forces, namely: (i) vertical shear force between the panel and the field-casted joint; and (ii) bending moment that puts the top half of the joint in compression and the bottom half in tension. The panel-to-panel connection has several shapes available in the literature including male-female (tongue/groove) shear key. However, cracking, spalling and leakage were observed in such joints in practice. Other panel-to-panel connections included female-to-female shear key which comes into bulb shape and diamond shape. Splicing longitudinal reinforcement was introduced into overlapping U-bars or using HS spirals, or using open or closed steel tubes (PCI, 2011a, 2011b). Grouting materials to fill the shear pockets and transverse joints have common properties, namely: (i) high strength at young age, (ii) small shrinkage deformation, (iii) superior bonding, and (iv) low permeability (Badie et al., 2006). Steel reinforced lap-splice joints exploit bonding performance with the joint-field materials made of UHPFRC (Hwang and Park, 2014).

Chapter II of this Thesis included conducting pullout tests on GFRP bars embedded into UHPFRC blocks to determine their development length. This led to developing three precast panel connection details that were considered for qualifying tests as one-way slab system under flexural loading in Chapters III and IV for precast FDDPs made of normal concrete and highperformance concrete, respectively. This Chapter reports the experimental program to test the three developed joint details in a real-world situation. Two precast FDDPs were constructed for each developed joint detail and then connected to an available braced twin-steel girder system. For each joint details, one precast FDDP system was tested under CAF loading followed by loading it monotonically to-collapse, and the other one was tested under VAF loading directly tocollapse. Test results are analyzed to examine the fatigue performance and the ultimate load carrying capacity of the developed jointed precast slabs.

\subsection{New Connection Details}

Three details for the joints between precast panels were proposed incorporating GFRP bars as depicted in Figs. 5.2 through 5.9. The first proposed joint has a 200-mm wide closure strip as shown in Fig. 5.2 In this joint, both the top and bottom GFRP bars in the precast slab project into 
the joint with a 175-mm anchorage length. In this joint, the precast panel has projected slab of 90 mm length at the bottom of the joint to hold UHPFRC within the closure strip during casting. A foam-type packing rod is inserted in the 20-mm gap between the two projected slabs at the bottom of the closure strip to avoid material leakage. This joint is called "Angle-shape" joint or "A-joint" in this research. Figure 5.2 shows view of the angle-joint cast for testing. Figure 5.3 shows a plan of the arrangement of FDDPs side-by-side over girders with closure strips oriented transversally between them and shear pockets to connect them to the supporting girders.

The second proposed joint has a 200-mm wide closure strip as shown in Fig. 5.4. This joint is identical to the first proposed joint but without the $90-\mathrm{mm}$ projecting slab. Both the top and bottom GFRP bars in the precast slab project into the joint with a 175-mm anchorage length. It is assumed that temporary form work will be used to close the bottom of the closure strip to hold UHPFRC materials before hardening. This joint is called " $\mathrm{C}$-shape" joint or " $\mathrm{C}$-joint" in this research. Figure 5.4 shows view of the $\mathrm{C}$-joint cast for testing. Figure 5.5 shows a plan of the arrangement of FDDPs side-by-side over girders with closure strips oriented transversally between them and shear pockets to connect them to the supporting girders. The vertical shear key is expected to provide vertical shear friction resistance between the precast concrete and the UHPFRC filling to allow for vertical shear continuity of the slab across the joint.

Figure 5.6 depicts the trapezoidal zigzag-shape panel-to-panel connection with vertical femaleto-female shear key. The slab thickness of $200 \mathrm{~mm}$ is divided vertically into equally four layers. The clear joint width between the ends of the jointed panels is $100 \mathrm{~mm}$, while the zigzag-shape (i.e. trapezoidal tooth-shape) allows for an extension of the joint width of other $100 \mathrm{~mm}$ into the precast panel. So a GFRP bar from the end of one panel at its wide width of the trapezoidal shape will project into the joint with a length of $175 \mathrm{~mm}$ in $200 \mathrm{~mm}$ joint width in the same bar direction (i.e. 100 into the closure strip and $75 \mathrm{~mm}$ into the grooved trapezoidal shape in the adjacent panel). The pullout strength of the embedded GFRP in the joint will be resisted by the bond between its surface and the surrounding UHPFRC filling in addition to the bearing pressure between the UHPFRC filling and the precast concrete at the included surface of the trapezoidal shape at the interface between the two concretes. Such bearing pressure expects to be resisted by the concrete surface normal to the joint at the narrow end of the trapezoidal shape and the GFRP bar projecting through it from the adjacent panel. A vertical shear key is introduced along the 
side of the precast panel as depicted in Figure 5.6.a. Figure 5.7 shows view of the expanded polystyrene foam cut to form the zigzag connection. While Fig. 5.8 shows plan of the GFRP bar arrangement in the zigzag joint along with a view of the joint cast for testing. Figure 5.9 shows a plan of the arrangement of FDDPs side-by-side over girders with closure strips oriented transversally between them and shear pockets to connect them to the supporting girders

\subsection{Experimental Program}

The experimental program included testing two laterally-restrained precast FDDPs supported over braced twin-steel girder bridge system shown in Figs. 5.10 and 5.11. The steel I-girders were $7500 \mathrm{~mm}$ in length and made of W610x241. They were placed over 330x330x25 mm elastomeric pads that were supported over steel pedestals, making the clear spacing of the girder equal $7000 \mathrm{~mm}$. Transverse cross-type bracings were installed at the two ends of the steel girders to provide lateral restraints to the deck slab as specified into the CHBDC empirical design method. The spacing of the twin girders was $2000 \mathrm{~mm}$ measured center-to-center of the girders. Figure 5.13.a shows cross-section details of the precast FDDP resting over the twin girders and connected to it using shear connectors. The M25 high-strength bolts, shown in Fig. 5.12, are used for the panel-to-girder connection. The width of the precast FDDP was taken $2500 \mathrm{~mm}$ so that it can be supported over the twin girders to produce slab span of $2000 \mathrm{~mm}$ as depicted in Figure 5.13. The precast FDDPs were of $200 \mathrm{~mm}$ thickness and were made of $35 \mathrm{MPa}$ normal strength concrete (NSC) with $10 \mathrm{~mm}$ nominal size aggregate, $150 \mathrm{~mm}$ slump with added superplasticizer, and no air-entrant.

Straight-ended, 16M ribbed-surface, HM GFRP bars were used to reinforce the precast FDDP per CHBDC requirements. The bottom and top transverse reinforcement of the slab was taken 16M bars@140 mm and 16M bars@ 200 mm, respectively. While the slab was reinforced with 16M bars@ $200 \mathrm{~mm}$ in the bottom and top longitudinal direction (i.e. parallel to the girder). Materials properties of the HM GFRP bars are listed in Table 5.1. The specified modulus of elasticity and ultimate tensile strength of the GFRP bars were $64 \mathrm{GPa}$ and $1188 \mathrm{MPa}$, respectively (Schoeck, 2013). To form the joint between the precast FDDPs, two precast FDDPs were formed first. The first precast FDDP was of $200 \mathrm{~mm}$ thickness, $2400 \mathrm{~mm}$ length in girder direction and $2500 \mathrm{~mm}$, while the second precast FDDP was of $200 \mathrm{~mm}$ thickness, $1000 \mathrm{~mm}$ length in girder direction and 2500 width. Those two FDDP segments are shown as FDDP 1 and 
FDDP 2 in Fig. 5.13.b. This made the final length of the jointed slab $3700 \mathrm{~mm}$ in the direction of traffic. It should be noted that the short precast FDDP of $1000 \mathrm{~mm}$ was introduced beside the large precast FDDP in Fig. 5.13.b to ensure deck slab continuity beyond the joint.

The panel-to-girder connection was made using shear pockets to achieve the full composite action. Shear bolts were used to establish such full composite action between the girder and the precast panel every $1200 \mathrm{~mm}$. Figures 5.14 through 5.16 shows cross-sections and views of the shear pockets between, as well as at, the transverse closer strips. UHPFRC (Ductal joint-fill JS1000 produced by Lafarge Canada Inc.) as filling materials in the shear pockets and the closure strips. The ultimate strengths of the UHPFRC were 140, 30 and $8 \mathrm{MPa}$ in compression, flexural and direct tension, respectively, while its modulus of elasticity was $50 \mathrm{GPa}$.

The experimental program included testing two precast FDDPs supported over twin-steel girder bridge system using the available force-control hydraulic actuator system. The first precast FDDP system was tested under high-cycle constant-amplitude fatigue (CAF) loading followed by increasing monotonic loading to-collapse. While the second precast FDDP system was tested under low-cycle incremental step fatigue loading of variable amplitude (VAF) to collapse. Table 5.2 presents a summary of fatigue-tested slab configurations. The actuator system generates sinusoidal harmonic force, $p_{t}=p_{\text {avg }}+p_{o} \sin 2 \pi f t$, where $p_{\text {avg }}=p_{\text {static }}$ is the average load of the max and min load, $p_{o}$ is the amplitude of applied load, $f$ is the frequency of the applied load and $t$ is the time. Before performing fatigue tests, a crack was initiated in the tested slab by applying monotonic loading equal to 3 times the applied wheel load for serviceability limit state design per CHBDC $\left(\mathrm{SLS}_{1}=87.5 \mathrm{kN}\right.$ x $\left.1.4 \times 0.9=110.25 \mathrm{kN}\right)$. This applied wheel load $(87.5$ $\mathrm{kN}$ ) equals the heaviest wheel load in the specified CHBDC truck, multiplied with the 1.4 to include the dynamic load allowance (DLA) and 0.9 as the load factor for fatigue limit state design. This makes this load 3 times $\mathrm{SLS}_{1}=110.25 \times 3=330.75 \mathrm{kN}$. The footprint of the applied wheel load on top of the tested slab measured $600 \mathrm{~mm}$ wide by $250 \mathrm{~mm}$ long, and was decided to locate it just beside the joint as depicted in Figure 5.13.b.

The constant amplitude fatigue (CAF) loading was applied under force control with sinusoidal shape to represent the fatigue limit state (FLS) load specified into the CHBDC as FLS $=87.5 \mathrm{x}$ $1.4 \times 1.0=122.5 \mathrm{kN}$ at the frequency of $4 \mathrm{~Hz}$ for 4 million cycles. To prevent rattling of the test setup under cyclic loading, the loading cycle started with $15 \mathrm{kN}$ applied load that increased by 
$122.5 \mathrm{kN}$. Thus, the sinusoidal cyclic CAF ended up with loading range of upper and lower absolute values of $137.5 \mathrm{kN}$ and $15 \mathrm{kN}$, respectively with sample rate of $20.013 \mathrm{~Hz}$. Figure 5.17 shows the CAF loading history applied to the test specimens. Monotonic test at 1.5 time the applied FLS load (i.e. $122.5 \mathrm{kN}$ x $1.5=183.75 \mathrm{kN}$ ) was conducted after each 250,000 cycles to assess the degradation of the FDDP system due to fatigue loading. The force-control monotonic test had a ramp segment shape at loading and unloading rate of $5 \mathrm{kN} / \mathrm{min}$. and $10 \mathrm{kN} / \mathrm{min}$., respectively, with collecting data points every $0.049967 \mathrm{sec}$, per Table 5.3. After the end of the 4 million cycles of CAF loading, the FDDP system was monotonically loaded to-collapse using a hydraulic jack with $1,300 \mathrm{kN}$ capacity. The resulting ultimate load was compared to CHBDC factored design load that was taken as $\mathrm{P}_{\mathrm{f}}=87.5 \times 1.4 \times 1.7=208.25 \mathrm{kN}$, where $87.5 \mathrm{kN}$ is the heaviest wheel load in CHBDC truck, the 1.4 is the dynamic load allowance and 1.7 is the live load factor for ultimate limit state design.

The incremental step variable amplitude fatigue (VAF) loading was applied under force control with sinusoidal shape to different 7 absolute peak levels of 1.0, 1.5, 2.0, 2.5, 3.0, 3.5, and 4.0 times the FLS load of $122.5 \mathrm{kN}$ plus $15 \mathrm{kN}$ as the absolute load lower level. The corresponding peak loads of the 7 incremental step VAF loading were 137.5, 198.75, 260, 321.25, 382.5, 443.75 and $505 \mathrm{kN}$. Each load level was applied for 100,000 cycles at the range of $2 \mathrm{~Hz}$ to 0.5

$\mathrm{Hz}$ depending on the stiffness of the FDDP system and the steel loading frame system, with lowest frequency used when approaching failure of the slab. Data was collected at a sample rate of $20.013 \mathrm{~Hz}$. Monotonic test was performed after each 100,000 cycles with the same setting of the CAF monotonic test. After finishing with 7 absolute peak levels mentioned earlier, the VAF loading testing continued with the highest peak value till collapse. Figure 5.18 shows the VAF loading history considered in this study.

\subsection{Test Results}

This section discusses the structural behavior of the tested specimens in the form of slab vertical deflection, and crack pattern. As mentioned earlier, fatigue precracking was conducted under force control. The first hair flexural crack was observed at about 2.5 times the FLS loading $(275.625 \mathrm{kN})$ underneath the wheel footprint area at the mid-span in the longitudinal direction (parallel to the supporting girders). Load was increased to 3 times the FLS load $(330.75 \mathrm{kN})$ to increase the crack propagation beyond the wheel footprint area. The flexural crack width was 
found to be $80 \mu \mathrm{m}$ at that static load. CHBDC specifies that design factored ultimate limit state (ULS) load of the deck slab is the multiplication of CHBDC truck wheel load of $87.5 \mathrm{kN}$, load factor of 1.7 and dynamic load allowance of 1.40. This makes the factored design applied load $\mathrm{ULS}_{1}=87.5 \times 1.4 \times 1.7=208.25 \mathrm{kN}$. It is interesting to mention that the precracking monotonic load of $330.75 \mathrm{kN}$, at which a minor flexural crack appeared, is about $59 \%$ greater than the CHBDC factored design load of $208.25 \mathrm{kN}$.

\subsubsection{Behavior of the A-Jointed Precast FDDP}

Figure 5.19 depicts the construction of the precast FDDP with the A-shape connection type along with the precast FDDPs placed side-by-side before placing UHPFRC in the closure strip. Figure 5.20 views the test setup for the fatigue loading for specimens S1 and S2, while Figure 5.21 views the test setup for the monotonic loading-to-collapse for slab S1.

\subsubsection{Constant Amplitude Fatigue Loading}

For the tested specimen under CAF loading, the compressive strength of the concrete cylinders taken from the concrete mix were $60.76,59.83$, and $54.26 \mathrm{MPa}$, with an average value of 58.28 MPa. The tested cylinders for the UHPFRC resulted in compressive strengths of 161.94, 163.30, 170.54 and 159.20 MPa, with an average value of 161.48 MPa. During the initiation of fatigue precracking procedure, at a static load of $220.5 \mathrm{kN}$, flexural crack propagated from underneath the mid-point of wheel footprint about $100 \mathrm{~mm}$ towards the middle shear pockets shown at the middle of the precast slab segment shown in Figure 5.19.a. When the applied load increased to $275.625 \mathrm{kN}$, the flexural crack propagated further another $300 \mathrm{~mm}$. However, when the applied load reached $330.75 \mathrm{kN}$, the flexural crack propagated diagonally from underneath the mid-point of the wheel footprint to the closest corner of the middle shear pocket. The maximum recorded flexural crack width at that point measured $80 \mu \mathrm{m}$. No more flexural cracks were observed during the CAF test that last over 16 days. After each 250,000 cycles, the slab was subjected to monotonic loading to observe the change in slab flexural stiffness through deflection measurements. Figure 5.22.a depicts the load-deflection relationship for the slab at the centre of the footprint of the wheel load. It can be observed that the slope of the curves after each group of fatigue cycles appeared unchanged and maintained linear. After the 4-million fatigue cycles, the slab was subjected to monotonic load to-collapse. The precast FDDP failed due to punching 
shear at a jacking load of $930.92 \mathrm{kN}$. Figure 5.22.b. depicts recorded slab deflection at the midlength of the free edge of the short slab shown as location of LVDT 1 in Fig. 13.b, noted as "Free end" curve in Figure 5.22.b. Such deflection reached $10.68 \mathrm{~mm}$ at failure. On the other hand, deflection under the wheel footprint shown as location of LVDT 2 in Fig. 5.13, denoted as "Point load" in Fig. 5.22.b, were recorded as 23.05 and $23.88 \mathrm{~mm}$ at failure since 2 LVDTs were installed at this location. The deflection at the centre of the long precast slab shown at location of LVDT 3 in Fig. 5.13.b, denoted as "Mid-span" in Fig. 5.22.b was recorded as $13.09 \mathrm{~mm}$. The maximum deflection of the long precast slab at the mid length of the edge joint shown as location of LVDT 4 in Fig. 5.13.b, denoted as "Fixed end" in Figure 5.22.b was recorded as 6.80 $\mathrm{mm}$ at failure.

It is interesting to mention that such failure load is about 4.47 times the CHBDC factored design wheel load. Figure 5.23.a presents top view of the slab showing punching shear failure at the footprint of the wheel load. While Figure 5.23.b shows bottom view of the slab showing crack pattern after failure due to the fan mechanism. One may observe the radial cracks starting from the location of the footprint of the wheel load and propagating towards the support line in a fan shape. At failure, concrete spalling appeared in some parts of the bottom side of the slabs as signs for punching shear failure. However, such concrete spalling appeared only in the large FDDP slab but it did not extend to the short FDDP segment in the other side of the closure strip.

\subsubsection{Variable Amplitude Fatigue Loading}

The second precast FDDP specimen underwent sinusoidal waveform fatigue load cycles with incremental step low cycle fatigue loading. The compressive strengths of concrete cylinders for the normal strength concrete (NSC) used to cast this slab were 54.29, 57.22, 59.98, 46.54, 65.84, 64.7 MPa, with an average value of 58.10 MPa. The splitting tensile test for the NSC resulted in tensile strength of $3.53,5.73,5.31,4.3$ and $4.7 \mathrm{MPa}$, with an average value of $4.71 \mathrm{MPa}$. The compressive strengths of the concrete cylinder for the UHPFRC used to fill the joints were 154.17, 188.12, 184.61 and 181.91 MPa, with an average value of 179.52 MPa. The splitting

tensile test for the UHPFRC resulted in tensile strength of $15.12,12.14,15.76 \mathrm{MPa}$, with an average value of $14.42 \mathrm{MPa}$. 
The first 501,002 fatigue load cycles were performed at a frequency of $2 \mathrm{~Hz}$, then followed by 160,242 cycles at $1 \mathrm{~Hz}$, and finally followed by 130,139 cycles at $0.5 \mathrm{~Hz}$ leading to punching shear failure at a total number of cycles of 809,493. Figure 5.24 depicts the 60-mm-deep punching shear failure at wheel footprint on top of the slab. While Figure 5.25.a depicts the crack pattern at the bottom surface of the slab at failure. One may observe the radial cracks starting from the location of the footprint of the wheel load and propagating towards the support line in a fan shape. At failure, concrete spalling appeared in some parts of the bottom side of the large slab on one side only of the closure strip as a sign for punching shear failure. However, ocncrete spalling appeared to deviate from the tradional shape for punching failure (close to circular shape at the end of diagonal cracks through slab thickness). However, major flexural crack appeared at failure just under the wheel load, extending in the direction of the girder towards the free end of the small FDDP segment while crossing the closure strip as depcited in 5.24.c. This precast FDDP failed at a jacking load of $487.50 \mathrm{kN}$ and a maximum slab deflection of $32.46 \mathrm{~mm}$. It is interesting to mention that such failure load is about 2.34 times the CHBDC factored design wheel load. Figure 5.25 depicts the monotonic load-deflection relationship of the slab after each 100,000 fatigue load cycles. It can be observed the slope of the curve decreased, leading to a reduction in slab flexural stiffness, with increase in number of VAF load cycles. Table 5.4 summarizes test data for specimens $\mathrm{S} 1$ and S2 with A-joint under CAF and VAF loading, respectively.

\subsubsection{Stiffness Degradation}

The stiffness degradation of precast FDDPs under flexural-shear loading was calculated in the form of spring stiffness, $\mathrm{k}$. $\mathrm{k}$ is considered as the ratio between the applied monotonic load, $\mathrm{F}$, in $\mathrm{kN}$ and the corresponding slab deflection, $\mathrm{d}$, in $\mathrm{mm}$. Stiffness degradation in reinforced concrete elements is the result of cracking, loss of bond, and interaction with higher shear or flexural stresses. The level of stiffness degradation depends on the characteristics of the structure as of material properties (P-delta effect), geometry level, level of connection ductility, the loading history as well as the combination of them. Table 5.5 summarizes the results for the CAF loading and for the VAF loading. Figures 5.26.a and 5.26.b depict the relationship between the spring stiffness and the number of fatigue cycles and slab deflection, respectively. One may observe that the first specimen's stiffness degraded by about $53.8 \%$ after 4 million cycles of constant 
amplitude fatigue loading. On the other hand, the second specimen's stiffness degraded by $86.86 \%$ when subjected to variable amplitude fatigue loading before complete collapse.

\subsubsection{Behavior of the C-Jointed Precast FDDP}

Figures 5.27 and 5.28 show schematics diagrams and photos for the construction of the precast FDDP with the C-shape connection type. While Fig. 5.29 shows details of reinforcement in the precast slab. Figures 5.30 and 5.31 show the precast FDDPs placed side-by-side on the top of the twin-girder and ready for the placement of the UHPFRC in the closure strip and shear pockets. Figure 5.32 view the test setup for the fatigue loading for slabs S3 and S4, while Fig. 5.33 views the test setup for the monotonic loading-to-collapse of slab S3.

\subsubsection{Constant Amplitude Fatigue Loading}

For the tested specimen under CAF loading, the compressive strength of the concrete cylinders taken from the concrete mix were 58.10, 57.08, and 54.87 MPa, with an average value of 56.68 $\mathrm{MPa}$. The tested cylinders for the UHPFRC, that were cast 10 days before the start of the fatigue testing, resulted in compressive strengths of 168.03, 162.33, 170.54 and 149.22 $\mathrm{MPa}$, with an average value of $162.53 \mathrm{MPa}$.

During the initiation of fatigue precracking procedure, at a static load of $220.5 \mathrm{kN}$, flexural crack propagated from underneath the mid-point of wheel footprint about $100 \mathrm{~mm}$ towards the middle shear pockets shown at the middle of the precast slab segment shown in Figure 5.27(a). When the applied load increased to $275.625 \mathrm{kN}$, the flexural crack propagated further another $300 \mathrm{~mm}$. However, when the applied load reached $330.75 \mathrm{kN}$, the flexural crack propagated diagonally from underneath the mid-point of the wheel footprint to the closest corner of the middle shear pocket. The maximum recorded flexural crack width at that point measured $80 \mu \mathrm{m}$. No more flexural cracks were observed during the CAF test that last over 16 days. After each 250,000 cycles, the slab was subjected to monotonic loading to observe the change in slab flexural stiffness through deflection measurements. Figure 5.34.a depicts the load-deflection relationship for the slab at the centre of the footprint of the wheel load. It can be observed that the slope of the curves after each group of fatigue cycles appeared unchanged and maintained linear. After the 4-million fatigue cycles, the slab was subjected to monotonic load to-collapse. The precast FDDP failed due to punching shear at a jacking load of $973 \mathrm{kN}$. It is interesting to mention that 
such failure load is about 4.67 times the CHBDC factored design wheel load. Figure 5.35.a presents the top view of the slab showing punching shear failure at the footprint of the wheel load. While Fig. 5.35.b presents bottom view of the slab showing crack pattern after failure. One may observe the radial cracks starting from the location of the footprint of the wheel load and propagating towards the support line in a fan shape. At failure, concrete spalling appeared in some parts of the bottom side of the slabs on one side of the closure strip as a sign for punching shear failure. However, this concrete spalling did not extend through the closure strip to the adjacent FDDP. Fig. 5.35.b shows that concrete diagonal crack at the end of the bottom perimeter of the punching shear plane appeared passing through UHPFRC on one side of the closure strip. In addition, fan-shape cracks at the bottom surface of the slab passed through the UHPFRC but less inetnsive as those in the precast slab. Figure 5.34.b depicts the load-deflection relationship for the tested slab under static loading to-collapse. Deflections values were recorded at the mid-length of the free edge of the short slab shown in Fig. 5.13.b , noted as "Free end" curve in Figure 5.34.b. Such deflection reached $2.54 \mathrm{~mm}$ at failure. On the other hand, deflection under the wheel footprint, denoted as "Point load" in Figure 5.33.b was recorded as $25.98 \mathrm{~mm}$ at failure. The deflection at the centre of the long precast slab, denoted as "Mid-span" in Figure 5.33.b was recorded as $17.83 \mathrm{~mm}$. The maximum deflection of the long precast slab at the mid length of the edge joint, denoted as "Fixed end" in Figure 5.34.b was recorded as $3.73 \mathrm{~mm}$ at failure.

\subsubsection{Variable Amplitude Fatigue}

The second precast FDDP specimen with C-joint underwent sinusoidal waveform fatigue load cycles with incremental step low cycle fatigue loading. The compressive strengths of concrete cylinders for the NSC used to cast this slab were 67.78, 64.93, 65.63, and 67.81 MPa, with an average value of $66.54 \mathrm{MPa}$. The compressive strengths of the concrete cylinder for the UHPFRC used to fill the joints were 147.60, 150.69, and 151.45 MPa, with an average value of 149.91 MPa. The first 500,000 fatigue load cycles were performed at a frequency of $2 \mathrm{~Hz}$, then followed by 100,000 cycles at $1 \mathrm{~Hz}$, and finally followed by 92,866 cycles at $1 \mathrm{~Hz}$ leading to punching shear failure at a total number of cycles of 692,866. Figure 5.36.a depicts the punching

shear failure at wheel footprint on top of the slab. While Figure 5.36.a depicts the crack pattern at the bottom surface of the slab at failure. A fan-shape crack pattern was observed at the bottom 
surface similar to those developed for the slab tested to collapse after passing the CAF loading. However, Figure 5.36.b shows greater concrete spalling along the perimeter on the punching shear plane at the bottom of the slab but only from one side of the closure strip. At failure of the specimen, major flexural crack appeared at the wheel load location and extended to the free edge of the short FDDP segment as depicted in Fig. 5.36.b. This led to the conclusion that the failure mode is primarily punching shear combined with flexural failure in the adjacent short FDDP. It should be noted that very few flexural cracks appeared at the bottom of UHPFRC in slab S4 tested under VAF loading, as depicted in Fig. 5.36.b, when compared to intensive cracks through UHPFRC in slab S3 tested under CAF loading, as depicted in Fig. 5.35.b. This slab failed at a jacking load of $495.69 \mathrm{kN}$ and a maximum slab deflection of $40.89 \mathrm{~mm}$. It is interesting to mention that such failure load is about 2.38 times the CHBDC factored design wheel load. Figure 5.37 depicts the monotonic load-deflection relationship of the slab after each 100,000 fatigue load cycles. It can be observed the slope of the curve decreased, leading to a reduction in slab flexural stiffness, with increase in number of VAF load cycles.

\subsubsection{Stiffness Degradation}

The stiffness degradation of precast FDDPs under flexural loading was calculated in the form of spring stiffness $(\mathrm{k}) . \mathrm{k}$ is considered as the ration between the applied monotonic load, $\mathrm{F}$, in $\mathrm{kN}$ and corresponding slab deflection, $\mathrm{d}$, in $\mathrm{mm}$. Table 5.6 summarizes the results for the CAF loading and for the VAF loading. Figures 5.38.a and 5.38.b depict the relationship between the spring stiffness and the number of fatigue cycles and slab deflection, respectively. One may observe that the first specimen's stiffness degraded by about $50.73 \%$ after 4 million cycles of constant amplitude fatigue loading. On the other hand, the second specimen's stiffness degraded by $63.24 \%$ when subjected to variable amplitude fatigue loading before complete collapse.

\subsubsection{Behavior of the Z-Jointed Precast FDDP}

Figures 5.39 through 5.42 depict the construction of the precast FDDP with the Z-shape connection type. Figure 5.43 shows view of the FDDPs placed side-by-side to form the closure strip before casting UHPFRC into the joint. Figure 5.44 view the test setup for the fatigue loading, while Figure 5.45 views the test setup for the monotonic loading-to-collapse. 


\subsubsection{Constant Amplitude Fatigue Loading}

For the tested specimen under CAF loading, compressive strength of the concrete cylinders taken from the concrete mix were 41.16, 35.32 and $35.028 \mathrm{MPa}$, with an average value of $37 \mathrm{MPa}$. The tested cylinders for the UHPFRC, which were cast 10 days before the start of the fatigue testing, resulted in compressive strengths of $130.90,136.43,114.96 \mathrm{MPa}$, with an average value of 127 $\mathrm{MPa}$. The splitting tensile tests for the NSC resulted in tensile strength of concrete of 3.37, 2.72, and 3.61 $\mathrm{MPa}$, with an average value of 3.23 MPa. During the initiation of fatigue precracking procedure, at a static load of $220.5 \mathrm{kN}$, flexural crack propagated from underneath the mid-point of wheel footprint about $100 \mathrm{~mm}$ towards the middle shear pockets shown at the middle of the precast slab segment shown in Fig. 5.39.a. When the applied load increased to $275.625 \mathrm{kN}$, the flexural crack propagated further another $300 \mathrm{~mm}$. However, when the applied load reached $330.75 \mathrm{kN}$, the flexural crack propagated diagonally from the mid-point of the wheel footprint to the closest corner of the middle shear pocket. The maximum recorded flexural crack width at that point measured $80 \mu \mathrm{m}$. No more flexural cracks were observed during the CAF test that last over 16 days.

After each 500,000 cycles, the slab was subjected to monotonic loading to observe the change in slab flexural stiffness through deflection measurements. Figure 5.46.a depicts the load-deflection relationship for the slab at the centre of the footprint of the wheel load. It can be observed that the slope of the curves after each group of fatigue cycles appeared unchanged and maintained linear. After the 4-million fatigue cycles, the slab was subjected to monotonic load to-collapse. The precast FDDP failed due to punching shear at a jacking load of $931 \mathrm{kN}$. It is interesting to mention that such failure load is about 4.47 times the CHBDC factored design wheel load. Figure 5.46.b depicts the load-deflection relationship for the tested slab under static loading tocollapse. Deflections values were recorded at the mid-length of the free edge of the short slab shown in Figure 5.39.a, noted as "Free end" curve in Figure 5.47(b). Such deflection reached $1.78 \mathrm{~mm}$ at failure. On the other hand, deflections under the wheel footprint, denoted as "Under load 1 and Under load 2" in Figure 5.45.b were recorded as 30.29 and $28.94 \mathrm{~mm}$ at failure, respectively. The deflection at the centre of the long precast slab, denoted as "Mid-span" in Fig. 5.45.b was recorded as $19.30 \mathrm{~mm}$. The maximum deflection of the long precast slab at the mid length of the edge joint, denoted as "Fixed end" in Figure 5.46.b was recorded as $2.65 \mathrm{~mm}$ at 
failure. Figure 5.47.a presents top view of the slab showing punching shear failure at the footprint of the wheel load. While Fig. 5.47.b presents bottom view of the slab showing crack pattern after failure. One may observe the radial cracks starting from the location of the footprint of the wheel load and propagating towards the support line in a fan shape. At failure, concrete spalling appeared in some parts of the bottom side of the slabs as signs for punching shear failure.

\subsubsection{Variable Amplitude Fatigue Loading}

The second precast FDDP specimen underwent sinusoidal waveform fatigue load cycles with incremental step low cycle fatigue loading. The compressive strengths of concrete cylinders for the NSC used to cast this slab were 43.26, 68.16, 64.99, 65.74 MPa, with an average value of 60 MPa. The compressive strengths of the concrete cylinder for the UHPFRC used to fill the joints were 163.35, 183.31, 153.28 MPa, with an average value of $167 \mathrm{MPa}$. The splitting tensile test for the UHPFRC resulted in tensile strength of 18.30, 20.47 and $21.69 \mathrm{MPa}$, with an average value of $20 \mathrm{MPa}$. The first 895,000 fatigue load cycles were performed at a frequency of $2 \mathrm{~Hz}$, then followed by 21,736 cycles at $1 \mathrm{~Hz}$, and finally followed by 44,804 cycles at $0.5 \mathrm{~Hz}$ leading to punching shear failure at a total number of cycles of 961,540. Figure 5.48.a depicts the punching shear failure at wheel footprint on top of the slab. While Figure 5.48.b depicts the crack pattern at the bottom surface of the slab at failure. A fan-shape crack pattern was observed at the bottom surface similar to those developed for the slab tested to collapse after passing the CAF loading. However, greater concrete spalling appeared along the perimeter on the punching shear plane at the bottom of the slab but only from one side of the closure strip, when compared to the failure mode shown in Fig. 5.47.b for slab S5 subjected to CAF loading. This precast FDDP failed at a jacking load of $488.43 \mathrm{kN}$ and a maximum slab deflection of $37.03 \mathrm{~mm}$. It is interesting to mention that such failure load is about 2.35 times the CHBDC factored design wheel load. Figure 5.49 depicts the monotonic load-deflection relationship of the slab after each 100,000 fatigue load cycles. It can be observed the slope of the curve decreased, leading to a reduction in slab flexural stiffness, with increase in number of VAF load cycles. 


\subsubsection{Stiffness Degradation}

The stiffness degradation of slabs under flexural loading was calculated in the form of spring stiffness $(\mathrm{k}) . \mathrm{k}$ is considered as the ratio between the applied monotonic load, $\mathrm{F}$, in $\mathrm{kN}$ and corresponding slab deflection, $\mathrm{d}$, in $\mathrm{mm}$. Table 5.7 summarizes the results for the CAF loading and for the VAF loading. Figures 5.50.a and 5.50.b depict the relationship between the spring stiffness and the number of fatigue cycles and slab deflection, respectively. One may observe that the first specimen's stiffness degraded by about $21.9 \%$ after 4 million cycles of constant amplitude fatigue loading. On the other hand, the second specimen's stiffness degraded by $71.32 \%$ when subjected to variable amplitude fatigue loading before complete collapse. Figure 5.51 shows comparison of slab degradation under CAF and VAF loading.

\subsection{Life Estimation of Fatigue of GFRP-Reinforced Precast FDDP}

Realistic representation of the service loads are usually variable amplitude that should consider the accurate measure of the applied load on existing structure and predict loads on the structure that does not exist yet. Loads can be obtained from real-life histories or through simplified segmental loading. The fatigue cycle counting methods is to compare the effect of the variable amplitude fatigue load histories to the fatigue data and curves obtained with the simple constant amplitude fatigue loading cycles. Applying linear damage rule where cumulative linear damage, $\mathrm{D},=1.0$ that requires the knowledge of the mean and amplitude of load to which the damaging event is compared. One approach to the variable load histories is the concept of the damage, known as fraction life or cycle ratio. These fractions are added together with the sum of 1.0 as defined into Equation 5.5 by the linear damage rule as proposed by Palmgren and later again by Miner.

$$
D=\sum \frac{n_{i}}{N_{f i}}=1
$$

Where $\mathrm{n}$ is the number of cycles, and $\mathrm{N}_{\mathrm{f}}$ is the number of repetitions of the same cycle that equals life to failure. The damaging effect of $\mathrm{n}_{1}$ cycles at $\mathrm{P}_{1}$ load amplitude is assume to be $n_{1} D_{1}=n_{1} / N_{f 1}$, while the damaging effect of $\mathrm{n}_{2}$ cycles at $\mathrm{P}_{2}$ load amplitude is assumed to be $n_{2} D_{2}=n_{2} / N_{f 2}$. Similarly, the cycle ratio or damage caused by $n_{i}$ cycles at $P_{i}$ load amplitude is $n_{i} D_{i}=n_{i} / N_{f i}$. Failure is predicted when the sum of all ratios becomes 1 or $100 \%$. The 
assumption of the linear damage depends on the rate of damage accumulation and load amplitude, that leads to $n_{i} / N_{f i} \neq 1$ for a low-to-high or a high-to-low loading sequences. Miner's Rule doesn't account for overload or high stress which may occur in compressive residual stress that lead to retarding of the crack growth. High-to-low stresses may have less damage due to the presence of compressive residual stress. However, it is widely used because of simplicity and hardly to achieve better agreement with the current experimental data. Nonlinear damage theories proposed $D=\sum\left(n_{i} / N_{f i}\right)^{\alpha_{l}}$ where $\alpha_{l}$ depends on the load level. When considering the change of load level to be $P / P_{u}$, the author proposes the nonlinearity of the damaging effect to the step loading through Equation 5.2, as result of the observed stiffness degradations, keeping the linearity of $D=1$ and solving for the $\eta$ using the nonlinear least square regression analysis (NLREG).

$$
N_{f}=e^{\eta\left(1-P / P_{u}\right)}
$$

Where $\eta$ equals to 25.86, 24.32 and 25.609 for the A-Joint, C-Joint and Z-Joint, respectively. Table 5.8 through 5.11 illustrate the fatigue data where $\mathrm{D}=1$ for all type of joints of the precast FDDPs. The proposed model to determine P-N effect is shown in Equation 5.3, where K equals to $0.039,0.041$ and 0.039 for the A-Joint, C-Joint and Z-Joint, respectively. For static failure $\mathrm{N}=$ 1, the model yields to $P / P_{u}=1$.

(5.3) $\frac{P}{P_{u}}=-K \ln \left(N_{f}\right)+1$

Figures 5.52 and 5.53 show representation of the P-N fatigue curves for the developed model compared to the recent developed models on log-scale graphs compared to other fatigue models (Khalafalla, 2014; El-Ragaby et al, 2007; Memon, 2005; Mufti et al., 2002). Equations 5.4 to 5.7 show the recent fatigue models. The common observations that these model by its current parameters are over estimating the fatigue life since $D \neq 1$.

Khalafalla's model

$$
\begin{array}{ll}
\text { (5.4.a) } & \frac{P}{P_{u}}=1-\frac{\ln (N)}{29} \\
\text { (5.4.b) } & N=e^{-29\left(P / P_{u}\right)+29}
\end{array}
$$


El-Ragaby et al. model

$$
\frac{P}{P_{u}}=0.0034(\log (N))^{2}-0.1187 \log (N)+1.0752
$$$$
N=e^{40.19-0.0147 \sqrt{-2.826 \times 10^{5}+7.21 \times 10^{6}\left(P / P_{u}\right)}}
$$

Memon's model

$$
\log N=5.737 \sqrt{\frac{1-R}{R}}
$$

Mufti et al. model

$$
N=10^{5.737} \sqrt{\frac{1-R}{R}}
$$

\subsection{Punching Shear Strength}

The punching shear strength of the two-way slab system without shear reinforcement rely on six principals, namely: (a) the concrete strength; (b) the ratio of the side length of the loaded area to the effective depth of the slab (c/d); (c) the ratio of the shear-to-moment near the critical section (V/M); (d) the load shape in terms of the ratio, $\beta_{c}$, of the long side to the short side of the rectangular load; (e) lateral restraints of the slab as stiff beams along the boundaries; and (f) the rate of loading (Wang and Salmon, 2002). The failure modes due to concentrated load over a slab may be in the form of: (a) shear-compression failure, due to typical deep section of short span (low a/d ratios) forming inclined cracks; (b) flexural failure after inclined cracks formed (typically with low a/d ratio); and (c) diagonal tension failure, known as punching shear, that occurs with medium span-to-average depth sections (intermediate values of a/d), on which the slab fails due to inclined cracks around the perimeter of the concentrated load, known as the 
critical section at $\mathrm{d} / 2$ up to $1.5 \mathrm{~d}$ per data available in the literature; and (d) flexure failure before the formation of the inclined cracks (large values of a/d).

The nominal punching shear strength $V_{n}=V_{c}$ for slabs with longitudinal steel bars and without shear reinforcement has been introduced by different design standards. The literature review revealed few equations to determine the punching shear capacity of reinforced concrete slabs (among them: CSA.S6-14; CSA S806-12; ACI 318-08; Jacobson et al., 2007; ACI 440.1R-06; El-Gamal et al., 2005; Ospina et al. 2003; Matthys and Taerwe, 2000; El-Ghandour et al, 1999).

ACI 318-08 provided the following equation to determine the punching shear capacity for steelreinforced slab, considering the critical section for punching shear plane at $0.5 \mathrm{~d}$ from the face of the column of applied patch loading area.

$$
V_{c}=\min \left\{\begin{array}{c}
0.33 \sqrt{f_{c}^{\prime}} b_{0.5} d \\
\left(0.167+\frac{0.33}{\beta_{c}}\right) \sqrt{f_{c}^{\prime}} b_{0.5} d \\
\left(0.167+\frac{3.32 d}{b_{o}}\right) \sqrt{f_{c}^{\prime}} b_{0.5} d
\end{array}\right.
$$

Where $d$ is the effective depth of the slab to the tension reinforcement, $b_{0.5}$ is the perimeter of the critical section for punching shear plane located at distance $0.5 \mathrm{~d}$ from the side of the column or applied load area and $b_{0}$ is the perimeter of the column or applied load area.

El-Ghandour et al. (2000) modifies the ACI 318 punching shear equation as in equation 5.9 by introducing the ratio of the modulus of elasticity of FRP, $\mathrm{E}_{\mathrm{f}}$, to that for steel reinforcement, $\mathrm{E}_{\mathrm{s}}$, powered to $1 / 3$, considering the critical section for punching shear at $0.5 \mathrm{~d}$ from the face of the column or the applied load area.

$$
V_{c}=0.33 \sqrt{f_{c}^{\prime}}\left(\frac{E_{f}}{E_{s}}\right)^{1 / 3} b_{0.5} d
$$

Matthys and Taerwe (2000) proposed the following modification to the BS 8110 punching shear equation for the two-way slabs reinforced with steel bars to account for the FRP bars, considering the critical section for punching shear at $1.5 \mathrm{~d}$ from the face of the column or applied load area. 


$$
V_{c}=1.36\left[100 \rho_{f} f_{c}^{\prime}\left(\frac{E_{f}}{E_{s}}\right)\right]^{1 / 3}\left(\frac{1}{d}\right)^{1 / 4} b_{1.5} d
$$

Opsina et al. (2003) refined Matthys and Taerwe's equation as follows by adjusting the coefficient to fit with the test data, while maintaining the critical section for punching shear at $1.5 \mathrm{~d}$ from the face of the column or applied load area.

$$
V_{c}=2.77\left(\rho_{f} f_{c}^{\prime}\right)^{1 / 3} \sqrt{\frac{E_{f}}{E_{s}}} b_{1.5} d
$$

El-Gamal et al. (2005) modified the ACI 318 punching shear equation by adding the effect of the flexural stiffness of the main bottom reinforcement, noted as $\alpha$, and considering the effect of the continuity of the slabs, noted as $\mathrm{N}$ where $\mathrm{N}=0$ for simple-span slab in both directions, 1 for slab continuous in one direction and 2 for slab continuous in two directions.

$$
\begin{aligned}
& V_{c}=0.33 \sqrt{f_{c}^{\prime}} b_{0.5} d \alpha(1.2)^{N} \\
& \alpha=0.62\left(\rho_{f} E_{f}\right)^{\frac{1}{3}}\left(1+\frac{8 d}{b_{o}}\right)
\end{aligned}
$$

Where $E_{f}$ is the modulus of elasticity of the FRP reinforcement in GPa and $\rho_{\mathrm{f}}$ is the FRP reinforcement ratio.

The ACI 440.1R.06 (2006) provided the following punching shear equation, considering the critical section for punching shear at $0.5 \mathrm{~d}$ from the face of the column or applied load area.

$$
\begin{aligned}
& V_{c}=\frac{4}{5} \sqrt{f_{c}^{\prime}} b_{0.5} c \\
& k=\sqrt{2 \rho_{f} n_{f}+\left(\rho_{f} n_{f}\right)^{2}}-\rho_{f} n_{f} \\
& n_{f}=E_{f} / E_{c} \text { and } \rho_{f}=\frac{A_{f}}{b d} \times 100 \\
& E_{c}=4500 \sqrt{f_{c}^{\prime}} \\
& c=k d
\end{aligned}
$$


The CSA S806-12 (2012) provided the following punching shear equation to determine the punching shear capacity for FRP reinforced slab, critical section for punching shear at $0.5 \mathrm{~d}$ from the face of the column or applied load area.

$$
V_{c}=\min \left\{\begin{array}{c}
\left(0.028+\frac{0.056}{\beta_{c}}\right)\left(E_{f} \rho_{f} f_{c}^{\prime}\right)^{1 / 3} b_{0.5} d \\
\left(0.028+\frac{0.588 d}{b_{o}}\right)\left(E_{f} \rho_{f} f_{c}^{\prime}\right)^{1 / 3} b_{0.5} d \\
0.056\left(E_{f} \rho_{f} f_{c}^{\prime}\right)^{1 / 3} b_{0.5} d
\end{array}\right.
$$

Jacobson et al. (2007) developed punching shear model for double layer glass/vinylester mechanically-connected FRP double-layer pultruded grating-reinforced concrete slab, with some level of edge restraint. This is model is an empirical modification of Matthys and Taerwe's model, considering the critical section for punching shear at $1.5 \mathrm{~d}$ from the face of the column or applied load area.

$$
V_{c}=4.5 \frac{\left(\rho_{f} f_{c}^{\prime}\right)^{1 / 3}}{d^{1 / 4}} b_{1.5} d
$$

The Japan Society of Civil Engineering (JSCE, 1997) provided an empirically-based code equation for the punching shear of FRP reinforced concrete deck, as follow:

$$
\begin{aligned}
& V_{c}=\beta_{d} \beta_{p} \beta_{r} \frac{f_{p c d}}{\gamma_{b}} b_{0.5} d \\
& \beta_{d}=\sqrt[4]{\frac{1}{d}} \leq 1.5 \quad \beta_{p}=\sqrt[3]{100 \rho_{f} \frac{E_{f}}{E_{s}}} \leq 1.5 \quad \beta_{r}=1+\frac{1}{\left(1+0.25 \frac{b_{o}}{d}\right)} \\
& f_{p c d}=0.2 \sqrt{f_{c d}} \leq 1.2 \mathrm{MPa} \\
& \gamma_{b}=1.3 \text { and } 1.5 \text { for } f_{c d}<50 \mathrm{MPa} \text { and }>50 \mathrm{MPa}, \text { respectively. }
\end{aligned}
$$

It should be noted that to compare the experimental data with this equation, the partial safety factor, $\gamma_{b}$, was set to 1.0 to get the unfactored prediction capacity, and the limitations of $\beta_{d}, \beta_{p}$, $f_{p c d}$ were removed and actual values were used. 
CHBDC (CSA-S6, 2014) clause 8.9.4.3 provided the following equation for the punching shear resistance of the concrete section reinforced with steel bars while CHBDC Chapter 16 did not refer to punching shear strength or two-way actions in slabs reinforced with FRP bars.

$$
\begin{aligned}
& V_{c}=\phi_{c} f_{c r} b_{0.5 d} d \\
& f_{c r}=0.4 \sqrt{f_{c}^{\prime}} \text { and } \phi_{c}=0.75
\end{aligned}
$$

To correlate the punching shear strength values obtained from the above mentioned equations with those obtained experimentally, all resistance factors in these equations were taken as 1 . To obtain the punching shear angle at each side of the loaded area, slabs S1, S3 and S5 were sliced at the load location in both the transverse direction (normal to the steel girders) and the longitudinal direction. Figures 5.54 and 5.55 show photos of the punching shear failure in the tested slab when sliced longitudinally and transversally at the wheel load location. While Table 5.12 and Fig. 5.56 depict the location of the critical section due to punching shear failure in the tested slabs. It can be observed that the average punching shear angles were $21.78^{\circ}$ in the transverse direction, and $13.33^{\circ}$ and $15.30^{\circ}$ at each side of the loaded area in the longitudinal direction. To be in the conservative side, the punching shear angle will be considered $21.78^{\circ}$ all around the perimeter of the applied load. In this case, the critical section of punching shear failure will be located at $2.5 \mathrm{~d}$ from the face of the loaded area. However, for the sake of comparing experimental findings with available punching shear strength equations, the later were calculated with critical punching shear perimeter per code the equation requirement (i.e. using either $0.5 \mathrm{~d}$ or $1.5 \mathrm{~d}$ ). Table 5.13 shows the correlation between the punching shear strength of the tested slabs obtained experimentally and from available equation in the literature. One may observe that the available equations in the literature are generally conservative in predicting the punching shear capacity of the tested slab with significant margin in many cases. As such, a new punching shear strength model was developed herein to better predict the capacity of the tested slabs considering the critical perimeter for punching shear failure at $2.5 \mathrm{~d}$ from the face of the loaded area as shown in the next section. 


\subsubsection{Developed Punching Shear Model}

Based on the experimental findings, the following equation for punching shear strength of the FDDPs was developed. The developed punching shear model accounts for the two-way GFRP reinforcement by considering the average of the bottom reinforcement ratios in the transverse and longitudinal directions and average effective slab depth calculated from the top surface of the slab to the bottom reinforcement in each direction (i.e. $d_{\text {avg }}=146 \mathrm{~mm}$ in the tested slabs).

$$
\begin{aligned}
& V_{c}=\eta\left(f_{c}^{\prime}\right)^{1 / 3} \rho_{\text {avg }} b_{2.5} d_{\text {avg }} / 1000 \\
& b_{2.5}=2[(600+2 \times 2.5 d)+(250+2 \times 2.5 d)] \\
& d_{\text {avg }}=\frac{d_{\text {transverse }}+d_{\text {longitudinal }}}{2} \\
& \rho_{\text {avg }}=\frac{\rho_{\text {trans }}+\rho_{\text {long }}}{2} \times 100
\end{aligned}
$$

Using a statistical package for best fit of the experimental findings, the constant $\eta$ in equation 5.18.a is taken as 0.427 . Table 5.14 shows correlation between the experimental findings and the developed equation on which good agreement was observed.

\subsection{Conclusions}

Three joint details were developed for transverse closure strips between precast FDDPs to accelerate bridge construction. Actual-size FDDPs were constructed and tested under CAF and VAF loading. Also, the panels tested under CAF loading were loaded under static loading to collapse. The following subsections summarize the experimental findings.

\subsubsection{A-Jointed Precast FDDP}

Based on the experimental results, it can be concluded that the developed transverse panel-topanel connection with projecting straight-ended high-modulus GFRP bars can provide a continuous force transfer in the transverse joints for the FDDPs. Experimental results also indicated that precast FDDPs reinforced with high-modulus GFRP ribbed-surface bars showed high fatigue performance as there was no observed fatigue damage when subjected to 4,000,000 
cycles under high-cyclic CAF loading of $122.5 \mathrm{kN}$ specified in CHDBC. The tested precast FDDP under high-cyclic CAF loading sustained a failure load about 4.47 times the CHBDC factored design wheel load of $208.25 \mathrm{kN}$. While the tested precast FDDP under low-cyclic incremental step VAF loading sustained a failure load about 2.34 times the CHBDC factored design wheel load. The two laterally-restrained precast FDDPs failed in punching shear mode. Finally, the first precast FDDP specimen's stiffness degraded by about 53\% after 4 million cycles of constant amplitude fatigue (CAF) loading. On the other hand, the second precast FDDP specimen's stiffness degraded by $86.86 \%$ when subjected to low-cyclic variable amplitude fatigue (VAF) loading before complete collapse. Based on experimental findings, a mathematical model was proposed to determine the cumulative fatigue damage (CFD) and fatigue resistance (P-N effect) for the GFRP-reinforced FDDPs with transvers joint. The cumulative linear damage, D, was successfully maintained to be 1 for transverse jointed FDDPs. It can be noticed that the magnification factor to the fatigue loading is inversely proportional to the number of the repetitions of the same cycle that equals life to failure.

\subsubsection{C-Jointed Precast FDDP}

Based on the experimental results, it can be concluded that the developed transverse panel-topanel C-shape joint with projecting straight-end HM GFRP bars can provide a continuous force transfer in the transverse joint for precast FDDPs. Experimental results also indicated that precast high-modulus, GFRP-reinforced, FDDP showed high fatigue performance as there was no observed fatigue damage after being subjected to 4,000,000 cycles of high-cyclic CAF loading of $122.5 \mathrm{kN}$ specified in CHDBC. The tested precast FDDP under CAF loading followed with increasing monotonic wheel load to-collapse sustained a failure load about 4.67 times the CHBDC factored ULS design wheel load. While the tested precast FDDP under low-cyclic incremental step VAF loading sustained a failure load about 2.38 times the CHBDC factored $\mathrm{ULS}_{1}$ design wheel load. The two precast FDDPs failed in punching shear mode. Finally, the first precast FDDP specimen's stiffness degraded by about $50.73 \%$ after 4 million cycles of highcyclic constant amplitude fatigue (CAF) loading. On the other hand, the second precast FDDP specimen's stiffness degraded by $63.42 \%$ when subjected to low-cyclic variable amplitude fatigue (VAF) loading before complete collapse. 


\subsubsection{The Z-Jointed Precast FDDP}

Based on the experimental results, it can be concluded that the developed transverse panel-topanel connection with projecting straight-ended high-modulus GFRP bars can provide a continuous force transfer in the transverse joints for the FDDPs. Experimental results also indicated that precast FDDP reinforced with high-modulus GFRP ribbed-surface bars showed high fatigue performance and there was no fatigue damage when subjected to 4,000,000 cycles under high-cyclic CAF loading of $122.5 \mathrm{kN}$ specified in CHDBC. The tested precast FDDP under high-cyclic CAF loading sustained a failure load about 4.47 times the CHBDC factored design wheel load of $208.25 \mathrm{kN}$. While the tested precast FDDP under low-cyclic incremental step VAF loading sustained a failure load about 2.35 times the CHBDC factored design wheel load. The two laterally restrained precast FDDPs failed in punching shear mode. Finally, the first precast FDDP specimen's stiffness degraded by about $21.9 \%$ after 4 million cycles of constant amplitude fatigue (CAF) loading. On the other hand, the second precast FDDP specimen's stiffness degraded by $71.32 \%$ when subjected to low-cyclic variable amplitude fatigue (VAF) loading before complete collapse.

\subsubsection{Developed Punching Shear Model}

Based on the experimental findings, and empirical equation for punching shear strength of the FDDPs was developed, considering the critical section for punching shear failure at distance $2.5 \mathrm{~d}$ from the face of the loaded area.

\section{References}

AASHTO, 2012. AASHTO-LRFD Bridge Design Specifications, Fifth Edition. American Association of State Highway and Transportation Officials, Washington, D.C.

ACI Committee 440. 2006. Guide for the Design and Construction of Structural Concrete Reinforced with FRP Bars, ACI 440.1R-06. American Concrete Institute, Farmington Hills, MI, USA.

Adimi, M., Rahman, A., and Benmokrane, B. 2000. New Method for Testing Fibre-Reinforced Polymers Rods under Fatigue. Journal of Composites for Construction, 4(4): 206-213. 
Ahmad, S. H., Zia, P., Yu, T., and Xie, Y. 1993. Punching Shear Tests of Slabs Reinforced with 3-D Carbon Fiber Fabric. Concrete International, 16: 36-41.

Badie, S., and Tadros, M. 2008. Full-Depth Precast Concrete Bridge Deck Panel Systems. NCHRP Report 584, Transportation Research Board, Washington, D.C.

Badie, S., Tadros, M., and Girgis, A., 2006. Full-Depth, Precast-Concrete Bridge Deck Panel Systems. Report No. NCHRP 12-65, Transportation Research Board, Washington, D.C.

Bakht, B. 1993. Less steel in bridge decks saves \$1 Million a year. Research and Development Branch Research Report, 1.

Bakht, B. and Csagoly, R.P. 1972. Bridge testing. Ontario Ministry of Transportation and Communications, SRR-79-10.

Banthia, N., Al-Asaly, M., and Ma, S. 1995. Behavior of Concrete Slabs Reinforced with FiberReinforced Plastic Grid. ASCE Journal of Materials in Civil Engineering, 7(4): 643-652.

Benmokrane, B., El-Salakawy, E., El-Ragaby, A., and Lackey, T. 2006. Designing and Testing of Concrete Bridge Decks Reinforced with Glass FRP Bars. ASCE Journal of Bridge Engineering, 11(2): 217-229.

Braimah, A., Green, M., and Soudki, K., 1998. Polypropylene FRC Bridge Deck Slabs Transversely Prestressed with CFRP Tendons. ASCE Journal of Composite for Construction, 2(4): 149-157.

BS 8110. 1997. Structural Use of Concrete, Part 1, Code of Practice for Design and Construction. British Standards Institution, London.

CAN/CSA-S806-12. 2012. Design and Construction of Building Structures with FibreReinforced Polymers. Rexdale, Ontario, Canada, 37 pp.

Clumo, M., 2011. Accelerated Bridge Construction - Experience in Design, Fabrication and Erection of Prefabricated Bridge Elements and Systems, FHWA-HIF-12-013, Federal Highway Administration, McLean, VA.

CSA. 2014. Canadian Highway Bridge Design Code, CAN/CSA-S6-14. Canadian Standards Association, Mississauga, Ontario, Canada.

Dulude, C., Hassan, M., Ahmed, E., and Benmokrance, B. 2013. Punching Shear Behavior of Flat Slabs Reinforced with Glass Fibre Reinforced Polymer Bars. ACI Structural Journal, 110(5): 723-733. 
Edalatmanesh, R., and Newhook, J. 2013a. Residual Strength of Precast Steel-Free Panels. ACI Structural Journal, 110(5): 715-721.

Edalatmanesh, R., and Newhook, J. 2013b. Investigation of Fatigue Damage in Steel-Free Bridge Decks with Application to Structural Monitoring. ACI Structural Journal, 110(4): 557-564.

El-Gamal, S. 2005. Behaviour of Restrained Concrete Bridge Deck Slabs Reinforced with FRP Reinforcing Bars under Concentrated Loads. Ph.D. Thesis, University of Sherbrook, Quebec, Canada.

El-Gamal, S., El-Salakawy, E., and Benmokrane, B. 2005. Behavior of Concrete Bridge Deck Slabs Reinforced with Fiber-Reinforced Polymer Bars under Concentrated Loads. ACI Structural Journal, 102(5): 727-735.

El-Ghandour, A., Pilakoutas, K., and Waldron, P. 1999. New Approach for Punching Shear Capacity Prediction of Fiber Reinforced Polymer Reinforced Concrete Flat Slabs. ACI Structural Journal, 188(13):135-144.

El-Ghandour, A., Pilakoutas, K., and Waldron, P. 2003. Punching Shear Behaviour of Fiber Reinforced Polymers Reinforced Concrete Flat Slabs: Experimental Study. ASCE Journal of Composite for Construction, 7(3): 258-265.

El-Ragaby, A., El-Salakawy, E., and Benmokrane, B., 2007. Fatigue Life Evaluation of Concrete Bridge Deck Slabs Reinforced with Glass FRP Composite Bars. Journal of Composite for Construction, 11(3): 258-268.

El-Salakawy, E., Benmokrane, B., El-Ragaby, A., and Nadeau, D. 2005. Field Investigation on the First Bridge Deck Slab Reinforced with Glass FRP Bars Constructed in Canada. Journal of Composites for Construction, 9(6): 470-479.

Gar, S., Head, M., Hurlebaus, S., and Mander, J. 2014. Experimental Performance of AFRP Concrete Bridge Deck Slab with Full-Depth Precast Prestressed Panels. Journal of Bridge Engineering, 04013018: 1-10.

Graybeal, B. 2010. Behavior of Field-Cast Ultra-High Performance Concrete Bridge Deck Connections under Cyclic and Static Structural Loading. Report No. FHWA-HRT-11-023, Federal Highway Administration, 116 pages.

Hassan, M., Ahmed, E., and Benmokrane, B. 2013. Punching Shear Strength of Normal and High-Strength Two-Way Concrete Slabs Reinforced with GFRP Bars. ASCE Journal of Composites for Construction, 04013003: 1-12. 
Hassan, T., Rizkalla, S., Abdelrahman, A., and Tadros, G. 2000a. Fibre Reinforced Polymer Reinforcing Bars for Bridge Decks. Canadian Journal of Civil Engineering, 27: 839-849.

Hassan, T., Rizkalla, S., Adelrahman, A., and Tadros, G. 2000b. Design Recommendations for Bridge Deck Slabs Reinforced by Fibre Reinforced Polymers. ACI SP, pp. 188-29.

Hwang, H., and Park, S. 2014. A Study on the Flexural Behavior of Lap-spliced Cast-in-place Joints under Static Loading in Ultra-high Performance Concrete Bridge Deck Slabs. Canadian Journal of Civil Engineering, 41: 615-623.

Hwang, H., Yoon, H., Joh, C., and Kim, B. 2010. Punching and Fatigue Behavior of Long-Span Prestressed Concrete Deck Slabs. Engineering Structures, 32: 2861-2872.

Jacobson, D., Bank, L., Oliva, M., and Russell, J. 2005. Punching Shear Capacity of Double Layer FRP Grid Reinforced Slabs. Proceedings of the $7^{\text {th }}$ International Conference on Fiber Reinforced Plastics for Reinforced Concrete Structures, American Concrete Institute, New Orleans, La., pp. 857-871.

Japan Society of Civil Engineers (1997). "Recommendation for Design and Construction of Concrete Structures Using Continuous Fiber Reinforcing Materials," Concrete Engineering Series 23, ed. by A. Machida, JSCE, Tokyo, Japan.

Karunananda, K., Ohga, K., Dissanayake, R., and Siriwardane, S. 2010. A Combined High and Low Cycle Fatigue Model to Estimate Life of Steel Bridges. Journal of Engineering and Technology Research, 2(8): 144-160.

Katz, A. 2000. Bond to Concrete of FRP Rebars After Cyclic Loading. Journal of Composites for Construction, 4(3): 137-144.

Khalafalla, I. 2014. Development of Sustainable Concrete Bridge Deck Slab Systems using Corrosion-Resistant GFRP Bars, Ph.D. Thesis, Ryerson University, Toronto, Ontario.

Khanna, O., Mufti, A., and Bakht, B. 2000. Experimental Investigation of the Role of Reinforcement in the Strength of Concrete Deck Slabs. Canadian Journal of Civil Engineering, 27: 475-480.

Klowak, C., Memon, A., and Mufti, A. 2007. Static and Fatigue Investigation of Innovative Second Generation Steel-Free Bridge Decks. Canadian Journal of Civil Engineering, 34: 331-339.

Kumar, J., and GangaRao, H. 1992. Fatigue Response of Concrete Decks Reinforced with FRB Rebars. ACI Structural Journal, 89(1): 13-19. 
Matsui, S., Tokai, D., Higashiyama, H. and Mizukoshi, M. 2001. Fatigue Durability of FibreReinforced Concrete Decks under Running Wheel Load. Proceedings of the 3rd International Conference on Concrete Under Severe Conditions, University of British Columbia, Vancouver, Canada, pp 982-991.

Matthys, S., and Taerwe, L. 2000. Concrete Slabs Reinforced with FRP Grid. II: Punching Resistance. ASCE Journal of Composite for Constructions, 4(3): 154-161.

Memon, A.H. 2005. Compressive Fatigue Performance of Steel Reinforced and Steel-Free Bridge Deck Slabs. PhD Thesis, Department of Civil Engineering, University of Manitoba, Wennipg, Canada, 125 p.

Miner, M.A., "Cumulative Damage in Fatigue," Journal of Applied Mechanics, Vol. 67, pp. A159-164, 1945.

Mufti, A., Jaeger, L., Bakht, B., and Wegner, L. 1993. Experimental Investigation of FRC Slabs without Internal Steel Reinforcement. Canadian Journal of Civil Engineering, 20(3): 389406.

Muniz, J.A. 2013. Empirical Deck for Phased Construction and Widening, Master Thesis. Retreived from http://diginole.lib.fsu.edu/etd/8609/

Ospina C.E., Alexander S.D.B., and Cheng J.J.R. 2003. Punching of Two-Way Concrete Slabs with Fiber-Reinforced Polymer Reinforcing Bars or Grids. ACI Structural Journal, 100(5): 589-598.

Palmgren, A. Die Lebensdauer von Kugellagern. Verfahrenstechinik. Berlin, Vol. 68, pp 338$341,1924$.

PCI. 2011a. State-of-the-Art Report on Full-Depth Precast Concrete Bridge Deck Panels (SOA01-1911), PCI Committee on Bridges and the PCI Bridge Producers Committee, Precast/Prestressed Concrete Institute, USA.

PCI 2011b. Full Depth Deck Panels Guidelines for Accelerated Bridge Deck Replacement or Construction. Report Number PCINER-11-FDDP, Precast/Prestressed Concrete Institute, USA.

Perdikaris, P., and Beim, S. 1988. RC Bridge Decks under Pulsating and Moving Load. Journal of Structural Engineering, 114(3): 591-607. 
Rahman, A., Kingsley, C., and Kobayashi, K. 2000. Service and Ultimate Load Behaviour of Bridge Deck Reinforced with Carbon FRP Grid. ASCE Journal of Composite of Construction, 4(1): 16-23.

Russell, H., and Graybeal, B. 2013. Ultra-High Performance Concrete: A State-of-the-Art Report for the Bridge Community, Publication No. FHWA-HRT-13-060, Federal Highway Administration, McLean, VA.

Schoeck Canada Inc. ComBar Product Manual. [Online] Available at: www.schoeckcanada.com [Accessed 2011].

Sonoda, K., and Horikawa, T. 1982. Fatigue Strength of Reinforced Concrete Slabs under Moving Load. Zurich, Switzerland, pp. 455-462.

Taylor, S.E. 2000. Compressive membrane action in high strength concrete bridge deck slab, $\mathrm{PhD}$ dissertation. Queen's University, Belfast, Northern Ireland, UK.

Thorburn, J. 1998. A study of Externally Reinforced Fibre-Reinforced Concrete Bridge Decks on Steel Girders. Ph.D. Thesis, Dalhousie University, Halifax, Nova Scotia, Canada.

You, Y., Kim, J., Park, Y., and Choi, J. 2015. Fatigue Performance of Bridge Deck Reinforced with Cost-to-Performance Optimized GFRP Rebar with $900 \mathrm{MPa}$ Guaranteed Tensile Strength. Journal of Advanced Concrete Technology, 13: 252-262.

Zhu, P., Ma, Z., Cao, Q., and French, C. 2012. Fatigue Evaluation of Longitudinal U-Bar Joint Details for Accelerated Bridge Construction. Journal of Bridge Engineering, 17: 201-210. 


\section{Tables}

Table 5.1 Mechanical properties of GFRP bars used in this study (Schoeck, 2013)

\begin{tabular}{llllll}
\hline Product type & Bar Size & $\begin{array}{l}\text { Bar area } \\
\left(\mathrm{mm}^{2}\right)\end{array}$ & $\begin{array}{l}\text { Guaranteed tensile } \\
\text { strength }(\mathrm{MPa})\end{array}$ & $\begin{array}{l}\text { Modulus pf } \\
\text { elasticity (GPa) }\end{array}$ & $\begin{array}{l}\text { Strain at } \\
\text { failure }\end{array}$ \\
\hline Ribbed-surface & $15 \mathrm{M}(\# 5)$ & 201 & 1188 & 64 & $2.6 \%$ \\
\hline
\end{tabular}

Table 5.2 Summary of fatigue tested slab configurations

\begin{tabular}{|c|c|c|c|c|c|c|}
\hline \multicolumn{3}{|c|}{ Slab Name } & \multicolumn{2}{|c|}{$\begin{array}{l}\text { Transverse reinforcement } \\
\text { (normal to girder) }\end{array}$} & \multicolumn{2}{|c|}{$\begin{array}{l}\text { Longitudinal reinforcement } \\
\text { (parallel to girder) }\end{array}$} \\
\hline $\begin{array}{l}\text { Slab } \\
\text { number }\end{array}$ & $\begin{array}{l}\text { Joint } \\
\text { type }\end{array}$ & $\begin{array}{l}\text { Test } \\
\text { type* }\end{array}$ & Bottom & Top & Bottom & Top \\
\hline $\begin{array}{l}\text { S1 } \\
\text { S2 }\end{array}$ & A & $\begin{array}{l}\text { CAF } \\
\text { VAF }\end{array}$ & $\begin{array}{l}\text { Straight end } \\
\text { No.16 @ } 140 \\
\mathrm{~mm}\end{array}$ & $\begin{array}{l}\text { Straight end } \\
\text { No. } 16 @ 200 \\
\text { mm }\end{array}$ & $\begin{array}{l}\text { Straight end } \\
\text { No. } 16 @ 200 \\
\text { mm }\end{array}$ & $\begin{array}{l}\text { Straight end } \\
\text { No. } 16 @ 200 \\
\text { mm }\end{array}$ \\
\hline $\begin{array}{l}\mathrm{S} 3 \\
\mathrm{~S} 4\end{array}$ & $\mathrm{C}$ & $\begin{array}{l}\text { CAF } \\
\text { VAF }\end{array}$ & $\begin{array}{l}\text { Straight end } \\
\text { No.16@140 } \\
\text { mm }\end{array}$ & $\begin{array}{l}\text { Straight end } \\
\text { No.16@200 } \\
\text { mm }\end{array}$ & $\begin{array}{l}\text { Straight end } \\
\text { No.16@200 } \\
\text { mm }\end{array}$ & $\begin{array}{l}\text { Straight end } \\
\text { No.16@200 } \\
\text { mm }\end{array}$ \\
\hline $\begin{array}{l}\text { S5 } \\
\text { S6 }\end{array}$ & $\mathrm{Z}$ & $\frac{\mathrm{CAF}}{\mathrm{VAF}}$ & $\begin{array}{l}\text { Straight end } \\
\text { No.16@140 } \\
\text { mm }\end{array}$ & $\begin{array}{l}\text { Straight end } \\
\text { No.16@200 } \\
\text { mm }\end{array}$ & $\begin{array}{l}\text { Straight end } \\
\text { No.16@200 } \\
\text { mm }\end{array}$ & $\begin{array}{l}\text { Straight end } \\
\text { No.16@200 } \\
\text { mm }\end{array}$ \\
\hline
\end{tabular}

Table 5.3 Static Load Configuration

\begin{tabular}{cc}
\hline Loading & Unloading \\
\hline Segment shape: ramp function & Segment shape: ramp function \\
Rate: $5 \mathrm{kN} /$ min & Rate: $10 \mathrm{kN} / \mathrm{min}$ \\
Control mode: Force & Control mode: force \\
Absolute end level (machine max.load for test purpose) $: 183.75 \mathrm{kN}$ & Absolute end level: zero kN \\
\hline
\end{tabular}


Table 5.4 Summary of test results

a) Loading data

\begin{tabular}{lllllllll}
\hline $\begin{array}{l}\text { Slab } \\
\text { number }\end{array}$ & $\begin{array}{l}\text { Joint } \\
\text { type }\end{array}$ & Test* & $\begin{array}{l}\text { Peak } \\
\text { cyclic } \\
\text { load }(\mathrm{kN})\end{array}$ & $\begin{array}{l}\text { Frequency } \\
(\mathrm{Hz})\end{array}$ & $\begin{array}{l}\text { No. of } \\
\text { load } \\
\text { cycles }\end{array}$ & $\begin{array}{l}\text { Ultimate } \\
\text { load } \\
(\mathrm{kN})\end{array}$ & $\begin{array}{l}\text { Ultimate } \\
\text { deflection } \\
(\mathrm{mm})\end{array}$ & $\begin{array}{l}\text { Failure } \\
\text { Mode }\end{array}$ \\
\hline S1 & A & CAF + SUL & 137.5 & 4 & $4,000,000$ & 930.92 & 23.47 & Punching \\
\hline S2 & A & VAF & 500.0 & $2-0.5$ & 809,493 & 487.50 & 32.46 & Punching \\
\hline S3 & C & CAF + SUL & 137.5 & 4 & $4,000,000$ & 973 & 26.09 & Punching \\
\hline S4 & C & VAF & 500 & $2-0.5$ & 692,866 & 495.69 & 40.89 & Punching \\
\hline S5 & Z & CAF + SUL & 137.5 & 4 & $4,000,000$ & 931 & 29.62 & Punching \\
\hline S6 & Z & VAF & 500 & $2-0.5$ & 961,540 & 488.43 & 37.03 & Punching \\
\hline
\end{tabular}

$* \mathrm{CAF}=$ constant amplitude fatigue loading; $\mathrm{SUL}=$ static ultimate load; $\mathrm{VAF}=$ variable amplitude fatigue load

b) Deflection data after SUL

\begin{tabular}{ccccl}
\hline Monitoring direction & \multicolumn{3}{c}{ Deflection, mm } & \multirow{2}{*}{ Comments } \\
\cline { 1 - 4 } Longitudinal direction & A-Shape & C-Shape & Z-Shape & \\
\hline Free-end & 7.48 & 8.78 & 9.00 & Small segment \\
\hline Point-load 1 & 23.05 & 25.98 & 30.29 & Actual segment \\
\hline Point-load 2 & 23.88 & 26.20 & 28.94 & Actual segment \\
\hline Mid-span & 13.39 & 16.03 & 18.06 & Actual segment \\
\hline Fixed-end & 2.44 & 8.28 & 7.47 & Rear joint \\
\hline Transverse direction & & & & \\
\hline Steel-girder 1 & 10.68 & 2.54 & 1.78 & Twin girder \\
\hline 0.25 Transverse-span & 13.09 & 17.86 & 19.32 & Actual segment \\
\hline Point-load 1 & 23.05 & 25.98 & 30.29 & Actual segment \\
\hline Point-load 2 & 23.88 & 26.20 & 28.94 & Actual segment \\
\hline Steel-girder 2 & 6.8 & 3.73 & 2.65 & Twin girder \\
\hline
\end{tabular}


Table 5.5 Stiffness degradation of the tested slabs with A-type joint

\begin{tabular}{cccc|cccc}
\hline \multicolumn{3}{c|}{ Slab S1 with CAF loading* } & \multicolumn{3}{c}{ Slab S2 with VAF loading* } \\
\hline $\begin{array}{c}\text { Cumulative } \\
\text { cycles }(\mathrm{N})\end{array}$ & $\begin{array}{c}\text { Load } \\
(\mathrm{kN})\end{array}$ & $\begin{array}{c}\text { Deflection } \\
(\mathrm{mm})\end{array}$ & $\mathrm{k}=\mathrm{F} / \mathrm{d}^{* *}$ & $\begin{array}{c}\text { Cumulative } \\
\text { cycles, } \mathrm{N}\end{array}$ & $\begin{array}{c}\text { Load } \\
(\mathrm{kN})\end{array}$ & $\begin{array}{c}\text { Deflection } \\
(\mathrm{mm})\end{array}$ & $\mathrm{k}=\mathrm{F} / \mathrm{d}^{* *}$ \\
\hline 0 & 183.76 & 1.40 & 131.26 & 0 & 183.76 & 1.24 & 148.19 \\
\hline 250,000 & 183.76 & 2.90 & 63.37 & 100,000 & 183.75 & 2.37 & 77.53 \\
\hline 500,000 & 183.76 & 2.89 & 63.59 & 200,000 & 183.76 & 2.47 & 74.40 \\
\hline 750,000 & 183.75 & 2.91 & 63.15 & 300,000 & 183.76 & 2.76 & 66.58 \\
\hline $1,000,000$ & 183.75 & 2.92 & 62.93 & 400,000 & 183.76 & 3.80 & 48.36 \\
\hline $1,250,000$ & 183.76 & 2.93 & 62.72 & 500,000 & 183.76 & 5.38 & 34.16 \\
\hline $1,500,000$ & 183.76 & 2.94 & 62.50 & 600,000 & 183.78 & 7.41 & 24.80 \\
\hline $1,750,000$ & 183.76 & 2.95 & 62.29 & 715,381 & 183.76 & 9.44 & 19.47 \\
\hline $2,000,000$ & 183.77 & 2.96 & 62.08 & 809,493 & & & \\
\hline $2,250,000$ & 183.76 & 2.97 & 61.87 & & & & \\
\hline $2,500,000$ & 183.75 & 2.99 & 61.46 & & & & \\
\hline $2,750,000$ & 183.76 & 3.01 & 61.05 & & & & \\
\hline $3,000,000$ & 183.75 & 2.99 & 61.46 & & & & \\
\hline $3,250,000$ & 183.76 & 3.02 & 60.84 & & & & \\
\hline $3,500,000$ & 183.76 & 3.02 & 60.85 & & & & \\
\hline $3,750,000$ & 183.75 & 3.01 & 61.05 & & & & \\
\hline $4,000,000$ & 183.76 & 3.03 & 60.65 & & & & \\
\hline
\end{tabular}

* CAF = constant amplitude fatigue loading; VAF = variable amplitude fatigue load.

$* * \mathrm{k}$ is the spring constant in $\mathrm{kN}$ per $\mathrm{mm}, \mathrm{F}$ is the ultimate load in $\mathrm{kN}$, and $\mathrm{d}$ is the deflection in $\mathrm{mm}$. 
Table 5.6 Stiffness degradation of the tested slabs with C-type joint

\begin{tabular}{cccc|cccc}
\hline \multicolumn{3}{c|}{ Slab S3 with CAF loading* } & \multicolumn{3}{c}{ Slab S4 with VAF loading* } \\
\hline $\begin{array}{c}\text { Cumulative } \\
\text { cycles }\end{array}$ & $\begin{array}{c}\text { Load } \\
(\mathrm{kN})\end{array}$ & $\begin{array}{c}\text { Deflection } \\
(\mathrm{mm})\end{array}$ & $\mathrm{k}=\mathrm{F} / \mathrm{d}^{* *}$ & $\begin{array}{c}\text { Cumulative } \\
\text { cycles }\end{array}$ & $\begin{array}{c}\text { Load } \\
(\mathrm{kN})\end{array}$ & $\begin{array}{c}\text { Deflection } \\
(\mathrm{mm})\end{array}$ & $\mathrm{k}=\mathrm{F} / \mathrm{d}^{* *}$ \\
\hline 0 & 183.75 & 1.35 & 136.11 & 100,000 & 183.76 & 3.58 & 51.33 \\
\hline 500,000 & 183.76 & 2.65 & 69.35 & 200,000 & 183.77 & 3.72 & 49.40 \\
\hline $1,000,000$ & 183.76 & 2.67 & 68.82 & 300,000 & 183.75 & 4.09 & 44.93 \\
\hline $1,250,000$ & 183.77 & 2.66 & 69.09 & 400,000 & 183.75 & 5.43 & 33.84 \\
\hline $1,500,000$ & 183.76 & 2.68 & 68.57 & 500,000 & 183.77 & 6.89 & 26.67 \\
\hline $1,750,000$ & 183.77 & 2.72 & 67.56 & 600,000 & 183.75 & 9.74 & 18.87 \\
\hline $2,000,000$ & 183.76 & 2.7 & 68.059 & 692,866 & & & \\
\hline $2,250,000$ & 183.76 & 2.71 & 67.81 & & & & \\
\hline $2,500,000$ & 183.76 & 2.73 & 67.31 & & & & \\
\hline $2,750,000$ & 183.76 & 2.74 & 67.07 & & & & \\
\hline $3,000,000$ & 183.75 & 2.71 & 67.81 & & & & \\
\hline $3,250,000$ & 183.76 & 2.77 & 66.34 & & & & \\
\hline $3,500,000$ & 183.77 & 2.77 & 66.34 & & & & \\
\hline $3,750,000$ & 183.77 & 2.76 & 66.58 & & & & \\
\hline $4,000,000$ & 183.76 & 2.74 & 67.07 & & & & \\
\hline
\end{tabular}

* $\mathrm{CAF}=$ constant amplitude fatigue loading; $\mathrm{VAF}=$ variable amplitude fatigue load.

** $\mathrm{k}$ is the spring constant in $\mathrm{kN}$ per $\mathrm{mm}, \mathrm{F}$ is the ultimate load in $\mathrm{kN}$, and $\mathrm{d}$ is the deflection in $\mathrm{mm}$. 
Table 5.7 Stiffness degradation of the tested slabs with Z-shape joint

\begin{tabular}{|c|c|c|c|c|c|c|c|}
\hline \multicolumn{4}{|c|}{ Slab S5 with CAF loading* } & \multicolumn{4}{|c|}{ Slab S6 with VAF loading* } \\
\hline $\begin{array}{c}\text { Cumulative } \\
\text { cycles }\end{array}$ & $\begin{array}{l}\text { Load } \\
(\mathrm{kN})\end{array}$ & $\begin{array}{l}\text { Deflection } \\
(\mathrm{mm})\end{array}$ & $\mathrm{k}=\mathrm{F} / \mathrm{d}^{* *}$ & $\begin{array}{c}\text { Cumulative } \\
\text { Cycles }\end{array}$ & $\begin{array}{l}\text { Load } \\
(\mathrm{kN})\end{array}$ & $\begin{array}{l}\text { Deflection } \\
(\mathrm{mm})\end{array}$ & $\mathrm{k}=\mathrm{F} / \mathrm{d}^{*} *$ \\
\hline 0 & 184.55 & 1.78 & 103.68 & 100,000 & 183.74 & 3.12 & 58.89 \\
\hline 500,000 & 183.76 & 1.99 & 92.34 & 200,000 & 183.76 & 3.2 & 57.42 \\
\hline $1,000,000$ & 183.75 & 2.07 & 88.77 & 300,000 & 183.76 & 3.5 & 52.5 \\
\hline $1,250,000$ & 183.75 & 2.09 & 87.92 & 400,000 & 183.75 & 4.55 & 40.38 \\
\hline $1,500,000$ & 183.76 & 2.06 & 89.2 & 500,000 & 183.76 & 6.19 & 29.68 \\
\hline $1,750,000$ & 183.75 & 2.14 & 85.86 & 600,000 & 183.75 & 7.64 & 24.05 \\
\hline $2,000,000$ & 183.77 & 2.14 & 85.87 & 700,000 & 183.75 & 8.77 & 20.95 \\
\hline $2,250,000$ & 183.75 & 2.15 & 85.46 & 800,000 & 183.83 & 10.88 & 16.89 \\
\hline $2,500,000$ & 183.75 & 2.17 & 84.68 & 895,000 & -- & & \\
\hline $2,750,000$ & 183.75 & 2.18 & 84.29 & 916,736 & -- & & \\
\hline $3,000,000$ & 183.75 & 2.22 & 82.77 & 961,540 & -- & & \\
\hline $3,250,000$ & 183.75 & 2.18 & 84.29 & & & & \\
\hline $3,500,000$ & 183.76 & 2.26 & 81.31 & & & & \\
\hline $3,750,000$ & 183.75 & 2.27 & 80.94 & & & & \\
\hline $4,000,000$ & 183.76 & 2.27 & 80.95 & & & & \\
\hline
\end{tabular}

* CAF = constant amplitude fatigue loading; VAF = variable amplitude fatigue load ** $\mathrm{k}$ is the spring constant in $\mathrm{kN}$ per $\mathrm{mm}, \mathrm{F}$ is the ultimate load in $\mathrm{kN}$, and $\mathrm{d}$ is the deflection in $\mathrm{mm}$.

Table 5.8. Fatigue parameters

\begin{tabular}{lcc}
\hline Joint Pattern & $\eta$ & $\mathrm{k}$ \\
\hline A-Jointed Precast FDDP & 25.86 & 0.039 \\
\hline C-Jointed Precast FDDP & 24.32 & 0.041 \\
\hline Z-Jointed Precast FDDP & 25.61 & 0.039 \\
\hline
\end{tabular}


Table 5.9. Loading history of the tested slab S2 of type A-joint with equivalent constant amplitude fatigue load segments

\begin{tabular}{|c|c|c|c|c|c|c|c|c|c|c|c|c|c|c|}
\hline \multirow[t]{2}{*}{ Segment } & \multirow[t]{2}{*}{$\mathrm{P}_{\mathrm{u}}$} & \multicolumn{3}{|c|}{ FLS } & \multirow[t]{2}{*}{$\mathrm{P}_{\min }$} & \multirow[t]{2}{*}{$\mathrm{P}_{\max }$} & \multirow[t]{2}{*}{$\mathrm{P}_{\mathrm{amp}}$} & \multirow[t]{2}{*}{$\mathrm{P}_{\text {mean }}$} & \multirow[t]{2}{*}{$\mathrm{R}$} & \multirow[t]{2}{*}{ A } & \multirow{2}{*}{$\mathrm{P}_{\max } / \mathrm{P}_{\mathrm{u}}$} & \multirow[t]{2}{*}{$\mathrm{n}$} & \multirow[t]{2}{*}{$\mathrm{N}_{\mathrm{f}}$} & \multirow{2}{*}{$\mathrm{n} / \mathrm{N}_{\mathrm{f}}$} \\
\hline & & MF & WL & FLS1 & & & & & & & & & & \\
\hline 1 & 930.92 & 1.0 & 87.5 & 122.5 & 15 & 137.50 & 61.25 & 76.25 & 0.109 & 0.803 & 0.148 & 100,000 & $3,740,491,266$ & $2.673 \mathrm{E}-05$ \\
\hline 2 & 930.92 & 1.5 & 87.5 & 183.8 & 15 & 198.75 & 91.88 & 106.88 & 0.075 & 0.860 & 0.213 & 100,000 & $682,217,686$ & 0.0001466 \\
\hline 4 & 930.92 & 2.5 & 87.5 & 306.3 & 15 & 321.25 & 153.13 & 168.13 & 0.046 & 0.911 & 0.345 & 100,000 & $22,694,030$ & 0.0044064 \\
\hline 5 & 930.92 & 3.0 & 87.5 & 367.5 & 15 & 382.50 & 183.75 & 198.75 & 0.039 & 0.925 & 0.411 & 100,000 & $4,139,100$ & 0.0241598 \\
\hline \multirow[t]{2}{*}{8} & 930.92 & 4.0 & 87.5 & 490.0 & 15 & 505.00 & 245.00 & 260.00 & 0.030 & 0.942 & 0.542 & 94,112 & 137,688 & 0.6835187 \\
\hline & & & & & & & & & & & Total & 809,493 & $\Sigma \mathrm{n} / \mathrm{N}$ & 0.9999995 \\
\hline
\end{tabular}

Notes: $\mathrm{P}_{\text {mean }}=\left(\mathrm{P}_{\max }+\mathrm{P}_{\min }\right) / 2 ; \mathrm{R}=\mathrm{P}_{\min } / \mathrm{P}_{\max } ; \mathrm{A}=\mathrm{P}_{\mathrm{a}} / \mathrm{P}_{\mathrm{m}} ; \mathrm{P}_{\mathrm{a}}=\left(\mathrm{P}_{\max }-\mathrm{P}_{\min }\right) / 2$

Table 5.10. Loading history of the tested slab S4 of type C-joint with equivalent constant amplitude fatigue load segments

\begin{tabular}{|c|c|c|c|c|c|c|c|c|c|c|c|c|c|c|}
\hline \multirow[t]{2}{*}{ Segment } & \multirow[t]{2}{*}{$\mathrm{P}_{\mathrm{u}}$} & \multicolumn{3}{|c|}{ FLS } & \multirow[t]{2}{*}{$\mathrm{P}_{\text {min }}$} & \multirow{2}{*}{$\mathrm{P}_{\max }$} & \multirow{2}{*}{$\mathrm{P}_{\mathrm{amp}}$} & \multirow{2}{*}{$\mathrm{P}_{\text {mean }}$} & \multirow[t]{2}{*}{$\mathrm{R}$} & \multirow[t]{2}{*}{ A } & \multirow{2}{*}{$\mathrm{P}_{\max } / \mathrm{P}_{\mathrm{u}}$} & \multirow[t]{2}{*}{$\mathrm{n}$} & \multirow[t]{2}{*}{$\mathrm{N}_{\mathrm{f}}$} & \multirow{2}{*}{$\mathrm{n} / \mathrm{N}_{\mathrm{f}}$} \\
\hline & & MF & WL & FLS1 & & & & & & & & & & \\
\hline 1 & 973 & 1.0 & 87.5 & 122.5 & 15 & 137.50 & 61.25 & 76.25 & 0.109 & 0.803 & 0.141 & 100,000 & $1,176,908,558$ & 8.497E-05 \\
\hline 2 & 973 & 1.5 & 87.5 & 183.8 & 15 & 198.75 & 91.88 & 106.88 & 0.075 & 0.860 & 0.204 & 100,000 & $254,549,386$ & 0.0003929 \\
\hline 3 & 973 & 2.0 & 87.5 & 245.0 & 15 & 260.00 & 122.50 & 137.50 & 0.058 & 0.891 & 0.267 & 100,000 & $55,055,586$ & 0.0018163 \\
\hline 4 & 973 & 2.5 & 87.5 & 306.3 & 15 & 321.25 & 153.13 & 168.13 & 0.047 & 0.911 & 0.330 & 100,000 & $11,907,778$ & 0.0083979 \\
\hline 5 & 973 & 3.0 & 87.5 & 367.5 & 15 & 382.50 & 183.75 & 198.75 & 0.039 & 0.925 & 0.393 & 100,000 & $2,575,491$ & 0.0388275 \\
\hline 6 & 973 & 3.5 & 87.5 & 428.8 & 15 & 443.75 & 214.38 & 229.38 & 0.034 & 0.935 & 0.456 & 100,000 & 557,044 & 0.1795191 \\
\hline \multirow[t]{2}{*}{7} & 973 & 4.0 & 87.5 & 490.0 & 15 & 505.00 & 245.00 & 260.00 & 0.030 & 0.942 & 0.519 & 92,886 & 120,481 & 0.7709595 \\
\hline & & & & & & & & & & & Total & 692,886 & $\Sigma \mathrm{n} / \mathrm{N}$ & 0.9999981 \\
\hline
\end{tabular}

Notes: $\mathrm{P}_{\text {mean }}=\left(\mathrm{P}_{\max }+\mathrm{P}_{\min }\right) / 2 ; \mathrm{R}=\mathrm{P}_{\min } / \mathrm{P}_{\max } ; \mathrm{A}=\mathrm{P}_{\mathrm{a}} / \mathrm{P}_{\mathrm{m}} ; \mathrm{P}_{\mathrm{a}}=\left(\mathrm{P}_{\max }-\mathrm{P}_{\min }\right) / 2$ 
Table 5.11. Loading history of the tested slab S6 of type Z-joint with equivalent constant amplitude fatigue load segments

\begin{tabular}{|c|c|c|c|c|c|c|c|c|c|c|c|c|c|c|}
\hline \multirow[t]{2}{*}{ Segment } & \multirow[t]{2}{*}{$\mathrm{P}_{\mathrm{u}}$} & \multicolumn{3}{|c|}{ FLS } & \multirow[t]{2}{*}{$\mathrm{P}_{\min }$} & \multirow[t]{2}{*}{$\mathrm{P}_{\max }$} & \multirow[t]{2}{*}{$\mathrm{P}_{\mathrm{amp}}$} & \multirow[t]{2}{*}{$\mathrm{P}_{\text {mean }}$} & \multirow[t]{2}{*}{$\mathrm{R}$} & \multirow[t]{2}{*}{ A } & \multirow[t]{2}{*}{$\mathrm{P}_{\max } / \mathrm{P}_{\mathrm{u}}$} & \multirow[t]{2}{*}{$\mathrm{n}$} & \multirow[t]{2}{*}{$\mathrm{N}_{\mathrm{f}}$} & \multirow[t]{2}{*}{$\mathrm{n} / \mathrm{N}_{\mathrm{f}}$} \\
\hline & & MF & WL & FLS1 & & & & & & & & & & \\
\hline 1 & 931 & 1.0 & 87.5 & 122.5 & 15 & 137.50 & 61.25 & 76.25 & 0.109 & 0.803 & 0.147 & 100,000 & $3,015,323,194$ & $3.316 \mathrm{E}-05$ \\
\hline 2 & 931 & 1.5 & 87.5 & 183.8 & 15 & 198.75 & 91.88 & 106.88 & 0.075 & 0.860 & 0.213 & 100,000 & $559,277,378$ & 0.0001788 \\
\hline 3 & 931 & 2.0 & 87.5 & 245.0 & 15 & 260.00 & 122.50 & 137.50 & 0.057 & 0.890 & 0.279 & 100,000 & $103,733,884$ & 0.000964 \\
\hline 4 & 931 & 2.5 & 87.5 & 306.3 & 15 & 321.25 & 153.13 & 168.13 & 0.046 & 0.911 & 0.345 & 100,000 & $19,240,397$ & 0.0051974 \\
\hline 5 & 931 & 3.0 & 87.5 & 367.5 & 15 & 382.50 & 183.75 & 198.75 & 0.039 & 0.925 & 0.411 & 100,000 & $3,568,678$ & 0.0280216 \\
\hline 6 & 931 & 3.5 & 87.5 & 428.8 & 15 & 443.75 & 214.38 & 229.38 & 0.034 & 0.935 & 0.477 & 100,000 & 661,913 & 0.1510773 \\
\hline 7 & 931 & 4.0 & 87.5 & 490.0 & 15 & 505.00 & 245.00 & 260.00 & 0.030 & 0.942 & 0.542 & 100,000 & 122,771 & 0.8145276 \\
\hline 8 & 931 & 4.0 & 87.5 & 490.0 & 15 & 505.00 & 245.00 & 260.00 & 0.030 & 0.942 & 0542 & 100,000 & 122,771 & 0.8145276 \\
\hline 9 & 931 & 4.0 & 87.5 & 490.0 & 15 & 505.00 & 245.00 & 260.00 & 0.030 & 0.942 & 0.542 & 95,000 & 122,771 & 0.7738013 \\
\hline 10 & 931 & 4.0 & 87.5 & 490.0 & 15 & 505.00 & 245.00 & 260.00 & 0.030 & 0.942 & 0.542 & 21,736 & 122,771 & 0.1770457 \\
\hline \multirow[t]{2}{*}{11} & 931 & 4.0 & 87.5 & 490.0 & 15 & 505.00 & 245.00 & 260.00 & 0.030 & 0.942 & 0.542 & 44,804 & 122,771 & 0.364941 \\
\hline & & & & & & & & & & & Total & 961,540 & $\Sigma \mathrm{n} / \mathrm{N}$ & 0.9999999 \\
\hline
\end{tabular}

Notes: $\mathrm{P}_{\text {mean }}=\left(\mathrm{P}_{\max }+\mathrm{P}_{\min }\right) / 2 ; \mathrm{R}=\mathrm{P}_{\min } / \mathrm{P}_{\max } ; \mathrm{A}=\mathrm{P}_{\mathrm{a}} / \mathrm{P}_{\mathrm{m}} ; \mathrm{P}_{\mathrm{a}}=\left(\mathrm{P}_{\max }-\mathrm{P}_{\min }\right) / 2$ 
Table 5.12. Critical sections due to punching shear

\begin{tabular}{|c|c|c|c|c|c|c|c|c|c|c|}
\hline \multirow{3}{*}{$\begin{array}{l}\text { Direction } \\
\text { Slab Type } \\
\text { Specimen }\end{array}$} & \multirow{2}{*}{\multicolumn{4}{|c|}{$\begin{array}{r}\text { Transverse crack length } \\
\text { First Precast FDDP }\end{array}$}} & \multicolumn{6}{|c|}{ Longitudinal crack length } \\
\hline & & & & & \multicolumn{3}{|c|}{ Second Precast FDDP } & \multicolumn{3}{|c|}{ First Precast FDDP } \\
\hline & $\mathrm{d}^{*}$ & $\begin{array}{c}\text { East/West } \\
\text { direction }\end{array}$ & $\frac{\mathrm{d} \text {.critical }}{\mathrm{d}}$ & $\beta$ & $\begin{array}{c}\text { North } \\
\text { direction }\end{array}$ & $\frac{\mathrm{d} \text {.critical }}{\mathrm{d}}$ & $\beta$ & $\begin{array}{c}\text { South } \\
\text { direction }\end{array}$ & $\frac{\mathrm{d} . \text { critical }}{\mathrm{d}}$ & $\beta$ \\
\hline & $\mathrm{mm}$ & $\mathrm{mm}$ & ratio & Degrees & $\mathrm{mm}$ & ratio & Degrees & $\mathrm{mm}$ & ratio & Degrees \\
\hline S1 (A-CAF) & 146 & 375.87 & 2.57 & 21.23 & 554.57 & 3.80 & 14.75 & 837.43 & 5.74 & 9.89 \\
\hline S2 (A-VAF) & 146 & 393.75 & 2.70 & 20.34 & 597.21 & 4.09 & 13.74 & 288.69 & 1.98 & 26.83 \\
\hline S3 (C-CAF) & 146 & 266.14 & 1.82 & 28.75 & 492.52 & 3.37 & 16.51 & 690 & 4.73 & 11.94 \\
\hline S4 (C-VAF) & 146 & 371.66 & 2.55 & 21.45 & 639.15 & 4.38 & 12.87 & 693.5 & 4.75 & 11.89 \\
\hline S5 (Z-CAF) & 146 & 438.2 & 3.00 & 18.43 & 735.6 & 5.04 & 11.23 & 557.44 & 3.82 & 14.68 \\
\hline S6 (Z-VAF) & 146 & 390.92 & 2.68 & 20.48 & 760.82 & 5.21 & 10.86 & 489.99 & 3.36 & 16.59 \\
\hline Average & & & 2.55 & 21.78 & & 4.31 & 13.33 & & 4.06 & 15.30 \\
\hline
\end{tabular}

*See Figure 5.56 for definitions of $d, d_{\text {critical }}$ and Beta 
Table. 5.13. Correlation between the punching shear strength of the tested slabs obtained experimentally and from available equations in the literature

\begin{tabular}{|c|c|c|c|c|c|c|}
\hline & Precast FDDP Name & S1 & $\mathrm{S} 3$ & S5 & \multirow[t]{2}{*}{$\begin{array}{l}\text { Critical } \\
\text { depth }\end{array}$} & \multirow[t]{2}{*}{$\begin{array}{l}\text { Critical } \\
\text { perimeter }\end{array}$} \\
\hline & $V_{\text {exp }}, \mathrm{kN}$ & 930.92 & 973.00 & 931.00 & & \\
\hline & $f_{c}^{\prime}, \mathrm{MPa}$ & 58.26 & 56.68 & 37.00 & & \\
\hline \multirow{9}{*}{ 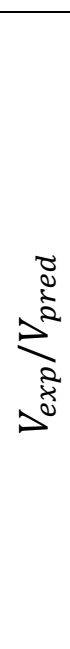 } & CSA S806-12 & 1.55 & 1.64 & 1.81 & $0.5 \mathrm{~d}$ & 2316 \\
\hline & ACI 440.1R-06 & 1.64 & 2.64 & 2.84 & $0.5 \mathrm{~d}$ & 2316 \\
\hline & El-Ghandour et al., 2000 & 1.51 & 1.61 & 1.90 & $0.5 d$ & 2316 \\
\hline & Matthys \& Taerwe, 2000 & 1.70 & 1.79 & 1.98 & $1.5 \mathrm{~d}$ & 3548 \\
\hline & Opsina et al., 2003 & 1.32 & 1.40 & 1.55 & $1.5 \mathrm{~d}$ & 3548 \\
\hline & El-Gamal et al., 2005 & 1.08 & 1.14 & 1.35 & $0.5 \mathrm{~d}$ & 2316 \\
\hline & Jacobson et al., 2007 & 1.63 & 1.72 & 1.90 & $1.5 \mathrm{~d}$ & 3548 \\
\hline & JSCE, 1997 & 1.53 & 1.62 & 1.92 & $0.5 \mathrm{~d}$ & 2316 \\
\hline & CHBDC, 2014 & 0.85 & 0.91 & 1.07 & $0.5 \mathrm{~d}$ & 2316 \\
\hline
\end{tabular}

N.B.: Loaded area $=250 \times 600 \mathrm{~mm} ; \mathrm{d}=154 \mathrm{~mm} ; E_{f}=64000 \mathrm{MPa}$ and $\rho_{f}=0.938 \%$

Table. 5.14. Comparison between the developed punching shear equation and experimental data

\begin{tabular}{|c|c|c|c|c|c|c|c|c|}
\hline \multirow[t]{2}{*}{ Name } & \multirow[t]{2}{*}{$\begin{array}{l}V_{\text {exp }} \\
\mathrm{kN}\end{array}$} & \multirow[t]{2}{*}{$\begin{array}{c}f_{c}^{\prime} \\
\mathrm{MPa}\end{array}$} & \multicolumn{2}{|c|}{$\begin{array}{l}\text { Critical } \\
\text { perimeter }\end{array}$} & \multicolumn{2}{|c|}{$\begin{array}{l}\text { Bottom } \\
\text { reinforcement ratio }\end{array}$} & \multirow[t]{2}{*}{$\begin{array}{c}V_{\text {pred }} \\
\mathrm{kN}\end{array}$} & \multirow[t]{2}{*}{$\frac{V_{\text {exp }}}{V_{\text {pred }}}$} \\
\hline & & & $d_{\text {avg }}$ & $b_{2.5}$ & $\rho_{\text {trans }}$ & $\rho_{\text {long }}$ & & \\
\hline $\mathrm{S} 1$ & 930.92 & 58.26 & 146 & 4620 & 0.938 & 0.728 & 930.92 & 1.00 \\
\hline S3 & 973 & 56.68 & 146 & 4620 & 0.938 & 0.728 & 922.43 & 1.05 \\
\hline S5 & 931 & 37.00 & 146 & 4620 & 0.938 & 0.728 & 800.18 & 1.16 \\
\hline
\end{tabular}




\section{Figures}

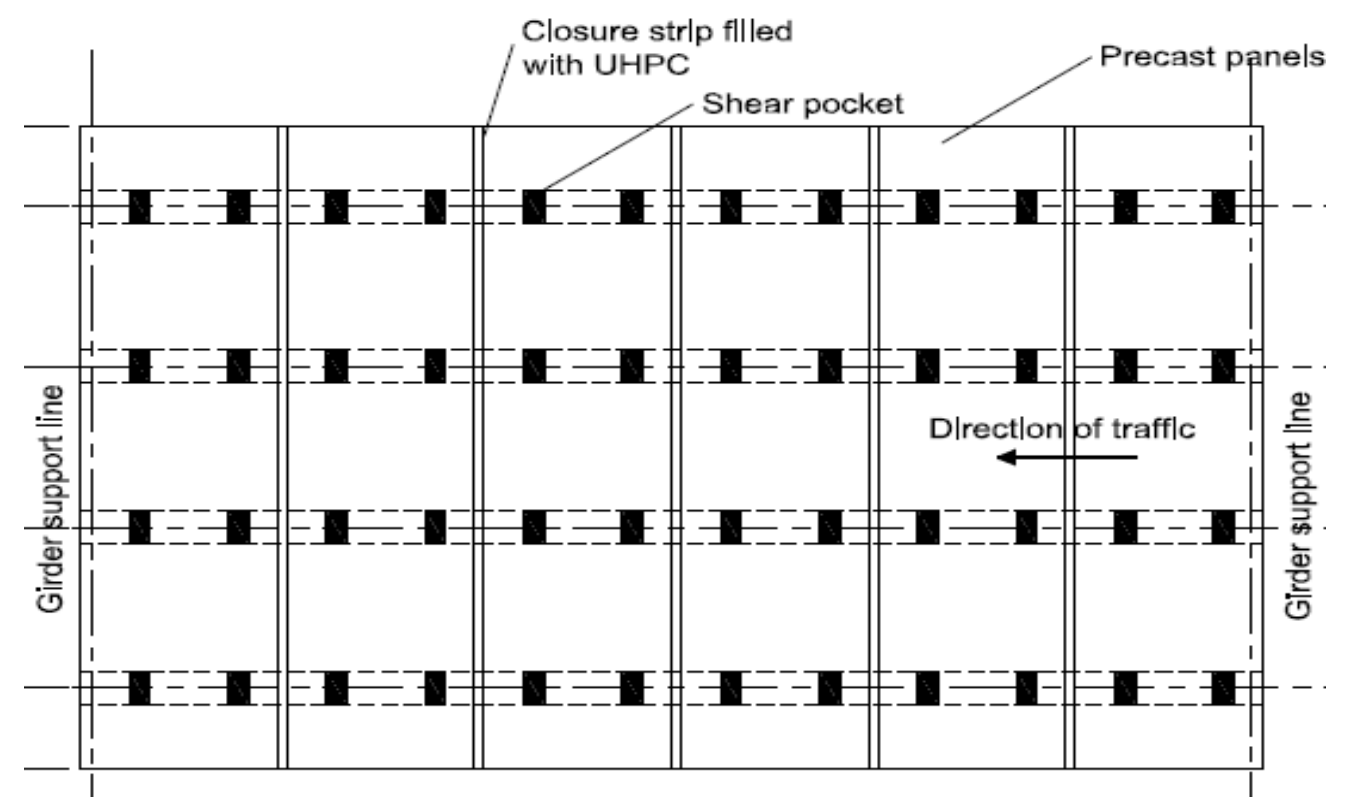

a) Top view of precast transverse full-depth deck panels (FDDPs) resting over longitudinal girders

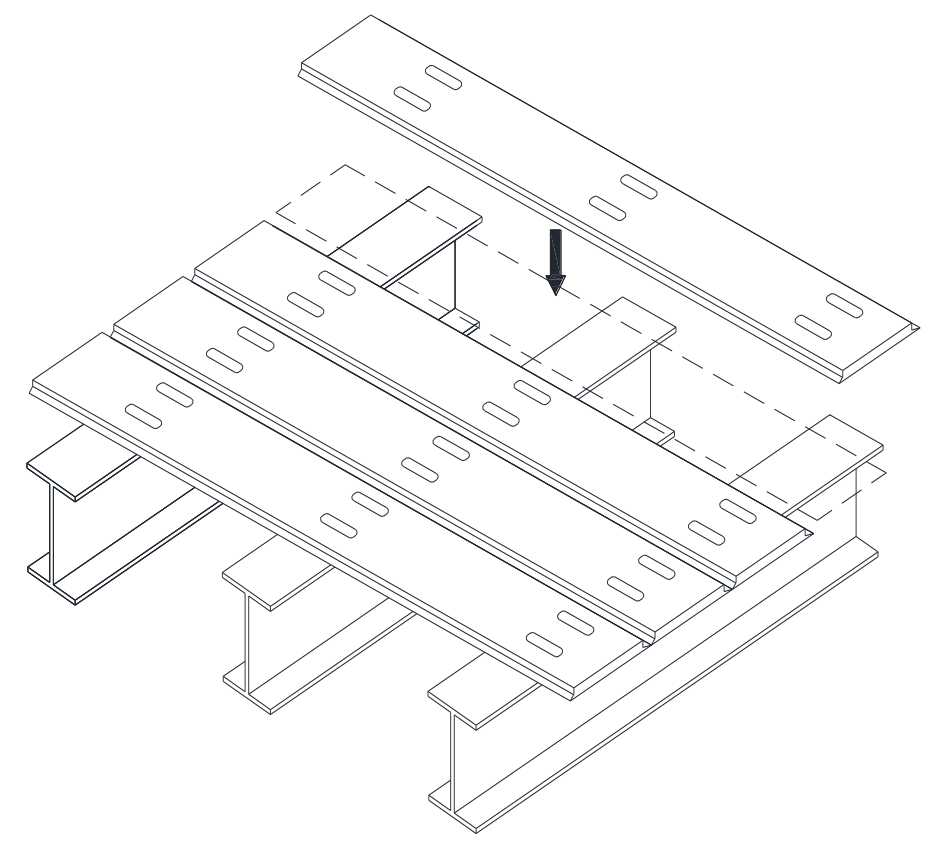

b) Isometric view of precast transverse full-depth deck panels (FDDPs) resting over longitudinal girders

Figure 5.1 Isometric views of precast full-depth, full width, deck panels placed transversally over girders 


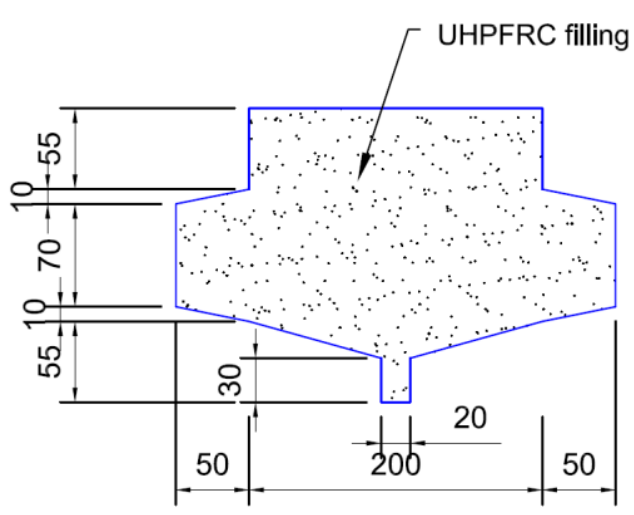

a) Cross-section of closure strip

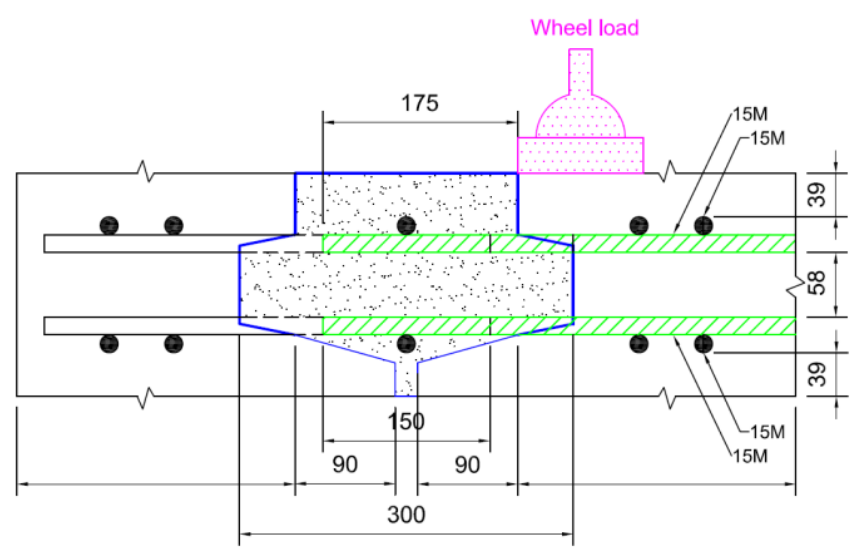

b) Reinforcement detailing

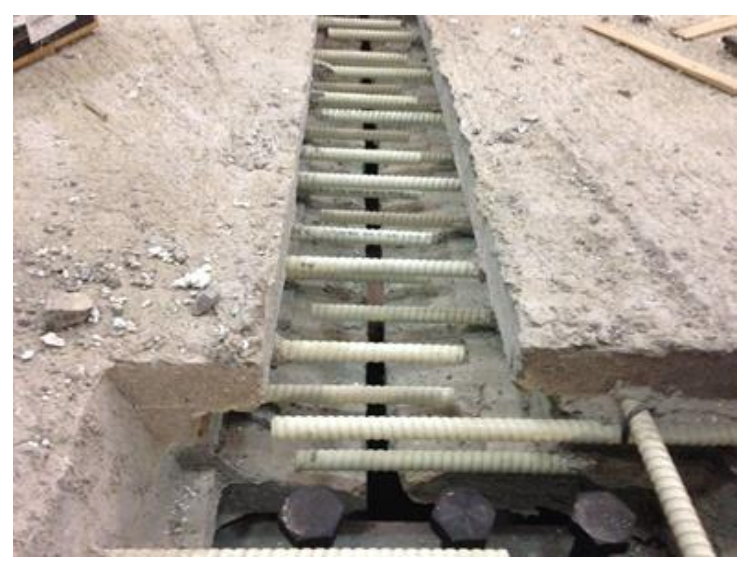

c) Photo of A-joint before adding tranverse bars

Figure 5.2 Cross-sections at the developed A-shape joint for precast slabs S1 and S2

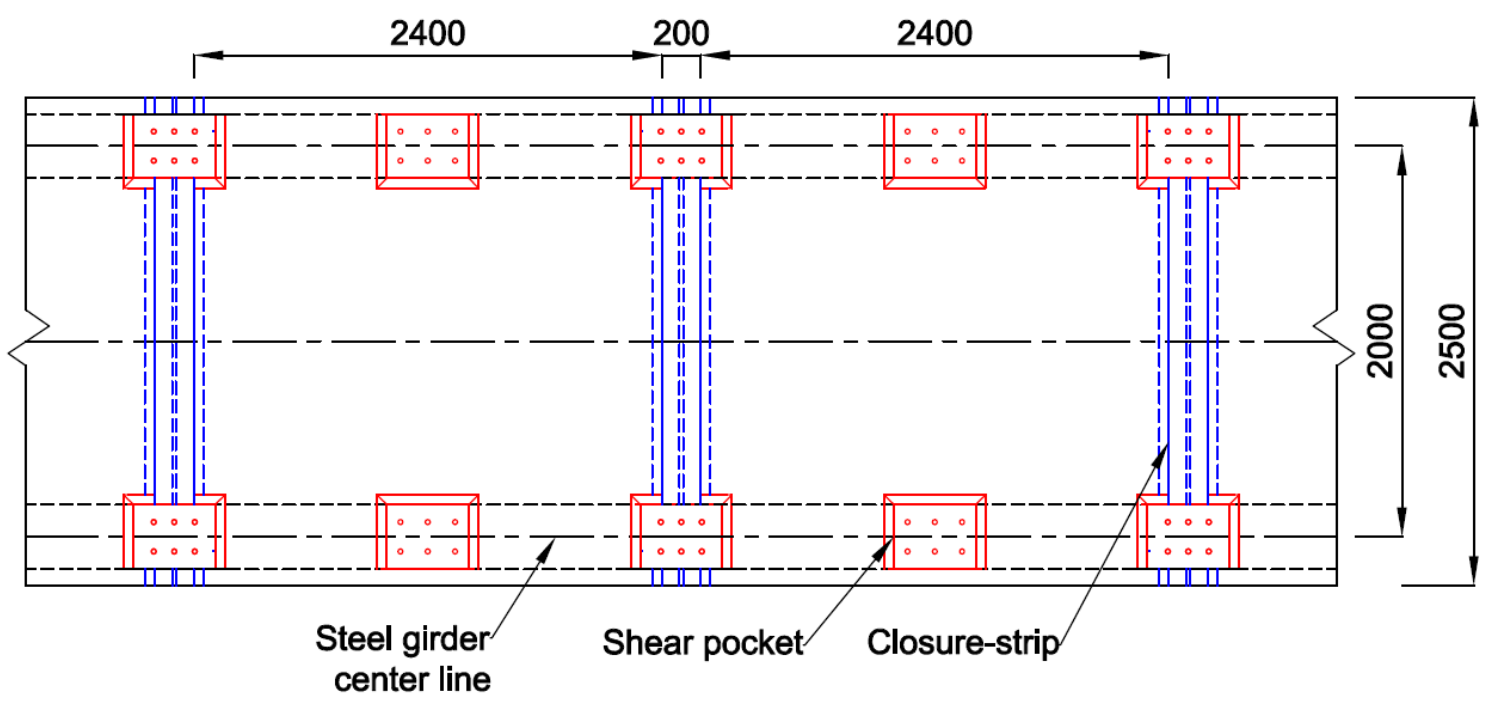

Figure 5.3 Plan of precast FDDP arrangement for A-shape joints 


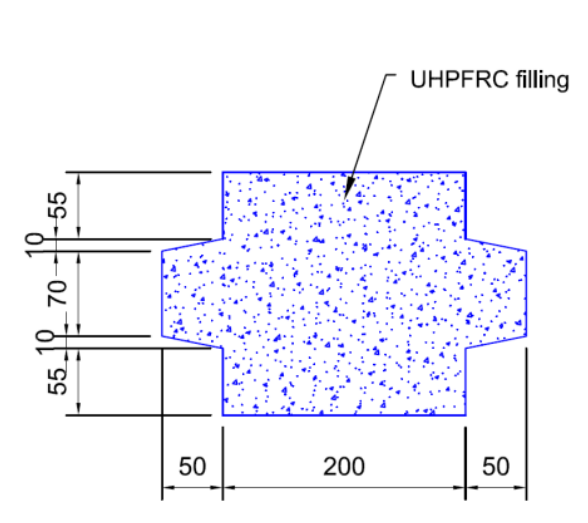

a) Cross-section of closure strip

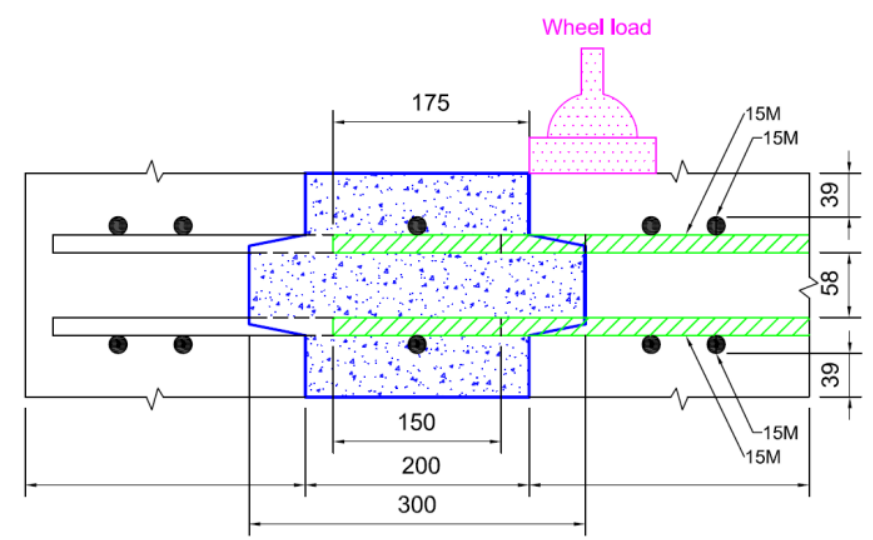

b) Reinforcement detailing

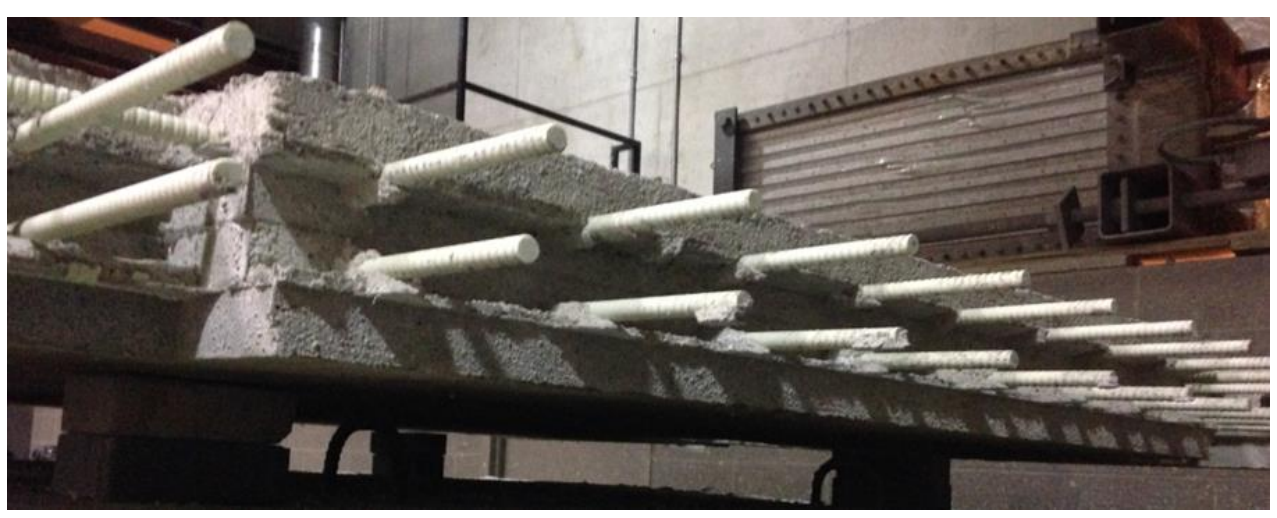

c) Photo of C-joint with projecting GFRP bars

Figure 5.4 Cross-sections at the developed C-shape joint for precast slabs S3 and S4

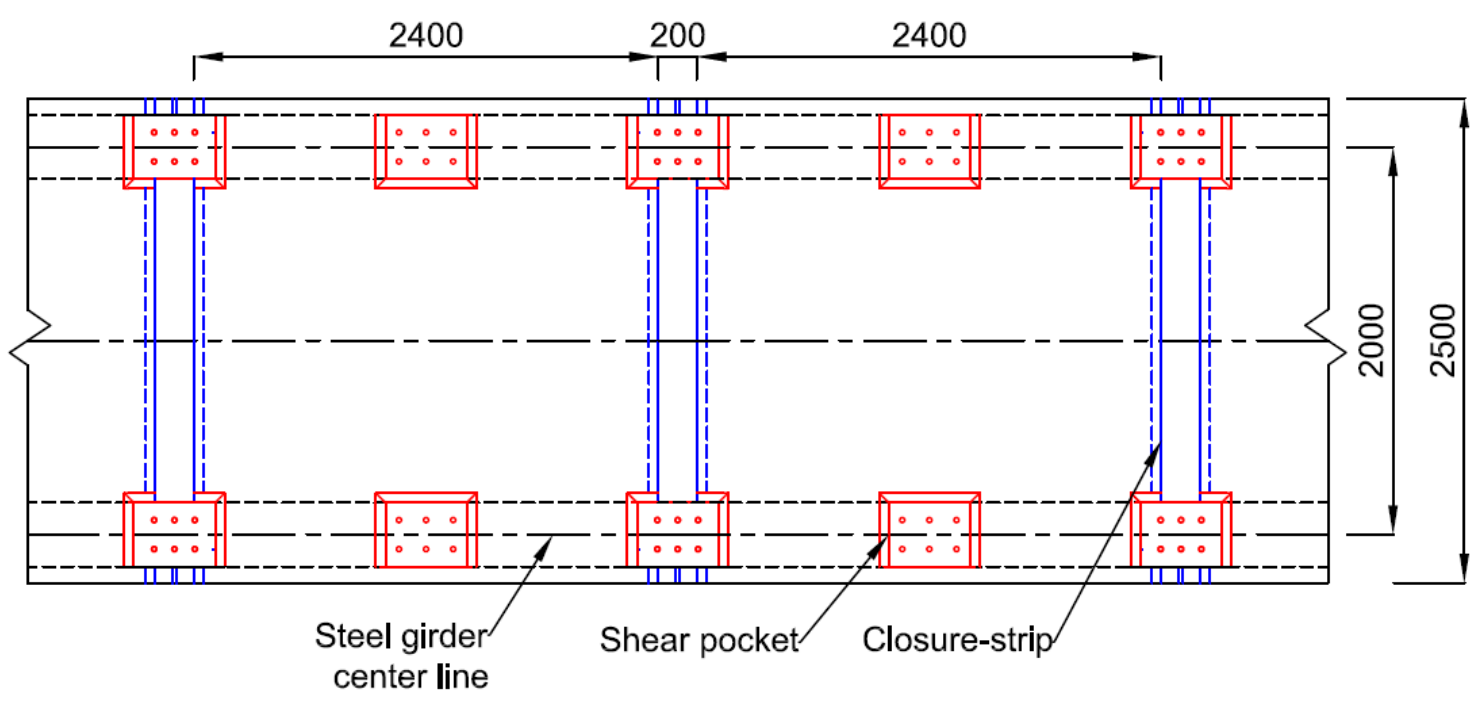

Figure 5.5 Plan of precast FDDP arrangement for C-shape joints 


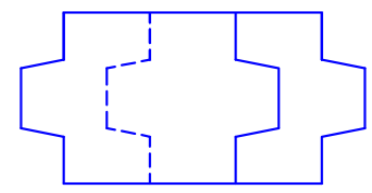

a. Cross-section A-A in the closure strip

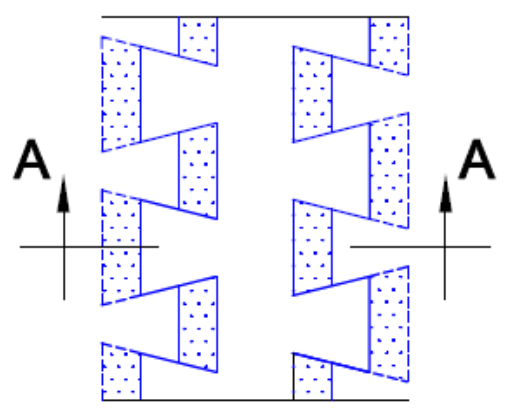

b. Top view of closure strip

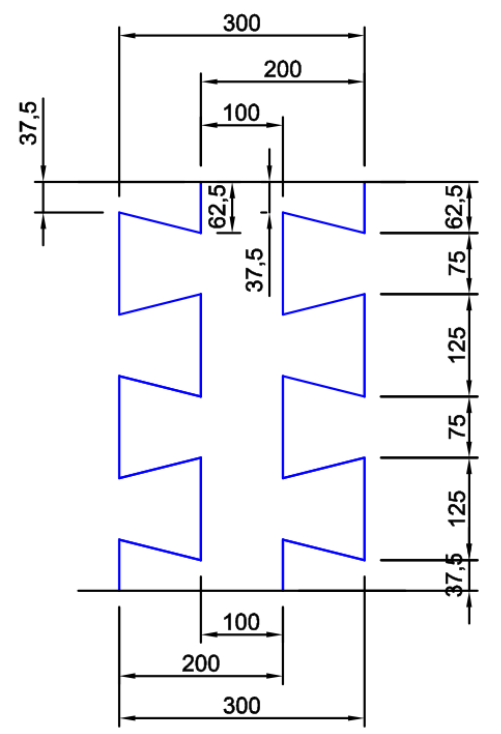

c. Sectional plan at top of slab depth

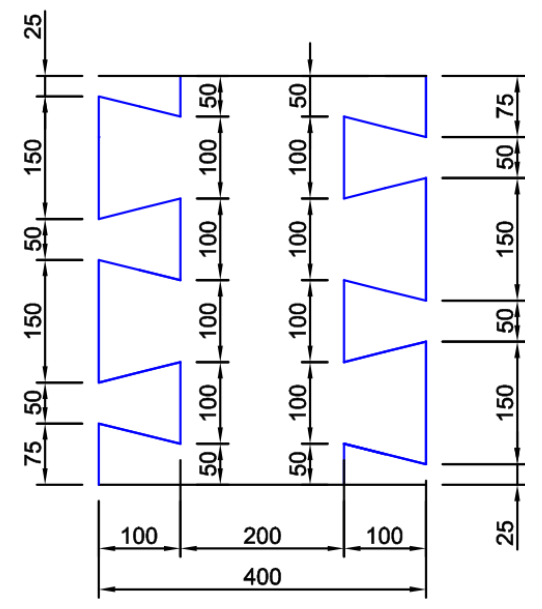

d. Sectional plan at mid-

Figure 5.6 Plan views of the developed zigzag-shape joint (Z-joint) for slabs S5 and S6
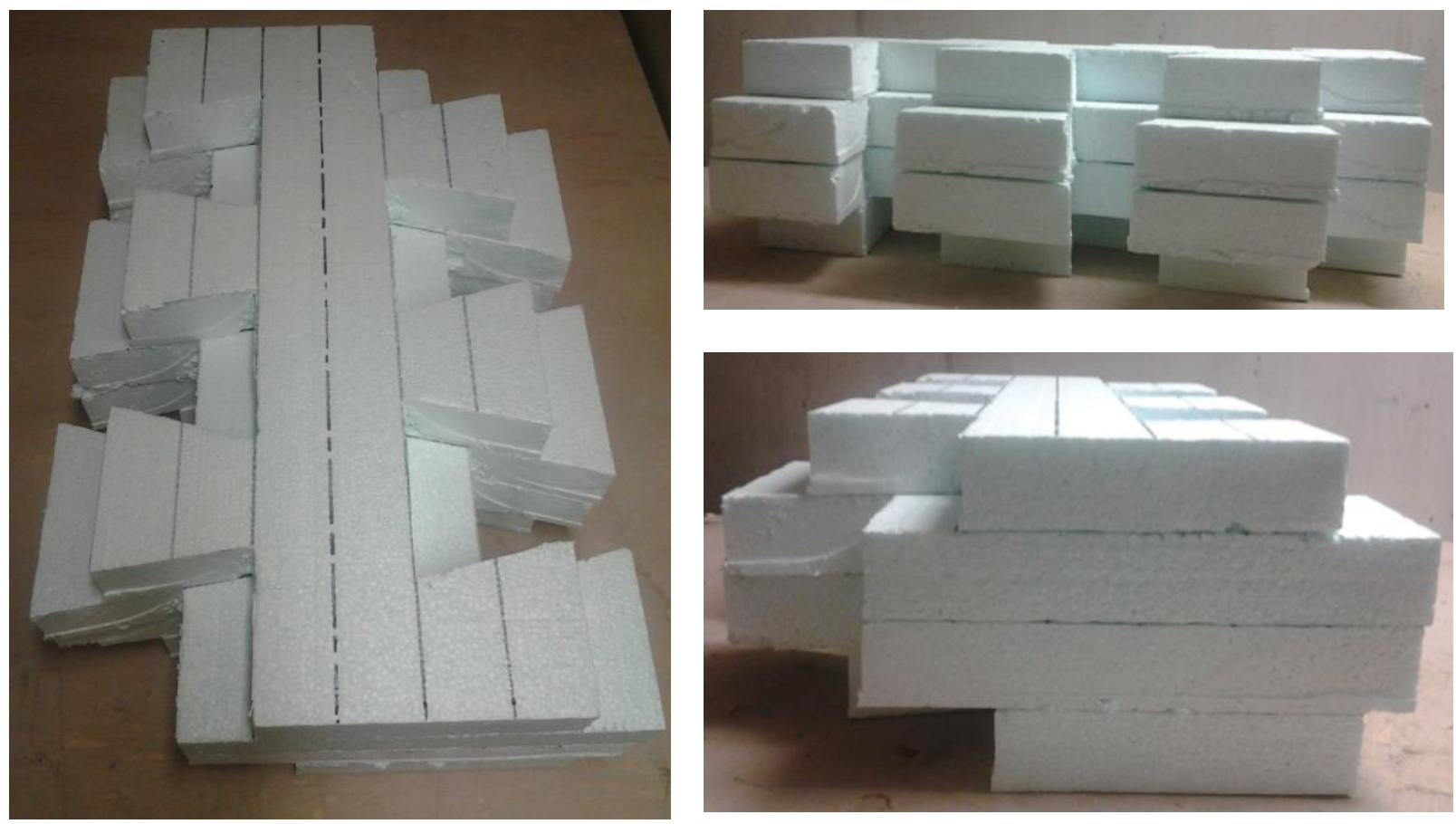

Figure 5.7 Styrofoam prepared to form the zigzag-shape joint 


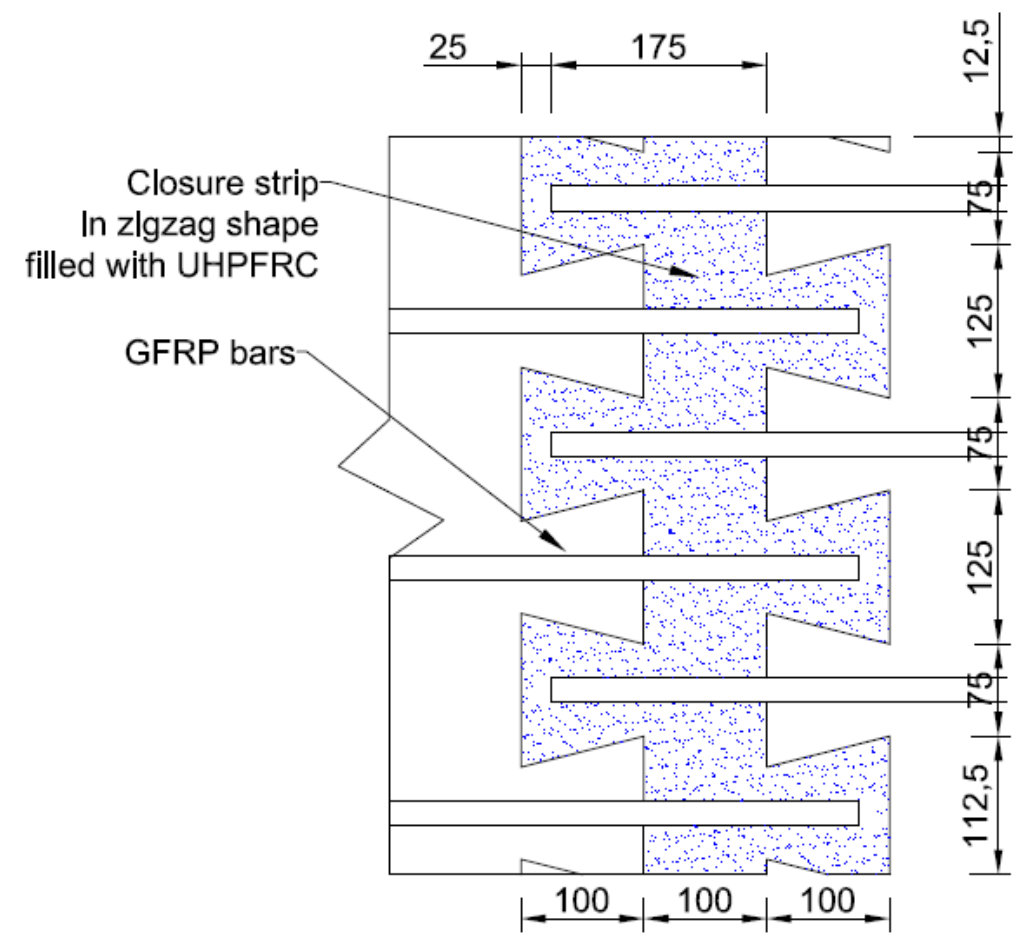

a) Arrangment of GFRP bars projecting into the joint

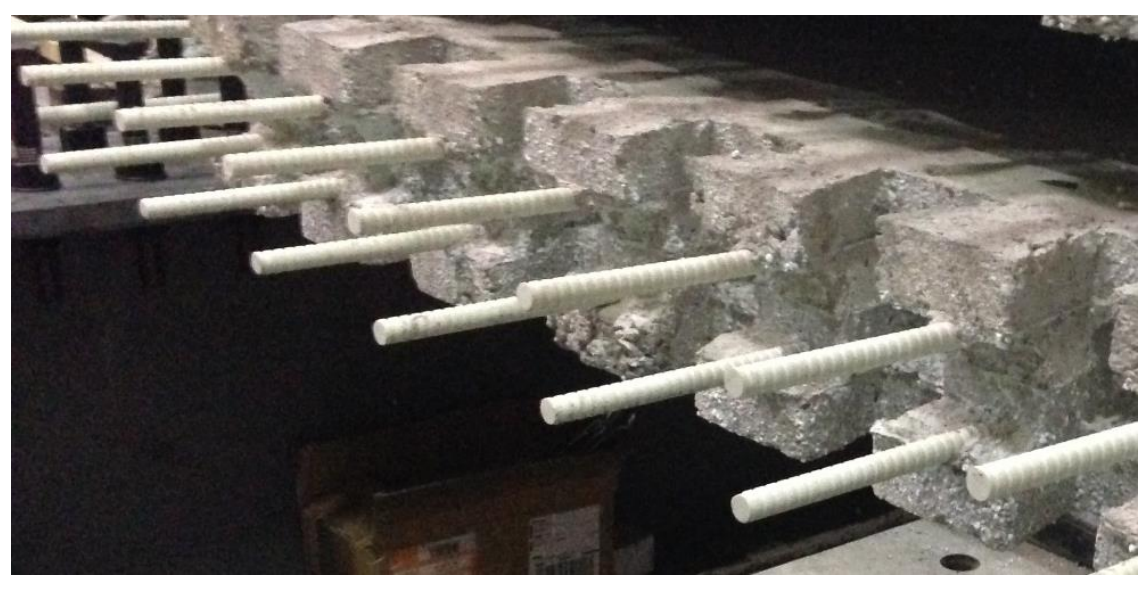

b) Photo of Z-joint

Figure 5.8 Arrangement of GFRP bars projecting into the zigzag-shape joint (Z-shape) for slabs S5 and S6 


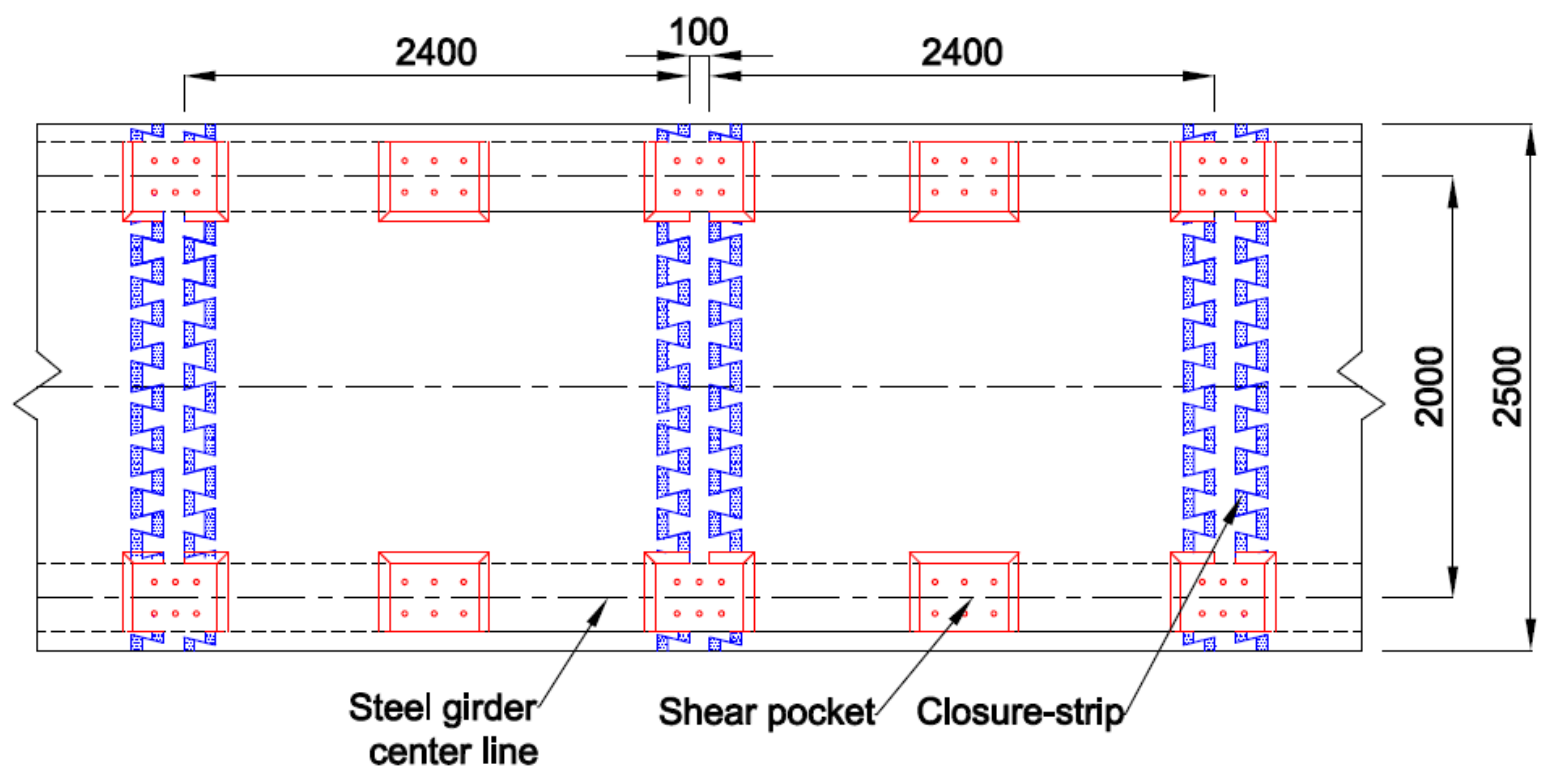

Figure 5.9 Plan of precast FDDP arrangement for Z-shape joints

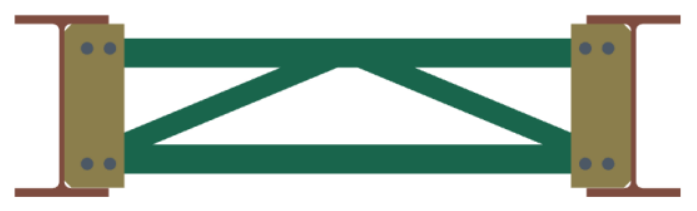

a) North / south elevation

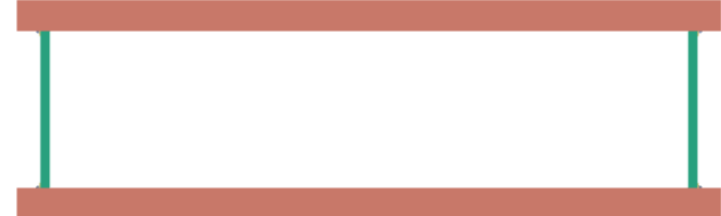

b) Top plan

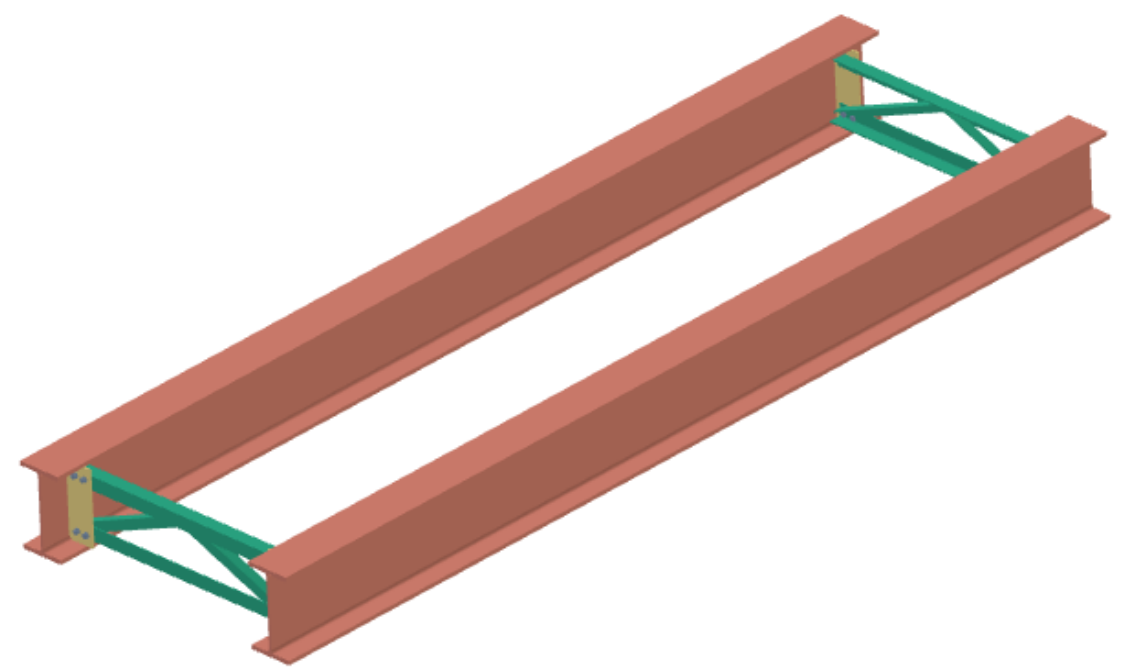

c) Twin-girder of W610x241 shape and $7500 \mathrm{~mm}$ length

Figure 5.10 Schematic diagrams of the steel twin-girder system supporting the precast FDDPs 


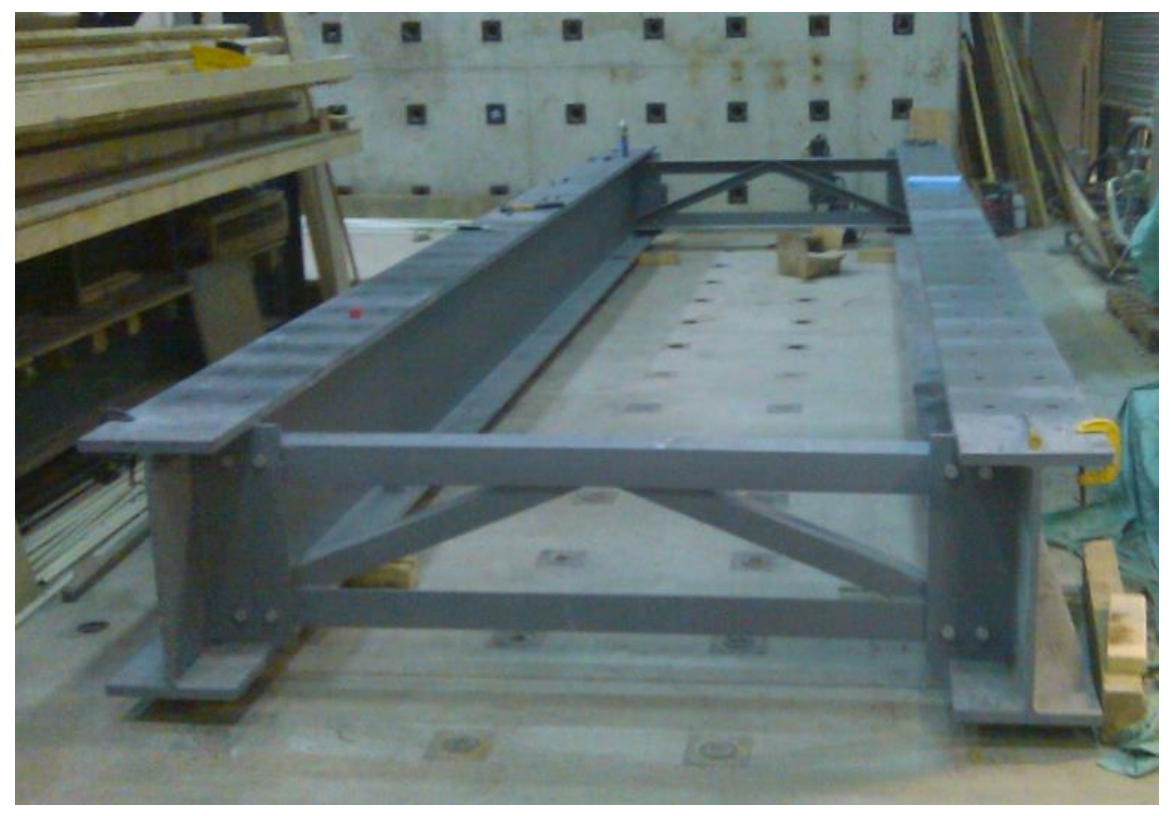

Figure 5.11 View of the steel twin-girder system supporting the precast FDDPs

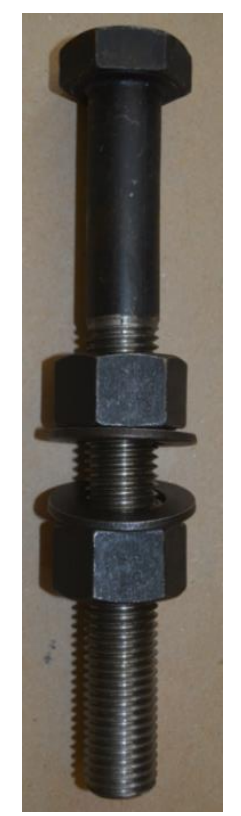

a) Assembled shear stud

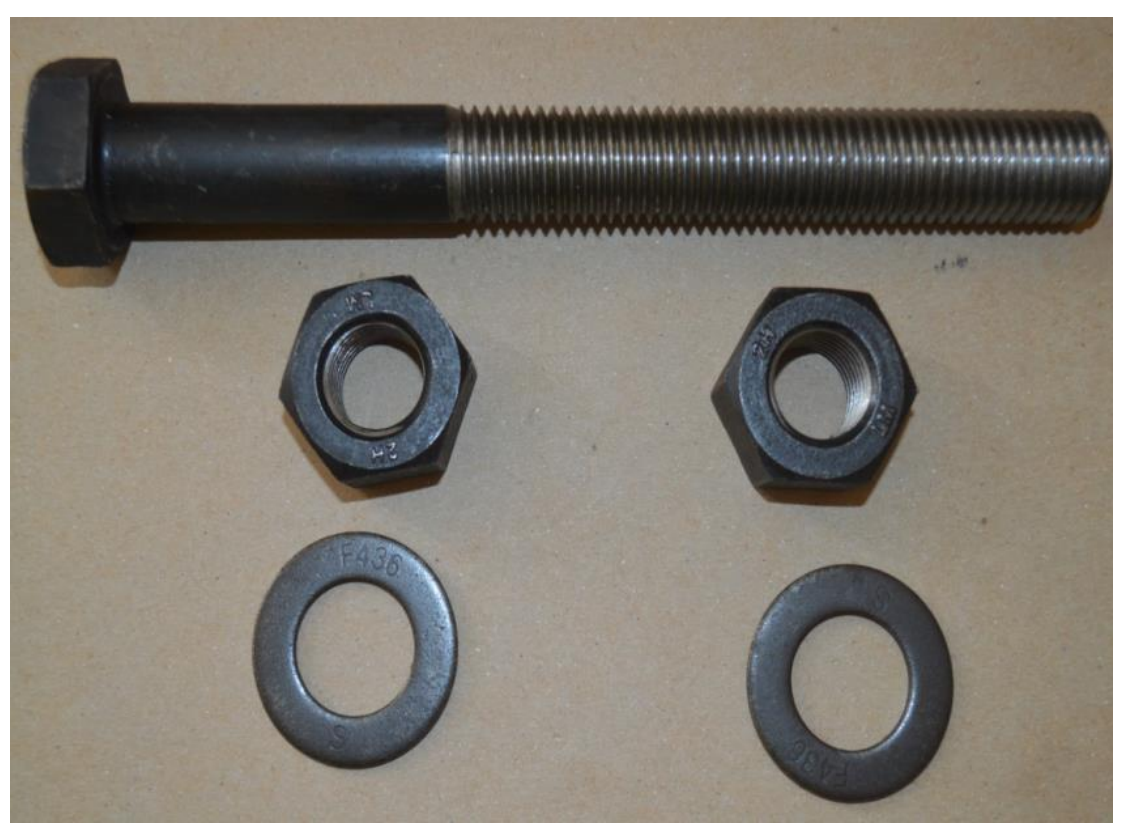

b) Assembly of the shear stud

Figure 5.12 Views of M25 high-strength headed bolt and its nuts and washers used to connect the precast FDDPs to the steel girders through shear pockets 


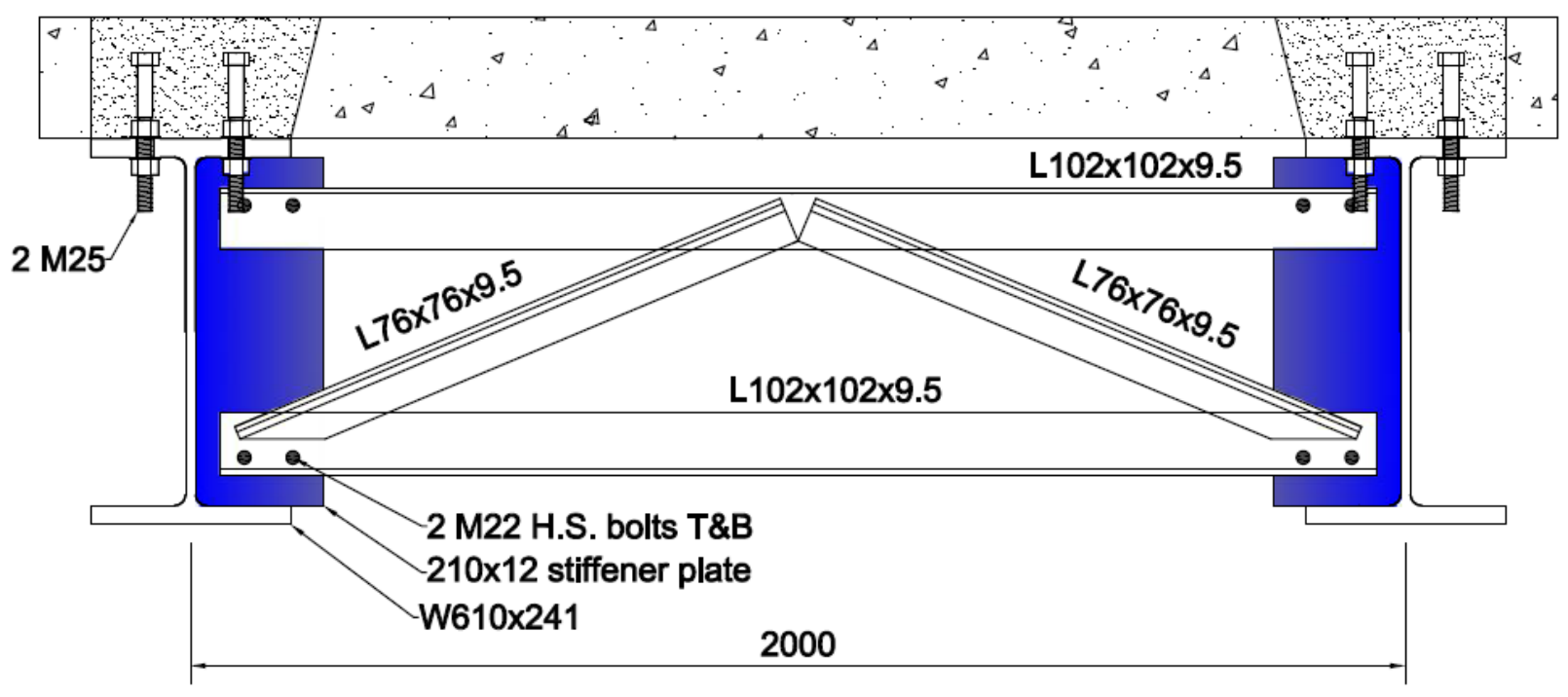

a) Cross-section details of the precast FDDP and the twin-girder system

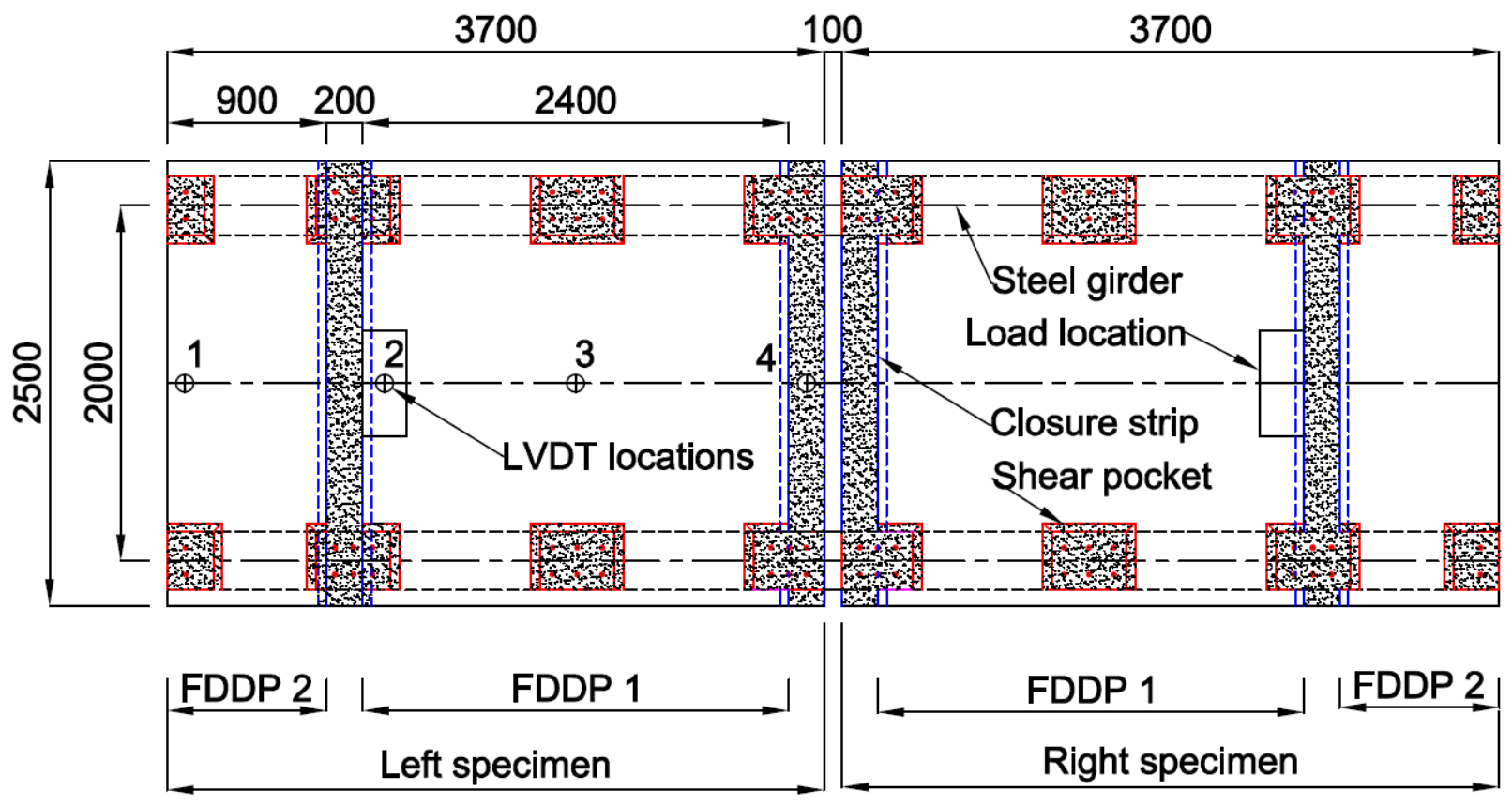

b) Plan view for the arrangement of the precast FDDPs and locations of displacement sensors (LVDT 1 is located at the free-end; LVDT 2 at load location; LVDT 3 at the mid-span; and LVDT 4 at the fixed-end)

Figure 5.13 Composite precast FDDP section and plan 

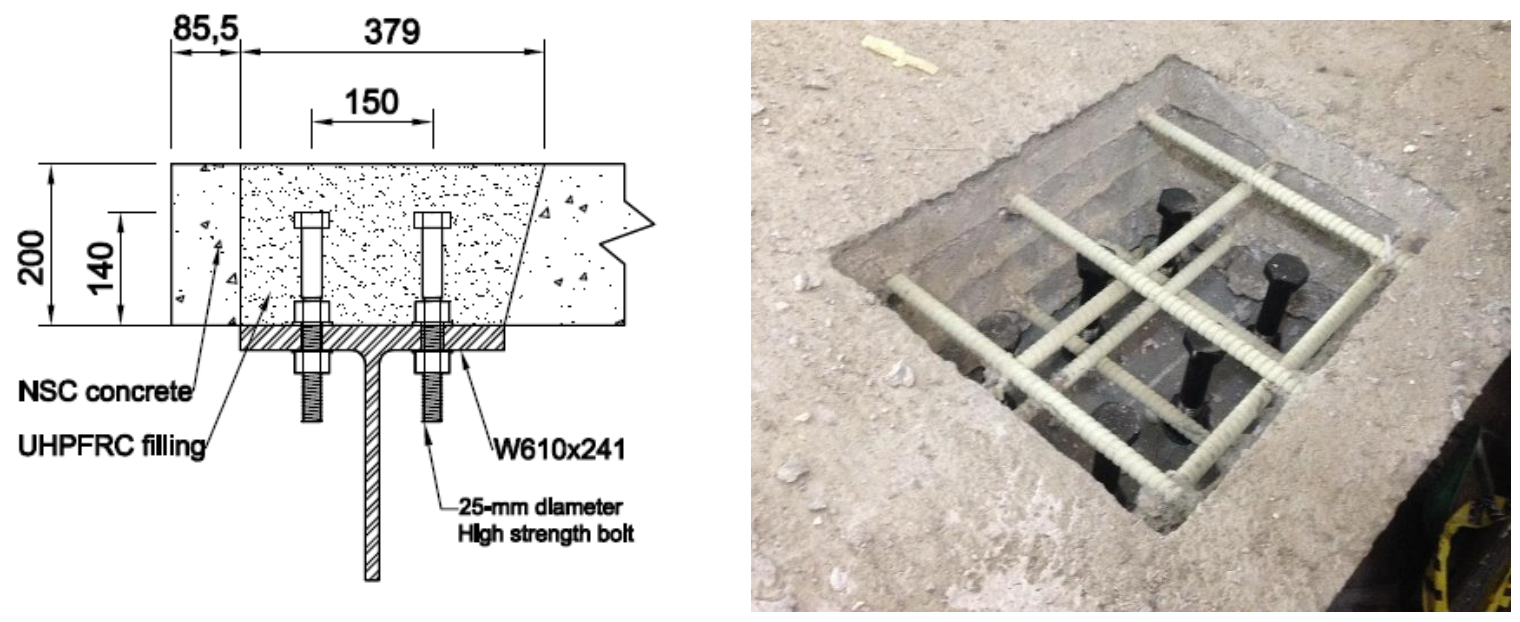

Figure 5.14 Panel-to-girder connection in the transverse direction
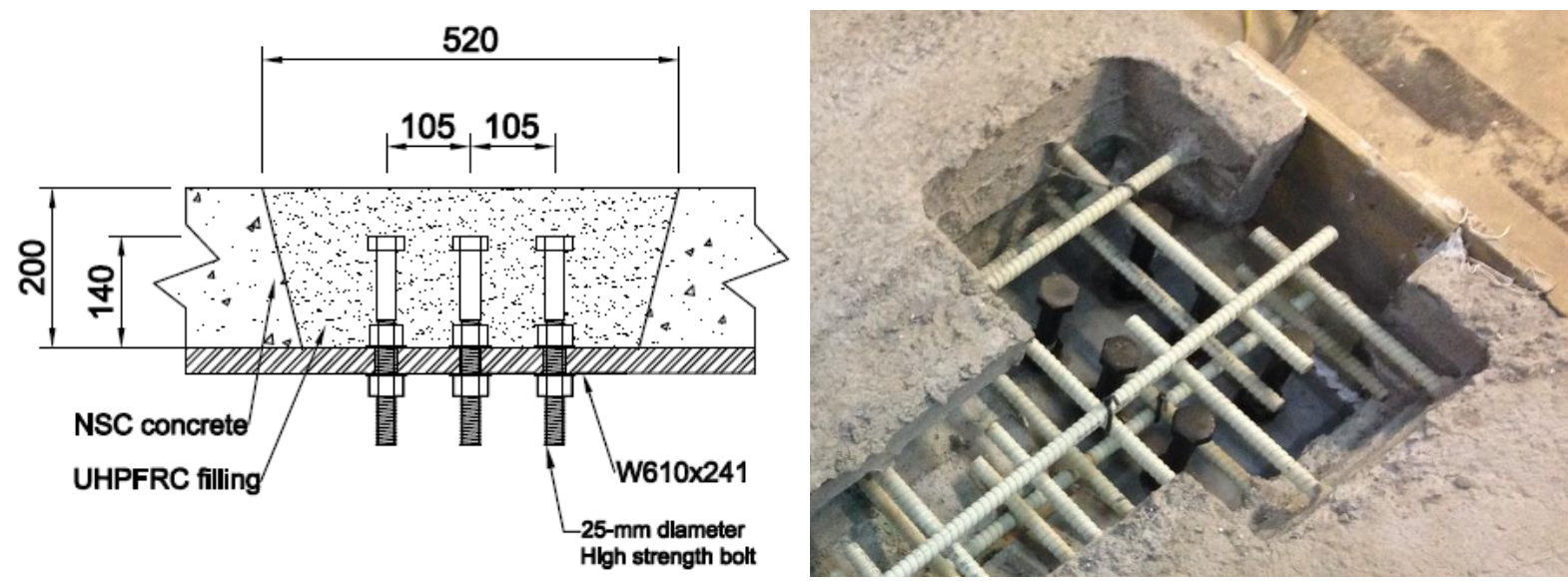

Figure 5.15 Panel-to-girder connection in the longitudinal direction at the edges of the precast FDDP 

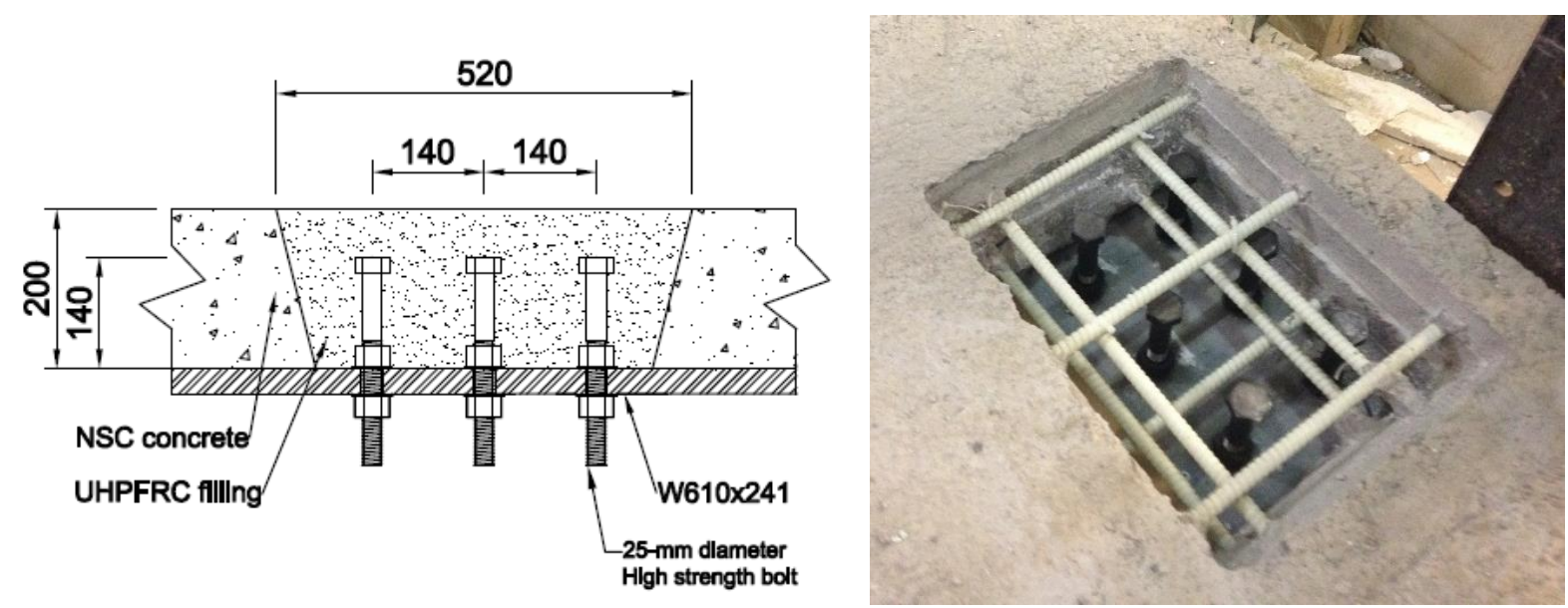

Figure 5.16 Panel-to-girder connection in the longitudinal direction at the mid-width of the precast FDDP 


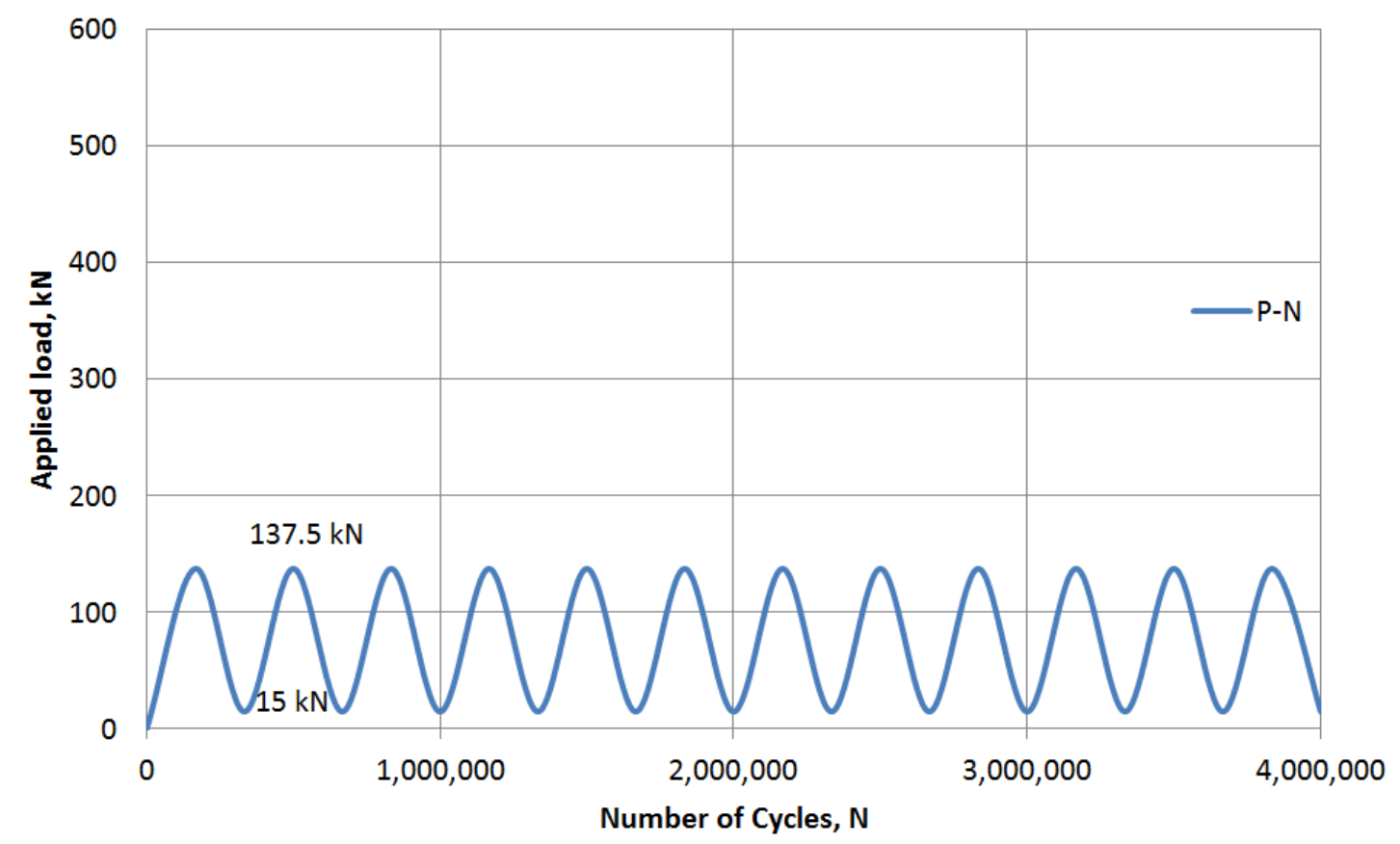

Figure 5.17 Constant amplitude fatigue (CAF) loading history

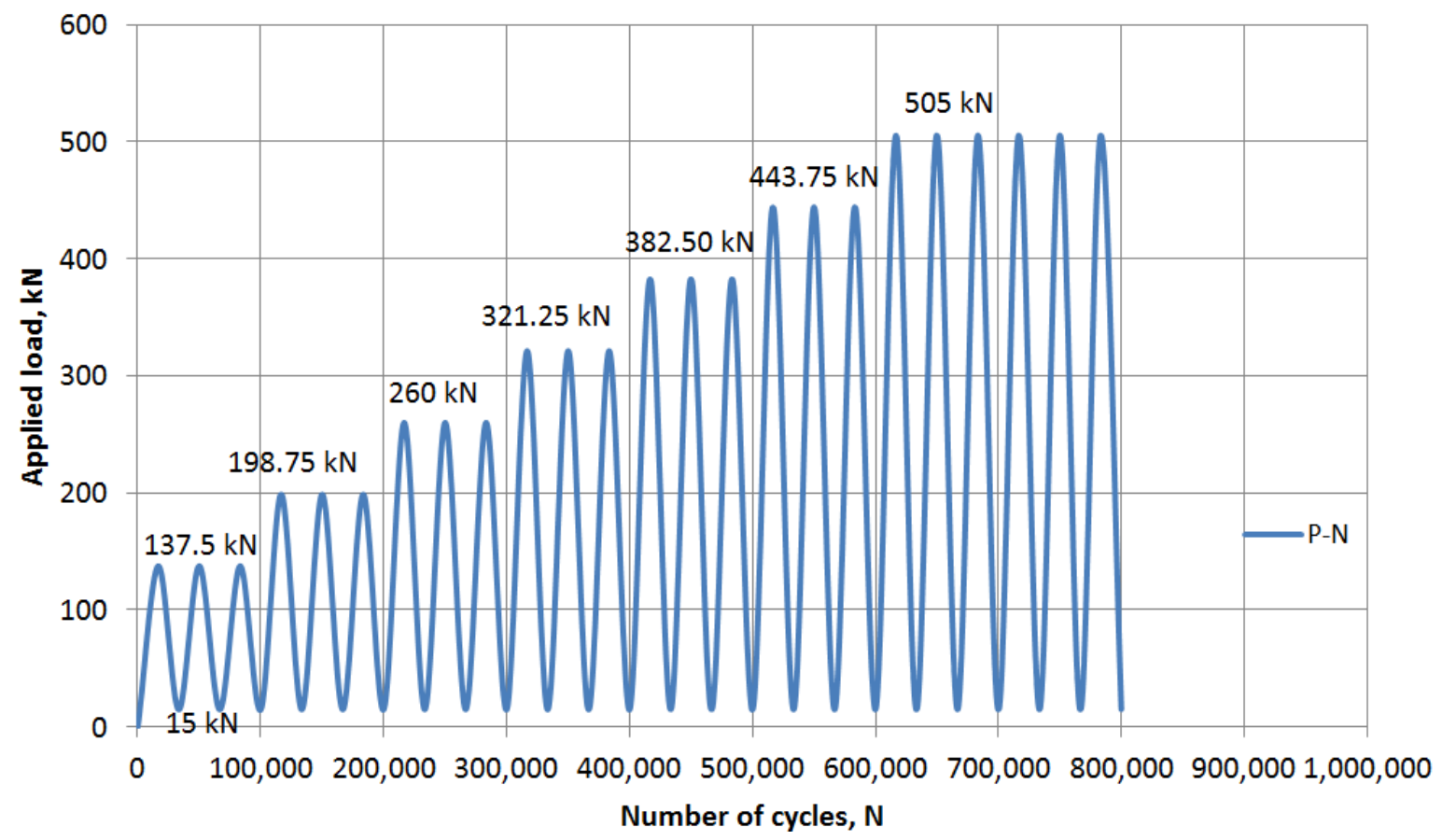

Figure 5. 18 Variable amplitude fatigue (VAF) loading history 


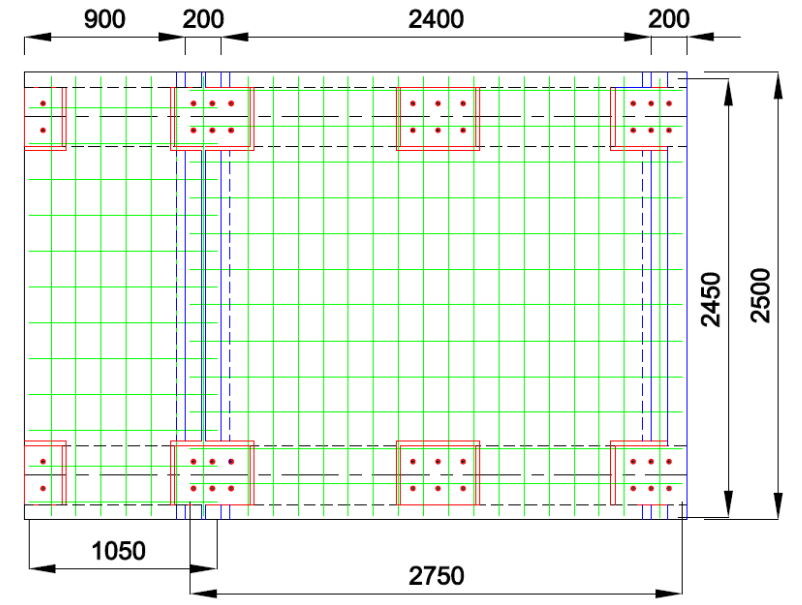

a) Plan view of bottom reinforcement

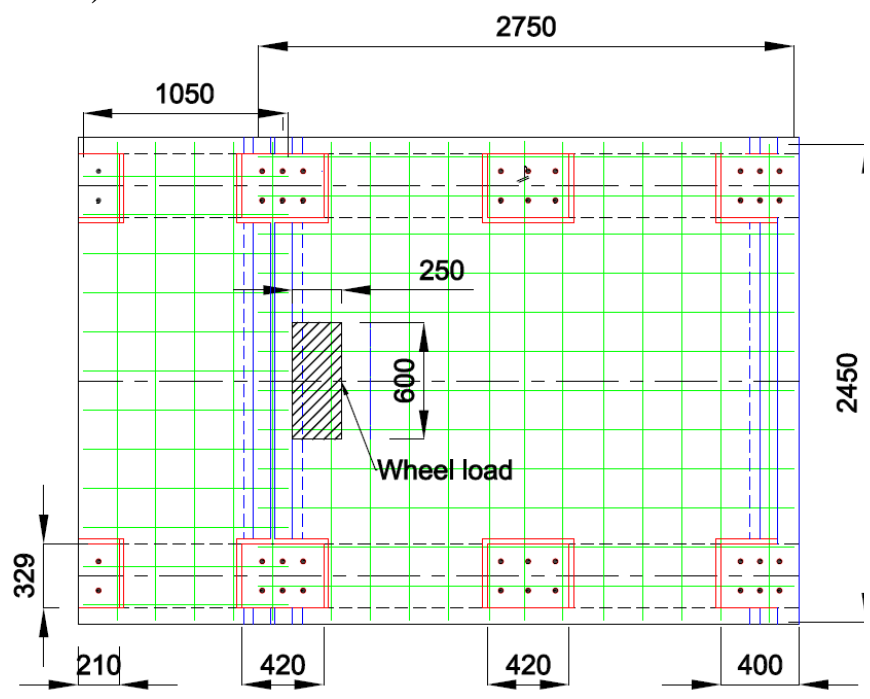

b) Plan view of top reinforcement and wheel load position

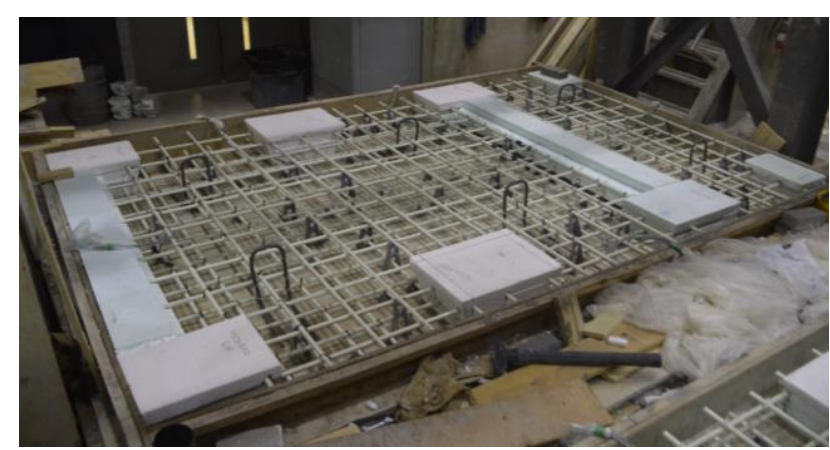

c) View of GRPP reinforcement and and styrofoam to form the joints

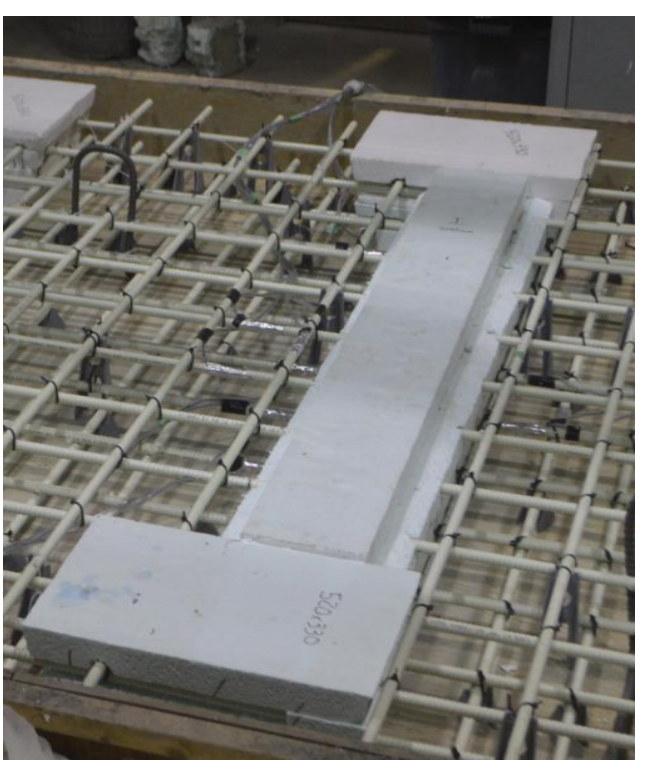

d) View of styrofoam forming the joint

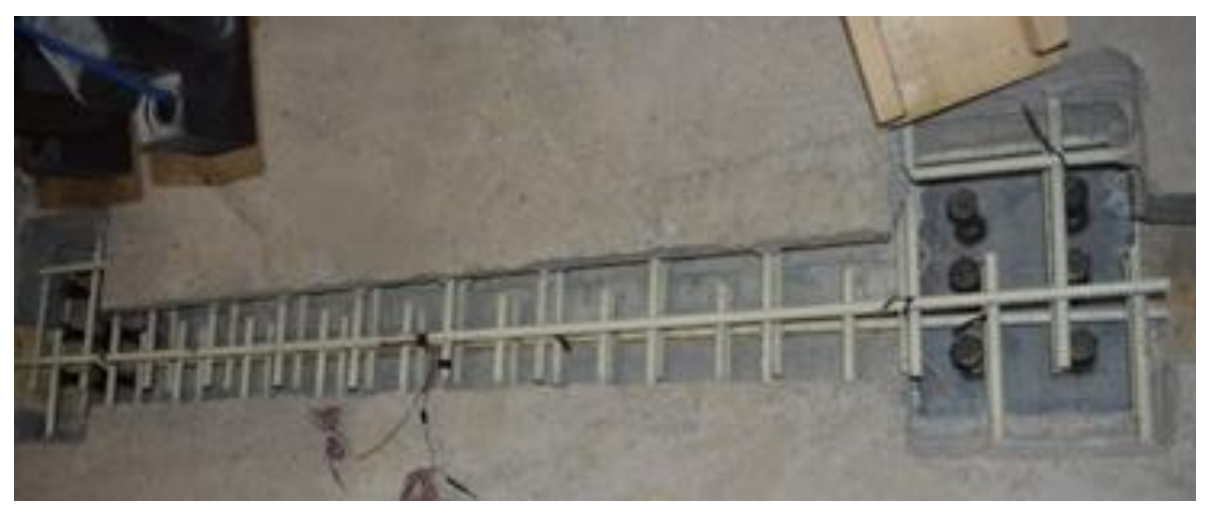

e) Photo of the closure strip for the A-joint before casting

Figure 5.19. Construction of the precast FDDP with A-shape join (slabs S1 and S2) 


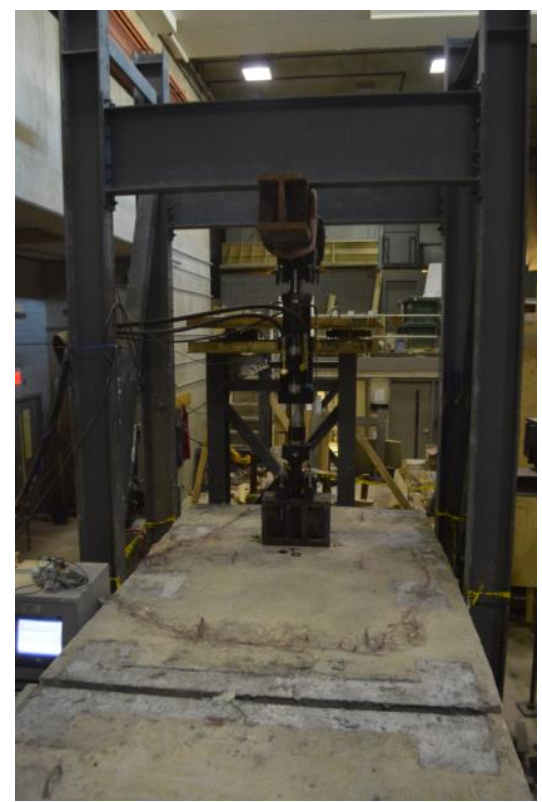

a) Slab-girder bridge system during CAF and VAF loading

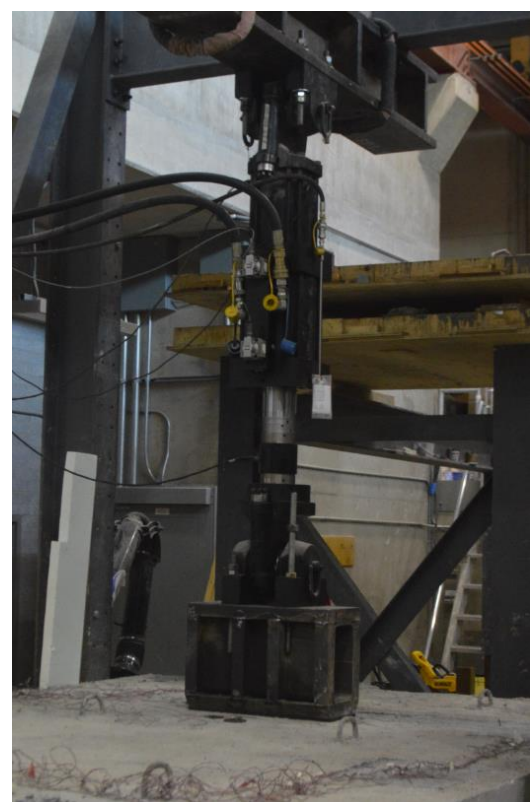

b) View of the actuator

Figure 5.20. View of the test setup for fatigue loading for specimens S1 and S2
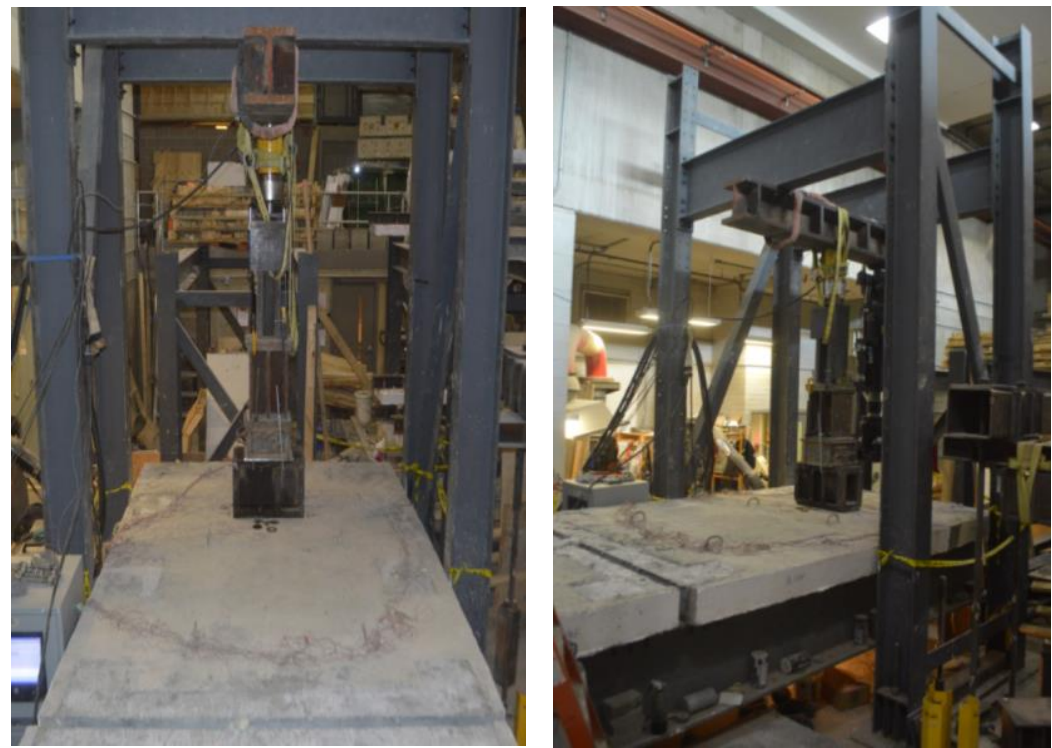

a) Slab-girder bridge system during monotonic loading

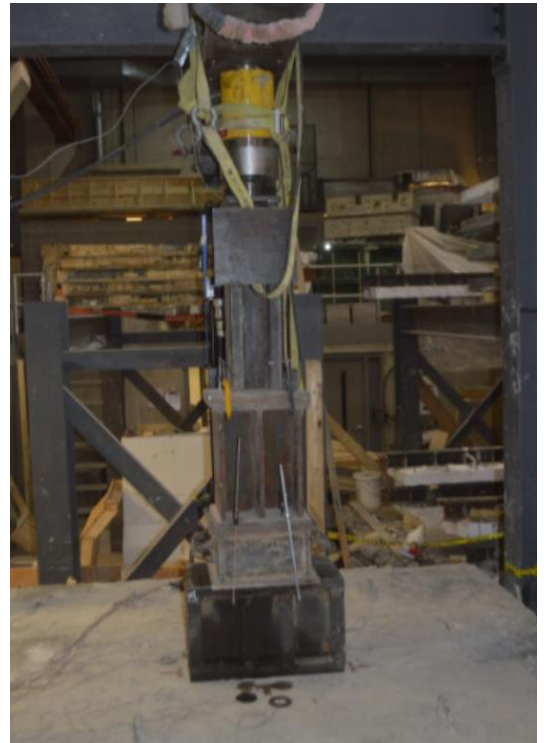

b) View of the loaded area

Figure 5.21. View of the test setup for the monotonic loading for slab S1 


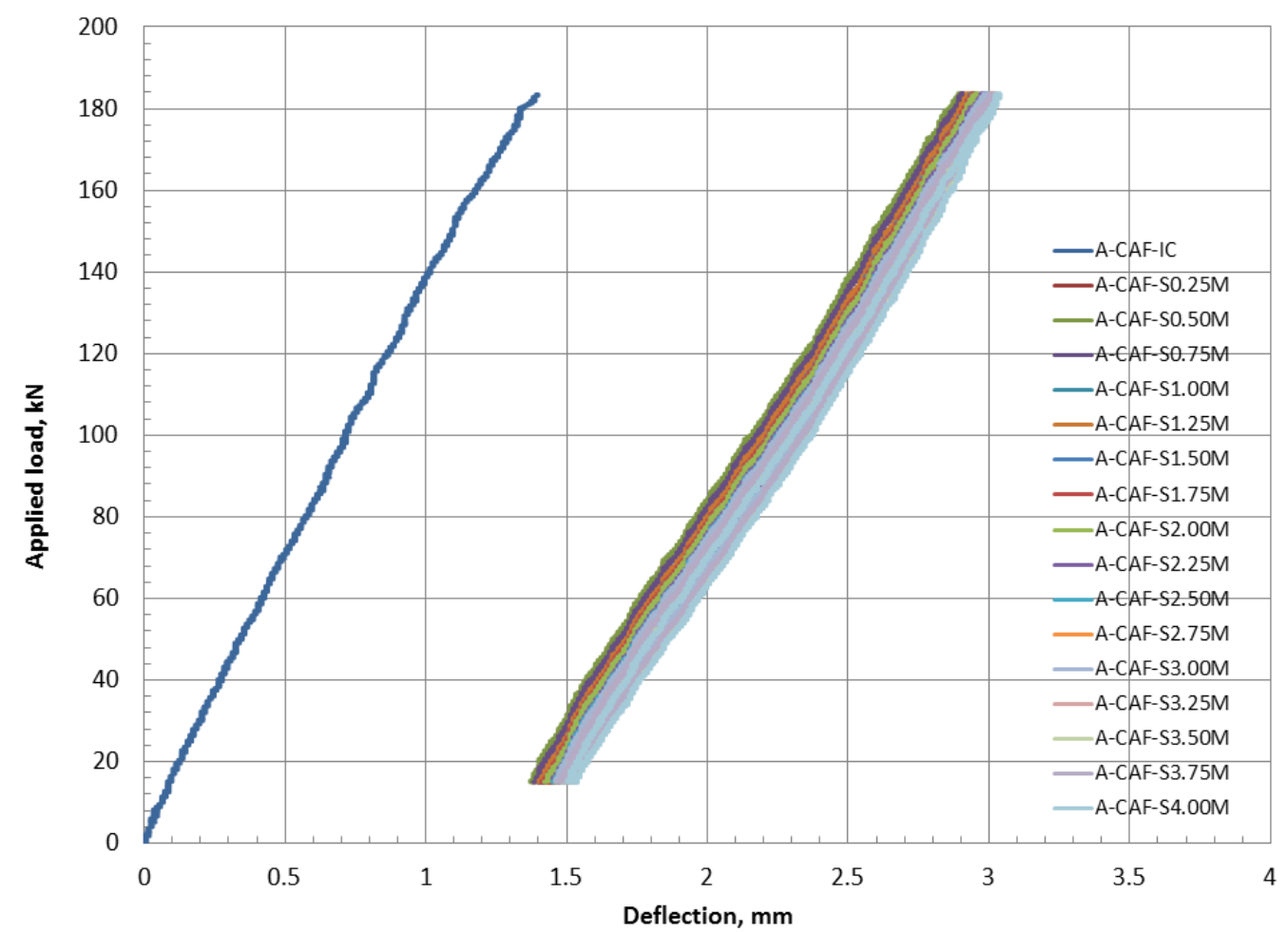

.a) Load-deflection curves under static load after each 250,000 CAF cycles for slab S1

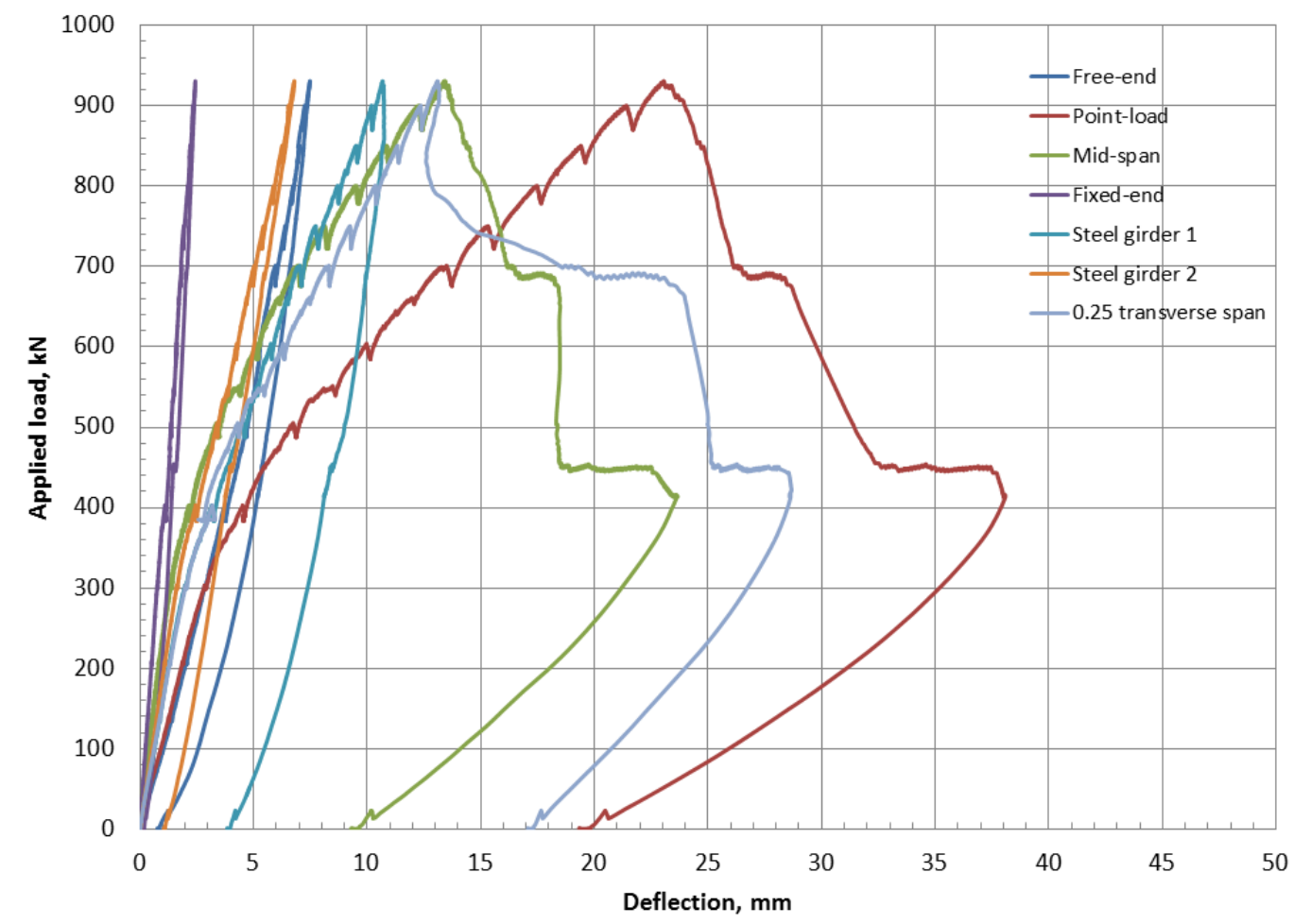

b) Load-deflection curves under static load to-collapse

Figure 5.22. Static load-deflection curves for slab S1 after being subjected to CAF loading 


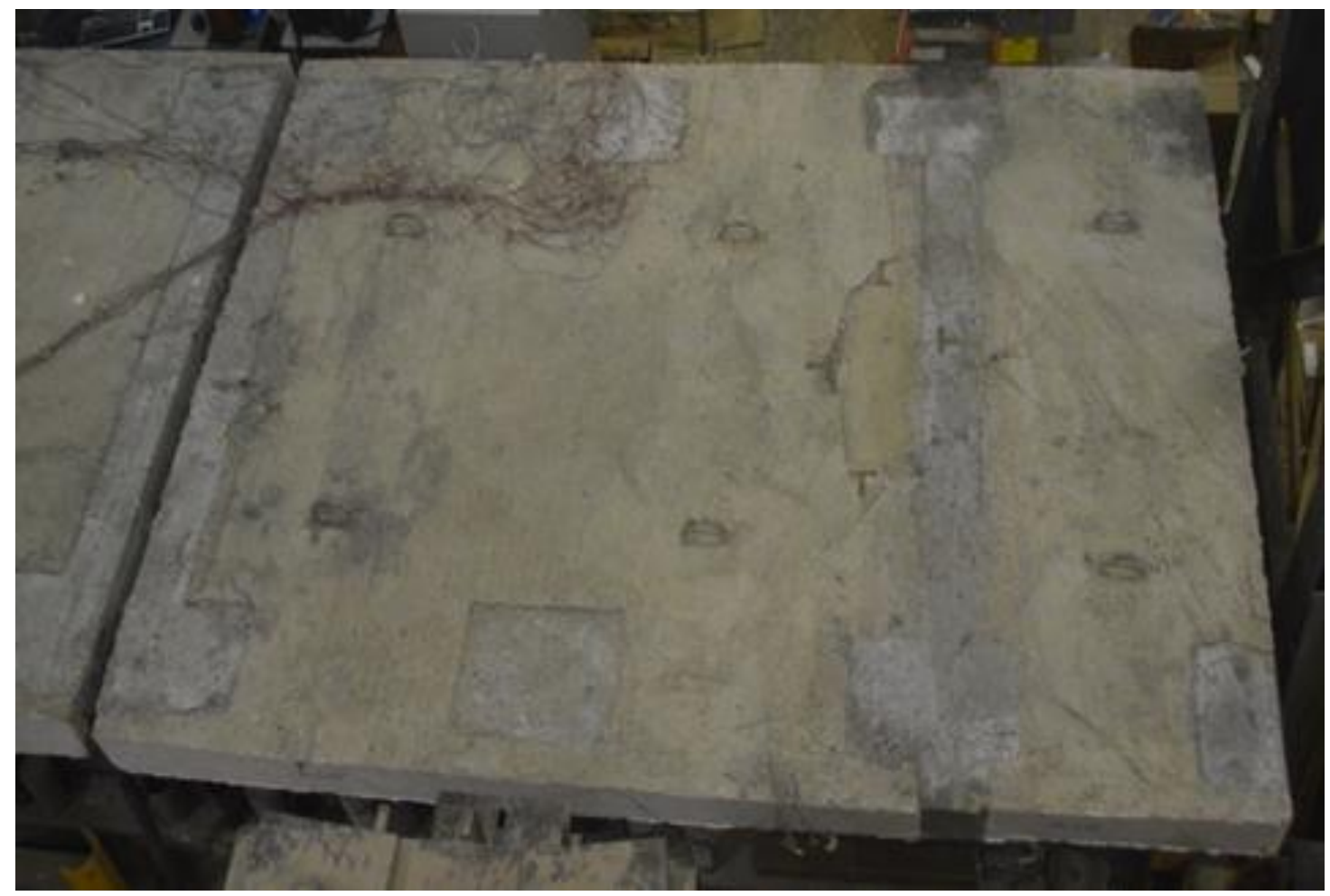

a) Top view of the slab showing punching shear failure

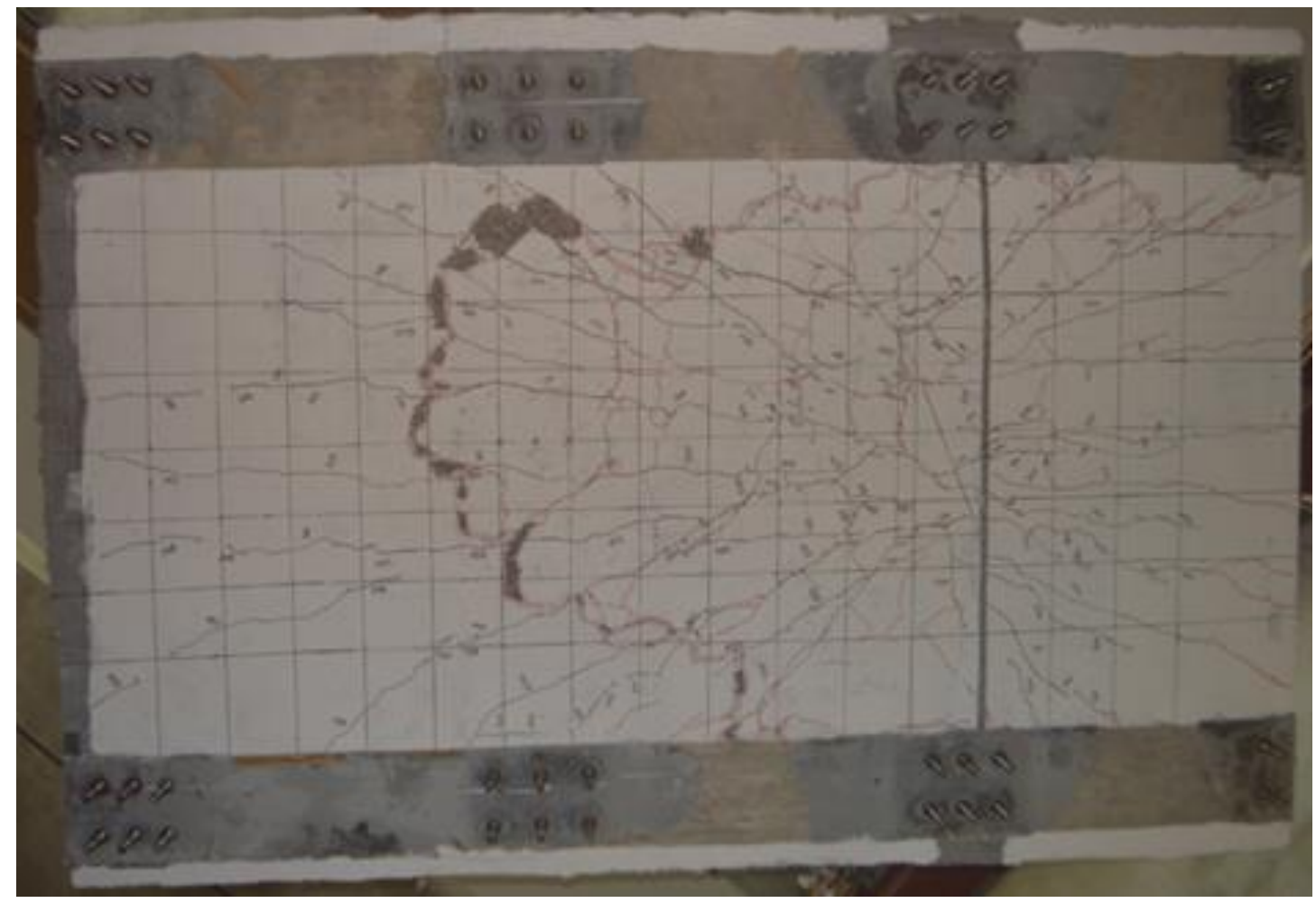

b) Bottom view of the slab showing crack pattern after failure

Figure 5.23. Crack pattern after failure of slab S1 after testing under static load to-collapse 


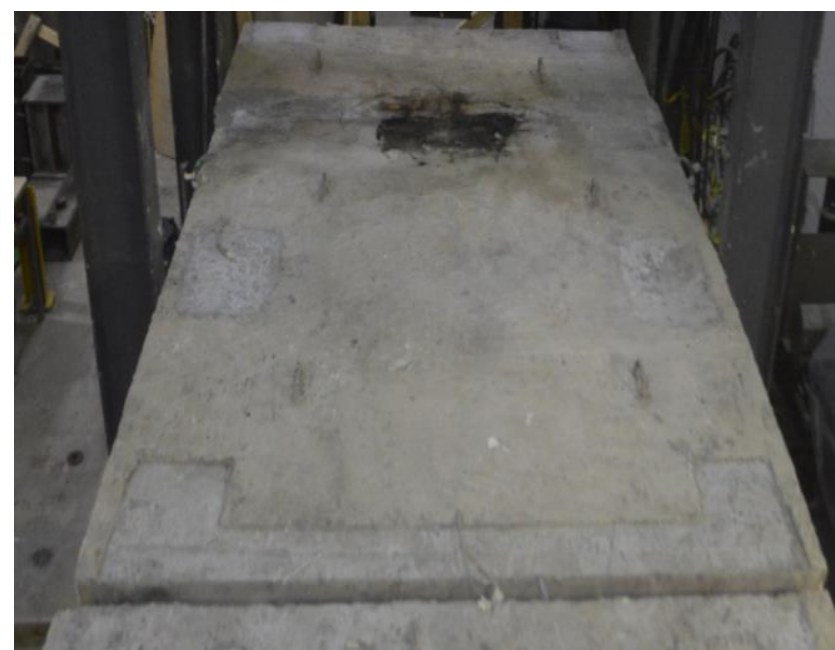

a) Top view showing punching shear failure at wheel footprint

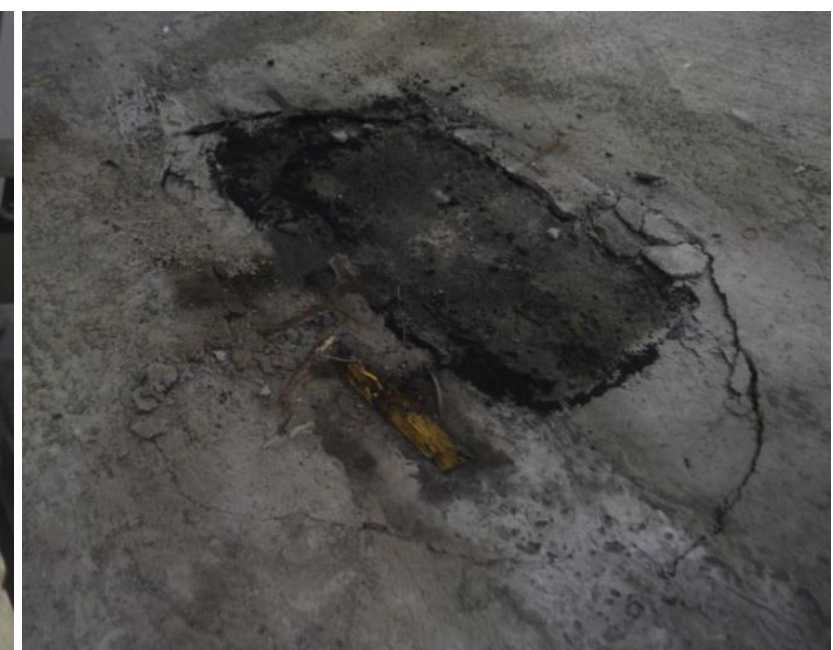

b) Close-up top view for the punching shear failure at the wheel footprint

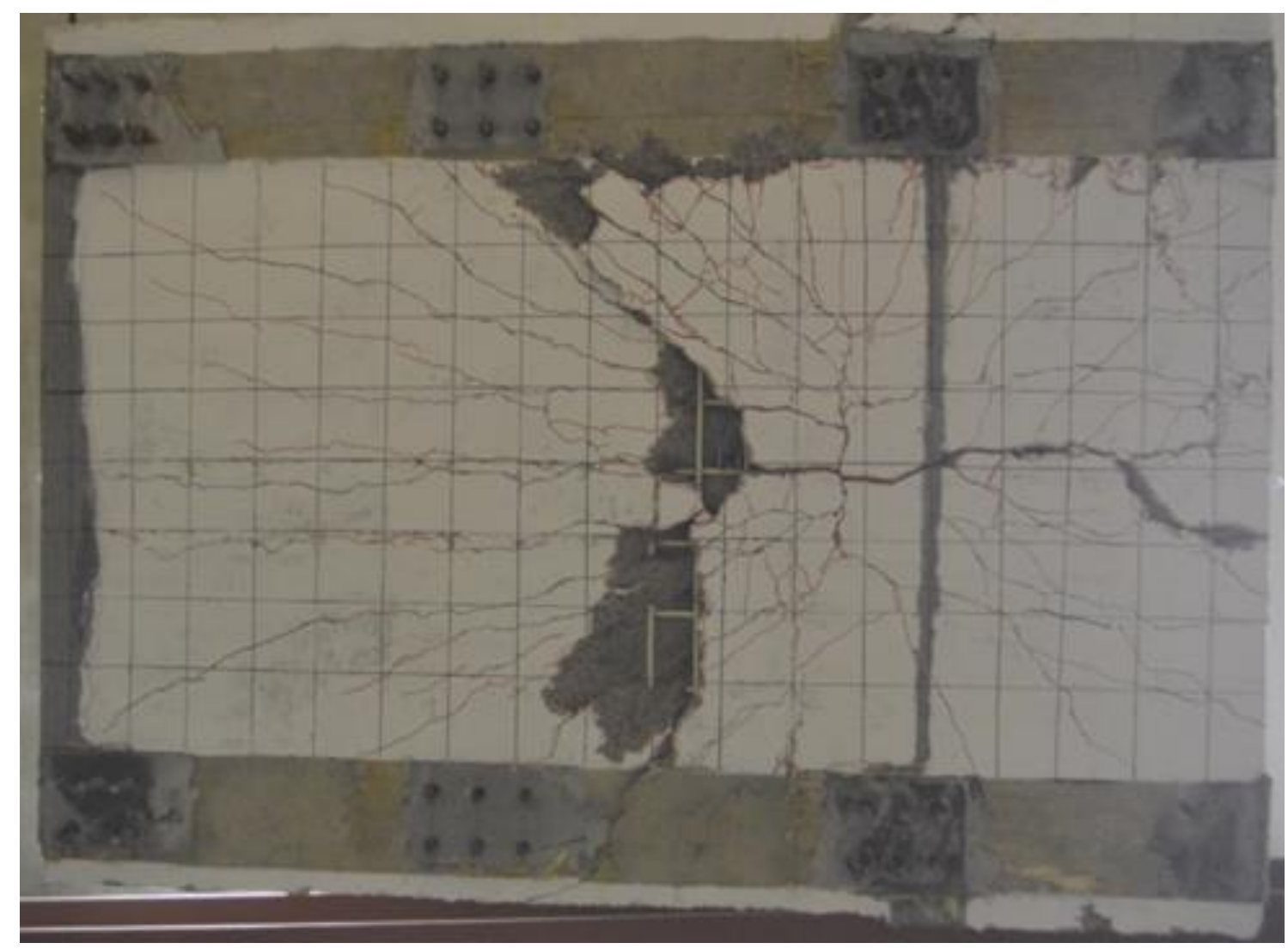

c) Bottom view showing crack pattern

Figure 5.24. Views of punching shear failure of slab S2 tested under VAF loading to-collapse 


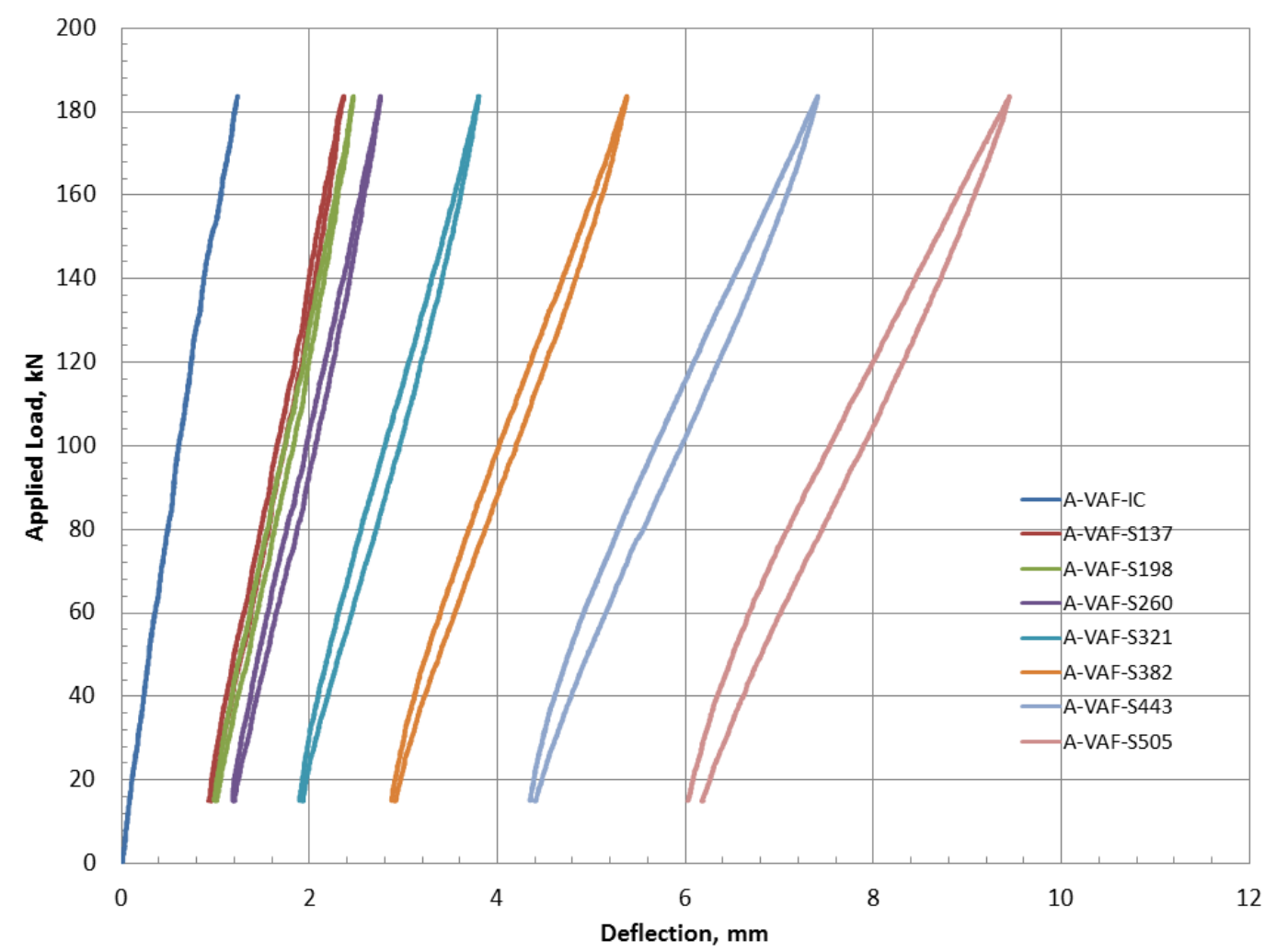

Figure 5.25. Static load-deflection curves of the slab S2 with A-joint after every 100,000 cycles of VAF loading 


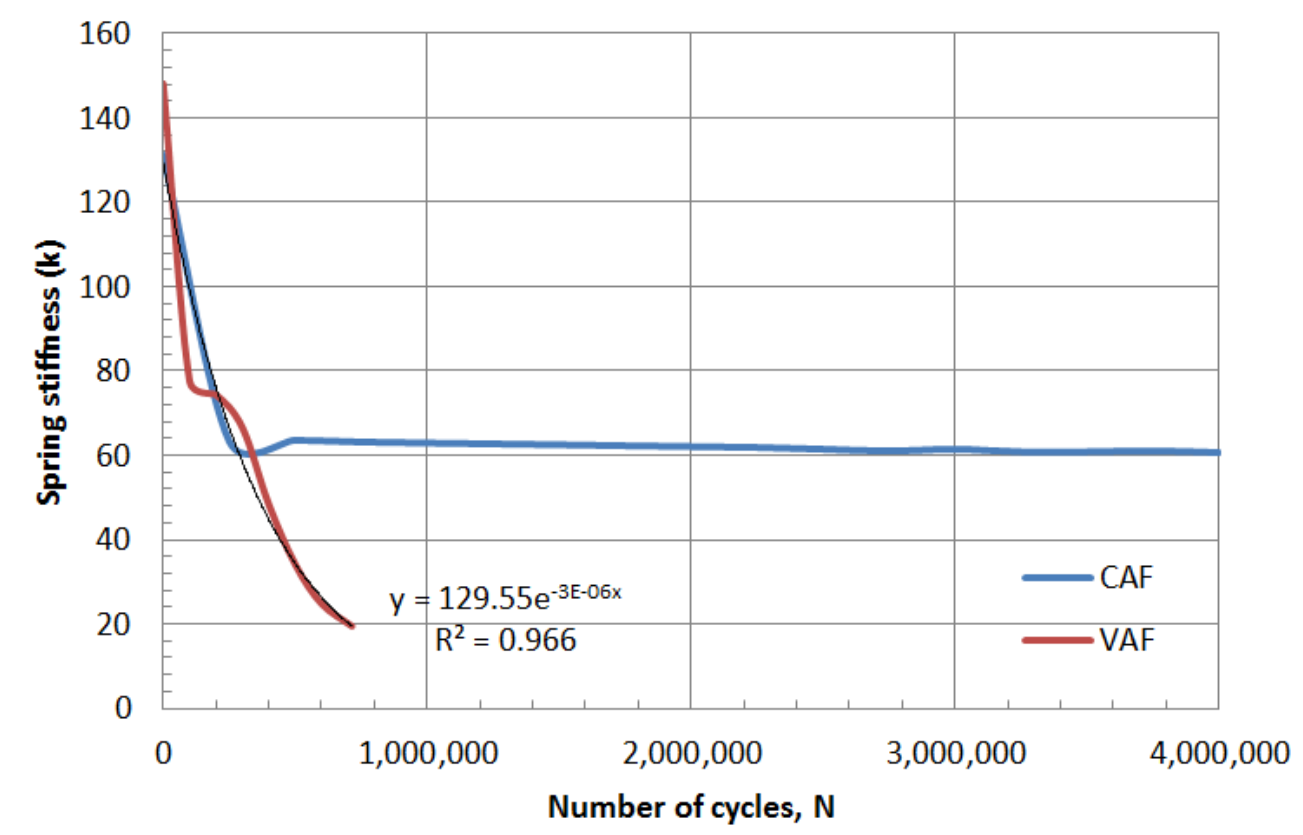

a) Spring stiffness-number of cycles curves

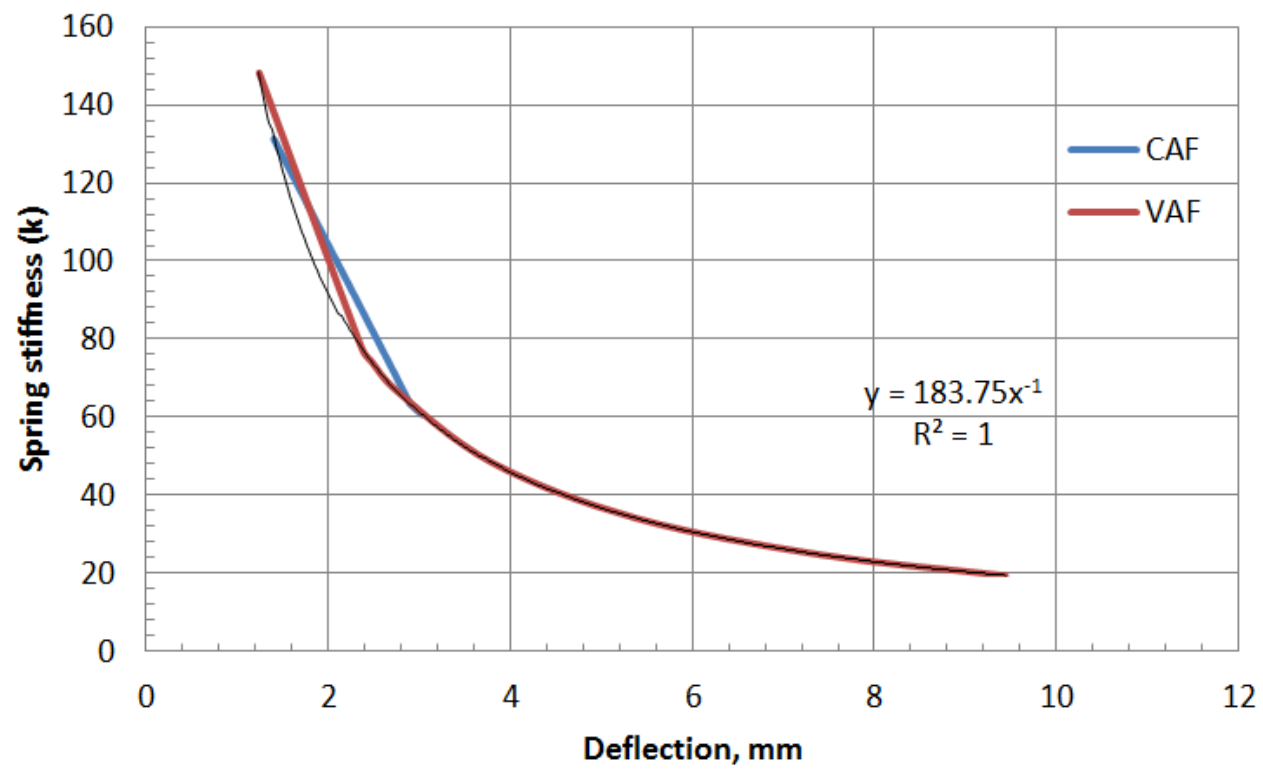

b) Spring stiffness-deflection curves

Figure 5.26. Degradation of the precast FDDPs with A-joint details under CAF and VAF loading 


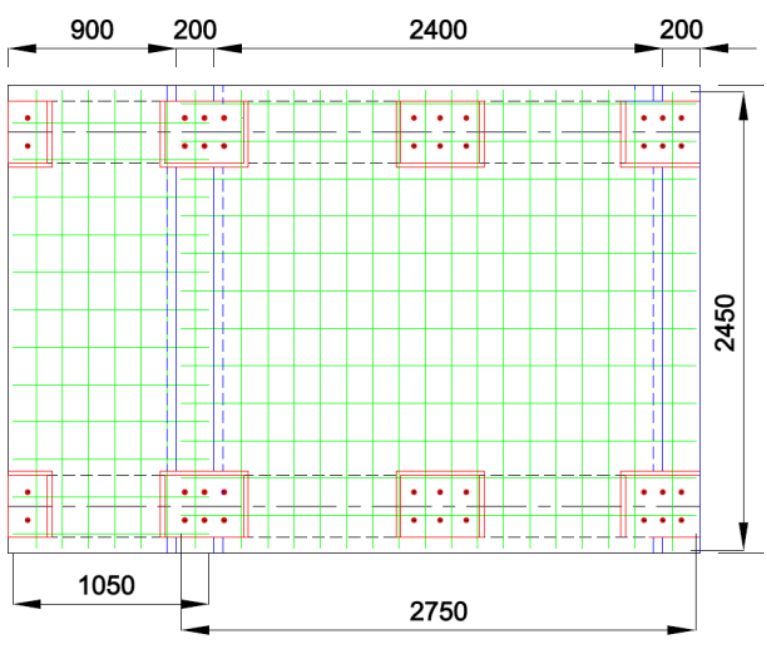

a) Place of bottom reinforcement

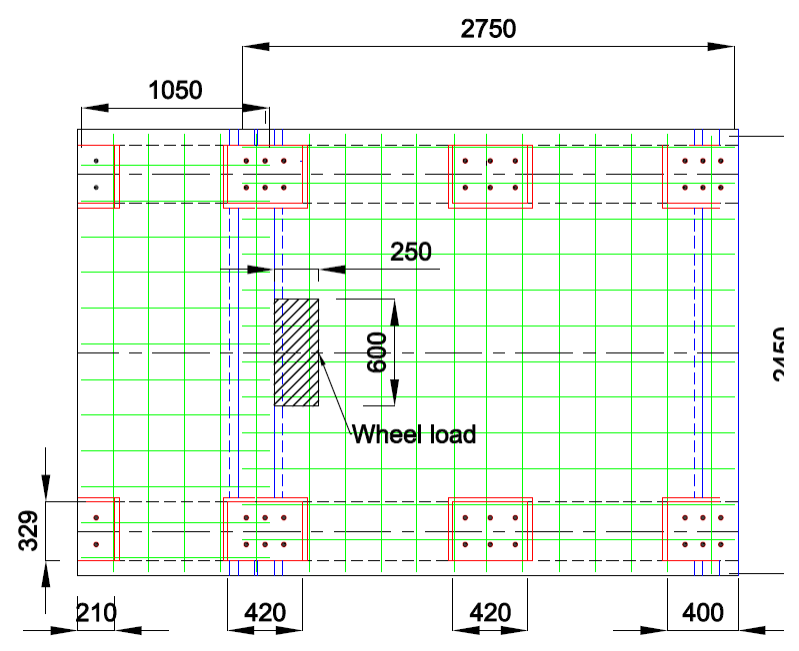

b) Plan of top reinforcement and wheel load position

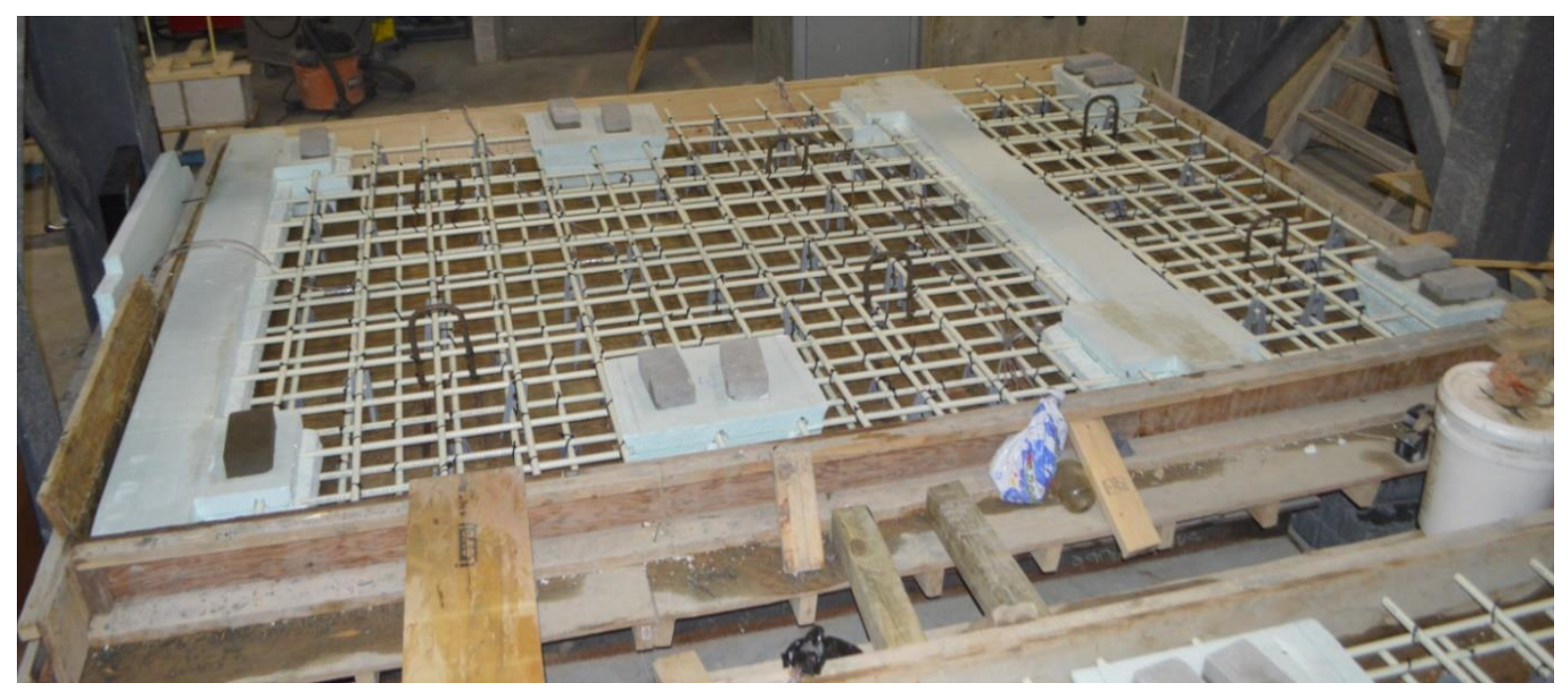

c) View of GFRP bars and styrofoam to form the joints

Figure 5.27 Construction of the precast FDDP with C-shape joint for slabs S3 and S4 


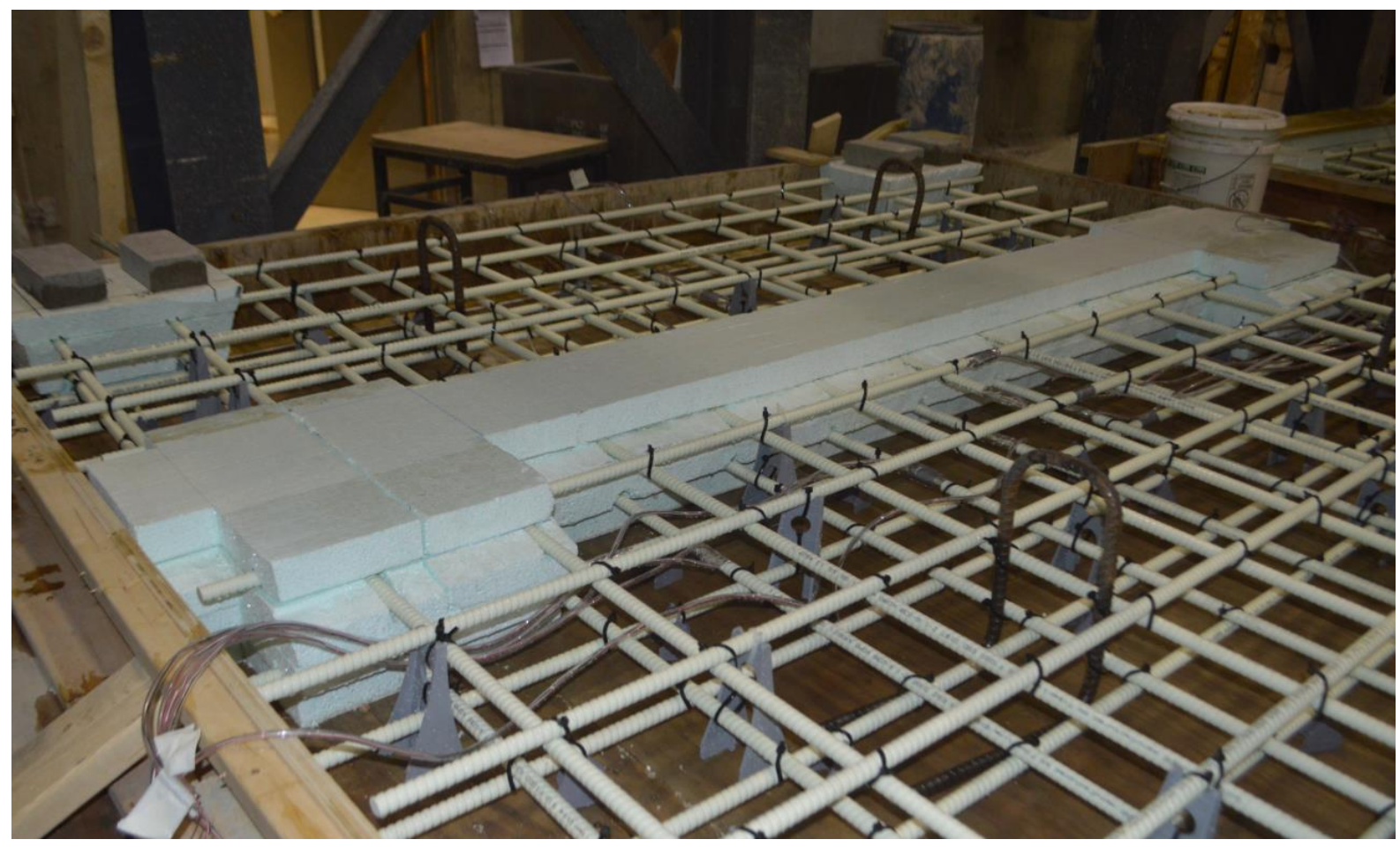

Figure 5.28 Close-up view of forming the C-joint using styrofoam

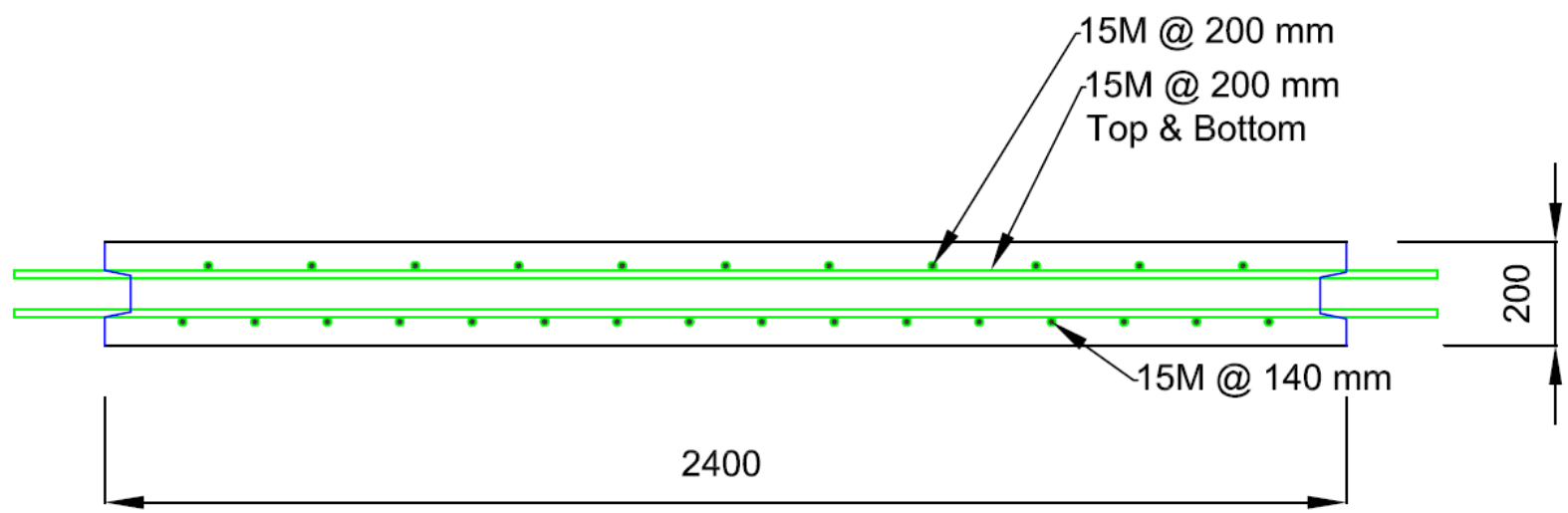

Figure 5.29 Longitudinal cross-section in the precast FDDP with C-joint showing details of GFRP arrangement 


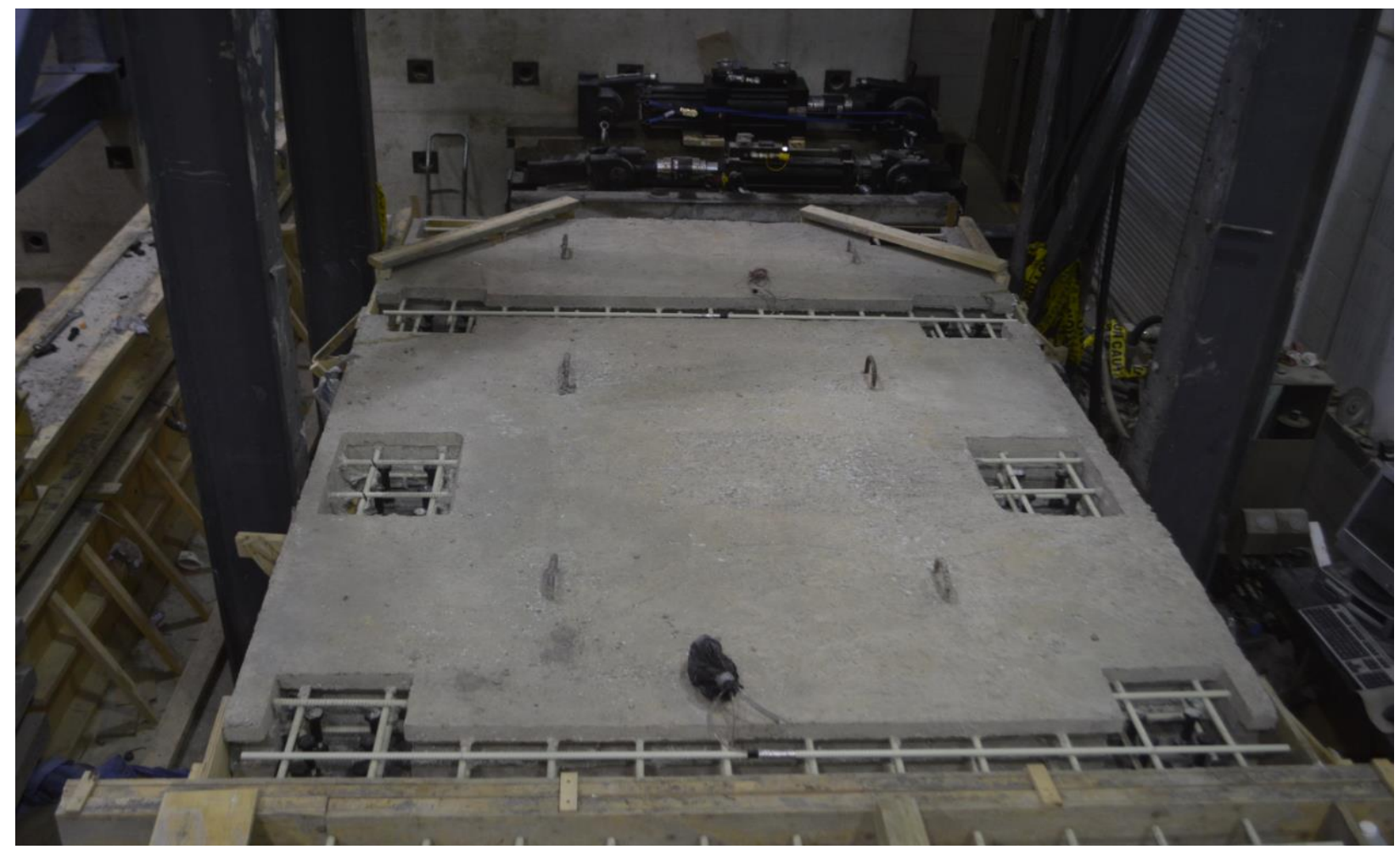

Figure 5.30 View of slab S3 with precast FDDP segments placed side by side normal the steel girders

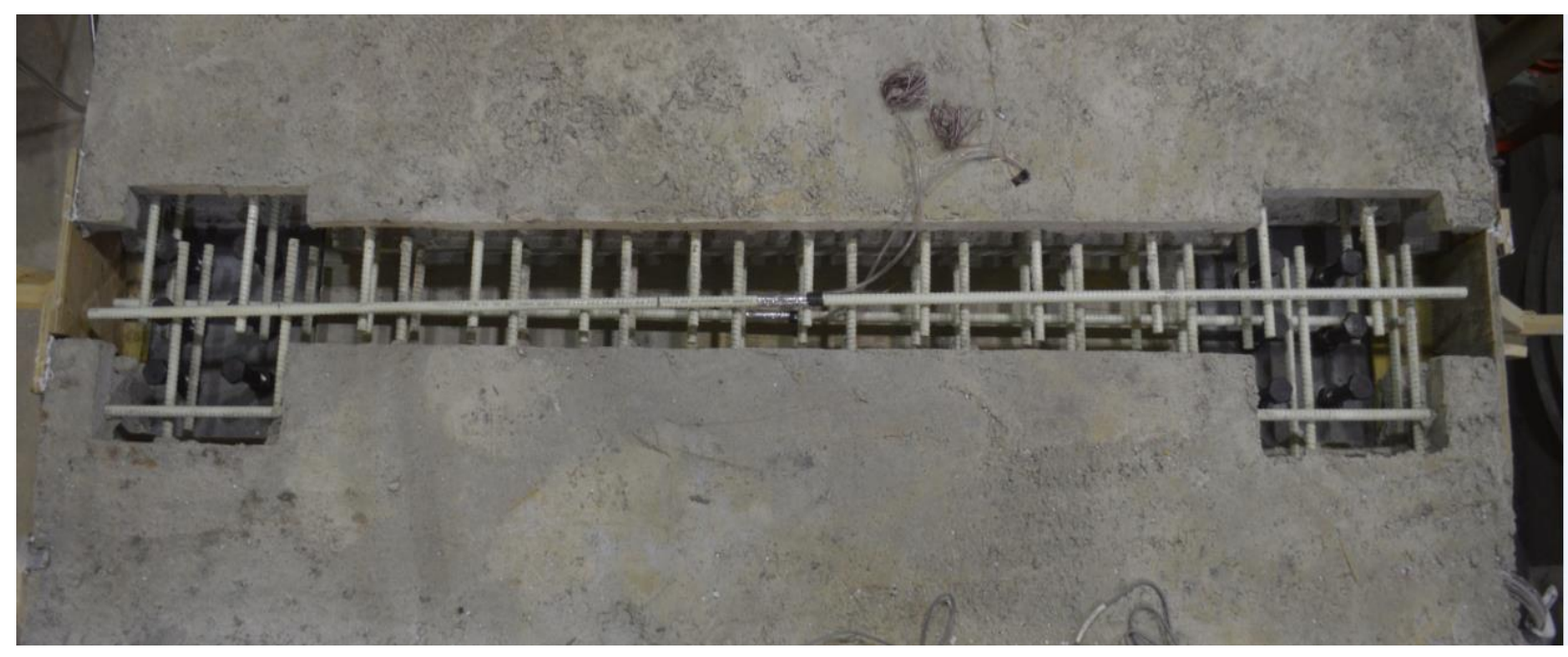

Figure 5.31 Photo of the closure strip for the $\mathrm{C}$-joint before casting 


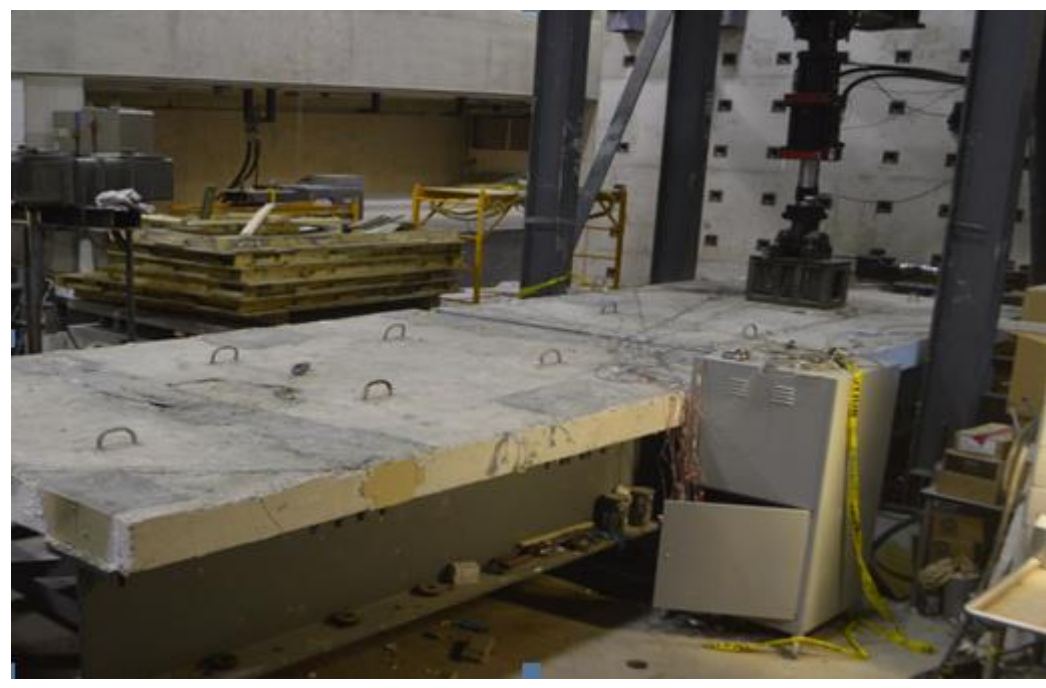

a) Slab-girder bridge system during CAF and VAF loading

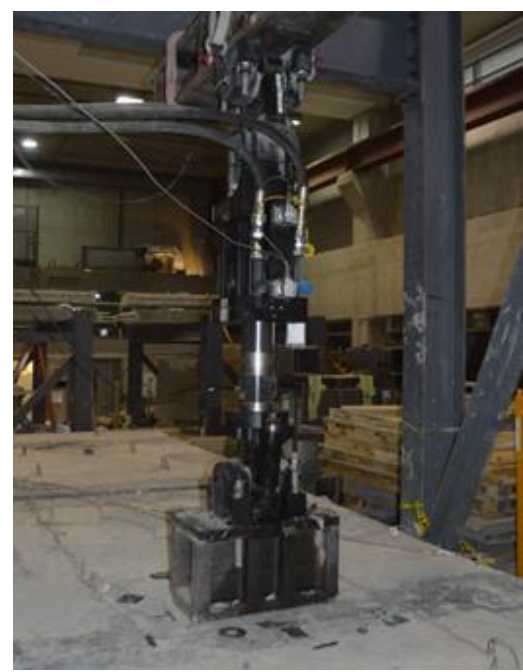

b) Close-up view for the actuator

Figure 5.32 View of the test setup for fatigue loading for slabs S3 and S4

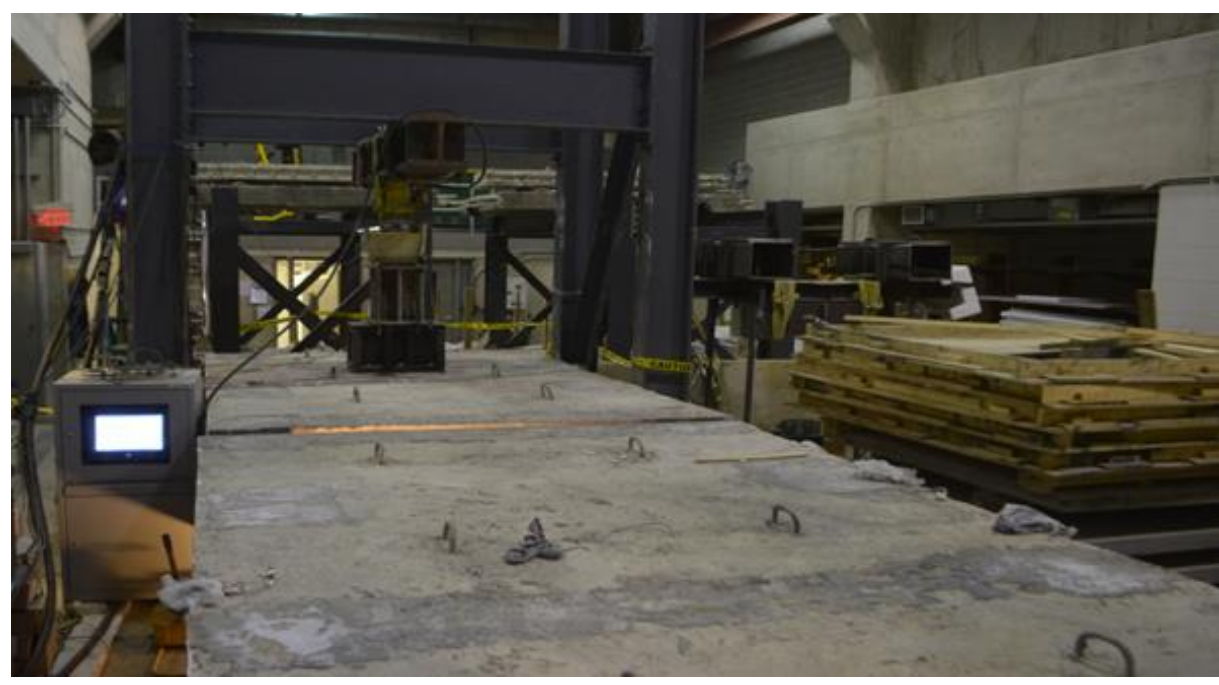

a) Slab-girder bridge system during static load test

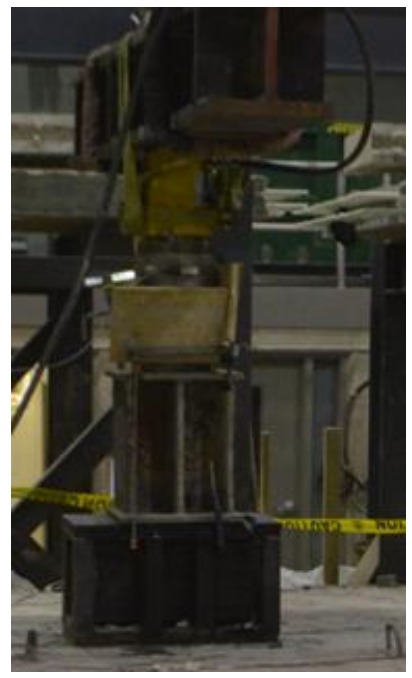

b) View of of the loaded area

Figure 5. 33. View of the test setup for the static load test for slab S3 with C-joint 


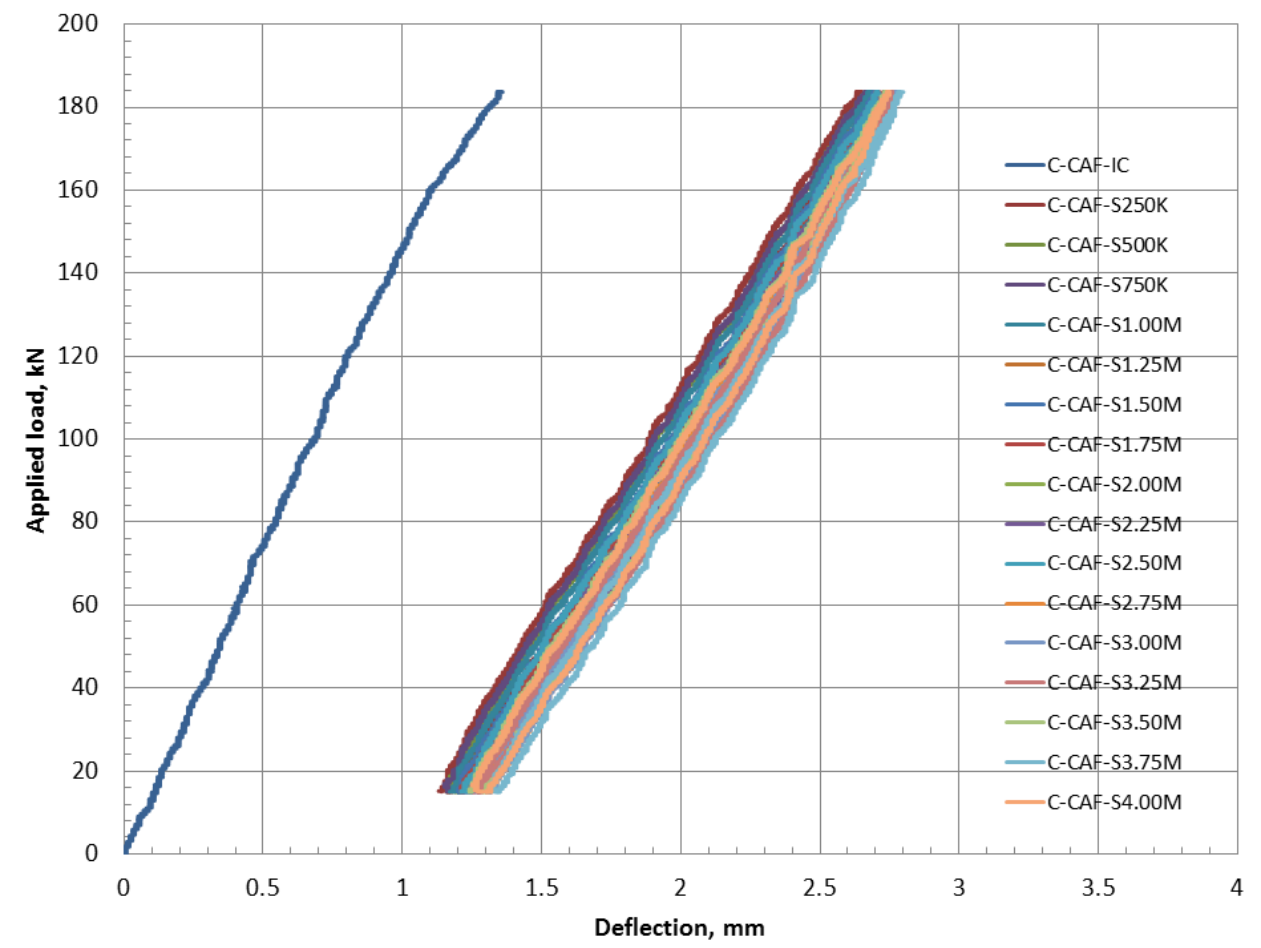

a) Load-deflection curves under static load after each 250,000 CAF cycles for slab S3

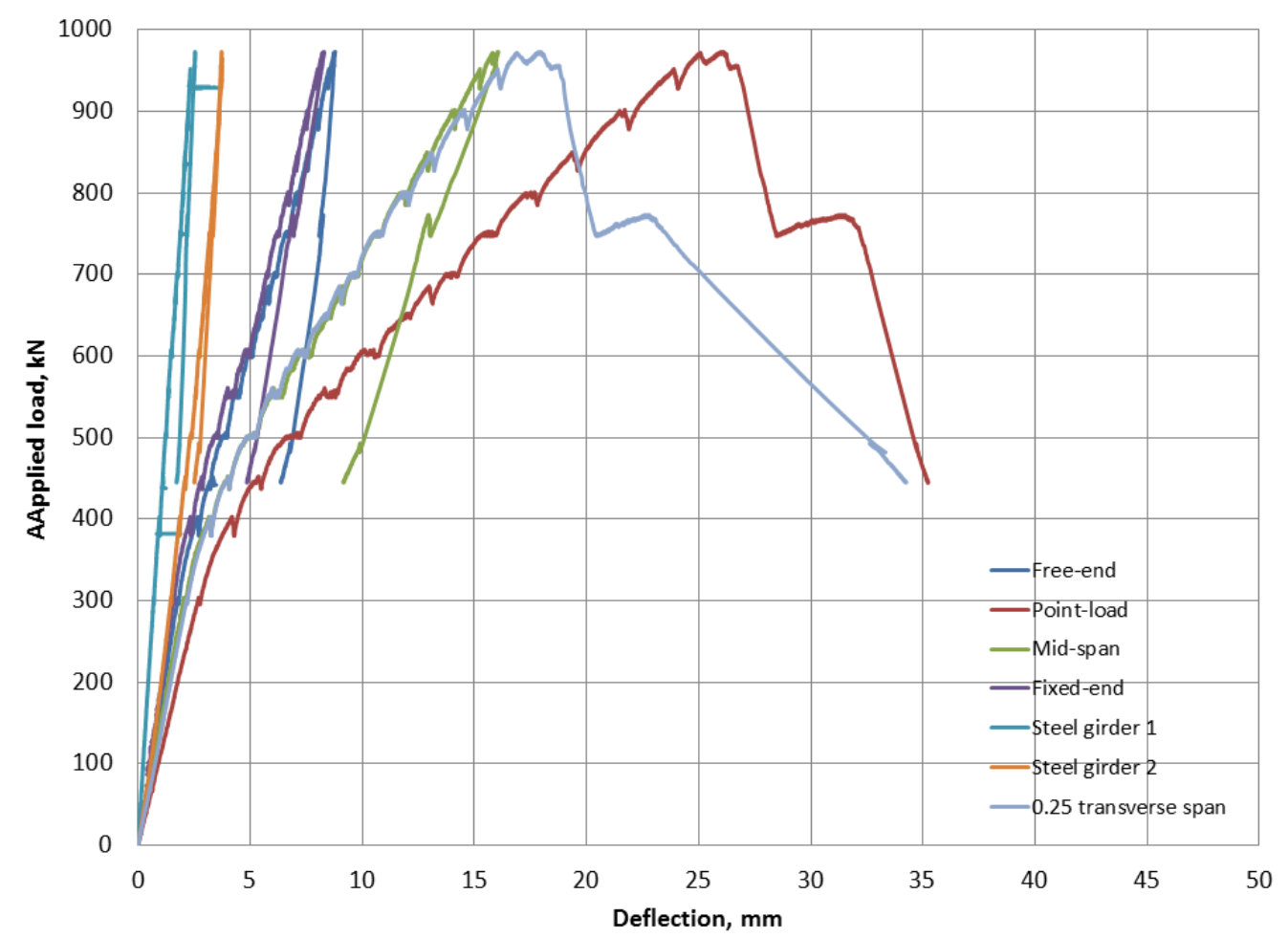

b) Load-deflection cures under static load to-collapse for slab S3 after condcting the CAF test

Figure 5.34. Monotonic load-deflection curves for slab S3 during CAF loading and static load test to-collapse 


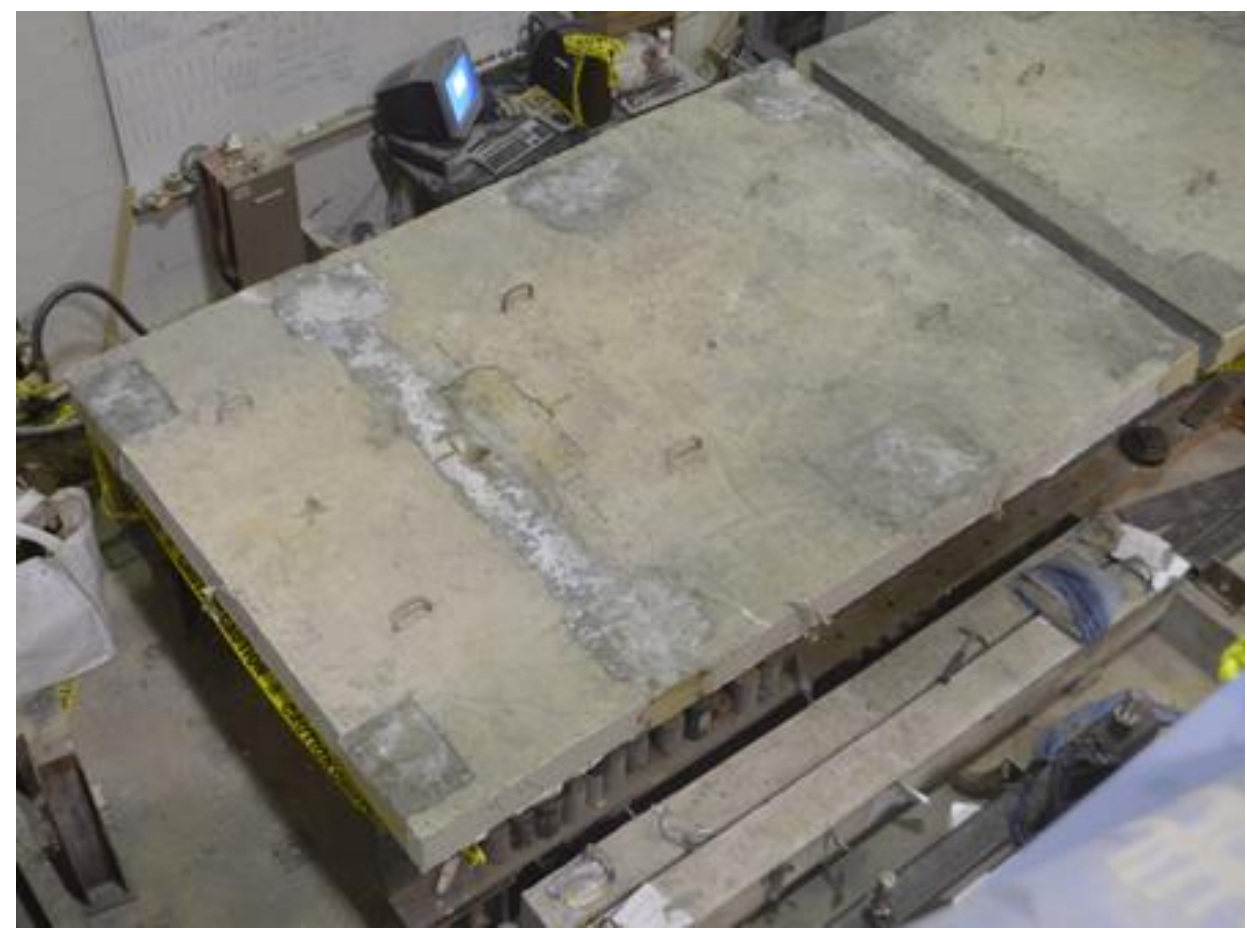

a) Top view of the slab showing punching shear failure at the footprint of the wheel load

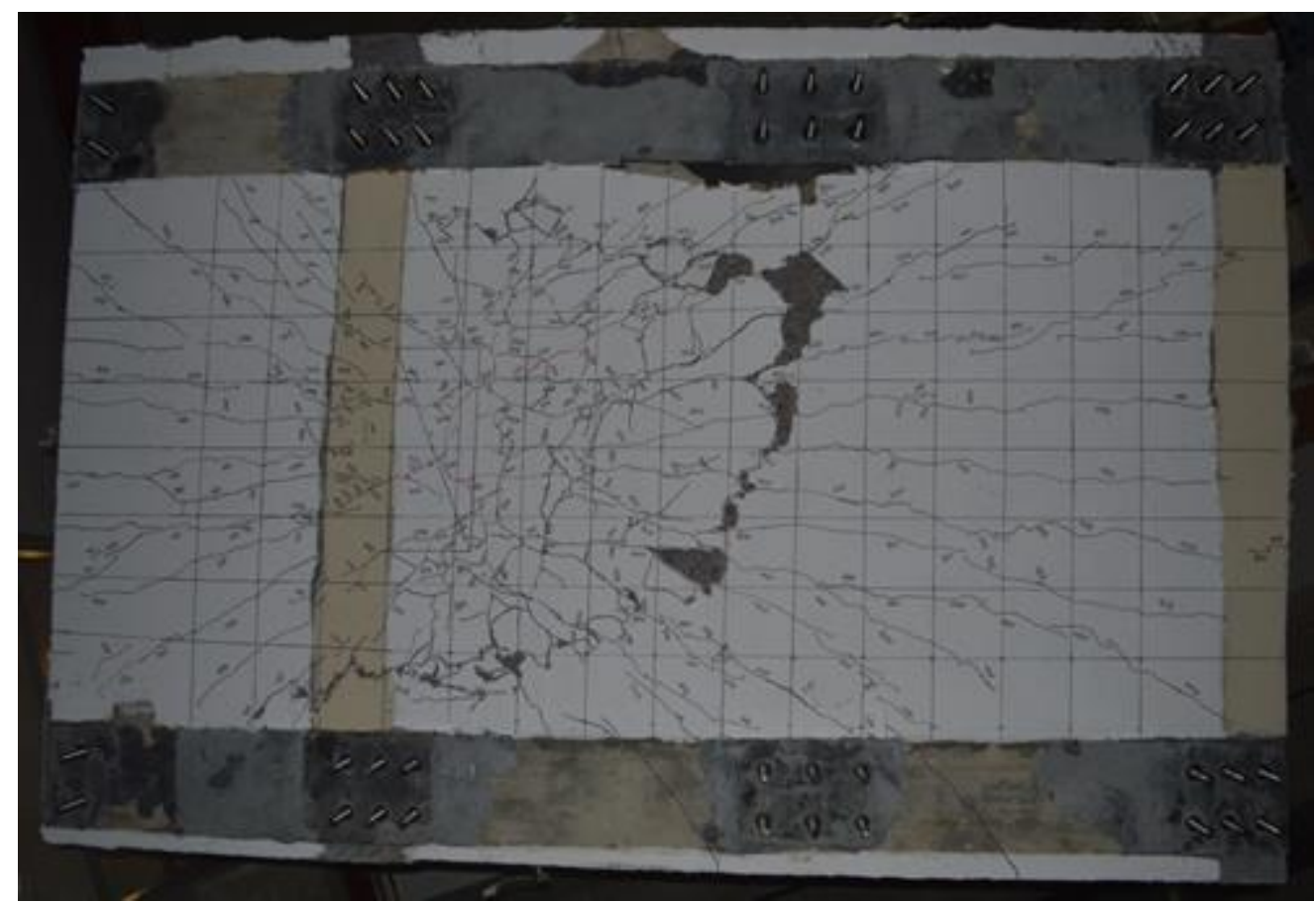

b) Bottom view of the slab showing crack pattern after failure

Figure 5.35. Crack pattern after failure of slab S3 with C-joint tested under CAF loading followed by static load test to-collapse 


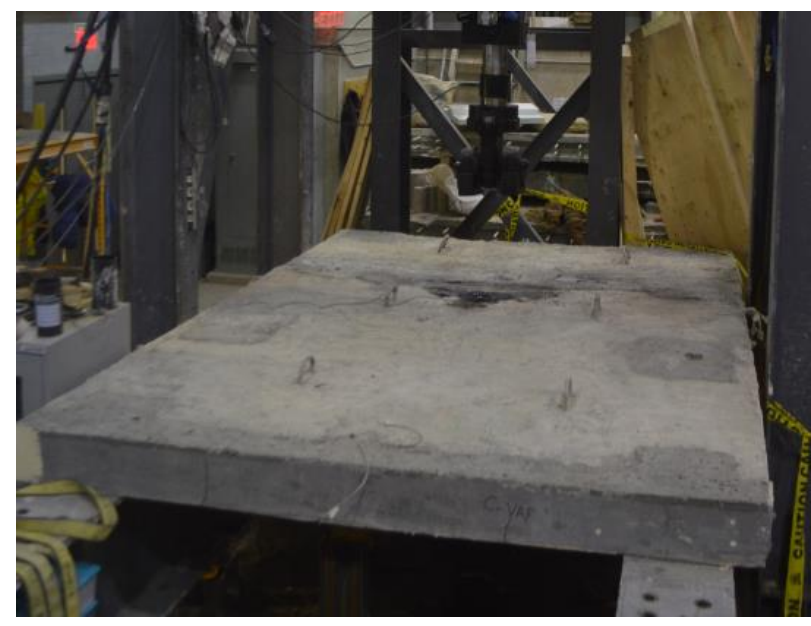

a) Top view showing punching shear failure at wheel footprint

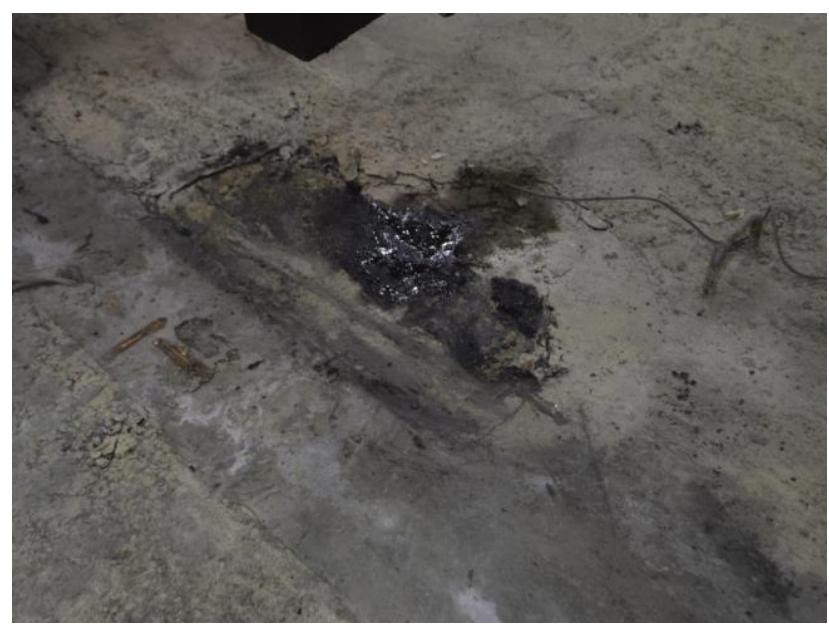

b ) Close-up view for the punching shear failure at the wheel footprint

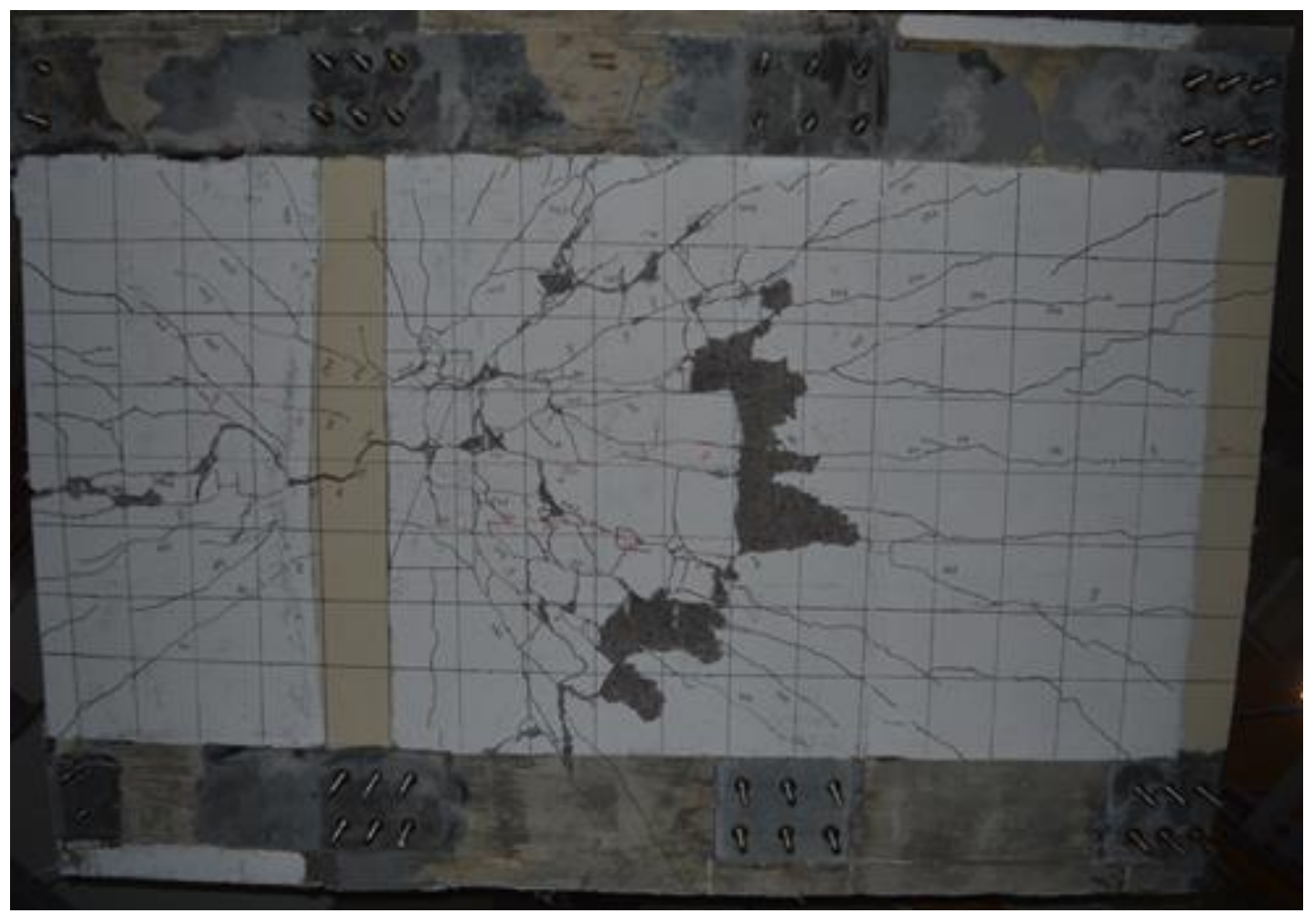

c) Bottom view showing crack pattern

Figure 5.36. Views of punching shear failure of slab S4 tested under VAF loading to-collapse 


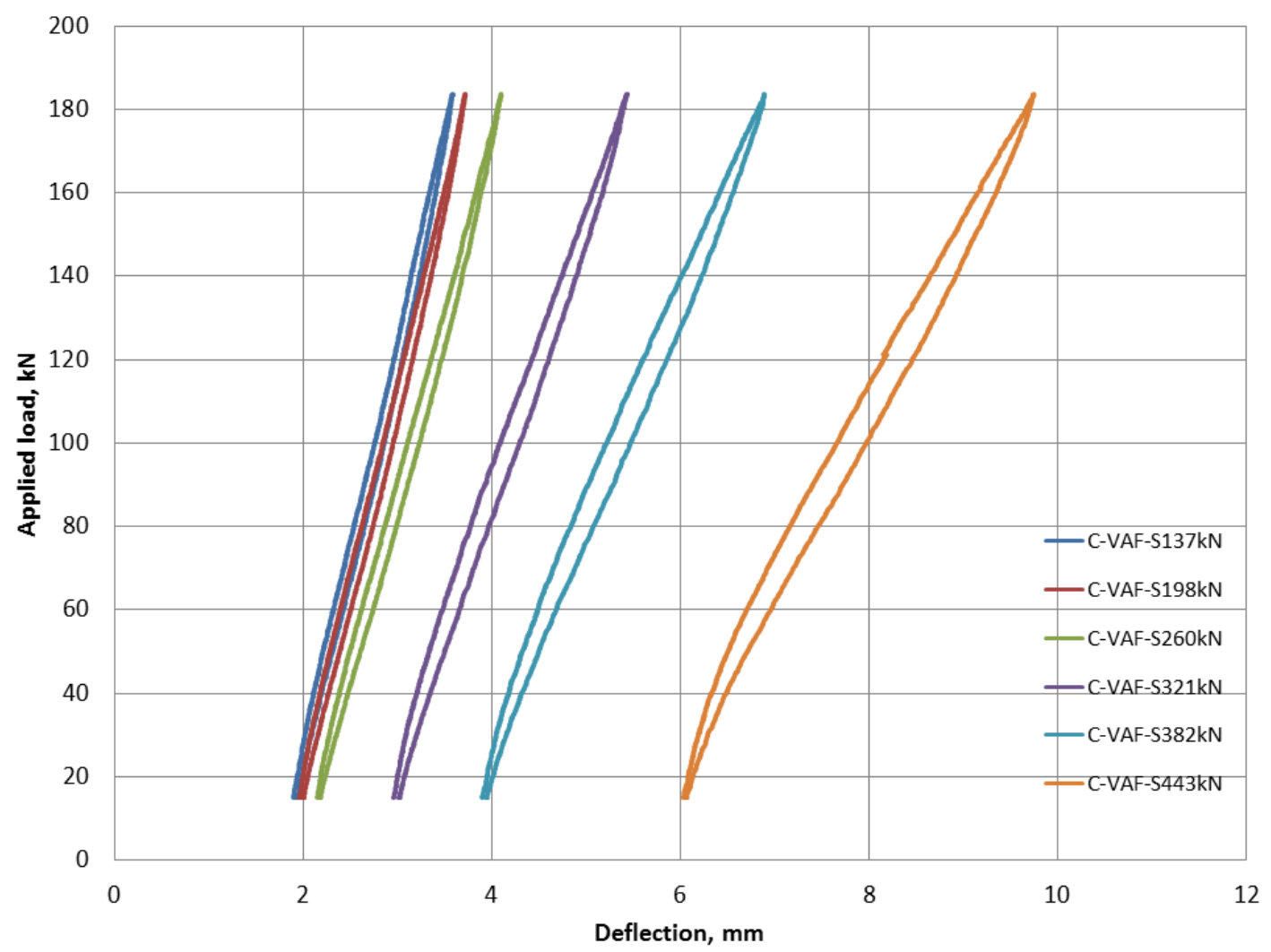

Figure 5.37. Monotonic load-deflection curves for slab S4 tested after each 100,000 cycles of VAF loading 


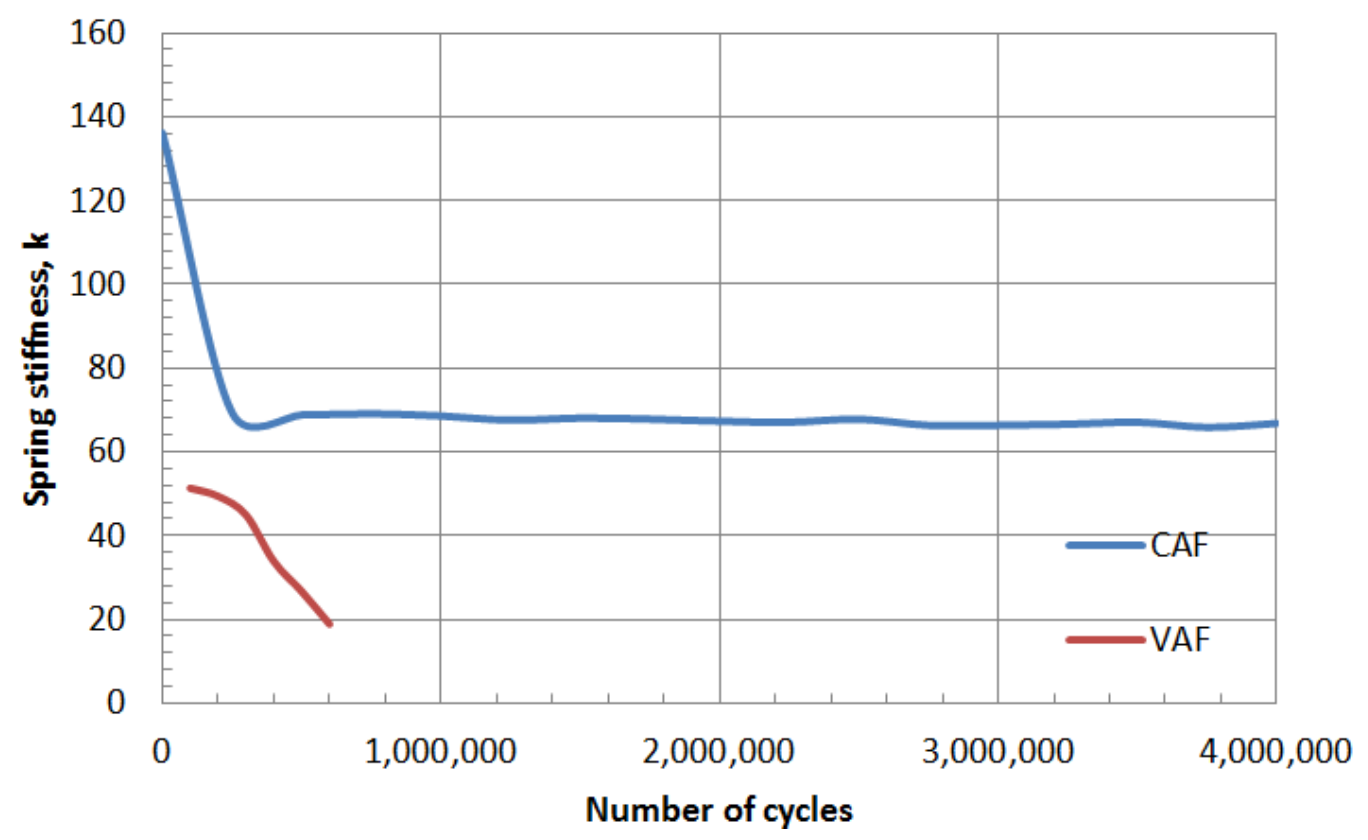

a) Spring stiffness-number of cycles curves

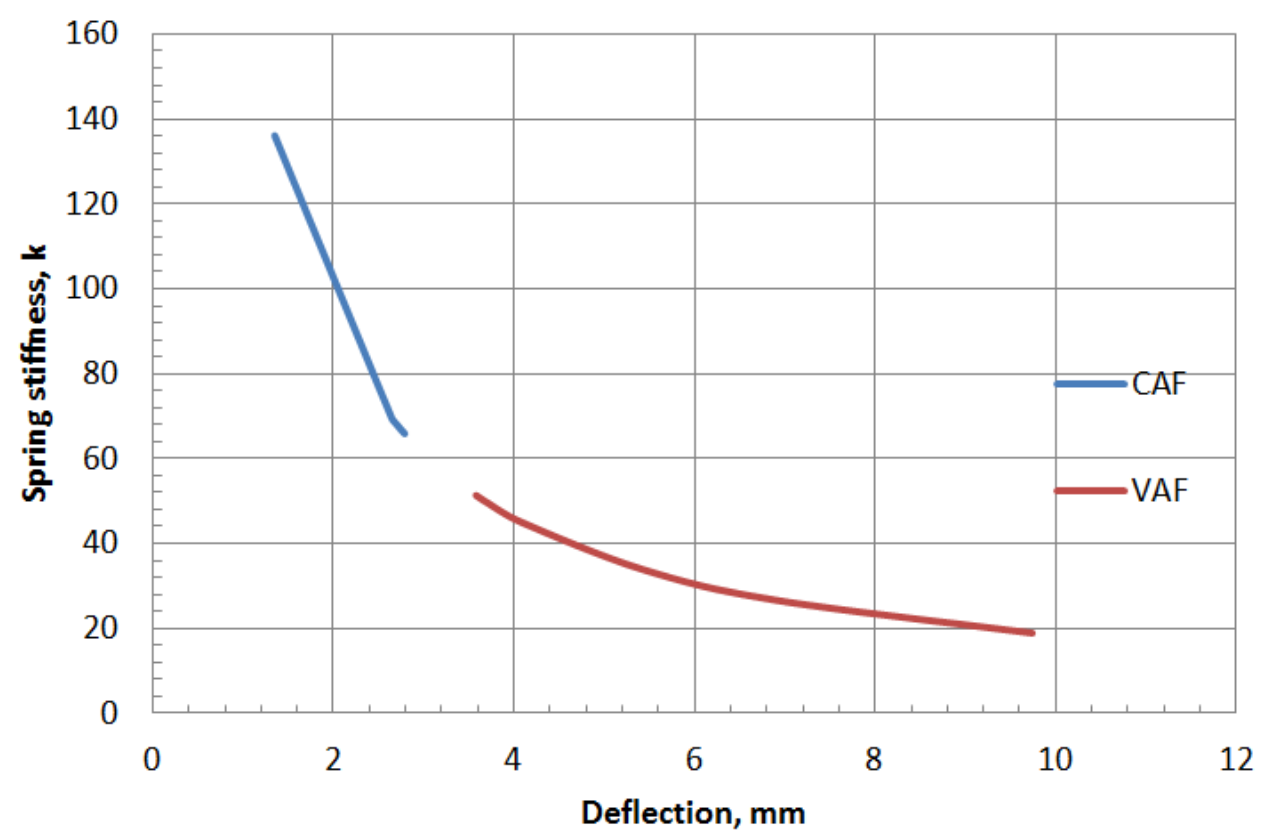

b) Spring stiffness-deflection curves 


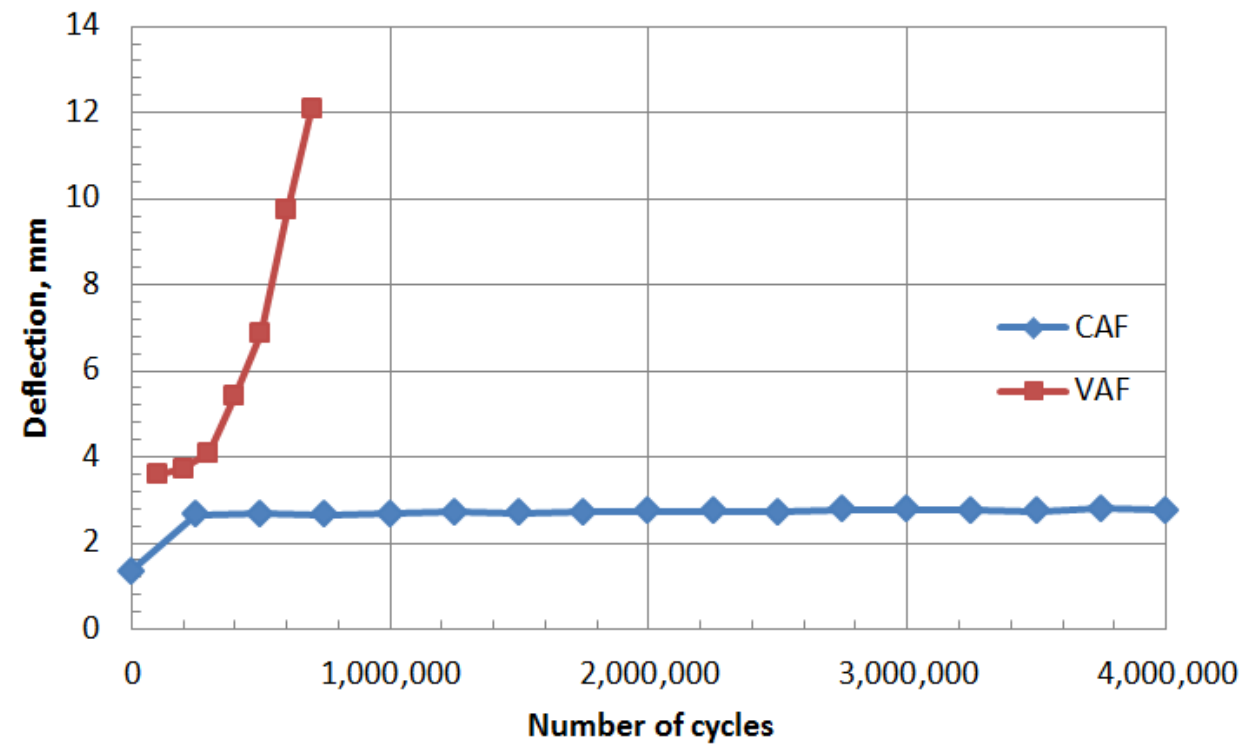

c) Deflection-number of cycles curves

Figure 5.38 Degradation of the precast FDDPs subjected to CAF and VAF loading 


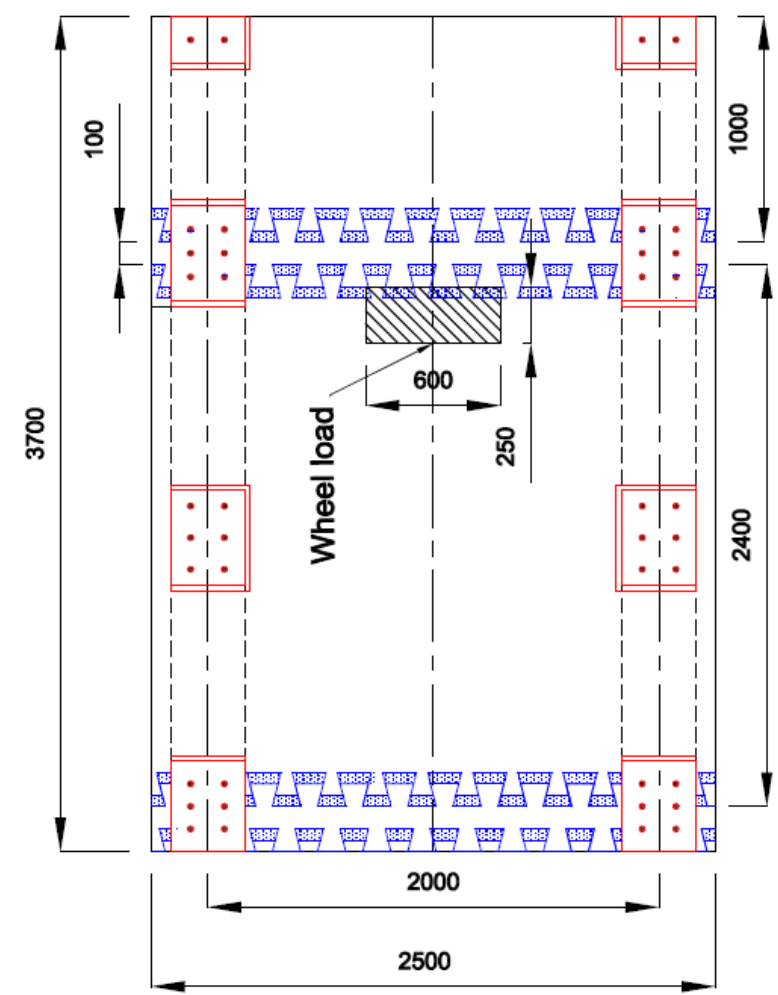

a) Plan view showing location of the wheel footprint beside the closure strip

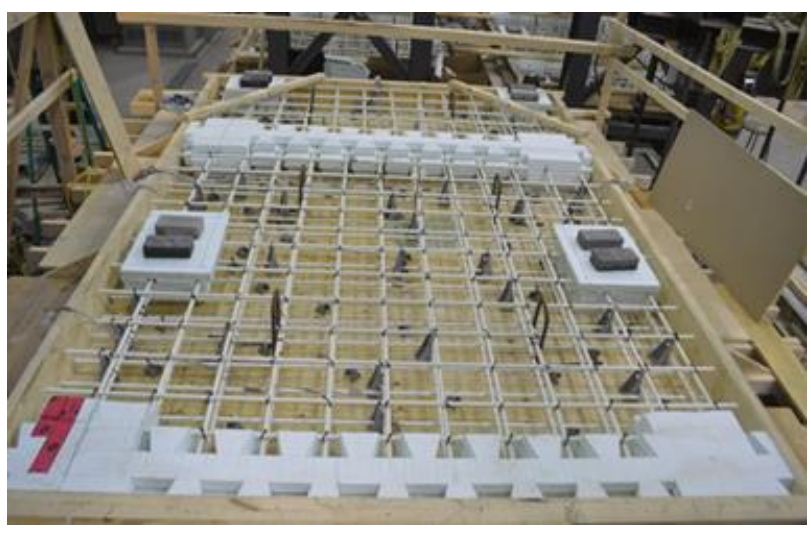

b) Placement of styrofoam to form the joints

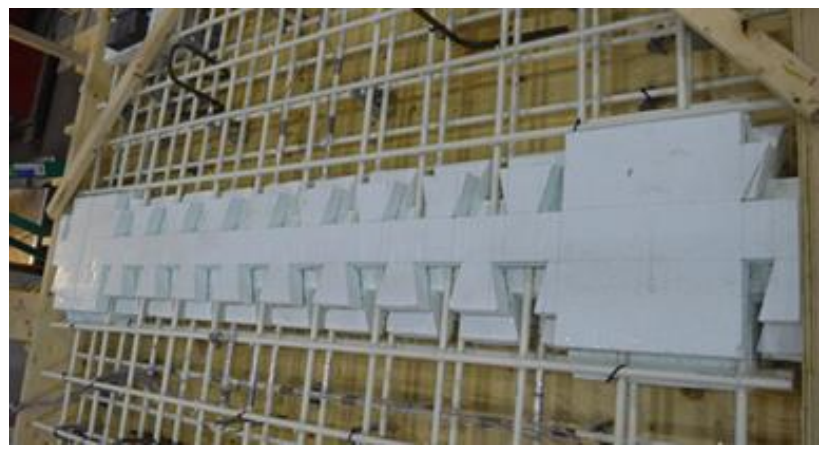

c) Close-up view of the styrofoam forming the joint

Figure 5.39 Construction of the precast FDDP with zigzag-shape joint for slabs S5 and S6

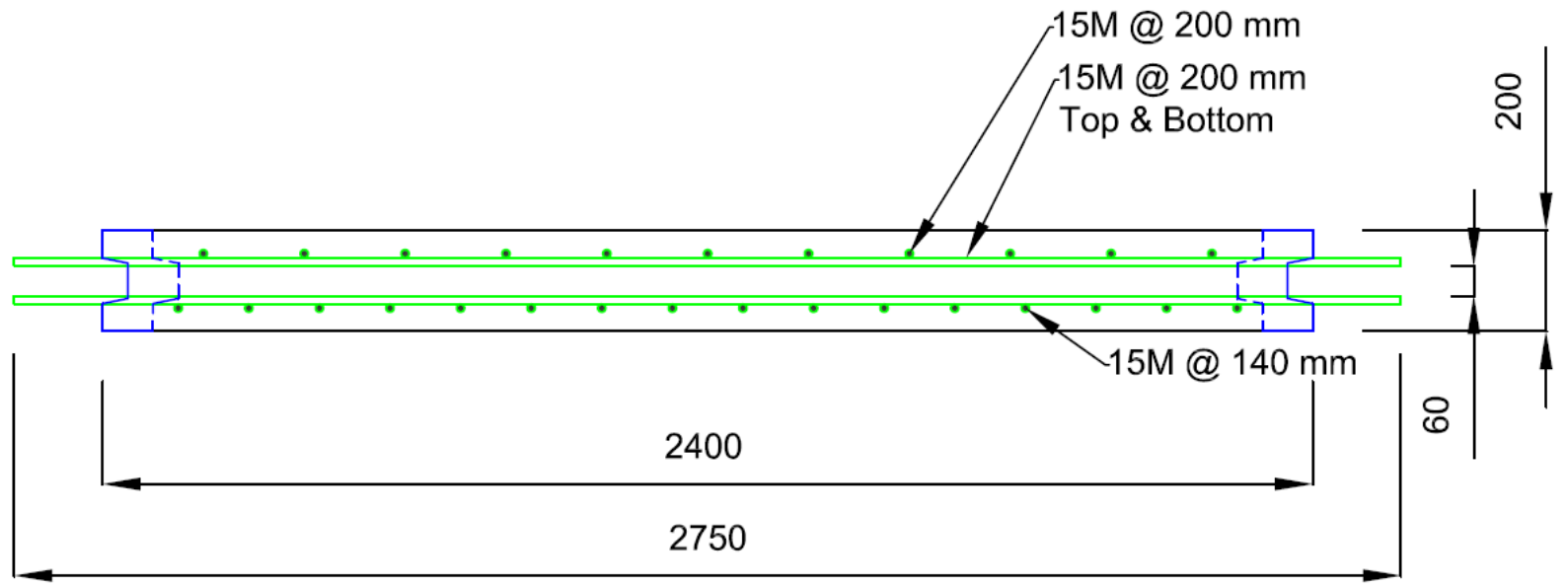

Figure 5.40 Longitudinal cross-section for the precast FDDP with zigzag joint 


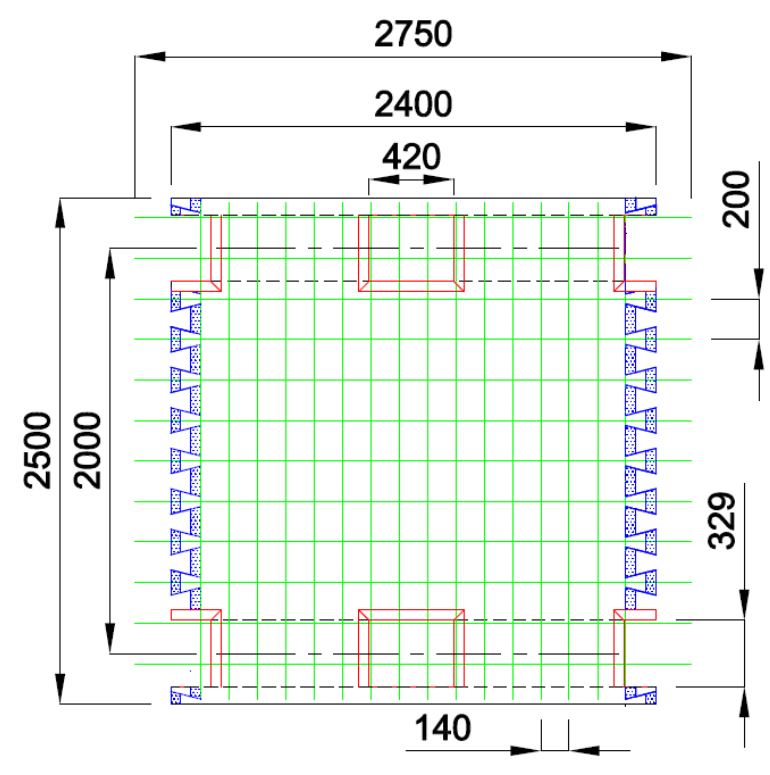

a) Bottom reinforcement

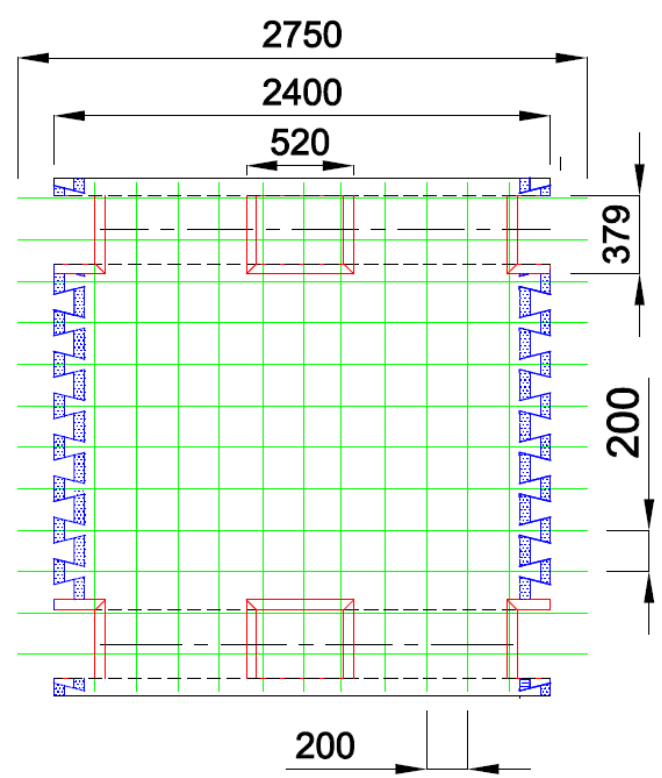

b) Top reinforcement

Figure 5.41 Placing of reinforcement for the precast FDDP with zigzag joint

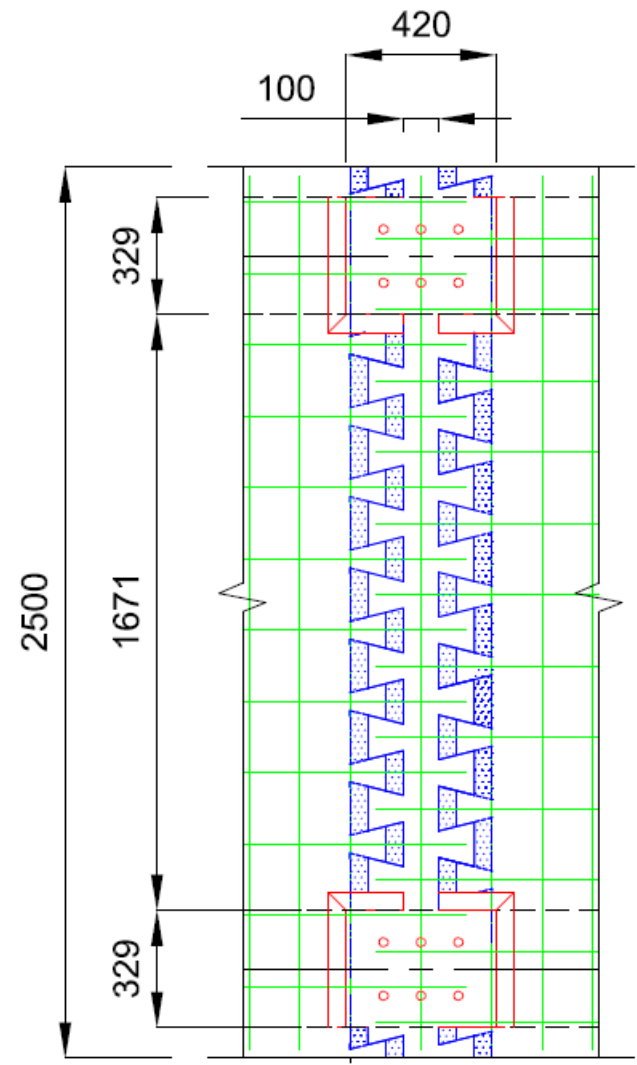

a) Bottom reinforcement

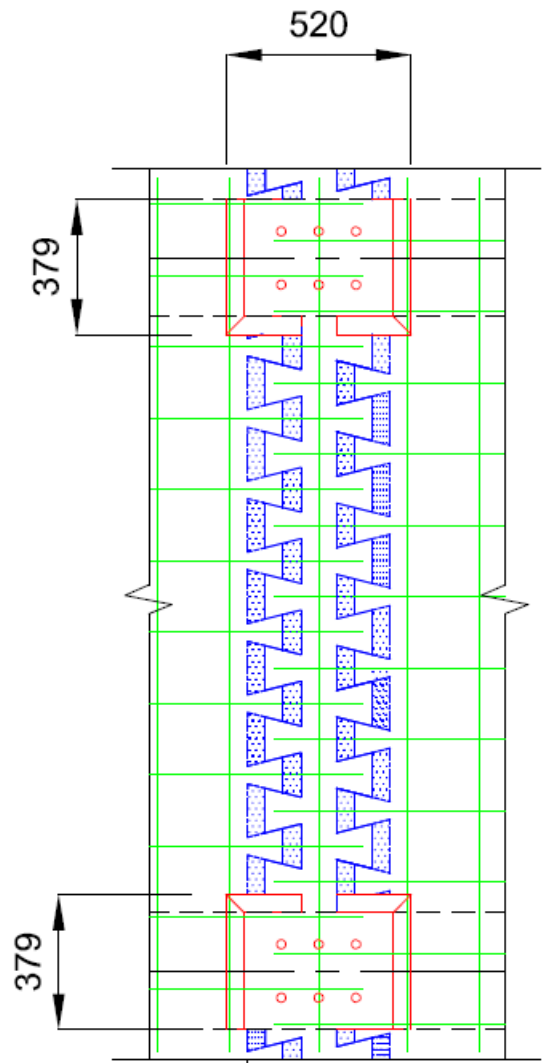

b) Top reinforcement

Figure 5.42 Close-up view for the reinforcement through the zigzag connection 


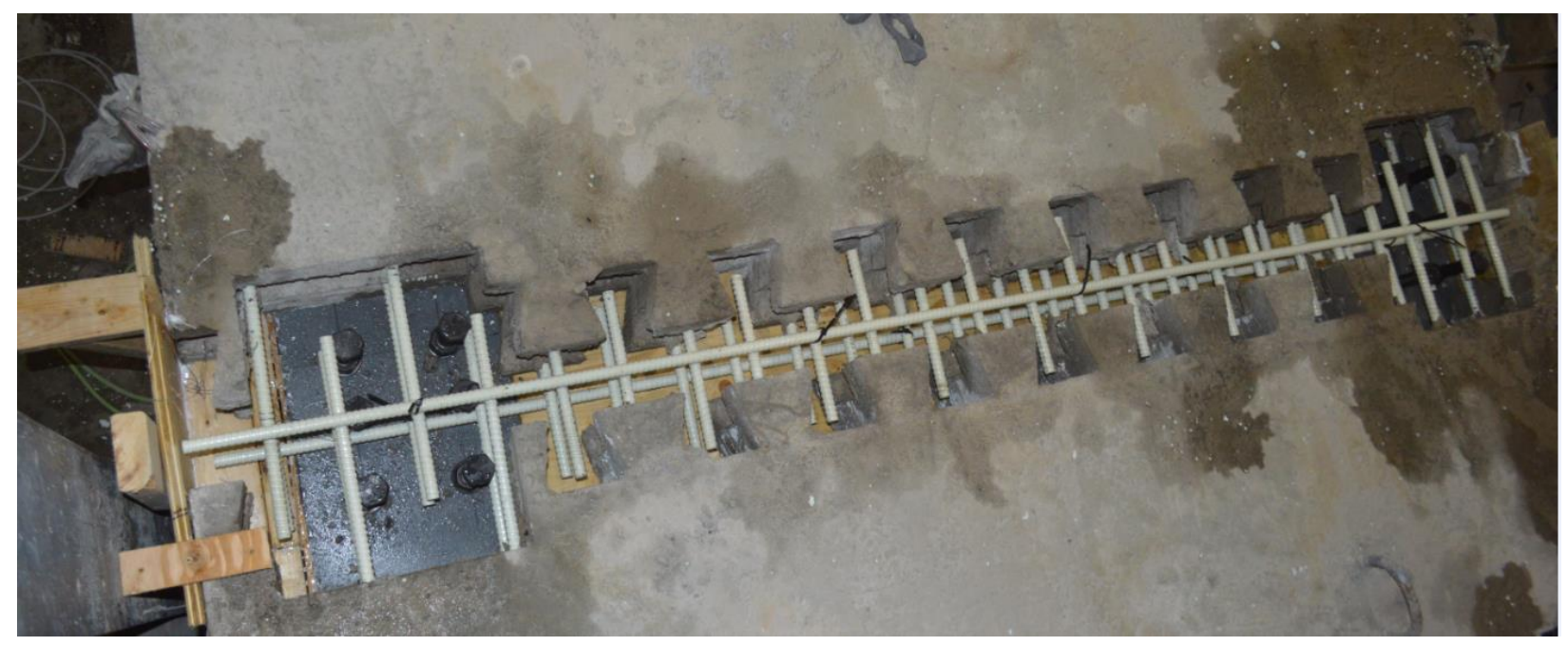

Figure 5.43 Close-up view for the zigzag joint before casting

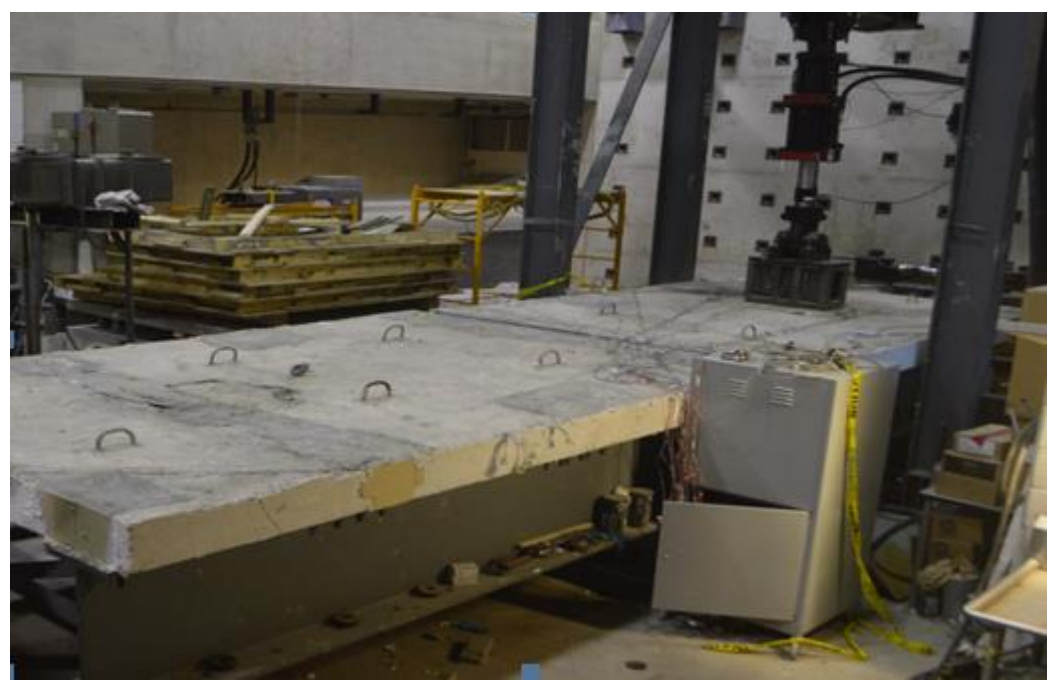

a) Slab-girder bridge system during CAF and VAF loading

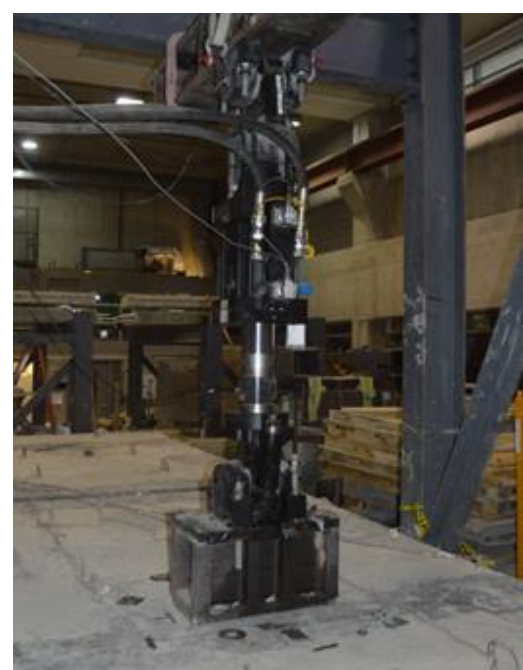

b) close-up view of the actuator

Figure 5. 44 View of the test setup for fatigue loading for slabs S5 and S6 


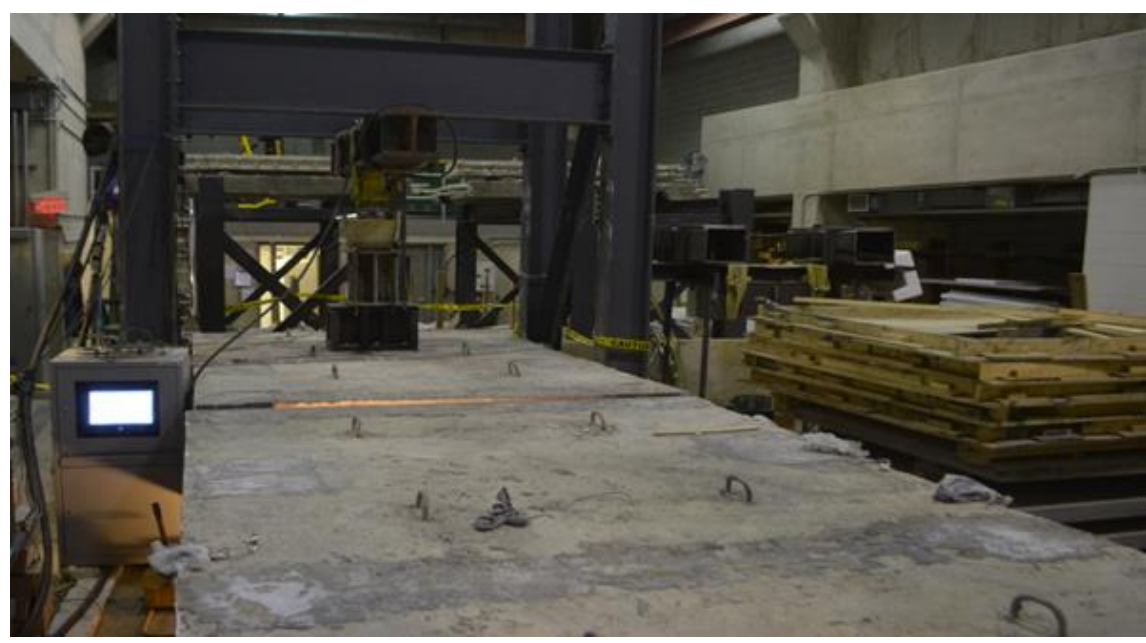

a) Slab-girder bridge system during monotonic loading

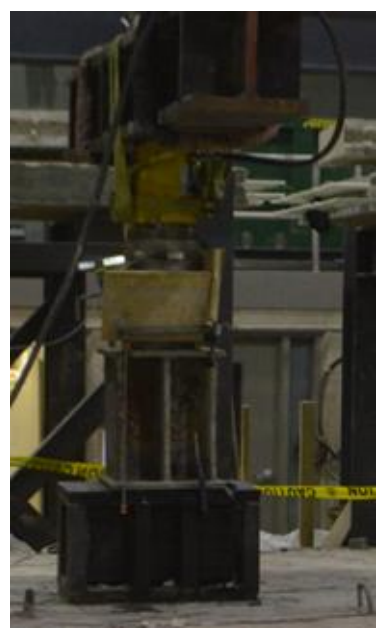

b) View of loaded area

Figure 5.45 View of the test setup for the monotonic loading for slab S5 with Z-shape joint 


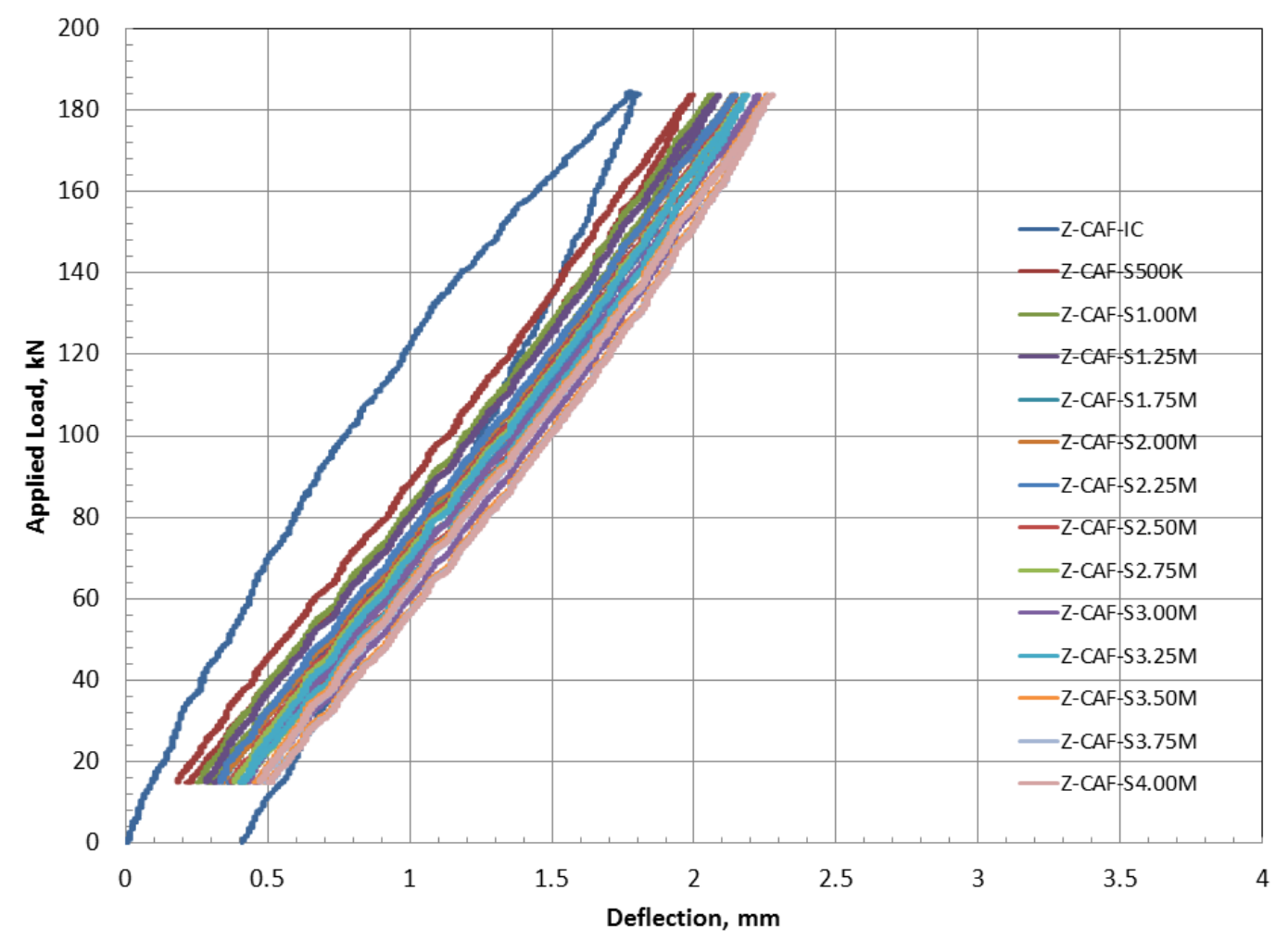

a) Load-deflection curves under static load after each 500,000 fatigue cycles

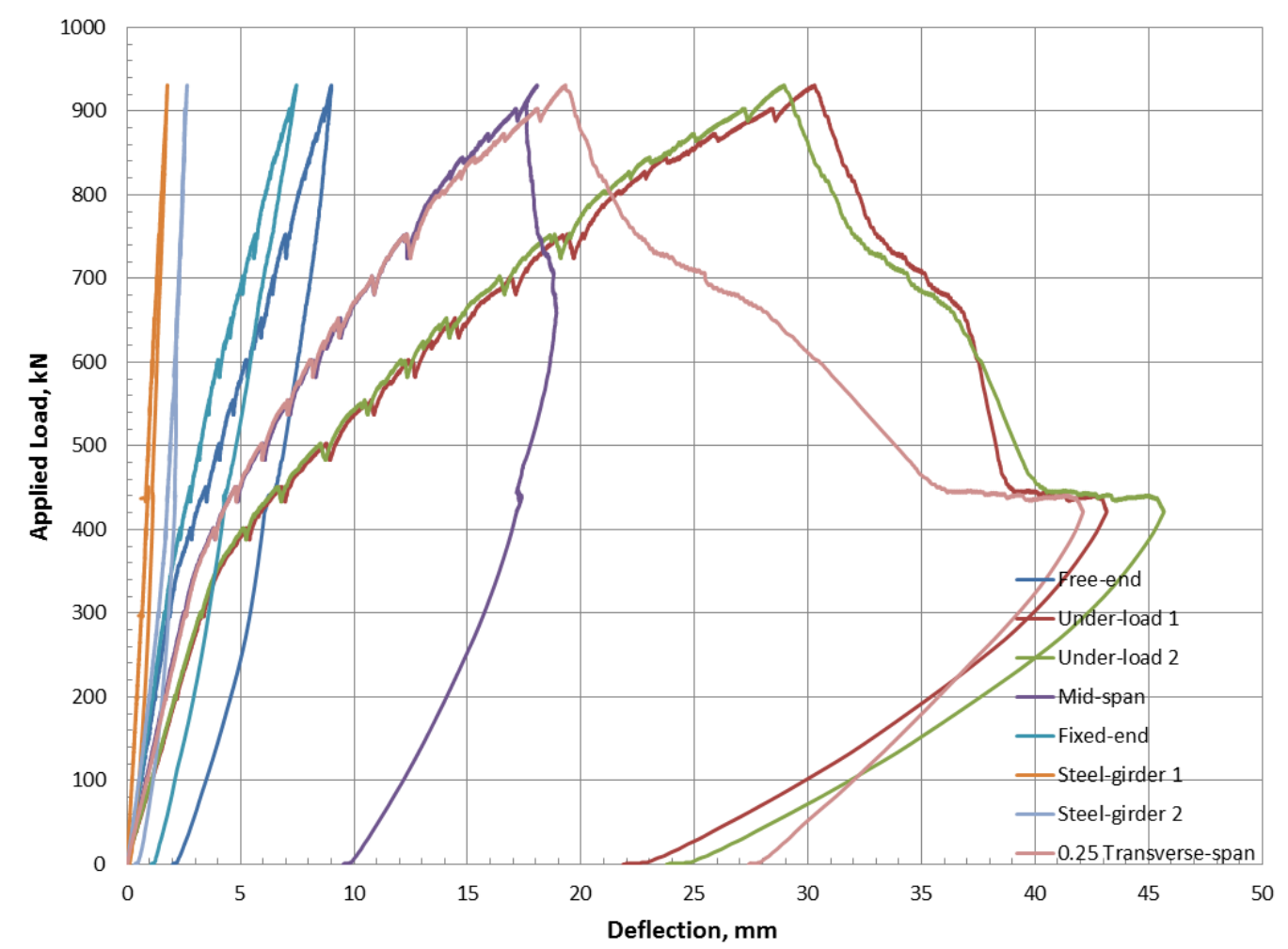

b) Load-deflection curves under static load to-collapse

Figure 5.46 Monotonic load-deflection history for slab S5 under CAF loading 


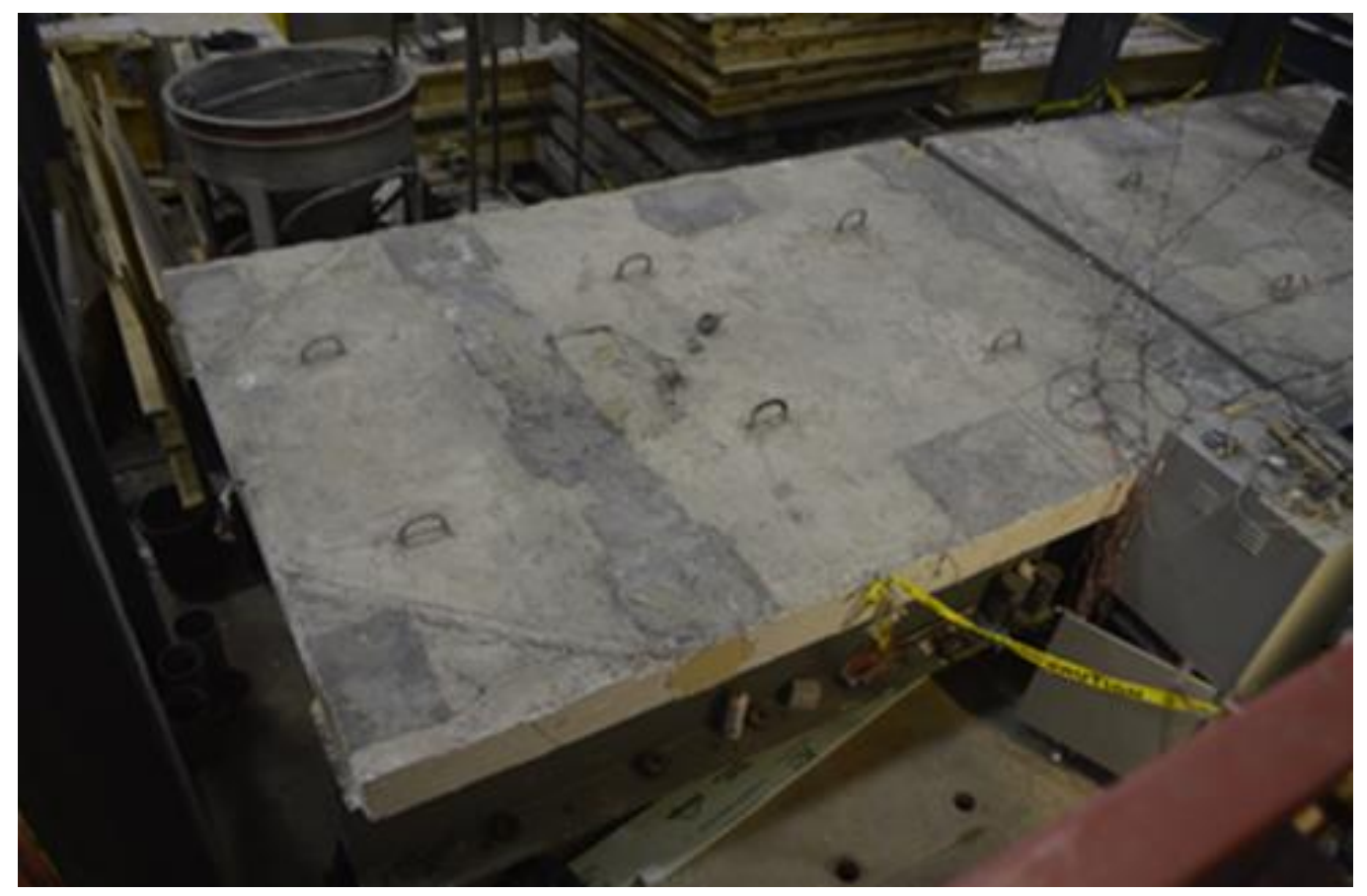

a) Top view of the slab showing punching shear failure at the footprint of the wheel load

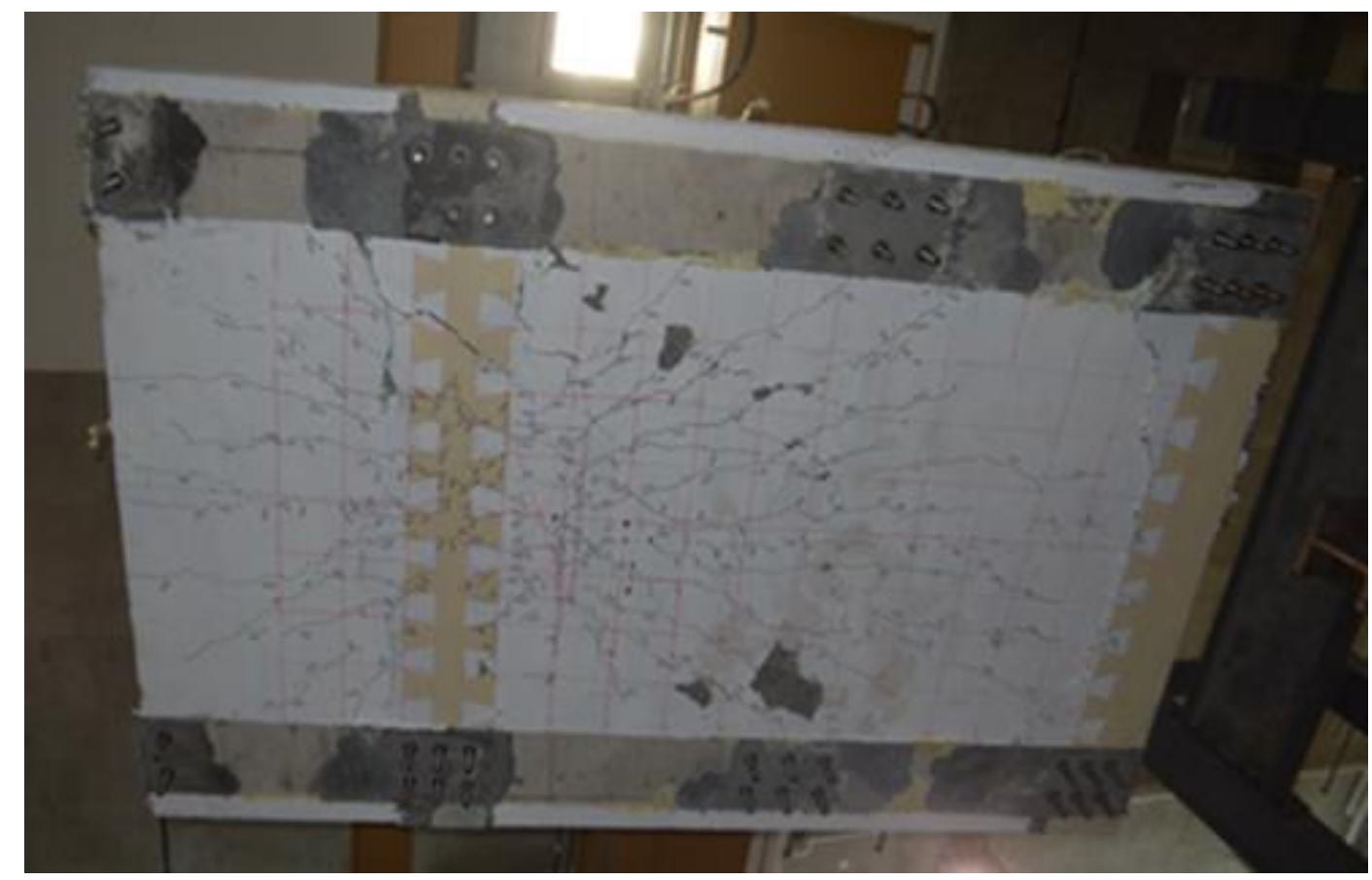

b) Bottom view of the slab showing crack pattern after failure

Figure 5.47 Crack pattern after failure of slab S5 under CAF loading, followed by static load test to-collapse 


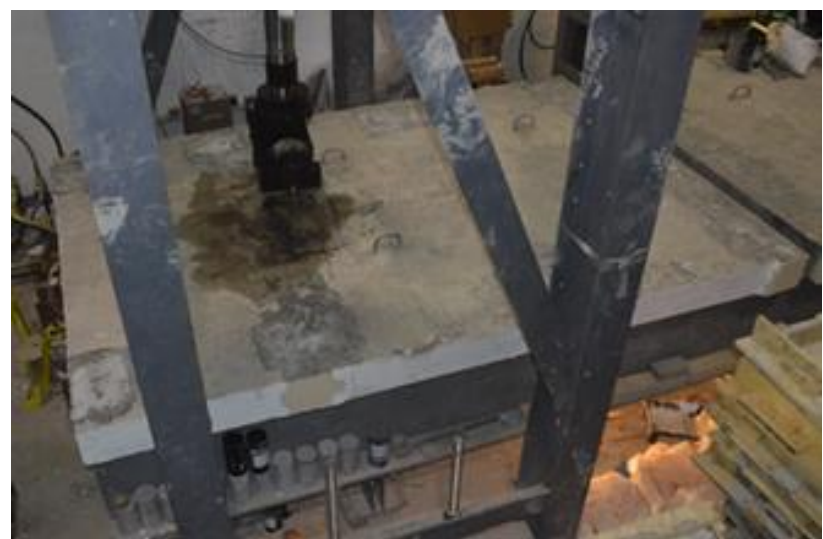

a) Top view showing punching shear failure at wheel footprint

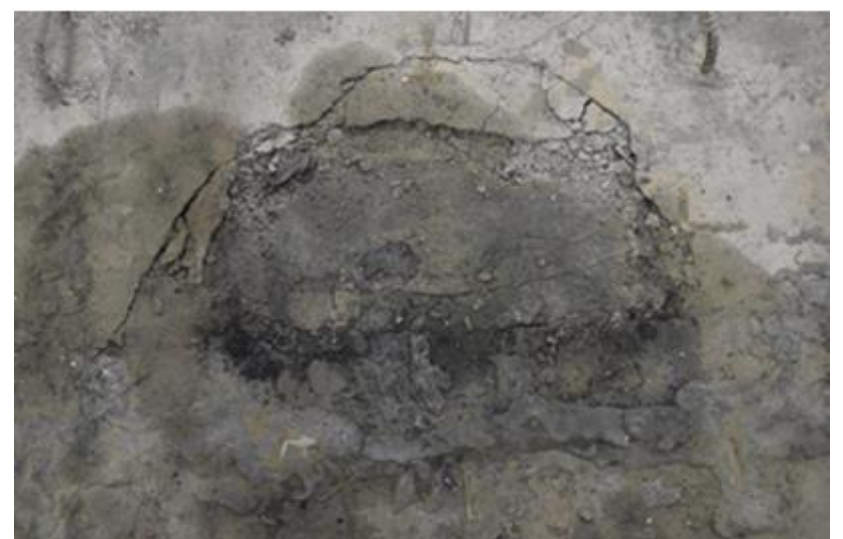

b) Close-up view for the punching shear failure at the wheel footprint

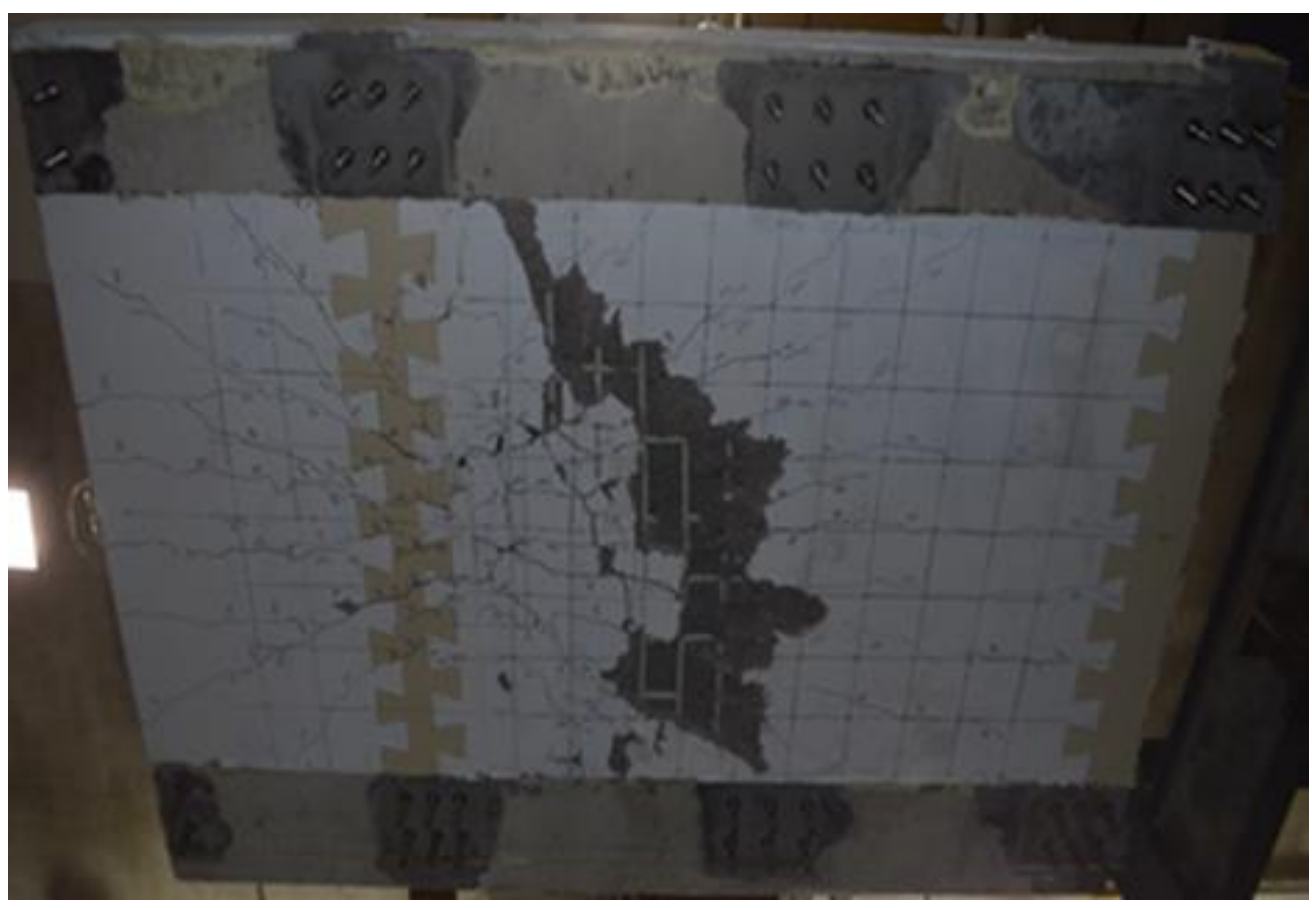

c) Bottom view showing crack pattern

Figure 5.48 Views of punching shear failure of slab S6 tested under VAF loading 


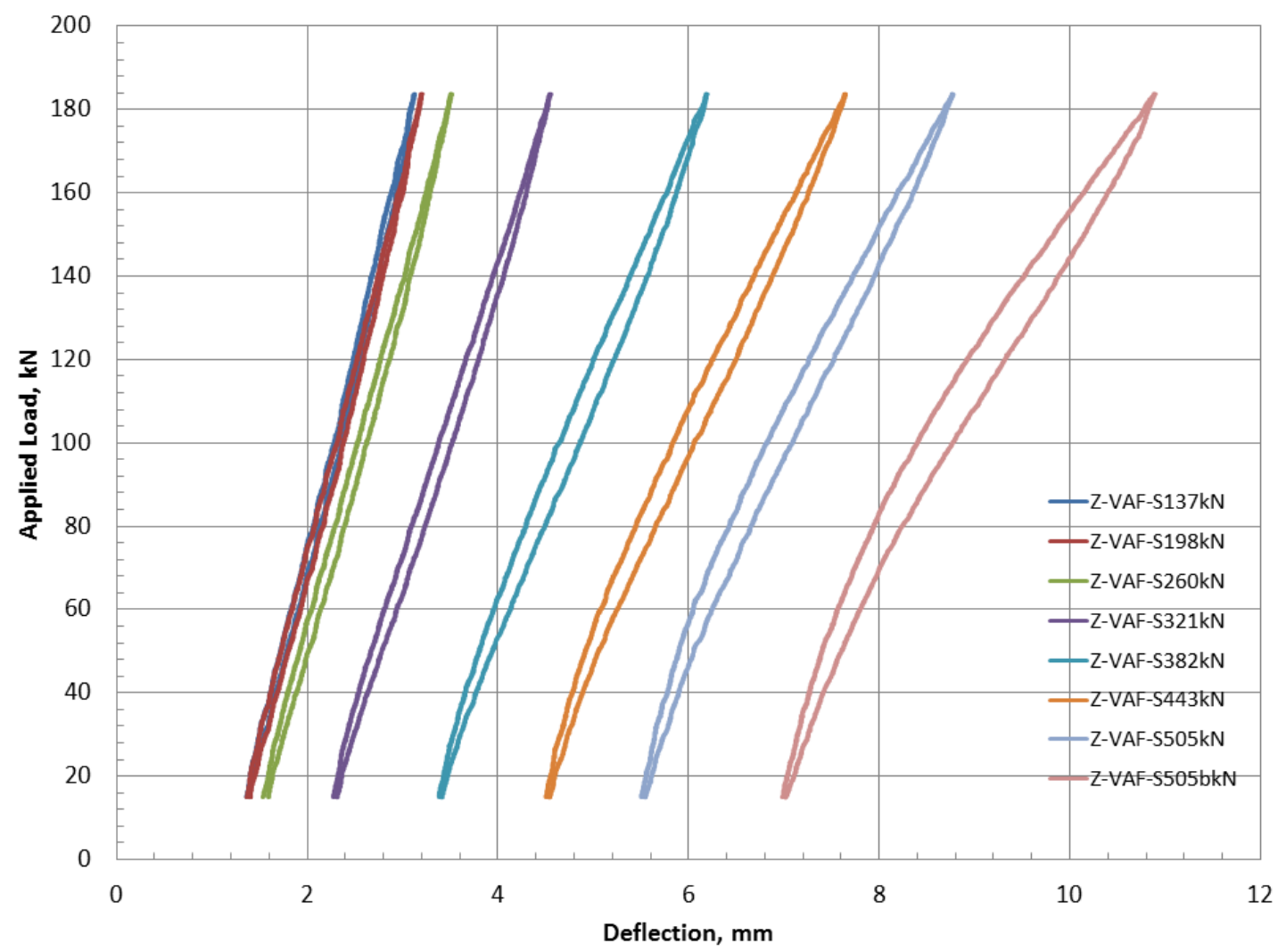

Figure 5.49 Monotonic load-deflection history for slab S6 at every 100,000 cycles of VAF loading

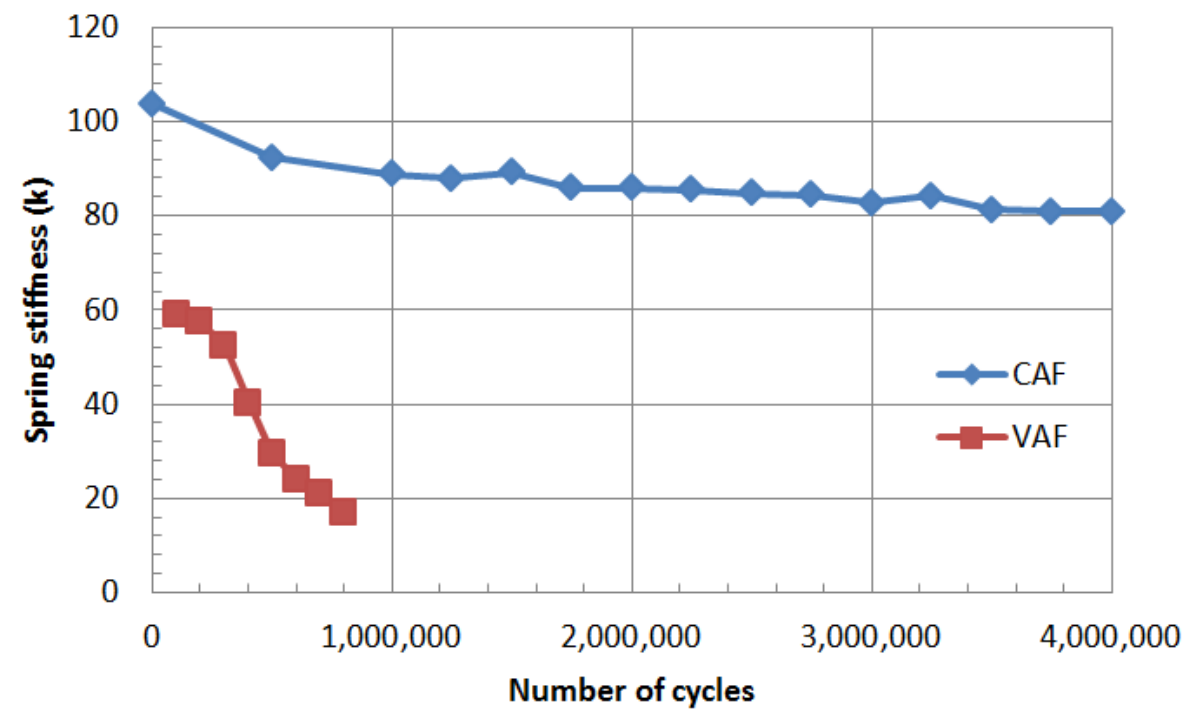

a) Spring stiffness-number of cycles curves 


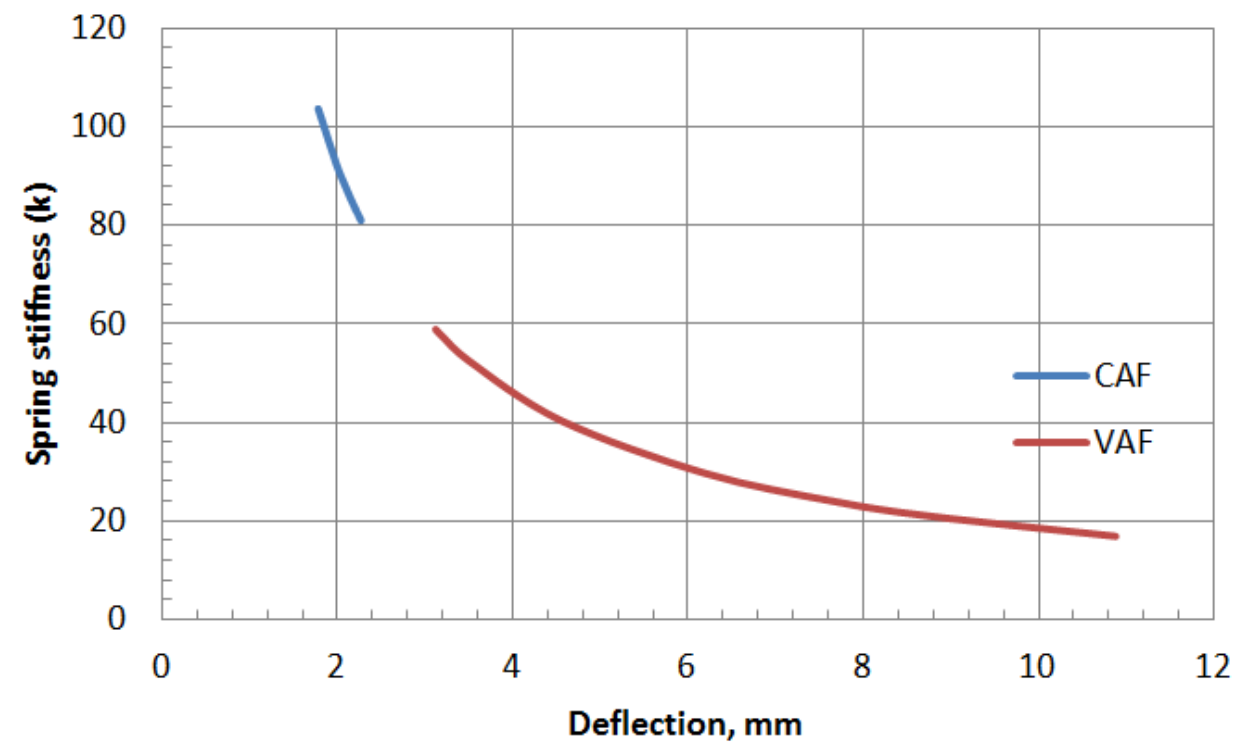

b) Spring stiffness-deflection curves

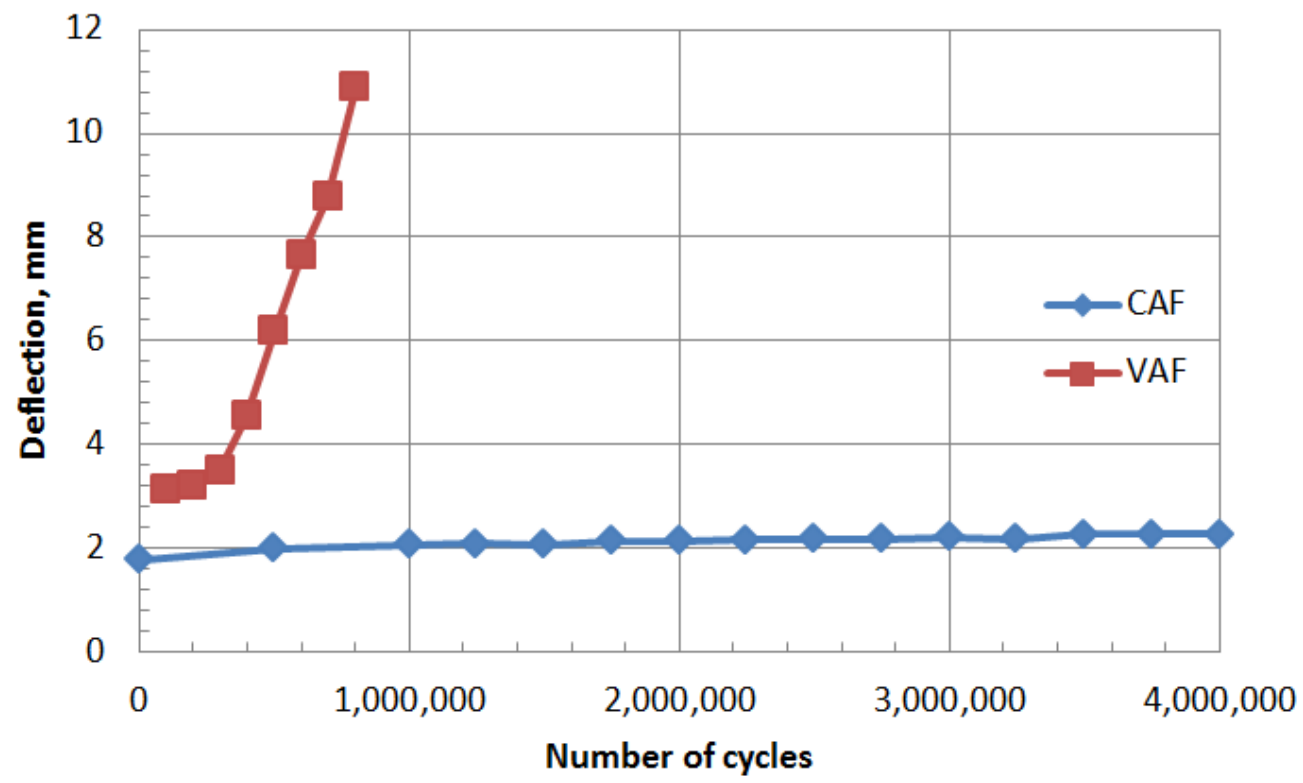

c) Deflection-number of cycles curves

Figure 5.50 Degradation of the precast FDDP with Z-shape joint under CAF and VAF loading 


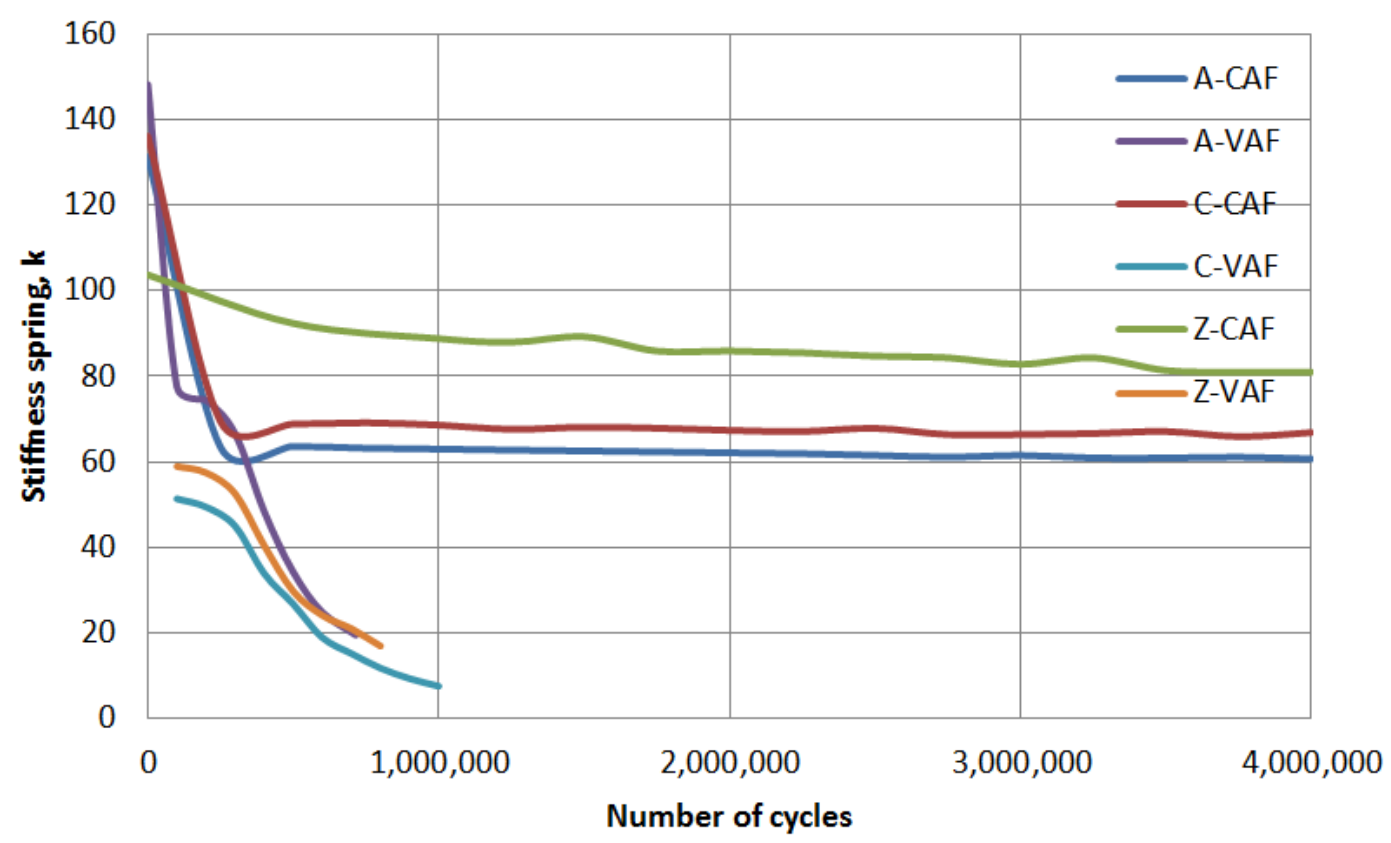

a) Spring stiffness-number of cycles

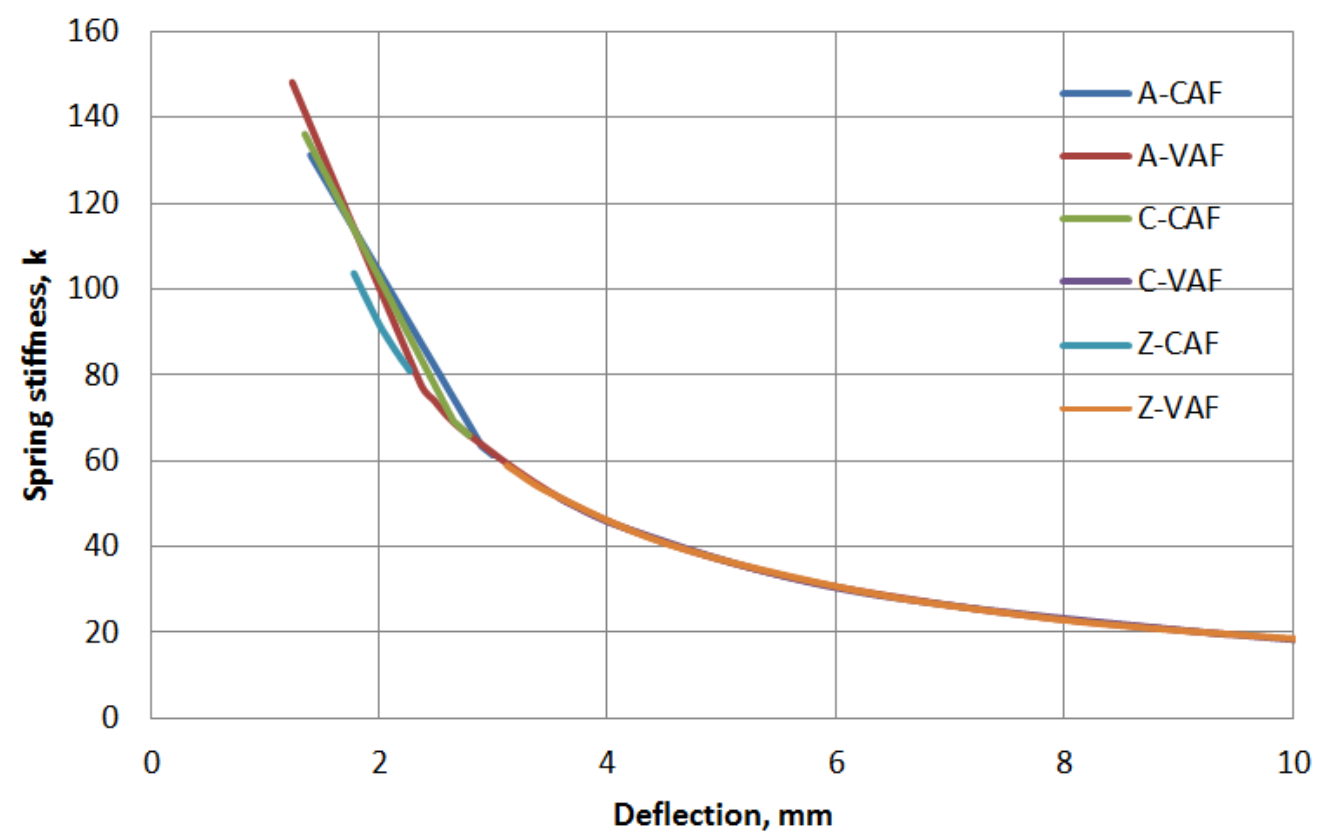

b) Spring stiffness-deflection curves 


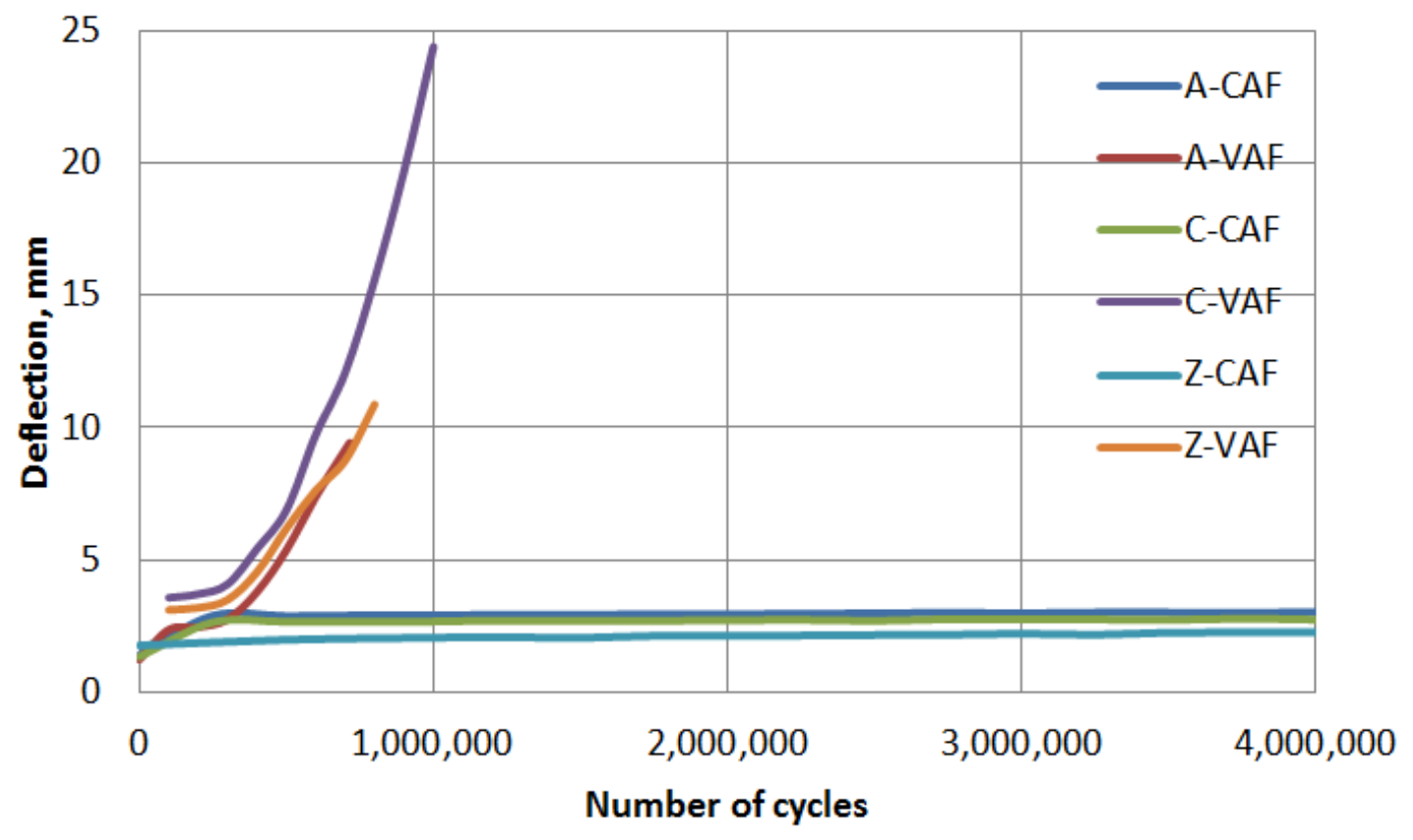

c) Deflection-number of cycles curves

Figure 5. 51. Degradation of the precast FDDPs under CAF and VAF loading 


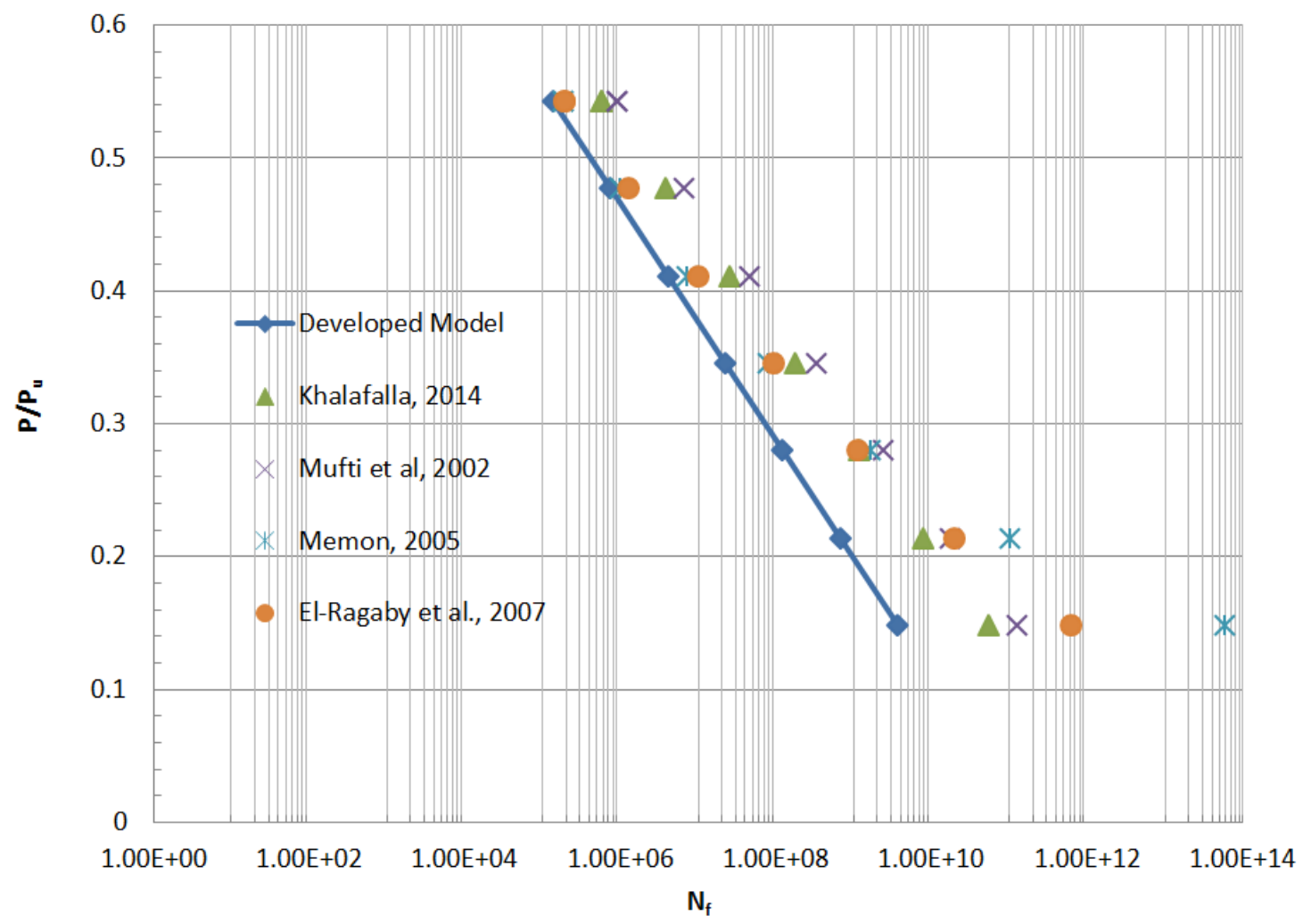

a) The P-N fatigue models for the precast FDDP with A-shape joint b)

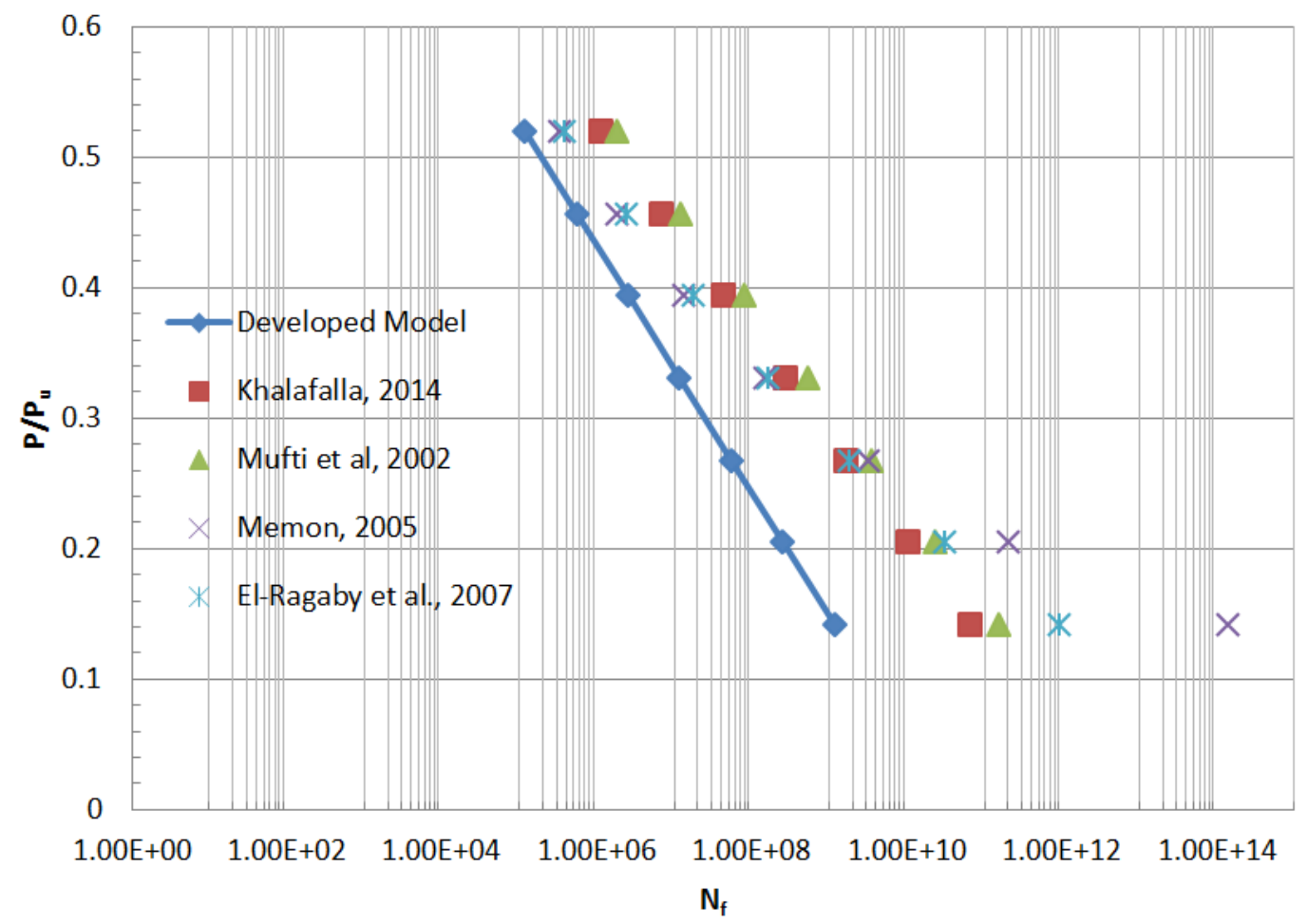

c) The P-N fatigue models for the precast FDDP with C-shape joint 


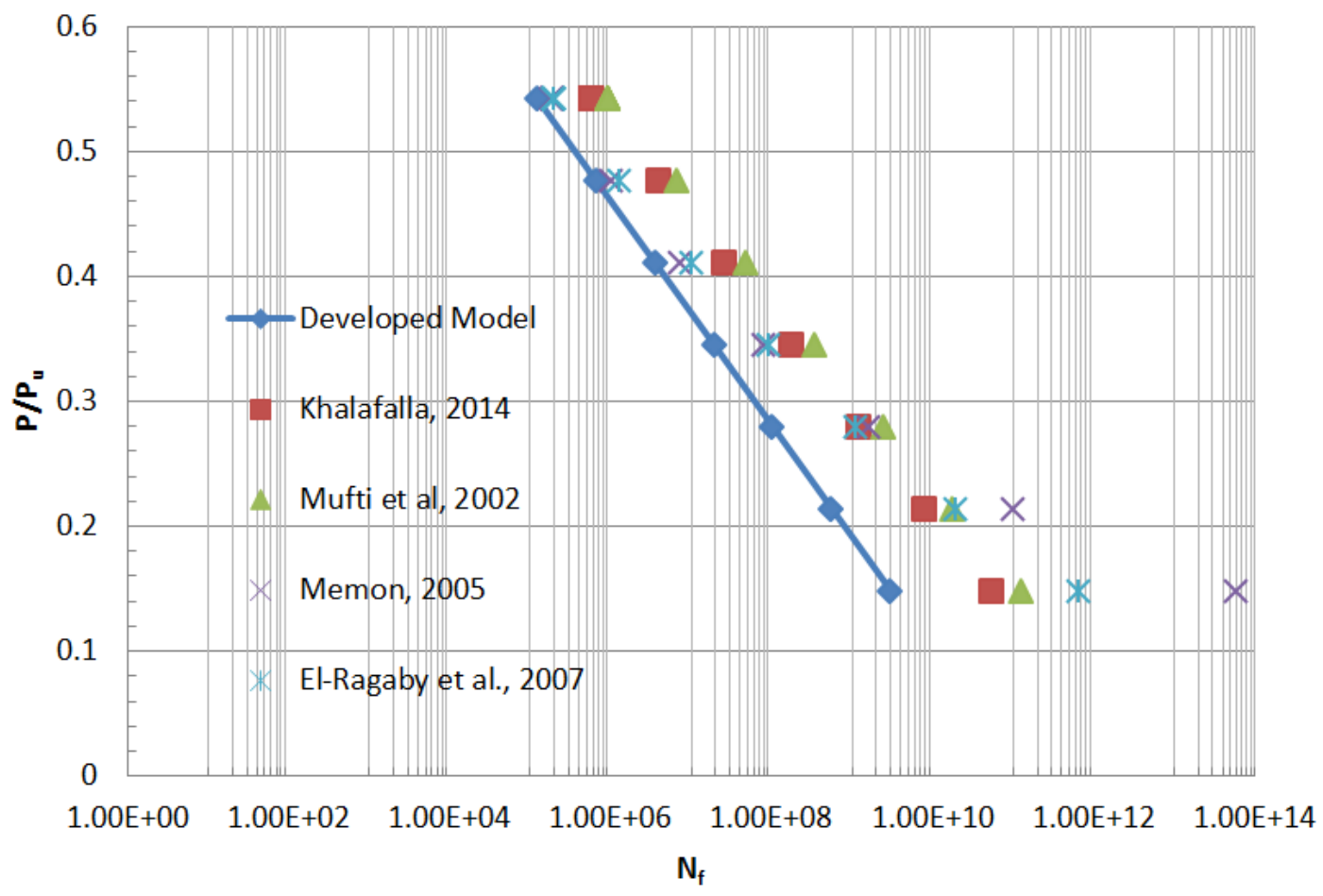

d) The P-N fatigue models for the precast FDDP with Z-shape joint

Figure 5.52. The P-N fatigue models for the precast FDDPs on log-graphs 


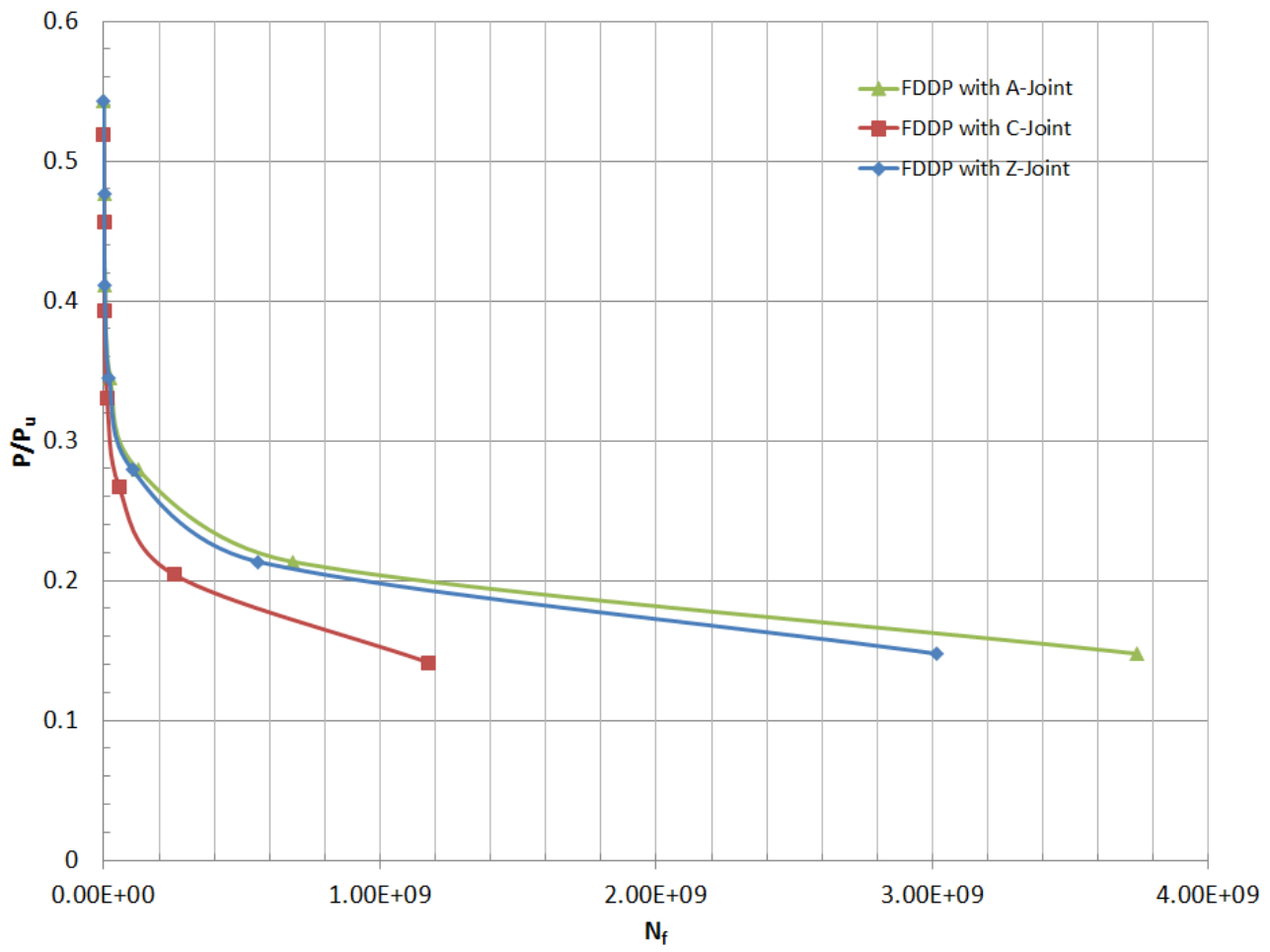

a) P-N curve on normal-scale graph

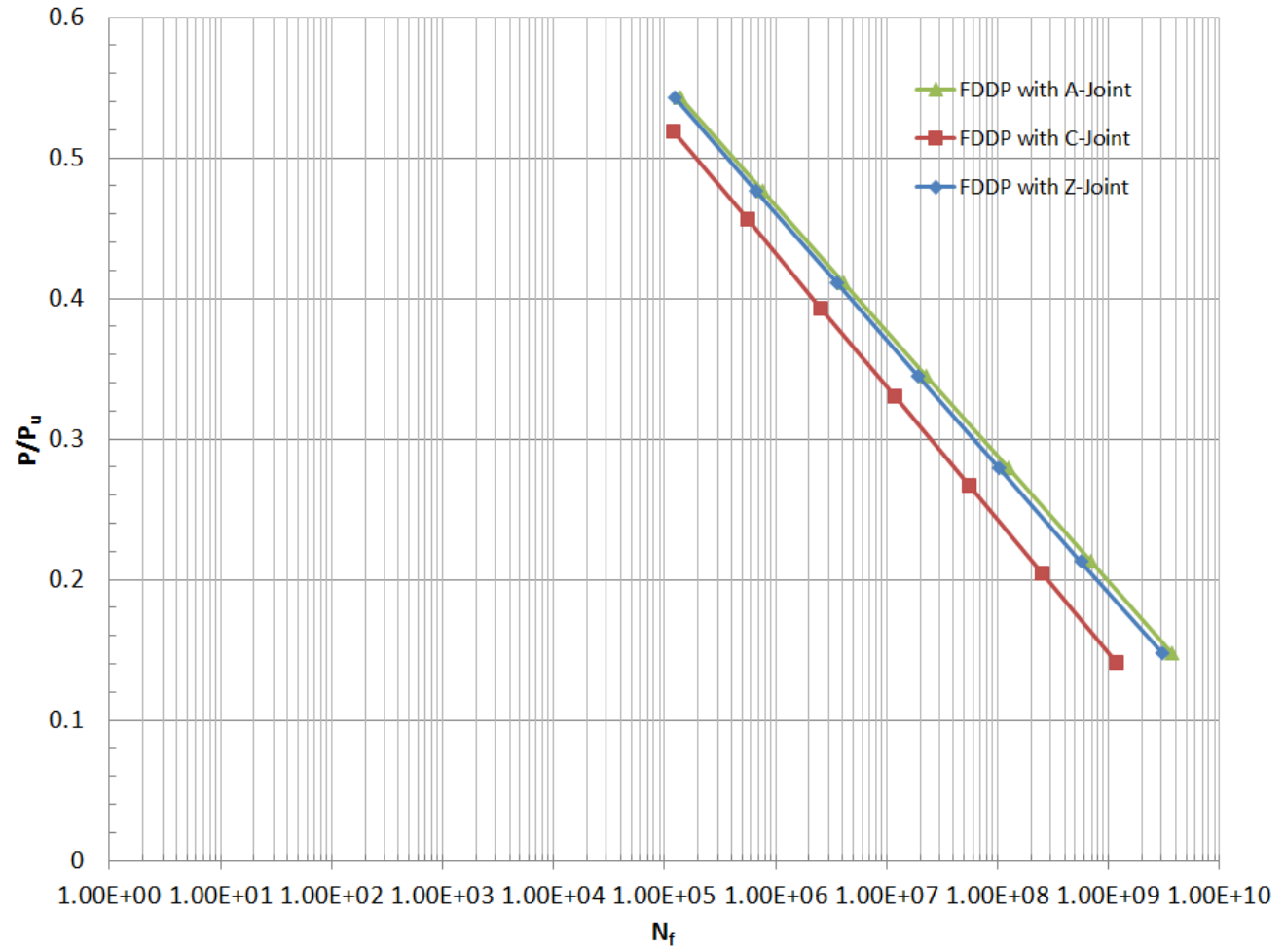

b) P-N curve on logarithmic graph

Figure 5.53. P-N curves for the ribbed-surface GFRP-reinforced precast FDDPs 


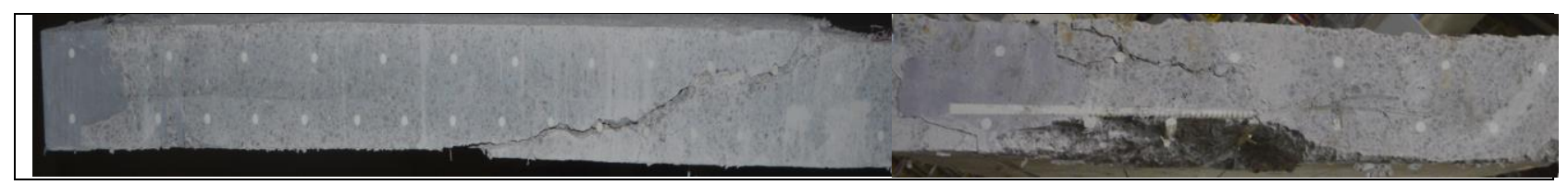

a) Longitudinal direction for slab S1 (A-CAF)
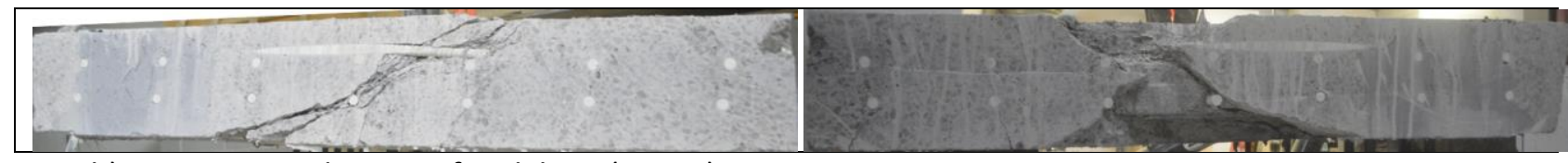

b) Transverse direction for slab S1 (A-CAF)

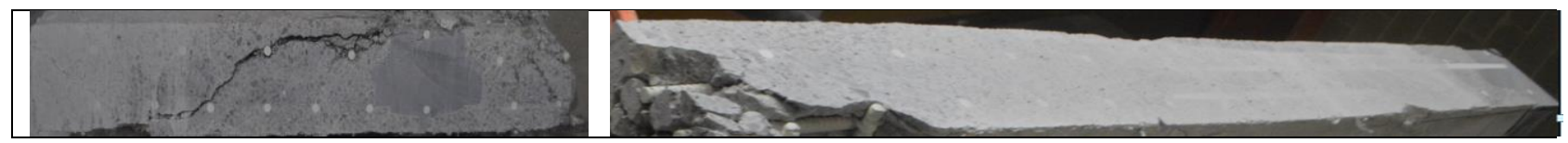

c) Longitudinal direction for slab S2 (A-VAF)

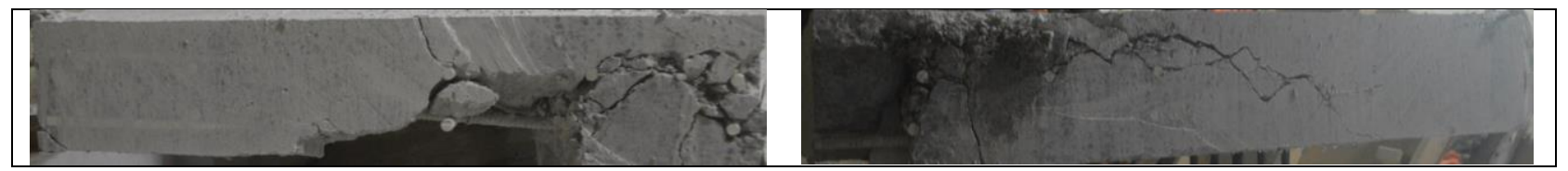

d) Transverse direction for slab S2 (A-VAF)

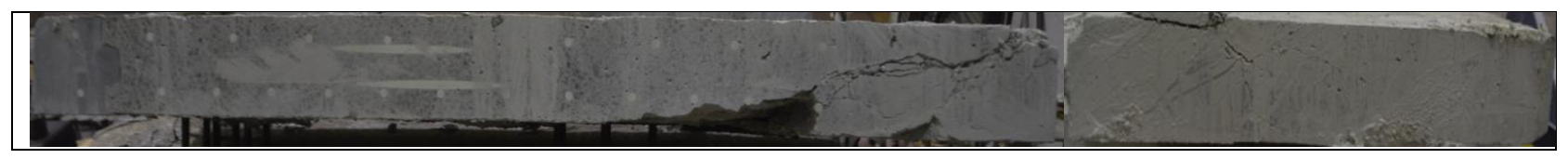

e) Longitudinal direction for slab S3 (C-CAF)

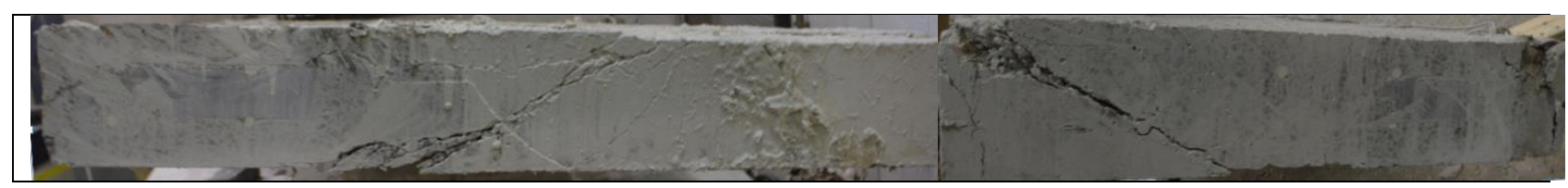

f) Transverse direction for slab S3 (C-CAF) 


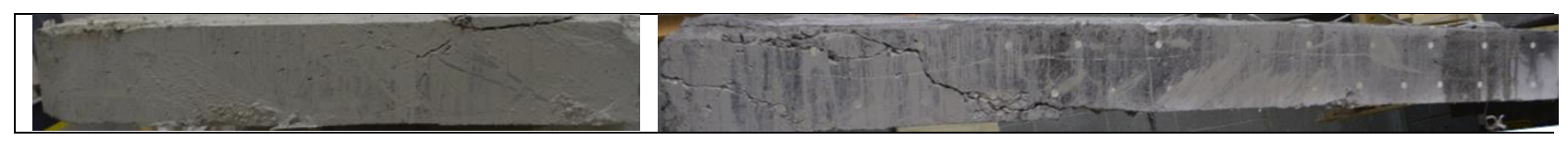

g) Longitudinal direction for slab S4 (C-VAF)
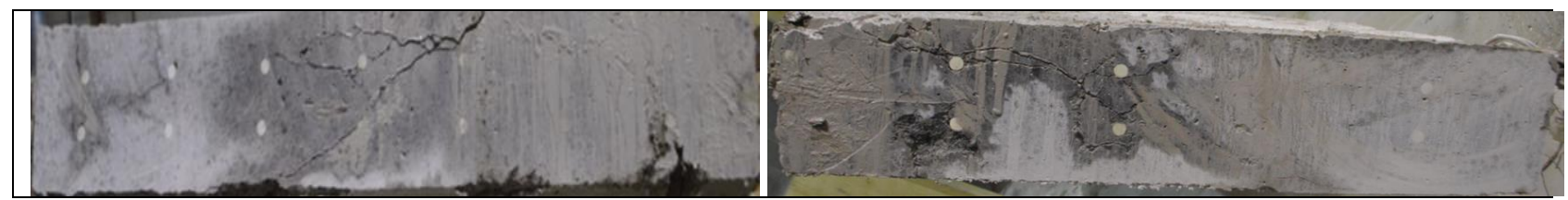

h) Transverse direction for slab S4 (C-VAF)
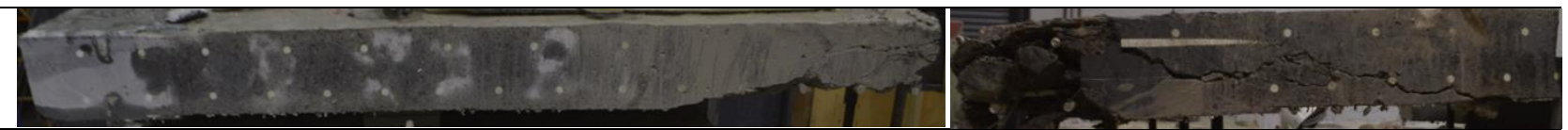

i) Longitudinal direction for slab S5 (Z-CAF)
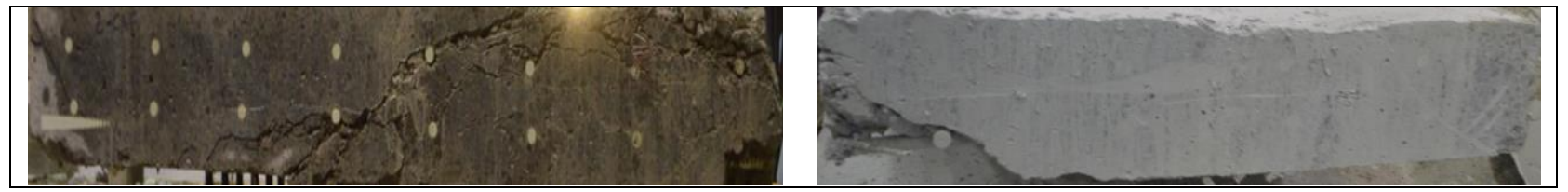

j) Transverse direction for slab S5 (Z-CAF)

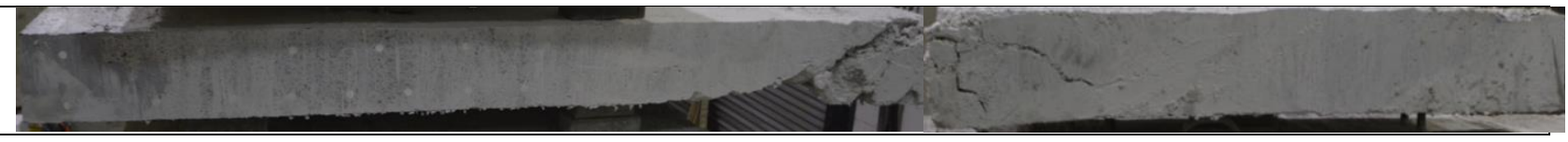

k) Longitudinal direction for slab S6 (Z-VAF)

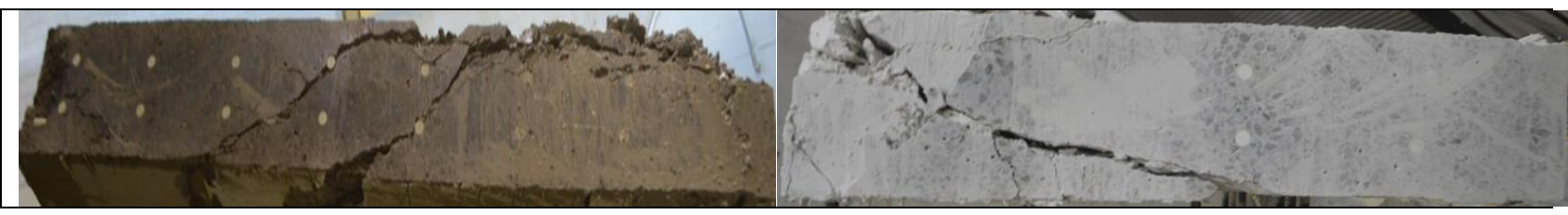

1) Transverse direction for slab S6 (Z-VAF)

N.B. Not-to-scale photos

Figure 5.54. Views of punching shear failure in tested slabs after slicing them at wheel load location 


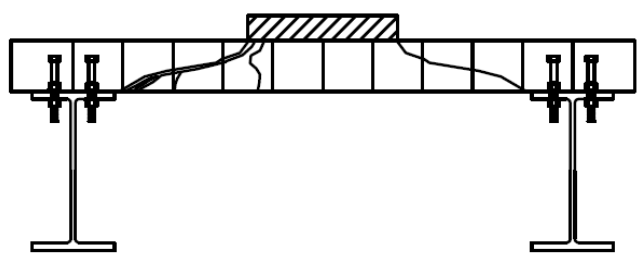

a) Cross-section of slab S1 (A-CAF)

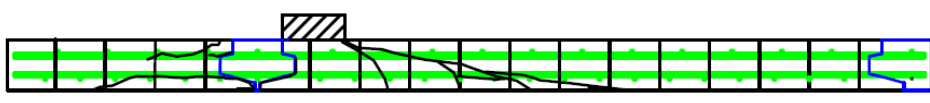

b) Long. Section of slab S1 (A-CAF)
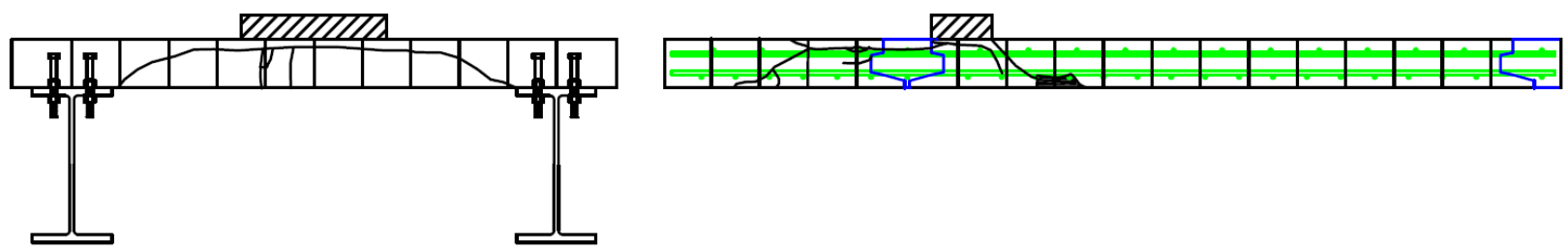

c) Cross-section of slab S2 (A-VAF)

d) Long. Section of slab S2 (A-VAF)
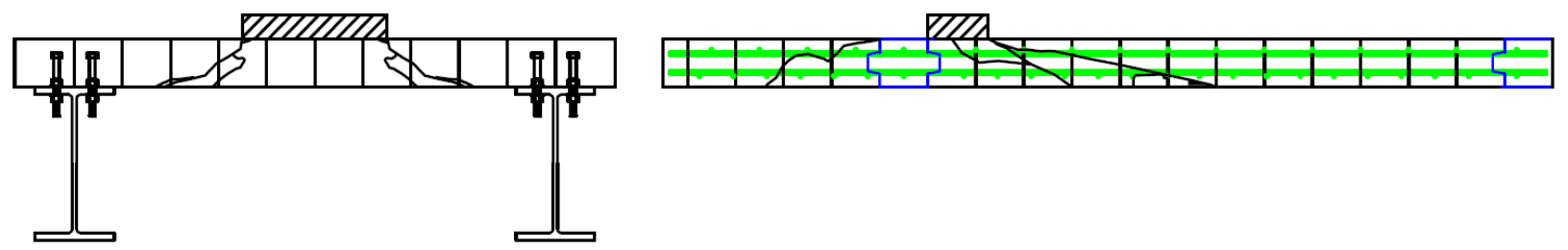

e) Cross-section of slab S3 (C-CAF)

f) Long. Section of slab S3 (C-CAF)
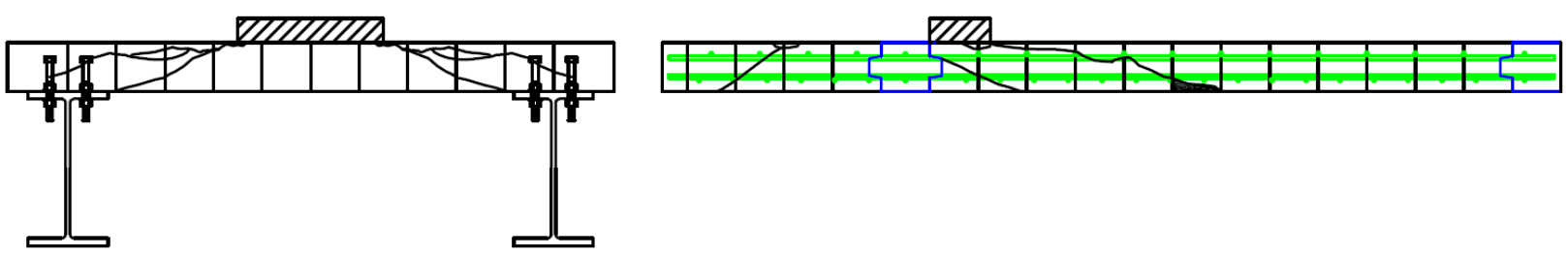

g) Cross-section of slab S4 (C-VAF)

h) Long. Section of slab S4 (C-VAF)
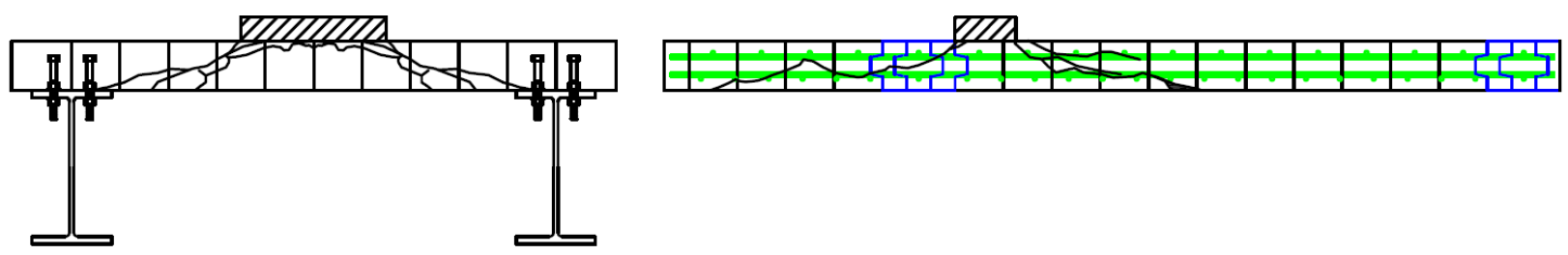

i) Cross-section of slab S5 (Z-CAF)

j) Long. Section of slab S5 (Z-CAF)
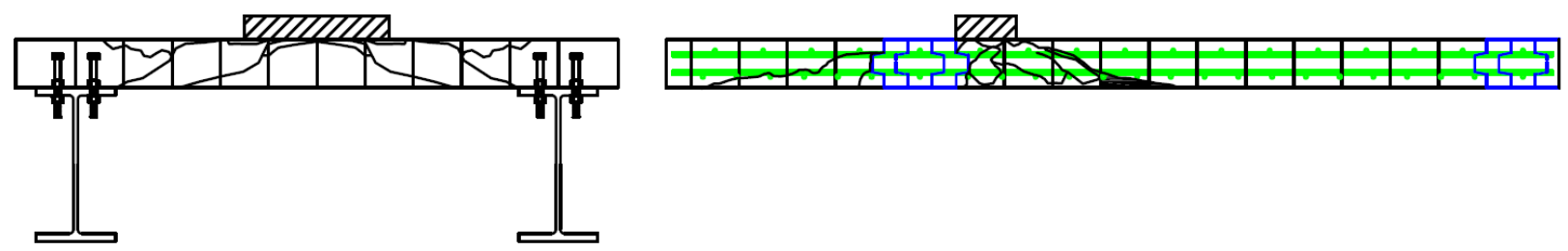

k) Cross-section of slab S6 (Z-VAF)

1) Long. Section of slab S6 (Z-VAF)

Figure 5.55. Schematic diagrams of the punching shear crack patterns for the tested slabs 


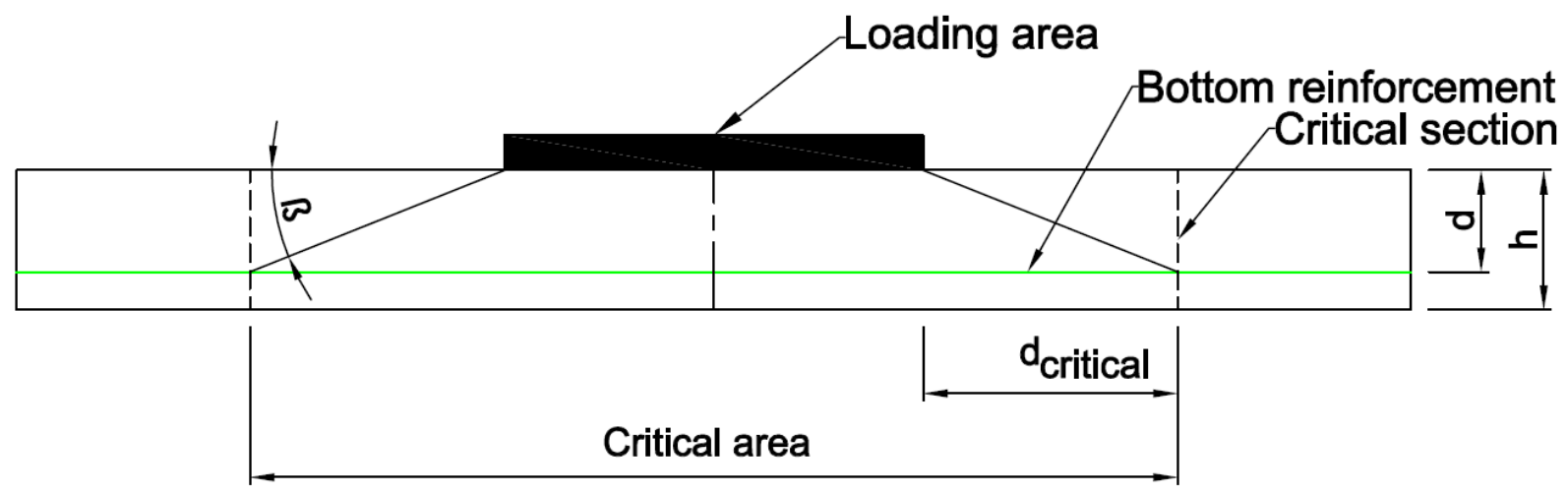

N.B.: $\beta=\arctan (\mathrm{d} / \mathrm{d} . c r i t i c a l)$

a) cross-sectional view for the critical section for the punching shear

B

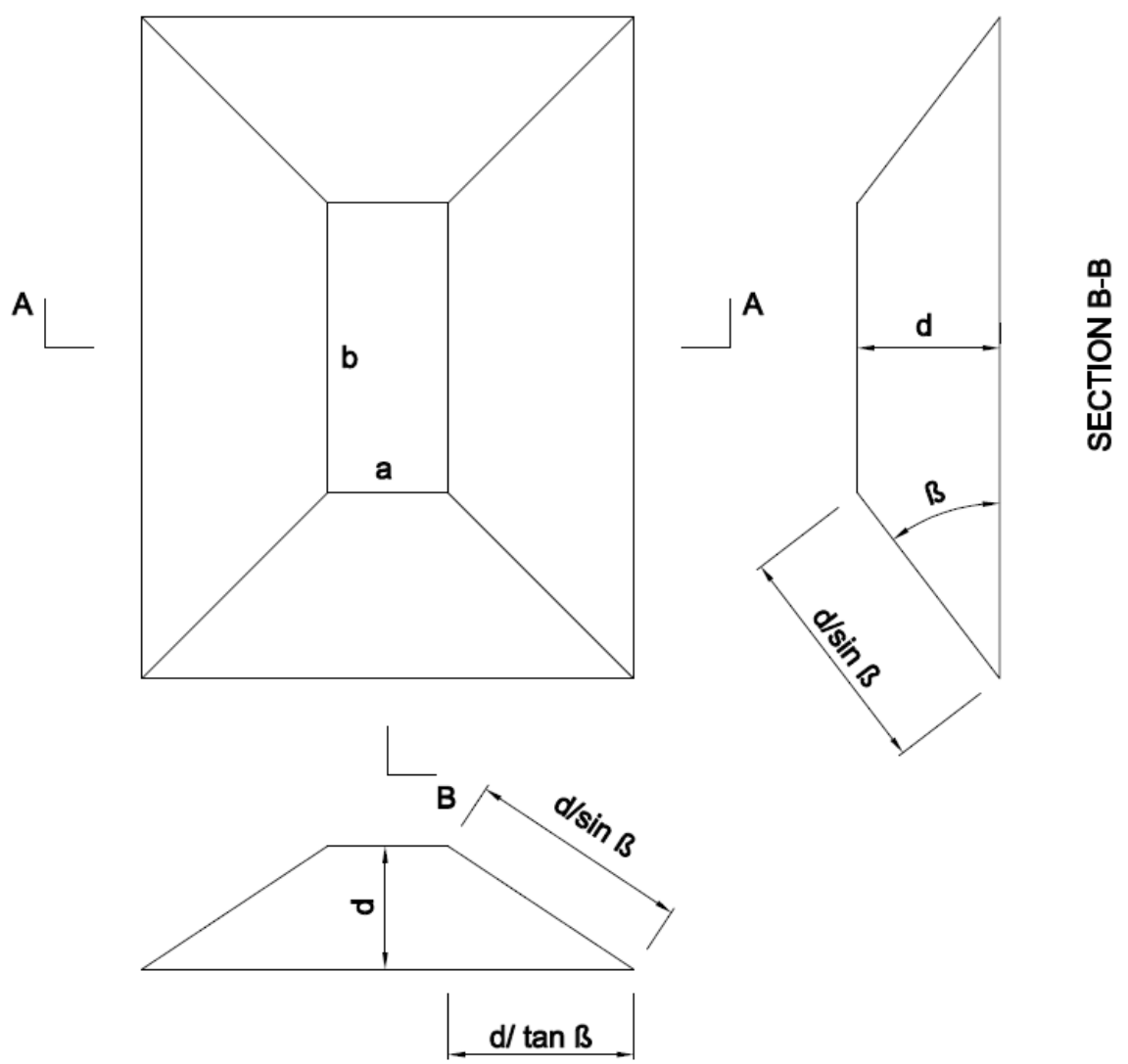

SECTION A-A

b) Plan and sectional view of failure surface for general punching shear model Figure 5.56. Schematic diagram for the critical section of the punching shear 


\section{Appendix: Experimental Data}

Table A5.1. Fatigue Equations for the d-N for the precast FDDPs

\begin{tabular}{lll}
\hline Number & \multicolumn{1}{c}{ Equations } & Comments \\
\hline$[1]$ & $N_{f}=e^{\eta\left(1-d / d_{u}\right)}$ & The Cumulative Fatigue Damage (CMD) \\
\hline$[2]$ & $\frac{d}{d_{u}}=-K \ln \left(N_{f}\right)+1$ & The d-N Fatigue \\
\hline
\end{tabular}

Table A5.2. Fatigue parameters for the d-N for the precast FDDPs

\begin{tabular}{lcc}
\hline Joint Pattern & $\eta$ & $\mathrm{k}$ \\
\hline A-Jointed Precast FDDP & 17.45 & -0.057 \\
\hline C-Jointed Precast FDDP & 18.73 & -0.053 \\
\hline Z-Jointed Precast FDDP & 17.3 & -0.058 \\
\hline
\end{tabular}

Table A5.3. Fatigue Equations for the $\epsilon-\mathrm{N}$ for for the precast FDDPs

\begin{tabular}{lll}
\hline Number & Equations & Comments \\
\hline$[1]$ & $N_{f}=e^{\eta\left(1-\epsilon / \epsilon_{u}\right)}$ & The Cumulative Fatigue Damage (CMD) \\
\hline$[2]$ & $\frac{\epsilon}{\epsilon_{u}}=-K \ln \left(N_{f}\right)+1$ & The $\epsilon-\mathrm{N}$ Fatigue \\
\hline
\end{tabular}

Table A5.4. Fatigue parameters for the $\epsilon-\mathrm{N}$ for concrete and GFRP bars embedded into the precast FDDPs

\begin{tabular}{lcc}
\hline Joint Pattern & $\eta$ & $\mathrm{k}$ \\
\hline A-Jointed Precast FDDP (L) & 14.38 & -0.070 \\
\hline A-Jointed Precast FDDP (T) & 21.04 & -0.048 \\
\hline A-Jointed Precast FDDP - Concrete & 19.25 & -0.052 \\
\hline C-Jointed Precast FDDP (L) & 14.37 & -0.070 \\
\hline C-Jointed Precast FDDP (T) & 18.08 & 0.055 \\
\hline C-Jointed Precast FDDP - Concrete & 13.87 & -0.072 \\
\hline Z-Jointed Precast FDDP (L) & 17.18 & -0.058 \\
\hline Z-Jointed Precast FDDP (T) & 22.09 & -0.045 \\
\hline Z-Jointed Precast FDDP - Concrete & 14.81 & -0.068 \\
\hline
\end{tabular}


Table A5.5. A-jointed precast FDDP deflection history for the equivalent constant amplitude fatigue load segments

\begin{tabular}{|c|c|c|c|c|c|c|c|c|c|c|c|c|c|c|}
\hline \multirow[t]{2}{*}{ Segment } & \multirow[t]{2}{*}{$\mathrm{du}$} & \multicolumn{3}{|c|}{ FLS } & \multirow[t]{2}{*}{$\mathrm{d}_{\min }$} & \multirow[t]{2}{*}{$\mathrm{d}_{\max }$} & \multirow[t]{2}{*}{$\mathrm{d}_{\mathrm{amp}}$} & \multirow[t]{2}{*}{$d_{\text {mean }}$} & \multirow[t]{2}{*}{$\mathrm{R}$} & \multirow[t]{2}{*}{ A } & \multirow[t]{2}{*}{$\mathrm{d}_{\max } / \mathrm{d}_{\mathrm{u}}$} & \multirow[t]{2}{*}{$\mathrm{n}$} & \multirow[t]{2}{*}{$\mathrm{N}_{\mathrm{f}}$} & \multirow[t]{2}{*}{$\mathrm{n} / \mathrm{N}_{\mathrm{f}}$} \\
\hline & & MF & WL & FLS1 & & & & & & & & & & \\
\hline & $\mathrm{mm}$ & & $\mathrm{kN}$ & $\mathrm{kN}$ & $\mathrm{mm}$ & $\mathrm{mm}$ & & & & & & & & \\
\hline 1 & 23.47 & 1 & 87.5 & 122.5 & 1.24 & 1.24 & 0.00 & 1.24 & 1 & 0 & 0.0528334 & 100,000 & $15,068,618$ & 0.0066363 \\
\hline 2 & 23.47 & 1.5 & 87.5 & 183.8 & 1.24 & 2.37 & 0.57 & 1.81 & 0.523207 & 0.313019 & 0.10098 & 100,000 & $6,504,233$ & 0.0153746 \\
\hline 3 & 23.47 & 2 & 87.5 & 245 & 1.24 & 2.47 & 0.62 & 1.86 & 0.502024 & 0.331536 & 0.1052407 & 100,000 & $6,038,180$ & 0.0165613 \\
\hline 4 & 23.47 & 2.5 & 87.5 & 306.3 & 1.24 & 2.76 & 0.76 & 2.00 & 0.449275 & 0.38 & 0.1175969 & 100,000 & $4,867,041$ & 0.0205464 \\
\hline 5 & 23.47 & 3 & 87.5 & 367.5 & 1.24 & 3.80 & 1.28 & 2.52 & 0.326316 & 0.507937 & 0.1619088 & 100,000 & $2,246,201$ & 0.0445196 \\
\hline 6 & 23.47 & 3.5 & 87.5 & 428.8 & 1.24 & 5.38 & 2.07 & 3.31 & 0.230483 & 0.625378 & 0.2292288 & 100,000 & 693,851 & 0.1441232 \\
\hline 7 & 23.47 & 4 & 87.5 & 490 & 1.24 & 7.41 & 3.09 & 4.33 & 0.167341 & 0.713295 & 0.3157222 & 115,381 & 153,383 & 0.7522387 \\
\hline 8 & 23.47 & 4 & 87.5 & 490 & 1.24 & 9.44 & 4.10 & 5.34 & 0.131356 & 0.76779 & 0.4022156 & 94,112 & 33,907 & 2.7755813 \\
\hline & & & & & & & & & & & Total & 809,493 & $\Sigma \mathrm{n} / \mathrm{N}$ & 1 \\
\hline
\end{tabular}

Table A5.6. C-jointed precast FDDP deflection history for the equivalent constant amplitude fatigue load segments

\begin{tabular}{|c|c|c|c|c|c|c|c|c|c|c|c|c|c|c|}
\hline \multirow[t]{2}{*}{ Segment } & \multirow[t]{2}{*}{$\mathrm{du}$} & \multicolumn{3}{|c|}{ FLS } & \multirow[t]{2}{*}{$\mathrm{d}_{\min }$} & \multirow[t]{2}{*}{$\mathrm{d}_{\max }$} & \multirow[t]{2}{*}{$\mathrm{d}_{\mathrm{amp}}$} & \multirow[t]{2}{*}{$d_{\text {mean }}$} & \multirow[t]{2}{*}{$\mathrm{R}$} & \multirow[t]{2}{*}{ A } & \multirow[t]{2}{*}{$\mathrm{d}_{\max } / \mathrm{d}_{\mathrm{u}}$} & \multirow[t]{2}{*}{$\mathrm{n}$} & \multirow[t]{2}{*}{$\mathrm{N}_{\mathrm{f}}$} & \multirow[t]{2}{*}{$\mathrm{n} / \mathrm{N}_{\mathrm{f}}$} \\
\hline & & $\mathrm{MF}$ & WL & FLS1 & & & & & & & & & & \\
\hline & $\mathrm{mm}$ & & $\mathrm{kN}$ & $\mathrm{kN}$ & $\mathrm{mm}$ & $\mathrm{mm}$ & & & & & & & & \\
\hline 1 & 25.98 & 1 & 87.5 & 122.5 & 1.35 & 3.58 & 1.12 & 2.47 & 0.377095 & 0.452333 & 0.1377983 & 100,000 & $10,305,645$ & 0.0097034 \\
\hline 2 & 25.98 & 1.5 & 87.5 & 183.8 & 1.35 & 3.72 & 1.19 & 2.54 & 0.362903 & 0.467456 & 0.1431871 & 100,000 & $9,316,300$ & 0.0107339 \\
\hline 3 & 25.98 & 2 & 87.5 & 245 & 1.35 & 4.09 & 1.37 & 2.72 & 0.330073 & 0.503676 & 0.1574288 & 100,000 & $7,135,140$ & 0.0140151 \\
\hline 4 & 25.98 & 2.5 & 87.5 & 306.3 & 1.35 & 5.43 & 2.04 & 3.39 & 0.248619 & 0.60177 & 0.2090069 & 100,000 & $2,715,628$ & 0.0368239 \\
\hline 5 & 25.98 & 3 & 87.5 & 367.5 & 1.35 & 6.89 & 2.77 & 4.12 & 0.195936 & 0.67233 & 0.265204 & 100,000 & 947,912 & 0.105495 \\
\hline \multirow[t]{2}{*}{6} & 25.98 & 3.5 & 87.5 & 428.8 & 1.35 & 9.74 & 4.20 & 5.55 & 0.138604 & 0.756537 & 0.3749038 & 100,000 & 121,473 & 0.8232287 \\
\hline & & & & & & & & & & & Total & 600,000 & $\Sigma \mathrm{n} / \mathrm{N}$ & 1 \\
\hline
\end{tabular}


Table A5.7. Z-jointed precast FDDP deflection history for the equivalent constant amplitude fatigue load segments

\begin{tabular}{|c|c|c|c|c|c|c|c|c|c|c|c|c|c|c|}
\hline \multirow[t]{2}{*}{ Segment } & \multirow[t]{2}{*}{$\mathrm{du}$} & \multicolumn{3}{|c|}{ FLS } & \multirow[t]{2}{*}{$\mathrm{d}_{\min }$} & \multirow[t]{2}{*}{$\mathrm{d}_{\max }$} & \multirow[t]{2}{*}{$\mathrm{d}_{\mathrm{amp}}$} & \multirow[t]{2}{*}{$d_{\text {mean }}$} & \multirow[t]{2}{*}{$\mathrm{R}$} & \multirow[t]{2}{*}{ A } & \multirow[t]{2}{*}{$\mathrm{d}_{\max } / \mathrm{d}_{\mathrm{u}}$} & \multirow[t]{2}{*}{$\mathrm{n}$} & \multirow[t]{2}{*}{$\mathrm{N}_{\mathrm{f}}$} & \multirow[t]{2}{*}{$\mathrm{n} / \mathrm{N}_{\mathrm{f}}$} \\
\hline & & MF & WL & FLS1 & & & & & & & & & & \\
\hline & $\mathrm{mm}$ & & $\mathrm{kN}$ & $\mathrm{kN}$ & $\mathrm{mm}$ & $\mathrm{mm}$ & & & & & & & & \\
\hline 1 & 29.62 & 1 & 87.5 & 122.5 & 1.78 & 3.12 & 0.67 & 2.45 & 0.570513 & 0.273469 & 0.1053342 & 100,000 & $5,273,922$ & 0.0189612 \\
\hline 2 & 29.62 & 1.5 & 87.5 & 183.8 & 1.78 & 3.2 & 0.71 & 2.49 & 0.55625 & 0.285141 & 0.1080351 & 100,000 & $5,033,157$ & 0.0198682 \\
\hline 3 & 29.62 & 2 & 87.5 & 245 & 1.78 & 3.5 & 0.86 & 2.64 & 0.508571 & 0.325758 & 0.1181634 & 100,000 & $4,224,165$ & 0.0236733 \\
\hline 4 & 29.62 & 2.5 & 87.5 & 306.3 & 1.78 & 4.55 & 1.39 & 3.17 & 0.391209 & 0.437599 & 0.1536124 & 100,000 & $2,287,664$ & 0.0437127 \\
\hline 5 & 29.62 & 3 & 87.5 & 367.5 & 1.78 & 6.19 & 2.21 & 3.99 & 0.287561 & 0.553325 & 0.2089804 & 100,000 & 877,770 & 0.113925 \\
\hline 6 & 29.62 & 3.5 & 87.5 & 428.8 & 1.78 & 7.64 & 2.93 & 4.71 & 0.232984 & 0.622081 & 0.2579338 & 100,000 & 376,328 & 0.265726 \\
\hline 7 & 29.62 & 4 & 87.5 & 490 & 1.78 & 8.77 & 3.50 & 5.28 & 0.202965 & 0.662559 & 0.2960837 & 100,000 & 194,502 & 0.5141335 \\
\hline \multirow[t]{2}{*}{8} & 29.62 & 4 & 87.5 & 490 & 1.78 & 10.88 & 4.55 & 6.33 & 0.163603 & 0.718799 & 0.3673194 & 100,000 & 56,714 & 1.763234 \\
\hline & & & & & & & & & & & Total & 800,000 & $\Sigma \mathrm{n} / \mathrm{N}$ & 1 \\
\hline
\end{tabular}

Table A5.8. A-jointed precast FDDP longitudinal bar strain history for the equivalent constant amplitude fatigue load segments

\begin{tabular}{|c|c|c|c|c|c|c|c|c|c|c|c|c|c|c|}
\hline \multirow[t]{2}{*}{ Segment } & \multirow[t]{2}{*}{$\mathrm{\epsilon u}$} & \multicolumn{3}{|c|}{ FLS } & \multirow[t]{2}{*}{$\epsilon_{\min }$} & \multirow[t]{2}{*}{$\epsilon_{\max }$} & \multirow[t]{2}{*}{$\epsilon_{\mathrm{amp}}$} & \multirow[t]{2}{*}{$\epsilon_{\text {mean }}$} & \multirow[t]{2}{*}{$\mathrm{R}$} & \multirow[t]{2}{*}{ A } & \multirow[t]{2}{*}{$\epsilon_{\max } / \epsilon_{\mathrm{u}}$} & \multirow[t]{2}{*}{$\mathrm{n}$} & \multirow[t]{2}{*}{$\mathrm{N}_{\mathrm{f}}$} & \multirow[t]{2}{*}{$\mathrm{n} / \mathrm{N}_{\mathrm{f}}$} \\
\hline & & MF & WL & FLS1 & & & & & & & & & & \\
\hline & $\mu \mathrm{m}$ & & $\mathrm{kN}$ & $\mathrm{kN}$ & $\mu \mathrm{m}$ & $\mathrm{Mm}$ & & & & & & & & \\
\hline 1 & 8707 & 1 & 87.5 & 122.5 & 108 & 253.00 & 72.50 & 180.50 & 0.426877 & 0.401662 & 0.0290571 & 100,000 & $1,157,994$ & 0.0863562 \\
\hline 2 & 8707 & 1.5 & 87.5 & 183.8 & 105 & 263.00 & 79.00 & 184.00 & 0.39924 & 0.429348 & 0.0302056 & 100,000 & $1,139,027$ & 0.0877943 \\
\hline 3 & 8707 & 2 & 87.5 & 245 & 124 & 303.00 & 89.50 & 213.50 & 0.409241 & 0.419204 & 0.0347996 & 100,000 & $1,066,212$ & 0.09379 \\
\hline 4 & 8707 & 2.5 & 87.5 & 306.3 & 154 & 353.00 & 99.50 & 253.50 & 0.436261 & 0.392505 & 0.0405421 & 100,000 & 981,704 & 0.1018637 \\
\hline 5 & 8707 & 3 & 87.5 & 367.5 & 202 & 485.00 & 141.50 & 343.50 & 0.416495 & 0.411936 & 0.0557023 & 100,000 & 789,410 & 0.1266769 \\
\hline 6 & 8707 & 3.5 & 87.5 & 428.8 & 252 & 712.00 & 230.00 & 482.00 & 0.353933 & 0.477178 & 0.0817733 & 100,000 & 542,606 & 0.1842958 \\
\hline 7 & 8707 & 4 & 87.5 & 490 & 322 & 958.00 & 318.00 & 640.00 & 0.336117 & 0.496875 & 0.1100264 & 115,381 & 361,442 & 0.3192238 \\
\hline \multirow[t]{2}{*}{8} & 8707 & 4 & 87.5 & 490 & 389 & $1,198.00$ & 404.50 & 793.50 & 0.324708 & 0.509767 & 0.1375904 & 94,112 & 243,163 & 0.3870331 \\
\hline & & & & & & & & & & & Total & 809,493 & $\Sigma \mathrm{n} / \mathrm{N}$ & 1 \\
\hline
\end{tabular}


Table A5.9. A-jointed precast FDDP transverse bar strain history for the equivalent constant amplitude fatigue load segments

\begin{tabular}{|c|c|c|c|c|c|c|c|c|c|c|c|c|c|c|}
\hline \multirow[t]{2}{*}{ Segment } & \multirow[t]{2}{*}{$\epsilon \mathrm{G}$} & \multicolumn{3}{|c|}{ FLS } & \multirow[t]{2}{*}{$\epsilon_{\min }$} & \multirow[t]{2}{*}{$\epsilon_{\max }$} & \multirow[t]{2}{*}{$\epsilon_{\mathrm{amp}}$} & \multirow[t]{2}{*}{$\epsilon_{\text {mean }}$} & \multirow[t]{2}{*}{$\mathrm{R}$} & \multirow[t]{2}{*}{ A } & \multirow[t]{2}{*}{$\epsilon_{\max } / \epsilon_{\mathrm{u}}$} & \multirow[t]{2}{*}{$\mathrm{n}$} & \multirow[t]{2}{*}{$\mathrm{N}_{\mathrm{f}}$} & \multirow[t]{2}{*}{$\mathrm{n} / \mathrm{N}_{\mathrm{f}}$} \\
\hline & & $\mathrm{MF}$ & WL & FLS1 & & & & & & & & & & \\
\hline & $\mu \mathrm{m}$ & & $\mathrm{kN}$ & $\mathrm{kN}$ & $\mu \mathrm{m}$ & $\mathrm{Mm}$ & & & & & & & & \\
\hline 1 & 8707 & 1 & 87.5 & 122.5 & 371 & 675.00 & 152.00 & 523.00 & 0.54963 & 0.290631 & 0.0775238 & 100,000 & $267,943,290$ & 0.0003732 \\
\hline 2 & 8707 & 1.5 & 87.5 & 183.8 & 377 & 714.00 & 168.50 & 545.50 & 0.528011 & 0.308891 & 0.082003 & 100,000 & $243,848,376$ & 0.0004101 \\
\hline 3 & 8707 & 2 & 87.5 & 245 & 437 & 847.00 & 205.00 & 642.00 & 0.515939 & 0.319315 & 0.0972781 & 100,000 & $176,832,419$ & 0.0005655 \\
\hline 4 & 8707 & 2.5 & 87.5 & 306.3 & 675 & $1,282.00$ & 303.50 & 978.50 & 0.526521 & 0.310169 & 0.1472379 & 100,000 & $61,817,701$ & 0.0016177 \\
\hline 5 & 8707 & 3 & 87.5 & 367.5 & 1062 & $1,886.00$ & 412.00 & $1,474.00$ & 0.563097 & 0.279512 & 0.2166073 & 100,000 & $14,365,831$ & 0.006961 \\
\hline 6 & 8707 & 3.5 & 87.5 & 428.8 & 1532 & $2,686.00$ & 577.00 & $2,109.00$ & 0.570365 & 0.273589 & 0.3084874 & 100,000 & $2,079,142$ & 0.0480968 \\
\hline 7 & 8707 & 4 & 87.5 & 490 & 2223 & $3,858.00$ & 817.50 & $3,040.50$ & 0.576205 & 0.26887 & 0.4430918 & 115,381 & 122,488 & 0.9419746 \\
\hline \multirow[t]{2}{*}{8} & 8707 & 4 & 87.5 & 490 & 2906 & $5,010.00$ & $1,052.00$ & $3,958.00$ & 0.58004 & 0.265791 & 0.5753991 & 94,112 & 7,573 & 12.426615 \\
\hline & & & & & & & & & & & Total & 809,493 & $\Sigma \mathrm{n} / \mathrm{N}$ & 1 \\
\hline
\end{tabular}

Table A5.10. A-jointed precast FDDP concrete strain history for the equivalent constant amplitude fatigue load segments

\begin{tabular}{|c|c|c|c|c|c|c|c|c|c|c|c|c|c|c|}
\hline \multirow[t]{2}{*}{ Segment } & \multirow[t]{2}{*}{$\mathrm{\epsilon u}$} & \multicolumn{3}{|c|}{ FLS } & \multirow[t]{2}{*}{$\epsilon_{\min }$} & \multirow[t]{2}{*}{$\epsilon_{\max }$} & \multirow[t]{2}{*}{$\epsilon_{\mathrm{amp}}$} & \multirow[t]{2}{*}{$\epsilon_{\text {mean }}$} & \multirow[t]{2}{*}{$\mathrm{R}$} & \multirow[t]{2}{*}{ A } & \multirow{2}{*}{$\epsilon_{\max } / \epsilon_{\mathrm{u}}$} & \multirow[t]{2}{*}{$\mathrm{n}$} & \multirow[t]{2}{*}{$\mathrm{N}_{\mathrm{f}}$} & \multirow[t]{2}{*}{$\mathrm{n} / \mathrm{N}_{\mathrm{f}}$} \\
\hline & & MF & WL & FLS1 & & & & & & & & & & \\
\hline & $\mu \mathrm{m}$ & & $\mathrm{kN}$ & $\mathrm{kN}$ & $\mu \mathrm{m}$ & $\mu \mathrm{m}$ & & & & & & & & \\
\hline 1 & 3354 & 1 & 87.5 & 122.5 & 64 & 389.00 & 162.50 & 226.50 & 0.164524 & 0.717439 & 0.1159809 & 100,000 & $24,508,742$ & 0.0040802 \\
\hline 2 & 3354 & 1.5 & 87.5 & 183.8 & 61 & 396.00 & 167.50 & 228.50 & 0.15404 & 0.733042 & 0.118068 & 100,000 & $23,543,755$ & 0.0042474 \\
\hline 3 & 3354 & 2 & 87.5 & 245 & 75 & 422.00 & 173.50 & 248.50 & 0.177725 & 0.698189 & 0.1258199 & 100,000 & $20,280,516$ & 0.0049308 \\
\hline 4 & 3354 & 2.5 & 87.5 & 306.3 & 143 & 547.00 & 202.00 & 345.00 & 0.261426 & 0.585507 & 0.1630888 & 100,000 & $9,898,199$ & 0.0101028 \\
\hline 5 & 3354 & 3 & 87.5 & 367.5 & 237 & 717.00 & 240.00 & 477.00 & 0.330544 & 0.503145 & 0.2137746 & 100,000 & $3,731,515$ & 0.0267988 \\
\hline 6 & 3354 & 3.5 & 87.5 & 428.8 & 355 & $1,011.00$ & 328.00 & 683.00 & 0.351137 & 0.480234 & 0.3014311 & 100,000 & 690,532 & 0.144816 \\
\hline 7 & 3354 & 4 & 87.5 & 490 & 699 & $1,285.00$ & 293.00 & 992.00 & 0.543969 & 0.295363 & 0.3831246 & 115,381 & 143,326 & 0.8050239 \\
\hline \multirow[t]{2}{*}{8} & 3354 & 4 & 87.5 & 490 & 930 & $1,621.00$ & 345.50 & $1,275.50$ & 0.57372 & 0.270874 & 0.4833035 & 94,112 & 20,843 & 4.5153642 \\
\hline & & & & & & & & & & & Total & 809,493 & $\Sigma \mathrm{n} / \mathrm{N}$ & 1 \\
\hline
\end{tabular}


Table A5.11. C-jointed precast FDDP longitudinal bar strain history for the equivalent constant amplitude fatigue load segments

\begin{tabular}{|c|c|c|c|c|c|c|c|c|c|c|c|c|c|c|}
\hline \multirow[t]{2}{*}{ Segment } & \multirow[t]{2}{*}{$\mathrm{\epsilon u}$} & \multicolumn{3}{|c|}{ FLS } & \multirow[t]{2}{*}{$\epsilon_{\min }$} & \multirow[t]{2}{*}{$\epsilon_{\max }$} & \multirow[t]{2}{*}{$\epsilon_{\mathrm{amp}}$} & \multirow[t]{2}{*}{$\epsilon_{\text {mean }}$} & \multirow[t]{2}{*}{$\mathrm{R}$} & \multirow[t]{2}{*}{ A } & \multirow[t]{2}{*}{$\epsilon_{\max } / \epsilon_{\mathrm{u}}$} & \multirow[t]{2}{*}{$\mathrm{n}$} & \multirow[t]{2}{*}{$\mathrm{N}_{\mathrm{f}}$} & \multirow[t]{2}{*}{$\mathrm{n} / \mathrm{N}_{\mathrm{f}}$} \\
\hline & & MF & WL & FLS1 & & & & & & & & & & \\
\hline & $\mu \mathrm{m}$ & & $\mathrm{kN}$ & $\mathrm{kN}$ & $\mu \mathrm{m}$ & $\mu \mathrm{m}$ & & & & & & & & \\
\hline 1 & 8647 & 1 & 87.5 & 122.5 & 143 & 279 & 68.00 & 211.00 & 0.512545 & 0.322275 & 0.0322655 & 100,000 & $1,095,013$ & 0.0913231 \\
\hline 2 & 8647 & 1.5 & 87.5 & 183.8 & 146 & 303 & 78.50 & 224.50 & 0.481848 & 0.349666 & 0.0350411 & 100,000 & $1,052,199$ & 0.0950391 \\
\hline 3 & 8647 & 2 & 87.5 & 245 & 169 & 383 & 107.00 & 276.00 & 0.441253 & 0.387681 & 0.0442928 & 100,000 & 921,212 & 0.1085526 \\
\hline 4 & 8647 & 2.5 & 87.5 & 306.3 & 306 & 672 & 183.00 & 489.00 & 0.455357 & 0.374233 & 0.0777148 & 100,000 & 569,876 & 0.1754768 \\
\hline 5 & 8647 & 3 & 87.5 & 367.5 & 464 & 962 & 249.00 & 713.00 & 0.482328 & 0.349229 & 0.1112525 & 100,000 & 351,949 & 0.2841325 \\
\hline \multirow[t]{2}{*}{6} & 8647 & 3.5 & 87.5 & 428.8 & 504 & 874 & 185.00 & 689.00 & 0.576659 & 0.268505 & 0.1010755 & 100,000 & 407,372 & 0.2454759 \\
\hline & & & & & & & & & & & Total & 600,000 & $\Sigma \mathrm{n} / \mathrm{N}$ & 1 \\
\hline
\end{tabular}

Table A5.12. C-jointed precast FDDP transverse bar strain history for the equivalent constant amplitude fatigue load segments

\begin{tabular}{|c|c|c|c|c|c|c|c|c|c|c|c|c|c|c|}
\hline \multirow[t]{2}{*}{ Segment } & \multirow[t]{2}{*}{$\mathrm{\epsilon u}$} & \multicolumn{3}{|c|}{ FLS } & \multirow[t]{2}{*}{$\epsilon_{\min }$} & \multirow[t]{2}{*}{$\epsilon_{\max }$} & \multirow[t]{2}{*}{$\epsilon_{\mathrm{amp}}$} & \multirow[t]{2}{*}{$\epsilon_{\text {mean }}$} & \multirow[t]{2}{*}{$\mathrm{R}$} & \multirow[t]{2}{*}{$\mathrm{A}$} & \multirow{2}{*}{$\epsilon_{\max } / \epsilon_{\mathrm{u}}$} & \multirow[t]{2}{*}{$\mathrm{n}$} & \multirow[t]{2}{*}{$\mathrm{N}_{\mathrm{f}}$} & \multirow[t]{2}{*}{$\mathrm{n} / \mathrm{N}_{\mathrm{f}}$} \\
\hline & & $\mathrm{MF}$ & WL & FLS1 & & & & & & & & & & \\
\hline & $\mu \mathrm{m}$ & & $\mathrm{kN}$ & $\mathrm{kN}$ & $\mu \mathrm{m}$ & $\mu \mathrm{m}$ & & & & & & & & \\
\hline 1 & 5985 & 1 & 87.5 & 122.5 & 456 & 793 & 168.50 & 624.50 & 0.575032 & 0.269816 & 0.1324979 & 100,000 & $6,481,998$ & 0.0154273 \\
\hline 2 & 5985 & 1.5 & 87.5 & 183.8 & 455 & 835 & 190.00 & 645.00 & 0.54491 & 0.294574 & 0.1395155 & 100,000 & $5,709,610$ & 0.0175143 \\
\hline 3 & 5985 & 2 & 87.5 & 245 & 423 & 859 & 218.00 & 641.00 & 0.492433 & 0.340094 & 0.1435255 & 100,000 & $5,310,304$ & 0.0188313 \\
\hline 4 & 5985 & 2.5 & 87.5 & 306.3 & 710 & 1303 & 296.50 & $1,006.50$ & 0.544896 & 0.294585 & 0.2177109 & 100,000 & $1,388,699$ & 0.0720099 \\
\hline 5 & 5985 & 3 & 87.5 & 367.5 & 880 & 1694 & 407.00 & $1,287.00$ & 0.519481 & 0.316239 & 0.2830409 & 100,000 & 426,217 & 0.2346223 \\
\hline \multirow[t]{2}{*}{6} & 5985 & 3.5 & 87.5 & 428.8 & 1124 & 2027 & 451.50 & $1,575.50$ & 0.554514 & 0.286576 & 0.33868 & 100,000 & 155,865 & 0.6415828 \\
\hline & & & & & & & & & & & Total & 600,000 & $\Sigma \mathrm{n} / \mathrm{N}$ & 1 \\
\hline
\end{tabular}


Table A5.13. C-jointed precast FDDP concrete strain history for the equivalent constant amplitude fatigue load segments

\begin{tabular}{|c|c|c|c|c|c|c|c|c|c|c|c|c|c|c|}
\hline \multirow[t]{2}{*}{ Segment } & \multirow[t]{2}{*}{$\mathrm{\epsilon u}$} & \multicolumn{3}{|c|}{ FLS } & \multirow[t]{2}{*}{$\epsilon_{\min }$} & \multirow[t]{2}{*}{$\epsilon_{\max }$} & \multirow[t]{2}{*}{$\epsilon_{\mathrm{amp}}$} & \multirow[t]{2}{*}{$\epsilon_{\text {mean }}$} & \multirow[t]{2}{*}{$\mathrm{R}$} & \multirow[t]{2}{*}{ A } & \multirow[t]{2}{*}{$\epsilon_{\max } / \epsilon_{\mathrm{u}}$} & \multirow[t]{2}{*}{$\mathrm{n}$} & \multirow[t]{2}{*}{$\mathrm{N}_{\mathrm{f}}$} & \multirow[t]{2}{*}{$\mathrm{n} / \mathrm{N}_{\mathrm{f}}$} \\
\hline & & MF & WL & FLS1 & & & & & & & & & & \\
\hline & $\mu \mathrm{m}$ & & $\mathrm{kN}$ & $\mathrm{kN}$ & $\mu \mathrm{m}$ & $\mu \mathrm{m}$ & & & & & & & & \\
\hline 1 & 2559 & 1 & 87.5 & 122.5 & 0 & 35 & 17.50 & 17.50 & 0 & 1 & 0.0136772 & 100,000 & 874,679 & 0.1143277 \\
\hline 2 & 2559 & 1.5 & 87.5 & 183.8 & 7 & 59 & 26.00 & 33.00 & 0.118644 & 0.787879 & 0.0230559 & 100,000 & 767,979 & 0.1302119 \\
\hline 3 & 2559 & 2 & 87.5 & 245 & 44 & 95 & 25.50 & 69.50 & 0.463158 & 0.366906 & 0.0371239 & 100,000 & 631,830 & 0.1582704 \\
\hline 4 & 2559 & 2.5 & 87.5 & 306.3 & 81 & 122 & 20.50 & 101.50 & 0.663934 & 0.20197 & 0.0476749 & 100,000 & 545,806 & 0.1832152 \\
\hline 5 & 2559 & 3 & 87.5 & 367.5 & 100 & 146 & 23.00 & 123.00 & 0.684932 & 0.186992 & 0.0570535 & 100,000 & 479,225 & 0.2086704 \\
\hline \multirow[t]{2}{*}{6} & 2559 & 3.5 & 87.5 & 428.8 & 120 & 143 & 11.50 & 131.50 & 0.839161 & 0.087452 & 0.0558812 & 100,000 & 487,081 & 0.2053045 \\
\hline & & & & & & & & & & & Total & 600,000 & $\Sigma \mathrm{n} / \mathrm{N}$ & 1 \\
\hline
\end{tabular}

Table A5.14. Z-jointed precast FDDP longitudinal bar strain history for the equivalent constant amplitude fatigue load segments

\begin{tabular}{|c|c|c|c|c|c|c|c|c|c|c|c|c|c|c|}
\hline \multirow[t]{2}{*}{ Segment } & \multirow[t]{2}{*}{$\mathrm{\epsilon u}$} & \multicolumn{3}{|c|}{ FLS } & \multirow[t]{2}{*}{$\epsilon_{\min }$} & \multirow[t]{2}{*}{$\epsilon_{\max }$} & \multirow[t]{2}{*}{$\epsilon_{\mathrm{amp}}$} & \multirow[t]{2}{*}{$\epsilon_{\text {mean }}$} & \multirow[t]{2}{*}{$\mathrm{R}$} & \multirow{2}{*}{$\mathrm{A}$} & \multirow{2}{*}{$\epsilon_{\max } / \epsilon_{\mathrm{u}}$} & \multirow[t]{2}{*}{$\mathrm{n}$} & \multirow[t]{2}{*}{$\mathrm{N}_{\mathrm{f}}$} & \multirow[t]{2}{*}{$\mathrm{n} / \mathrm{N}_{\mathrm{f}}$} \\
\hline & & $\mathrm{MF}$ & WL & FLS1 & & & & & & & & & & \\
\hline & $\mu \mathrm{m}$ & & $\mathrm{kN}$ & $\mathrm{kN}$ & $\mu \mathrm{m}$ & $\mu \mathrm{m}$ & & & & & & & & \\
\hline 1 & 4550 & 1 & 87.5 & 122.5 & 287 & 582 & 147.50 & 434.50 & 0.493127 & 0.339471 & 0.1279121 & 100,000 & $3,042,923$ & 0.0328631 \\
\hline 2 & 4550 & 1.5 & 87.5 & 183.8 & 317 & 617 & 150.00 & 467.00 & 0.513776 & 0.321199 & 0.1356044 & 100,000 & $2,667,502$ & 0.0374883 \\
\hline 3 & 4550 & 2 & 87.5 & 245 & 391 & 718 & 163.50 & 554.50 & 0.544568 & 0.29486 & 0.1578022 & 100,000 & $1,824,238$ & 0.0548174 \\
\hline 4 & 4550 & 2.5 & 87.5 & 306.3 & 490 & 875 & 192.50 & 682.50 & 0.56 & 0.282051 & 0.1923077 & 100,000 & $1,010,555$ & 0.0989555 \\
\hline 5 & 4550 & 3 & 87.5 & 367.5 & 579 & 1073 & 247.00 & 826.00 & 0.539609 & 0.299031 & 0.2358242 & 100,000 & 479,788 & 0.2084255 \\
\hline 6 & 4550 & 3.5 & 87.5 & 428.8 & 634 & 1079 & 222.50 & 856.50 & 0.587581 & 0.259778 & 0.2371429 & 100,000 & 469,079 & 0.2131838 \\
\hline 7 & 4550 & 4 & 87.5 & 490 & 756 & 1214 & 229.00 & 985.00 & 0.622735 & 0.232487 & 0.2668132 & 100,000 & 282,273 & 0.3542664 \\
\hline \multirow[t]{2}{*}{8} & 4550 & 4 & 87.5 & 490 & 1005 & 1394 & 194.50 & $1,199.50$ & 0.720947 & 0.162151 & 0.3063736 & 100,000 & 143,407 & 0.6973179 \\
\hline & & & & & & & & & & & Total & 800,000 & $\Sigma \mathrm{n} / \mathrm{N}$ & 1 \\
\hline
\end{tabular}


Table A5.15. Z-jointed precast FDDP transverse bar strain history for the equivalent constant amplitude fatigue load segments

\begin{tabular}{|c|c|c|c|c|c|c|c|c|c|c|c|c|c|c|}
\hline \multirow[t]{2}{*}{ Segment } & \multirow[t]{2}{*}{$\mathrm{\epsilon u}$} & \multicolumn{3}{|c|}{ FLS } & \multirow[t]{2}{*}{$\epsilon_{\min }$} & \multirow[t]{2}{*}{$\epsilon_{\max }$} & \multirow[t]{2}{*}{$\epsilon_{\mathrm{amp}}$} & \multirow[t]{2}{*}{$\epsilon_{\text {mean }}$} & \multirow[t]{2}{*}{$\mathrm{R}$} & \multirow[t]{2}{*}{ A } & \multirow[t]{2}{*}{$\epsilon_{\max } / \epsilon_{\mathrm{u}}$} & \multirow[t]{2}{*}{$\mathrm{n}$} & \multirow[t]{2}{*}{$\mathrm{N}_{\mathrm{f}}$} & \multirow[t]{2}{*}{$\mathrm{n} / \mathrm{N}_{\mathrm{f}}$} \\
\hline & & MF & WL & FLS1 & & & & & & & & & & \\
\hline & $\mu \mathrm{m}$ & & $\mathrm{kN}$ & $\mathrm{kN}$ & $\mu \mathrm{m}$ & $\mu \mathrm{m}$ & & & & & & & & \\
\hline 1 & 6432 & 1 & 87.5 & 122.5 & 411 & 761 & 175.00 & 586.00 & 0.540079 & 0.298635 & 0.1183147 & 100,000 & $288,215,017$ & 0.000347 \\
\hline 2 & 6432 & 1.5 & 87.5 & 183.8 & 408 & 768 & 180.00 & 588.00 & 0.53125 & 0.306122 & 0.119403 & 100,000 & $281,367,772$ & 0.0003554 \\
\hline 3 & 6432 & 2 & 87.5 & 245 & 399 & 824 & 212.50 & 611.50 & 0.484223 & 0.347506 & 0.1281095 & 100,000 & $232,132,703$ & 0.0004308 \\
\hline 4 & 6432 & 2.5 & 87.5 & 306.3 & 587 & 1135 & 274.00 & 861.00 & 0.517181 & 0.318235 & 0.1764614 & 100,000 & $79,763,086$ & 0.0012537 \\
\hline 5 & 6432 & 3 & 87.5 & 367.5 & 860 & 1658 & 399.00 & $1,259.00$ & 0.518697 & 0.316918 & 0.2577736 & 100,000 & $13,231,725$ & 0.0075576 \\
\hline 6 & 6432 & 3.5 & 87.5 & 428.8 & 1220 & 2278 & 529.00 & $1,749.00$ & 0.535558 & 0.302459 & 0.3541667 & 100,000 & $1,573,009$ & 0.0635724 \\
\hline 7 & 6432 & 4 & 87.5 & 490 & 1680 & 3058 & 689.00 & $2,369.00$ & 0.549379 & 0.29084 & 0.4754353 & 100,000 & 107,936 & 0.9264758 \\
\hline \multirow[t]{2}{*}{8} & 6432 & 4 & 87.5 & 490 & 2240 & 3998 & 879.00 & $3,119.00$ & 0.56028 & 0.281821 & 0.6215796 & 100,000 & 4,275 & 23.392654 \\
\hline & & & & & & & & & & & Total & 800,000 & $\Sigma \mathrm{n} / \mathrm{N}$ & 1 \\
\hline
\end{tabular}

Table A5.16. Z-jointed precast FDDP concrete strain history for the equivalent constant amplitude fatigue load segments

\begin{tabular}{|c|c|c|c|c|c|c|c|c|c|c|c|c|c|c|}
\hline \multirow[t]{2}{*}{ Segment } & \multirow[t]{2}{*}{$\mathrm{\epsilon u}$} & \multicolumn{3}{|c|}{ FLS } & \multirow[t]{2}{*}{$\epsilon_{\min }$} & \multirow[t]{2}{*}{$\epsilon_{\max }$} & \multirow[t]{2}{*}{$\epsilon_{\mathrm{amp}}$} & \multirow[t]{2}{*}{$\epsilon_{\text {mean }}$} & \multirow[t]{2}{*}{$\mathrm{R}$} & \multirow[t]{2}{*}{ A } & \multirow[t]{2}{*}{$\epsilon_{\max } / \epsilon_{\mathrm{u}}$} & \multirow[t]{2}{*}{$\mathrm{n}$} & \multirow[t]{2}{*}{$\mathrm{N}_{\mathrm{f}}$} & \multirow[t]{2}{*}{$\mathrm{n} / \mathrm{N}_{\mathrm{f}}$} \\
\hline & & MF & WL & FLS1 & & & & & & & & & & \\
\hline & $\mu \mathrm{m}$ & & $\mathrm{kN}$ & $\mathrm{kN}$ & $\mu \mathrm{m}$ & $\mu \mathrm{m}$ & & & & & & & & \\
\hline 1 & 2506 & 1 & 87.5 & 122.5 & 45 & 111 & 33.00 & 78.00 & 0.405405 & 0.423077 & 0.0442937 & 100,000 & $1,408,735$ & 0.0709857 \\
\hline 2 & 2506 & 1.5 & 87.5 & 183.8 & 54 & 131 & 38.50 & 92.50 & 0.412214 & 0.416216 & 0.0522745 & 100,000 & $1,251,648$ & 0.0798947 \\
\hline 3 & 2506 & 2 & 87.5 & 245 & 261 & 198 & -31.50 & 229.50 & 1.318182 & -0.13725 & 0.0790104 & 100,000 & 842,304 & 0.1187219 \\
\hline 4 & 2506 & 2.5 & 87.5 & 306.3 & 261 & 204 & -28.50 & 232.50 & 1.279412 & -0.12258 & 0.0814046 & 100,000 & 812,952 & 0.1230085 \\
\hline 5 & 2506 & 3 & 87.5 & 367.5 & 261 & 312 & 25.50 & 286.50 & 0.836538 & 0.089005 & 0.1245012 & 100,000 & 429,329 & 0.2329218 \\
\hline 6 & 2506 & 3.5 & 87.5 & 428.8 & 255 & 293 & 19.00 & 274.00 & 0.870307 & 0.069343 & 0.1169194 & 100,000 & 480,363 & 0.2081758 \\
\hline 7 & 2506 & 4 & 87.5 & 490 & 226 & 255 & 14.50 & 240.50 & 0.886275 & 0.060291 & 0.1017558 & 100,000 & 601,353 & 0.1662917 \\
\hline \multirow[t]{2}{*}{8} & 2506 & 4 & 87.5 & 490 & 237 & 272 & 17.50 & 254.50 & 0.871324 & 0.068762 & 0.1085395 & 100,000 & 543,856 & 0.183872 \\
\hline & & & & & & & & & & & Total & 800,000 & $\Sigma \mathrm{n} / \mathrm{N}$ & 1 \\
\hline
\end{tabular}




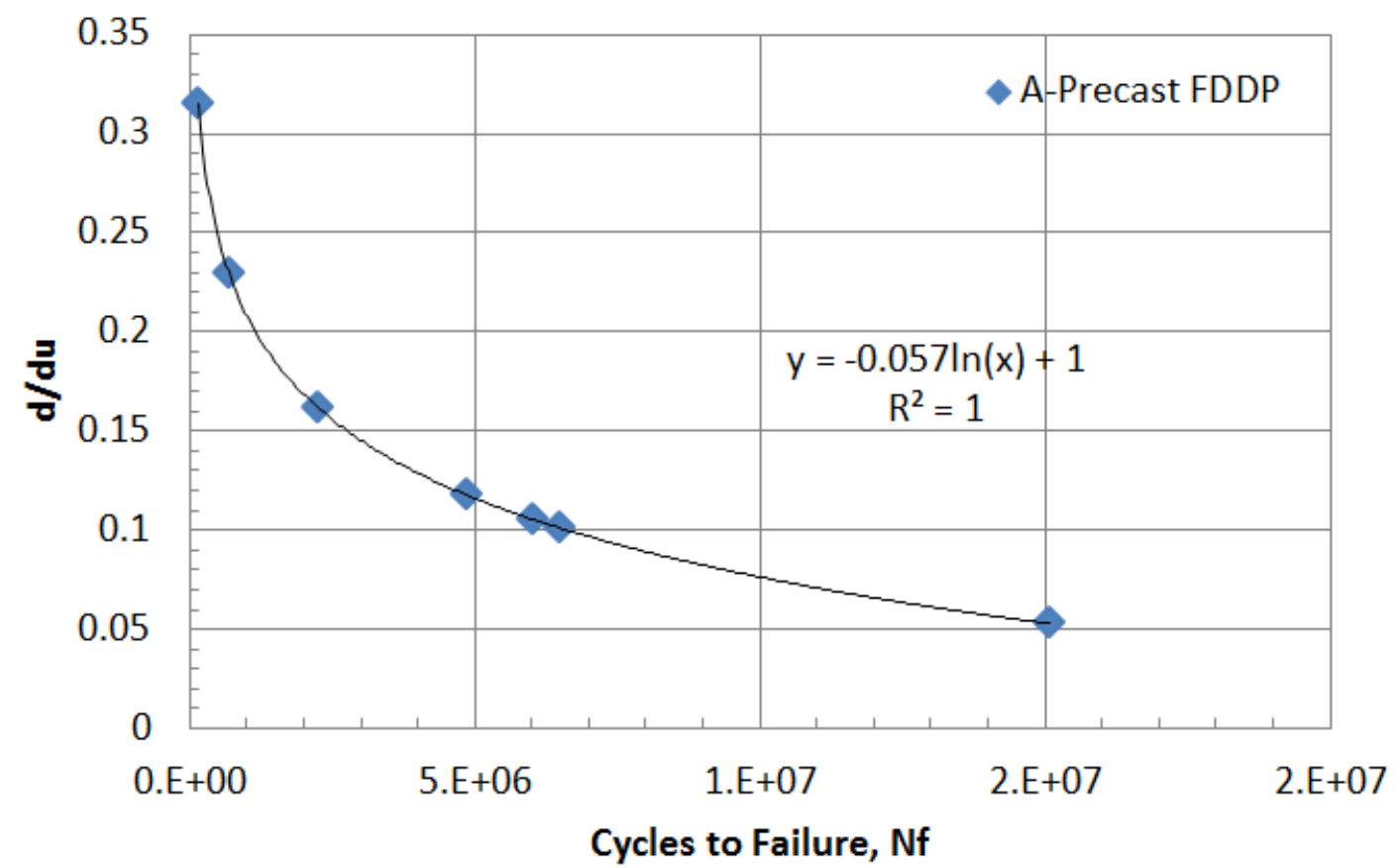

Figure A5.1. d-N curve for deflection of the A-Jointed precast FDDP

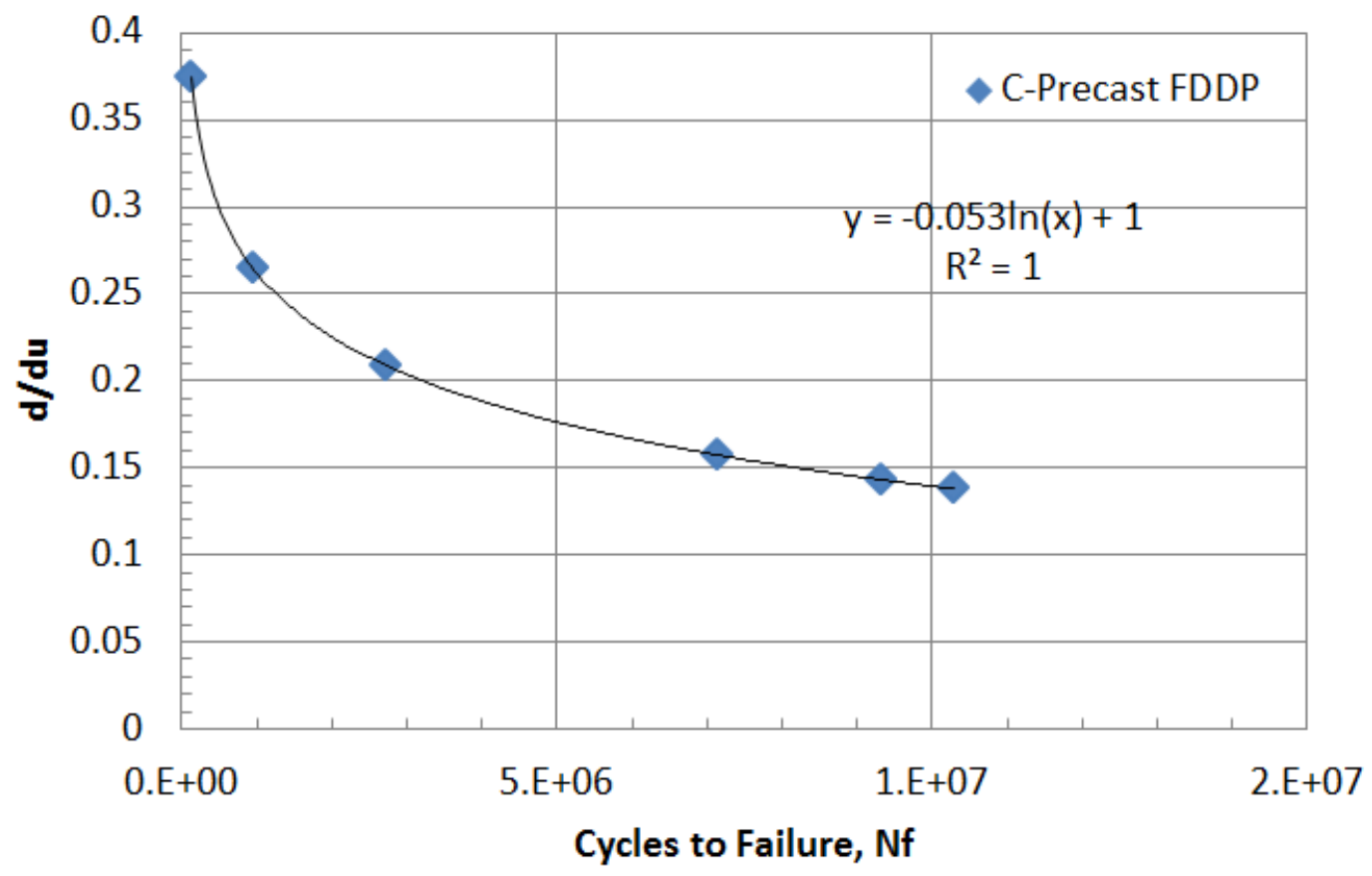

Figure A5.2. d-N curve for deflection of the C-Jointed precast FDDP 


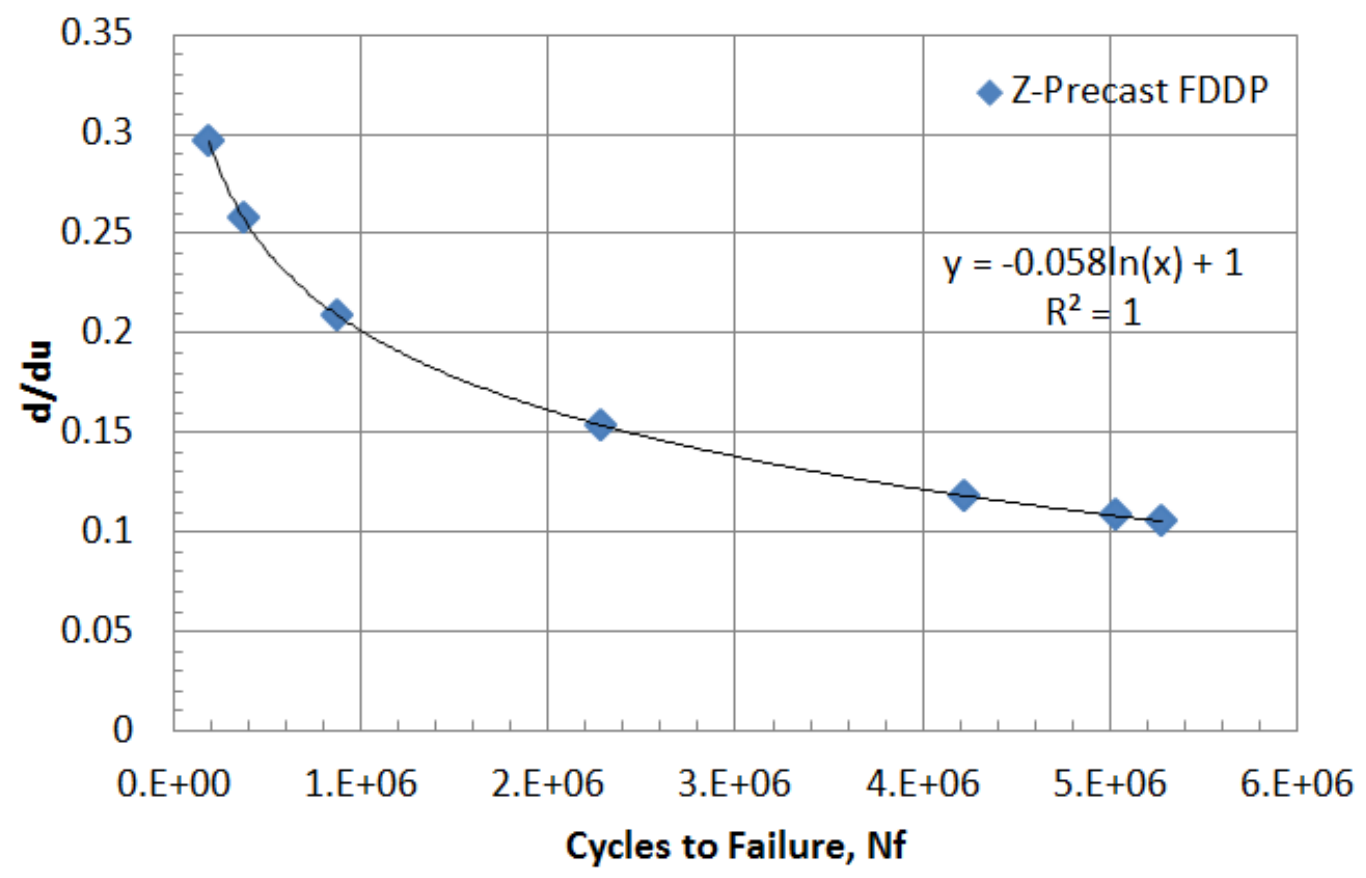

Figure A5.3. d-N curve for deflection of the Z-Jointed precast FDDP

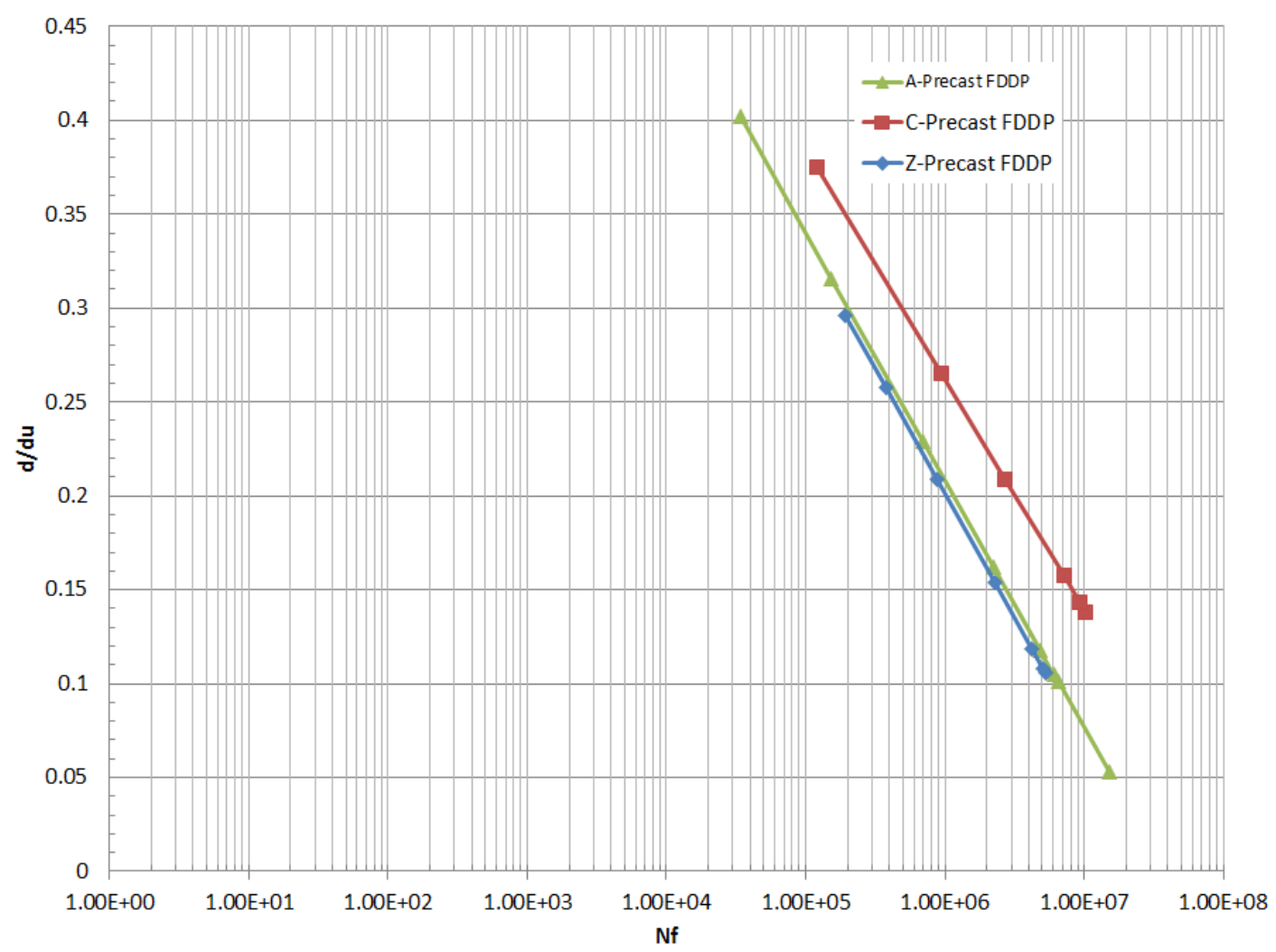

Figure A5.4. ACZ jointed precast FDDPs - deflection versus number of cycles 


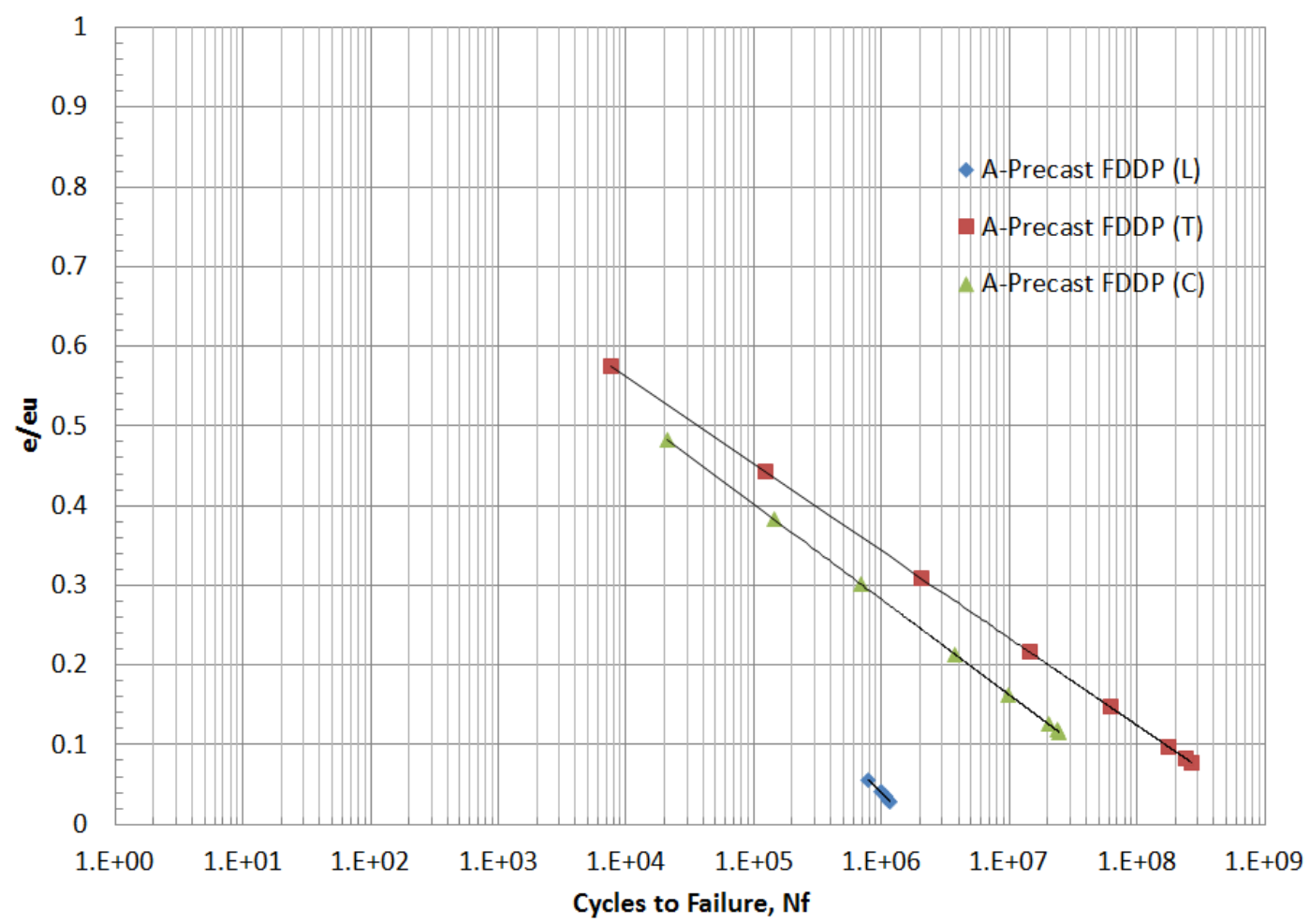

Figure A5.5. The A-Precast FDDP's concrete, longitudinal bar and transverse bar strain versus load cycles

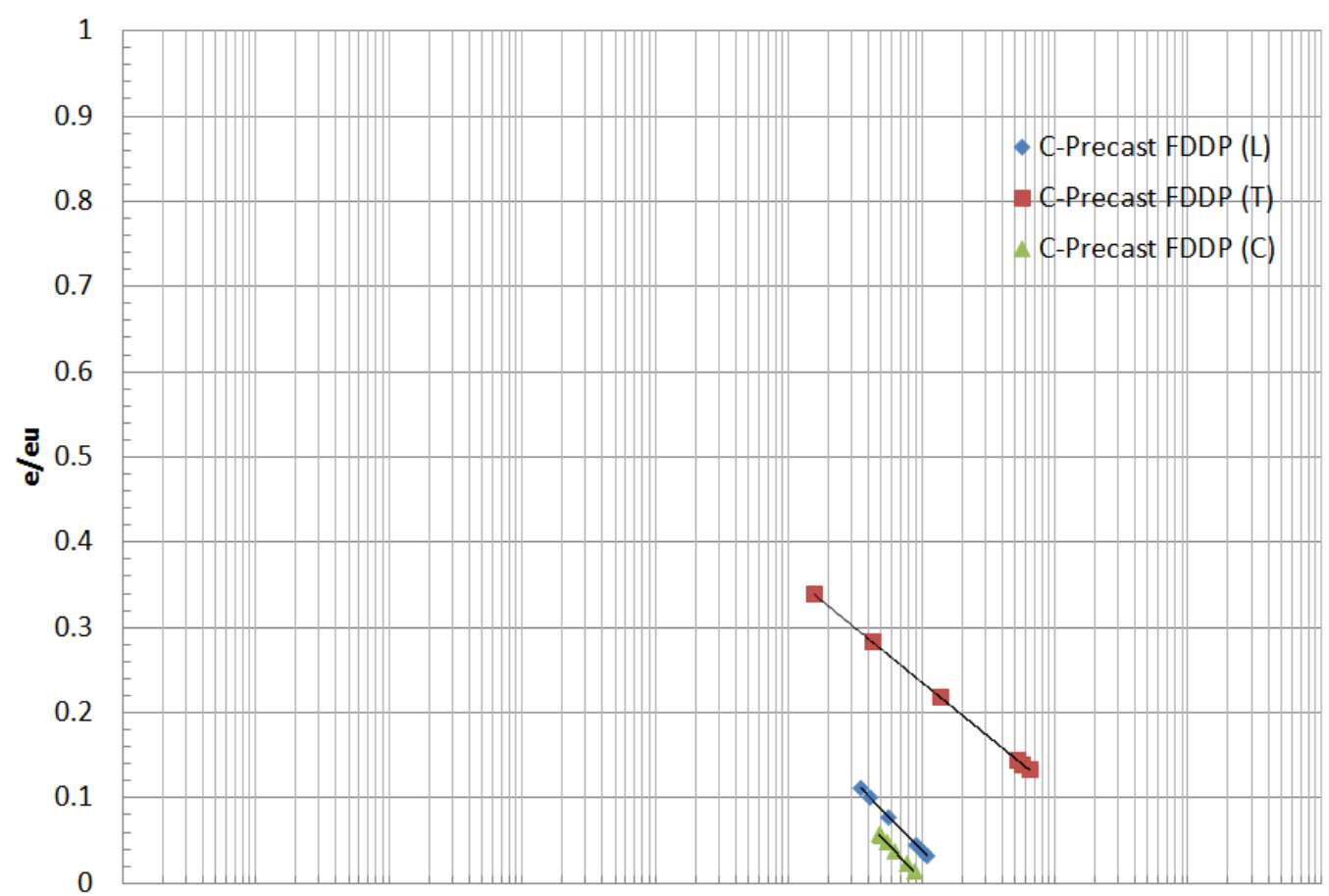

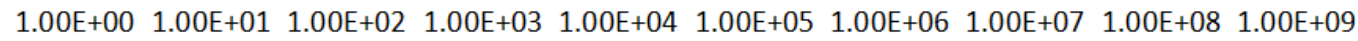

$\mathrm{Nf}$

Figure A5.6. The C-Precast FDDP's concrete, longitudinal bar and transverse bar strain versus load cycles 


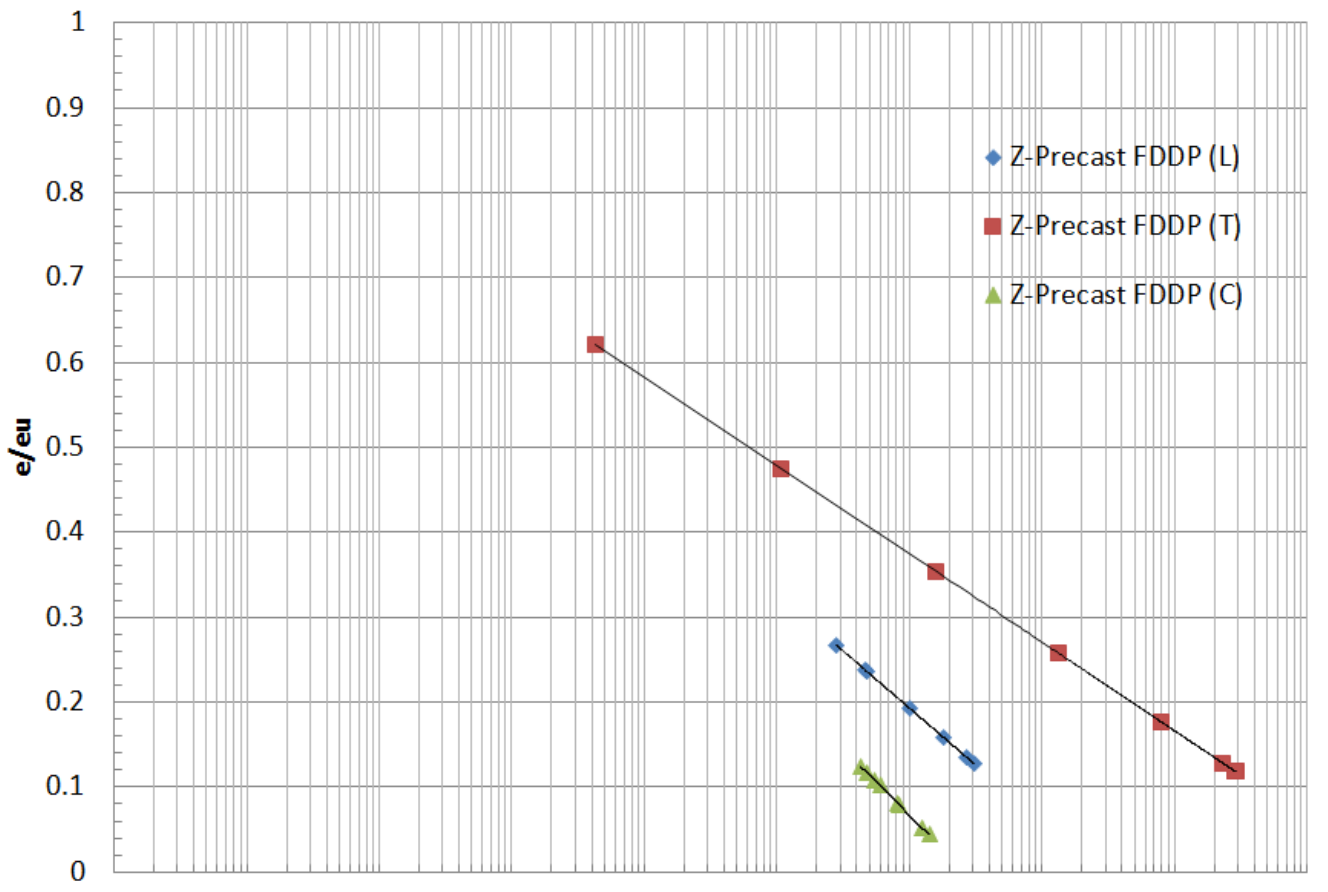

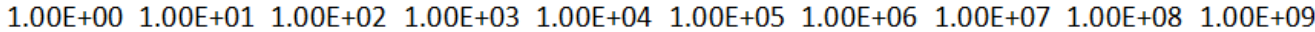
$\mathrm{Nf}$

Figure A5.7. The Z-Precast FDDP's concrete, longitudinal bar and transverse bar strain versus load cycles 


\section{Chapter VI}

\section{Conclusions and Recommendations for Future Research}

\subsection{Summary}

Research on the materials between precast bridge components has emerged in the last decade, including non-shrink cementitous grouts, ultra-high performance concrete (UHPFRC), epoxy grout, magnesium ammonium phosphate grout and post-tensioning cable grout. UHPFRC can exhibit exceptional bond when cast against previously cast concrete and can significantly shorten the development length of embedded discrete GFRP bars. Based on literature survey, the minimum development length of GFRP bars produced by different manufacturers, with either straight or headed-end, embedded in UHPFRC is as yet unavailable. Phase I of this research investigated the pullout strength for the straight and headed-end, ribbed-surface, high modulus GFRP bars embedded into UHPFRC. The results from Phase II led to the development of 3 joint details between precast deck panels with possible applications as transverse joints between precast full-depth, full-width, deck panels, FDDPs, supported over longitudinal girders or longitudinal joints between precast bulb-tee girders. Phase II investigated the structural behavior of the GFRP-reinforced precast FDDPs made of (i) normal strength concrete (NSC) and (ii) high performance concrete (HPC). Precast FDDPs were joint-filled with UHPFRC. NSC is the traditional concrete composed of cement, coarse aggregate, fine aggregate and water. While HPC has same components as of the NSC but with more fine binding materials including Silica Fume, finely-grinding granulated blast furnace slag (GGBFS) and high range water reducer (HRWR) to turn HPC into self-consolidated concrete (SCC). UHPFRC has micro particle size distributions with cement, Silica Fume, very fine aggregates, water, HRWR and steel fibre reinforcement (SFR). Phase III involved application of the developed joint details in actual bridge configuration on which FDDP are supported over braced steel girders and connected to them using intermittent shear connectors. The shear connectors were embedded into shear pockets filled with UHFRPC. The closure strip between FDDP were also filled with UHFPRC. The following subsections 
summarizes the test program for each phase of research, followed by the conclusions drawn from this research. The chapter ends with recommendations for future research.

\subsubsection{Phase I: Pullout Strength GFRP Bars Embedded into UHPFRC}

In phase I, two types of GFRP bars were tested under direct tension pullout embedded into unconfined UHPFRC blocks to determine their adequate development length to ensure rupture in the bar in tension before bond failure. Testing included ribbed-surface and sand-coated GFRP bars with straight-end and headed-end. Test results were able to determine the adequate development length needed to design for the lap-splice embedded into the closure strip between adjacent precast FDDPs.

\subsubsection{Phase II: Development and Study of Closure Strip Details Incorporating UHPFRC as Joint-Filling Materials and GFRP Bars Projecting into the Joint}

In phase II, two groups of actual-size one-way deck slabs were constructed and tested. Each of the 2 groups has 7 slabs in total. One group was cast using normal-strength concrete (NSC), while the second group was cast using high-performance concrete (HPC). Each slab has $200 \mathrm{~mm}$ thickness, $2500 \mathrm{~mm}$ length, and $600 \mathrm{~mm}$ width in the direction of the traffic, representing the one-way slab action in the design of deck slab using AASHTO-LRFD Specification as well as the flexural design method specified in CHBDC. FDDPs were constructed with 200-mm wide joint with GFRP bars projecting $175 \mathrm{~mm}$ into the joint and filled with UHPFRC. All slabs were tested under static load to collapse to examine their structural adequacy for the proposed joint configurations.

\subsubsection{Phase III: Monotonic and Cyclic Load Tests on Laterally-Restrained Deck Slabs}

In phase III, 6 actual-size GFRP-reinforced precast FDDPs were constructed and then tested under monotonic and cyclic loading testing to determine their structural behaviour under CHBDC truck wheel loading. For each test, two segments of FDDPs were placed beside each other to form a closure strip than was then filled with UHPFRC. The first FDDP had $200 \mathrm{~mm}$ thickness, $2500 \mathrm{~mm}$ width and 2400 length in the direction of the traffic, while the second FDDP was of the same size except that the length in the direction of traffic was taken as $900 \mathrm{~mm}$. The first specimen in each pair of precast FDDPs was tested under constant amplitude fatigue loading 
followed with monotonic loading-to-collapse, while the second specimen was tested under multistep incremental variable amplitude fatigue loading to-collapse.

\subsection{Conclusions}

Based on the experimental results generated form this research, the following conclusions were drawn:

\section{Phase I: Pullout Strength GFRP Bars Embedded into UHPFRC}

1. The allowable slips at loaded-end and free-end of the HM GFRP bars should be limited with acceptable range as bar fails suddenly due to interlaminar shear failure of the GFRP bar, with shear failure of the ribbed-surface or sand coated surface for the straight bars and bar slippage from the anchor head for the headed-end bars.

2. For straight HM GFRP bars embedded into UHPFRC, it is recommended to use a minimum development length of 9.5 times the bar diameter or $152 \mathrm{~mm}$, whichever is greater, to ensure bar reaching its tensile strength before anchorage failure.

3. For headed-end HM GFRP bars embedded into UHPFRC, it is recommended to use minimum development length, including the headed-end length, of 7 times the bar diameter or $111 \mathrm{~mm}$, whichever is greater, to ensure bar reaching its tensile strength before anchorage failure.

4. The Modification Factor for bar locations within UHPFRC cross-section can be taken in the range of 1 to 1.3 representing the range from bar centric location to the $40 \mathrm{~mm}$ concrete cover for eccentric location, respectively.

\section{Phase II: Development and Study of Closure Strip Details Incorporating UHPFRC as Joint-Filling Materials and GFRP Bars Projecting into the Joint}

For FDDPs constructed using NSC, the following conclusions were drawn:

1- The ultimate load capacity of GFRP-reinforced cast-in-place slab was about $15 \%$ less than the capacity of a similar slab reinforced with steel bars in accordance with the CHBDC and having the same cross-sectional area as the GFRP bars. 
2- GFRP-reinforced jointed slab with a $200 \mathrm{~mm}$ wide joint width with a zigzag-shape exhibited a flexural strength that was about $1.4 \%$ less than that for a similar cast-in-place slab.

3- Comparing jointed precast slabs with different applied load patterns, it was observed that the ultimate load of the jointed slab with a vertical shear key increased by $15 \%$ as a result of using eccentric loading rather than the concentric loading at the closure strip.

4- The precast slab with a $200-\mathrm{mm}$ wide closure strip and projected bottom slab segment proved to have flexural capacity less than those for the steel-reinforced cast-in-place slab and the GFRP-reinforced cast-in-place slab by $25 \%$ and $12 \%$, respectively.

5- In case, of the effect of load location on this slab, it was observed that the eccentric wheel loading, when compared to the concentric wheel loading, increased the load carrying capacity by $7.8 \%$. As such, concentric loading will be used in further tests to examine the fatigue life of the successful control joints under simulated vehicular wheel loading in case of bulb-tee girder system. However, in case of FDDPs placed transversally over the girders, eccentric loading may be the proper choice given the distribution of wheel loads within the NSC panel is more critical than that when the wheel load is located over the UHPFRC-filled closure strip.

6- The failure mode in all tested one-way slab specimens was either pure flexural of combined flexural and shear in the NSC panel rather than (i) in the UHPFRC-filled joint or (ii) bond failure of GFRP bars embedded into the joint. This conclusion would allow Bridge Design Engineers design the precast jointed NSC-built deck slabs using the oneway slab action specified in AASHTO-LRFD Specification or the flexural design method specified in CHBDC. In this case, Bridge Design Engineers will adopt one of the developed UHPFRC-filled joint configurations without design and consider the design of the precast slab under factored applied wheel load specified in the code per meter width to prevent pure flexure failure or combined shear-flexural failure outside the joint.

For FDDPs constructed using HPC, the following conclusions were drawn:

1. The ultimate load capacity of GFRP-reinforced cast-in-place slab was about $5 \%$ less than the capacity of a similar slab reinforced with steel bars in accordance with the CHBDC and having the same cross-sectional area as the GFRP bars. 
2. The GFRP-reinforced jointed slab with a $200 \mathrm{~mm}$ wide joint width with vertical shear keys exhibited a flexural strength about $8 \%$ less than that for a similar cast-in-place slab.

3. Comparing jointed precast slabs with different applied load patterns, it was observed that the slab ultimate load slightly increased (i.e. by 3\%) as a result of using eccentric loading rather than concentric loading at the closure strip, while the slab ultimate deflection decreased by about $41 \%$.

4. The precast slab with a zigzag-shaped joint appeared to be as good as the steel-reinforced slab (i.e. $4 \%$ difference) and GFRP-reinforced slab (i.e. $0.7 \%$ difference) with respect to ultimate strength.

5. The precast slab with a $200-\mathrm{mm}$ wide closure strip and projected bottom slab segment proved to have flexural capacity less than those for the steel-reinforced cast-in-place slab and the GFRP-reinforced cast-in-place slab 34\% and 31\%, respectively.

6- The failure mode in all tested one-way slab specimens was either pure flexural of combined flexural and shear in the HPC panel rather than (i) in the UHPFRC-filled joint or (ii) bond failure of GFRP bars embedded into the joint. This conclusion would allow Bridge Design Engineers design the precast jointed NSC-built deck slabs using the oneway slab action specified in AASHTO-LRFD Specification or the flexural design method specified in CHBDC. In this case, Bridge Design Engineers will adopt one of the developed UHPFRC-filled joint configurations without design and consider the design of the precast slab under factored applied wheel load specified in the code per meter width to prevent pure flexure failure or combined shear-flexural failure outside the joint.

\section{Phase III: Monotonic and Cyclic Load Tests on Laterally-Restrained Deck Slabs}

\section{For the A-jointed precast FDDPs, the following conclusions were drawn:}

1. Precast FDDPs reinforced with high-modulus GFRP ribbed-surface bars showed high fatigue performance as there was no observed fatigue damage when subjected to 4,000,000 cycles under high-cyclic CAF loading of $122.5 \mathrm{kN}$ specified in CHDBC.

2. The tested precast FDDP under high-cyclic CAF loading sustained a failure load about 4.47 times the CHBDC factored design wheel load of $208.25 \mathrm{kN}$.

3. The tested precast FDDP under low-cyclic incremental step VAF loading sustained a failure load about 2.34 times the CHBDC factored design wheel load. 
4. The two laterally-restrained precast FDDPs failed in punching shear mode. However, the transverse UHPFRC-filled joint did not allow for the punching shear failure plane to form in all direction around the location of the wheel load. Due to its very high strength compared to NSC, UHPFRC deviated the radial tensile stresses to fully form on the adjacent precast FDDP in the other side of the joint.

5. The first precast FDDP specimen's stiffness degraded by about $53 \%$ after 4 million cycles of constant amplitude fatigue (CAF) loading. On the other hand, the second precast FDDP specimen's stiffness degraded by $86.86 \%$ when subjected to low-cyclic variable amplitude fatigue (VAF) loading before complete collapse.

6. Based on experimental findings, a mathematical model was proposed to determine the cumulative fatigue damage (CFD) and fatigue resistance (P-N effect) for the GFRPreinforced FDDPs with transverse joint. The cumulative linear damage, D, was successfully maintained to be 1 for transverse jointed FDDPs. It can be noticed that the magnification factor to the fatigue loading is inversely proportional to the number of the repetitions of the same cycle that equals life to failure.

\section{For the C-jointed precast FDDPs, the following conclusions were drawn:}

1. Precast high-modulus GFRP-reinforced FDDP showed high fatigue performance as there was no observed fatigue damage after being subjected to 4,000,000 cycles of high-cyclic CAF loading of $122.5 \mathrm{kN}$ specified in CHDBC.

2. The tested precast FDDP under CAF loading followed with increasing monotonic wheel load to-collapse sustained a failure load about 4.67 times the CHBDC factored ULS design wheel load.

3. The tested precast FDDP under low-cyclic incremental step VAF loading sustained a failure load about 2.38 times the CHBDC factored design wheel load.

4. The two precast FDDPs failed in punching shear mode. In the specimens tested under static load to-collapse after being tested under CAF loading, the very high strength of UHPFRC-filled transverse joint did not allow the punching shear failure to fullydevelop on the side of wheel load location towards the adjacent FDDP. In case, of the specimen tested under VAF loading, major flexural crack appeared at failure just under the wheel load, extending into the adjacent FDDP thin the direction of the 
girder towards the free end of the small FDDP segment through the UHPFRC-filled joints.

5. The first precast FDDP specimen's stiffness degraded by about $50.73 \%$ after 4 million cycles of high-cyclic constant amplitude fatigue (CAF) loading. On the other hand, the second precast FDDP specimen's stiffness degraded by $63.42 \%$ when subjected to low-cyclic variable amplitude fatigue (VAF) loading before complete collapse.

\section{For the Z-jointed precast FDDPs, the following conclusions were drawn:}

1. The precast FDDP reinforced with high-modulus GFRP ribbed-surface bars showed high fatigue performance and there was no fatigue damage when subjected to 4,000,000 cycles under high-cyclic CAF loading of $122.5 \mathrm{kN}$ specified in CHDBC.

2. The tested precast FDDP under high-cyclic CAF loading sustained a failure load about 4.47 times the CHBDC factored design wheel load of $208.25 \mathrm{kN}$.

3. The tested precast FDDP under low-cyclic incremental step VAF loading sustained a failure load about 2.35 times the CHBDC factored design wheel load.

4. The two laterally-restrained precast FDDPs failed in punching shear mode. The punching shear failure plane and associated radial cracks at the bottom of the slab was more intensive in the loaded FDDP than the adjacent FDDP on the other side of the closure strip. In case of the specimen loaded using VAF loading to-failure, punching shear in the loaded FDDP was accompanied by major flexural crack appeared at the wheel load location and extended into the adjacent FDDP on the other side of the closure strip. This led to the conclusion that the failure mode is primarily punching shear combined with flexural failure in the adjacent short FDDP. However, this may not be a general conclusion as long as the adjacent FDDP was of short length in the direction of traffic (i.e. $900 \mathrm{~mm}$ ) rather than full panel length of $2400 \mathrm{~mm}$ as the loaded one.

5. The first precast FDDP specimen's stiffness degraded by about $21.9 \%$ after 4 million cycles of constant amplitude fatigue (CAF) loading. On the other hand, the second precast FDDP specimen's stiffness degraded by $71.32 \%$ when subjected to low-cyclic variable amplitude fatigue (VAF) loading before complete collapse. 


\subsection{Recommendations for Future Research}

Based on the outcome of this research, the following recommendations for future research can be made:

1. Perform series of testing for high modulus Glass FRP bars with thermoplastic resins, which allows the bars to be reheated and shaped for the structural needs, and later can be reheated and returned to the liquid state for recycling purpose.

2. Perform series of flexural bond strength tests to accurately determine the modification factor for bar location in UHPFRC beam cross-section based on the amount of concrete cover.

3. Perform series of shakedown testing for precast FDDPs with different contact and noncontact lap-splices, with different projecting lengths embedded into confined and unconfined joint-fill materials as the UHPFRC, and with different slab thickness.

4. Study the monotonic and cyclic pushover effect of the shear pockets, and shear stud distribution for the GFRP-reinforced panel-to-girder connection.

5. Perform series of monotonic and cyclic testing over composite sections with GFRPreinforced precast FDDP resting concrete girders, where shear pockets are filled with UHPFRC.

6. Conduct static and fatigue tests on a composite girder incorporating the developed zigzag-shape joint as a transverse joint subjected to pure tension resulting from global negative bending moment on the continuous bridge girders. 